\title{
A Catalog of Cultivated Woody Plants of the Southeastern United States
}

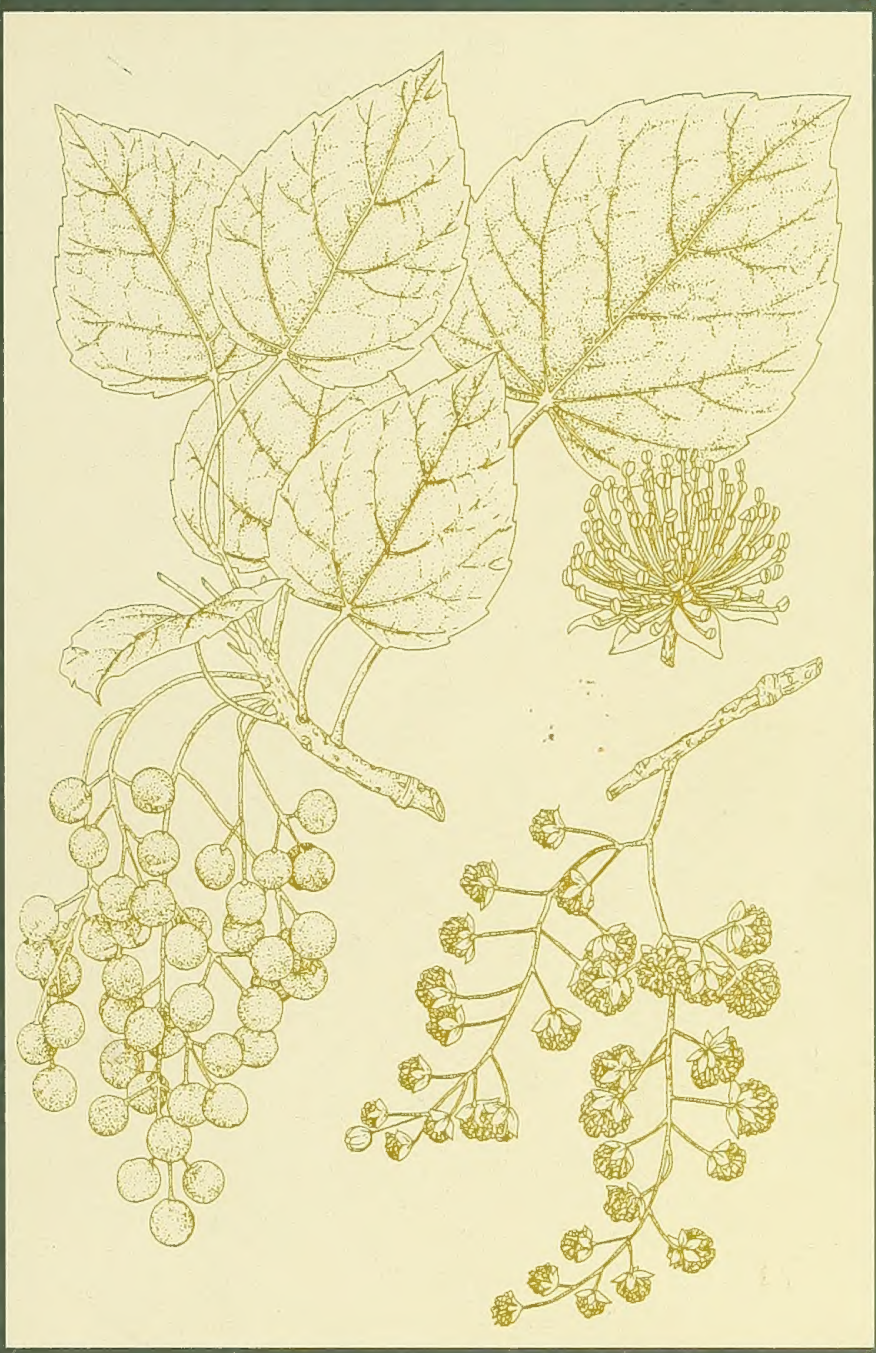

United States

Department of Agriculture

\section{Agricultural}

Research Service

United States

National

Arboretum

Contribution

Number 7 


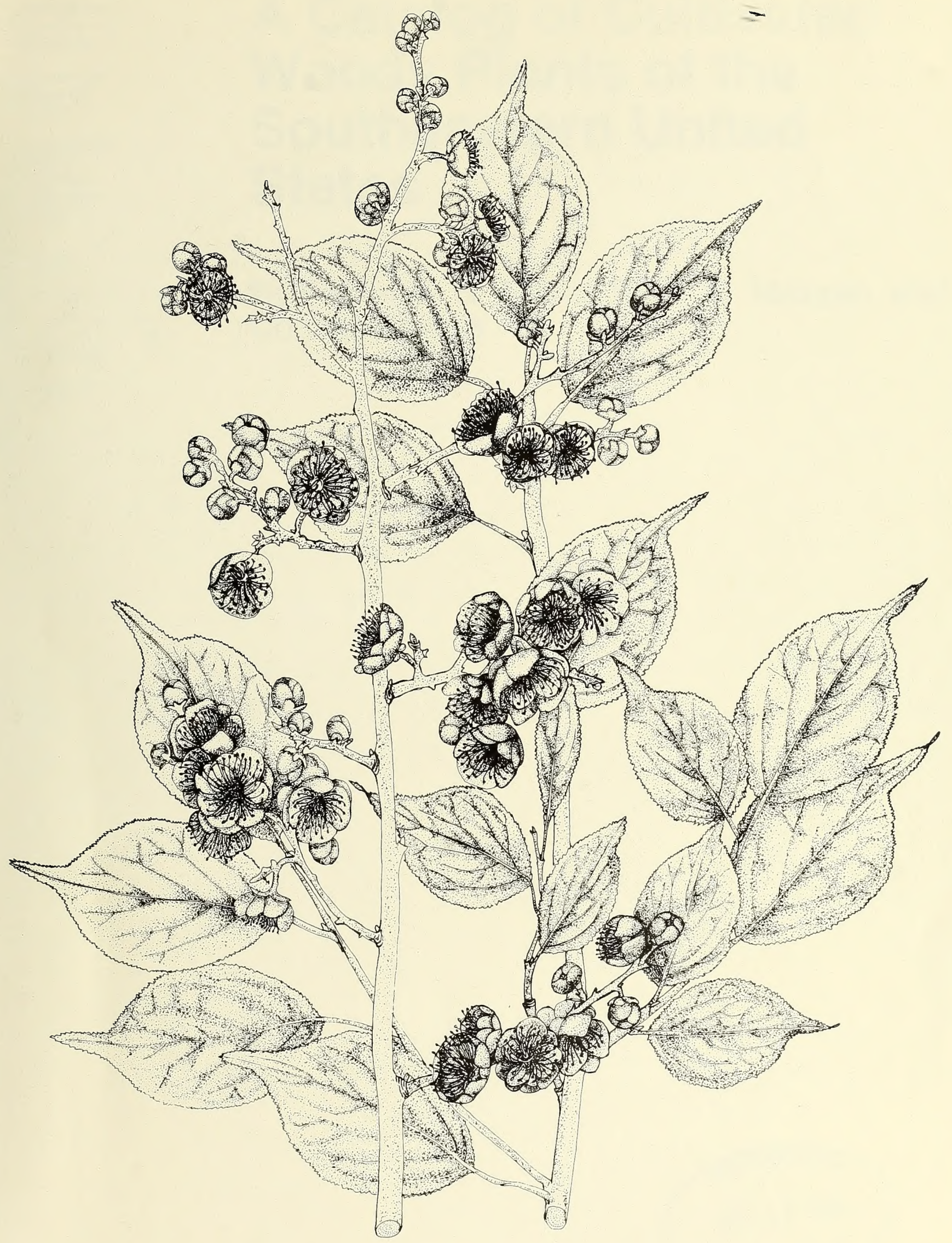

PRUNUS mume Sieb. \& Zucc.

'Peggy Clarke' [illustrator Lillian Nicholson Meyer] 



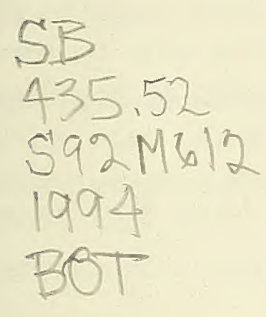

By Frederick G. Meyer, Peter M. Mazzeo, and Donald H. Voss

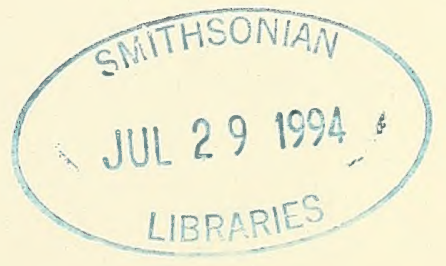


Meyer, Frederick G., Peter M. Mazzeo, and Donald H. Voss. 1993. A Catalog of Cultivated Woody Plants of the Southeastern United States. U.S. Department of Agriculture, Agricultural Research Service, U.S. National Arboretum Contribution No. 7, 338 pp.

This work catalogs cultivated landscape trees, shrubs, and woody climbers in a 13-state area of the southeastern United States, together with the District of Columbia. Included are approximately 5,000 entries-covering species, subspecies, varieties, forms, and cultivars-based on more than 14,000 documented voucher specimens. The burgeoning nursery business, along with the continued introduction of new cultivated plants, has added to the need for accurate application of botanical nomenclature. Many plants are misidentified in the nursery trade and among researchers, students, and home gardeners. The catalog serves as a reference guide for these groups, as well as for horticulturists, botanists, extension agents, educators, germplasm conservators, and those who work in botanic gardens and arboreta.

Keywords: climbing plants, cultivars, gardens, horticulture, landscaping, names (botanical), nurseries, plant geography, plant names, plant nomenclature, plant taxonomy, shrubs, trees, woody vines.

While supplies last, single copies of this publication may be obtained at no cost from the Curator of the Herbarium, U.S. National Arboretum, 3501 New York Avenue, N.E., Washington, DC 200021958.

Copies of the publication may be purchased from the National Technical Information Service, 5285 Port Royal Road, Springfield, VA 22161.
The United States Department of Agriculture (USDA) prohibits discrimination in its programs on the basis of race, color, national or igin, sex, religion, age, disability, political beliefs, and marital or familial status. (Not all prohibited bases apply to all prog rams.) Persons with disabilities who require alternative means for communication of program information (Braille, large print, audio tape, etc.) should contact the USDA Office of Communications at (202) 720-5881 (voice) or (202) 720-7808 (TDD).

To file a complaint, write the Secretary of Agriculture, U.S. Department of Agriculture, Washington, DC, 20250, or call (202) 720-7327 (voice) or (202) 720-1127 (TDD). USDA is an equal employment opportunity employer. 


\section{Contents}

Foreword ..vii

Acknowledgments vii

Introduction .1

Symbols and abbreviations 11

Catalog of plants and their collection sites

Appendix A: Authors cited 218

Appendix B: Plant collection sites, cited by acronym 237

Appendix C: Glenn Dale azalea cultivars documented in the U.S. National Arboretum Herbarium 248

Appendix D: Plants listed by botanical names and cultivar names 249

Appendix E: Vernacular names 313

Genera 313

Species and cultivars 314

Selected bibliography 326

Illustrations

IDESIA polycarpa cover PRUNUS mume 'Peggy Clarke' .........frontispiece ACTINIDIA arguta ........................................22

AKEBIA quinata ..........................................24

BIGNONIA capreolata ...................................... 31

BRUNFELSIA australis ................................33

CALYCANTHUS floridus ................................38

CHIMONANTHUS praecox ..........................50

DAVIDIA involucrata var. vilmoriniana .........63

DISANTHUS cercidifolius ............................66

FOTHERGILLA major .....................................74

HALESLA carolina .........................................77

HAMAMELIS mollis .......................................79

HOVENIA dulcis ............................................8

HYDRANGEA anomala ssp. petiolaris .........89

ILLICIUM floridanum ...................................110

ILLICIUM parviflorum .................................111

JASMINUM nudiflorum ..............................113

JUGLANS regia .........................................114

KOELREUTERIA bipinnata ........................120
LAURUS nobilis ............................................123

MAGNOLIA ashei (fruit) ...........................129

MAGNOLIA macrophylla (fruit) ...................129

MAGNOLIA tripetala ..................................133

MICHELIA figo ..........................................138

PARROTIOPSIS jacquemontiana ................143

PSEUDOLARIX amabilis ............................161

SCHIZOPHRAGMA hydrangeoides .............191

STYRAX japonicus …...............................197

SYCOPSIS sinensis ..................................198

TOONA sinensis .......................................206

ULMUS parvifolia ......................................209 

Errata for

A Catalog of Cultivated

Woody Plants of the

Southeastern United States

Page 27, in Arecastrum: = Syagrus

romanzoffianum should read = Syagrus romanzoffiana.

Page 32, in Buddleja: farreri Balf.f. \& W.W.Sm. should read farreri Balf.f. \& W.W.Sm. = crispa Benth. var. farreri (Balf.f. \& W.W.Sm.) Hand.-Mazz.

Page 54, in Cornus: 'Gold Star' [C. kousa Hance] should read 'Gold Star' (Buerger ex Miq.) Hance.

Page 69, under Euodia daniellii (Benn.) Hemsl.: include the note $E$. daniellii still appears widely in horticultural works, but T.G. Hartley's revision of the genus Tetradium moves it to Tetradium daniellii (Benn.) Hartley.

Page 128, in Magnolia: include the entry foetida Sarg. = M. grandiflora (L.) after 'Elizabeth' [M. acuminata $\times M$. denudata].

Page 134, in Malus 'Aldenhamensis': 'Niedzwetzkeyana' should read 'Niedzwetzkyana'.

Page 135, in Malus: include the entry niedzwetzkyana Dieck $=M$. domestica 'Niedzwetzkyana' after 'Naragansett' [M. ] hybrid \#28/ $\times$ M. 'Wintergold'].

Page 136, under Malus $\times$ purpurea: 'Niedzwetzkeyana' should read 'Niedzwetzkyana'.

Page 156, in Prunus: 'Albo-plena' [P. glandulosa Thunb.] should read 'Albo-plena' [P. glandulosa Thunb. ex J.A. Murr.].

Page 160, in Prunus 'Yae murasaki zakura': (Yato zakura group) should read (Sato zakura group).

Page 160, in Pseudotsuga: include the entry taxifolia (Lamb.) Britton $=P$. menziesii (Mirb.) Franco after 'Oudemansii' [P. menziesii (Mirb.) Franco var. glauca (Beissn.) Francol.
Page 180, in Rosa: banksiae Ait. should read banksiae Ait.f.

Page 188, under Salix $\times$ laestadiana Hartm.: S. cineria should read $S$. cinerea.

Page 188, under Salix $\times$ pontederana Willd.: S. cineria should read $S$. cinerea.

Page 196, in Syagrus: romanzoffianum should read romanzoffiana.

Page 199, in Syringa: 'Charles Giant' should read 'Clarke's Giant'.

Page 199, under Syringa $\times$ chinensis Willd.: [S. $\times$ persica $\times S$. vulgaris] should read [S. persica $\times S$. vulgaris].

Page 203, in Thuja: 'Cuprea' [T. plicata D.Don] should read 'Cuprea' [T. plicata J.Donn ex D.Don].

Page 203, in Thuja: plicata J.Donn ex G.Don should read plicata J.Donn ex D.Don.

Page 203, in Thuja: 'Rogersii' [T. plicata D.Don] should read 'Rogersii' [T. plicata J.Donn ex D.Don].

Page 203, in Thuja: 'Zebrina' [T. plicata D.Don] should read 'Zebrina' [T. plicata J.Donn ex D.Don].

Page 212, in Viburnum 'Mt. Fuji': [V. plicatum Fort. f. tomentosum (Thunb. ex J.A.Murr.) Rehd.] should read [V. plicatum Thunb. f. tomentosum (Thunb. ex J.A.Murr.) Rehd.].

Page 213, in Viburnum 'Shoshoni': [V. plicatum Fort. f. tomentosum (Thunb. ex J.A.Murr.) Rehd.] should read [V. plicatum Thunb. f. tomentosum (Thunb. ex J.A.Murr.) Rehd.].

Page 255, under Buddleja: farteri Balf.f. \& W.W.Sm. should read farreri Balf.f. \& W.W.Sm. = crispa Benth. var. farreri (Balf.f. \& W.W. Sm.) Hand.-Mazz.

Page 273, under Hibiscus: syriacus 'Tamausagi' should read syriacus 'Tama usagi'.

Page 275, under Mlex: aquifolium 'Scotia' should read aquifolium 'Scotica'. 
Page 301, in Rosa: include the entry Xodorata (Andr.) Sweet 'Old Blush' after odorata (Andre.) Sweet 'Fun Jwan Lo'.

Page 301 , in Rosa: include a $\times$ before the three odorata entries.

Page 302, in Rosa: ir 'ude the entry 'The Fairy' after 'Sombreuil'.

Page 305, in XSorbopyrus: 'Bulbiformis' should be indented under auricularis.

Page 306, under Syagrus: romanzoffianum should read romanzoffiana.

Page 307, under Syringa: 'Charles Giant' should read 'Clarke's Giant'.

Page 321, under Palm, Queen: Syargus romanzoffianum should read Syargus romanzoffiana.

Page 264, under cotoneaster racemiflorus, soongoricus should read songoricus. 
The herbarium of the U. S. National Arboretum is one of the few major herbaria to emphasize cultivated plants together with their wild progenitors. Its specimens support scientific research on these plants not only within the Department of Agriculture but also worldwide through a loan program. The ongoing work of the herbarium staff in addressing problems in plant taxonomy and nomenclature assists researchers, germplasm conservators, and others in plant identification and contributes to the efforts of nurseries to identify correctly the plant material they propagate and sell.

A Catalog of Cultivated Woody Plants of the Southeastern United States represents the culmination of more than 20 years of field work begun in 1967 that covers 13 southeastern states and the District of Columbia. The catalog has been prepared as a reference guide for nurseries, landscape architects, horticulturists, botanical and horticultural libraries, botanists, extension agents, educators, germplasm conservators, botanical gardens and arboreta, propagators, students, various units of the U.S. Department of Agriculture, plant societies, and the gardening public. It will be useful not only in the southeastern states, but also in other parts of the United States where many of the plants listed can be found in cultivation.

The catalog is based on more than 14,000 documented voucher specimens critically reviewed for correctness of identification and nomenclature. With about 5,000 entries, both botanical names and cultivar names, the catalog is a comprehensive enumeration of woody plants cultivated in the southeastern United States.

The catalog is unique, being based wholly on documented material deposited in the U.S. National Arboretum herbarium and including a guide to sites where collections were made of plants growing in cultivation. It is hoped that the catalog will, by example, encourage similar documentation of the woody landscape plants in other areas of the country and the deposit in appropriate herbaria of voucher specimens collected for permanent reference.
Many individuals, too numerous to mention, generously facilitated our collection efforts at botanical gardens and arboreta, experiment stations, college campuses, nurseries, and private gardens.

We specifically acknowledge the invaluable assistance of the following specialists for their help in identifying plant specimens: W.A. Anderson (Malpighiaceae), George Argus (Salix), Helen L. Blake (old roses), Barbara Briggs (Callistemon), C.E. Codd (Carissa macrocarpa), David W. Hall (miscellaneous Florida plants), James W. Hardin, Jr. (Aesculus), Elizabeth McClintock (Hydrangea and Myrtaceae), A. Edward Murray, Jr. (Acer), Eliane Norman (Buddleja), J.B. Phipps (Crataegus), the late Sigmund Solymosy (miscellaneous Louisiana plants), and Charles A. Walker (old roses). T. R. Dudley contributed many voucher specimens cited in the catalog and compiled the lists of Mex and Caprifoliaceae.

We express appreciation to the Friends of the National Arboretum (FONA) and to the Southern Nurserymen's Association for financial support that greatly enhanced our ability to collect specimens in the southeastern United States.

We also thank Carol A. Rahbar and Franziska Walczak as volunteer collaborators for their substantive assistance in coordinating data related to the computer data base, the list of site acronyms, and attending to many other details that contributed enormously to the accuracy of the catalog.

The authors are grateful for the privilege of using artwork by the late Lillian Nicholson Meyer, Susan M. Johnston, and Peggy K. Duke.

\author{
Allan K. Stoner \\ Acting Director, U. S. National Arboretum
}





\title{
A Catalog of Cultivated Woody Plants of the Southeastern United States
}

\author{
by Frederick G. Meyer, Peter M. Mazzeo, and Donald H. Voss
}

\section{Scope}

This catalog presents a comprehensive reference list, based on documented material, of the trees, shrubs, suffruticose plants, and woody climbers cultivated in the southeastern United States. A list of acronyms identifies the collection sites for each entry. While the southeastern United States is well served by botanical manuals and other floristic works that identify native plants, such works on the cultivated woody plants (indigenous and introduced) of the southeastern states have been much neglected.

The catalog contains about 5,000 entriesspecies, subspecies, varieties, forms, and cultivars-in 112 plant families and 493 genera. These are based on more than 14,000 voucher herbarium specimens, most collected specifically for this project. The primary focus of the project has been a critical review for correct identification and nomenclature of material in the list. This material, now permanently deposited in the herbarium of the U. S. National Arboretum, was collected at nearly 700 sites in Alabama, Arkansas, Delaware, the District of Columbia, northern Florida, Georgia, Louisiana, Maryland, Mississippi, North Carolina, South Carolina, Tennessee, southeastern Texas, and Virginia. This area covers about one-sixth of the continental United States and spans Plant Hardiness Zones 6b through 9a (U.S. Department of Agriculture 1990). The collection sites include botanical gardens and arboreta, cemeteries, college campuses, experiment stations, nurseries, private gardens, and roadway plantings.

While comprehensiveness was an objective, the catalog makes no claim to completeness, since new plants are constantly being introduced to horticulture; and from a practical standpoint we were unable to visit every possible site. In genera such as Camellia, Rosa, Rhododendron, Hedera, and some others with a vast number of cultivars, completeness was simply not possible. However,

Meyer is a supervisory botanist (retired) and Mazzeo is a botanist, U.S. National Arboretum, Agricultural Research Service, U.S. Department of Agriculture, Washington, DC 20002; Voss, of Vienna, Virginia, prepared the computer data base and programming for the catalog and assisted in the checking of nomenclature. a large number of plants not previously listed or reported as cultivated in the United States are included as a result of recent introductions.

The catalog includes many unusual or rare plants from the living collections of the U. S. National Arboretum not well represented elsewhere in this country. Between 1961 and 1990, 125 cultivars in 18 genera were released by the National Arboretum to the horticultural industry-cultivars of Buxus, Camellia, Clematis, $\times$ Cupressocyparis, Eurya, Hibiscus, Ilex, Kalmia, Lagerstroemia, Magnolia, Malus, Metasequoia, Platanus, Pyracantha, Pyrus, Rhododendron, Ulmus, and Viburnum. The catalog also lists for the first time most of the dwarf and slow-growing conifers in the Gotelli Collection at the U.S. National Arboretum. The list of Mex, the largest of any genus in the catalog, is based primarily on the extensive collections of living plants and voucher herbarium specimens at the U.S. National Arboretum.

The authors are solely responsible for the names and identifications of the plants listed in the catalog, but in difficult genera such as Acer, Aesculus, Callistemon, Crataegus, Hydrangea, Ilex, Salix, and Viburnum, we had the expertise of specialists who generously provided names and verified identifications. In this introduction, most of the botanical names are cited without authorities, since these are properly listed with the names in the catalog; but sometimes authorities are cited to substantiate a point.

The catalog is arranged alphabetically by the internationally accepted botanical and cultivar names, including some synonyms. Vernacular, or common, names are also included for many entries. When a species is subdivided, the epithet for the infraspecific taxon that includes the type of the species name is an exact repetition of the specific epithet (an autonym) and is cited without the author's name. The list of collection-site acronyms in Appendix B identifies the sources of the documented voucher specimens, thereby providing information on the abundance, distribution, and hardiness range of the plants listed. 
Although we have not indicated the geographic origins of the plants, the cultivated woody flora of the southeastern states represents a wide diversity of germplasm from many countries, in particular the southeastern United States, Europe, and eastern Asia. In lesser numbers, the catalog lists plants from temperate areas of South America (southern Brazil, Uruguay, Paraguay, northern Argentina, and northern Chile), including Araucaria araucana, Brunfelsia australis, Butia capitata, Calliandra tweediei, Erythrina crista-galli, Sesbania punicea, Tipuana tipu, and others. The diversity of woody landscape plants now in cultivation from South America and Central America suggests that renewed germplasm exploration in these areas would no doubt turn up other plants worth introducing for landscape use in the southeastern United States. Other areas represented in the catalog include Mexico, with Antigonon leptopus, Dioon edule, Parkinsonia aculeata, and some others; South Africa, with Carissa macrocarpa, Ochna atropurpurea, Phygelius capensis, and Tecomaria capensis; and Australia, with Casuarina cunninghamiana, Callistemon (six species), Callitris columellaris, Eucalyptus (six species), and Westringia rosmariniformis.

\section{Need for the Catalog}

In recent years, the nursery industry has become increasingly active in offering new plant introductions, as a result of renewed interest in plant exploration and germplasm diversity. Each year introductions appear in ever-increasing numbers from all parts of the world. People are now planting a greater diversity of woody landscape plants than ever before in U.S. history. The U.S. National Arboretum has contributed significantly to this flow of new introductions from its plant breeding program and from expeditions to Japan, the People's Republic of China, and the Republic of Korea. Some woody plants totally unknown in cultivation only a few years ago are now bestsellers in garden centers; these include Pyracantha 'Mohave' and Hibiscus syriacus 'Diana', both of which resulted from the U.S. National Arboretum plant breeding program.

The influx of new plants has compounded the problems of plant identification and nomenclature in the nursery trade, as well as among researchers, students, and home gardeners. Many plants found in the nursery trade are misidentified. The same plant may appear under various names in different nursery catalogs, a problem that leads to nomenclatural confusion.
We have observed, for example, that dwarf and slow-growing conifers are frequently misnamed in the nursery trade and by the specialists who grow them. Because of this confusion, the need is pressing to investigate the taxonomy and nomenclature not only of landscape plants but also of food, medicinal, and agronomic plants as a much-neglected aspect of horticultural botany. Access to voucher herbarium specimens makes identification of these plants much easier and far more accurate.

Unfortunately, too few technically qualified botanists or horticulturists are available and willing to tackle the identification and nomenclature of landscape plants, leaving many plant groups in a state of perpetual taxonomic and nomenclatural confusion. Although botanical gardens and arboreta often maintain small herbaria to document the plants in their collections, very few of the world's major herbaria are willing to incorporate specimens of cultivated plants for scientific study. The value of voucher herbarium specimens has not been sufficiently appreciated by horticulturists. Many problems related to the taxonomy and nomenclature of the plants in this catalog simply could not have been effectively investigated and resolved without the benefit of herbarium specimens.

\section{Plant Origins}

The area covered by the catalog encompasses much the same range as J.K. Small's Manual of the Southeastern Flora (1933). This vast region supports the richest ligneous flora in temperate North America and may be compared only with the highly diverse woody flora of the People's Republic of China. More than 250 species of indigenous trees occur in the southeastern United States, many of them grown as landscape plants. One of the best known and admired is Magnolia grandiflora, which ranks among the most valuable indigenous landscape trees of the United States. Now widely cultivated abroad, this magnificent flowering tree is one of America's great gifts to the gardens of the world.

Because of climatic similarities between eastern Asia and the eastern United States, countries such as Japan, Korea, and the People's Republic of China, with Taiwan, are heavy contributors of woody landscape plants now widely cultivated in the southeastern United States. Some wellknown plants of east Asian origin include 
Camellia japonica, Cryptomeria japonica, Ilex crenata, Juniperus chinensis, Lagerstroemia indica, Ligustrum japonicum, L. lucidum, Magnolia denudata, Rhododendron (many species), Ternstroemia gymnanthera, Wisteria floribunda, and $W$. sinensis.

From a horticultural perspective, the southeastern United States is one of the most interesting areas in the country. The oldest landscaped gardens in the United States, dating from the late 17 th and early 18th centuries, are found in Virginia, the Carolinas, and Georgia. Since plants follow people, we know that many of the earlier introductions were brought by European immigrants to their new homes in America. Early plantation life in the southern colonies promoted an interest in the cash crops of tobacco, rice, and indigo. By the end of the 17th century, ornamental plants such as boxwood (Buxus sempervirens) and garden flowers introduced from Europe were cultivated. The gardens at Middleton Place near Charleston, SC, date from about 1730 as one of the oldest landscaped gardens in America. About the same period, the earliest nurseries were established in Charleston. The first plant-introduction garden in America, called the "Founder's Garden," was established at Savannah soon after the founding of the Georgia colony in 1733 by James Oglethorpe.

It is recorded that about 20 to 25 exotic trees and shrubs were already in cultivation in gardens at Williamsburg, VA, by the middle of the 18th century, including cornelian cherry (Cornus mas), fig (Ficus carica), littleleaf linden (Tilia cordata), weeping willow (Salix babylonica), and the common lilac (Syringa vulgaris). At the same time, about 80 species of native American trees and shrubs were cultivated at Williamsburg, including flowering dogwood (Cornus florida), American beech (Fagus grandifolia), common catalpa (Catalpa bignonioides), red buckeye (Aesculus pavia), Carolina cherry laurel (Prunus caroliniana), southern live oak (Quercus virginiana), and American holly (Ilex opaca).

The introduction of the parasol tree (Firmiana simplex) about 1780 at Charleston, SC, is attributed to André Michaux, a French plant explorer who lived near Charleston. American ships returning from China often landed at Charleston, and Michaux may have obtained seeds of the parasol tree from one of these ships. From the evidence at hand, it is doubtful that the common camellia (C. japonica) came directly to America on board a ship returning from the Orient. Michaux probably did not introduce the camellia to Middleton Place in 1785 as long assumed. We have identified one of the four original plants attributed to him on this site as C. japonica 'Anemoniflora', a cultivar that only reached England in the first decade of the 19th century and was illustrated in Curtis's Botanical Magazine, t. 1654, 1814. It is more likely that the camellias at Middleton Place reached America from Great Britain after 1814 and not earlier.

\section{Categories of Ligneous Plants in the Catalog}

\section{Trees}

The distinction between trees and shrubs is often controversial from various points of view. No two definitions totally agree. We know that some shrubs become treelike under certain growing conditions. A tree, by standard dictionary definition, is a perennial woody plant usually having a single self-supporting trunk and ranging in height from 10 to more than 300 feet. Many trees fit this definition, but others do not. Indigenous species such as Cornus florida and Viburnum prunifolium rarely attain the stature of a tree in their native woodland habitats, but in an open sunny location these same species often become treelike. In another example, the red buckeye (Aesculus pavia) may grow to 30 feet as a singletrunked tree in the open but not more than a shrub 5 to 6 feet high in a woodland setting.

Examples of indigenous trees of the southeastern United States, with their maximum height, include:

Acer saccharinum, silver maple, $70 \mathrm{ft}$ Aesculus flava, yellow buckeye, $70 \mathrm{ft}$ Fagus grandifolia, American beech, $70 \mathrm{ft}$ Fraxinus americana, American ash, $80 \mathrm{ft}$ Ilex opaca, American holly, $100 \mathrm{ft}$

Liriodendron tulipifera, yellow poplar, tulip tree, $100 \mathrm{ft}$

Magnolia grandiflora, southern magnolia, $90 \mathrm{ft}$ Magnolia macrophylla, bigleaf magnolia, $50 \mathrm{ft}$ Platanus occidentalis, American sycamore, $80 \mathrm{ft}$ Guercus alba, white oak, $70 \mathrm{ft}$

Quercus phellos, willow oak, $70 \mathrm{ft}$ Quercus velutina, black oak, $70 \mathrm{ft}$ Quercus virginiana, southern live oak, $70 \mathrm{ft}$. 
Examples of trees introduced into the region, with their maximum height, include:

Ailanthus altissima, tree-of-heaven, $70 \mathrm{ft}$, People's Republic of China

Broussonetia papyrifera, paper mulberry, 40

$\mathrm{ft}$, eastern Asia to Polynesia

Cedrus deodara, deodar cedar, $100 \mathrm{ft}$, Nepal and adjacent People's Republic of China

Cunninghamia lanceolata, China fir, $70 \mathrm{ft}$,

People's Republic of China, with Taiwan

Firmiana simplex, parasol tree, $60 \mathrm{ft}$, People's Republic of China

Morus alba, white mulberry, $40 \mathrm{ft}$, People's Republic of China

Paulownia tomentosa, princess tree, $50 \mathrm{ft}$, People's Republic of China

Platanus $\times$ acerifolia, London plane, $60 \mathrm{ft}$, garden origin

Salix babylonica, weeping willow, $60 \mathrm{ft}$,

People's Republic of China(?)

Sapium sebiferum, Chinese tallow tree, $80 \mathrm{ft}$,

People's Republic of China

Sequoia sempervirens, coast redwood, $100 \mathrm{ft}$, California.

Examples of smaller trees, both native and introduced, include:

Albizia julibrissin, mimosa tree, $30 \mathrm{ft}$, Iran to People's Republic of China, Japan, and Korea

Amelanchier arborea, serviceberry, $35 \mathrm{ft}$, eastern United States

Asimina triloba, pawpaw, $35 \mathrm{ft}$, eastern United States

Buxus sempervirens, common boxwood, $25 \mathrm{ft}$, Europe

Cercis canadensis, North American redbud, $25 \mathrm{ft}$, eastern United States

Chionanthus retusus var. serrulatus, $25 \mathrm{ft}$,

People's Republic of China, with Taiwan

Cornus florida, flowering dogwood, $30 \mathrm{ft}$, eastern United States

Cotinus obovatus, American smoke tree, $40 \mathrm{ft}$, eastern United States

Cyrilla racemiflora, titi, $25 \mathrm{ft}$, southeastern United States

Magnolia ashei, Florida bigleaf magnolia, $25 \mathrm{ft}$, northern Florida.

Trees are deciduous, evergreen, or sometimes semi-evergreen, depending upon climate and species. A well-known tree indigenous to the southeastern United States is the southern live oak (Quercus virginiana), usually regarded as evergreen. In fact, this oak is not truly evergreen in the strict sense. As the new leaves emerge in the spring, the previous year's leaves are lost, and the tree becomes essentially leafless for a short period until the new leaves have fully expanded. But some oaks are, indeed, permanently evergreen, such as Quercus myrsinifolia, Q. glauca, and Q. acuta - all from Japan. Another well-known tree, Magnolia grandiflora, remains fully evergreen throughout the year. In this species, leaves normally remain on the tree for 2 years before falling in spring of the second year, but the tree is never leafless.

\section{Shrubs}

Typically, shrubs differ from trees primarily in being multitrunked from the base and shorter than trees. Shrubs are seldom more than 20 feet in height; some of the largest may be singletrunked and treelike. Stature alone is not an absolute measure of shrub classification. A good example is illustrated by Magnolia virginiana. The northern aspect of this species, distributed from Massachusetts to North Carolina, is typically deciduous, multitrunked, and shrubby, with a spreading habit that fits the stature of a large shrub. In cultivation and in the wild, it sometimes attains a height of 30 feet but seldom more. The southern aspect, often referred to as var. australis, occurs from North Carolina to southern Florida and west to Tennessee, Arkansas, and southeastern Texas, where it becomes a lofty evergreen tree with one or two trunks and sometimes reaches 100 feet in height. In cultivation, the southern plant consistently remains evergreen and treelike at least as far north as Washington, DC, even in the coldest winters.

Although the distinction of deciduous or evergreen habit and the differences in stature can be used to separate the northern and southern aspects in the living state, no discernable taxonomic characters can be found to consistently distinguish these variants morphologically. Moreover, the two variants of $M$. virginiana are often intermediate as to evergreenness and stature, factors that further complicate their identification. For these reasons, var. australis is not recognized in this catalog as a distinct entity.

Examples of common native and introduced shrubs cultivated in the southeastern United States include:

Abelia $\times$ grandiflora, glossy abelia, 6-8 ft, a hybrid of Chinese parentage Agarista populifolia, pipe plant, 12-15 ft, southeastern United States 
Camellia japonica, common camellia, $20 \mathrm{ft}$, Japan

Chionanthus virginicus, old-man's beard, $12 \mathrm{ft}$, eastern United States

Kalmia latifolia, mountain laurel, $20 \mathrm{ft}$, eastern United States.

\section{Subshrubs}

A subshrub, or undershrub, is an intermediate category of a half-woody, or suffruticose, plant. The lower part of the stem, from which the new growth emerges each year, is woody and persistent. These are not simply half-hardy plants but represent a stage between herbaceous and the truly woody condition. Examples of subshrubs in the catalog include:

Chrysanthemum nipponicum, Nippon daisy, Japan

Indigofera spp., indigo (listed species)

Lespedeza spp., bush clover (listed species)

Nierembergia scoparia, tall cup-flower, Uruguay and Argentina

Pachysandra procumbens, Allegheny spurge, southeastern United States

Pachysandra terminalis, Japanese spurge, Japan, People's Republic of China, and Korea

Teucrium chamaedrys, European germander, Europe

Vinca major, greater periwinkle, Europe

Vinca minor, common periwinkle, Europe.

\section{Woody climbers, or lianes}

The term scandent, from the Latin scandens, refers to woody and herbaceous plants that climb by various means. The term liana, or liane, refers to a woody climbing plant, especially in the tropics, but lianes occur in all climates except polar areas. Woody climbers include plants in five categories according to their mode of climbing, as follows:

\section{Root-climbers with holdfast roots and disc- like attachments}

Campsis radicans, trumpet vine, eastern United States

Decumaria barbara, wood-vamp, southeastern United States

Euonymus fortunei, wintercreeper spindlebush, Japan, People's Republic of China, and Korea

Hedera helix, English ivy, Europe

Hydrangea anomala ssp. petiolaris, climbing hydrangea, Japan.
Tendril-climbers with auxiliary filiform leafless and usually brancfied organs that attach to another body and curl around it for support

Morphologically, a tendril may be a modified stem, a modified branch, a petiole, a stipule, or a leaflet of a compound leaf. Occasionally, as in Parthenocissus tricuspidata, the filiform branch tips are provided with terminal cups called holdfasts. Examples include:

Ampelopsis arborea, peppervine, eastern United States

Ampelopsis glandulosa var. brevipedunculata, porcelain-berry, Japan and People's Republic of China

Bignonia capreolata, cross vine, southeastern United States

Parthenocissus quinquefolia, Virginia creeper, eastern United States

Parthenocissus tricuspidata, Boston ivy, Japan and People's Republic of China

Vitis spp., grape vine.

\section{Twiners that climb spirally for support}

Actinidia arguta, tara vine, Japan, Korea, and northeastern Asia

Actinidia deliciosa var. deliciosa, Chinese gooseberry or kiwi fruit, People's Republic of China

Akebia quinata, chocolate vine, Japan, People's Republic of China, and Korea.

Berchemia scandens, Alabama supplejack, eastern United States

Celastrus orbiculatus, Oriental bittersweet, Japan, Korea, and People's Republic of China

Gelsemium sempervirens, Carolina jessamine, southeastern United States

Kadsura japonica, kadsura vine, Japan, People's Republic of China, with Taiwan

Lonicera sempervirens, trumpet honeysuckle, eastern United States

Trachylospermum jasminoides, Confederate jasmine, Japan, Korea, and People's Republic of China

Wisteria spp. (species twine either to the right or left).

Scramblers with long straggling branches that support themselves on other branches without fastening in any active manner; prickles or thorns may assist climbing, as in roses and brambles

Rosa laevigata, Cherokee rose, People's Republic of China

Rubus calycinoides, Taiwan

Rubus cockburnianus, People's Republic of China. 
Leaf-petiole climbers that hold the stem in place by twining around an adjacent branch

Clematis spp. and hybrids.

\section{Plant Nomenclature}

The guiding principles for the regulation of plant nomenclature are embodied in two contemporary documents: (1) the International Code of Botanical Nomenclature, 1988 edition (called the Botanical Code) and (2) the International Code of $\mathrm{No}^{-}$ menclature for Cultivated Plants, 1980 edition (called the Cultivated Code). The Botanical Code "governs the use of botanical names in Latin form for both cultivated and wild plants, except for graft chimaeras" (Brickell et al. 1980: 11). The Cultivated Code regulates nomenclatural matters related exclusively to agricultural, horticultural, and silvicultural cultivars.

Reasons for changing plant names are rooted in the rule of priority stated in Article 11 of the Botanical Code. For a genus, the correct epithet is the earliest legitimate one at the same rank, except for a conserved name (nomen conservandum); for example, Pseudolarix Gordon (1858) not Chrysolarix H.E. Moore (1965). In categories below the rank of genus, the correct name is the earliest legitimate name at the same rankspecies, subspecies, varietas, or forma-to which the plant is assigned. A plant has only one correct name, generally a binary name or binomial, that consists of a genus name (for example, Magnolia) followed by a specific epithet (for example, grandiflora). To this binary name is appended the name of the authority(ies) who published the name. The correct citation of the southern magnolia is Magnolia grandiflora L. ("L." stands for Carl Linnaeus, who first described this species in 1759). Other names for this plant are treated as synonyms (Magnolia foetida Sarg., for example) or homonyms but are illegitimate for purposes of scientific nomenclature. The binary name must be in Latin form regardless of its derivation. Plant nomenclature contains many names adapted from other languages, especially from Greek.

Names of plants are based on nomenclatural types, usually type specimens, which are documented, dried, and pressed specimens deposited in an herbarium for permanent reference. In the absence of a specimen, an accurate illustration may be substituted for purposes of typification. Botanical names published since 1935 must be accompanied by a Latin diagnosis to be botanically legitimate.

\section{Hierarchy of Rank Categories}

\section{Genus}

Genus (plural, genera) is the first major taxonomic category under the family. The catalog is arranged alphabetically by genus, beginning with Abelia R.Br., assigned to the family Caprifoliaceae and its common name, honeysuckle family. Genera of Caprifoliaceae in the catalog include: Abelia, Diervilla, Dipelta,

Heptacodium, Kolkwitzia, Lonicera, Sambucus, Symphoricarpos, Viburnum, and Weigela. Some families are monotypic, comprising a single genus, such as Ginkgo in the Ginkgoaceae and Cercidiphyllum in the Cercidiphyllaceae. For the convenience of the user, the species, subspecies, varietas, forma, and cultivar names are listed alphabetically under each genus without regard to botanical rank. A supplementary list (Appendix D) groups the names according to botanical rank.

\section{Species}

Biologically, the species is the basic taxonomic unit employed in the recognition and classification of plants. For purposes of this catalog, Cronquist's general definition of species is useful: "Species are the smallest groups that are consistently and persistently distinct, and distinguishable by ordinary means" (1988, p.71). Criteria for the recognition of species may include factors of reproductive isolation, chromosome number, phenetic or morphological discontinuity, geographic isolation, and ecogeographic coherence.

As the basic unit of biology, the species is subordinate to the genus. As the next higher major category, the genus consists of one or more species. The number of species varies from genus to genus. The monotypic genus Ginkgo, for example, consists of a single species, biloba, but the genus Quercus consists of many species, perhaps 450 worldwide, and some genera are even larger. While botanists argue the relative importance of species criteria, the viability of the species as a fundamental biological unit is attested by the presence of over 1,000 Linnean names in the catalog more than 200 years after the death of Linnaeus.

The word species is either singular or plural (specie refers to coinage). It is abbreviated sp. when singular and spp. when plural. In this catalog, species and cultivar names are cited in strict alphabetical order under the name of the 
genus. The entries under the genus Abelia, for example, include the species chinensis, followed by the cultivar 'Edward Goucher', the hybrid $\times$ grandiflora, and the cultivars 'Prostrata' and 'Sherwoodii'.

In horticultural works, authorities for cultivar names are not required according to the Cultivated Code. In nursery lists and in many publications on landscape plants, authorities for botanical names are usually omitted, but this is a mistake and leads to confusion. The citation of authorities clearly identifies the botanical name with its correct author(s), for example, Aesculus flava Solander, not of Aiton. In another example, the name Acer parviflorum was applied by Ehrhart in 1789 to A. spicatum Lamarck (1786) and later by Franchet \& Savatier in 1879 to the plant now known as A. nipponicum Hara. Because of Ehrhart's earlier use of the specific epithet (though illegitimate because of Lamarck's priority), the Franchet \& Savatier name was a "later homonym" and therefore illegitimate. Without an author citation, a reference to the name $A$. parviflorum would not inform the reader whether $A$. spicatum or $A$. nipponicum was meant.

\section{Subspecies}

The subspecies, abbreviated ssp. or subsp., follows the rank of species and is often used to categorize geographic or ecological variants of a polymorphic species into generally disjunct morphological units. These units may be isolated from each other by latitude, longitude, altitude, and by ecological or geographical factors, as well as by morphological characters. Botanists still disagree on the application of the subspecies category, especially when the distinctions among variants are ill defined. Examples of subspecies in the catalog include Acer negundo L. ssp. mexicanum (DC.) Wesmael, Hydrangea anomala D. Don ssp. petiolaris (Sieb. \& Zucc.) McClint., and Koelreuteria elegans (Seemann) A.C. Sm. ssp. formosana (Hayata) F.G. Meyer.

\section{Varietas}

The category varietas, or botanical variety (abbreviated var.) was long used as the only infraspecific category generally recognized by botanists. Because of difficulties in evaluating morphological characters in plants, the categories subspecies and varietas have been used interchangeably by different workers. There is no common agreement among botanists on the application of these categories. As indicated by Cronquist, "Nowadays one taxonomist's subspecies is likely to be another's variety" (1988, p. 99). Varieties are recognized in many genera in this catalog. In the genus Acer, for example, we recognize Acer rubrum L. var. trilobum T. \& G. ex K. Koch, A. rubrum L. var. drummondii (Nutt.) Sarg., A. palmatum Thunb. ex J.A. Murr. var. amoenum (Carr.) Ohwi, and A. saccharum Marsh. var. rugelii (Pax) Rehd.

\section{Forma}

The category forma (abbreviated f.) has long been applied to distinguish transitory variation without persistent biological significance that is found randomly within populations of wild plants. Such variation includes flower color, leaf size, leaf shape, plant habit, vesture (hairiness, for example), and other characters. The following examples illustrate the use of forma in three wellknown native woody plants cultivated in the southeastern United States: pink flowering dogwood (Cornus florida L. f. rubra (Weston) Schelle), yellow-fruited flowering dogwood (Cornus florida L. f. xanthocarpa Rehd.), and the yellow-fruited American holly (Ilex opaca Ait. f. xanthocarpa Rehd.).

Before the term cultivar was adopted by the Cultivated Code in 1953, the category forma had long been in use for many garden plants and also for some wild plants. Examples include Sophora japonica L. f. pendula (Sweet) Zabel and Pseudotsuga menziesii (Mirb.) Franco var. glauca (Beissn.) Franco f. glauca pendula Rueppel that were automatically given cultivar status after 1953. These plants are now properly cited as Sophora japonica L. 'Pendula' and Pseudotsuga menziesii (Mirb.) Franco var. glauca (Beissn.) Franco 'Glauca Pendula'.

\section{Cultivar}

The 1980 edition of the Cultivated Code provides guidelines "to promote uniformity, accuracy, and fixity in the naming of agricultural, horticultural, and silvicultural cultivars (varieties)." The term cultivar (derived from cultivated variety) was included when the Cultivated Code was drawn up in 1953; it denotes "an assemblage of cultivated plants which is clearly distinguished by any characters (morphological, physiological, cytological, chemical, or others) and which, when reproduced (sexually or asexually), retains its distinguishing characters." 
Cultivars are without rank in the hierarchy of botanical nomenclature. In woody plants, cultivars are usually perpetuated as clones, that is as a genetically uniform assemblage of plants derived originally from a single individual and reproduced asexually as cuttings, divisions, or grafts, or by obligate apomixis. Seed-reproduced cultivars occur infrequently among woody plants (for example, in Acer and Betula). They are, however, well known in herbaceous genera, for example, Impatiens, Phlox drummondii 'Sternenzauber', and Zea mays.

Cultivar names, to be legitimate, must be registered through the appropriate international registration authority or published with an adequate description in a recognized publication, such as a dated nursery catalog or journal. Cultivar names simply listed in a nursery catalog or other publication are without valid status. These practices are important for ensuring the validity and stability of cultivar names. Cultivar names published on or after January 1, 1959, may no longer be in Latin form but must be fancy names in the vernacular, that is, in English or another modern language. Cultivar names may be designated by the abbreviation $\mathrm{cv}$. preceding the name, or, as in this catalog, by single quotation marks as in the following examples: Abelia 'Edward Goucher', Hibiscus syriacus 'Diana', and Magnolia 'Galaxy'. However, cultivar names in Latin form published before January 1, 1959, such as Acer palmatum 'Atropurpureum' and Mex aquifolium 'Argentea Marginata', must be retained, according to the Cultivated Code.

Our method of listing cultivar names alphabetically facilitates easy identification of the binary name (Latin scientific name) associated with a cultivar name, especially in those genera for which the user of the catalog does not know the species of a particular cultivar. The following examples from the genus Acer illustrate the usual method of citing cultivars, first listing the cultivar name and, in square brackets, identifying the species: 'Akaji Nishiki' [A. palmatum Thunb. ex J.A. Murr.]; 'Schwedleri' [A. platanoides L.]; and 'Temple's Upright' [A. saccharum Marsh.]. In some cases, the cultivar is identified only as to genus.

\section{Sexual hybrids}

In interspecific hybrids, a multiplication sign is placed before the hybrid epithet as in Abelia $\times$ grandiflora, a hybrid of $A$. chinensis and $A$. uniflora. In intergeneric hybrids, a multiplication sign is placed in front of the hybrid genus name as in $\times$ Cupressocyparis leylandii, a hybrid of Chamaecyparis nootkatensis and Cupressus macrocarpa.

\section{Graft chimeras}

As defined in the Cultivated Code, "Graft-chimaeras are composed of tissues in intimate association from two different plants. They originate by grafting and are not sexual hybrids." There is no combining of cell contents or nuclei as in the case of hybrids; thus the word hybrid is inappropriate for these plants. In a graft chimera, the tissue of a shoot originating at the callus of the graft union contains at least one cell from the scion and one from the root stock. Periclinal chimeras, in which cells from the scion and cells from the stock are in different layers, are persistent when propagated. Chimeras may exhibit characters typical of the scion or the stock, or a range of intermediate forms.

The graft chimeras listed in the catalog are of the periclinal type. Graft chimeras are most unusual in the simultaneous production of flowers from the scion and from the stock. They are known worldwide by only a very few welldocumented examples; two are listed in the catalog, designated with a plus (+) sign before the name, as follows:

- Camellia +'Daisy Eagleson' = C. sasanqua 'Maiden's Blush' + C. japonica. Camellia sasanqua 'Maiden's Blush' is the rootstock and C. japonica, the scion. At flowering time, this evergreen shrub displays simultaneously on different branches the single flowers of $C$. sasanqua and the semi-double flowers of $C$. japonica.

- +Laburnocytisus adamii = Cytisus purpureus + Laburnum anagyroides. This small deciduous tree combines species of two genera of the bean family (Fabaceae). Branches of the yellow flowers of $L$. anagyroides and the dull purple flowers of C. purpureus bloom simultaneously on the same plant. 


\section{How Plant Names Change}

The names of plants are not fixed in time but are subject to change for various reasons. It is not difficult to find examples of plants long known under established names that are incorrect according to the Botanical Code. Name changes for some well-known woody landscape plants listed in the catalog are discussed below.

The name Plumbago auriculata Lam. is the correct binary name for a now widely cultivated South African species. Published by Lamarck in 1786, this name has an 8-year priority over the name Plumbago capensis Thunb. that was long used for this plant. In another example, the name Cladrastis lutea (Michx.f.) K. Koch dates from 1813 and is well known; but in 1811 Dumont de Courset had published the specific epithet kentukea for the same plant. According to the rule of priority, the name Cladrastis kentukea (Dum.-Cours.) Rudd has date priority as the correct name for yellow-wood.

Changes in plant names sometimes may not conform with the facts, causing more confusion than existed before the change was made. Two examples illustrate name changes too hastily made. Under Magnolia in Hortus Third (Bailey 1976), the entries M. heptapeta (Buc'hoz) Dandy and $M$. quinquepeta (Buc'hoz) Dandy are listed for two well-known magnolias, $M$. denudata and $M$. liliiflora, respectively. In 1779, Buc'hoz published Lassonia heptapeta and L. quinquepeta for these taxa. In 1934, Dandy transferred the Buc'hoz epithets to the genus Magnolia. In a paper by F. G. Meyer and E. McClintock published in 1987, the evidence shows that the combinations of Dandy were totally misapplied and without botanical validity. The original Chinese illustrations on which these names were based are artist's renditions, probably of Magnolia, but the details are totally incorrect and the drawings may not be properly assigned to the genus Magnolia or, indeed, to any known living plant. Meyer and McClintock concluded that the earliest valid names for these magnolias, $M$. denudata and $M$. lilifflora, were correctly published by Desrousseaux in 1791 and stand as the correct names for these plants.

The name Toona sinensis (Endl.) M.J. Roem. applies to a tree native to eastern Asia, replacing the name Cedrela sinensis Juss. This follows an earlier taxonomic decision that the name Toona should apply to the Asiatic species, while the New World species (although closely related) are correctly placed in the genus Cedrela.
Until relatively recently, two species of Sequoia were recognized. A detailed study by J.T. Buchholz showed that only the coast redwood (Sequoia sempervirens (D. Don) Endl.) should be retained in the genus, while the giant sequoia of the high Sierras of California (formerly known as Sequoia gigantea Lindl.) belongs to the closely related genus Sequoiadendron with the binary name Sequoiadendron giganteum (Lind1.) J. Buchholz.

From time to time, various specific epithets have been applied to the Douglas fir-including Pseudotsuga douglasii Carr. (1867), P. lindleyana Carr. (1868), P. taxifolia (Lamb.) Britton (1889), and $P$. mucronata (Raf.) Sudworth (1895). But all of these must give way to the name Pseudotsuga menziesii (Mirb.) Franco (1950), based on Pinus menziesii Mirb. (1825), as the valid name for this well-known tree of the western United States.

A well-known tree indigenous to the southeastern United States, the yellow buckeye, should be cited as Aesculus flava Sol. (1784), not A. flava Ait. (1789). This may appear as a small detail, but it illustrates the application of the rule of priority that exists to promote stability and accuracy in plant nomenclature. The name $A$. octandra Marsh. (1785), published later and long used for this tree, is a synonym without taxonomic validity of $A$. flava Sol.

Confusion reigns in the names applied to two evergreen shrubs planted in the southeastern states, one of them commonly grown and the other one rare. The well-known Florida native Ilicium parviflorum Michx. ex Vent. is often misidentified in nurseries as I. anisatum L., a Japanese plant. Although the Japanese species is barely known in most areas, its name has long been associated with the wrong plant.

Another common shrub in nurseries, and one widely cultivated in the southeastern United States, is Ternstroemia gymnanthera (Wight \& Arn.) Sprague of the tea family (Theaceae). It has long been sold in nurseries under the name Cleyera japonica Thunb., a different though closely related plant. Many nurseries are now aware of the correct identification of these plants and are labeling them accordingly. The true Cleyera japonica is an attractive, small evergreen tree with fragrant white flowers and black fruit, but it is rarely cultivated.

The name Anisostichus capreolatus (L.) Bur. (1884) can no longer be used for the cross vine, because the earlier name Bignonia capreolata L. 
(1753) is a nomen conservandum, or conserved name, for this woody climber of the southeastern United States.

For more than a century, nurseries confused two commonly planted species in the genus

Ligustrum-L. japonicum Thunb., Japanese privet; and $L$. lucidum Ait.f., wax-leaf privet. The name $L$. lucidum was misapplied to the Japanese privet, while the name L. japonicum was mistakenly applied to the wax-leaf privet. These errors were repeated year after year in nursery catalogs until Roland Jefferson (1976) corrected them. The problem was a simple case of applying the right name to the right plant.

\section{Homonyms}

According to the Botanical Code, "A name, unless conserved (Art. 14) or sanctioned under Art. 13.1(d), is illegitimate if it is a later homonym, that is, if it is spelled exactly like a name based on a different type that was previously and validly published for a taxon of the same rank." Many homonyms exist among plant names found in the literature. For example, Linnaeus in 1753 published Ilex aquifolium as the name for English holly (in the Aquifoliaceae, or holly family). In 1784, Thunberg used Ilex aquifolium for a Japanese plant subsequently recognized as being in the Oleaceae, or olive family. Placing the Japanese plant in the genus Osmanthus of the Oleaceae, Siebold in 1846 adopted the specific epithet from Thunberg's name and published Osmanthus aquifolium. But this name is illegitimate, because Thunberg's name is a later homonym, and the nomenclatural type for llex aquifolium is the English holly named by

Linnaeus. Because the earliest available specific epithet for the Japanese plant is that from G. Don's Ilex heterophylla (1832), the legitimate name for the familiar holly olive is Osmanthus heterophyllus (G.Don) P.S.Green var. heterophyllus.

Another significant confusion occurred in the names of two well-known species in the genus Rhododendron, one an elepidote rhododendron and the other a deciduous azalea. In 1826 , Blume applied the name Hymenanthes japonica to the elepidote rhododendron. In 1835, Siebold and Zuccarini transferred the name Hymenanthes to Rhododendron as a generic synonym and named the species in question Rhododendron metternichii Sieb. \& Zucc.; however, by citing the earlier name $H$. japonica Blume as a synonym they rendered $R$. metternichii illegitimate. In 1909, Camillo Schneider transferred the earlier name Hymenanthes japonica Blume to Rhododendron japonicum (Blume) Schneider. This was, in fact, the correct name until very recently the Committee for Spermatophyta of the International Botanical Congress determined that the specific epithet japonicum correctly applies to the deciduous azalea. The name $R$. degronianum ssp. heptamerum (Maxim.) Hara is now the correct name for the elepidote rhododendron long known as $R$. metternichii. The illegitimate name R. metternichii Sieb. \& Zucc., however, continues to be used widely in horticultural circles.

The deciduous azalea involved in this confusion of names was described by Asa Gray as Azalea japonica, in the Luteum subseries from Japan. In 1908, Suringar transferred the specific epithet to Rhododendron as R. japonicum (A.Gr.) Suring. Suringar's publication of this combination was, however, interpreted by the author of a revision of the genus as provisional, making the name illegitimate as applied to the azalea and requiring its use for the elepidote rhododendron. Because the resulting name changes would have undesirable consequences, a formal proposal was made to reject $R$. japonicum (Blume) Schneider. After detailed re-examination of Suringar's work, the Committee for Spermatophyta concluded that the Suringar combination (using japonicum for the azalea) was indeed validly published. The name $R$. japonicum (Blume) Schneider for the elepidote rhododendron is therefore illegitimate as a later homonym and the proposal to reject became moot.

As noted, a later homonym may be conserved by formal action under the Botanical Code. For example, the genus name, Torreya Arn. (1838) of the yew family (Taxaceae) is conserved, and the earlier homonym Torreya of Rafinesque (1818) applied to a plant in the mint family (Lamiaceae) is rejected.

\section{Synonyms}

Many plants have been repeatedly redescribed by different botanists, thus creating a long, complicated synonymy that is often difficult to reconcile. The synonyms of Magnolia denudata Desr. (1791), for example, include $M$. conspicua Salisb. (1806), M. precia Correa ex Vent. (1803), and $M$. yulan Desf. (1809). The name Negundo fraxinifolium de Vos (1887) is a synonym and invalid, because its nomenclatural type is the same as that of Acer negundo L. (1753), boxelder, a common North American tree that spans the continent east to west and is cultivated worldwide. 
Common taxonomic synonyms are listed in the catalog to identify invalid names found in nursery catalogs and in the horticultural and botanical literature, such as the following:

Cladrastis lutea (Michx.f.) K. Koch =

C. kentukea (Dum.-Cours.) Rudd Magnolia foetida (L.) Sarg. = M. grandiflora L. Rhododendron nudiflorum (L.) Torr. =

$\boldsymbol{R}$. periclymenoides (Michx.) Shinners Rhododendron metternichii Sieb. \& Zucc. = R. degronianum Carr. ssp. heptamerum (Maxim.) Hara

Rhododendron roseum (Loisel.) Rehd. = R.prinophyllum (Small) Millais.

\section{Vernacular (Common) Names}

The botanical (Latin-form) names and registered cultivar names of plants have universal application in the world scientific community. They are, moreover, instructive in many ways. By contrast, vernacular names are simply names of convenience for local application. Over a wide area some plants may have several common names, all of equal value, as in laurel magnolia, bay, sweet bay, swamp bay, beaver tree, and laurel for Magnolia virginiana. Some plants, especially the exotics, are often without a common name until one is invented. In this catalog we include one or two common names in general use for many of the listed plants. These names are summarized in Appendix E.

While there are problems associated with common names, many people prefer the vernacular. Some complain about Latin names of plants as too awkward and difficult to pronounce. Approached with an open mind, scientific names are not difficult. Familiar names used in the vernacular, such as anemone, catalpa, chrysanthemum, gladiolus, iris, magnolia, petunia, phlox, rhododendron, sassafras, wisteria, yucca, and zinnia are, in fact, Latin genus names that have entered vernacular usage.

Once established, common names are often useful and convenient for trees such as Douglas fir, deodar cedar, white oak, and American elm. However, unlike botanical names, common names are sometimes misleading and confusing and may impede clear communication and understanding, since nothing about these names relates to name stability or to classification. In the southeastern United States, for example, the native deciduous azaleas are commonly known as "honeysuckle," but to many people honey- suckle refers only to species of Lonicera of the honeysuckle family (Capritoliaceae). The common name for Rhododendron periclymenoides is pinxterbloom in Maryland, purple honeysuckle in the Carolinas, and election pink in some other areas of the southeast. In the United States, the indigenous species of Tilia are called basswood or linden, while in the United Kingdom members of this genus are called lime trees. The use of common names should be discouraged because of the endless possibilities for misidentification and confusion.

\section{Symbols and Abbreviations}

Acronyms are cited alphabetically under each plant entry to identify the collection sites, for example, DCNA = District of Columbia/National Arboretum; FLUF = Florida/University of Florida, Gainesville; and GAUG = Georgia/University of Georgia.

Cultivar names are spelled with initial capital letters in roman type and are enclosed with single quotation marks: Abelia 'Edward Goucher', Magnolia 'Galaxy'.

\section{Other symbols and abbreviations follow.}

ssp. = subspecies: Hydrangea anomala D. Don ssp. petiolaris (Sieb. \& Zucc.) McClint.

var. = varietas (botanical variety): Acer palmatum Thunb. ex J.A. Murr. var. amoenum (Carr.) Hara

$x=$ multiplication sign used for hybrids: interspecific hybrids are identified with the multiplication sign placed in front of the hybrid epithet, as Abelia $\times$ grandiflora (Andre) Rehd.; in bigeneric hybrids the multiplication sign is placed in front of the hybrid genus name, as $\times$ Cupressocyparis leylandii (Dallim. \& Jacks.) Dallim.

$+=$ symbol for graft chimera, as Camellia +'Daisy Eagleson'

[] enclose botanical names of cultivars and names of parents of hybrids and cultivars

$\star$ designates plant introductions from the U. S. National Arboretum breeding program

? indicates uncertainty as to parentage. 
ABELIA R.Br.

CAPRIFOLIACEAE Honeysuckle Family

(Contributed by T. R. Dudley)

chinensis $\mathrm{R} . \mathrm{Br}$.

Chinese A.

DCNA MDFM SCWI

'Edward Goucher' [A. $\times$ grandiflora $\times$

A. schumannii]

$\begin{array}{llll}\text { ALBH } & \text { GAUG } & \text { SCCU } & \text { TXRS } \\ \text { GAJS } & \text { MDGC } & \text { TNFN } & \end{array}$

$\times$ grandiflora (Andre) Rehd.

Glossy A.
[A. chinensis $\times$
DCCG $\times$. Uniflora]
MDJH MSEP
FLMG MDKN NCBE
LASL MDLT NCDU
MDDF MDNA NCGP
NCSM
SCCU
TNBM

'Prostrata' [A. × grandiflora (Andre)

Rehd.] [A. chinensis $\times$ A. uniflora]
DCNA

'Sherwoodii' [A. × grandiflora (Andre) Rehd.]

[A. chinensis $\times$ A. uniflora]

GAUG SCBR

\section{ABELIOPHYLLUM Nakai OLEACEAE}

Olive Family

distichum Nakai

White Forsythia

DCNA MDLT

ABIES Mill.

Fir

PINACEAE

Pine Family

alba Mill.

Silver F.

GABS NCTE VAWP

balsamea (L.) Mill.

Balsam F.

DCNA

brachyphylla Maxim.

= A. homolepis

cephalonica Loud.

Greek F.

$$
\text { DCNA MDLT }
$$

chensiensis Tieghem ssp. salouenensis

(Bord.-Rey. \& Gaussen) Rushforth

Shensi $F$. DCNA

cilicica (Ant. \& Kotschy) Carr.

Cilician F. VAMP

concolor (Gord. \& Glend.) Lindl. ex Hildebr. White F.

$$
\text { MDLT NCBE VAMP }
$$

ernestii Rehd.

= A. chensiensis ssp. salouenensis

firma Sieb. \& Zucc.

Momi F.

$\begin{array}{lll}\text { ALOS } & \text { MDLT } & \text { TNWF } \\ \text { DCNA } & \text { NCBE }\end{array}$

fraseri (Pursh) Poir.

Fraser F.

$$
\text { NCAS TNBV VAFR VASG }
$$

'Glauca' [A. pinsapo Boiss.] DCNA

holophylla Maxim.

Manchurian F.

DCNA

homolepis Sieb. \& Zucc.

Nikko F.

DCNA

homolepis Sieb. \& Zucc. var. umbellata (Mayr) Wils.

= A. $\times$ umbellata

koreana Wils.

Korean F.

DCNA

nobilis (Dougl.) Lindl.

= A. procera

nordmanniana (Steven) Spach

Nordmann F.

$$
\begin{array}{lll}
\text { DCAE } & \text { NCBE } & \text { VABF } \\
\text { MDLT } & \text { NCKK } &
\end{array}
$$

numidica de Lannoy ex Carr.

Algerian $\mathrm{F}$.

DCNA

pectinata $\mathrm{DC}$.

= A. alba

pinsapo Boiss.

Spanish F.

DCSH VAMP 
procera Rehd.

Noble F.

DCNA

$\times$ umbellata (Mayr) Liu

[A. firma $\times$ A. homolepis] DCNA

$\times$ vilmorinii Mast.

[A. cephalonica $\times$ A. pinsapo]

DCNA

$\begin{array}{lr}\text { ABUTILON Mill. } & \begin{array}{r}\text { Flowering Maple } \\ \text { Mallow Family }\end{array} \\ \text { MALVACEAE } & \text { Mate }\end{array}$

pictum (Gillies ex Hook. \& Arn.) Walp. LAMP

striatum Dicks. ex Lindl.

= A. pictum

'Thompsonii' [A. pictum (Gillies ex Hook. \& Arn.) Walp.] GACG

ACACIA Mill. FABACEAE (Mimosoideae)

Wattle, Mimosa Bean Family

berlandieri Benth. TXSE

farnesiana (L.) Willd.

Sweet A.

$\begin{array}{lll}\text { FLUF } & \text { LAMP } & \text { TXJS } \\ \text { LAHG } & \text { SCKS } & \text { TXSE }\end{array}$

rigidula Benth. LASL

roemeriana Scheele TXSE

wrightii Benth. TXPS

ACALYPHA L. EUPHORBIACEAE

hispida Burm.f.

Chenille Plant ALBG

sanderi N.E.Br.

$=A$. hispida
ACANTHOPANAX (Becne. \& Planch.)

Miq.

= ELEUTHEROCOCCUS

ACCA O.Berg

MYRTACEAE

Myrtle Family

sellowiana (O.Berg) Burret

Pineapple Guava

$\begin{array}{llll}\text { FLMG } & \text { LAHG } & \text { NCGP } & \text { SCBY } \\ \text { FLRH } & \text { MSEP } & \text { NCOP } & \text { SCLO } \\ \text { FLUF } & \text { MSRN } & \text { NCWM } & \text { VANB }\end{array}$

sellowiana (O.Berg) Burret

(Leaves variegated)

$\begin{array}{llll}\text { DCCG } & \text { GAJS } & \text { MSEN } & \text { VAWP } \\ \text { DCNA } & \text { MDNA } & \text { VACW } & \\ \text { FLUF } & \text { MDRP } & \text { VASC } & \end{array}$

ACER L.

ACERACEAE

Maple

Maple Family

'Aconitifolium' [A. japonicum Thunb. ex

J.A.Murr.]

DCNA GACG MDJS MDPJ

'Akaji nishiki' [A. palmatum Thunb. ex

J.A.Murr.]

DCNA

'Aka washi no o' [A. palmatum Thunb. ex

J.A.Murr.]

='Ornatum'

'Akikaze nishiki' [A. truncatum Bunge] DCNA

'Albo-limbatum' [A. rufinerve Sieb. \& Zucc.] = 'Hatsuyuki'

'Albo-variegatum' [A. palmatum Thunb. ex J.A.Murr.]

$=$ 'Versicolor'

'Almira' [A. platanoides L.] DCNA

amplum Rehd.

= A. longipes ssp. amplum

'Aokii' [A. palmatum Thunb. ex J.A.Murr.]

$=$ 'Versicolor'

'Aoyagi' [A. palmatum Thunb. ex J.A.Murr.] DCNA

'Argenteo-variegatum' [A. palmatum Thunb. ex J.A.Murr.]

$=$ 'Versicolor' 
'Asahi zuru' [A. palmatum Thunb. ex J.A.Murr.] DCNA

'Atropurpureum' [A. palmatum Thunb. ex J.A.Murr.]

Bloodleaf M.

(Atropurpureum group)

$\begin{array}{llll}\text { ALEH } & \text { GACD } & \text { MDJH } & \text { NCOP } \\ \text { ALTD } & \text { GACG } & \text { MDJS } & \text { NCPL } \\ \text { ARSN } & \text { GATS } & \text { MDLA } & \text { TNMB } \\ \text { DCNA } & \text { GAUG } & \text { MDMM } & \text { TNWF } \\ \text { DEWG } & \text { MDDF } & \text { MDNA } & \text { VAAC } \\ \text { FLMG } & \text { MDGG } & \text { NCBE } & \text { VACW } \\ \text { GAAB } & \text { MDHN } & \text { NCDU } & \text { VAGW }\end{array}$

'Atropurpureum' [ A. pseudoplatanus L.]

Purpleleaf Sycamore M.

DCNA

'Atropurpureum Superbum' [A. palmatum Thunb. ex J.A.Murr.]

(Atropurpureum group) DCNA

'Aureo-variegatum' [A. palmatum Thunb. ex J.A.Murr.] DCNA

'Aureum' [A. cappadocicum Gleditsch] Golden Colosseum M. TNTV

'Aureum' [A. japonicum Thunb. ex J.A.Murr.]

Golden Full-moon M. MDKN SCCC

'Aureum' [A. palmatum Thunb. ex J.A.Murr.] MDDF

'Autumn Glory' [A. palmatum Thunb. ex J.A.Murr.] DCNA

'Bloodgood' [A. palmatum Thunb. ex J.A.Murr.] DCNA GAEC GAFN VATA

'Brilliantissimum' [A. pseudoplatanus L.] DCNA

buergerianum Miq.

Trident M.

$\begin{array}{llll}\text { DCWR } & \text { GAUG } & \text { TXCC } & \text { VATA } \\ \text { GACG } & \text { TNFE } & \text { TXMS } & \\ \text { GAFN } & \text { TNPW } & \text { VAAC } & \\ \text { GAME } & \text { TNUT } & \text { VABF } & \end{array}$

'Burgundy Lace' [A. palmatum Thunb. ex J.A.Murr.]
DCNA
GACG

'Butterfly' [A. palmatum Thunb. ex J.A.Murr.]

MDBG MDJS MDKN TXHL

campbellii Hiern ssp. flabellatum (Rehd.) E.Murr.

DCNA

campbellii Hiern ssp. oliverianum (Pax)

E.Murr.

= A. oliverianum ssp. oliverianum

campestre $\mathrm{L}$.

Hedge $M$.

$\begin{array}{llll}\text { DCCG } & \text { DCWH } & \text { TNDR } & \text { VASC } \\ \text { DCLC } & \text { GAUG } & \text { VAAC } & \\ \text { DCNA } & \text { NCBE } & \text { VACW } & \end{array}$

campestre L. var. leiocarpum (Opiz)

Wallroth

Hedge $\mathrm{M}$.

$\begin{array}{lll}\text { MDCP } & \text { NCCA } & \text { VABF } \\ \text { NCBE } & \text { NCDU } & \end{array}$

capillipes Maxim.

DCNA TNPW

cappadocicum Gleditsch

Colosseum M.

DCCG DCGP DCNA

cappadocicum Gleditsch ssp. truncatum

(Bunge) E.Murr.

$=$ A. truncatum

carpinifolium Sieb. \& Zucc.

Hornbeam M. TNWF

'Chas. F. Irish' [A. platanoides L.] DCNA

'Chirimen nishiki' [A. palmatum Thunb. ex J.A.Murr.] DCNA

'Chitoseyama' [A. palmatum Thunb. ex J.A.Murr.] DCNA

cissifolium (Sieb. \& Zucc.) K.Koch $\mathrm{VABF}$

'Compactum' [A. campestre L.]

= 'Nanum' 
ACER

'Corallinum' [A. palmatum Thunb. ex

J.A.Murr.]

DCNA

'Crimson King' [A. platanoides L.] DCNA MDJH TNSC VATA

'Crimson Queen' [A. palmatum Thunb. ex J.A.Murr.]

(Dissectum group) DCNA

davidii Franch.

David's M.

DCNA MDBG TNRT

GATS TNFE

diabolicum Bl. ex K.Koch

Devil M.

DCNA VABF

'Discolor Versicolor' [A. palmatum Thunb. ex J.A.Murr.]

='Versicolor'

dissectum Thunb. ex J.A.Murr.

= A. palmatum 'Dissectum'

'Dissectum' [A. palmatum Thunb. ex J.A.Murr.]

Laceleaf $M$.

(Dissectum group; green-leaved) DCCG GACG TNDR DCNA GAEC

'Dissectum Flavescens' [A. palmatum

Thunb. ex J.A.Murr.] DCNA

'Dissectum Palmatifidum' [A. palmatum

Thunb. ex J.A.Murr.] DCNA SCCC

'Dissectum Paucum' [A. palmatum Thunb. ex J.A.Murr.]

= 'Dissectum Palmatifidum'

'Dissectum Rubrifolium' [A. palmatum

Thunb. ex J.A.Murr.] DCNA DCWH MDJS NCBE

'Dissectum Variegatum' [A. palmatum

Thunb. ex J.A.Murr.] MDKN

'Drummondii' [A. platanoides L.] DCNA

'Faassen's Black' [A. platanoides L.] DCCG DCNA
'Filicifolium' [A. japonicum Thunb. ex J.A.Murr.]

= 'Aconitifolium'

'Filigree' [A. palmatum Thunb. ex J.A.Murr.] $\mathrm{SCCC}$

flabellatum Rehd.

= A. campbellii ssp. flabellatum

'Flavescens' [A. palmatum Thunb. ex J.A.Murr.]

= 'Dissectum Flavescens'

$\times$ freemanii E.Murr.

[A. rubrum $\times$ A. saccharinum] DCNA

'Garnet' [A. palmatum Thunb. ex J.A.Murr.]

(Dissectum group) GAEC

'Gerling' [A. rubrum L.] DCNA

ginnala Maxim.

Amur M.

$\begin{array}{lll}\text { DCNA } & \text { SCCU } & \text { TNSC } \\ \text { GAUG } & \text { TNCT } & \text { VACW } \\ \text { MDJH } & \text { TNDR } & \text { VATA }\end{array}$

'Globosum' [A. campestre L.] = 'Nanum'

griseum (Franch.) Pax

Paperbark M.

$\begin{array}{llll}\text { DCNA } & \text { GAWH } & \text { MDLT } & \text { VAGS } \\ \text { DEWG } & \text { MDBG } & \text { TNHT } & \text { VATA } \\ \text { GALA } & \text { MDGD } & \text { TNSN } & \end{array}$

'Hagaromo' [A. palmatum Thunb. ex J.A.Murr.] DCNA MDKN MDMG SCCC

'Hatsuyuki' [A. rufinerve Sieb. \& Zucc.] DCNA MDBG

'Heptalobum' [A. palmatum Thunb. ex J.A.Murr.]

= A. palmatum var. amoenum

'Hessei' [A. palmatum Thunb. ex J.A.Murr.]

DCNA MDJS MDKN

'Higasayama' [A. palmatum Thunb. ex J.A.Murr.]

DCNA

GACG MDJS MDKN 
'Hogyoku' [A. palmatum Thunb. ex

J.A.Murr.]

GACG

'Ichigyo ji' [A. palmatum Thunb. ex J.A.Murr.]

DCNA

'Iijima sunago' [A. palmatum Thunb. ex J.A.Murr.] DCNA

'Inaba shidare' [A. palmatum Thunb. ex J.A.Murr.]

(Dissectum group) DCNA

'Itaya' [A. japonicum Thunb. ex J.A.Murr.] DCNA MDKN

'Itaya meigetsu' [A. japonicum Thunb. ex J.A.Murr.I

= A. japonicum

japonicum Thunb. ex J.A.Murr.

Japanese M., Full-moon M.

DCNA MDJS

'Jiro shidare' [A. palmatum Thunb. ex J.A.Murr.]

DCNA

'Kagiri nishiki' [A. palmatum Thunb. ex J.A.Murr.]

DCNA

'Killarney' [A. palmatum Thunb. ex

J.A.Murr.]

DCNA

'Kingsville Red' [A. palmatum Thunb. ex J.A.Murr.]

DCNA

'Kinugasayama' [A. japonicum Thunb. ex J.A.Murr.]

(Sometimes placed in A. sieboldianum Miq.) DCNA

'Kocho nishiki' [A. palmatum Thunb. ex J.A.Murr.]

= 'Butterfly'

'Kohauchina kaido' [A. japonicum Miq.] DCNA

'Koshimino' [A. palmatum Thunb. ex J.A.Murr.]

MDMG

'Kurabeyama' [A. palmatum Thunb. ex J.A.Murr.]

DCNA
'Laceleaf [A. palmatum Thunb. ex

J.A.Murr.]

$=$ 'Filigree'

'Linearilobum' [A. palmatum Thunb. ex J.A.Murr.] DCNA MDBG

longipes Franch. ssp. amplum (Rehd.) Jong DCNA

'Lutescens' [A. palmatum Thunb. ex J.A.Murr.] MDGD MDJS

'Maiko' [A. palmatum Thunb. ex J.A.Murr.] DCNA

'Mai kujaku' [A. japonicum Thunb. ex J.A.Murr.]

= 'Aconitifolium'

mandshuricum Maxim.

Manchurian M.

MDKN

'Masukagami' [A. palmatum Thunb. ex J.A.Murr.] DCNA

'Matsukaze' [A. palmatum Thunb. ex J.A.Murr.] DCNA

maximowiczianum Miq.

Nikko M. DCNA MDDF VAHM

'Mikasayama' [A. sieboldianum Miq.] DCNA

'Mino yatsufusa' [A. buergerianum Miq.] TNSN

'Mioun' [A. palmatum Thunb. ex J.A.Murr.] (Dissectum group) DCNA

miyabei Maxim.

Miyabe M. MDGD

'Miyasama' [A. buergerianum Miq. ssp. formosanum (Hayata) E.Murr. \& Lauener]

DCNA MDKN TNSN

'Mizu kuguri' [A. palmatum Thunb. ex J.A.Murr.]

DCNA 
mono Maxim.

= A. truncatum

monspessulanum $\mathrm{L}$.

Montpellier M.

$\mathrm{VABF}$

'Monumentale' [A. saccharum Marsh.]

= 'Temple's Upright'

'Monzukushi' [A. palmatum Thunb. ex

J.A.Murr.]

DCNA

'Moonfire' [A. palmatum Thunb. ex

J.A.Murr.]

DCNA

'Mure hibari' [A. palmatum Thunb. ex J.A.Murr.]

DCNA

'Musashino' [A. palmatum Thunb. ex

J.A.Murr.]

= 'Nomura'

'Nanum' [A. campestre L.]

Dwarf Hedge $M$.

$\begin{array}{lll}\text { DCNA } & \text { TNDR } & \text { TNTV } \\ \text { GACG } & \text { TNSN } & \text { VAGS }\end{array}$

'Naruo nishiki' [A. palmatum Thunb. ex J.A.Murr.]

DCNA

'Nauto kaede' [A. buergerianum Miq.] TNSN

negundo $\mathrm{L}$.

Boxelder

ALAU DCNA DEEM

negundo L. ssp. latifolium (Pax) Schwerin

= A. negundo ssp. negundo var. texanum

negundo L. ssp. mexicanum (DC.) Wesmael MDGD

negundo L. ssp. negundo var. texanum Pax

FLUF MSEN TXHA

MDSJ NCCA

'Newton Sentry' [A. saccharum Marsh.] MDJS

nigrum Michx.

= A. saccharum ssp. nigrum

nikoense Maxim.

= A. maximowiczianum
'Nomura' [A. palmatum Thunb. ex

J.A.Murr.]

DCNA

'Nomura nishiki' [A. palmatum Thunb. ex J.A.Murr.] DCNA

oblongum Wall. ex DC.

Evergreen M.

FLUF

'October Glory' [A. rubrum L.] DCNA

'Oekonomierat Stoll' [A. platanoides L.] DCNA

'Ogino nagare' [A. palmatum Thunb. ex J.A.Murr.] DCNA

'Ogon sarasa' [A. palmatum Thunb. ex J.A.Murr.] DCNA

'O isami' [A. japonicum Thunb. ex J.A.Murr.] DCNA

'O kagami' [A. palmatum Thunb. ex J.A.Murr.] DCNA

'Okushimo' [A. palmatum Thunb. ex J.A.Murr.] DCNA MDBG MDKN GACG MDJS

oliverianum Pax DCNA FLUF GAIS

'Omato' [A. palmatum Thunb. ex J.A.Murr.] DCNA

'Oo shi rini' [A. palmatum Thunb. ex J.A.Murr.] DCNA

'Oregon Sunset' [A. palmatum Thunb. ex J.A.Murr.] DCNA

'Orido nishiki' [A. palmatum Thunb. ex J.A.Murr.] DCNA

'Ornatum' [A. palmatum Thunb. ex J.A.Murr.]

DCCG DCNA
DCWH GACG

DEWG 
'Osakazuki' [A. palmatum Thunb. ex J.A.Murr.] DCNA MDKN

'Oshio beni' [A. palmatum Thunb. ex J.A.Murr.] DCNA GACG

'Oshu shidare' [A. palmatum Thunb. ex J.A.Murr.] DCNA

'O take' [A. japonicum Thunb. ex J.A.Murr.] DCNA

\section{'Palmatum' [A. saccharinum L.]} FLUF

palmatum Thunb. ex J.A.Murr.

var. amoenum (Carr.) Ohwi

$\begin{array}{llll}\text { ALBH } & \text { GAUG } & \text { SCCC } & \text { VACW } \\ \text { DCNA } & \text { LAHG } & \text { SCHS } & \\ \text { GATS } & \text { MDJS } & \text { TNDR } & \end{array}$

palmatum Thunb. ex J.A.Murr.

var. heptalobum Rehd.

= A. palmatum var. amoenum

palmatum Thunb. ex J.A.Murr.

var. matsumurae (Koidz.) Makino DCNA

palmatum Thunb. ex J.A.Murr.

var. palmatum

Japanese M.

$\begin{array}{llll}\text { ARWS } & \text { GAEC } & \text { MDCP } & \text { SCCC } \\ \text { DCCG } & \text { GAOH } & \text { MDHN } & \text { SCNR } \\ \text { DCNA } & \text { GAUG } & \text { MDJS } & \text { VACW } \\ \text { FLMG } & \text { GAWP } & \text { MSEP } & \text { VATA } \\ \text { GACG } & \text { MDBG } & \text { NCBE } & \end{array}$

pensylvanicum L.

Striped Maple

VAGW

pictum Thunb. ex J.A.Murr.

= Kalopanax septemlobus

'Pixie' [A. palmatum Thunb. ex J.A.Murr.]

(Name of doubtful status)

DCNA

platanoides L.

Norway Maple

$\begin{array}{llll}\text { ALAU } & \text { DCWR } & \text { MDNA } & \text { VACW } \\ \text { DCCG } & \text { DEMC } & \text { MDPJ } & \text { VATA } \\ \text { DCNA } & \text { MDHN } & \text { MDSJ } & \text { VAWR } \\ \text { DCWH } & \text { MDJS } & \text { SCKG } & \end{array}$

pseudoplatanus L.

Sycamore Maple
DCCG
DESR
DCNA
MDLA
TNFE
VACS
VARO

pseudosieboldianum (Pax) Komar.

Purplebloom Maple
DCNA
MDJS

$\underset{\text { DCSE }}{\text { 'Pyramidale' [A. rubrum L.] }}$

'Reticulatum' [A. palmatum Thunb. ex J.A.Murr.]

= 'Shigitatsu sawa'

'Ribbon Leaf' [A. palmatum Thunb. ex J.A.Murr.]

(Atropurpureum group)

DCNA

'Roseo-maculatum' [A. palmatum Thunb. ex J.A.Murr.]

$=$ 'Versicolor'

'Roseo-marginatum' [A. palmatum Thunb. ex J.A.Murr.]

$=$ 'Kagiri nishiki'

'Rubrifolium' [A. palmatum Thunb. ex J.A.Murr.]

= 'Dissectum Rubrifolium'

rubrum L.

Red Maple

$\begin{array}{llll}\text { DCCG } & \text { DCWH } & \text { MDLA } & \text { VAGG } \\ \text { DCNA } & \text { DEWG } & \text { VACW } & \end{array}$

rubrum L. var. drummondii (Nutt.) Sarg. Drummond Maple

$$
\text { FLUF SCMP TXCT }
$$

rubrum L. var. tridens A.Wood

= A. rubrum var. trilobum

rubrum L. var. trilobum T. \& G. ex K.Koch

$\begin{array}{lll}\text { ALAU } & \text { MDHN } & \text { VACW } \\ \text { ALOS } & \text { SCUC } & \text { VAGW } \\ \text { FLMG } & \text { VAAC } & \text { VAWR }\end{array}$

rufinerve Sieb. \& Zucc.

Redvein Maple

$$
\text { DCNA GAEC }
$$

saccharinum L.

Silver Maple

$\begin{array}{llll}\text { ALAU } & \text { DCNA } & \text { GAFN } & \text { TNWF } \\ \text { ARRB } & \text { DEMC } & \text { GAVI } & \text { VACM } \\ \text { DCCG } & \text { FLUF } & \text { SCEM } & \text { VACW }\end{array}$


saccharum Marsh. ssp. floridanum

(Chapm.) Desm.

Florida Maple
ALEH
GAAB TXLL
FLUF
NCSM TXMS

saccharum Marsh. ssp. leucoderme (Small) Desm.

Chalk Maple
ALEH
GAEC
GAWD

saccharum var. monumentale (Temple)

Rehd.

= 'Temple's Upright'

saccharum Marsh. ssp. nigrum (Michx.f.) Desm.

Black Maple
DCNA
TNWF VAMP

saccharum Marsh. var. rugelii (Pax) Rehd. DCCG TNSC VAWD

TNHG VACW VAWR

saccharum Marsh. ssp. saccharum

Sugar Maple

$\begin{array}{llll}\text { ARRB } & \text { MDHN } & \text { TNWF } & \text { VAHC } \\ \text { DCCG } & \text { MDMM } & \text { VABF } & \text { VAWR } \\ \text { DCNA } & \text { MDNA } & \text { VAGG } & \end{array}$

'Sagara nishiki' [A. palmatum Thunb. ex J.A.Murr.] DCNA

'Sango kaku' [A. palmatum Thunb. ex J.A.Murr.]

$\begin{array}{lll}\text { DCEL } & \text { FLMG } & \text { MDKN } \\ \text { DCNA } & \text { MDBG } & \text { SCCC }\end{array}$

'Sanguineum' [A. palmatum Thunb. ex J.A.Murr.]

= A. palmatum var. palmatum

'Sazanami' [A. palmatum Thunb. ex J.A.Murr.]

DCNA

'Schlesingeri' [A. rubrum L.] DCNA

'Schwedleri' [A. platanoides L.]

Schwedler M.

$\begin{array}{llll}\text { DCEL } & \text { GAOH } & \text { MDNA } & \text { VACW } \\ \text { GALA } & \text { MDCP } & \text { TNFE } & \text { VAWR }\end{array}$

'Scolopendrifolium' [A. palmatum Thunb. ex J.A.Murr.]

= 'Linearilobum'

'Seigen' [A. palmatum Thunb. ex J.A.Murr.] DCNA
'Seiryu' [A. palmatum Thunb. ex J.A.Murr.] (Dissectum group) DCNA

'Sessilifolium’ [A. palmatum Thunb. ex J.A.Murr.]

= 'Hagoromo'

'Sherwood Flame' [A. palmatum Thunb. ex J.A.Murr.] DCNA

'Shigitatsu sawa' [A. palmatum Thunb. ex J.A.Murr.] DCNA MDJS MDKN

'Shigurezome' [A. palmatum Thunb. ex J.A.Murr.] DCNA

'Shino buga oka' [A. palmatum Thunb. ex J.A.Murr.] MDJS

shirasawanum Koidz. MDJS TNSN

'Shirigosan' [A. palmatum Thunb. ex J.A.Murr.]

(Linearilobum group) DCNA

'Shishigashira' [A. palmatum Thunb. ex J.A.Murr.] DCNA MDJS MDKN SCCC

'Shojo' [A. palmatum Thunb. ex J.A.Murr.] DCNA

sieboldianum Miq.

Siebold Maple DCNA DEWG

spicatum Lam.

Mountain Maple GAEC

'Stollii' [A. platanoides L.]

= 'Oekonomierat Stoll'

'Sweet Shadow Cut-Leaf' [A. saccharum Marsh.] DCCG

'Tamukeyama' [A. palmatum Thunb. ex J.A.Murr.]

(Dissectum group) DCNA

tataricum L.

Tatarian Maple DCNA GACG VATA 
'Tatsuta' [A. palmatum Thunb. ex

J.A.Murr.]

DCNA

tegmentosum Maxim.

Manchurian Striped M.

DCNA DCPP MDLT TNTV

'Temple's Upright' [A. saccharum Marsh.] DCNA DEWG MDJS MDPJ

'The Bishop' [A. palmatum Thunb. ex

J.A.Murr.]

DCNA MDKN

'Tilford' [A. rubrum L.]

DCNA

'Tokiwa nishiki' [A. truncatum Bunge] DCNA

'Toyama nishiki' [A. palmatum Thunb. ex J.A.Murr.]

(Dissectum group) DCNA

trifidum Hook. \& Arn.

= A. buergerianum

'Trompenburg' [A. palmatum Thunb. ex J.A.Murr.]

DCNA

truncatum Bunge

Shantung Maple

$\begin{array}{llll}\text { DCNA } & \text { MDKN } & \text { TNUT } & \text { VABP } \\ \text { GAUG } & \text { TNDR } & \text { VABF } & \end{array}$

truncatum Bunge f. dissectum Wesmael DCNA

'Tsuchi uno' [A. palmatum Thunb. ex J.A.Murr.] DCNA

'Tsuku bane' [A. palmatum Thunb. ex J.A.Murr.] DCNA

'Tsukushi gata' [A. palmatum Thunb. ex J.A.Murr.]

DCNA

'Tsuri nishiki' [A. palmatum Thunb. ex J.A.Murr.] DCNA

'Ukon' [A. palmatum Thunb. ex J.A.Murr.] = 'Aoyagi'

'Usugumo' [A. truncatum Bunge] DCNA
'Utsu semi' [A. palmatum Thunb. ex

J.A.Murr.]

DCNA

'Variegatum' [A. negundo L.]

ALTD LAGN MDKN VANP

'Variegatum' [A. palmatum Thunb. ex

J.A.Murr.]

= 'Dissectum Variegatum'

'Variegatum' [A. rufinerve Sieb. \& Zucc.] MDBG

velutinum Boiss. var. vanvolxemii (Mast.)

Rehd.

Velvet M.

DCNA

'Versicolor' [A. palmatum Thunb. ex J.A.Murr.]

GACG MDKN

'Vitifolium' [A. japonicum Thunb. ex J.A.Murr.] DCNA

'Waka momiji' [A. palmatum Thunb. ex J.A.Murr.] $\mathrm{MDOF}$

'Washi no o' [A. palmatum Thunb. ex J.A.Murr]

= 'Dissectum Palmatifidum'

'Waterfall' [A. palmatum Thunb. ex J.A.Murr.] $\mathrm{SCCC}$

wilsonii Rehd. DCNA

'Yatsubusa' [A. palmatum Thunb. ex J.A.Murr.]

DCNA GACG MDPJ

'Yayoigasa' [A. japonicum Thunb. ex J.A.Murr.]

DCNA

'Yezo nishiki' [A. palmatum Thunb. ex J.A.Murr.] DCNA GACG

'Yukigumi' [A. palmatum Thunb. ex J.A.Murr.]

DCNA

$\times$ zoeschense Pax

[A. campestre $\times$ A. lobellii] DCNA 
ACOELORRHAPHE H.Wendl. ARECACEAE

Palm Family

wrightii (Griseb. \& H.Wendl.) H.Wendl. ex Becc.

FLUF

ACTINIDIA Lindl.

ACTINIDIACEAE

Actinidia Family

arguta (Sieb. \& Zucc.) Planch. ex Miq.

Tara Vine

DCDO DCNA MDGD VATA

'Chico' [A. deliciosa (A.Chev.) C.F.Liang \&

A.R.Ferguson var. deliciosa]

VATA

chinensis Planch.

= A. deliciosa var. deliciosa

chinensis Planch. var. deliciosa (A.Chev.)

A.Chev.

= A. deliciosa var. deliciosa

deliciosa (A.Chev.) C.F.Liang \&

A.R.Ferguson var. deliciosa

Kiwi Fruit, Chinese Gooseberry

GAAB NCDU

$\times$ fairchildii Rehd.

[A. arguta $\times$ A. chinensis]
MDGD VATA

kolomikta (Maxim. \& Rupr.) Maxim. VAGS

polygama (Sieb. \& Zucc.) Maxim.

Silver Vine DCNA

purpurea Rehd. VATA

\section{ADINA Salisb.}

\section{RUBIACEAE}

Madder Family

rubella Hance

Chinese Buttonbush
ALAU
$\mathrm{SCCU}$
VAHM
LASL
VACW

AESCULUS L. Horsechestnut, Buckeye HIPPOCASTANACEAE

Buckeye Family

'Baumanii' [A. hippocastanum L.]

Double-flowered $\mathrm{H}$.
DCNA
MDBG
NCBE
DCSE MDCP NCDU

'Briotii' [A. $\times$ carnea Hayne]

[A. hippocastanum $\times$ A. pavia] DCNA DEWG MDPJ DCWR MDKN VACW

$\times$ bushii Schneid.

[A. glabra $\times$ A. pavia] MDBP

californica (Spach) Nutt.

California B.

$$
\text { DCNA SCWI }
$$

$\times$ carnea Hayne

Red H.

$\begin{array}{cc}\text { [A. hippocastanum } \times \text { A. pavia] } \\ \text { DCPL } & \text { MDBG } \\ \text { DCSE } & \text { MDCP }\end{array}$

$\times$ dupontii Sarg.

[A. flava $\times($ A. pavia $\times$ A. sylvatica)] DEEM DEWG

flava Sol.

Yellow B.

$\begin{array}{llll}\text { DCCG } & \text { MDHN } & \text { NCTE } & \text { VAKH } \\ \text { DCNA } & \text { MDJH } & \text { TNFE } & \text { VAMP } \\ \text { DEEM } & \text { MDNA } & \text { TNSC } & \text { VAWP } \\ \text { MDBP } & \text { NCBE } & \text { VACP } & \\ \text { MDCP } & \text { NCKH } & \text { VACW } & \end{array}$

flava Ait.

= A. flava Sol.

glabra Willd.

Ohio B.

$\begin{array}{llll}\text { ARHS } & \text { DCWR } & \text { MDJH } & \text { TNFE } \\ \text { DCNA } & \text { DEWG } & \text { MDLS } & \text { VAMP } \\ \text { DCWH } & \text { MDHN } & \text { SCWI } & \end{array}$

$\times$ glaucescens Sarg.

[A. flava $\times$ A. sylvatica]

DEWG

hippocastanum L.

H.

$\begin{array}{llll}\text { DCCG } & \text { DEWG } & \text { MDMM } & \text { VAPS } \\ \text { DCNA } & \text { MDBG } & \text { VACW } & \\ \text { DCSE } & \text { MDCP } & \text { VAGW } & \\ \text { DCWH } & \text { MDLA } & \text { VAMP } & \end{array}$




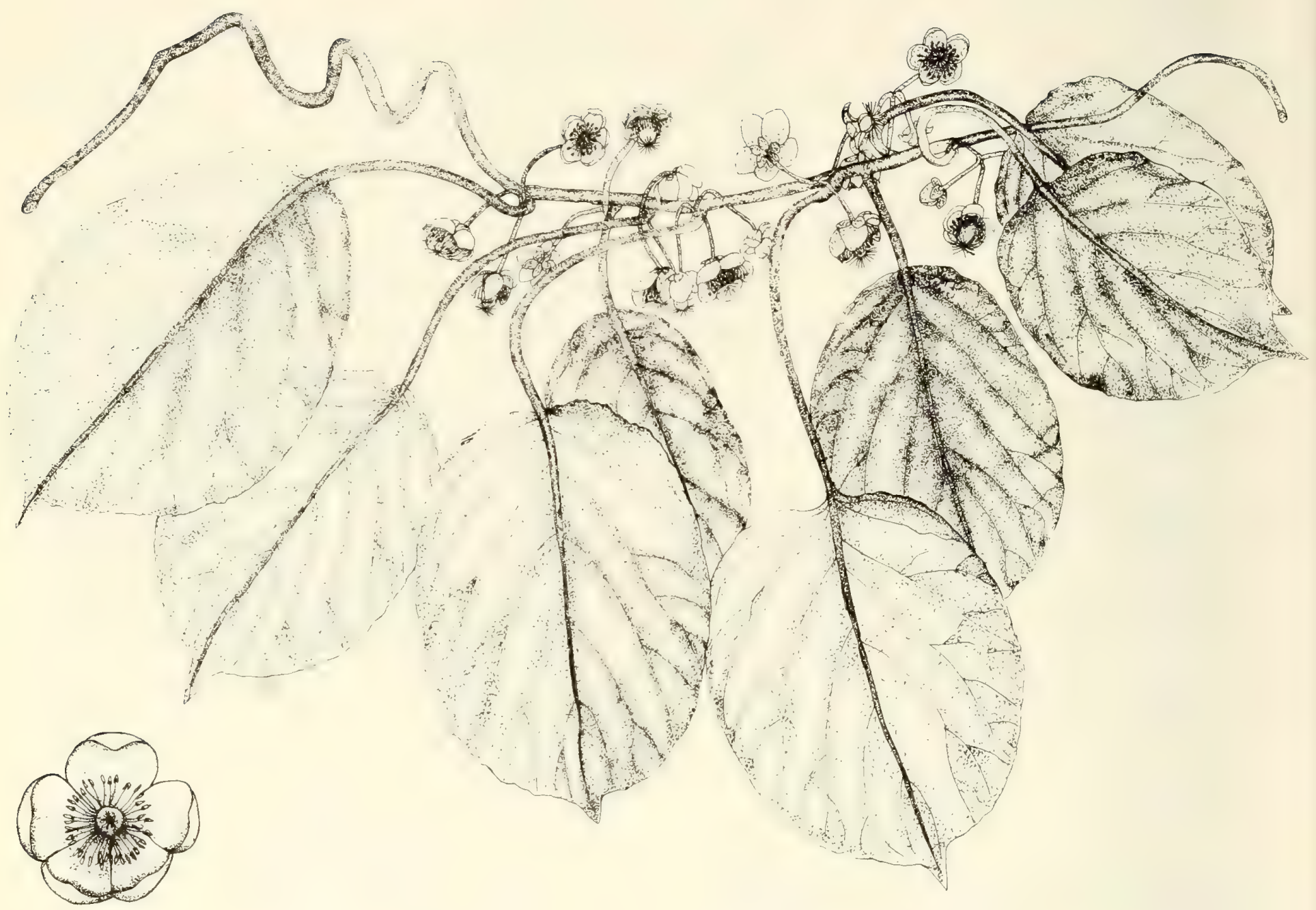

ACTINIDLA arguta (Sieb. \& Zucc.) Planch. ex Miq.

[illustrator Lillian Nicholson Meyer] 
$\times$ hybrida DC.

[A. flava $\times$ A. pavia]

$\begin{array}{lll}\text { DCNA } & \text { DEWG } & \text { VAGW } \\ \text { DCWH } & \text { VAAL } & \text { VAWP }\end{array}$

$\times$ marilandica Booth ex Kirchn.

[A. glabra $\times$ A. flava]
DCSE
DEEM

maxima Drake

= A. flava Sol.

\section{'Memmingeri' [A. hippocastanum L.]} DCNA

$\times$ mutabilis (Spach) Scheele

[A. sylvatica $\times$ A. pavia]
GACG GAEC

octandra Marsh.

= A. flava Sol.

parviflora Walt.

Bottlebrush Buckeye

$\begin{array}{llll}\text { DCNA } & \text { LARP } & \text { MDKN } & \text { TNNE } \\ \text { DCWR } & \text { MDAM } & \text { MDLT } & \text { TNUT } \\ \text { GACG } & \text { MDHN } & \text { MDRT } & \end{array}$

parviflora Walt. var. serotina Rehd. DCNA

pavia $\mathrm{L}$.

Red Buckeye

$\begin{array}{llll}\text { ALBG } & \text { LAAL } & \text { MDKN } & \text { TNHT } \\ \text { DEWG } & \text { LALG } & \text { NCTP } & \text { TNMB } \\ \text { FLUF } & \text { MDBG } & \text { SCBR } & \text { TXMS } \\ \text { GACG } & \text { MDIH } & \text { SCKG } & \text { VACW } \\ \text { GASM } & \text { MDJH } & \text { SCMG } & \text { VAWR }\end{array}$

pavia L. var. flavescens (Sarg.) Correll TXLL

$\times$ plantierensis Andre

[A. hippocastanum $\times$ A. $\times$ carnea] DCNA DEWG

sylvatica Bartram
DCNA
GAEC
NCCA SCWI

$\times$ woerlitzensis Koehne
[A. flava $\times$
DEWG

AGARISTA D.Don ex G.Don

ERICACEAE

Heath Family

populifolia (Lam.) D.Don ex Judd

Pipe Plant, Pipe-stem Wood
ALAU
MDLT
SCBR
SCWI
LAAL
MDPJ
SCCU
VAGS
LALG
NCBE
SCMP
VAPH

AGAVE L.

Century Plant

AGAVACEAE

Agave Family

americana L.

FLUF

'Marginata' [A. americana L.]

Variegated C. P.

FLUF

\section{AILANTHUS Desf. SIMAROUBACEAE}

Quassia Family

altissima (Mill.) Swingle

Tree-of-heaven

$$
\begin{array}{lll}
\text { DEEM } & \text { MDHN } & \text { MDSJ } \\
\text { MDCP } & \text { MDLT } & \text { VABP }
\end{array}
$$

glandulosa Desf.

= A. altissima

AKEBIA Decne. Akebia LARDIZABALACEAE Lardizabala Family

quinata (Thunb. ex Houtt.) Decne.

Chocolate Vine

$$
\begin{array}{lll}
\text { DCNA } & \text { MDFM } & \text { VASS } \\
\text { MDBG } & \text { NCDU } &
\end{array}
$$

'Shirobana' [A. quinata (Thunb. ex Houtt.) Decne.] MDBG

ALBIZLA Durazz. FABACEAE (Mimosoideae) Bean Family

julibrissin Durazz.

Silk Tree, Mimosa Tree
DCNA MSHB
FLUF NCDU
MSBN NCOP TNTV 


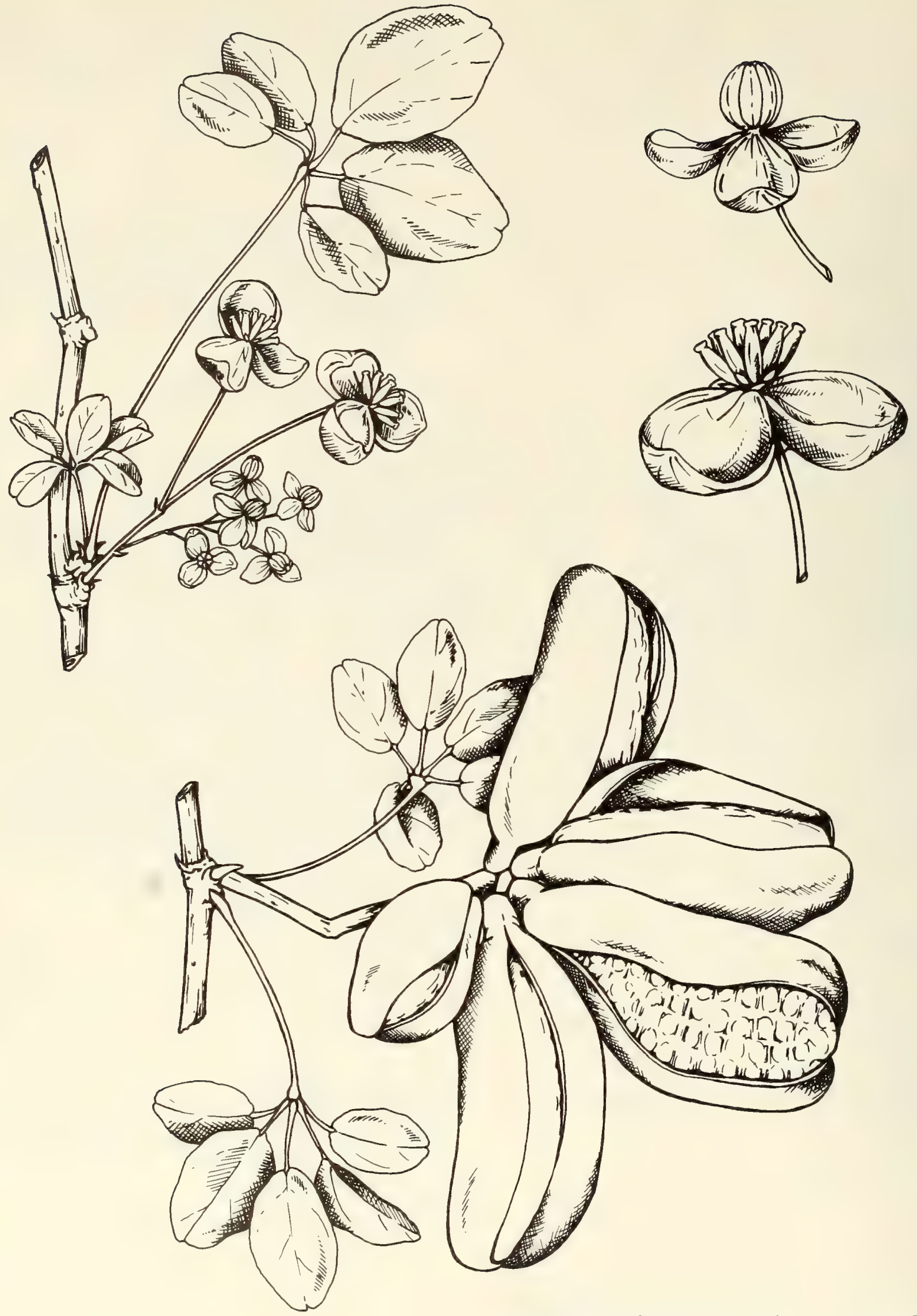

AKEBIA quinata, (Thunb. ex Houtt.) Decne. [illustrator Susan M. Johnston] 
kalkora (Roxb.) Prain

$\begin{array}{llll}\text { DCNA } & \text { NCBE } & \text { NCTR } & \text { VATA } \\ \text { GAIS } & \text { NCDU } & \text { VABF } & \end{array}$

ALEURITES J.R. \& J.G. Forst. EUPHORBLACEAE

Spurge Family

fordii Hemsl.

Tung-oil Tree

$\begin{array}{lll}\text { ALBG } & \text { FLUF } & \text { MSMN } \\ \text { FLLE } & \text { GATS } & \text { NCOP } \\ \text { FLMG } & \text { LAAL } & \text { SCFW }\end{array}$

montana (Lour.) Wils.

Mu-oil Tree

FLUF

\section{ALLAMANDA L. APOCYNACEAE \\ Dogbane Family}

cathartica L.

ALBG

\section{ALNUS Mill.}

Alder

BETULACEAE

Birch Family

formosana (Burkw.) Makino MDJC TXLL

glutinosa (L.) Gaertn.

European Alder, Black Alder
DCCG
DCNA
VATA

hirsuta (Spach) Rupr. var. sibirica

(Spach) Schneid.

DCNA

japonica (Thunb.) Steud.

MDGD

maritima (Marsh.) Nutt.

Seaside Alder

VACW

serrulata (Ait.) Willd.

Smooth Alder
ALAU
$\mathrm{NCDU}$
LAHG
SCPL

ALOYSLA JusS. VERBENACEAE

Verbena Family

gratissima (Gillies \& Hook.) Troncoso

Lemon Verbena

LASL
AMELANCHIER Medîk.

Shadbush ROSACEAE

Rose Family

alnifolia (Nutt.) Nutt.

MDGD

arborea (Michx.f.) Fern.

Common S., Downy Serviceberry

$\begin{array}{llll}\text { ALEH } & \text { DCSG } & \text { MDBG } & \text { SCUC } \\ \text { DCEL } & \text { DEMC } & \text { MDLT } & \text { VACW } \\ \text { DCNA } & \text { GACG } & \text { SCBR } & \text { VAGW }\end{array}$

asiatica (Sieb. \& Zucc.) Endl. DCNA MDKN

canadensis (L.) Medik. DCNA SCBR VAGW MDWJ VACW

laevis Wieg.

Allegheny S., Smooth S. DCNA GAEC MDLM VASK

oblongifolia T. \& G.

$=A$. canadensis

'Rosea' [A. laevis Wieg.] DCNA

sanguinea (Pursh) DC. VAGS

spicata (Lam.) K.Koch DCNA GAUG TNUT

stolonifera Wieg.

= A. spicata

'Success'

MDOF

AMORPHA L.

FABACEAE (Faboideae) Bean Family

fruticosa L.

Indigo Bush

$\begin{array}{lll}\text { DCNA } & \text { TNDR } & \text { VAGW } \\ \text { MDNA } & \text { VABF } & \end{array}$

glabra Desf. ex Poir.

Mountain Indigo

ALTR

AMPELOPSIS Michx.

VITACEAE

Grape Family

arborea (L.) Koehne

DCNA TXLL 
brevipedunculata (Maxim.) Traut.

= A. glandulosa var. brevipedunculata

glandulosa (Wall.) Momiy.

var. brevipedunculata (Maxim.) Momiy.

Porcelain-berry
GAAB
MDBG VAWP

'Elegans' [A. glandulosa

var. brevipedunculata (Maxim.) Momiy.] DCNA

AMYRIS P.Br.
RUTACEAE

texana (Buckl.) P.Wilson

Texas Torchwood

TXLL

\section{ANDROMEDA L. \\ ERICACEAE}

Heath Family

'Nana' [A. polifolia L.]

MDLT

polifolia L.

NCFR

\section{ANISACANTHUS Nees \\ ACANTHACEAE \\ Acanthus Family}

thurberi (Torr.) A.Gr.

TXCT

\section{ANISOSTICHUS Bur. \\ = BIGNONIA}

\section{ANTIGONON Endl.}

POLYGONACEAE

Buckwheat Family

leptopus Hook. \& Arn.

Mexican Creeper

ALBG FLMG

\section{APHANANTHE Planch. \\ ULMACEAE \\ Elm Family}

aspera (Thunb. ex J.A.Murr.) Planch.

GAME GANG GATC SCHS

ARALIA L.

ARALIACEAE

Ginseng Family

elata (Miq.) Seemann

DCNA DEMC

spinosa $\mathrm{L}$.

Devil's Walking Stick

ALAU MDLT SCBR VAWP

ARAUCARIA JusS.

ARAUCARIACEAE Araucaria Family

araucana (Mol.) K.Koch

Monkey Puzzle

MDJR

bidwillii Hook.

Bunya-Bunya Tree
FLUF
LAHS

\section{ARBUTUS L. ERICACEAE}

Heath Family

unedo $\mathrm{L}$.

Strawberry Tree

$\begin{array}{lll}\text { FLUF } & \text { NCEL } & \text { SCWI } \\ \text { LASL } & \text { SCMP } & \text { VANB }\end{array}$

ARCTOSTAPHYLOS Adans. ERICACEAE

Heath Family

uva-ursi (L.) Spreng.

Bearberry, Kinnikinnick

DCNA MDLT

ARDISIA O.Swartz

MYRSINACEAE

Myrsine Family

'Alba' [A. crenata Sims] FLUF

crenata Sims

Coralberry

$\begin{array}{llll}\text { ALBG } & \text { FLUF } & \text { GADG } & \text { TXDC } \\ \text { FLMG } & \text { GACH } & \text { GATS } & \end{array}$

crispa (Thunb. ex J.A.Murr.) A.DC. Coral A.

ALBG FLDG GACG LAAL 
japonica (Thunb.) Bl.

Japanese A.

$\begin{array}{lll}\text { ALFS } & \text { LAAL } & \text { MSMN } \\ \text { FLDG } & \text { LAHP } & \text { SCLM } \\ \text { GACG } & \text { LAPA } & \text { SCMG }\end{array}$

\section{ARECASTRUM (Drude) Becc.}

romanzoffianum (Cham.) Becc.

= Syagrus romanzoffianum

ARISTOLOCHIA L.

ARISTOLOCHIACEAE Aristolochia Family

elegans Mast.

Calico Dutchman's Pipe TXPO

ARONIA Medik.
ROSACEAE

arbutifolia (L.) Ell.

Red C.

$\begin{array}{llll}\text { DCNA } & \text { MDMM } & \text { VADW } & \text { VAPO } \\ \text { GAEC } & \text { TXSE } & \text { VAFB } & \\ \text { MDLT } & \text { VABP } & \text { VAGW } & \end{array}$

melanocarpa (Michx.) Ell.

Black C.

$$
\begin{array}{lll}
\text { DCNA } & \text { VACW } & \text { VAPO } \\
\text { MDGD } & \text { VAGS } &
\end{array}
$$

melanocarpa (Michx.) Ell. var. elata Rehd.

\section{= A. melanocarpa}

prunifolia (Marsh.) Rehd.

Purplefruit C.

MDGJ

\section{ARTEMISIA L. ASTERACEAE}

Aster Family

\section{abrotanum L.}

Southernwood

DCNA

ARUINDINARIA Michx.

POACEAE

Grass Family

'Variegata' [A. pygmaea (Miq.) Mitf.] SCBR

\section{ASCYRUM L. $=$ \\ HYPERICACEAE St. John's-wort Family}

hypericoides $\mathrm{L}$.

St. Andrew's Cross

NCEG SCWI

stans Michx.

= Hypericum stans

ASIMINA Adans.

ANNONACEAE Custard-apple Family

triloba (L.) Dunal

Pawpaw

$\begin{array}{llll}\text { DEWG } & \text { NCCA } & \text { VACM } & \text { VAGW } \\ \text { MDLT } & \text { TXLL } & \text { VACW } & \end{array}$

ASTER L.

ASTERACEAE Aster Family

carolinianus Walt.

Carolina Aster

SCWI

AUCUBA Thunb.

CORNACEAE

Dogwood Family

chinensis Benth.

DCNA

'Goldieana' [A. japonica Thunb.] VAPH

japonica Thunb.

Japanese Aucuba
ALEH
GACG
MDLT
SCNS
ARWS
GAJS
MDPJ
VACW
DCCG
LAAL
SCMG
VAWR
FLMG MDHN SCMP

japonica Thunb. var. borealis Miyabe \& Kudo DCNA

'Limbata' [A. japonica Thunb.] DCNA

'Longifolia' [A. japonica Thunb.] DCNA VAPH

'Meigetsu' [A. japonica Thunb.] MDMG

'Nana' [A. japonica Thunb.] MDTD SCMG 
'Salicifolia' [A. japonica Thunb.] DCNA

'Variegata' [A. japonica Thunb.] Gold-dust Shrub

$\begin{array}{llll}\text { ALBG } & \text { GAHC } & \text { MDPJ } & \text { SCFW } \\ \text { ARPT } & \text { GAJS } & \text { MSMN } & \text { TNUT } \\ \text { DCCG } & \text { LALG } & \text { NCOP } & \text { VACW } \\ \text { FLMG } & \text { MDLT } & \text { SCBR } & \end{array}$

BACCHARIS L.

Groundsel

ASTERACEAE Aster Family

'Dauphin Island' [B. halimifolia L.] SCWI

glomeruliflora Pers.

Southern B. $\mathrm{SCWI}$

halimifolia L.

Sea Myrtle, G. Tree GACG

\section{BAMBUSA Schreber} POACEAE

Bamboo Grass Family

'Alphonse Karr' [B. multiplex (Lour.)

Raeusch.]

GAIS

'Fernleaf' [B. multiplex (Lour.) Raeusch.] FLDG SCCY TXVO

glaucescens (Willd.) Sieb. ex Munro

= B. multiplex

multiplex (Lour.) Raeusch.

Hedge Bamboo FLDG FLMG LAAL NCOP

'Silverstripe' [B. multiplex (Lour.)

Raeusch.] SCCY

tuldoides Munro FLUF

'Variegata' [B. multiplex (Lour.) Raeusch.] LAAL
BAUHINIA L.
FABACEAE (Caesalpinioideae)

Bean Family

congesta (Britton \& Rose) Lundell

$=B$. lunarioides

divaricata $\mathrm{L}$. LASL

lunarioides A.Gr. ex S.Wats.

Texas B.

TXMS TXSE

saigonensis Pierre ex Gagnep.

= B. yunnanensis

yunnanensis Franch.

TXPS

BEFARIA Mutis ex L.

ERICACEAE

Heath Family

racemosa Vent.

Tarflower

ALAU

BELOPERONE Nees

ACANTHACEAE Acanthus Family

guttata Brandegee

= Justicia brandegeana

BERBERIS L.

BERBERIDACEAE

Barberry

Barberry Family

'Atropurpurea' [B. koreana Palib.]

Redleaf Korean B. VAGS

'Atropurpurea' [B. thunbergii DC.] Redleaf Japanese B.
ALBH
MDBG
TNDR
FLMN
NCBE
VAPH
FLRH
SCCU
VAWR

'Atropurpurea Nana' [B. thunbergii DC.]

Dwarf Redleaf Japanese B.
$\mathrm{NCBE}$
VACW
VAPH

'Aurea' [B. thunbergii DC.]

Yellowleaf Japanese B.

$$
\text { MDBG TNTV VAGS }
$$

'Byers' [B. julianae Schneid.]

Byers Wintergreen B.

$\mathrm{SCCU}$ 


\section{'Chenault' [B. $\times$ hybrido-gagnepainii}

Suring.]

[B. gagnepainii $\times$ B. verruculosa] VAWR

$\times$ chenaultii Ahrendt

= B. $\times$ hybrido-gagnepainii 'Chenault'

'Crimson Pygmy' [B. thunbergii DC.]

$\begin{array}{lll}\text { DCNA } & \text { MDBG } & \text { TNTV } \\ \text { GACG } & \text { TNSN } & \text { VAGS }\end{array}$

hookeri Lem.

Hooker B.

FLUF

hookeri Lem. var. viridis Schneid.

Hooker B.

$\mathrm{SCCU}$

julianae Schneid.

Wintergreen B.

$\begin{array}{llll}\text { ALAU } & \text { GACG } & \text { MDNA } & \text { SCUC } \\ \text { ALBH } & \text { GAHC } & \text { NCBE } & \text { VACW } \\ \text { ALIT } & \text { GAJY } & \text { NCDU } & \text { VAMP } \\ \text { ALTD } & \text { GAUG } & \text { NCEG } & \text { VATA } \\ \text { FLMG } & \text { LAHG } & \text { SCCU } & \text { VAWR }\end{array}$

'Kobold' [B. thunbergii DC.]

GACG

$\times$ mentorensis H.Schultz \& Horvath

ex L.M.Ames

Mentor B.

[B. julianae $\times$ B. thunbergii]

(Plant Patent No. 99)
ALAU
ALIT
SCCU
ALBH
NCBE VATA

mouillacana Schneid.

DCNA

'Parkjuweel' [B. $\times$ media Grootend.]

[B. hybrido-gagnepainii 'Chenault' $\times B$.

thunbergii]

DCNA

sargentiana Schneid.

Sargent B.
ALAU
GABS

soulieana Schneid.

Soulie B.

GAJS

'Sparkle' [B. thunbergii DC.]
VATA

'Special' [B. thunbergii DC.]

DCNA thunbergii DC.

Japanese B.

$\begin{array}{llll}\text { DEWG } & \text { MDLT } & \text { SCCU } & \text { VAWL } \\ \text { FLMG } & \text { MDNA } & \text { VAGG } & \end{array}$

triacanthophora Hort. not Fedde

= B. $\times$ wisleyensis

verruculosa Hemsl. \& Wils.

Warty B.

DCNA TNTV VAMP VATA

'William Penn' [B. × gladwynensis Li]

[B. julianae $\times$ B. verruculosa]

MDBG VATA

$\times$ wisleyensis Ahrendt

(Parentage unknown; sometimes labeled triacanthophora)

$\begin{array}{llll}\text { MDBG } & \text { NCDU } & \text { SCCU } & \text { VAWR } \\ \text { MDPJ } & \text { NCWC } & \text { VAMP } & \end{array}$

BERCHEMIA Necker ex DC.

RHAMNACEAE Buckthorn Family

'Issai' [B. racemosa Sieb. \& Zucc.] MDBG

racemosa Sieb. \& Zucc.

Japanese Supplejack

TNTV

scandens (J.Hill) K.Koch

Alabama Supplejack

SCBR TNSN

BETULA L.

Birch

BETULACEAE

Birch Family

alleghaniensis Britton

Yellow B. VACW

costata Traut. MDBG VATA

'Dalecarlica' [B. pendula Roth] MDNA TNSN VAJL

davurica Pall. DCNA

fontinalis Sarg.

$=B$. occidentalis

jaquemontii Spach

DCNA 
lenta $\mathrm{L}$.

Sweet B.

DCNA

lutea Michx.f.

$=$ B. alleghaniensis

maximowicziana Regel VATA

nigra $\mathrm{L}$.

River B.

$\begin{array}{llll}\text { DCDO } & \text { NCCA } & \text { SCBR } & \text { VACW } \\ \text { DCWR } & \text { NCDU } & \text { SCCU } & \text { VAMP } \\ \text { FLUF } & \text { NCTP } & \text { TXDM } & \end{array}$

occidentalis Hook. DCNA

papyrifera Marsh.

Paper B.

$\begin{array}{lll}\text { DCNA } & \text { MDGD } & \text { NCBE } \\ \text { MDBG } & \text { MDLM }\end{array}$

$\underset{\mathrm{VAGM}}{\operatorname{papyrifera}} \times \boldsymbol{B}$. maximowicziana

pendula Roth

European White B.

$\begin{array}{llll}\text { ALBH } & \text { MDLT } & \text { TNCT } & \text { VATA } \\ \text { DCNA } & \text { NCDU } & \text { TNFN } & \text { VAWR } \\ \text { DCWH } & \text { SCCU } & \text { TNSM } & \end{array}$

pendula $\times$ B. nigra

VATA

platyphylla Sukachev var. japonica (Miq.)

Hara

Japanese White B.
DCNA
$\mathrm{SCCU}$

populifolia Marsh.

Gray B.

$\begin{array}{lll}\text { DCCG } & \text { MDBG } & \text { NCWC } \\ \text { DCNA } & \text { MDJS } & \text { TNBO }\end{array}$

potaninii Batal.

DCNA

pubescens Ehrh.

Hairy B.

DCNA

'Purpurea' [B. pendula Roth] TNTV

uber (Ashe) Fern.

Virginia Roundleaf B.

DCNA SCWI

verrucosa Ehrh.

$=B$. pendula
'Youngii' [B. pendula Roth] $\mathrm{MDBG}$

BIGNONIA L.

BIGNONIACEAE

Bignonia Family

capreolata L.

Cross Vine

FLMG GAJI SCWR

FLUF MDFM VACW

unguis-cati $\mathrm{L}$.

= Macfadyena unguis-cati

BIOTA (D.Don) Endl.

= PLATYCLADUS

BREYNIA J.R. \& J.G. Forst. EUPHORBIACEAE

Spurge Family

disticha J.R. \& J.G. Forst.

Snowbush LAHG

nivosa (W.G.Sm.) Small

$=$ B. disticha

BROUSSONETIA L'Her. ex Vent.

MORACEAE

Mulberry Family

papyrifera (L.) Vent.

Paper Mulberry

$\begin{array}{llll}\text { DCNA } & \text { MSRN } & \text { TNSG } & \text { VABF } \\ \text { MDJH } & \text { SCUC } & \text { TNTV } & \text { VACW }\end{array}$

'Variegata' [B. papyrifera (L.) Vent.] SCWI

BRUCKENTHALIA Reichenb.

ERICACEAE

Heath Family

spiculifolia (Salisb.) Reichenb.

Spike Heath

DEWG

BRUGMANSIA Pers.

SOLANACEAE : Nightshade Family

suaveolens (Humboldt\& Bonpland ex Willd.) Bercht.\& J.Presl

Angel's Trumpet

ALBG MSHB 


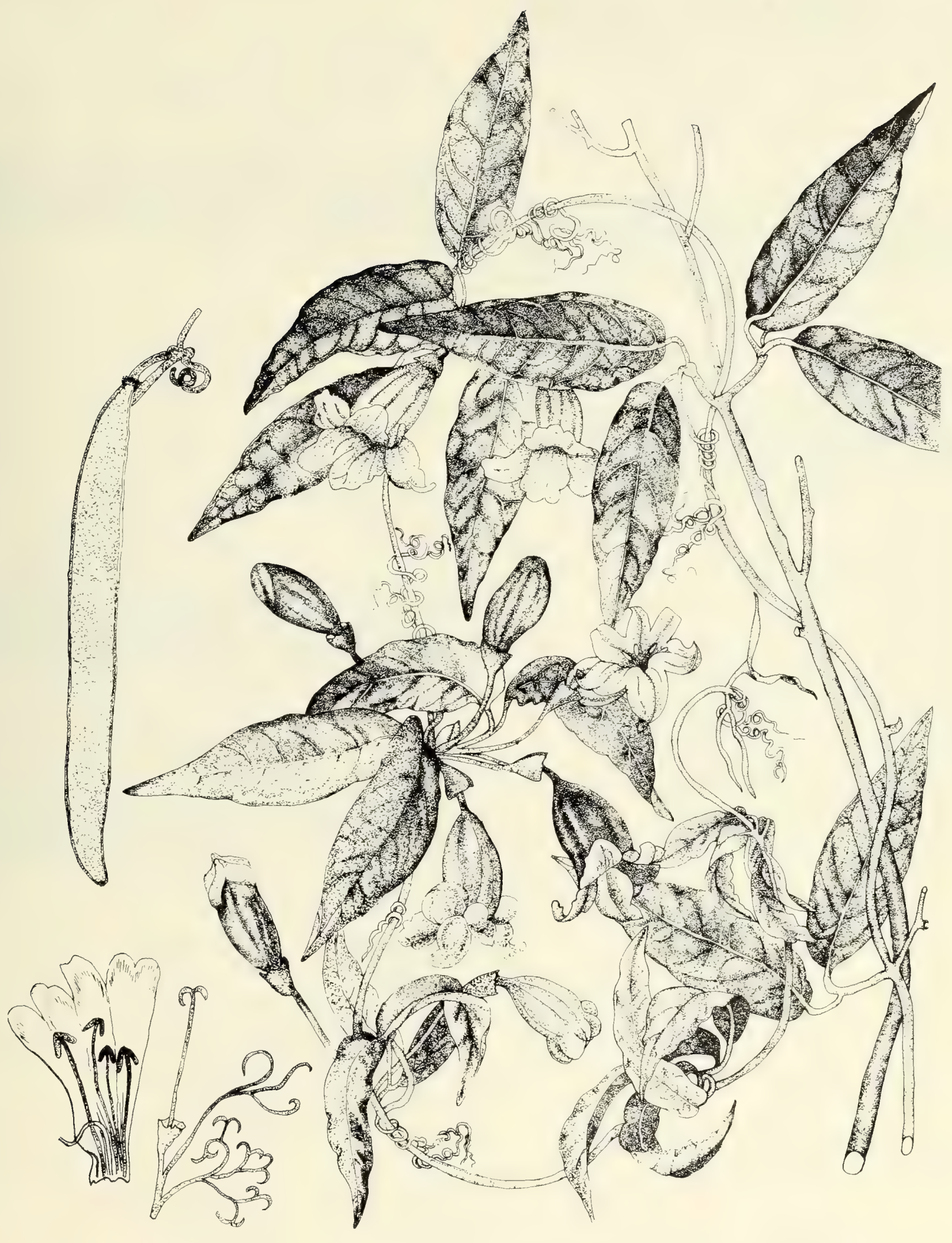

BIGNONIA capreolata L.

[illustrator Lillian Nicholson Meyer] 


\section{BRUNFELSLA L. \\ SOLANACEAE Nightshade Family}

australis Benth.

Yesterday-today-tomorrow
FLCG
GATS TXAV
FLUF
LALG TXLL
GAJI
LASI
TXMK

BUCKLEYA Torr.

SANTALACEAE

Sandalwood Family

distichophylla (Nutt.) Torr.

Piratebush

GAEC NCBE

\section{BUDDLEJA L. Butterfly Bush BUDDLEJACEAE Buddleja Family}

'Alba' [B. davidii Franch.] MDWP

alternifolia Maxim.

Fountain B.

DEWG

crispa Benth. var. farreri (Balf.f. \&

W.W.Sm.JHand.-Mazz.

$$
\text { DCNA }
$$

davidii Franch.

Orange-eye $\mathrm{B}$.

$$
\begin{array}{lll}
\text { MDBG } & \text { NCBE } & \text { SCCU } \\
\text { MDWP } & \text { NCOP }
\end{array}
$$

fallowiana Balf.f. \& W.W.Sm. GATS

farreri Balf.f. \& W.W.Sm.

$$
\text { DCNA }
$$

japonica Hemsl.

DCNA

lindleyana Fort. ex Lindl.

$$
\begin{array}{lll}
\text { GATN } & \text { LARP } & \text { MSHB } \\
\text { LAAL } & \text { MSEN } &
\end{array}
$$

'Sungold' [B. $\times$ weyeriana Weyer]
[B. davidii $\times$ B. globosa]

VAGS

venenifera Makino

DCNA $\times$ weyeriana Weyer

[B. davidii $\times$ B. globosa]

SCJF

$\times$ whiteana R.J.Moore

[B. alternifolia $\times$ B. asiatica]

DCNA

BUMELIA O.Swartz

SAPOTACEAE

Sapodilla Family

lacuum Small

= B. tenax

lanuginosa (Michx.) Pers.

Chittamwood SCMG TXMS

lycioides (L.) Pers.

Southern Buckthorn SCBR VACW

tenax (L.) Willd.

Ironwood

SCWI

BUTIA (Becc.) Becc.

ARECACEAE

Palm Family

capitata (Mart.) Becc.

Jelly Palm

FLMG

FLUF LAAL

BUXUS L.

BUXACEAE

Box

Boxwood Family

'Abilene' [B. sempervirens L.] DCNA

'Agram' [B. sempervirens L.] DCNA

'Albo-marginata' [B. sempervirens L.]

= 'Marginata'

'Angustifolia' [B. sempervirens L.]

Willow-leaved B.
DCNA
MSMN TNLW
FLDG
NCCA

'Arborescens' [B. sempervirens L.] Tree B.
DCNA
DEMC TNLW VACW

'Arborescens Decussata' [B. sempervirens L.]

DCNA 


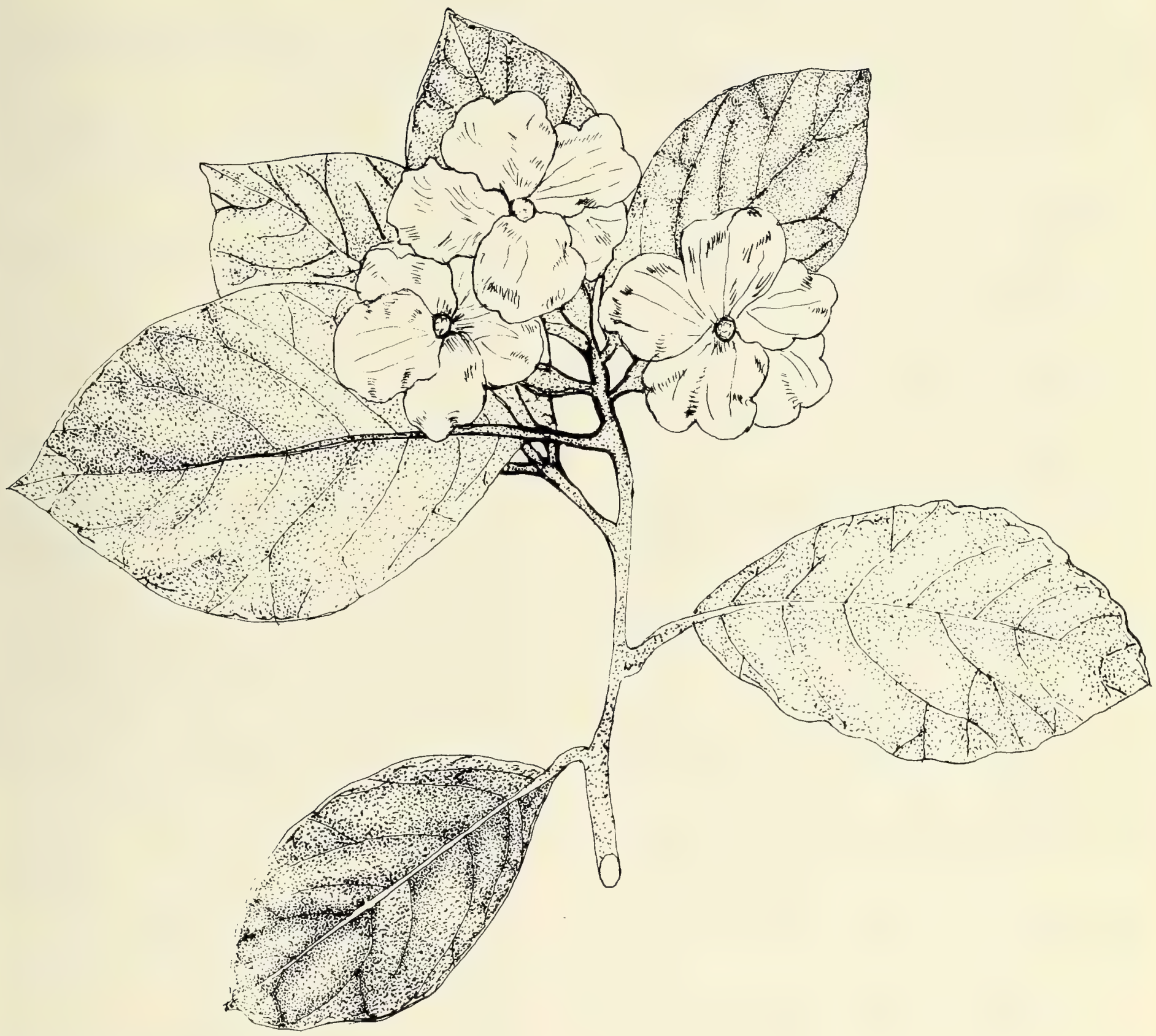

BRUNFELSIA australis Benth.

[Illustrator Lillian Nicholson Meyer] 
'Argenteo-variegata' [B. sempervirens L.] DCNA LARP VAGS

'Aristocrat' [B. sempervirens L.] DCNA

'Aurea Maculata' [B. sempervirens L.]

= 'Aureo-variegata'

'Aurea Marginata' [B. sempervirens L.]

= 'Marginata'

'Aurea Pendula' [B. sempervirens L.] DCNA

'Aureo-variegata' [B. sempervirens L.] DCNA

balearica Lam.

Balearic B.

DCNA SCMG

'Belleville' [B. sempervirens L.] DCNA

'Bullata' [B. sempervirens L.] ARRB DCNA GAHC VAAC

'Butterworth' [B. sempervirens L.] DCNA

'Compacta' [B. microphylla Sieb. \& Zucc.]
ALHC
NCFR
VATA
ALTD
MDBG
VACW
VATW

'Curly Locks' [B. microphylla Sieb. \&

Zucc.] DCNA

'Denmark' [B. sempervirens L.] DCNA

'Edgar Anderson' [B. sempervirens L.] DCNA

'Elegantissima' [B. sempervirens L.] DCNA VAGS

'Fastigiata' [B. sempervirens L.]

Columnar B. DCNA VABF

'Glauca' [B. sempervirens L.] DCNA

'Grace Hendrick Phillips' [B. microphylla Sieb. \& Zucc.] DCNA

'Graham Blandy' [B. sempervirens L.] DCNA
'Green Gem'

(? Hybrid) DCNA

'Green Mountain'

(? Hybrid)

DCNA

'Green Pillow' [B. microphylla Sieb. \&

Zucc.] DCNA

'Green Velvet'

(? Hybrid) DCNA

'Handsworthiensis' [B. sempervirens L.] Handsworth B. DCNA TNSN TNTV

'Hardwickensis' [B. sempervirens L.] DCNA

harlandii Hance

Harland B.

$\begin{array}{llll}\text { ALBG } & \text { FLMG } & \text { MSEN } & \text { VAGS } \\ \text { ALTD } & \text { GADG } & \text { MSEP } & \text { VATA } \\ \text { ARHS } & \text { LALG } & \text { NCTP } & \text { VAWM } \\ \text { ARRB } & \text { LATU } & \text { SCFW } & \\ \text { DCNA } & \text { MDKN } & \text { SCJM } & \end{array}$

'Heinrich Bruns' [B. sempervirens L.] DCNA

'Helen Whiting' [B. microphylla Sieb. \& Zucc.] DCNA

'Henry Hohman' [B. microphylla Sieb. \& Zucc.] DCNA

'Henry Shaw' [B. sempervirens L.] DCNA

himalayensis Hort.

= B. wallichiana

'Inglis' [B. sempervirens L.] DCNA

'Ipek' [B. sempervirens L.] DCNA

'Joe Gable' [B. sempervirens L.] DCNA TNSN TNTV

'John Baldwin' [B. microphylla Sieb. \& Zucc.] DCNA 
'Joy' [B. sempervirens L.] DCNA

'Kingsville Dwarf [B. microphylla Sieb. \& Zucc.]

$=$ 'Compacta'

'Latifolia Macrophylla' [B. sempervirens L.]

DCNA

'Latifolia Nova' [B. sempervirens L.] DCNA

'Lynnhaven' [B. sempervirens L.] DCNA

'Macrophylla' [B. sempervirens L.] DCNA

'Marginata' [B. sempervirens L.] DCNA MDHN

'Memorial' [B. sempervirens L.] DCNA

microphylla Sieb. \& Zucc.

Japanese B.
ARHS
DEEM MDJS
DCCG
DEWG TNSN
DCNA
GAEC
TNUT

microphylla Sieb. \& Zucc. var. insularis Nakai

= B. sinica var. insularis

microphylla Sieb. \& Zucc. var. japonica (Muell.-Arg.) Rehd. \& Wils.

Japanese Box

$\begin{array}{llll}\text { ALBG } & \text { FLCG } & \text { LASL } & \text { SCMP } \\ \text { ALTD } & \text { FLMG } & \text { SCCU } & \text { TNCT } \\ \text { ARHS } & \text { FLUF } & \text { SCEA } & \\ \text { DCCG } & \text { GATS } & \text { SCJM } & \\ \text { DCNA } & \text { LAAL } & \text { SCMG } & \end{array}$

microphylla Sieb. \& Zucc. var. koreana Nakai ex Rehd.

$=B$. sinica var. insularis

'Morris Dwarf' [B. microphylla Sieb. \& Zucc. var. japonica (Muell.-Arg.) Rehd. \& Wils.]

DCNA

'Morris Midget' [B. microphylla Sieb. \& Zucc. var. japonica (Muell.-Arg.) Rehd. \& Wils.] DCNA

'Myrtifolia' [B. sempervirens L.] ALTD

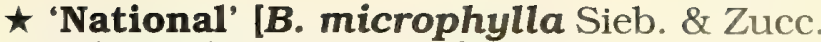

var. japonica (Muell.-Arg.) Rehd. \& Wils.] DCNA MDBG

'Nish' [B. sempervirens L.] DCNA

'Northern Find' [B. sempervirens L.] DCNA

'Northern New York' [B. sempervirens L.] DCNA

'Northland' [B. sempervirens L.] DCNA

'Pendula' [B. sempervirens L.]

Weeping B. DCCG DCNA

'Pincushion' [B. sinica (Rehd. \& Wils.) M.Cheng var. insularis (Nakai) M.Cheng] DCNA

'Ponteyi' [B. sempervirens L.] DCNA

'Prostrata' [B. sempervirens L.] DCNA

'Pyramidalis' [B. sempervirens L.] DCNA TNTV

'Richard' [B. harlandii Hance] ARWS DCNA LASL

'Rotundifolia' [B. sempervirens L.] DCNA LARP TNLW VAHM

'Ste. Genevieve' [B. sempervirens L.] DCNA

'Salicifolia' [B. sempervirens L.] DCNA

'Salicifolia Elata' [B. sempervirens L.] DCNA

sempervirens L.

Common B.

$\begin{array}{llll}\text { ALBG } & \text { GABG } & \text { NCDU } & \text { SCMP } \\ \text { ARRB } & \text { GACG } & \text { NCTP } & \text { TNCT } \\ \text { DCCG } & \text { GAHC } & \text { SCBR } & \text { TNRU } \\ \text { DCNA } & \text { GAKN } & \text { SCCU } & \text { TNUT } \\ \text { DCWH } & \text { GAOH } & \text { SCFW } & \text { VAGS } \\ \text { DEEM } & \text { GATN } & \text { SCJM } & \text { VAGW } \\ \text { FLMG } & \text { MDHN } & \text { SCMG } & \text { VAWR }\end{array}$

sinica (Rehd. \& Wils.) M.Cheng var. insularis (Nakai) M.Cheng Korean B. ARHS DCNA SCCU VATA 
stenophylla Hance

DCNA

'Suffruticosa' [B. sempervirens L.]

Dwarf English B.

$\begin{array}{llll}\text { DCNA } & \text { GAKN } & \text { NCSM } & \text { TNCT } \\ \text { DCWH } & \text { GAOH } & \text { NCTE } & \text { VACW } \\ \text { FLCM } & \text { MDJS } & \text { SCBR } & \text { VAGU } \\ \text { FLDG } & \text { MSEN } & \text { SCCU } & \text { VATA } \\ \text { GAHC } & \text { NCDU } & \text { SCMP } & \text { VAWR }\end{array}$

'Sunnyside' [B. microphylla Sieb. \& Zucc.] DCNA

'Tall Boy' [B. sinica (Rehd. \& Wils.)

M.Cheng var. insularis (Nakai) M.Cheng] DCNA

'Tide Hill' [B. sinica (Rehd. \& Wils.)

M.Cheng var. insularis (Nakai) M.Cheng] VATA

'Vardar Valley' [B. sempervirens L.]

Jugoslavian B.
DCNA
GACG TNPW

'Varifolia' [B. sempervirens L.]

DCNA

wallichiana Baill.

DCNA

'Welleri' [B. sempervirens L.]

DCNA GACG

'Winter Beauty' [B. sinica (Rehd. \& Wils.)

M.Cheng var. insularis (Nakai) M.Cheng] DCNA

'Wintergreen' [B. sinica (Rehd. \& Wils.)

M.Cheng var. insularis (Nakai) M.Cheng] SCCU

CAESALPINLA L.

FABACEAE (Caesalpinioideae)

Bean Family

gilliesii Hook.

SCWI

CALLIANDRA Benth.

FABACEAE (Mimosoideae) Bean Family

tweediei Benth.

Mexican Flamebush

GACH LAAL TXDC TXPS
CALLICARPA L.

Beautyberry

VERBENACEAE

Verbena Family

americana $\mathrm{L}$.

American B.
ALAU
GACG
NCDU
VAGS
ALBG
LAAL
SCBR
FLUF NCAG TXRS

bodinieri Lev.

Bodinier B.

MDPJ

bodinieri Lev. var. giraldii Rehd.

DCBG DCNA SCCU

dichotoma (Lour.) K.Koch

Purple B.

MDBG SCCU VACW

japonica Thunb. ex J.A.Murr.

Japanese B.
DCNA
MDKN SCWI
GAEH SCCU TNUT

'Lactea' [C. americana L.]

White-fruited B.
GACG
SCBR
SCWI
TXRS

'Leucocarpa' [C. dichotoma (Lour.) K.Koch] DCNA

macrophylla Vahl

FLUF

tosaensis Makino

VATA

CALLISTEMON R.Br.

Bottlebrush

MYRTACEAE

Myrtle Family

citrinus (Curtis) Skeels

ALFS FLUF LASL

FLCS GASM NCOP

linearis (Schrad. \& J.C.Wendl.) DC.

Narrowleaf B.

FLUF LASL

rigidus $\mathrm{R} . \mathrm{Br}$.

Stiff B.

FLUF

rugulosus Miq.

SCWI

salignus (Sm.) DC.

Willow B.

ALBG LASL 
CALLISTEMON

viminalis (Sol. ex Gaertn.) G.Don

FLMG FLUF GACG

CALLITRIS Vent.

CUPRESSACEAE

Cypress Family

columellaris F.Muell.

FLUF

CALLUNA Salisb.

ERICACEAE

Heather

Heath Family

'August Beauty' [C. vulgaris (L.) Hull] MDLT

'Aureafolia' [C. vulgaris (L.) Hull] MDLT

'H. E. Beale' [C. vulgaris (L.) Hull] MDLT

'Juno' [C. vulgaris (L.) Hull] MDLT

'Plena' [C. vulgaris (L.) Hull] MDLT

'Silver Queen' [C. vulgaris (L.) Hull] MDLT

'Tib' [C. vulgaris (L.) Hull] MDLT

vulgaris (L.) Hull

Scotch $\mathrm{H}$.

DCNA

CALOCEDRUS KurZ

CUPRESSACEAE

Cypress Family

decurrens (Torr.) Florin

Incense Cedar

$\begin{array}{llll}\text { ALFN } & \text { GAMP } & \text { NCCA } & \text { TNUT } \\ \text { DCPC } & \text { GAWJ } & \text { NCTE } & \text { VAMP } \\ \text { FLUF } & \text { MDBG } & \text { SCHS } & \\ \text { GAHC } & \text { MDJH } & \text { TNPC } & \\ \text { GAHS } & \text { MDJS } & \text { TNTV } & \end{array}$

CALYCANTHUS L.

\section{CALYCANTHACEAE Calycanthus Family}

'Athens' [C. floridus L.]

$$
\text { GACG GAMD GAPG }
$$

fertilis Walt.

$=$ C. floridus floridus L.

Sweetshrub, Carolina Allspice

$\begin{array}{llll}\text { ALAU } & \text { GAVA } & \text { NCBE } & \text { TNDG } \\ \text { ALBH } & \text { LASL } & \text { NCCA } & \text { VACW } \\ \text { DEEM } & \text { MDBM } & \text { NCTP } & \text { VADW } \\ \text { DEWG } & \text { MDJS } & \text { SCCU } & \text { VAGW } \\ \text { GACG } & \text { MDLA } & \text { SCDB } & \text { VANB } \\ \text { GAEC } & \text { MDNA } & \text { SCHW } & \text { VAWP } \\ \text { GAFF } & \text { MDWP } & \text { SCMP } & \\ \text { GAUG } & \text { MSEP } & \text { SCWC } & \end{array}$

floridus L. var. laevigatus (Willd.) T. \& G.

= C. floridus

'Margarita' [C. floridus L.]

GAEC VAPH

CAMELLIA L.

THEACEAE

Tea Family

$\star$ 'Ack-Scent' [C. japonica 'Kramer's

Supreme' $\times$ C. 'Fragrant Pink Improved'] DCNA

$\star$ 'Ack-Scent Pink' [C. japonica 'Fragrant Star' $\times$ C. 'Fragrant Pink'] DCNA

$\star$ 'Ack-Scent Red' [C. japonica 'Arabella'

$\times$ C. 'Fragrant Pink'] DCNA

$\star$ 'Ack-Scent Sno' [C. japonica 'Frost Queen' $\times$ C. 'Fragrant Pink'] DCNA

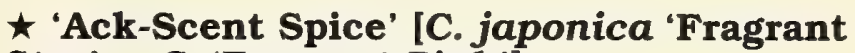
Star' $\times$ C. 'Fragrant Pink'] DCNA

$\star$ 'Ack-Scent Star' [C. japonica 'Fragrant Star' $\times$ C. 'Fragrant Pink'] DCNA

$\star$ 'Ack-Scent White' [C. japonica 'Frost Gueen' $\times$ C. 'Fragrant Pink'] DCNA

'Alice Morrison' [C. japonica L.] ALBG

'Anemoniflora' [C. japonica L.]

Warratah C. SCMP

'Annette' [C. sasanqua Thunb. ex J.A.Murr.] DCNA

assimilis Champ. ex Benth. MDGD 


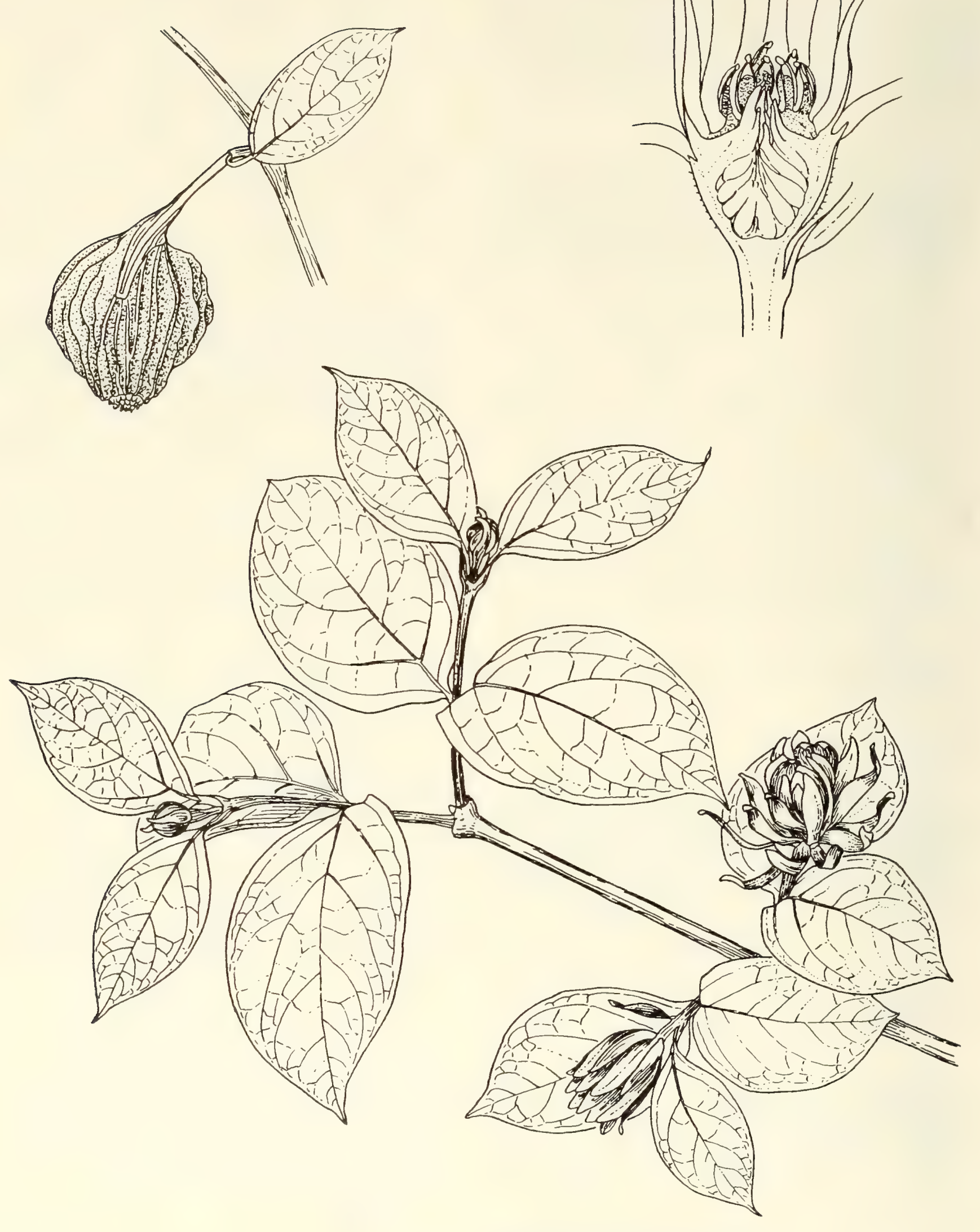

CALYCANTHUS floridus L.

[illustrator Peggy K. Duke] 
'Aunt Jetty' [C. japonica L.]

FLMG

'Barbara Morgan' [C. japonica L.] DCNA

'Capt. Rawes' [C. reticulata Lindl.] LAGN

'Chandleri Elegans' [C. japonica L.] VACW

'Cherokee' [C. sasanqua Thunb. ex J.A.Murr.] DCNA

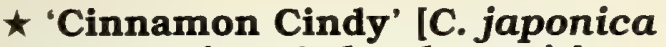
'Kenyo-Tai' $\times$ C. lutchuensis] DCNA

'Cleopatra' [C. sasanqua Thunb.

ex J.A.Murr.] VATA

crapnelliana Tutch. DCNA MDGD

'Crimson Bride' [C. sasanqua Thunb. ex J.A.Murr.] DCNA

cuspidata (Kochs) J.G.Veitch SCMG

+'Daisy Eagleson' [C. sasanqua 'Maiden's Blush' + C. japonica]

(Graft chimera) DCNA

'Daitairin' [C. japonica L.] ALBG

'Dawn' [C. vernalis (Makino) Makino] MDGD

'Doctor Tinsley' [C. japonica L.] ARRB

'Donation' [C. $\times$ williamsii W.W.Sm.]

[C. japonica $\times$ C. saluenensis] ALBG

'Donation Sport' [C. $\times$ williamsii W.W.Sm.]

[C. japonica $\times$ C. saluenensis] DCNA

drupifera (Lour.) Pierre FLIF

\footnotetext{
$\star$ 'Fragrant Joy' [C. rusticana $\times$

C. Iutchuensis] DCNA
}

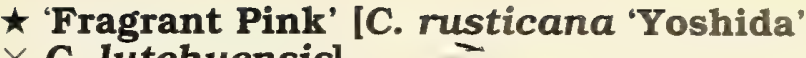

$\times$ C. lutchuensis] DCNA

$\star$ 'Fragrant Pink Improved'

(Polyploid of C. 'Fragrant Pink') DCNA

fraterna Hance ALTD LASL MDGD

$\star$ 'Frost Prince' [C. hiemalis

'Shishigashira' $\times$ C. oleifera] DCNA MDLT

Ł 'Frost Princess' [C. hiemalis 'Billy

Wylam' $\times$ C. oleifera] DCNA

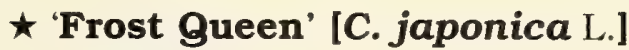
DCNA

'Gov. Mouton Variegated' [C. japonica L.] SCMP

granthamiana Sealy MDGD SCMG VANB

'Guilio Nuccio' [C. japonica L.] TNMB

'Inspiration' [C. reticulata $\times$

C. saluenensis] VAGS

japonica L.

Common C.

$\begin{array}{llll}\text { ALSH } & \text { FLUF } & \text { SCBR } & \text { SCMP } \\ \text { DCNA } & \text { MDBG } & \text { SCMG } & \text { VACW }\end{array}$

kissii Wall. MDGD

'Kumasaka' [C. japonica L.] ALBG

'Leucantha' [C. japonica L.] DCWH

'Little Princess' [C. fraterna $\times$ C. japonical ALBG

lutchuensis Ito MDGD

'Marie Wood' [C. japonica L.] VANB

miyagii (Koidz.) Makino \& Nemoto

= C. sasanqua 
'November Pink' [C. $\times$ williamsii W.W.Sm.]

[C. japonica $\times$ C. saluenensis]

MDGD

oleifera Abel

Oil C.

$\begin{array}{lll}\text { DCNA } & \text { GAEC } & \text { MDLT } \\ \text { GACG } & \text { MDGD } & \text { VACW }\end{array}$

$\star$ 'Polar Ice' [C. 'Frost Princess' $\times$

C. oleifera]

DCNA

'Professor Sargent' [C. japonica L.] DCWH

'Reverend John G. Drayton'

[C. japonica L.]

SCMG

saluenensis Stapf

NCEL

'Sarah Frost' [C. japonica L.]

ALRH NCTP

sasanqua Thunb. ex J.A.Murr.

Sasanqua C.

$\begin{array}{llll}\text { ALBG } & \text { FLUF } & \text { NCAG } & \text { SCMG } \\ \text { ARRB } & \text { GAWH } & \text { NCDU } & \text { SCWP } \\ \text { DCNG } & \text { MDGD } & \text { SCKG } & \end{array}$

'Sayehime' [C. vernalis (Makino) Makino] DCNA

'Sharon Elizabeth' [C. sasanqua Thunb. ex J.A.Murr.]

DCNA

'Sieboldii' [C. japonica L.]

DCWH

sinensis (L.) O.Ktze.

Tea Plant

$\begin{array}{llll}\text { DCNA } & \text { GAJS } & \text { MSEP } & \text { SCMP } \\ \text { FLIF } & \text { MDFM } & \text { NCTE } & \text { VAGS } \\ \text { GACG } & \text { MDLT } & \text { SCBR } & \text { VAMP } \\ \text { GAEH } & \text { MDRP } & \text { SCKG } & \end{array}$

$\star$ 'Snow Flurry' [C. 'Frost Princess' $\times$

C. oleifera]

DCNA

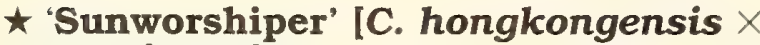

C. rusticana]

DCNA

'Tama no ura' [C. japonica L.]

DCNA

tenuifolia (Hayata) Cohen-Stuart

FLIF MDGD
'Texas Star' [C. sasanqua Thunb.

ex J.A.Murr.]

ALCW

'Tiny Princess'

VAPH

tsaii H.H.Hu

MDGD

$\star$ 'Two Marthas' [C. sasanqua 'Onishiki' $\times$

C. Kissii]

DCNA

'Ville de Nantes' [C. japonica L.] FLUF

‡ 'Winter's Charm' [C. oleifera $\times$

C. sasanqua 'Takara-awase'] DCNA

$\star$ 'Winter's Hope' [C. 'Frost Princess' $\times$

C. oleifera]

DCNA

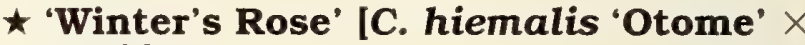

C. oleifera]

DCNA

$\star$ 'Winter's Star' [C. 'Frost Prince' $\times$

C. hiemalis]

DCNA

CAMPSIS Lour.

BIGNONLACEAE

Bignonia Family

grandiflora (Thunb. ex J.A.Murr.) Schum.

Chinese Trumpet-creeper MDFM TNSC

'Mme. Galen' [C. $\times$ tagliabuana (Vis.) Rehd.] [C. grandiflora $\times$ C. radicans]

DCNA MDFM TNSC

DCRS SCCU VAGS

radicans (L.) Seemann ex Bur.

Trumpet-creeper, Trumpet Vine
DCNA
MDLT
SCBR
VACW
LAAL NCBE SCCU VAMW

'Yellow Trumpet' [C. radicans (L.)

Seemann ex Bur.]

$\mathrm{SCCU} \quad \mathrm{VAGS}$

CAMPYLOTROPIS Bunge

FABACEAE (Papilionoideae) Bean Family

macrocarpa Bunge

MDSC 
CARAGANA Fabr.

Pea Shrub

FABACEAE (Faboideae)

arborescens Lam.

P.S.

DEWG

chamlagu Lam.

= C. sinica

'Lorbergii' [C. arborescens Lam.] MDKN

'Pendula' [C. arborescens Lam.] DCNA TNSN

sinica (Buc'hoz) Rehd.

Chinese P.S. DCNA GAEC MDKN

'Sylvatica' [C. frutex (L.) K.Koch] DCNA

CARISSA L. APOCYNACEAE Dogbane Family

macrocarpa (Eckl. \& Zeyh.) A.DC. LALG LASL

CARPINUS L. BETULACEAE

Hornbeam Birch Family

'Ascendens' [C. caroliniana Walt.] TNLW

betulus $\mathrm{L}$.

European $\mathrm{H}$.

DCCG DCNA DEMC MDTD

caroliniana Walt.

American H., Ironwood

$\begin{array}{llll}\text { ALAU } & \text { FLUF } & \text { NCCA } & \text { TXMS } \\ \text { DCCG } & \text { MDHN } & \text { SCBR } & \text { VACW } \\ \text { DEWG } & \text { MDWP } & \text { SCMP } & \end{array}$

cordata $\mathrm{Bl}$.

Heartleaf $\mathrm{H}$.

MDGD TNDG

'Fastigiata' [C. betulus L.]

Columnar $\mathrm{H}$.

$\begin{array}{lll}\text { DCCG } & \text { TNFN } & \text { TNTV } \\ \text { DCNA } & \text { TNSN } & \text { VACW }\end{array}$

japonica $\mathrm{Bl}$.

Japanese $\mathrm{H}$.

DCNA_ TNSN TNTV VACW

DCNA - TNSN TNTV VACW

orientalis Mill.

DCNA

'Purpurea' [C. betulus L.]

Purple-leaf H.

TNTV

CARYA Nutt.

Hickory

JUGLANDACEAE

Walnut Family

aquatica (Michx.f.) Nutt.

Bitter Pecan, Water H.

SCBR

cordiformis (Wang.) K.Koch

Bitternut

DCWM

glabra (Mill.) Sweet

Pignut

$\begin{array}{llll}\text { ALAU } & \text { FLWC } & \text { TNBM } & \text { VASC } \\ \text { FLUF } & \text { SCBR } & \text { VAGG } & \end{array}$

'Glover' [C. ovata (Mill.) K.Koch] $\mathrm{MDAB}$

'Hines' [C. ovata (Mill.) K.Koch] MDAB

illinoinensis (Wang.) K.Koch

Pecan

$\begin{array}{llll}\text { ALCG } & \text { GAIS } & \text { MDHN } & \text { SCWC } \\ \text { DCCG } & \text { LATU } & \text { SCBR } & \text { SCWI } \\ \text { DCJS } & \text { MDBR } & \text { SCMP } & \text { VAGW }\end{array}$

illinoinensis $\times$ C. $\mathrm{sp}$.

Hican TNSN

'Koon' [C. illinoinensis $\times$ C. sp.] MDGD

laciniosa (Michx.f.) Loud.

Shellbark $\mathrm{H}$. FLMG NCCP

'Lake' [C. ovata (Mill.) K.Koch] $\mathrm{MDAB}$

'Lingenfelter' [C. ovata (Mill.) K.Koch] MDAB

'Mehan' [C. illinoinensis (Wang.) K.Koch] Mehan Pecan ALBH

ovalis (Wang.) Sarg.

Sweet Pignut ALAU SCBR 
ovata (Mill.) K.Koch

Shagbark $\mathrm{H}$.

DEEM VAGG

ovata (Mill.) K.Koch var. pubescens Sarg. TNHG

pallida (Ashe) Engl. \& Graebn.

Sand $\mathrm{H}$.

$$
\text { ALAU VASC }
$$

'Romig' [C. ovata (Mill.) K.Koch] $\mathrm{MDAB}$

'Schaul' [C. ovata (Mill.) K.Koch] $\mathrm{MDAB}$

'Schinnerling' [C. ovata (Mill.) K.Koch] MDAB

'Stuart' [C. illinoinensis (Wang.) K.Koch] Stuart Pecan
ALBH
DCNA
FLUF
NCCA

tomentosa (Lam.) Nutt.

Mockernut $\mathrm{H}$

$\begin{array}{llll}\text { DCCG } & \text { FLUF } & \text { SCBR } & \text { VAMP } \\ \text { FLMG } & \text { NCWK } & \text { TNBM } & \end{array}$

'Vest' [C. ovata (Mill.) K.Koch] $\mathrm{MDAB}$

\section{CARYOPTERIS Bunge \\ VERBENACEAE Verbena Family \\ $\times$ clandonensis Simmonds \\ [C. incana $\times$ C. mongholica] MDBG \\ incana (Thunb. ex Houtt.) Miq. DCNA}

CASIMIROA Llave \& LeX. RUTACEAE

Citrus Family

edulis Llave \& Lex.

White Sapote FLUF

pringlei (S.Wats.) Engelm. TXMS TXPS
CASSIA L.

FABACEAE (Caesalpinioideae)

Senna

Bean Family

alata $\mathrm{L}$.

Ringworm S. ALBG NCOP

coluteoides Coll.

ALBG FLMG LAAP

FLDJ GATS SCCL

corymbosa Lam.

FLUF

CASTANEA Mill.

Chestnut

FAGACEAE Beech Family

'Argenteo-variegata' [C. sativa L.] DCNA

'Crane' [C. mollissima Bl.] MDAB

crenata Sieb. \& Zucc.

Japanese C.

DCCT

'Meiling' [C. mollissima Bl.] MDAB

mollissima $\mathrm{Bl}$.

Chinese $\mathrm{C}$.

$\begin{array}{llll}\text { DCNA } & \text { GAIS } & \text { TNSC } & \text { VAWR } \\ \text { DCWH } & \text { MDHN } & \text { TNWF } & \\ \text { DEMC } & \text { MDLT } & \text { VACM } & \end{array}$

'Nanking' [C. mollissima Bl.] $\mathrm{MDAB}$

'Orrin' [C. mollissima Bl.] MDAB

sativa Mill.

Common C., European C. MDBR

vesca Gaertn.

= C. sativa

CASTANOPSIS (D.Don) Spach

Chinquapin

FAGACEAE Beech Family

cuspidata (Thunb. ex J.A.Murr.) Schottky Japanese C.

ALON GAIS LAGN


cuspidata (Thunb. ex J.A.Murr.) Schottky

var. sieboldii (Makino) Nakai

FLMG

sclerophylla Schottky

GAIS

CASUARINA Adans.

CASUARINACEAE

Casuarina Family

cunninghamiana Miq.

Australian Pine, Beefwood

FLDH FLGA

CATALPA Scop.

BIGNONIACEAE

Bignonia Family

bignonioides Walt.

Common C., Indian Bean

DCSH MDHN VAWR

GAMC SCGB

bungei C.A.Mey.

Manchurian C.

TNRW

ovata G.Don

Chinese C.

DCCG DCJJ DCPC SCCU

speciosa Warder ex Engelm.

Western C.

DEWG NCCA VACM

GABS TNHG

\section{CEANOTHUS L.}

RHAMNACEAE

Buckthorn Family

americanus L.

New Jersey Tea

DCNA VAMP

$\times$ delilianus Spach

[C. americanus $\times$ C. caeruleus]

VAGS

'Gloire de Versailles' [C. $\times$ delilianus

Spach]

[C. americanus $\times$ C. caeruleus]

SCWI

'Marie Simon' [C. $\times$ pallidus Lindl.]

[C. ? $\times$ delilianus $\times$ C. ovatus]

DCNA DEWG

microphyllus Michx.

SCWI velutinus Dougl.

Snowbrush C.

SCJF

CEDRELA P.Br.

MELIACEAE

Mahogany Family

sinensis Juss.

= Toona sinensis

CEDRUS Trew

Cedar

PINACEAE

Pine Family

atlantica (Endl.) Manetti ex Carr.

Atlas C.

$\begin{array}{lll}\text { DCNA } & \text { NCCA } & \text { VABF } \\ \text { GAUG } & \text { NCTP } & \text { VACW } \\ \text { MDJS } & \text { TNMB } & \text { VARH }\end{array}$

'Aurea' [C. atlantica (Endl.) Manetti ex Carr.]

DCNA MDJS

'Aurea' [C. deodara (Roxb.) G.Don]

DCNA MDBG

brevifolia (Hook.f.) A.Henry

= C. libani ssp. brevifolia

deodara (Roxb.) G.Don

Deodar C.

$\begin{array}{llll}\text { ALBG } & \text { GACG } & \text { MDHN } & \text { TNSN } \\ \text { ARUM } & \text { GAJI } & \text { NCBE } & \text { VAMP } \\ \text { DCCG } & \text { GAOH } & \text { NCTE } & \text { VARH } \\ \text { DCNA } & \text { GAUG } & \text { SCCU } & \\ \text { FLMG } & \text { GAWS } & \text { SCHG } & \end{array}$

'Fastigiata' [C. atlantica (Endl.) Manetti ex Carr.] DCNA

'Fastigiata' [C. deodara (Roxb.) G.Don] DCNA

'Glauca' [C. atlantica (Endl.) Manetti ex Carr.]

Blue Atlas C.

$\begin{array}{lll}\text { DCNA } & \text { MDCP } & \text { TNSG } \\ \text { MDBG } & \text { MDLT }\end{array}$

'Glauca Pendula' [C. atlantica (Endl.)

Manetti ex Carr.]

DCNA MDBG MDLT

libanensis Juss. ex Mirb.

= C. libani 
libani A.Rich.

Cedar-of-Lebanon
ALAU
ALTD
MDBG
ALFN
GALS
MDHN

libani A.Rich. ssp. brevifolia (Hook.f.)

Meikle

Cyprus Cedar

DCNA

libani A.Rich. var. stenocoma (O.Schwarz)

Davis

$$
\text { DCNA MDDF }
$$

libanotica Trew ex Pilger ssp. brevifolia

(Hook.f.) O.Schwarz

= C. libani ssp. brevifolia

'Limelight' [C. deodara (Roxb.) G.Don] DCNA

'Nana' [C. libani A.Rich.] DCNA

'Pendula' [C. atlantica (Endl.) Manetti ex Carr.]

DCNA

'Pendula' [C. deodara (Roxb.) G.Don] DCNA

'Pygmy' [C. deodara (Roxb.) G.Don] DCNA

'Repandens' [C. deodara (Roxb.) G.Don] DCNA

CELASTRUS L. CELASTRACEAE

angulatus Maxim.

TNSN

orbiculatus Thunb. ex J.A.Murr.

Oriental B.

$\begin{array}{lll}\text { ALAU } & \text { NCBE } & \text { TNTV } \\ \text { MDGJ } & \text { SCBR } & \text { VACW } \\ \text { MDPJ } & \text { SCCU } & \text { VANB }\end{array}$

rosthornianus Loesn.

SCBR

CELTIS L. ULMACEAE

Hackberry Elm Family

australis $\mathrm{L}$.

DCNA GAIS caucasica Willd.

Caucasian $\mathrm{H}$.

GAIS

laevigata Willd.

Sugarberry

$\begin{array}{llll}\text { ALTD } & \text { FLUF } & \text { SCMP } & \text { VAMP } \\ \text { DCLP } & \text { LAAL } & \text { TXHN } & \end{array}$

occidentalis L.

Common H.

$\begin{array}{llll}\text { ALUA } & \text { GAHC } & \text { TNFE } & \text { TNWF } \\ \text { DCCG } & \text { MDLA } & \text { TNLW } & \text { VARR } \\ \text { DEMC } & \text { NCWT } & \text { TNUT } & \text { VASC }\end{array}$

pallida Torr.

Spiny $\mathrm{H}$.

TXPS

sinensis Pers.

Chinese $\mathrm{H}$.
DCGP
DCNA
DCPG
GAUG
TNFE

sinensis Pers. var. japonica (Planch.) Nakai VATA

tala Gillies ex Planch.

GAIS

\section{CEPHALANTHUS L. RUBLACEAE}

Madder Family

occidentalis $\mathrm{L}$.

Buttonbush

$\begin{array}{lll}\text { ALAU } & \text { LAMP } & \text { SCBR } \\ \text { FLUF } & \text { MDWP }\end{array}$

CEPHALOTAXUS Sieb. \& Zucc. ex Endl.

CEPHALOTAXACEAE Plum-yew Family

'Fastigiata' [C. harringtonia (J.Knight ex Forbes) K.Koch]
ALTD FLUF
FLMG GAOH SCMG

harringtonia (J.Knight ex Forbes) K.Koch Plum Yew

$\begin{array}{llll}\text { ALEH } & \text { GAEH } & \text { MDGD } & \text { VABR } \\ \text { DCNA } & \text { GAGR } & \text { NCBE } & \text { VAGG } \\ \text { GABG } & \text { GAIS } & \text { NCCA } & \text { VAMP }\end{array}$

'Nana' [C. harringtonia (J.Knight ex Forbes) K.Koch]

SCMG 
CERATIOLA Michx.

EMPETRACEAE

Crowberry Family

ericoides Michx.

Sandheath

SCBR

CERATOSTIGMA Bunge

PLUMBAGINACEAE Leadwort Family

plumbaginoides Bunge

DCNA DEMC

willmottianum Stapf

Chinese Plumbago

SCWI

CERCIDIPHYLLUM Sieb. \& Zucc.

\section{CERCIDIPHYLLACEAE}

Katsura-tree Family

japonicum Sieb. \& Zucc.

Katsura Tree

DCAC

DCNA

DEMC

MDJS

DCCG

DCWR

GAUG

TNHT

DCDO

DEEM

$\mathrm{MDJH}$

TNMB

'Pendula' [C. japonicum Sieb. \& Zucc.] MDBG

CERCIS L.

FABACEAE (Caesalpinioideae)

Bean Family

'Alba' [C. canadensis L.]

$\begin{array}{llll}\text { ALTD } & \text { DCNA } & \text { LASL } & \text { VAWR } \\ \text { ARPT } & \text { GAEC } & \text { TNCT } & \end{array}$

'Alba' [C. canadensis L. ssp. texensis

(S.Wats.) E.Murr.]

TNTV

'Alba' [C. chinensis Bunge]

DCNA

canadensis $\mathrm{L}$.

North American Redbud

$\begin{array}{llll}\text { ALBG } & \text { DEMC } & \text { MDBG } & \text { SCRL } \\ \text { ALEH } & \text { DEWG } & \text { MDJH } & \text { SCUC } \\ \text { ALTD } & \text { FLCG } & \text { MDJS } & \text { VACW } \\ \text { ARRB } & \text { FLMG } & \text { MSHB } & \text { VAGW } \\ \text { ARUM } & \text { FLUF } & \text { NCTP } & \text { VAMP } \\ \text { DCCG } & \text { GAVI } & \text { SCCU } & \text { VATA } \\ \text { DCWH } & \text { LASL } & \text { SCMP } & \end{array}$

chinensis Bunge -

Chinese Redbud
ALIT
GACG
MDBG
MDWP
DCCG
GACW
MDDF
VACW

'Forest Pansy' [C. canadensis L.]
DCNA
TNCT
TNSC
TNTV
GAUG
TNFN
TNSM
VACW

'Oklahoma' [C. canadensis L. ssp. texensis

(S.Wats.) E.Murr.]

MDBG TNCT

'Plena' [C. canadensis L.]

FLMG SCBR

reniformis Engelm.

= C. canadensis ssp. texensis

'Rubrum' [C. siliquastrum L.] DCNA

'Ruby Atkinson' [C. canadensis L.] TNCT

'Wither's Pink Charm' [C. canadensis L.] DEWG MDKN

\section{CESTRUM L.}

SOLANACEAE

Nightshade Family

nocturnum $\mathrm{L}$.

Night Jessamine

FLUF

CHAENOMELES Lindl.

ROSACEAE

Rose Family

(Chaenomeles is an orthographic correction of the name published by Lindley in 1821 as

Choenomeles.)

'Apple Blossom' [C. speciosa (Sweet) Nakai] DEWG GACG

'Candida' [C. speciosa (Sweet) Nakai] ARPT MDHN VAMP

DEMC VACW

'Cardinalis' [C. speciosa (Sweet) Nakai] GACG

'Corallina' [C. $\times$ superba (Frahm) Rehd.]

[C. japonica $\times$ C. speciosa]

SCBR 
'Crimson and Gold' [C. $\times$ superba (Frahm) Rehd.]

[C. japonica $\times$ C. speciosa] DEWG

'Hanazono' [C. speciosa (Sweet) Nakai] GACG

japonica (Thunb.) Lindl. ex Spach

Japanese Flowering Quince MSHB

'Knap Hill Scarlet' [C. $\times$ superba (Frahm) Rehd.]

[C. japonica $\times$ C. speciosa] DEWG

lagenaria (Loisel.) Koidz.

= C. speciosa

'Mandarin' [C. $\times$ superba (Frahm) Rehd.]

[C. japonica $\times$ C. speciosa] DCNA

'Marmorata' [C. speciosa (Sweet) Nakai] GACG

'Mt. Everest' [C. $\times$ vilmoriniana Weber] [C. cathayensis $\times$ C. speciosa] GACG

'Nivalis' [C. speciosa (Sweet) Nakai] GACG

'Perfecta' [C. $\times$ superba (Frahm) Rehd.]

[C. japonica $\times$ C. speciosa] DEWG

'Phyllis Moore' [C. speciosa (Sweet) Nakai] DEWG

'Pink Lady' [C. $\times$ superba (Frahm) Rehd.]

[C. japonica $\times$ C. speciosa] GACG

'Rosy Morn' [C. × californica Clarke \& Weber]

[C. cathayensis $\times$ C. $\times$ superba] GACG

'Roxana Foster' [C. × superba (Frahm) Rehd.]

[C. japonica $\times$ C. speciosa] GACG

'Rubra' [C. speciosa (Sweet) Nakai] GACG

'Ruby Glow' [C. $\times$ superba (Frahm) Rehd.] [C. japonica $\times$ C. speciosa] GACG
'Simonii' [C. speciosa (Sweet) Nakai] GACG

'Snow' [C. speciosa (Sweet) Nakai] DEWG GACG

speciosa (Sweet) Nakai

Japanese Quince, Flowering Quince

$\begin{array}{llll}\text { DCWH } & \text { MDHN } & \text { SCBR } & \text { VACW } \\ \text { FLDG } & \text { MDLA } & \text { SCPR } & \text { VAMP } \\ \text { GAGM } & \text { MSMN } & \text { SCSR } & \\ \text { MDBG } & \text { NCWC } & \text { TXAB } & \end{array}$

'Stanford Red' [C. × superba (Frahm)

Rehd.]

[C. japonica $\times$ C. speciosa] GACG

$\times$ superba (Frahm) Rehd.

[C. japonica $\times$ C. speciosa] DCNA FLCG FLMN SCFW

'Toyo nishiki' [C. speciosa (Sweet) Nakai] GACG

'Umbilicata' [C. speciosa (Sweet) Nakai] GACG

\section{CHAMAECYPARIS Spach}

CUPRESSACEAE

False Cypress Cypress Family

'Albospica' [C. obtusa (Sieb. \& Zucc.) Endl.] DCNA

'Allumii' [C. lawsoniana (A.Murr.) Parl.] VACR

'Andelyensis' [C. thyoides (L.) BSP.] DCNA MDBG

'Aurea Nana' [C. pisifera (Sieb. \& Zucc.) Endl.] MDLT

'Blom' [C. lawsoniana (A.Murr.) Parl.] DCNA

'Boulevard' [C. pisifera (Sieb. \& Zucc.) Endl.]

$$
\text { ALBH MDBG VATA }
$$

'Breviramea' [C. obtusa (Sieb. \& Zucc.) Endl.] DCNA

'Compacta' [C. pisifera (Sieb. \& Zucc.) Endl.1 DCNA 
CHAMAECYPARIS

'Contorta' [C. obtusa (Sieb. \& Zucc.) Endl.] DCNA

'Coralliformis' [C. obtusa (Sieb. \& Zucc.) Endl.] DCNA

'Crippsii' [C. obtusa (Sieb. \& Zucc.) Endl.] DCNA MDBG NCDU

GAEC MDJS

'Cyanoviridis' [C. pisifera (Sieb. \& Zucc.)

Endl.]

$=$ 'Boulevard'

'Ericoides' [C. thyoides (L.) BSP.]

$\begin{array}{llll}\text { ALSM } & \text { FLUF } & \text { NCTP } & \text { VATW } \\ \text { DCNA } & \text { GABC } & \text { SCFW } & \\ \text { FLMG } & \text { GAEC } & \text { SCRS } & \\ \text { FLRI } & \text { MDBG } & \text { SCTR } & \end{array}$

'Filicoides' [C. obtusa (Sieb. \& Zucc.) Endl.] GAWH MDBG MDJS NCDU

'Filifera' [C. pisifera (Sieb. \& Zucc.) Endl.] DCNA MDBG VAOD GAEH VAMP VATA

'Filifera Aurea' [C. pisifera (Sieb. \& Zucc.) Endl.] DCNA MDLT VAMP

'Filiformis' [C. Lawsoniana (A.Murr.) Parl.] DCNA

'Filiformis Aurea' [C. obtusa (Sieb. \& Zucc.) Endl.] MDLT

'Filiformis Compacta' [C. Lawsoniana (A.Murr.) Parl.] DCNA

funebris (Endl.) Franco

Mourning Cypress $\begin{array}{lll}\text { GAFN } & \text { LARP } \\ \text { LAAL } & \text { LATU }\end{array}$

'Gold Drop' [C. obtusa (Sieb. \& Zucc.)

Endl.] MDLT

'Gold Spangle' [C. pisifera (Sieb. \& Zucc.) Endl.] DCNA

'Golden Mop' [C. pisifera (Sieb. \& Zucc.) Endl.] MDBG

'Gracilis' [C. obtusa (Sieb. \& Zucc.) Endl.] DCNA _ MDJS VAGG henryae $\mathrm{Li}$

= C. thyoides

'Intermedia' [C. obtusa (Sieb. \& Zucc.) Endl.] DCNA

'Juniperoides Aurea' [C. pisifera (Sieb. \& Zucc.) Endl.] DCNA

'Kosteri' [C. obtusa (Sieb. \& Zucc.) Endl.] DCNA

lawsoniana (A.Murr.) Parl.

Lawson Cypress, Port Orford Cedar FLGS SCCU VAMP VAWM

'Lutea' [C. Lawsoniana (A.Murr.) Parl.] ALTD

'Lycopodioides' [C. Lawsoniana (A.Murr.) Parl.] DCNA

'Lycopodioides' [C. obtusa (Sieb. \& Zucc.) Endl.] DCNA MDBG

'Mariesii' [C. obtusa (Sieb. \& Zucc.) Endl.] DCNA

'Monstrosa' [C. pisifera (Sieb. \& Zucc.) Endl.]

DCNA

'Nana' [C. obtusa (Sieb. \& Zucc.) Endl.] DCNA VATA

'Nana Argentea' [C. obtusa (Sieb. \& Zucc.) Endl.] DCNA

'Nana Aurea' [C. obtusa (Sieb. \& Zucc.) Endl.] DCNA

'Nana Gracilis' [C. obtusa (Sieb. \& Zucc.) Endl.] DCNA MDBG MDLT NCGC

'Nana Miko' [C. pisifera (Sieb. \& Zucc.) Endl.] = 'Snow'

'Nana Pyramidalis' [C. obtusa (Sieb. \& Zucc.) Endl.] DCNA

nootkatensis (D.Don) Spach

Alaska Cedar, Nootka False Cypress DCNA MDBG MDLT MSHB 
CHAMAECYPARIS

obtusa (Sieb. \& Zucc.) Endl.

Hinoki False Cypress

$\begin{array}{llll}\text { DCNA } & \text { MDDF } & \text { VASK } & \text { VAWM } \\ \text { GAIS } & \text { VAMP } & \text { VATA } & \end{array}$

'Pembury Blue' [C. Lawsoniana (A.Murr.) Parl.] DCNA

'Pendula Vera' [C. Lawsoniana (A.Murr.) Parl.] TNSN

pisifera (Sieb. \& Zucc.) Endl.

Sawara False Cypress

$\begin{array}{llll}\text { ALBH } & \text { MDBG } & \text { MDLA } & \text { MDSM } \\ \text { ALTD } & \text { MDHN } & \text { MDLT } & \text { VAMP } \\ \text { DCNA } & \text { MDJS } & \text { MDSJ } & \end{array}$

'Plumosa' [C. pisifera (Sieb. \& Zucc.) Endl.] DCNA

'Plumosa Aurea' [C. pisifera (Sieb. \& Zucc.) Endl.] MDJS

'Plumosa Aurea Nana' [C. pisifera (Sieb. \& Zucc.) Endl.] DCNA MDBG VATA

'Plumosa Compacta' [C. pisifera (Sieb. \& Zucc.) Endl.] DCNA MDBG VATA

'Plumosa Compressa' [C. pisifera (Sieb. \& Zucc.) Endl.] DCNA

'Pygmaea' [C. obtusa (Sieb. \& Zucc.) Endl.] DCNA

'Pygmaea' [C. pisifera (Sieb. \& Zucc.) Endl.] DCNA

'Pygmaea Aurescens' [C. obtusa (Sieb. \& Zucc.) Endl.] DCNA

'Reis Dwarf' [C. obtusa (Sieb. \& Zucc.) Endl.] DCNA

'Repens' [C. obtusa (Sieb. \& Zucc.) Endl.] DCNA

'Rigid Dwarf' [C. obtusa (Sieb. \& Zucc.) Endl.] DCNA

'Sanderi' [C. obtusa (Sieb. \& Zucc.) Endl.] ALHC DCNA
'Snow' [C. pisifera (Sieb. \& Zucc.) Endl.] DCNA

'Spiralis' [C. obtusa (Sieb. \& Zucc.) Endl.] DCNA

'Squarrosa' [C. pisifera (Sieb. \& Zucc.) Endl.]

Moss False Cypress

$\begin{array}{llll}\text { DCSH } & \text { GAEC } & \text { SCCU } & \text { VATA } \\ \text { DCWR } & \text { MDHN } & \text { VAMP } & \\ \text { FLUF } & \text { MDJS } & \text { VASK } & \end{array}$

'Squarrosa Intermedia' [C. pisifera (Sieb. \& Zucc.) Endl.] DCNA MDBG VATA

'Squarrosa Minima' [C. pisifera (Sieb. \& Zucc.) Endl.] DCNA

'Stoneham' [C. obtusa (Sieb. \& Zucc.) Endl.] DCNA

'Tempelhof' [C. obtusa (Sieb. \& Zucc.) Endl.] DCNA

'Tetragona Aurea' [C. obtusa (Sieb. \& Zucc.) Endl.]

$\begin{array}{lll}\text { DCNA } & \text { MDLT } & \text { VAMP } \\ \text { MDBG } & \text { SCCC } & \text { VATA }\end{array}$

thyoides (L.) BSP.

Atlantic White Cedar MDBG SCBR SCWI VACW

'Tonia' [C. obtusa (Sieb. \& Zucc.) Endl.] DCNA

'Verdonii' [C. obtusa (Sieb. \& Zucc.) Endl.] DCNA

CHAMAECYTISUS Link

FABACEAE (Faboideae)

Bean Family

supinus (L.) Link MDSC

CHAMAEDAPHNE Moench ERICACEAE Heath Family

calyculata (L.) Moench

Leatherleaf

ALTD MDLT VACW 
CHAMAEROPS L.

ARECACEAE

Palm Family

humilis L.

European Fan Palm FLMG FLUF TXSE

CHILOPSIS D.Don

BIGNONIACEAE

Bignonia Family

linearis (Cav.) Sweet

Desert Willow

LASL

CHIMONANTHUS Lindl.

CALYCANTHACEAE

Calycanthus Family

'Luteus' [C. praecox (L.) Link] DCNA

'Mangetsu' [C. praecox (L.) Link] DCNA

nitens Oliv. $\mathrm{MDBG}$

praecox (L.) Link

Wintersweet

$\begin{array}{llll}\text { DCAC } & \text { GAUG } & \text { SCHW } & \text { VAGS } \\ \text { DCNA } & \text { LAHG } & \text { SCUC } & \text { VAGW } \\ \text { GAHC } & \text { MDBG } & \text { TXPS } & \text { VATW } \\ \text { GAOH } & \text { MSMN } & \text { VACW } & \end{array}$

CHIONANTHUS L. OLEACEAE
Fringe Tree

Olive Family retusus Lindl. \& Paxt.

Japanese F.
DCEL
DCNA MDDF

retusus Lindl. \& Paxt. var. serrulatus

(Hayata) Koidz.

Taiwan F.

$\begin{array}{llll}\text { ALAU } & \text { FLUF } & \text { TNSN } & \text { TXDC } \\ \text { DCNA } & \text { GAGB } & \text { TNUT } & \text { VACW } \\ \text { DEWG } & \text { TNBM } & \text { TXBA } & \end{array}$

virginicus $\mathrm{L}$.

American F.T., Old-man's Beard

$\begin{array}{llll}\text { DCNA } & \text { GACG } & \text { MDJS } & \text { VAGW } \\ \text { DEWG } & \text { LAAL } & \text { MSMN } & \text { VANB } \\ \text { FLMG } & \text { LASL } & \text { SCMG } & \\ \text { FLUF } & \text { MDBG } & \text { VACP } & \\ \text { GACC } & \text { MDJH } & \text { VACW } & \end{array}$

CHOSENIA Nakai

SALICACEAE

Willow Family

arbutifolia (Pall.) Skvortz.

DCNA

CHRYSANTHEMUM L.

ASTERACEAE

Aster Family

nipponicum (Franch. ex Maxim.) Matsum.

Nippon Daisy

DCNA MDFM

CHRYSOLARIX H.E.Moore

= PSEUDOLARIX

CINNAMOMUM Schaeffer

LAURACEAE

Laurel Family

camphora (L.) T.Nees \& Eberm.

Camphor Tree

$\begin{array}{llll}\text { ALTD } & \text { FLUF } & \text { MSEN } & \text { SCCL } \\ \text { FLCG } & \text { GAIS } & \text { MSHB } & \text { SCMP } \\ \text { FLPM } & \text { GAJI } & \text { NCOP } & \text { SCUC }\end{array}$

daphnoides Sieb. \& Zucc.

LASL

CISSUS L.

VITACEAE

Grape Family

incisa (Nutt.) Desmoul.

Marine Ivy, Marine Vine

LAIS

CISTUS L.

CISTACEAE

Rock-rose Family

corbariensis Pourr.

=C. $\times$ hybridus

$\times$ hybridus Pourr.

[C. populifolius $\times$ C. salvifolius] MDFM SCWI VACW

ladanifer L.

Laudanum SCWI VACW

$\times$ purpureus Lam.

[C. ladanifer $\times$ C. villosus] SCWI VACW 

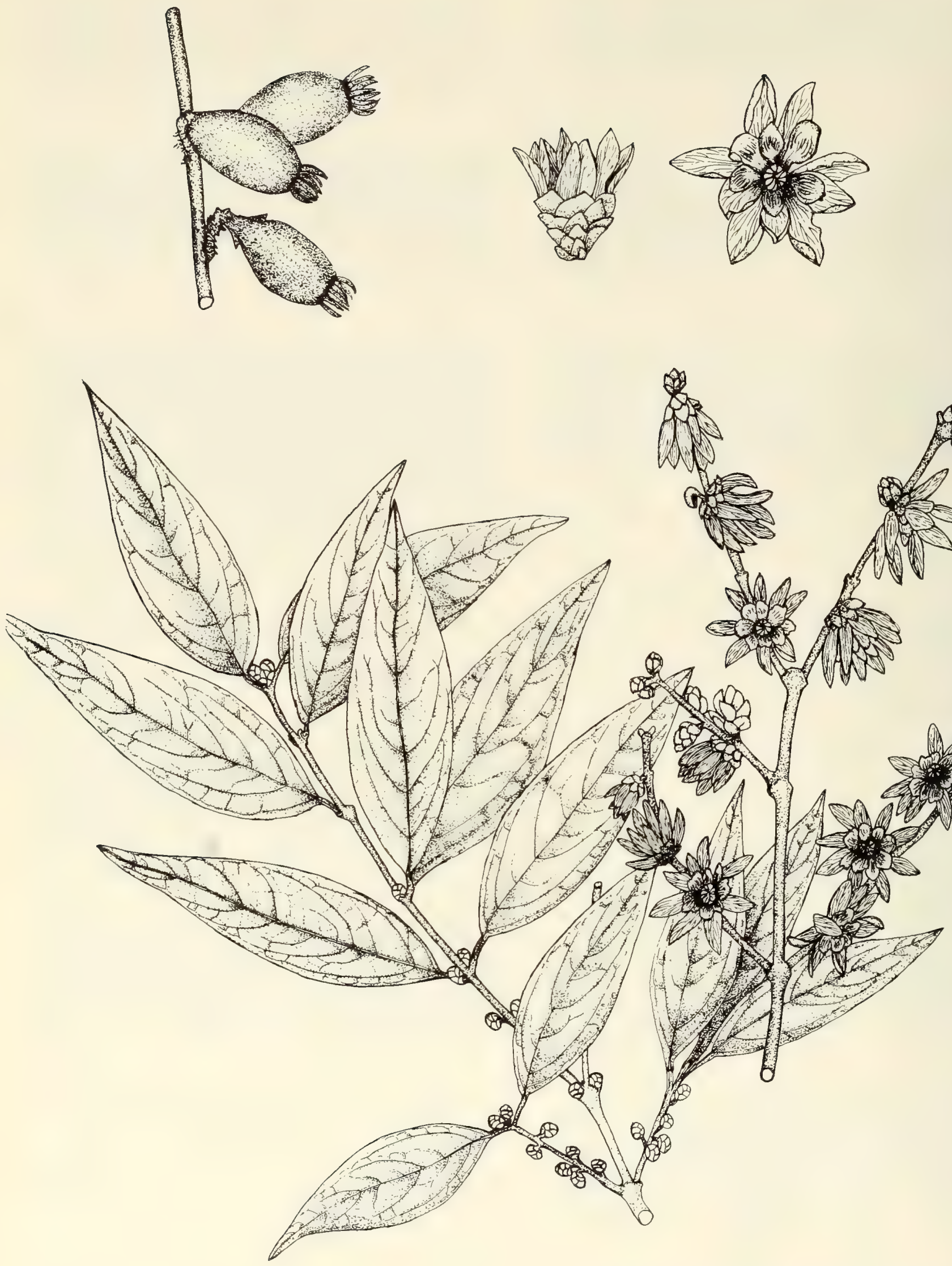

CHIMONANTHUS praecox (L.) Link

[illustrator Lillian Nicholson Meyer] 
CITHAREXYLUM L. VERBENACEAE

Verbena Family

berlandieri Robinson

Fiddlewood

TXLL

$\times$ CITROFORTUNELLA J.Ingram \&

H.E.Moore

RUTACEAE

Citrus Family

[Citrus $\times$ Fortunella]

mitis (Blanco) J.Ingram \& H.E.Moore

Calamondin

[Citrus reticulata $\times$ Fortunella ?margarita]

ALTD FLMG LALG

FLCG FLUF

$\times$ CITRONCIRUS J.Ingram \& H.E.Moore

RUTACEAE

Citrus Family

[Citrus $\times$ Poncirus]

webberi J.Ingram \& H.E.Moore

Citrange

[Citrus sinensis $\times$ Poncirus trifoliata] SCRP

CITRUS L.

RUTACEAE

Citrus Family

aurantium L.

Sour Orange, Seville Orange

FLUF

'Etrog' [C. medica L.]

Citron FLUF

hystrix DC.

FLUF

mitis Blanco

$=\times$ Citrofortunella mitis

$\times$ paradisi Macf.

Grapefruit

[C. maxima $\times$ C. sinensis]

FLUF TXJS

reticulata Blanco

Mandarin, Tangerine, Satsuma Orange

FLUF

'Thomasville Citrangequat'

[(Citrus sinensis $\times$ Poncirus trifoliata) $\times$

Fortunella sp.]

GAIS
CLADRASTIS Raf.

FABACEAE (Faboideae)

Bean Family

kentukea (Dum.-Cours.) Rudd

Yellow-wood

$\begin{array}{llll}\text { DCNA } & \text { DEWG } & \text { MDHC } & \text { VABF } \\ \text { DCWH } & \text { MDCR } & \text { TNHG } & \text { VACW } \\ \text { DEMC } & \text { MDDF } & \text { TNUT } & \text { VAMP }\end{array}$

lutea (Michx.f.) K.Koch

= C. kentukea

tinctoria Raf.

= C. kentukea

CLEMATIS L.

RANUNCULACEAE Buttercup Family

armandii Franch.

Evergreen C.

ALBG DCNA MDWP

'Betty Corning' [C. viticella L.]

DCNA MDFM MDTD

chrysocoma Franch. var. sericea (Franch.)

Schneid.

Goldwool C.

DCNA

crispa L

Blue Jasmine SCBR

dioscoreifolia Lev. \& Van.

= C. terniflora

'Farquhariana' [C. armandii Franch.]

Pink-flowered Evergreen C.

ALBG

'Grandiflora' [C. montana Buch.-Ham.] VAGS

$\times$ jackmanii T.Moore

[C. lanuginosa $\times$ C. viticella]
ALHC
MDJS
VAGS

maximowicziana Franch. \& Sav.

= C. terniflora

paniculata Thunb.

= C. terniflora

pitcheri T. \& G.

TXLL

'Rubro-marginata' [C. $\times$ triternata DC.]

[C. flammula $\times$ C. viticella]

DCNA 
spooneri Rehd. \& Wils.

= C. chrysocoma var. sericea

stans Sieb. \& Zucc. MDTD

'Superba' [C. ×jackmanii T.Moore]

[C. lanuginosa $\times$ C. viticella] MDBG MDJS

terniflora DC.

Virgin's Bower

$\begin{array}{llll}\text { ALBG } & \text { MDGD } & \text { NCOP } & \text { SCCU } \\ \text { DCNA } & \text { MSHB } & \text { SCBR } & \end{array}$

texensis Buckl.

Scarlet C.

$\mathrm{SCWI}$

versicolor Britton

Leatherflower

SCWI

viorna $\mathrm{L}$.

Vase Vine

DCNA SCBR

CLERODENDRUM L. VERBENACEAE

Verbena Family

bungei Steud. LAAL LASL VACW

fragrans (Vent.) R.Br.

= C. philippinum

indicum (L.) O.Ktze.

Tubeflower, Turk's Turban

ALBG SCBR

philippinum Schauer

Fragrant Glorybower

FLUF

thomsonae Balf.f.

Bleedingheart Glorybower

FLUF LALG

trichotomum Thunb.

Harlequin Glorybower

DCNA MDBG VATW

GAJS MDLT
CLETHRA L.

White Alder

CLETHRACEAE

White-alder Family

acuminata Michx.

DCNA GACG MDLT

alnifolia $\mathrm{L}$.

Sweet Pepperbush
DCNA
MDLT
NCOP
VATA
MDBG NCDB SCBR

barbinervis Sieb. \& Zucc.

Japanese Summersweet TNTV

fargesii Franch. DCNA

'Rosea' [C. alnifolia L.]

Pink Summersweet
DEMC
MDLT
VACW

tomentosa Lam.

Woolly Summersweet

$\begin{array}{lll}\text { ALAU } & \text { MDLT } & \text { SCBR } \\ \text { ALBG } & \text { NCBE }\end{array}$

CLEYERA Thunb.

THEACEAE

Tea Family

gymnanthera Wight \& Arn.

= Ternstroemia gymnanthera

japonica Thunb.

Japanese C.

(Thunberg originally included material from another plant, now known as Ternstroemia gymnanthera(Wight \& Arn.) Sprague, in his description of Cleyera japonica. As a result, these names have been confused in the nursery trade.)

$\begin{array}{lll}\text { ALEH } & \text { LAAL } & \text { VANB } \\ \text { GAIS } & \text { SCCU } & \text { VAWM }\end{array}$

ochnacea DC

= C. japonica

'Tricolor' [C. japonica Thunb.]

Variegated $C$. GACG

CLIFTONIA Banks ex C.F.Gaertn. CYRILLACEAE Cyrilla Family

monophylla (Lam.) Britton ex Sarg.

Buckwheat Tree, Titi

ALAU GAEC

ALBG LALG 


\section{CLINOPODIUM L.}

LAMIACEAE

Mint Family

ashei (Weatherby) Small SCWI

coccineum (Nutt.) O.Ktze. SCWI

dentatum (Chapm.) O.Ktze. SCWI

\section{CLYTOSTOMA Miers ex Bur. \\ BIGNONIACEAE Bignonia Family}

callistegioides (Cham.) Bur.

Argentine Trumpetvine

FLCG FLUF GACH TXMK

\section{CNEORUM L. \\ CNEORACEAE}

Spurge-olive Family

tricoccon $\mathrm{L}$.

Spurge-olive

SCWI

COCCULUS DC.

MENISPERMACEAE

Moonseed Family

laurifolius DC.

Laurel-leaf Snailseed
FLUF
LAAL
LALG LATU

trilobus (Thunb.) DC.

Coralbeads

GAGS

COLUTEA L.

FABACEAE (Faboideae) B Bean Family

arborescens L.

Bladder Senna

$\mathrm{VABF}$

COMPTONIA L'Her. ex Ait.

MYRICACEAE $\quad$ Wax-myrtle Family

peregrina (L.) Coult.

Sweetfern

MDWP VAHB

CONDALIA Cav. $\quad$ Buckthorn Family
RHAMNACEAE

hookeri M.C.Johnst.

TXSC

CONRADINA A.Gr.

LAMIACEAE

Mint Family

canescens (T. \& G.) A.Gr.

Bluesage C.
ALTD
GAJI
GACG SCWI
VAPH

glabra Shinners

SCWI

grandiflora Small SCWI

verticillata Jennison

Whorled C.

GAFG SCWI

CORDIA L.

BORAGINACEAE

Borage Family

boissieri A.DC.

Anacahuita

FLJH TXRE

CORIARIA L.

CORIARIACEAE Coriaria Family

japonica A.Gr.

Japanese C.

DCNA GAEC MDKN

CORNUS L.

Dogwood

CORNACEAE

Dogwood Family

alba L.

MDGD

alternifolia L.f.

Green Osier, Alternate-leaved D. MDJS TNUT VAHR

amomum Mill.

Silky D.

$\begin{array}{llll}\text { ALUA } & \text { MDLT } & \text { SCCU } & \text { VAPO } \\ \text { DCNA } & \text { NCDU } & \text { VAGW } & \end{array}$


'Argenteo-marginata' [C. alba L.]

Cream-edged D. MDBG VAGS

asperifolia Michx.

DCNA LAAL SCBR

'Aurea' [C. mas L.]

DCNA

australis C.A.Mey, var. koenigii (Schneid.) Wang.

DCNA

$$
\text { MDGD }
$$

'Bay Beauty' [C. florida L.]

Double-flowered D.

TXLL

'Cherokee Chief' [C. florida L.] DCNA

'Cherokee Princess' [C. florida L.] DCNA

'Cherokee Sunset' [C. florida L.] TNSN

'Cloud Nine' [C. florida L.] DCNA

controversa Hemsl.

Giant D.

$\begin{array}{lll}\text { DEEM } & \text { MDGJ } & \text { TNPW } \\ \text { GARW } & \text { MDMG } & \text { VAGS } \\ \text { MDBG } & \text { TNDG } & \text { VANB }\end{array}$

coreana Wang.

DCNA

drummondii C.A.Mey. TXCT

'Dwarf' [C. florida L.] DCNA

'First Lady' [C. florida L.] DCNA TNBO

'Flaviramea' [C. sericea L.] MDBG MDLT VATA

florida L.

Flowering D.

$\begin{array}{llll}\text { ALBG } & \text { FLUF } & \text { MDLT } & \text { SCUC } \\ \text { ARRB } & \text { GAJI } & \text { MDWP } & \text { SCWI } \\ \text { DCCG } & \text { GAKN } & \text { NCCS } & \text { TNMB } \\ \text { DCFM } & \text { LAAL } & \text { NCPR } & \text { VACW } \\ \text { DCNA } & \text { LAHG } & \text { NCTE } & \text { VAMP } \\ \text { DCWH } & \text { LASL } & \text { SCBR } & \text { VATA } \\ \text { FLMG } & \text { MDBG } & \text { SCFW } & \\ \text { FLRD } & \text { MDJS } & \text { SCMP } & \end{array}$

florida L. f. pluribracteata Rehd.

Double-flowered D.

$\begin{array}{llll}\text { DCCG } & \text { MDLT } & \text { NCGC } & \text { SCMG } \\ \text { FLMG } & \text { MDMM } & \text { NCPL } & \\ \text { GAEC } & \text { NCBE } & \text { SCDB } & \end{array}$

florida L. f. rubra (Weston) Schelle

Pink Flowering D.

$\begin{array}{llll}\text { ARSN } & \text { DCWH } & \text { SCPR } & \text { VAMP } \\ \text { DCCG } & \text { FLMG } & \text { VAGG } & \end{array}$

florida L. f. xanthocarpa Rehd.

Yellow-fruited Flowering D. MDCG

foemina Mill.

Stiff D.

GAUG TNHS

'Fragrant Cloud' [C. florida L.] DCNA

'Gold Star' [C. kousa Hance] DCNA

'Kesselringii' [C. alba L.] DCNA

kousa (Buerger ex Miq.) Hance Kousa D.

$\begin{array}{llll}\text { DCAC } & \text { GAEC } & \text { MDMG } & \text { TNSN } \\ \text { DCNA } & \text { GAUG } & \text { MDPJ } & \text { TNUT } \\ \text { DCPC } & \text { MDBG } & \text { MDSJ } & \text { VACW } \\ \text { DCWH } & \text { MDJH } & \text { NCBE } & \text { VAPH } \\ \text { DEWG } & \text { MDLT } & \text { TNSC } & \end{array}$

'Lustgarten Weeping' [C. Kousa (Buerger ex Miq.) Hance] MDBG TNSN

'Macrocarpa' [C. mas L.] DCNA

mas L.

Cornelian Cherry

$\begin{array}{llll}\text { DCCG } & \text { MDBG } & \text { TNCT } & \text { VAGW } \\ \text { DCNA } & \text { MDJH } & \text { TNTV } & \\ \text { DEWG } & \text { MDLT } & \text { VACW } & \end{array}$

'Milky Way' [C. kousa (Buerger ex Miq.) Hance] DCNA DEWG

'Nana' [C. mas L.] VAGS

nuttallii Audubon

Western D. DCNA

obliqua Raf.

Pale D.

GAUG NCDU SCAB TNUT


officinalis Sieb. \& Zucc.

Japanese Cornelian Cherry

$\begin{array}{lll}\text { DCNA } & \text { MDBG } & \text { TNTV } \\ \text { DEWG } & \text { MDLT } & \end{array}$

paucinervis Hance

DCNA

'Prosser Red' [C. florida L.] DCNA

racemosa Lam.

Gray D.

$$
\begin{array}{lll}
\text { DEWG } & \text { SCBR } & \text { VAHB } \\
\text { NCAS } & \text { VAGW } &
\end{array}
$$

'Rainbow' [C. florida L.] TNAB

'Royal Red' [C. florida L.] DCNA

'Rubra' [C. kousa (Buerger ex Miq.) Hance] DCNA

'Salicifolia' [C. florida L.] TNBO TNTV

sanguinea $\mathrm{L}$. DCNA

sericea L.

Red Osier

$\begin{array}{llll}\text { LARP } & \text { MSMN } & \text { TNTV } & \text { TXCT } \\ \text { MDLT } & \text { TNAB } & \text { TNUT } & \text { VACW }\end{array}$

'Sibirica' [C. alba L.]

Siberian D. ALBH

'Springtime' [C. florida L.] DCNA

'Steele's Fastigiata' [C. florida L.] DCNA

'Stokes' Pink' [C. florida L.] LAAU

stolonifera Michx.

= C. sericea

stricta Lam.

= C. foemina

'Sweetwater' [C. florida L.] DCNA

'Variegata' [C. kousa (Buerger ex Miq.) Hance] DEWG

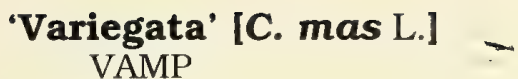

walteri Wang. DCNA

'Welch's Junior Miss' [C. florida L.] ALCW DCNA FLJU

'Welchii' [C. florida L.]

DCNA GARW MDBG

CORYLOPSIS Sieb. \& Zucc.

Winter Hazel

HAMAMELIDACEAE Witch-hazel Family

coreana Uyeki

= C. glabrescens

glabrescens Franch. \& Sav.

Fragrant W.

DCNA MDBG MDKN

gotoana Makino

= C. glabrescens

pauciflora Sieb. \& Zucc.

Buttercup W.
DCNA
MDBG VACW
DEWG MDKN VAGS

platypetala Rehd. \& Wils.

= C. sinensis var. calvescens

sinensis Hemsl. var. calvescens Rehd. \& Wils.

$\begin{array}{llll}\text { DCNA } & \text { DCPP } & \text { MDFM } & \text { VAGS } \\ \text { DCNC } & \text { MDBG } & \text { MDKN } & \end{array}$

sinensis Hemsl. var. calvescens Rehd. \& Wils. f. veitchiana (Bean) Morley \& Chao MDKN

sinensis Hemsl. var. sinensis Chinese W.
DCNA
DEWG MDFM
MDLT
DCNC
GACG MDGD VAGS

spicata Sieb. \& Zucc.

$\begin{array}{llll}\text { DCNA } & \text { MDBG } & \text { NCBE } & \text { VAPH } \\ \text { DCNC } & \text { MDKN } & \text { VACW } & \\ \text { DEWG } & \text { MDLT } & \text { VAGS } & \end{array}$

'Spring Purple' [C. sinensis Hems].

var. sinensis]

DCNA

veitchiana Bean

= C. sinensis var. calvescens $\mathrm{f}$. veitchiana 
wilmottiae Rehd.

$=\mathrm{C}$. sinensis var. sinensis

'Winterthur' [C. sinensis Hemsl.] DEWG

CORYLUS L.

Hazelnut, Filbert

BETULACEAE

Birch Family

americana Marsh.

American $\mathrm{H}$.
GAFN
MDBG SCCU

'Atropurpurea' [C. avellana L.]

= 'Fusco-rubra'

avellana $\mathrm{L}$.

European $\mathrm{H}$.

$\begin{array}{llll}\text { DCCG } & \text { DCNA } & \text { MDLA } & \text { TNUT } \\ \text { DCDO } & \text { MDGJ } & \text { MDPJ } & \end{array}$

chinensis Franch.

Chinese F. MDGD

colurna L.

Turkish F.

DCWR MDAB MDGD

'Contorta' [C. avellana L.]

Harry Lauder's Walking-stick
GAEC
MDBG VAGS
GAEH
TNUT VATA

cornuta Marsh.

Beaked F.

VAHR

'Cosford' [C. avellana L.]

$\mathrm{MDAB}$

'Fusco-rubra' [C. avellana L.]

Purple-leaved $\mathrm{H}$.

$\begin{array}{lll}\text { DCAE } & \text { DCNA } & \text { MDHN } \\ \text { DCCG } & \text { DEWG } & \text { TNSN }\end{array}$

'Potomac' [C. americana $\times$ C. avellana ] $\mathrm{MDAB}$

'Purpurea' [C. maxima Mill.]

Purple-leaved F.

TNUT

'Reed' [C. americana $\times$ C. avellana ] $\mathrm{MDAB}$

'Rush' [C. americana Walt.] MDAB

sieboldiana $\mathrm{Bl}$. DCNA MDGD

sieboldiana $\mathrm{Bl}$. var. mandschurica

(Maxim. \& Rupr.) Schneid.

DCNA

COTINUS Mill. Smoke Tree ANACARDIACEAE Cashew Family

americanus Nutt.

= C. obovatus

'Atropurpurea' [C. coggygria Scop.]

= 'Purpureus'

'Baby Doll' [C. coggygria Scop.] TNTV

coggygria Scop.

Smoke Tree

$\begin{array}{llll}\text { DCCG } & \text { MDHN } & \text { VABF } & \text { VAGW } \\ \text { DCNA } & \text { SCCU } & \text { VACP } & \text { VAMF } \\ \text { DEMC } & \text { TNSC } & \text { VACW } & \text { VAMP }\end{array}$

cotinoides (Nutt. ex Chapm.) Britton

= C. obovatus

'Flame' [C. coggygria Scop.] DCNA

'Nordine Red' [C. coggygria Scop.] DCNA TNSC

obovatus Raf.

American Smoke Tree

ALAU GAEC SCWI TNSN

ALBH MDDS TNSC

'Purpureus' [C. coggygria Scop.]

DCNA MDJS TNSC VACW

GAUG MDKN TNUT

'Royal Purple' [C. coggygria Scop.] TNSC

'Rubrifolius' [C. coggygria Scop.] DCNA

'Velvet Cloak' [C. coggygria Scop.] DCNA

COTONEASTER Medik.

ROSACEAE

Rose Family

acutifolius Turcz.

DEMC

adpressus Bois

Creeping $\mathrm{C}$.

DCNA NCWC VAGS 
COTONEASTER

ambiguus Rehd. \& Wils. VABF

apiculatus Rehd. \& Wils.

Cranberry C.

ALBH VATA

cochleatus (Franch.) Klotz.

= C. microphyllus var. cochleatus

congestus Baker VAGS

conspicuus Marq.

Wintergreen $\mathrm{C}$. DCNA GACG

'Cornubia' [C. $\times$ watereri Exell]

[C. frigidus $\times$ C. salicifolius] DCNA

dammeri Schneid. MDBG SCWI

'Decorus' [C. conspicuus Marq.] GACG SCCU

dielsianus Pritz. ex Diels

Diels C.

MDBG VABF

divaricatus Rehd. \& Wils.

Spreading C.

MDNA

floccosus (Rehd. \& Wils.) Flinck \& Hylmo DCNA NCTE

foveolatus Rehd. \& Wils. DEMC

franchetii Bois DCNA MDLT MDNA SCCU

henryanus Hort. not (Schneid.) Rehd. \& Wils.

= C. salicifolius

'Hessei' [C. adpressus Bois] DCNA

horizontalis Decne.

Rock C.

$\begin{array}{llll}\text { DCLC } & \text { GAUG } & \text { MDLT } & \text { VATA } \\ \text { DCNA } & \text { MDBG } & \text { SCCU } & \end{array}$

horizontalis Decne. var. perpusillus

Schneid.

DCNA

hupehensis Rehd. \& Wils.

= C. silvestri ignavus E.Wolf MDTD

integerrimus Medik.

European C. $\mathrm{MDJH}$

lacteus W.W.Sm. GATS GAUG GAWH

'Lowfast' [C. dammeri Schneid.] VATA

lucidus Schlechtend. TNSN

microphyllus Wall. ex Lindl. DCNA MDLT VATA

microphyllus Wall. ex Lindl. f. thymifolius (Lindl.) Koehne DCNA

microphyllus Wall. ex Lindl.

var. cochleatus (Franch.) Rehd. \& Wils. TNSN VACW

microphyllus Wall. ex Lindl. var. glacialis

Hook.f.

= C. congestus

nanshan Mottet

MDLT

nitens Rehd. \& Wils.

DEMC

nummularius Fischer \& C.A.Mey. DCNA

obscurus Rehd. \& Wils.

Bloodberry C.

SCCU VABF

'Parkteppich' [C. salicifolius Franch.] DCNA

racemiflorus (Desf.) K.Koch MDGD

racemiflorus (Desf.) K.Koch

var. songoricus (Regel \& Herd.) Schneid. DEMC

roseus Edgew. DEMC MDTD

'Royal Beauty' [C. dammeri Schneid.] GAAB VATA

salicifolius Franch.

DCNA GAAB GAWH MDBG 
salicifolius Franch. var. floccosus Rehd. \& Wils.

= C. floccosus

'Scarlet Leader' [C. salicifolius Franch.] MDBG

silvestrii Pamp.

DEWG MDTD

'Skogsholmen' [C. dammeri Schneid.] GAUG

splendens Flinck \& Hylmo

ALBH

sternianus (Turrill) Boom

DCNA MDTD

'Variegata' [C. horizontalis Decne.] MDBG MDLT

$\times$ watereri Exell

[C. frigidus $\times$ C. salicifolius] DCNA

wilsonii Nakai TNSN

zabelii Schneid. GAWH

$\times$ CRATAEGOSORBUS Makino ex

Pojark.

ROSACEAE

[Crataegus $\times$ Sorbus]

Rose Family

miczurinii Pojark.

[Crataegus sanguinea $\times$ Sorbus aucuparia] DCNA

\begin{tabular}{l}
$\begin{array}{l}\text { CRATAEGUS } \\
\text { ROSACEAE }\end{array}$ \\
\hline
\end{tabular}

aestivalis (Walt.) T. \& G.

May H.

$\begin{array}{ll}\text { DCNA } & \text { GAEC } \\ \text { FLUF } & \text { MSMN }\end{array}$

arnoldiana Sarg.

DEWG

'Autumn Glory' [C. laevigata (Poir.) DC.] DCNA

brachyacantha Sarg. \& Engelm.

Blueberry $\mathrm{H}$.

TXDM calpodendron (Ehrh.) Medik.

Pear $\mathrm{H}$.

TNGN

'Carrierei' [C. $\times$ lavallei Herincq ex Lav.]

[C. stipulacea $\times$ C. crus-galli]

DCSH VAMP

coccinioides Ashe

TNGN

compta Sarg.

MDAC

concinna Beadle

GACG

cordata Ait.

= C. phaenopyrum

crus-galli L.

Cockspur $\mathrm{H}$.

DCCG DCNG GANG TNLW

flava Ait.

Yellow $\mathrm{H}$.

FLUF

floridana Sarg.

FLUF

fulleriana Sarg.

DCRC DEEM DEWG

'Inermis' [C. monogyna Jacq.] DCNA

'Inermis' [C. punctata Jacq.] DCNA

intricata J.Lange

Thicket $\mathrm{H}$. VACW

lacrimata Small GAEC SCWI

laevigata (Poir.) DC.

English $\mathrm{H}$.

DCNA MDKN

$\times$ lavallei Herincq ex Lav.

[C. stipulacea $\times$ C. crus-galli]

DCSH VAMP

macrosperma Ashe

DCWH TNBO

marshallii Egglest.

Parsley H.

$\begin{array}{lll}\text { ARRB } & \text { LALG } & \text { TXSE } \\ \text { GACG } & \text { SCBR }\end{array}$




\section{CRATAEGUS}

mollis (T. \& G.) Scheele

Downy H.

DEMC TNGN

monogyna Jacq.

Common $\mathrm{H}$.

$\begin{array}{llll}\text { DCGW } & \text { MDBG } & \text { MDGB } & \text { VAGW } \\ \text { DCNA } & \text { MDCD } & \text { TNGN } & \text { VAMP } \\ \text { GAUG } & \text { MDCP } & \text { VACW } & \end{array}$

opaca Hook. \& Arn.

DCNA VATA

oxyacantha L.

$=$ C. laevigata

'Paul's Scarlet' [C. laevigata (Poir.) DC.] DEMC MDKN NCBE

pennsylvanica Ashe DEWG

phaenopyrum (L.f.) Medik.

Washington Thorn
DCBG
DEMC
MDAC
VAGW
DCNA
GAUG
MDLT
VATA

pinnatifida Bunge MDGD

'Plena' [C. laevigata (Poir.) DC.] MDKN

populifolia Walt. FLEM

'Praecox' [C. monogyna Jacq.] $\mathrm{DCNC}$

pringlei Sarg.

Pringle $\mathrm{H}$. MDAC

pruinosa (H.Wendl.) K.Koch var. leiophylla (Sarg.) Phipps DCCK DCNA

punctata Jacq.

Dotted $\mathrm{H}$. TNGN VAAH

schuettei Ashe DCCK

spathulata Michx.

Littlehip H. GAHC GANG TXHA

'Stricta' [C. monogyna Jacq.] DCNA
'Superba' [C. laevigata (Poir.) DC.] DCNA

'Toba' [C. $\times$ mordenensis Boom]

[C. laevigata 'Paul's Scarlet' $\times$ C. succulenta] DCNA

uniflora Moench ALAU FLUF

vaileae Britton MDCD MDGB

viridis $\mathrm{L}$.

Green H.

$\begin{array}{llll}\text { ARPT } & \text { GACG } & \text { MDUM } & \text { SCWP } \\ \text { DEWG } & \text { MDBP } & \text { SCBR } & \text { TNMB }\end{array}$

'Winter King' [C. viridis L.] DCNA

CROOKEA Small

= HYPERICUM microsepalum

CROTON L.

EUPHORBIACEAE

Spurge Family

alabamensis E.A.Sm.

Alabama C.

$\begin{array}{llll}\text { ALTD } & \text { GACG } & \text { MDLT } & \text { VACW } \\ \text { ALUA } & \text { GAEC } & \text { TNCT } & \\ \text { DCNA } & \text { MDJW } & \text { VACG } & \end{array}$

CRYPTOMERIA D.Don

TAXODIACEAE

Taxodium Family

'Bandai sugi' [C. japonica (Thunb. ex L.f.)

D.Don] MDPJ

'Cristata' [C. japonica (Thunb. ex L.f.)

D.Don] MDBG

'Dacrydioides' [C. japonica (Thunb. ex L.f.) D.Don] DCNA

'Elegans' [C. japonica (Thunb. ex L.f.)

D.Don]

MDJW MDWP VAGS 
'Enko sugi' [C. japonica (Thunb. ex L.f.)

D.Don]

DCNA

'Globosa' [C. japonica (Thunb. ex L.f.)

D.Don] LASL

'Globosa Nana' [C. japonica (Thunb. ex L.f.) D.Don] DCNA

japonica (Thunb. ex L.f.) D.Don

Japanese Cedar

$\begin{array}{llll}\text { ALBG } & \text { GAHC } & \text { MDHN } & \text { SCMP } \\ \text { ALEH } & \text { GAJI } & \text { MDJH } & \text { SCPP } \\ \text { ALFN } & \text { GATN } & \text { MDJS } & \text { TNMB } \\ \text { DCCG } & \text { LAAL } & \text { MSHB } & \text { TXAB } \\ \text { DCNA } & \text { LARP } & \text { NCDU } & \text { VAMP } \\ \text { FLMG } & \text { MDBG } & \text { SCBR } & \\ \text { FLUF } & \text { MDEF } & \text { SCDB } & \\ \text { GAAB } & \text { MDFM } & \text { SCMG } & \end{array}$

'Jindai sugi' [C. japonica (Thunb. ex L.f.)

D.Don]

$$
\text { ALFN DCNA }
$$

'Knaptonensis' [C. japonica (Thunb. ex

L.f.) D.Don]

MDBA

'Lobbii' [C. japonica (Thunb. ex L.f.) D.Don] SCCU

'Lycopodioides' [C. japonica (Thunb. ex

L.f.) D.Don]

MDJS

'Pygmaea' [C. japonica (Thunb. ex L.f.)

D.Don]

DCNA

'Vilmoriniana' [C. japonica (Thunb. ex L.f.)

D.Don]

DCNA FLUF MDBG VAGS

'Yoshino' [C. japonica (Thunb. ex L.f.)

D.Don]

DCNA

\section{CUDRANLA Trecul}

MORACEAE

Mulberry Family

tricuspidata (Carr.) Bur. ex Lav.

Silk-worm Tree

$$
\text { DCNA MDOF SCCU VABF }
$$

CUININGHAMIA R.Br.

China Fir

TAXODIACEAE

Taxodium Family

'Glauca' [C. lanceolata (Lamb.) Hook.f.]

Blue C.F.

$\begin{array}{llll}\text { GAAB } & \text { MDLT } & \text { MDPJ } & \text { VATW } \\ \text { MDJS } & \text { MDMG } & \text { TNHD } & \text { VAWR }\end{array}$

lanceolata (Lamb.) Hook.f.

China F.

$\begin{array}{llll}\text { ALTD } & \text { GACG } & \text { MSEN } & \text { TNMB } \\ \text { FLDG } & \text { GAHC } & \text { NCBE } & \text { TNSN } \\ \text { FLIF } & \text { GAIS } & \text { NCKH } & \text { TNTV } \\ \text { FLMG } & \text { GAVI } & \text { NCTE } & \text { VAMP } \\ \text { FLPS } & \text { LAAL } & \text { NCWK } & \\ \text { FLUF } & \text { LASL } & \text { SCCU } & \\ \text { GABG } & \text { MDBG } & \text { SCHA } & \end{array}$

CUPHEA P.Br.

LYTHRACEAE

Loosestrife Family

hyssopifolia HBK.

False Heather, Elfin Herb
FLUF
GACG
LAHG
LALG

micropetala HBK.

LAHG LASL

\section{$\times$ CUPRESSOCYPARIS Dallim. \\ CUPRESSACEAE \\ Cypress Family \\ [Chamaecyparis $\times$ Cupressus]}

'Leighton Green' [ $\times$ C. leylandii (Dallim. \& A.B.Jacks.) Dallim.]

[Chamaecyparis nootkatensis $\times$ Cupressus macrocarpa]

DCNA

leylandii (Dallim. \& A.B.Jacks.) Dallim. Leyland Cypress

[Chamaecyparis nootkatensis $\times$ Cupressus macrocarpa]

$\begin{array}{llll}\text { ALAU } & \text { MDLT } & \text { SCBR } & \text { VATA } \\ \text { MDBG } & \text { NCDU } & \text { SCCU } & \end{array}$

$\star$ Silver Dust $[\times$ C. leylandii (Dallim. \& A.B.Jacks.) Dallim.]

[Chamaecyparis nootkatensis $\times$ Cupressus macrocarpa]

(Sport of $\times$ C. leylandii 'Leighton Green')

$$
\begin{array}{lll}
\text { DCNA } & \text { GAEC } & \text { MDBG } \\
\text { GACG } & \text { LASL } & \text { VATA }
\end{array}
$$


CUPRESSUS L.

Cypress CUPRESSACEAE

Cypress Family

arizonica Greene

$\begin{array}{lll}\text { ALBG } & \text { GAAB } & \text { GAUG } \\ \text { ARHS } & \text { GAEC } & \text { SCCU } \\ \text { FLUF } & \text { GAPM } & \text { VATA }\end{array}$

funebris Endl.

= Chamaecyparis funebris

'Gareei' [C. arizonica Greene] FLUF

lusitanica Mill.

Portuguese C. FLMG FLUF

macrocarpa Hartw.

Monterey C. SCHA

sempervirens $\mathrm{L}$.

Italian C.

$\begin{array}{llll}\text { ALSH } & \text { GAJI } & \text { MSHB } & \text { VAGW } \\ \text { FLMG } & \text { GANG } & \text { SCMG } & \\ \text { FLUF } & \text { GASU } & \text { VACW } & \end{array}$

CYCAS L.
CYCADACEAE
Cycad Family

circinalis L.

Queen Sago

FLUF

revoluta Thunb.

Sago Palm

$\begin{array}{lll}\text { ALBG } & \text { FLMG } & \text { SCNS } \\ \text { ALSM } & & \\ & \text { FLUF } & \text { TXJS }\end{array}$

CYDONIA Mill.

Quince ROSACEAE

Rose Family

oblonga Mill.

Common $\mathrm{Q}$.

$\begin{array}{llll}\text { DCNA } & \text { MDGD } & \text { NCTP } & \text { VACW } \\ \text { MDAB } & \text { MSHB } & \text { SCUC } & \text { VASK }\end{array}$

sinensis Thouin

= Pseudocydonia sinensis
CYRILLA Gard. ex L. CYRILLACEAE

Cyrilla Family

racemiflora $\mathrm{L}$.

Leatherwood, Titi

$\begin{array}{lll}\text { ALAU } & \text { LALG } & \text { NCBE } \\ \text { FLMG } & \text { LASL } & \text { NCGP } \\ \text { GAIS } & \text { MDLT } & \text { SCBR }\end{array}$

CYTISUS Desf. Broom

FABACEAE (Faboideae) Bean Family

'Andreanus' [C. scoparius (L.) Link] DEMC VAGS

battandieri Maire DCNA

commutatus (Willd.) Briq. MDSC

decumbens (Durande) Spach MDSC

'Hollandia' [C. $\times$ praecox Bean]

[C. multiflorus $\times$ C. purgans] DCNA

'Moonlight' [C. $\times$ praecox Bean]

[C. multiflorus $\times$ C. purgans] MDBG

$\times$ praecox Bean

Warminster B.

[C. multiflorus $\times$ C. purgans] $\begin{array}{ll}\text { DEWG } & \text { MDKN } \\ \text { GACG } & \text { MDSC }\end{array}$

scoparius (L.) Link Scotch B.
DEMC
NCGR
VAMP
GACG
VAGS
VANB

DALBERGIA L.f.

FABACEAE (Faboideae)

Bean Family

hupeana Hance DCNA

sissoo Roxb. ex DC.

Sissoo

FLUF 
DANAE Medik.

LILIACEAE

Lily Family

racemosa (L.) Moench

Alexandrian Laurel
GACG
MDMG
NCWM
GAJS NCDU VAGW
GAJY NCOP VANB

DAPHNE L.

THYMELAEACEAE Mezereum Family

'Alba' [D. odora Thunb. ex J.A.Murr.] VAPH

'Albo-marginata' [D. odora Thunb. ex J.A.Murr.]

$\begin{array}{lll}\text { DCNA } & \text { GAHC } & \text { NCEG } \\ \text { FLDG } & \text { MDLT } & \text { VANB }\end{array}$

'Aureo-marginata' [D. odora Thunb. ex J.A.Murr.] SCMP

'Carol Mackie' [D. $\times$ burkwoodii Turrill]

[D. caucasica $\times D$. cneorum] VAPH

caucasica Pall. MDLT

cneorum L.

Garland Flower, Rose D. MDLT VAPH

genkwa Sieb. \& Zucc.

Lilac D.

$$
\text { DCNA MDLT VAGS }
$$

kamtschatica Maxim. var. jezoensis

(Maxim.) Ohwi

DCNA

'Manten' [D. × mantensiana T.M.C.Taylor \& F. Vrugtman]

[D. $\times$ burkwoodii $\times$ D. retusa $]$

DCNA MDLT

mezereum L.

February Daphne, Mezereon MDLT

odora Thunb. ex J.A.Murr.

Winter D.

$$
\begin{array}{lll}
\text { GACG } & \text { NCEG } & \text { SCMP } \\
\text { GATS } & \text { SCBR }
\end{array}
$$

'Ringmaster' [ $\boldsymbol{D}$. odora Thunb. ex J.A.Murr.]
'Ruby Glow' [D. cneorum L.] DEMC

'Somerset' [D. X burkwoodii Turrill]

[D. caucasica $\times$ D. cneorum] DEWG GACG VAPH

tangutica Maxim. MDLT

'Zuiko nishiki' [D. odora Thunb. ex J.A.Murr.] MDBG

DAPHNIPHYLLUM Bl. EUPHORBIACEAE

Spurge Family

macropodum Miq.

ALEH DCNA FLRP LAAL

DATURA L.

SOLANACEAE Nightshade Family

suaveolens Humboldt \& Bonpland ex Willd.

= Brugmansia suaveolens

DAUBENTONIA DC.

= SESBANIA

DAVIDIA Baill.

NYSSACEAE

Sour-gum Family

involucrata Baill. var. vilmoriniana (Dode)

Wang.

Dove Tree

$\begin{array}{lll}\text { DCNA } & \text { MDHA } & \text { VAAH } \\ \text { MDDF } & \text { MDHW } & \text { VAWR }\end{array}$

DECUMARIA L

SAXIFRAGACEAE (Hydrangeoideae)

Saxifrage Family

barbara L.

Wood-vamp

SCBR TNTV

DENDROPANAX Decne. \& Planch. ARALIACEAE

Ginseng Family

trifidus (Thunb. ex J.A.Murr.) Makino ex Hara SCWI 


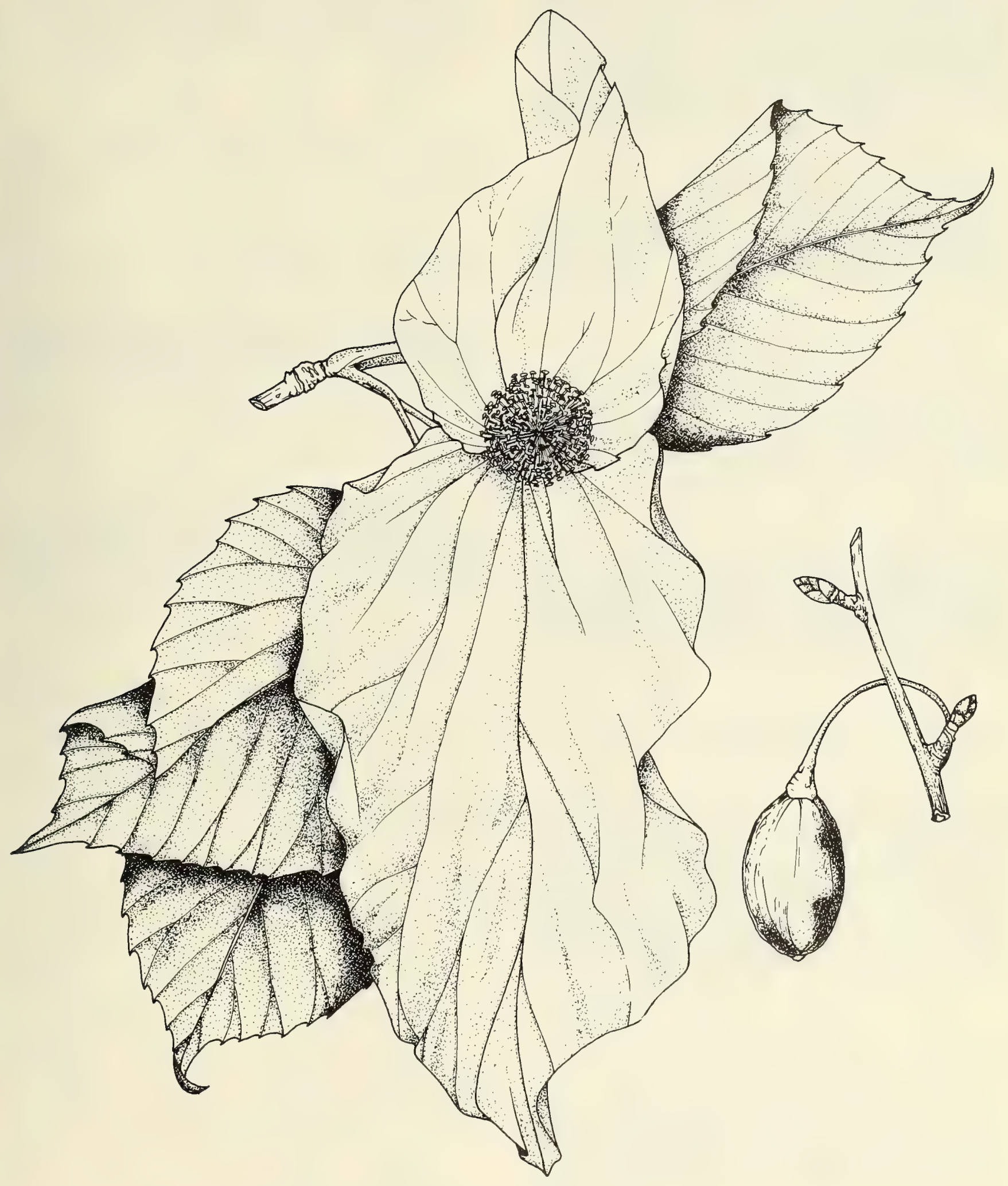

DAVIDIA involucrata var. vilmoriniana (Dode) Wang.

[illustrator Susan M. Johnston] 


\section{DESMOTHAMNUS Small}

= LYONLA

DEUTZIA Thunb.

SAXIFRAGACEAE (Hydrangeoideae)

Saxifrage Family

$\times$ candelabrum (Lem.) Rehd.

[D. gracilis $\times D$. sieboldiana]

DEWG

$\times$ candida (Lem.) Rehd.

[D. $\times$ lemoinei $\times$ D. sieboldiana] MDGD

'Candidissima' [D. scabra Thunb.]

$\begin{array}{llll}\text { DCSH } & \text { GAOH } & \text { LATU } & \text { MSHB } \\ \text { DEWG } & \text { GARW } & \text { MDGD } & \text { NCCA } \\ \text { FLDJ } & \text { GAUG } & \text { MDPJ } & \text { VABF } \\ \text { GACD } & \text { LAHG } & \text { MDSJ } & \end{array}$

'Carminea' [D. $\times$ rosea (Lem.) Rehd.]

[D. gracilis $\times D$. purpurascens]

DEWG MDSG

chunii H.H.Hu

DEWG

'Conspicua' [D. Xelegantissima (Lem.)

Rehd.]

[D. purpurascens $\times$ D. sieboldiana] MDGD

'Contraste' [D. $\times$ hybrida E.Lemoine]

$[D$. discolor $\times D$. longifolia] MDGD

discolor Hemsl. MDGD

'Eburnea' [D. $\times$ magnifica (Lem.) Rehd.]

[D. scabra $\times$ D. vilmoriniae] MDGD

$\times$ elegantissima (Lem.) Rehd.

[D. purpurascens $\times D$. sieboldiana] DEMC MDGD

'Eminens' [D. $\times$ magnifica (Lem.) Rehd.]

[D. scabra $\times$ D. vilmoriniae] MDGD

'Erecta' [D. $\times$ magnifica (Lem.) Rehd.]

[D. scabra $\times$ D. vilmoriniae] MDGD

'Formosa' [D. $\times$ magnifica (Lem.) Rehd.] [D. scabra $\times D$. vilmoriniae] MDGD glabrata Komar. DCNA

gracilis Sieb. \& Zucc.

$\begin{array}{llll}\text { DCWR } & \text { GAOH } & \text { MDJS } & \text { VADW } \\ \text { DEEM } & \text { GAUG } & \text { MDLT } & \text { VAGS } \\ \text { DEWG } & \text { LAHG } & \text { SCFR } & \\ \text { GAFG } & \text { MDCP } & \text { VACW } & \end{array}$

'Latiflora' [D. $\times$ magnifica (Lem.) Rehd.]

[D. scabra $\times$ D. vilmoriniae] MDGD

$\times$ lemoinei E.Lemoine ex Bois

[D. gracilis $\times$ D. parviflora] DCDO MSHB

'Magicien' [D. Xhybrida E.Lemoine]

[D. discolor $\times D$. longifolia] DEWG MDGD

$\times$ magnifica (Lem.) Rehd.

[D. scabra $\times$ D. vilmoriniae] DEWG MDGD MDPJ

'Mohican' [D. gracilis Sieb. \& Zucc.] MDBG

'Nikko' [D. gracilis Sieb. \& Zucc.] DCNA

'Plena' [D. scabra Thunb.]
DCSH MDPJ NCCW
GABS MDSJ

'Pride of Rochester' [D. scabra Thunb.] VAAC

$\times$ rosea (Lem.) Rehd.

[D. gracilis $\times D$. purpurascens] MDKN MDPJ

scabra Thunb.

Rough-leaved Deutzia

$\begin{array}{llll}\text { DCSH } & \text { GAOH } & \text { SCMG } & \text { VAWP } \\ \text { DEEM } & \text { LAAL } & \text { SCMP } & \\ \text { DEWG } & \text { NCBE } & \text { VAGW } & \end{array}$

schneideriana Rehd. DEWG

sieboldiana Maxim. DCNA LALG

'Summer Snow' [D. scabra Thunb.] MDBG MDMG

'Suspensa' [D. scabra Thunb.] MDGD

'Watereri' [D. scabra Thunb.] $\mathrm{GAOH}$ 
DIERVILLA Mill. Bush Honeysuckle CAPRIFOLIACEAE Honeysuckle Family (Contributed by T. R. Dudley)

lonicera Mill.

Dwarf Bush $\mathrm{H}$. MDWP NCDB VAGS

rivularis Gatt.

Georgia Bush H. $\mathrm{SCCU}$

Xsplendens (Carr.) Kirchn.

[D. lonicera $\times$ D. sessilifolia] DEWG

\section{DIOON Lindl.}

CYCADACEAE

Cycad Family

edule Lindl.

Chestnut D.

TXPS

DIOSPYROS L.

Persimmon

EBENACEAE

Ebony Family

'Hagabushi' [D. kaki L.f.] MDAB

kaki L.f.

Kaki, Japanese P. DCCA DCHM TXHL DCCL SCCC VANB

'Kyungsun bansi' [D. kaki L.f.] MDAB

sinensis Hemsl.

Chinese P.

$$
\text { DCNA GAIS }
$$

texana Scheele

Texas $\mathrm{P}$.

$$
\text { TXDC TXDM TXPS }
$$

virginiana $L$.

Common P.

$\begin{array}{llll}\text { ALAU } & \text { DEWG } & \text { MDLT } & \text { TNSN } \\ \text { DCCG } & \text { MDAB } & \text { MSEP } & \text { VAMP } \\ \text { DCSH } & \text { MDBP } & \text { SCBR } & \end{array}$

DIPELTA MaXim.

CAPRIFOLIACEAE

(Contributed by T. R. Dudley)

Honeysuckle Family

floribunda Maxim.

MDGD

yunnanensis Franch.

DCNA

DIRCA L.

THYMELAEACEAE Mezereum Family

palustris $\mathrm{L}$.

Leatherwood MDLT VACW

DISANTHUS MaXim.

HAMAMELIDACEAE Witch-hazel Family

cercidifolius Maxim.

MDBG MDFM MDJS MDKN

DISTYLIUM Sieb. \& Zucc.

HAMAMELIDACEAE Witch-hazel Family

'Akebono' [D. racemosum Sieb. \& Zucc.] MDBG

racemosum Sieb. \& Zucc.

ALTD FLRH GAIS TXPS

DCNA FLUF LASL VAWM

DOXANTHA Miers

= MACFADYENA

DURANTA L.

VERBENACEAE Verbena Family

repens $\mathrm{L}$.

Creeping Skyflower
FLMG
GACG
LAAL
FLUF GAJI LASL

EDGEWORTHIA Meisn.

THYMELAEACEAE Mezereum Family

chrysantha Lindl.

Paperbush

GACG GAEC MDFM

papyrifera Sieb. \& Zucc.

= E. chrysantha 


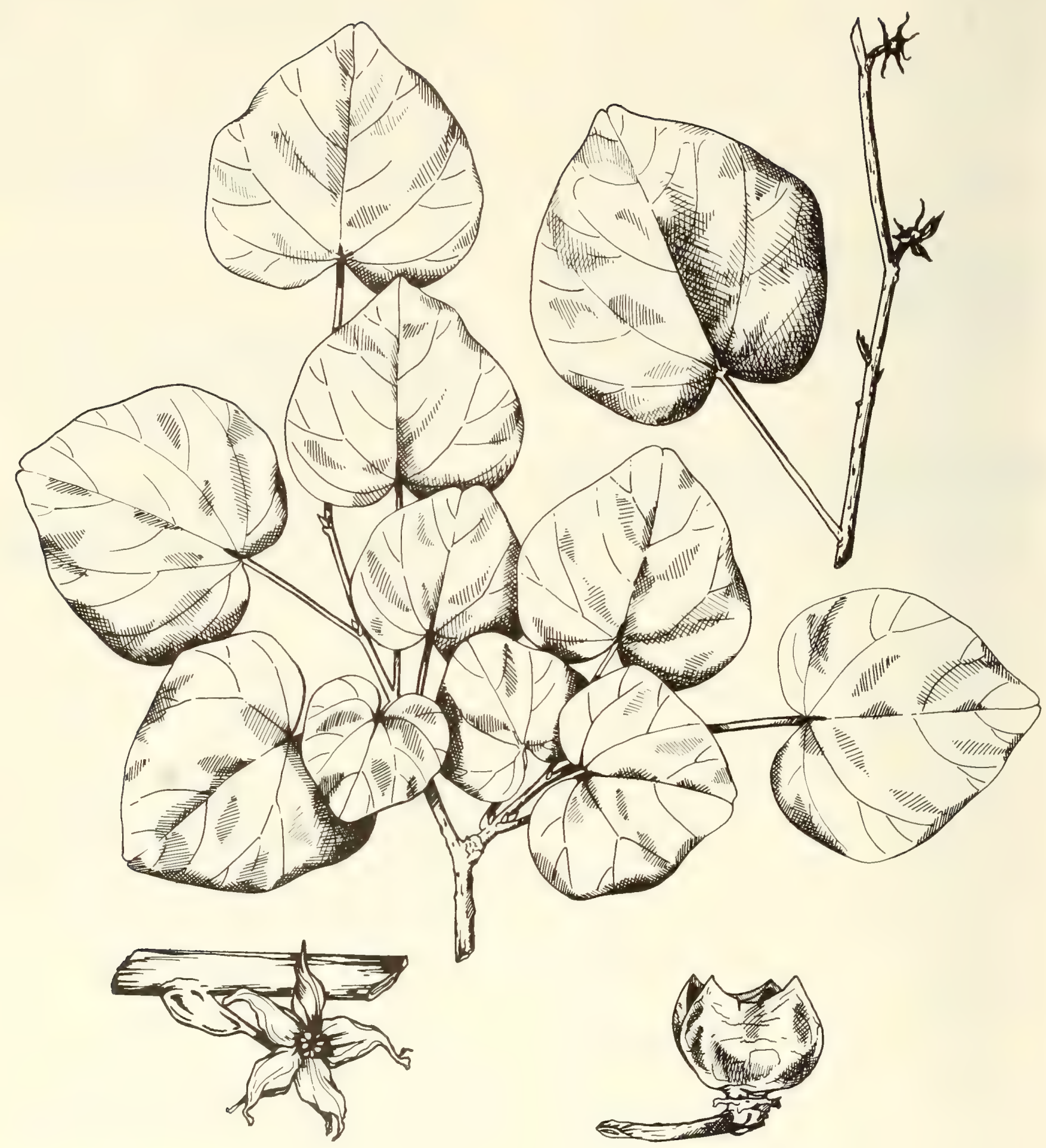

DISANTHUS cercidifolius Maxim.

[illustrator Susan M. Johnston] 
EHRETIA P.Br. BORAGINACEAE

Borage Family

acuminata R.Br. var. serrata (Roxb.)

I.M.Johnst. FLUF

anacua (Teran \& Berl.) I.M.Johnst. LAAL TXAB TXGM TXPS

thyrsiflora (Sieb. \& Zucc.) Nakai

= E. acuminata var. serrata

ELAEAGNUS L.

ELAEAGNACEAE

Oleaster Family

angustifolia L.

Russian Olive MDJS MDSJ

'Aurea' [E. pungens Thunb. ex J.A.Murr.] SCMA

'Aureo-variegata' [E. pungens Thunb. ex J.A.Murr.]

= 'Maculata'

$\times$ ebbingei Doorenbos

[E. macrophylla $\times$ E. pungens] VATA

'Ebbingei'

= E. Xebbingei

'Fruitlandii' [E. pungens Thunb. ex

J.A.Murr.]

GABS GAFN SCMP

glabra Thunb. ex J.A.Murr. SCBR

macrophylla Thunb.

MDBG

'Maculata' [E. pungens Thunb. ex J.A.Murr.] ALAU FLMG GACG

multiflora Thunb. ex J.A.Murr.

Cherry E., Gumi MDKN SCCU

pungens Thunb. ex J.A.Murr.

Thorny E.

$\begin{array}{llll}\text { ALAU } & \text { GAUG } & \text { SCCL } & \text { VANB } \\ \text { ALIT } & \text { MDLT } & \text { SCDB } & \\ \text { ARHS } & \text { NCBE } & \text { TNDG } & \\ \text { GAJS } & \text { NCDU } & \text { TNUT } & \end{array}$

umbellata Thunb. ex J.A.Murr.

Autumn Olive

$\begin{array}{llll}\text { ALON } & \text { LASL } & \text { SCBR } & \text { VAGW } \\ \text { ALTD } & \text { MDLT } & \text { SCCU } & \text { VAWR } \\ \text { DCNA } & \text { MDPJ } & \text { SCDB } & \\ \text { DEMC } & \text { MSHB } & \text { SCMM } & \\ \text { LAHG } & \text { NCBE } & \text { VACW } & \end{array}$

'Variegata' [E. pungens Thunb. ex J.A.Murr.] VATA

ELEUTHEROCOCCUS MaXim.

ARALIACEAE Ginseng Family

gracilistylus (W.W.Sm.) S.Y.Hu MDBG

sieboldianus (Makino) Koidz. VATA

trifoliatus (L.) S.Y.Hu

FLUF GATS

ELLIOTTIA Muhl. ex Ell. ERICACEAE

Heath Family

bracteata (Maxim.) Hook.f. MDLT

racemosa Muhl. ex Ell.

Southern Plume

GATS MDFM NCBE

ENKIANTHUS LOUR.

ERICACEAE

Heath Family

'Ambassador' [E. campanulatus (Miq.)

Nichols.] MDKN

campanulatus (Miq.) Nichols. DEWG MDBG MDLT NCBE GAEC MDJS MDMG

perulatus (Miq.) Schneid.

DCNA DEWG MDBG VAMP

ERICA L.

Heath

ERICACEAE Heath Family

carnea L.

Spring $\mathrm{H}$.

DCNA MDBG VATW 
'Silberschmelze' [E. $\times$ darleyensis Bean]

$[E$. carnea $\times$ E. mediterranea] MDWP

'Springwood Pink' [E. carnea L.] DCNA MDWP

'Springwood White' [E. carnea L.] DCNA MDBG

vagans $\mathrm{L}$.

Cornish $\mathrm{H}$.

NCBE VAGS

ERIOBOTRYA Lindl. Loquat ROSACEAE Rose Family

deflexa (Hemsl.) Nakai FLUF LASL

japonica (Thunb.) Lindl.

Loquat

$\begin{array}{llll}\text { ALBG } & \text { FLUF } & \text { NCEL } & \text { VACW } \\ \text { ALTM } & \text { GADG } & \text { SCBH } & \text { VANB } \\ \text { DCNA } & \text { GAJI } & \text { SCEM } & \\ \text { FLMG } & \text { GATS } & \text { SCNS } & \end{array}$

ERYTHRINA L.

FABACEAE (Faboideae)

Bean Family

arborea (Chapm.) Small

= E. herbacea

$\times$ bidwillii Lindl.

[E. crista-galli $\times$ E. herbacea]

LAMP

crista-galli L.

Cockspur Coral Tree

$$
\begin{array}{lll}
\text { ALTD } & \text { LAAL } & \text { LANR } \\
\text { FLUF } & \text { LAMP } & \text { MSHB }
\end{array}
$$

herbacea L.

Coral Bean

$$
\text { DCNA MSEP SCBR }
$$

herbacea L. var. arborea Chapm.

= E. herbacea
ESCALLONIA Mutis ex L.f.

SAXIFRAGACEAE (Escallonioideae)

Saxifrage Family

bifida Link \& Otto

FLUF SCBG VANB

GACH SCBR

montevidensis (Cham. \& Schlechtend.) DC.

$=$ E. bifida

rosea Griseb.

SCJF

rubra (Ruiz \& Pavon) Pers.

LASL MSHB

EUCALYPTUS L'Her.

MYRTACEAE

Myrtle Family

camaldulensis Dehnh.

FLIF

coccifera Hook.f.

Tasmanian Snow Gum

$\mathrm{SCJF}$

dalrympleana Maiden

Mountain Gum

SCJF

gunnii Hook.f.

Cider Gum

SCJF

robusta $\mathrm{Sm}$.

Swamp Mahogany

FLUF LAAP TXZH

viminalis Labill.

Manna Gum

ALEH

EUCOMMIA Oliv. EUCOMMIACEAE

Eucommia Family

ulmoides Oliv.

Chinese Rubber Tree

MDGD TNSN VABF VATA 
EUODIA J.R. \& J.G. Forst.

\section{RUTACEAE}

Citrus Family

daniellii (Benn.) Hemsl.

$\begin{array}{llll}\text { DCNA } & \text { MDGD } & \text { NCWM } & \text { VACW } \\ \text { GAEH } & \text { NCJP } & \text { VABF } & \text { VAPC }\end{array}$

EUONYMUS L.

Spindle Tree

CELASTRACEAE

Staff-tree Family

alatus (Thunb. ex J.A.Murr.) Sieb.

Winged Spindle T.

$\begin{array}{llll}\text { DCDO } & \text { MDGJ } & \text { MDMG } & \text { VACP } \\ \text { DEWG } & \text { MDJH } & \text { NCBE } & \text { VACW } \\ \text { GACG } & \text { MDJS } & \text { NCWC } & \text { VADW } \\ \text { LAHG } & \text { MDLA } & \text { SCCU } & \text { VAGW } \\ \text { MDBG } & \text { MDLT } & \text { TNMB } & \text { VASK }\end{array}$

alatus (Thunb. ex J.A.Murr.) Sieb.

f. apterus (Regel) Rehd.

MDDF

'Albo-marginatus' [E. japonicus Thunb.] ALHC VATW

'Aldenhamensis' [E. europaeus L.] TNSN TNTV

americanus $\mathrm{L}$.

Strawberry Bush

$\begin{array}{llll}\text { GABQ } & \text { MDPL } & \text { SCWI } & \text { TXSE } \\ \text { GAEC } & \text { NCDU } & \text { TXLL } & \\ \text { MDLT } & \text { SCBR } & \text { TXPS } & \end{array}$

'Argenteo-marginatus' [E. fortunei (Turcz.)

Hand.-Mazz.]

= 'Variegatus'

'Argenteo-variegatus' [E. japonicus

Thunb.]

LALG NCWM

'Aureo-marginatus' [E. japonicus Thunb.] FLMG LALG LASL TNCT

'Aureus' [E. japonicus Thunb.]

ALBG GAIS MSRN

FLMG LALG VADW

bungeanus Maxim.

Winterberry Spindle T.

$\begin{array}{llll}\text { DCNA } & \text { GAPN } & \text { MDHN } & \text { TXBA } \\ \text { FLUF } & \text { LASL } & \text { NCDU } & \text { VASK }\end{array}$

bungeanus Maxim. var. semipersistans (Rehd.) Schneid.

DEWG
'Compactus' [E. alatus (Thunb. ex

J.A.Murr.) Sieb.]

$\begin{array}{lll}\text { DCNA } & \text { GAUG } & \text { SCCU } \\ \text { GACG } & \text { MDLT } & \text { VATA }\end{array}$

'Duc d'Anjou' [E. japonicus Thunb.] FLUF

'Emerald Gaiety' [E. fortunei (Turcz.)

Hand.-Mazz.]

(Plant Patent No. 1,960)

GACG TNTV

europaeus L.

European Spindle T.

GAUG NCBE

fortunei (Turcz.) Hand.-Mazz.

Wintercreeper Spindlebush

ARWS GAJY NCEL VACP

DEWG NCDU NCWC VATA

fortunei (Turcz.) Hand.-Mazz. var. radicans (Miq.) Rehd. DCNA FLPM

'Gracilis' [E. fortunei (Turcz.) Hand.-Mazz.] ARSN DCNA GACD LALG

'Harlequin' [E. fortunei (Turcz.)

Hand.-Mazz.]

DCNA MDBG

japonicus Thunb.

Japanese Spindle T.

$\begin{array}{lll}\text { DCNA } & \text { MDNA } & \text { NCWC } \\ \text { GAIS } & \text { MDRT } & \text { SCCG } \\ \text { MDJH } & \text { MSHB } & \text { SCUC }\end{array}$

kiautschovicus Loesn.

$\begin{array}{llll}\text { GAEH } & \text { GAUG } & \text { SCCU } & \text { VABF } \\ \text { GAMM } & \text { MDPJ } & \text { SCUC } & \text { VAWR }\end{array}$

lanceolatus Yatabe

DCNA

'Longwood' [E. fortunei (Turcz.)

Hand.-Mazz.]

MDMG

maackii Rupr.

MDGD TXGS

'Macrophyllus' [E. japonicus Thunb.] DCNA

'Manhattan' [E. kiautschovicus Loesn.] DCNA GACG MDLT

'Microphyllus' [E. japonicus Thunb.] FLMN GAUG MSMN VACW GACG LAHG SCMG 
'Minima' [E. fortunei (Turcz.) Hand.-Mazz.] NCDU

nanus Bieb. VAGS

patens Rehd.

$=$ E. kiautschovicus

'Sarcoxie' [E. fortunei (Turcz.) Hand.-

Mazz.] DCNA

sieboldianus $\mathrm{Bl}$.
DCNA TNTV VABF

'Silver King' [E. japonicus Thunb.] FLUF

'Silver Queen' [E. fortunei (Turcz.) Hand.Mazz.] LALG MDET VATA

'Variegatus' [E. fortunei (Turcz.) Hand.Mazz.] GACB VAHF

wilsonii Sprague GACG

EUPHORBIA L. Spurge EUPHORBLACEAE Spurge Family

characias L. ssp. wulfenii (Hoppe ex

K.Koch) A.R.Sm. SCWI

leucocephala Lotsy

Pascuita FLUF

pulcherrima Willd.

Poinsettia FLUF

EUPTELEA Sieb. \& Zucc. EUPTELEACEAE Euptelea Family

polyandra Sieb. \& Zucc. MDMG

EURYA Thunb.

THEACEAE Tea Family acuminata DC. GAIS
'Confetti' [E. japonica Thunb.] MDBG

emarginata (Thunb. ex J.A.Murr.) Makino

$\begin{array}{llll}\text { ALEH } & \text { FLMG } & \text { LASL } & \text { SCBY } \\ \text { ALTD } & \text { FLUF } & \text { SCBP } & \\ \text { FLLE } & \text { LAAL } & \text { SCBR } & \end{array}$

'Harmony' [E. japonica Thunb.] MDBG

japonica Thunb.

ALEH ALTD DCNA LAAL

ochnacea (DC.) Szysz.

= Cleyera japonica

$\star$ 'Winter Wine' [E. japonica Thunb.] DCNA

EUSCAPHIS Sieb. \& Zucc.

STAPHYLEACEAE

Bladdernut Family

japonica (Thunb. ex J.A.Murr.) Kanitz DCNA

EVODIA Lam.

= EUODLA

EXOCHORDA Lindl.

ROSACEAE

Rose Family

giraldii Hesse

= E. racemosa

racemosa (Lindl.) Rehd.

Pearlbush

$\begin{array}{llll}\text { ALBH } & \text { MDAB } & \text { SCCU } & \text { VAGS } \\ \text { ALIT } & \text { MDAF } & \text { SCDB } & \text { VAGW } \\ \text { DCNA } & \text { MDJH } & \text { SCEI } & \text { VAMP } \\ \text { DEWG } & \text { MDLA } & \text { SCMM } & \text { VATA } \\ \text { GAHC } & \text { MDSS } & \text { SCMP } & \\ \text { LAAL } & \text { MSEP } & \text { TNRM } & \\ \text { LASL } & \text { NCOP } & \text { VACW } & \end{array}$

'The Bride' [E. $\times$ macrantha (V.Lemoine) Schneid.]

[E. korolkowii $\times$ E. racemosa]

DCNA DEWG MDLT VAPH

FAGUS L.

Beech

FAGACEAE Beech Family

'Asplenifolia' [F. sylvatica L.] DCFP DCWH DEMC NCKH 
'Atropunicea' [F. sylvatica L.]

= F. sylvatica f. purpurea

'Cristata' [F. sylvatica L.] MDAN

'Dawyck' [F. sylvatica L.]

Columnar Beech DEWG MDBG MDHC TNSN

'Fastigiata' [F. sylvatica L.]

= 'Dawyck'

grandifolia Ehrh.

American B.

$\begin{array}{llll}\text { ALBG } & \text { DEMC } & \text { MDWP } & \text { TNHD } \\ \text { ALMC } & \text { DEWG } & \text { NCBE } & \text { TNHG } \\ \text { ALSH } & \text { GAEH } & \text { NCTE } & \text { TNSC } \\ \text { DCCG } & \text { GAUG } & \text { NCTP } & \text { VAGG } \\ \text { DCDO } & \text { MDBG } & \text { SCBR } & \text { VAMP } \\ \text { DCNA } & \text { MDHN } & \text { SCKG } & \text { VAWR } \\ \text { DCWH } & \text { MDLA } & \text { SCMP } & \end{array}$

'Laciniata' [F. sylvatica L.]

Fernleaf Beech

TNSM

'Pendula' [F. sylvatica L.]

Weeping Beech DCAC DEWG VABR VAHC

'Purpurea Tricolor' [F. sylvatica L.] MDKN

'Quercifolia' [F. sylvatica L.] DCNA NCBE

'Rohanii' [F. sylvatica L.] DCNA MDAN TNSN TNTV

'Rotundifolia' [F. sylvatica L.] DCNA MDAN MDBG

sylvatica $\mathrm{L}$.

European B.

DCCG DCWH MDCP VASS

sylvatica L. f. purpurea (Ait.) Schneid. Purple B.

$\begin{array}{llll}\text { DCCG } & \text { DCWH } & \text { MDHC } & \text { MDLA } \\ \text { DCDO } & \text { DEMC } & \text { MDHN } & \text { NCBE } \\ \text { DCMS } & \text { MDBR } & \text { MDJS } & \end{array}$

'Tricolor' [F. sylvatica L.]

= 'Purpurea Tricolor'

'Zlatia' [F. sylvatica L.]

MDKN

\section{FARGESIA Franch.}

POACEAE

Grass Family

FALLUGLA Endl.

ROSACEAE

Rose Family

paradoxa (D.Don) Endl.

Apache Plume

VACW

spathacea Franch.

= Thamnocalamus spathaceus

$\times$ FATSHEDERA Guill.

ARALIACEAE
$[$ Fatsia $\times$ Hedera]

lizei (Cochet) Guill.

Tree Ivy

[Fatsia japonica 'Moseri' $\times$ Hedera helix

var. hibernica]

$$
\text { ALBG DCNA GACH LALG }
$$

FATSIA Decne. \& Planch.

ARALIACEAE

Ginseng Family

japonica (Thunb.) Decne. \& Planch.

Japanese Fatsia

$\begin{array}{llll}\text { ALBG } & \text { FLMG } & \text { GAWH } & \text { VANB } \\ \text { DCNA } & \text { FLUF } & \text { MSHB } & \\ \text { FLDG } & \text { GACH } & \text { SCBR } & \end{array}$

FEIJOA O.Berg

= ACCA sellowiana

FICUS L.

MORACEAE

Fig

Mulberry Family

carica $\mathrm{L}$.

Common $\mathrm{F}$.

$\begin{array}{llll}\text { DCCG } & \text { GAJS } & \text { MSEN } & \text { VAWP } \\ \text { DCNA } & \text { MDNA } & \text { VACW } & \\ \text { FLUF } & \text { MDRP } & \text { VASC } & \end{array}$

carica $\times$ F. pumila

SCWI

'Minima' [F. pumila L.] SCBR

palmata Forssk.

FLUF 
pumila L.

Creeping F.

$\begin{array}{llll}\text { ALBG } & \text { GAHC } & \text { LAHG } & \text { TXJS } \\ \text { FLDG } & \text { GAJI } & \text { MSRN } & \text { VANB } \\ \text { FLMM } & \text { GAJY } & \text { SCBR } & \\ \text { FLUF } & \text { GAUG } & \text { SCMP } & \end{array}$

tikoua Bur. LASL

FIRMLANA Marsili STERCULIACEAE

Sterculia Family

simplex (L.) W.F. Wight

Chinese Parasol Tree

$\begin{array}{llll}\text { ALAU } & \text { FLUF } & \text { LAAL } & \text { MSHB } \\ \text { DCNA } & \text { GAAB } & \text { LASL } & \text { SCMP }\end{array}$

platanifolia (L.f.) Marsili

=F. simplex

FONTANESIA Labill.

\section{OLEACEAE}

Olive Family

fortunei Carr.

$\begin{array}{llll}\text { DCNA } & \text { MDGJ } & \text { NCCA } & \text { TNSN } \\ \text { DCSH } & \text { NCBE } & \text { TNRG } & \text { VATA }\end{array}$

FORESTIERA Poir.

OLEACEAE

Olive Family

acuminata (Michx.) Poir.

Swamp Privet

TXRS

FORSYTHIA Vahl

OLEACEAE

Golden Bells

Olive Family

'Beatrix Farrand' [F. X intermedia Zab.]

= 'Karl Sax'

'Bronxensis' [F. viridissima Lindl.] DCNA

'Ilgwang' [F. koreana Nakai] MDBG

$\times$ intermedia Zab.

[F. suspensa $\times \boldsymbol{F}$. viridissima]

ALIT GACG VACW

DEWG SCUC
'Karl Sax' [F. Xintermedia Zab.]

[F. suspensa $\times$ F. viridissima] ALEH DEWG

'Lynwood' [F. $\times$ intermedia Zab.]

[F. suspensa $\times F$. viridissima]

DCNA DEWG MDLT

'Lynwood Gold' [F. $\times$ intermedia Zab.]

= 'Lynwood'

'New Hampshire Gold' [F. $\times$ intermedia Zab.]

[F. suspensa $\times$ F. viridissima] DCNA

'Ottawa' [F. ovata (? hybrid)] DEWG

ovata Nakai

Korean G.B.

DCNA SCCU

'Pallida' [F. suspensa (Thunb.) Vahl] DEWG

'Spectabilis' [F. $\times$ intermedia Zab.]

[F. suspensa $\times F$. viridissima] DEWG

'Spring Glory' [F. Xintermedia Zab.] [F. suspensa $\times$ F. viridissima] DCNA DCWH DEWG MDHN

suspensa (Thunb.) Vahl

Weeping G.B.

$\begin{array}{llll}\text { DCDO } & \text { MDLT } & \text { NCDU } & \text { VAPH } \\ \text { DEWG } & \text { MDPJ } & \text { VACW } & \end{array}$

suspensa (Thunb.) Vahl var. fortunei (Lindl.) Rehd.

DEWG

'Variegata' [F. $\times$ intermedia Zab.]

[F. suspensa $\times F$. viridissima] VATA

'Vermont Sun' [F. mandshurica Nakai] DCNA

viridissima Lindl.

$$
\begin{array}{lll}
\text { ALEH } & \text { NCEG } & \text { SCMP } \\
\text { NCBE } & \text { NCSM } & \text { VAMP }
\end{array}
$$

'Winterthur' [F. ovata $\times F$. $\times$ intermedia 'Spring Glory']

DEWG 
FORTUNEARIA Rehd. \& Wils.

HAMAMELIDACEAE Witch-hazel Family

sinensis Rehd. \& Wils. MDGD

FORTUNELLA Swingle RUTACEAE

Kumquat Citrus Family

$\times$ crassifolia Swingle

Meiwa K.

[F. margarita $\times F$. japonica]

LAGN

japonica (Thunb.) Swingle

Round K.

FLUF

\section{FOTHERGILLA L.}

HAMAMELIDACEAE Witch-hazel Family

gardenii J.A.Murr.

Witch Alder

$\begin{array}{llll}\text { GAEC } & \text { NCBE } & \text { SCBR } & \text { VAPH } \\ \text { MDWP } & \text { NCFR } & \text { SCWI } & \end{array}$

MDWP NCFR SCWI

major (Sims) Lodd.

$\begin{array}{llll}\text { DCEL } & \text { GAEC } & \text { MDLT } & \text { SCWI } \\ \text { DEMC } & \text { MDBG } & \text { MDPJ } & \text { VABF } \\ \text { DEWG } & \text { MDFM } & \text { MDWP } & \text { VAGS } \\ \text { GACG } & \text { MDKN } & \text { NCBE } & \end{array}$

major $\times$ F. gardenii

DEWG VAPH

monticola Ashe

$=\boldsymbol{F}$. major

FRANGULA Mill.

\section{RHAMNACEAE}

Buckthorn Family

alnus Mill.

DEMC VATA

FRANKLINLA Marsh.

THEACEAE

Tea Family

alatamaha Marsh.

Franklin Tree

$\begin{array}{llll}\text { DCCG } & \text { MDBD } & \text { MDLT } & \text { SCCU } \\ \text { DEMC } & \text { MDBG } & \text { NCBE } & \text { VACW } \\ \text { GARW } & \text { MDGD } & \text { NCDB } & \text { VAMP }\end{array}$
FRAXINUS
$=$
OLEACEAE
Ash
Olive Family

americana $\mathrm{L}$.

American A., White A.

$\begin{array}{llll}\text { DCCG } & \text { MDJS } & \text { TNFL } & \text { VAWR } \\ \text { FLUF } & \text { NCCA } & \text { TNSC } & \\ \text { MDBP } & \text { SCBR } & \text { VAMP } & \\ \text { MDHN } & \text { TNDR } & \text { VAPO } & \end{array}$

americana L. var. biltmoreana (Beadle)

J.Wright

Biltmore A.

MDCP MDHN

'Aurea' [F. excelsior L.]

DCNA

berlandieriana A.DC.

Berlandier A.

TXBA

chinensis Roxb. var. rhynchophylla

(Hance) Hemsl.

TNDR

'Doorenbos \#5' [F. excelsior L.]

DCNA

excelsior $\mathrm{L}$.

European A. MDSJ

holotricha Koehne DCNA

lanceolata Borkh.

= F. pennsylvanica var. subintegerrima

longicuspis Sieb. \& Zucc.

VATA

nigra Marsh.

Black A.

TNLW

ornus L.

Flowering A.

GACG MDLT

ornus $\times F$. excelsior

DCNA

pennsylvanica Marsh.

Red A.

$$
\text { VABF }
$$

pennsylvanica Marsh. var. subintegerrima (Vahl) Fern.

Green A.

MDHN 


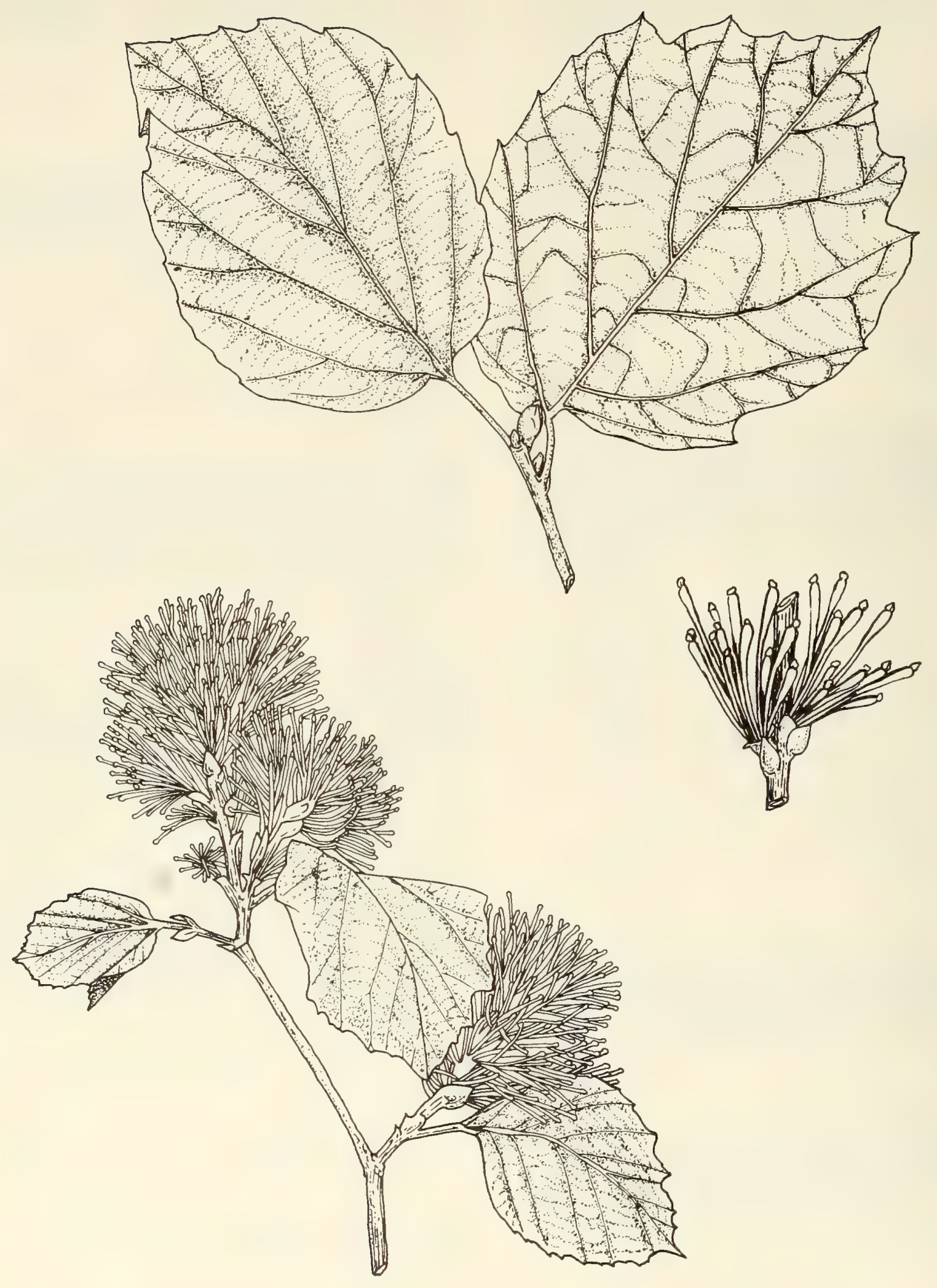

FOTHERGILLA major (Sims) Lodd.

[illustrator Susan M. Johnston] 
pubescens Lam.

= F. pennsylvanica

sieboldiana $\mathrm{Bl}$. DCNA VATA

toumeyi Britton

$=F$. velutina

velutina Torr. var. coriacea (S.Wats.) Rehd.

Leatherleaf A.

FLUF SCCU

velutina Torr. var. glabra Rehd.

Modesto A.

FLMM FLUF FLWG

GALPHIMIA Cav.

MALPIGHIACEAE

Malpighia Family

glauca Cav. ALBG

FLUF

GACG SCWI

GARDENIA Ellis

RUBIACEAE

Madder Family

jasminoides Ellis

Cape Jasmine

$\begin{array}{llll}\text { FLMG } & \text { LAAL } & \text { LARP } & \text { NCWC } \\ \text { GATS } & \text { LAHG } & \text { MSMN } & \text { VACM }\end{array}$

jasminoides Ellis var. radicans (Thunb.)

Makino

= 'Radicans'

'Radicans' [G. jasminoides Ellis]

LAHG LARP MSMN VANB

GAULTHERIA L.

ERICACEAE

Heath Family

procumbens $\mathrm{L}$.

Wintergreen, Tea Berry

DCNA
GAYLUSSACIA HBK. ERICACEAE

Huckleberry Heath Family baccata (Wang.) K.Koch

Black H.

VATG

brachycera (Michx.) A.Gr.

Box H.

NCDU

GELSEMIUM Juss. - Jessamine
LOGANLACEAE

'Plena' [G. sempervirens (L.) Ait.f.]

$=$ 'Pride of Augusta'

'Pride of Augusta' [G. sempervirens (L.)

Ait.f.]

Double-flowered Jessamine

$$
\begin{array}{lll}
\text { ALTD } & \text { VACW } \\
\text { LASL } & \text { VAGS }
\end{array}
$$

rankinii Small

SCWI VACW

sempervirens (L.) Ait.f.

Yellow J., Carolina J.

$\begin{array}{llll}\text { ALON } & \text { LAHG } & \text { TNCG } & \text { VAWM } \\ \text { ARPT } & \text { LASL } & \text { VACW } & \text { VAWR } \\ \text { ARSN } & \text { NCOP } & \text { VAGG } & \\ \text { GAEC } & \text { SCBR } & \text { VAGS } & \end{array}$

GENISTA L.

Broom

FABACEAE (Faboideae) Bean Family

lydia Boiss. VACW

'Prostrata' [G. germanica L.] DCNA

\section{GINKGO L.}

\section{GINKGOACEAE}

Ginkgo Family

biloba L.

Maidenhair Tree, Ginkgo

$\begin{array}{llll}\text { DCCG } & \text { LASL } & \text { NCDU } & \text { VABF } \\ \text { DCWH } & \text { MDHN } & \text { NCTE } & \text { VAMP } \\ \text { FLCG } & \text { MDJS } & \text { TNCT } & \text { VATA } \\ \text { GAGN } & \text { MSMN } & \text { TNFE } & \text { VAWR } \\ \text { GAMW } & \text { NCCA } & \text { TNPW } & \end{array}$

(The specimen at GAGN develops pendent mammillate projections (called "chi" in Japan) from the lower side of the main branches; this is the first specimen of its kind recorded in the United States.)

'Fastigiata' [G. biloba L.] GACD

'Lakeview' [G. biloba L.] TNPW

'Mayfield' [G. biloba L.] TNPW 
GLEDITSIA L. Honeylocust FABACEAE (Caesalpinioideae) Bean Family

\section{aquatica Marsh.}

Water Locust DCLC

japonica Miq.

Japanese $\mathrm{H}$. SCCU

'Shademaster' [G. triacanthos L.

f. inermis (Pursh) Schneid.] NCDU

'Sunburst' [G. triacanthos L. f. inermis (Pursh) Schneid.] NCCA TNSC VACW

triacanthos L.

Common $\mathrm{H}$. MDHN MDJH VACW

triacanthos L. f. inermis (Pursh) Schneid. Spineless H. ALBH NCCA TNBO

GLOCHIDION J.R. \& J.G.Forst.

EUPHORBIACEAE Spurge Family

puberum (L.) Hutch.

FLUF

\begin{tabular}{|c|c|}
\hline $\begin{array}{l}\text { GLYCOSMIS Correa } \\
\text { RUTACEAE }\end{array}$ & Citrus Family \\
\hline $\begin{array}{l}\text { citrifolia (Willd.) Lindl. } \\
\text { FLUF }\end{array}$ & \\
\hline
\end{tabular}

GLYPTOSTROBUS Endl.

TAXODIACEAE

Taxodium Family

heterophyllus (Brongn.) Endl.

$=G$. lineatus

lineatus (Poir.) Druce

Chinese Water-pine DCNA MDBG

pensilis (Staunt.) K.Koch

$=G$. lineatus
GORDONLA Ellis

THEACEAE

Tea Family

alatamaha Sarg.

= Franklinia alatamaha

axillaris (Roxb. ex Ker-Gawl.) D.Dietr. FLUF GACG

chrysandra Cowan FLUF

lasianthus (L.) Ellis

Loblolly Bay

$\begin{array}{lll}\text { ALAU } & \text { SCBR } & \text { VATA } \\ \text { DCNA } & \text { VANB }\end{array}$

GREWIA L.

TILIACEAE

Linden Family

biloba G.Don

GAIS VABF

GYMINOCLADUS Lam.

FABACEAE (Caesalpinioideae) Bean Family

dioica (L.) K.Koch

Kentucky Coffee-tree

$\begin{array}{llll}\text { DCCG } & \text { DEMC } & \text { VACW } & \text { VAMF } \\ \text { DCNA } & \text { MDHN } & \text { VAGW } & \\ \text { DCPP } & \text { MDJS } & \text { VAHC } & \end{array}$

HALESIA Ellis eX L.

Silverbell Tree STYRACACEAE

Storax Family

carolina L.

ALEH

DCCG

MDCA NCTE

VACP

GARW

MDGJ

NCTP

VAMP

GASM

MDJH

SCBR

VATA

GATS

MDJS

SCWI

VAWR

LALG

MDWP

TNMB

MDBG

MSMN

TNSC

TNSM

diptera Ellis

DCNA

FLMG

LAAL

LASL

NCTE

GAEC

LAHG

MSMN

TXLL

diptera Ellis var. magniflora Godfrey

DCNA FLMG NCBE

monticola Rehd. \& Sarg.

$=\boldsymbol{H}$. carolina

parviflora Michx.

ALEH 


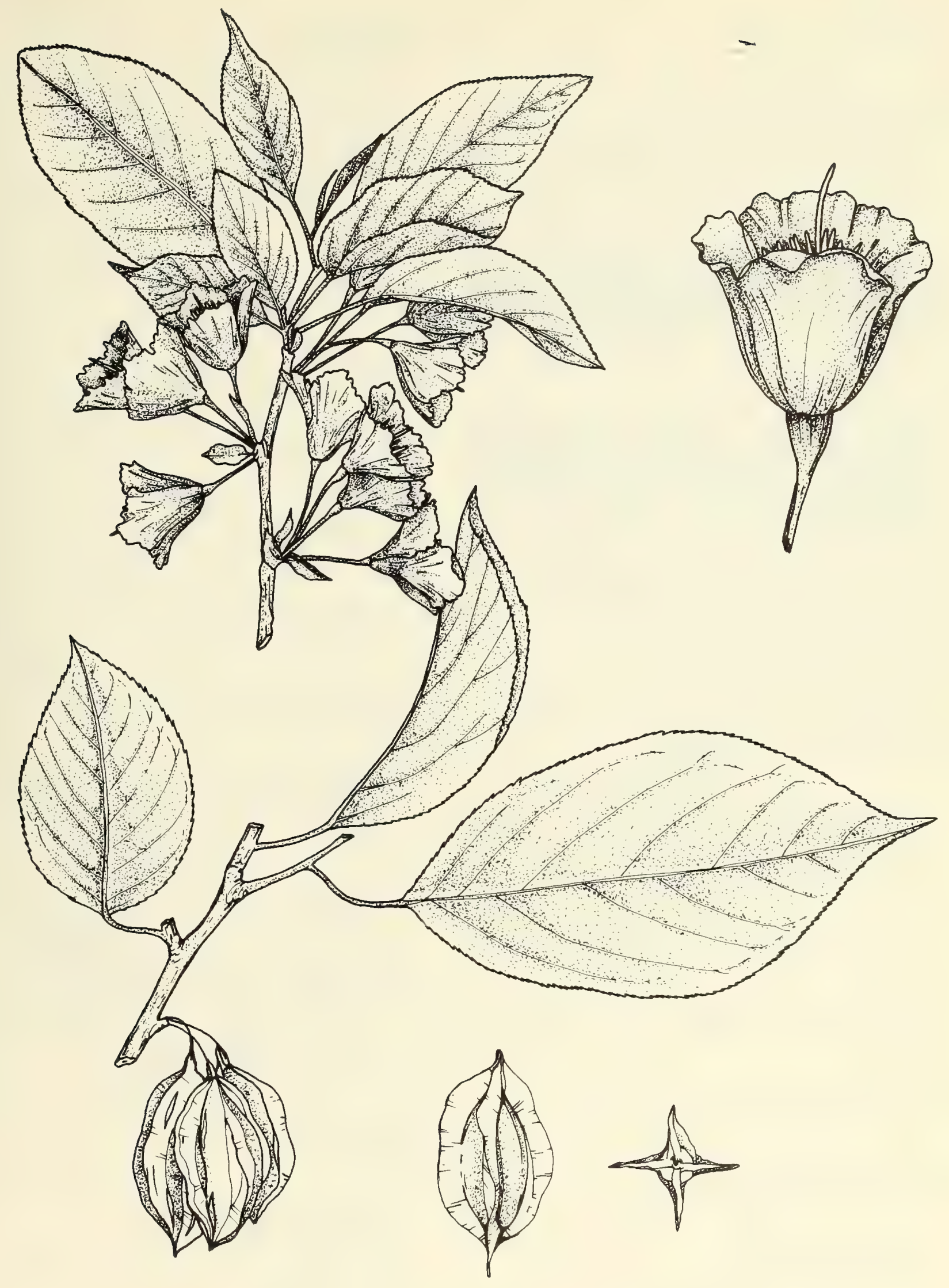

HALESIA carolina L.

[illustrator Susan M. Johnston] 
'Rosea' [H. carolina L.]

DCNA

tetraptera Ellis

$=\boldsymbol{H}$. carolina

tetraptera Ellis var. monticola (Rehd.) Reveal \& Seldin

$=\boldsymbol{H}$. carolina

\section{HALTMODENDRON Fischer ex DC. \\ FABACEAE (Faboideae) \\ Bean Family}

halodendron (L.) Voss

DCNA

HAMAMELIS L. Witch Hazel

HAMAMELIDACEAE Witch-hazel Family

arborea Ottol.

$=\boldsymbol{H}$. japonica

'Arnold Promise' [H. $\times$ intermedia Rehd.]

$[\boldsymbol{H}$. japonica $\times H$. mollis] DCNA MDBG

'Brevipetala' [H. mollis Oliv.] DCNA MDBG MDKN

'Carnea' [H. vernalis Sarg.] MDBG

'Christmas Cheer' [H. vernalis Sarg.] MDBG

'Copper' [H. vernalis Sarg.] MDBG

'Copper Beauty' [H. × intermedia Rehd.] $=$ 'Jelena'

'Feuerzauber' [H. $\times$ intermedia Rehd.] $[H$. japonica $\times$ H. mollis] DCNA

'Fire Charm' [H. $\times$ intermedia Rehd.]

$=$ 'Feuerzauber'

$\times$ intermedia Rehd.

[H. japonica $\times H$. mollis] DCNA MDGC MDKN

$\times$ japollis J.Lange

$=\boldsymbol{H}$. $\times$ intermedia

japonica Sieb. \& Zucc.

DCNA MDBG MDFM MDKN japonica Sieb. \& Zucc.

f. flavopurpurascens (Makino) Rehd. DCNA MDBG MDKN

'Jelena' [H. Xintermedia Rehd.]

[H. japonica $\times$ H. mollis] DCNA MDBG

'Lombarts Weeping' [H. vernalis Sarg.] DCNA MDBG MDFM

'Luna' [H. X intermedia Rehd.]

[H. japonica $\times$ H. mollis] DCNA MDBG

macrophylla Pursh

$=H$. virginiana

'Magic Fire' [H. $\times$ intermedia Rehd.]

= 'Feuerzauber'

mollis Oliv.

Chinese W.H.
ALBG
DEMC MDBG
DCNA
GAUG MDKN

'Orange Beauty' [H. $\times$ intermedia Rehd.]

[H. japonica $\times H$. mollis] DCNA

'Pallida' [H. $\times$ intermedia Rehd.]

[H. japonica $\times$ H. mollis] MDBG

'Primavera' [H. Xintermedia Rehd.]

[H. japonica $\times$ H. mollis] DCNA

'Rubra' [H. X intermedia Rehd.]

[H. japonica $\times$ H. mollis] DCNA

'Ruby Glow' [H. $\times$ intermedia Rehd.]

[H. japonica $\times H$. mollis]

(Sometimes listed as 'Adonis')

DCNA MDBG

'Sandra' [H. vernalis Sarg.] MDBG

vernalis Sarg.

DCNA MDKN MDWP

vernalis Sarg. f. tomentella Rehd.

$=H$. vernalis

vernalis Sarg. var. tomentella (Rehd.) Palm.

$=H$. vernalis

'Vesna' [H. $\times$ intermedia Rehd.]

[H. japonica $\times$ H. mollis]

DCNA 


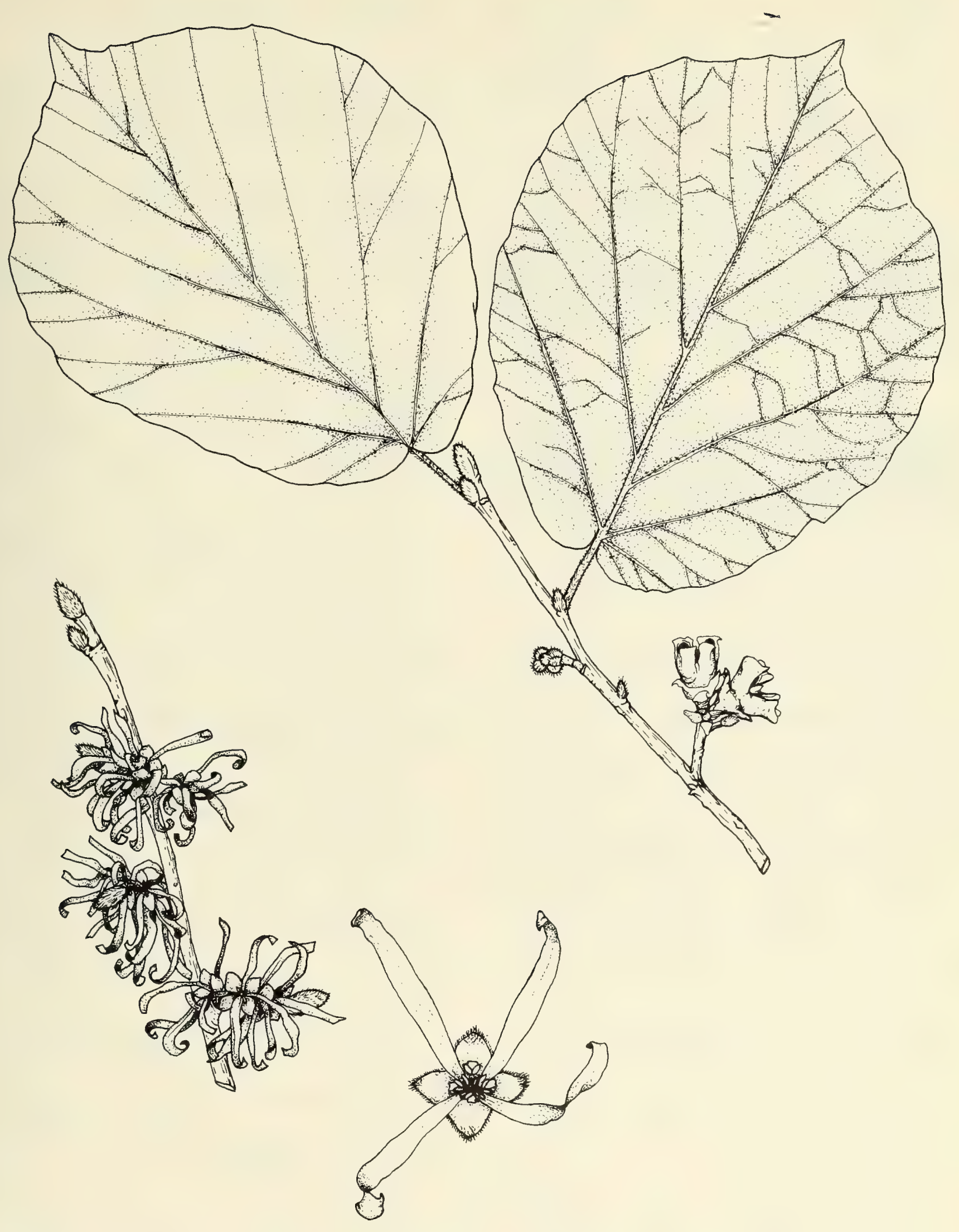

HAMAMELIS mollis Oliv.

[illustrator Susan M. Johnston] 
virginiana $\mathrm{L}$.

Common W. H.

$\begin{array}{llll}\text { ALBG } & \text { GAEH } & \text { MDBG } & \text { TXSE } \\ \text { DCNA } & \text { LALG } & \text { TXDC } & \text { VACW }\end{array}$

virginiana L. var. parvifolia Nutt.

$=\mathbf{H}$. virginiana

'Zuccariniana' [H. japonica Sieb. \& Zucc.] DCNA MDKN

HAMELIA Jacq.

RUBLACEAE

Madder Family

patens Jacq.

Scarlet Bush

TXRS

HEDERA L.

ARALIACEAE

Ivy

Ginseng Family

algeriensis Hibb.

$=\boldsymbol{H}$. canariensis

'Alpha' [H. helix L.]

SCWF

'Anchor' [H. helix L.]

SCWF

'Angularis Aurea' [H. helix L.] SCWF

Arborescens group [H. helix L.]

Shrub Ivy

(This group name applies to the adult flowering form of the species.)

$\begin{array}{llll}\text { ALAU } & \text { GAJY } & \text { MDNA } & \text { VACM } \\ \text { ALON } & \text { LASL } & \text { MDPJ } & \text { VACW } \\ \text { DCCG } & \text { MDBG } & \text { NCBE } & \\ \text { DCSE } & \text { MDFM } & \text { TNUT } & \end{array}$

'Arrowhead' [H. helix L.] SCWF

'Aurea Spectabilis' [H. helix L.] SCWF

'Baby Merion' [H. helix L.] GAAB

'Baccifer' [H. helix L.] SCWF

'Big Deal' [H. helix L.] SCWF

'Boskoop' [H. helix L.] SCWF
'Bulgaria' [H. helix L.] MDBG

'California' [H. helix L.] SCWF

'California Gold' [H. helix L.] SCWF

canariensis Willd.

Algerian I.

$\begin{array}{llll}\text { DEWG } & \text { GAJI } & \text { SCCL } & \text { SCNS } \\ \text { FLCG } & \text { LAHG } & \text { SCFW } & \text { VADW } \\ \text { FLMG } & \text { MDMG } & \text { SCMI } & \\ \text { FLUF } & \text { SCBR } & \text { SCMP } & \end{array}$

'Canary Cream' [H. canariensis Willd.] MDMG

'Carolina Crinkle' [H. helix L.] GAHC MDBG SCBR SCWF

'Cascade' [H. helix L.] SCWF

'Cathedral Wall' [H. helix L.] SCWF

'Cavendishii' [H. helix L.] ALHC FLCG

'Christian' [H. helix L.] GAAB

'Chrysantha' [H. helix L.] DCNA MDBG

'Chrysocarpa' [H. helix L.]

$=\boldsymbol{H}$. helix var. poetica

'Cockle Shell' [H. helix L.] GAAB SCWF

colchica K.Koch

Caucasus Ivy $\begin{array}{llll}\text { DCCC } & \text { NCEL } & \text { SCMP } & \text { VAGS } \\ \text { MDBG } & \text { SCBR } & \text { SCWF } & \end{array}$

'Conglomerata' [H. helix L.] GACG SCWF VAGS

'Crenata' [H. helix L.] DCDO

'Deltoidea' [H. helix L.] MDBG SCWF

'Dentata' [H. helix L.] SCWF

'Denticulata' [H. helix L.] SCWF 
'Digitata' [H. helix L.] ALHC SCWF

'Discolor' [H. helix L.] SCWF

'Dragon Claw' [H. helix L.] SCWF

'Edison' [H. helix L.] SCWF

'Emerald Beauty' [H. helix L.] GAFN

'Emerald Gem' [H. helix L.] SCWF

'Emerald Jewel' [H. helix L.] DCNA

'Erecta' [H. helix L.] VATA

'Erin' [H. helix L.] SCWF

'Eva' [H. helix L.] SCWF

'Fan' [H. helix L.] DCNA MDBG

'Fantasia' [H. helix L.] SCWF

'Ferney' [H. helix L.] SCWF

'Fleur' [H. helix L.] SCBR SCWF

'Fleur de Lis' [H. helix L.] SCWF

'Four Square' [H. helix L.] SCWF

'Garland' [H. helix L.] SCWF

'Gavotte' [H. helix L.] SCWF

'Ginkgo' [H. helix L.] SCWF

'Glacier' [H. helix L.] $\mathrm{SCWF}$

'Gladiator' [H. helix L.] SCWF
'Glymii' [H. helix L.] SCWF

'Goldcraft' [H. helix L.] SCWF

'Gold Dust' [H. helix L.] SCWF

'Gold Heart' [H. helix L.] MDBG SCWF

'Goods Selfbranching' [H. helix L.] SCWF

'Gracilis' [H. helix L.] SCWF

'Green Crown' [H. helix L.] SCWF

'Green Feather' [H. helix L.]

= 'Meagheri'

'Green Finger' [H. helix L.] SCWF

'Green Guartz' [H. helix L.] SCWF

'Green Ripples' [H. helix L.] GAFN SCWF

'Green Spear' [H. helix L.] SCWF

'Green Velvet' [H. helix L.] GAFN

'Hahn Selfbranching' [H. helix L.] SCWF

'Hahn Variegated' [H. helix L.] SCWF

'Harold' [H. helix L.] SCWF

'Harrison' [H. helix L.] SCWF

'Hebron' [H. helix L.] SCWF

helix L.

English I.

$\begin{array}{llll}\text { ALBG } & \text { GABW } & \text { NCEG } & \text { TXSH } \\ \text { FLBF } & \text { LAHG } & \text { NCOP } & \\ \text { FLCG } & \text { LASL } & \text { NCTP } & \\ \text { FLMG } & \text { NCDU } & \text { SCBP } & \end{array}$


helix L. var. poetica Weston

Italian I.

SCWF

'Helvetica' [H. helix L.] SCWF

'Heterophylla' [H. helix L.] SCWF

'Hibernica' [H. helix L.] SCWF

'Hite Miniature' [H. helix L.] SCWF

'Holly' [H. helix L.] SCWF

'Ideal' [H. helix L.] SCWF

'Imp' [H. helix L.] SCBR SCWF

'Itsy Bitsy' [H. helix L.] SCWF

'Iva Lace' [H. helix L.] MDBG SCWF

'Jack Frost' [H. helix L.] SCWF

japonica Tobl.

= H. rhombea

'Lady Kay' [H. helix L.] SCWF

'La Platta' [H. helix L.] SCWF

'Lobata Major' [H. helix L.] SCWF

'Lolla Rookh' [H. helix L.] MDBG 'Long Point' [H. helix L.]

'Lucida Aurea' [H. helix L.] SCWF

'Luzzi' [H. helix L.] SCWF

'Manda Crested' [H. helix L.] MDBG SCWF

'Manda Fringette' [H. helix L.] SCWF
'Manda's Star' [H. helix L.] SCWF

'Maple Queen' [H. helix L.] SCWF

'Marbled Dragon' [H. helix L.] SCWF

'Marginata' [H. helix L.] SCWF

'Meagheri' [H. helix L.] SCWF VATA

'Merion Beauty' [H. helix L.] GAFN VATA

'Merrie's Albany' [H. helix L.] SCWF

'Microphylla Variegata' [H. helix L.] SCWF

'Midget' [H. helix L.] SCWF

'Miniature Needlepoint' [H. helix L.] VATA

'Minima' [H. helix L.] SCWF

'Minor Marmorata' [H. helix L.] MDBG

'Mount Vernon' [H. helix L.] SCWF

'My Variegated' [H. helix L.] SCWF

'Needlepoint' [H. helix L.] SCBR SCWF

nepalensis K.Koch var. sinensis Tobl. SCWF

'Obscura' [H. helix L.] SCWF

'Palmata' [H. helix L.]

$=$ 'Digitata'

'Paper Doll' [H. helix L.] SCWF

'Parsley Crested' [H. helix L.] SCWF

'Pedata' [H. helix L.] SCWF 


\section{HEDERA}

'Perfection' [H. helix L.] SCWF

'Permanent Wave' [H. helix L.] SCWF

'Pin Oak' [H. helix L.] SCWF

'Pin Oak Improved' [H. helix L.] SCWF

'Pittsburgh' [H. helix L.] MDBG SCWF

'Pittsburgh Variegated' [H. helix L.] SCWF

'Pixie' [H. helix L.] SCWF

'Plume de Or' [H. helix L.] SCWF

'Poetica' [H. helix L.]

$=\boldsymbol{H}$. helix var. poetica

'Preston Tiny' [H. helix L.] SCWF

'Purpurea' [H. helix L.] SCWF

'Ralf' [H. helix L.] GAAB

'Rambler' [H. helix L.] SCWF

'Ray's Supreme' [H. helix L.] FLMG SCWF

rhombea (Miq.) Bean

Japanese I. DCNA

'Ripples' [H. helix L.] SCWF

'Rochester' [H. helix L.] SCWF

'Roehr's Minor' [H. helix L.] SCWF

'Rubaiyat' [H. helix L.] SCWF

'Russell Gold' [H. helix L.] MDBG SCWF

'Ruth' [H. helix L.] SCWF
'Sagittaefolia' [H. helix L.] SCWF

'Sea Foam' [H. helix L.] SCMP

'Shamrock' [H. helix L.]

= 'Meagheri'

'Shannon' [H. helix L.] SCWF

'Silver Queen' [H. helix L.] SCWF

'Sinclair Silverleaf' [H. helix L.] GAAB

'Small Deal' [H. helix L.] SCWF

'Spearpoint' [H. helix L.] SCWF

'Springtime Snow' [H. helix L.] SCWF

'Staghorn' [H. helix L.] SCWF

'Stardust' [H. helix L.] SCWF

'Stare' [H. helix L.] SCWF

'Sulphurea' [H. helix L.] SCWF

'Sulfur Heart' [H. colchica K.Koch var. dentata Hibb.] MDBG

'Susan Gibles' [H. helix L.] SCBR

'Suzanne' [H. helix L.] SCWF

'Sylvanian' [H. helix L.] SCWF

'Teardrop' [H. helix L.] SCWF

'Teena' [H. helix L.] SCWF

'Telecurl' [H. helix L.] MDBG SCWF

'Tesselata' [H. helix L.] SCWF 
'Thorndale' [H. helix L.] SCWF

'Tidal Wave' [H. helix L.] SCWF

'Tribairn' [H. helix L.] SCWF

'Triloba' [H. helix L.] SCWF

'Triton' [H. helix L.] MDTD SCWF

'Trustee' [H. helix L.] SCWF

'238th Street' [H. helix L.] SCWF

'Ustlers' [H. helix L.] SCWF

'Vanderhof' [H. helix L.] SCWF

'Variegata' [H. canariensis Willd.]

Gloire de Marengo I. GACG VANB

'Walthamensis' [H. helix L.] SCWF

'Weber's California' [H. helix L.] SCWF

'Wilson' [H. helix L.] LASL SCWF

'Woodsii' [H. helix L.] SCWF

'Yalta' [H. helix L. var. taurica Rehd.] SCWF

HELIANTHEMUM Mill.

CISTACEAE

Rock-rose Family

apenninum (L.) Mill. MDSC

apenninum (L.) Mill. var. roseum (Jacq.) Schneid. MDSC

'Buttercup' [H. nummularium (L.) Mill.] DEMC

'Fireball' [H. nummularium (L.) Mill.] MDSC nummularium (L.) Mill. MDSC

HELICHRYSUM Mill. Strawflower ASTERACEAE Aster Family

italicum (Roth) G.Don VAGS

HEMIPTELEA Planch.

ULMACEAE

Elm Family

davidii (Hance) Planch. VABF

HEPTACODIUM Rehd.

CAPRIFOLIACEAE Honeysuckle Family (Contributed by T. R. Dudley)

jasminoides Airy-Shaw

$=\mathrm{H}$. miconioides

miconioides Rehd.

Seven-sun Flower DCNA

\section{HETEROPTERIS HBK. \\ MALPIGHIACEAE Malpighia Family}

angustifolia Griseb. FLJH

syringifolia Griseb.
LAAL
LASL
TXSC

HIBISCUS L.

MALVACEAE

Mallow Family

'Admiral Dewey' [H. syriacus L.] DCNA

'Aka yae' [H. syriacus L.] DCNA

'Albo-laciniata' [H. rosa-sinensis L.] FLUF

'Albus Plenus' [H. syriacus L.] DCNA

'Amarantus' [H. syriacus L.] DCNA 
$\star$ 'Aphrodite' [H. syriacus L.]

[(H. syriacus 'Suminokura' / diploid/ $\times$

H. syriacus 'William R. Smith') $\times$ tetraploid seedling of $\boldsymbol{H}$. syriacus 'William R. Smith'] DCNA

'Ardens' [H. syriacus L.] DCNA

'Ardens Plena' [H. syriacus L.] DCNA

'Bicolor' [H. syriacus L.] DCNA

'Blue Bird' [H. syriacus L.] DCNA

'Blue Rouge' [H. syriacus L.] DCNA

'Boule de Feu' [H. syriacus L.] DCNA

'Brilliant' [H. rosa-sinensis L.] FLUF

'Caeruleus Plenus' [H. syriacus L.] DCNA

'Campanha' [H. syriacus L.] DCNA

'Celestial Blue' [H. syriacus L.] DCNA

'Colie Mullins' [H. syriacus L.] DCNA SCCU

'Comte de Flandre' [H. syriacus L.] DCNA

'Comte de Haimout' [H. syriacus L.] DCNA

'Dela Vaux' [H. syriacus L.] DCNA

'De La Veuve' [H. syriacus L.] DCNA

$\star$ 'Diana' [H. syriacus L.]

(Tetraploid sdlg. $\times$ diploid sdlg.) DCNA MDBG

'Double Light Pink' [H. syriacus L.] DCNA

'Duc de Brabant' [H. syriacus L.] DCNA
'Effie Riegel' [H. syriacus L.] SCCU

'Elegantissimus' [H. syriacus L.] DCNA

'Gion mamori' [H. syriacus L.] DCNA

'Grandiflorus Superbus' [H. syriacus L.] DCNA

'Hagan Hybrid \#2' [H. syriacus L.] DCNA

'Hamabo' [H. syriacus L.] DCNA

'Hanagasa' [H. syriacus L.]

= 'Shiro hanagasa'

$\star$ 'Helene' [H. syriacus L.] DCNA

'Hinomaru' [H. syriacus L.] DCNA

'Hitoe' [H. syriacus L.] DCNA

'Jeanne d'Arc' [H. syriacus L.] DCNA

'Koki yae' [H. syriacus L.] DCNA

'Kreider Blue' [H. syriacus L.] DCNA SCCU

'Lady Stanley' [H. syriacus L.] DCNA

'La Fleur' [H. syriacus L.] DCNA

'Leopoldii' [H. syriacus L.] DCNA

'Leopoldii Plenus' [H. syriacus L.] DCNA

'Lovely Pink' [H. syriacus L.] DCNA

'Lucy' [H. syriacus L.] DCNA

'Luteus Plenus' [H. syriacus L.] DCNA

'Martha Jane' [H. syriacus L.] DCNA 
'Mauve Gueen' [H. syriacus L.] DCNA

'Meehanii' [H. syriacus L.] DCNA

'Mimihara' [H. syriacus L.] DCNA

$\star$ 'Minerva' [H. syriacus L.]

$[\boldsymbol{H}$. syriacus 'Blue Bird' $\times \boldsymbol{H}$. syriacus

'Hanagasa'] DCNA

'Monstrosus' [H. syriacus L.] DCNA

'Monstrosus Plenus' [H. syriacus L.] DCNA

'Monstrosus Simple' [H. syriacus L.] DCNA

mutabilis $\mathrm{L}$.

Confederate Rose FLUF

'Oiseau Bleu' [H. syriacus L.] DCNA

paramutabilis Bailey VABF

'Perry's Purple' [H. syriacus L.] DCNA

'Pheasant Eye' [H. syriacus L.] DCNA

'Pink Delight' [H. syriacus L.] DCNA SCCU

'Plume' [H. syriacus L.] DCNA

'Pom Pom Rouge' [H. syriacus L.] DCNA

'Pompon' [H. syriacus L.] DCNA

'Pulcherrimus' [H. syriacus L.] DCNA

'Puniceus Plenus' [H. syriacus L.] DCNA

'Purpurea Semiplena' [H. syriacus L.] DCNA

'Purpureus Plenus' [H. syriacus L.] DCNA
'Ranunculiflorus' [H. syriacus L.] DCNA

'Ranunculiflorus Plenus' [H. syriacus L.] DCNA

'Rosalinda' [H. syriacus L.] DCNA

'Roseus Plenus' [H. syriacus L.] DCNA

'Roxanus' [H. syriacus L.] DCNA

'Rubis' [H. syriacus L.] DCNA

'Rubra Grandiflora' [H. syriacus L.] DCNA

'Rubra Plena' [H. syriacus L.] DCNA

'Shiro hanagasa' [H. syriacus L.] DCNA

'Shiro midare' [H. syriacus L.] DCNA

'Sir de Charles Breton' [H. syriacus L.] DCNA

'Snowdrift' [H. syriacus L.] DCNA

'Soft Pink' [H. syriacus L.] DCNA

'Sokobeni yae' [H. syriacus L.] DCNA

'Sonde' [H. syriacus L.] DCNA

'Souvenir de Charles Breton'

[H. syriacus L.] DCNA

'Speciosus' [H. syriacus L.] LAHP

'Spectabilis Plena' [H. syriacus L.] DCNA

'Suminokura' [H. syriacus L.] DCNA

'Suminokura hanagasa' [H. syriacus L.] DCNA 


\section{HIBISCUS}

'Suminokura yae' [H. syriacus L.] DCNA

syriacus L.

Rose-of-Sharon, Shrub Althea $\begin{array}{llll}\text { ALBG } & \text { FLUF } & \text { NCAS } & \text { VACW } \\ \text { DCCG } & \text { LAAL } & \text { SCSR } & \end{array}$

'Tama usagi' [H. syriacus L.] DCNA

'Totus Albus' [H. syriacus L.] DCNA

'Usu hitoe' [H. syriacus L.] DCNA

'Variegatus' [H. syriacus L.] DCNA

'Violaceus Plenus' [H. syriacus L.] DCNA

'Violet Clair' [H. syriacus L.] DCNA

'White Red Eye' [H. syriacus L.] DCNA

'White Supreme' [H. syriacus L.] DCNA

'William P. Smith' [H. syriacus L.] DCNA

'Woodbridge' [H. syriacus L.] DCNA

'Zulauf' [H. syriacus L.] DCNA

HOVENIA Thunb. RHAMNACEAE Buckthorn Family

dulcis Thunb.

Japanese Raisin-tree
DCNA
FLFB
GAGR
DCTB
FLUF
GAWM

HYDRANGEA L.

SAXIFRAGACEAE (Hydrangeoideae)

Saxifrage Family

acuminata Sieb. \& Zucc.

= H. macrophylla ssp. serrata

'Annabelle' [H. arborescens L.]

ALHC DEWG TNUT

DCNA MDBG VAGS anomala D.Don ssp. petiolaris (Sieb. \& Zucc.) McClint.

Climbing $\mathrm{H}$.

$\begin{array}{llll}\text { DCNA } & \text { MDBG } & \text { MDMG } & \text { VAPH } \\ \text { GAEC } & \text { MDLT } & \text { TNRW } & \text { VARC }\end{array}$

'Grandiflora' [H. arborescens L.]

= 'Annabelle'

'Grandiflora' [H. paniculata Sieb.]

Peegee $\mathrm{H}$. DCCG SCBR VARC

'Grayswood' [H. macrophylla (Thunb. ex J.A.Murr.) Ser.]

(Lacecap group) MDMG

'Harmony' [H. quercifolia Bartram] DCNA MDFM TNTV

macrophylla (Thunb. ex J.A.Murr.) Ser.

Lacecap

(Lacecap group)

$\begin{array}{lll}\text { DCNA } & \text { LASL } & \text { MSPR } \\ \text { LALG } & \text { MDBG } & \text { VAGS } \\ \text { LARP } & \text { MDBR } & \text { VAMP }\end{array}$

macrophylla (Thunb. ex J.A.Murr.) Ser. Hortensia, Garden Hydrangea

(Hortensia group)

$\begin{array}{llll}\text { ALBG } & \text { LARP } & \text { MSKR } & \text { VATA } \\ \text { DCNA } & \text { LASL } & \text { VAGS } & \\ \text { LAAL } & \text { MSEP } & \text { VAPH } & \end{array}$

macrophylla (Thunb. ex J.A.Murr.) Ser. ssp. serrata (Thunb. ex J.A.Murr.) Makino DCNA MDBG MDJS MDPJ DEWG MDGD MDMG

'Maculata' [H. macrophylla (Thunb. ex J.A.Murr.) Ser.]

(Lacecap group)

DCNA LASL

'Otaksa' [H. macrophylla (Thunb. ex J.A.Murr.) Ser.]

$=\boldsymbol{H}$. macrophylla (Hortensia group)

paniculata Sieb.

Peegee $\mathrm{H}$.

$\begin{array}{llll}\text { DCNA } & \text { MDGJ } & \text { NCAS } & \text { VABK } \\ \text { MDBG } & \text { MDLT } & \text { NCSM } & \text { VAMP }\end{array}$

'Prolifera' [H. macrophylla (Thunb. ex J.A.Murr.) Ser. ssp. serrata (Thunb. ex J.A.Murr.) Makino]

DCNA 


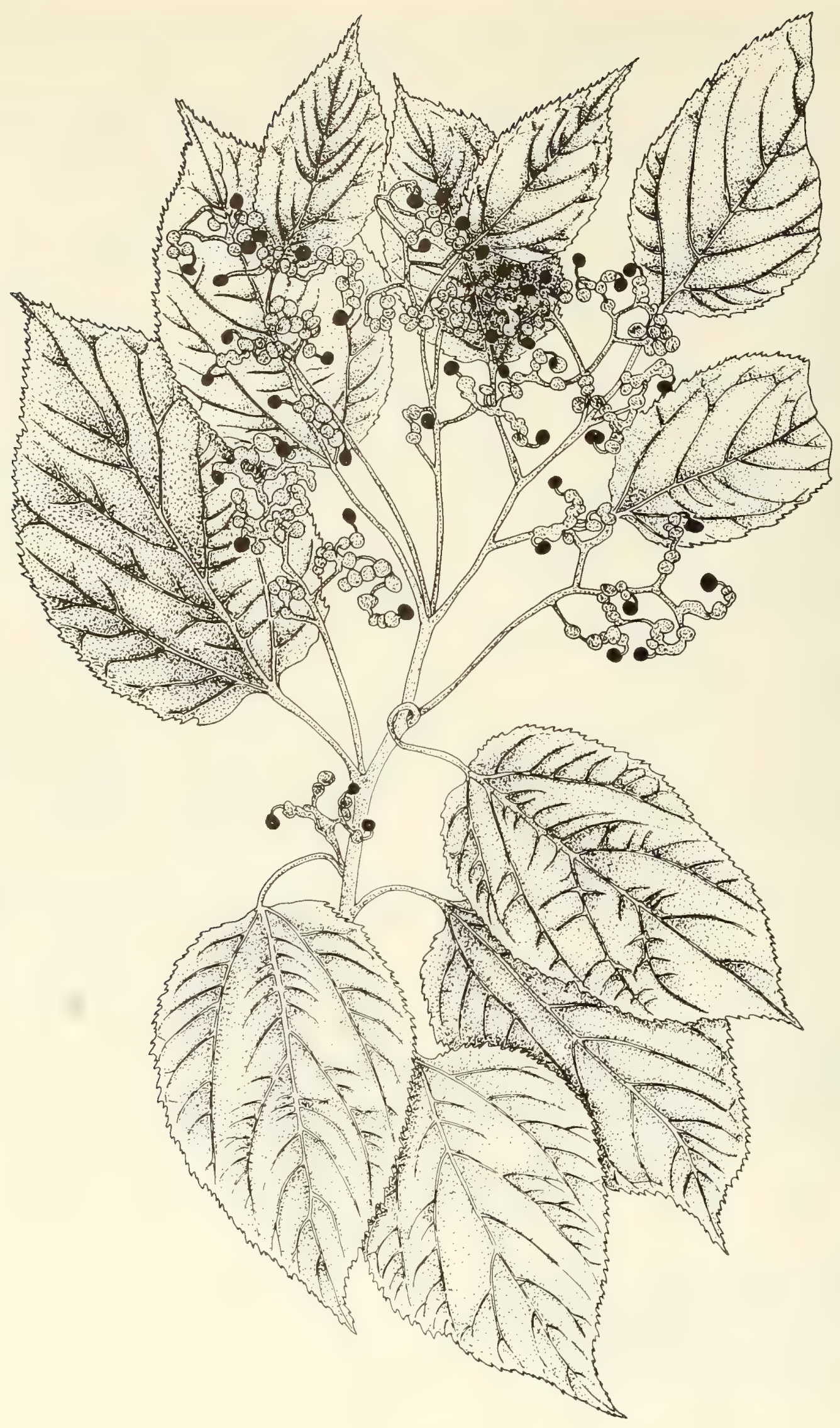

HOVENIA dulcis Thunb.

[illustrator Lillian Nicholson Meyer] 


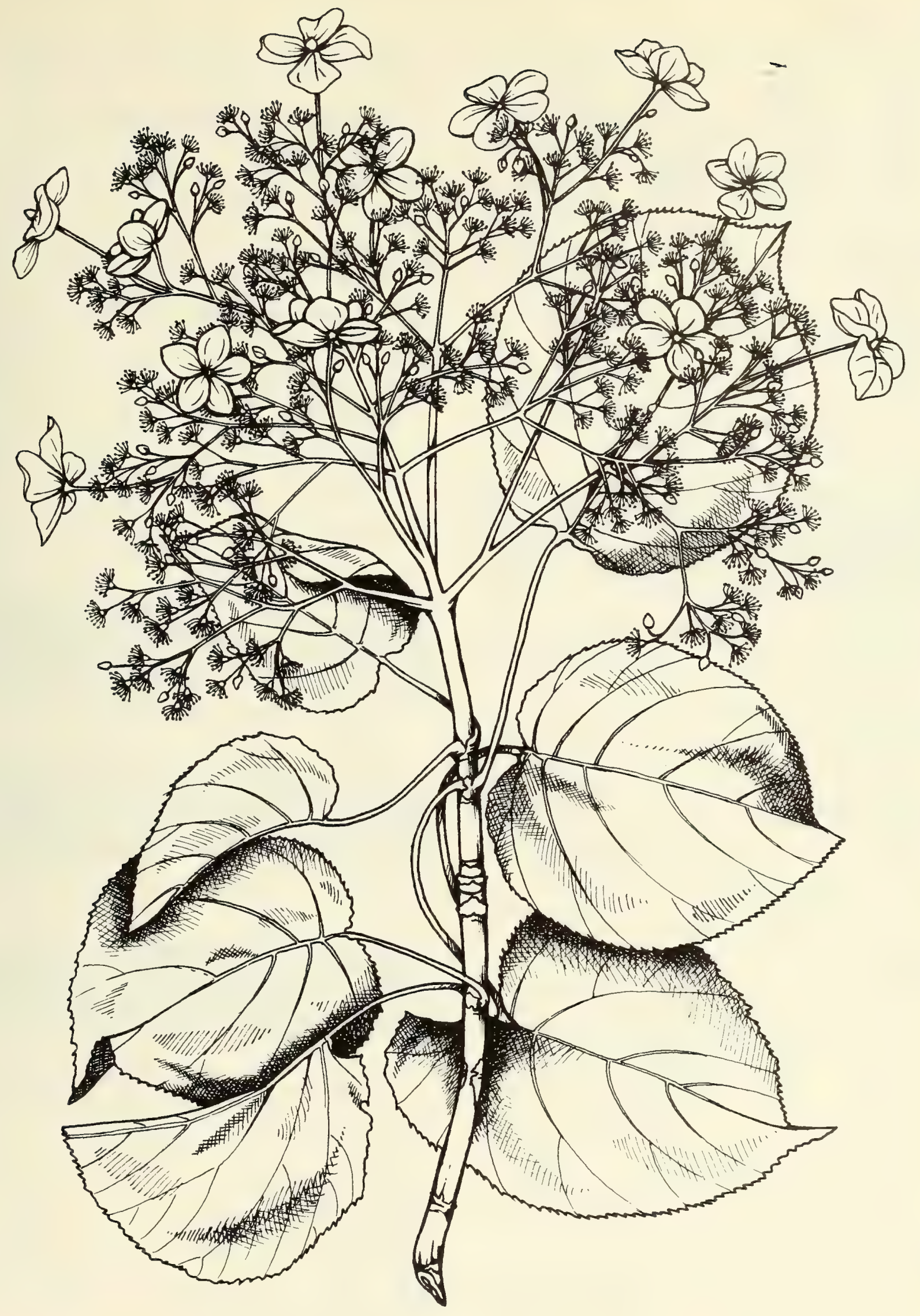

HYDRANGEA anomala ssp. petiolaris (Sieb. \& Zucc.) McClint.

[illustrator Susan M. Johnston] 
quercifolia Bartram

Oakleaf $\mathrm{H}$.

$\begin{array}{llll}\text { ALAU } & \text { LALG } & \text { MSRN } & \text { TNSC } \\ \text { ALHC } & \text { MDJH } & \text { NCBE } & \text { TNUT } \\ \text { DCNA } & \text { MDKN } & \text { NCSM } & \text { VAPH } \\ \text { FLUF } & \text { MDLT } & \text { SCBR } & \\ \text { GAUG } & \text { MDPJ } & \text { SCMP } & \\ \text { LAHG } & \text { MSMN } & \text { SCWI } & \end{array}$

scandens (L.f.) Ser. ssp. liukiuensis (Nakai) McClint.

GALH
'Snowflake' [H. quercifolia Bartram] MDBG MDMG VAPH MDFM SCWI
'Tardiva' [H. paniculata Sieb.] $\mathrm{SCCU}$

\begin{tabular}{|c|c|}
\hline HYPERICUM L. & $\begin{array}{l}\text { St. John's-wort } \\
\end{array}$ \\
\hline
\end{tabular}

brachyphyllum (Spach) Steud. SCWI

buckleyi M.A.Curtis MDJW

calycinum $\mathrm{L}$.

Aaron's Beard, Goldflower MDBG

fasciculatum Lam.

Sandweed SCWI

frondosum Michx. DCNA

galioides Lam. LALG SCBR SCWI

'Hidcote' [H. ?calycinum $\times H$. forrestii] MDLT NCBE TNUT VAWP

hircinum L. VAGS

hookerianum Wight \& Arn. DCNA

hypericoides (L.) Crantz

= Ascyrum hypericoides

kalmianum L. MDWP

\section{lissophloeus Adams} SCWI lloydii (Svenson) Adams MDJW

microsepalum (T. \& G.) A. Gr. SCWI

patulum Thunb. ex J.A.Murr. LARP

prolificum $\mathrm{L}$. NCBE SCBR SCWI

reductum (Svenson) Adams SCBR SCWI

stans (Michx.) Adams \& N.Robs.

St. Peter's-wort

SCBR SCWI

'Sunburst' [H. frondosum Michx.] DCNA

'Van Fleetii'

DCNA

HYSSOPUS L.

LAMIACEAE

Mint Family

officinalis $\mathrm{L}$.

DCNA

IDESIA Maxim.

FLACOURTIACEAE

Flacourtia Family

polycarpa Maxim.

ALON ALTD DCNA

ILEX L.

AgUIFOLIACEAE

(Contributed by T. R. Dudley)

(Because of a large number of cultivars in the hybrid species of $\boldsymbol{M l e x}$, parentage is not shown for each cultivar. Parentage is listed under the respective hybrid-species names.)

'Aalto' [I. opaca Ait.] NCSH

'Aalto \#2' [I. opaca Ait.]

$=$ 'Harriet'

'Aalto \#3' [I. opaca Ait.]

= 'Kate'

'Aalto \#5' [I. opaca Ait.] DCNA
Holly

Holly Family 
ILEX

'Aalto \#5A' [I. opaca Ait.]

DCNA

$\star$ 'Accent' [I. integra $\times$ I. pernyi] DCNA

'Afterglow' [I. verticillata (L.) A.Gr.] DCNA

'Aglo' [I. cornuta Lindl. \& Paxt.] DCCB

'Aka tsuge' [I. sugerokii Maxim.] DCNA

'Alagold' [I. Xattenuata Ashe] VATA

'Alice' [I. $\times$ altaclerensis (Loud.) Dallim.] DCNA

'Alice' [I. aquifolium]

='Alice' [I. Xaltaclerensis $]$

$\times$ altaclerensis (Loud.) Dallim.

Highclere $\mathrm{H}$.

[I. aquifolium $\times$ I. perado] DCNA MDLT MDWP VATA

ambigua (Michx.) Torr.

Carolina H., Ambiguous Winterberry VAGW

amelanchier M.A.Curtis

Sarvis H.
ALJG
GACG
TNFB
DCNA
LASL
VACW

'Andorra' [I. opaca Ait.] $\mathrm{NCSH}$

'Angustifolium' [I. aquifolium L.]

(Female and male clones of this cultivar exist) $\begin{array}{lll}\text { ALTD } & \text { GACG } & \text { MDLT } \\ \text { DCNA } & \text { MDBG } & \text { TNUT }\end{array}$

'Angyo' [I. crenata Thunb. ex J.A.Murr.] DCNA MDGD

'Anicet Delcambrie' [I. cornuta Lindl. \& Paxt.] DCNA LAMP

'Anna Mae' [I. cornuta Lindl. \& Paxt.] GACG LASL

'Anne Arundel' [I. opaca Ait.] MDMN

$\star$ 'Apollo' [I. serrata $\times$ I. verticillata] DCNA VATA
'Apricot' [I. aquifolium L.]

$\mathrm{NCSH}$

aquifolium L.

English $\mathrm{H}$.

$\begin{array}{llll}\text { DCCG } & \text { DCWH } & \text { TXHN } & \text { VATW } \\ \text { DCLC } & \text { GACG } & \text { VAGW } & \\ \text { DCNA } & \text { MDLT } & \text { VATA } & \end{array}$

aquifolium $\times$ I. spinigera DCNA

'Aquipern' [I. $\times$ aquipernyi Gable] DCNA DCWH GAIS MDTD

$\times$ aquipernyi Gable

[I. aquifolium $\times$ I. pernyi]

(Sometimes confused with I. bioritensis) DCNA GACG MDBG MDLT

'Arden' [I. opaca Ait.] DCNA

'Argentea Marginata' [I. aquifolium L.] DCNA MDLT NCSH

'Arthur Bruner' [I. cornuta 'Burfordii' $\times$ I. Latifolia] TNHH

asprella (Hook. \& Arn.) Champ. ex Benth. DCNA

'Astoria' [I. aquifolium L.]

= 'Beacon'

'Atlas' [I. aquifolium L.] DCNA

'Atlas' [I. cornuta $\times$ I. pernyi] GACG

$\times$ attenuata Ashe

Topel H., Attenuate-leaved H.

[I. cassine $\times$ I. opaca]

ALTD MDGD NCEG SCMP

DCNA MDLT SCBR

GAOH MSHB SCFR

'Audry' [I. cornuta $\times$ I. pernyi] MDPF

'Aurantiaca' [I. verticillata (L.) A.Gr.] DCNA TNFB

'Aurea Regina' [I. aquifolium L.]

= 'Golden Queen'

'Aureo-marginata' [I. aquifolium L.] DCNA VATA 
'Aurifodina' [I. aquifolium L.] GACG

'Autumn Glow' [I. serrata $\times$ I. verticillata] DCNA MDLT

'Avery Island' [I. cornuta Lindl. \& Paxt.] LAAL SCBR

'B. \& O.' [I. opaca Ait.]

DCNA MDBO

'Bacciflava' [I. aquifolium L.] DCNA GACG MDMG VATW

'Baldwin' [I. cassine L.] GACG

'Balearica' [I. $\times$ altaclerensis (Loud.)

Dallim.] ARSN DCCG DCNA

'Balkans' [I. aquifolium L.] DCNA

'Barclay' [I. opaca Ait.] DCNA

'Barnes' [I. aquifolium L.] DCNA

'Beacon' [I. aquifolium L.] DCNA GACG

beadlei Ashe

Beadle's H. TNFB

$\times$ beanii Rehd.

[I. aquifolium $\times$ I. dipyrena] DCNA MDTD

'Beauty Spra' [I. aquifolium L.] DCNA GACG

'Beauty Spra Espalier' [I. aquifolium L.] DCNA

'Belgica' [I. Xaltaclerensis (Loud.) Dallim.] DCNA LASL

'Belin's Weeping' [I. $\times$ altaclerensis (Loud.) Dallim.] DCNA

'Berigold' [I. aquifolium L.] DCNA GACG

'Beulah' [I. opaca Ait.] $\mathrm{NCSH}$
'Big Bull' [I. aquifolium L.]

$$
\text { DCNA GACG }
$$

bioritensis Hayata

(Sometimes confused with $I$. pernyi and

I. $\times$ aquipernyi)

DCNA FLMG GACG

DCWH FLUF NCSH

'Bleeg' [I. aquifolium L.] DCNA

'Blue Angel' [I. $\times$ meserveae S.Y.Hu] MDBG VATA

'Blue Boy' [I. Xmeserveae S.Y.Hu] DCNA MDBG

'Blue Girl' [I. $\times$ meserveae S.Y.Hu] DCNA

'Blue Maid' [I. $\times$ meserveae S.Y.Hu] MDLT

'Blue Prince' [I. $\times$ meserveae S.Y.Hu] MDTD VATA

'Blue Princess' [I. $\times$ meserveae S.Y.Hu] MDBG VATA

'Blue Stallion' [I. $\times$ meserveae S.Y.Hu] MDLT VATA

'Bob Bruner' [I. cornuta 'Burfordii' $X$

I. Latifolia] TNAB TNHH

'Bodley's Bleeg' [I. aquifolium L.] DCNA

'Bonanza' [I. aquifolium L.] GACG

'Bostic' [I. cornuta Lindl. \& Paxt.] GACG

'Boyce Thompson' [I. opaca Ait.] MDLT

'Boyce Thompson \#3' [I. opaca Ait.] DCNA

'Boyce Thompson Xanthocarpa' [I. opaca Ait. f. xanthocarpa Rehd.] DCNA MDBG

'Braddock Heights' [I. crenata Thunb. ex J.A.Murr.] MDAG

'Brawley' [I. cornuta Lindl. \& Paxt.] GACG 
'Brighter Shine' [I. cornuta $\times$ I. pernyi] GACG

'Bright Horizon' [I. verticillata (L.) A.Gr.] DCNA

'Brilliant' [I. aquifolium $\times$ I. ciliospinosa] ALBG MDBG VAGS DCNA SCWI VATA

'Bronze' [I. aquifolium L.] DCNA

'Brown \#3' [I. opaca Ait.]

= 'Judge Brown'

'Brown \#5' [I. opaca Ait.] $\mathrm{NCSH}$

'Brown \# 16' [I. opaca Ait.] DCNA

'Brownell' [I. aquifolium L.] GACG

buergeri Miq. ALTD DCNA

'Burfordii' [I. cornuta Lindl. \& Paxt.] Burford $\mathrm{H}$.

$\begin{array}{llll}\text { ARRB } & \text { FLUF } & \text { NCBE } & \text { TNSB } \\ \text { DCCG } & \text { GACG } & \text { NCEG } & \text { TXSH } \\ \text { DCNA } & \text { GAWH } & \text { NCGP } & \text { VACW } \\ \text { FLCG } & \text { MDLT } & \text { SCFW } & \text { VATA } \\ \text { FLMG } & \text { MSHB } & \text { SCUC } & \end{array}$

buswellii Small

Sand H., Buswell's H. DCNA TNFB

\section{'Butler' [I. aquifolium L.]} MDWA

'Buxifolia' [I. crenata Thunb. ex J.A.Murr.] (Name applied to numerous clones of different origins) DCNA

'Byers Golden' [I. decidua Walt.] ALBH DCNA GACG

'Cacapon' [I. verticillata (L.) A.Gr.] DCNA

'Cajun Gold' [I. cornuta Lindl. \& Paxt.] LAGN

'Callina' [I. cornuta $\times$ I. aquifolium] DCNA NCNB

'Calloway' [I. opaca Ait. f. xanthocarpa Rehd.] LASL
'Camelliifolia' [I. $\times$ altaclerensis (Loud.) Dallim.] DCLC DCNA MDBG MDTD

'Campus Variegated' [I. aquifolium L.] DCNA

canariensis Poir.

Canary Islands $\mathrm{H}$. DCNA

'Canary' [I. opaca Ait. f. xanthocarpa Rehd.] DCNA DEMC MDLT

'Cape Christmas' [I. opaca Ait.] MDTN

'Captain Bonneville' [I. aquifolium L.] GACG

'Carefree' [I. crenata Thunb. ex J.A.Murr.

var. paludosa (Nakai) Hara] SCCU

'Carissa' [I. cornuta Lindl. \& Paxt.] MDTD

'Casey's Dwarf' [I. cornuta Lindl. \& Paxt.] GACG SCMG

cassine L.

Dahoon, Cassine

$\begin{array}{lll}\text { DCNA } & \text { GACG } & \text { LASL } \\ \text { FLIF } & \text { GASM } & \text { SCBR } \\ \text { FLUF } & \text { LAHG } & \text { VANB }\end{array}$

cassine L. var. angustifolia Ait.

Narrow-leaved Dahoon

$\begin{array}{lll}\text { ALAU } & \text { FLIF } & \text { TNUT } \\ \text { DCNA } & \text { NCDU } & \end{array}$

cassine L. var. angustifolia

f. aurea-baccata Tarbox ex S.F.Blake

Yellow-berried Dahoon FLUF SCBR

cassine L. var. bryanii Tarbox ex S.F.Blake DCNA SCBR

cassine L. var. myrtifolia (Walt.) Sarg.

= I. myrtifolia

'Cetus' [I. cornuta $\times$ I. pernyi] GACG

'Changsha' [I. crenata Thunb. ex J.A.Murr.]

GACG

'Charles' [I. opaca Ait.] DCNA 
'Cherry Berry' [I. $\times$ altaclerensis (Loud.)

Dallim.]

DCNA

'Chief' [I. aquifolium L.]

$\mathrm{NCSH}$

'Chieftan' [I. Xkoehneana Loesn.] DCNA MDBO

'China Boy' [I. cornuta $\times$ I. rugosa] VATA

'China Girl' [I. cornuta $\times$ I. rugosa] VATA

chinensis (sensu S.Y.Hu \& nurseries, non Sims)

= I. purpurea

'Christmas Carol' [I. opaca Ait.] DCNA

'Christmas Cheer' [I. verticillata (L.) A.Gr.] DCNA

'Christmas Gem' [I. verticillata (L.) A.Gr.] MDJN

'Christmas Hedge' [I. opaca Ait.] DCNA

'Christmas Queen' [I. opaca Ait.]

= 'Cape Christmas'

ciliospinosa Loesn.

(Often confused with 'Brilliant')

DCNA MDBG MDTD

MDBA MDHS

ciliospinosa $\times$ I. fargesii

DCNA DEFC MDMN

cinerea Champ.

DCNA

'Clarendon' [I. opaca Ait.]

GACG

'Clarendon Batwing' [I. cornuta Lindl. \& Paxt.] NCCG

'Clarendon Small Leaf' [I. cornuta Lindl. \& Paxt.] DCNA GACG

'Clarendon Spreading' [I. opaca Ait.] DCNA NCCG TNTV

'Clarissa' [I. opaca Ait.] DCNA
'Clark' [I. opaca Ait.] DCNA

'Clouded Gold' [I. aquifolium L.] $\mathrm{NCSH}$

$\star$ 'Clusterberry'

[(I. cornuta $\times I$. aquifolium) 'Nellie R. Stevens'

$\times$ I. leucoclada] DCNA VATA

'Colburn' [I. $\times$ altaclerensis (Loud.)

Dallim.] DCNA

colchica Pojark.

Pontic H., Caucasian H. DCNA

'Coleman' [I. aquifolium L.] DCNA

collina Alex.

Appalachian $\mathrm{H}$.

DCNA MDPJ TNFB

'Compacta' [I. crenata Thunb. ex J.A.Murr.]

(Name applied to numerous clones of different origins)

$$
\text { DCNA GACG }
$$

'Compacta' [I. vomitoria Ait.]

(Name applied to numerous clones of different origins) VATA

'Conners' [I. crenata Thunb. ex J.A.Murr.] DCNA

'Convexa' [I. crenata Thunb. ex J.A.Murr.] DCNA FLMG MDMG SCMG FLCG GACG NCEG VATA

corallina Franch. DCNA MDGD

coriacea (Pursh) Chapm.

Large Gallberry

DCNA SCBR

cornuta Lindl. \& Paxt.

Chinese H., Horned $\mathrm{H}$.

$\begin{array}{llll}\text { ALBG } & \text { DCWH } & \text { LAMP } & \text { TNDR } \\ \text { ALTD } & \text { FLMG } & \text { MDTD } & \text { VAGF } \\ \text { ARRB } & \text { FLUF } & \text { NCDU } & \text { VATA } \\ \text { DCCG } & \text { GACG } & \text { SCBR } & \\ \text { DCNA } & \text { GAIS } & \text { SCEG } & \end{array}$


cornuta $\times$ I. pernyi

(CB hybrids originated at Kingsville Nursery,

Kingsville, MD; $\mathrm{CB}$ is an acronym for $\mathrm{I}$. cornuta

$\times$ I. cornuta 'Burfordii')

DCCG DCNA GACG

'Corpening \# 1' [I. opaca Ait.

f. xanthocarpa Rehd.] DCNA

'Cover Girl' [I. aquifolium L.] DCNA

crenata Thunb. ex J.A.Murr.

Japanese H., Box-leaved H.
FLCG
GACG
NCBE
DCNA
FLMG
MDLT
SCMG

crenata Thunb. ex J.A.Murr.

ssp. fukasawana (Makino) Murata DCNA

crenata Thunb. ex J.A.Murr.

var. mutchagara Makino

=I. maximowicziana var. kanahirae

crenata Thunb. ex J.A.Murr. var. paludosa

(Nakai) Hara

DCNA GACG

crenata Thunb. ex J.A.Murr.

var. thomsonii (Hook.f.) Loesn.

DCNA

crenata Thunb. ex J.A.Murr.

f. watanabeana Makino

DCNA GACG MDTD

'Crescent' [I. crenata Thunb. ex J.A.Murr.

var. paludosa (Nakai) Hara]

SCCU

'Crinkle Variegated' [I. aquifolium L.]

DCNA

'Crispa' [I. aquifolium L.]

Green Screw

DCNA GACG

'Crispa Aureo-picta' [I. aquifolium L.] GACG

'Croonenberg' [I. opaca Ait.] VAGF

'Cumberland' [I. opaca Ait.] DCNA

cumulicola Small

Dune H., Hummock H.

DCNA FLUF curtissii (Fern.) Small

Suwanee H. DCNA

GACG TNFB

TXDM

cyrtura Merr.

DCNA

'D'Or' [I. cornuta Lindl. \& Paxt.]

DCNA GAIS VACW

GACG LASL

'Daddyo' [I. aquifolium L.] DCNA

'Dan Fenton' [I. opaca Ait.] DCNA

'Dare County' [I. vomitoria Ait.] $\mathrm{NCMH}$

decidua Walt.

Possum Haw

$\begin{array}{llll}\text { DCNA } & \text { LAAP } & \text { NCTE } & \text { VACW } \\ \text { FLIF } & \text { LAMP } & \text { SCBR } & \\ \text { GACG } & \text { MDLT } & \text { TNUT } & \\ \text { GAEC } & \text { NCDU } & \text { TXPS } & \end{array}$

decidua Walt. var. curtissii Fern.

= I. curtissii

'Delaware Diamond' [I. crenata Thunb. ex J.A.Murr.] DCNA

'Deluxe' [I. aquifolium L.] DCNA

'Dengle Belles' [I. opaca Ait.

f. xanthocarpa Rehd.] MDBG

'Dewerth' [I. vomitoria Ait.] GACG

'Dick' [I. opaca Ait.]

(Originally 'Wheeler \# 1') $\mathrm{NCSH}$

dimorphophylla Koidz.

Okinawa $\mathrm{H}$. DCNA

dipyrena Wall. DCNA

'Divaricata' [I. crenata Thunb.

ex J.A.Murr.]

GACG

'Doctor Kassab' [I. cornuta $\times$ I. pernyi] DCNA MDBG MDMG 
'Dodd Special' [I. cornuta Lindl. \& Paxt.] GACG

'Dorsey' [I. opaca Ait.] MDWA

'Drace' [I. cornuta $\times$ I. pernyi] GACG

'Dragon Lady' [I. × aquipernyi Gable] MDLT VATA

'Dr. James Foret' [I. cornuta Lindl. \& Paxt.] LAAL

'Dr. John Creech' [I. cornuta Lindl. \& Paxt.] ALOS LAAL

'Dr. T. B. Symons' [I. opaca Ait.] DCNA

'Dude' [I. aquifolium L.] DCNA GACG

'Dumbarton Oaks' [I. aquifolium L.] DCNA

'Dwarf Burford' [I. cornuta Lindl. \& Paxt.] DCNA LAHG NCDU GACG MDBG

'Dwarf Cone' [I. crenata Thunb. ex J.A.Murr.] DCNA DECN

'Dwarf Pagoda' [I. crenata Thunb. ex J.A.Murr.] DCNA MDMN

'Eagleson' [I. $\times$ attenuata Ashe] TXEN

'E. A. McIlhenny' [I. cornuta Lindl. \& Paxt.] LAAL

'Earlibright' [I. verticillata (L.) A.Gr.] DCNA

'Early Cluster' [I. $\times$ altaclerensis (Loud.) Dallim.] DCNA

'East Palatka' [I. $\times$ attenuata Ashe] ALFN DCWH LASL VACW ARRB FLUF NCEG VATA DCCG GACG SCMG DCNA GAUG TNSB
'Edna Jean' [I. Xattenuata Ashe] TNML

'Edward J. Stevens' [I. cornuta $\times I$. aquifolium] DCNA

'Edwin Dozier' [I. crenata Thunb. ex J.A.Murr.] DCNA

'Eldridge' [I. Xaltaclerensis (Loud.) Dallim.] DCNA MDHA NCSH

'Elegance' [I. integra $\times I$. pernyi] DCNA FLUF

'Elegantissima' [I. aquifolium L.]

(Commercially known as 'Mistigold') DCNA GACG

'Elfin' [I. crenata Thunb.]

= 'Delaware Diamond'

'Elizabeth' [I. opaca Ait.] DCNA

'Emily' [I. opaca Ait.] DCNA

'Emily Bruner' [I. cornuta 'Burfordii' $X$ I. Latifolia] DCNA TNAB VATA GACG TNHH

'Erma Byrd' [I. Xattenuata Ashe] DCNA MDTD

'Escort' [I. aquifolium L.] DCNA

'Evangeline' [I. $\times$ altaclerensis (Loud.) Dallim.]

$=$ 'Hazel'

'Fairfax' [I. verticillata (L.) A.Gr.] DCNA

'Farage' [I. opaca Ait.] DCNA

fargesii Franch.

Farges' $\mathrm{H}$. DCNA GAEC

fargesii Franch. ssp. melanotricha (Merr.) S.Andrews DCNA 


\section{ILEX}

'Father Charles' [I. $\times$ altaclerensis (Loud.)

Dallim. ex Rehd.]

$$
\text { DCNA GACG }
$$

'Faulkner' [I. opaca Ait.] $\mathrm{MDHH}$

'Favorite' [I. aquifolium L.] DCNA GACG

'Felten's Selection' [I. opaca Ait.] DCNA

'Ferox' [I. aquifolium L.] DCNA GACG MDBG VADW

'Ferox Argentea' [I. aquifolium L.] DCNA

'Ferox Aurea Marginata' [I. aquifolium L.] VATA

ficoidea Hemsl.

Fig-leaved $\mathrm{H}$.

DCNA

'Fine Line' [I. cornuta Lindl. \& Paxt.] ALTD

'Fire Chief' [I. opaca Ait.] DCNA

'Firecracker' [I. aquifolium L.] GACG

'Firefly' [I. crenata Thunb. ex J.A.Murr.] DECN

'Firelight' [I. $\times$ altaclerensis (Loud.)

Dallim.] DCNA

'Flavescens' [I. aquifolium L.] GACG

'Flushing' [I. crenata Thunb. ex J.A.Murr.] DCNA

'Folsom's Weeping' [I. vomitoria Ait.

f. pendula Foret \& Solym.] GACG GAUG TNCT TXSE

'Formal' [I. opaca Ait.] $\mathrm{NCSH}$

'Formax' [I. cornuta $\times$ I. pernyi] GACG

'Fort McCoy' [I. cumulicola Small] ALEH
'Fort McCoy' [I. vomitoria Ait.]

(Cultivar name illegitimate) FLUF

'Foster \# 1' [I. Xattenuata Ashe] ALAU ALTD

'Foster \#2' [I. Xattenuata Ashe] ALAU GABS MDLT NCDU ALFN MDBG MDTD VATA

'Foster \# 3' [I. Xattenuata Ashe] ALFN GACG

'Foster \# 4' [I. Xattenuata Ashe] DCNA

'Foster No. 1' [I. crenata Thunb. ex J.A.Murr.]

(Cultivar name illegitimate) GACG

'Foster No. 2' [I. crenata Thunb. ex J.A.Murr.]

(Cultivar name illegitimate) GACG

'Foxii' [I. aquifolium L.] DCNA GACG

'Francis Lewis' [I. opaca Ait.] DEFL

'Freeman' [I. opaca Ait.] DCNA

'Frierson' [I. crenata Thunb. ex J.A.Murr.] GACG

'Fructo-1utea' [I. aquifolium L.] DCNA GACG

'Fruitland Nursery' [I. opaca Ait.

f. xanthocarpa Rehd.] GACG NCSH

'Gable' [I. Xaquipernyi Gable] DCNA

'Gayle' [I. crenata Thunb. ex J.A.Murr.

var. paludosa (Nakai) Hara] SCCU

'Gee' [I. opaca Ait.] DCNA

geniculata Maxim.

Furin $\mathrm{H}$. DCNA

georgei Comber

DCNA MDTD 
glabra (L.) A.Gr.

Inkberry

$\begin{array}{lll}\text { ALBG } & \text { GACG } & \text { MDLT } \\ \text { ALUA } & \text { GAUG } & \text { MDTD } \\ \text { DCNA } & \text { MDBG } & \text { VAWP }\end{array}$

glabra (L.) A.Gr. f. leucocarpa F.W.Woods

White-fruited Inkberry

DCNA GACG SCBR

'Glass' [I. crenata Thunb. ex J.A.Murr.] GACG VATA

'Glenwood' [I. cornuta Lindl. \& Paxt.] VAIG

'Globe' [I. aquifolium L.] DCNA

'Glory' [I. crenata Thunb. ex J.A.Murr.] DCNA

'Golden Beauty' [I. aquifolium L.]

$=$ 'Golden Milkmaid'

'Golden Butterfly' [I. aquifolium L.] DCNA GACG

'Golden Fleece' [I. opaca Ait.] DCNA

'Golden Gate' [I. aquifolium]

= 'Rubricaulis Aurea'

'Golden Gem' [I. crenata Thunb. ex J.A.Murr.]

DCNA

'Golden Girl' [I. $\times$ meserveae S.Y.Hu]

(Commercially known as 'Mesglog") DCNA

'Golden Heller' [I. crenata Thunb. ex J.A.Murr.] VATA

'Golden Milkboy' [I. aquifolium L.] DCNA

'Golden Milkmaid' [I. aquifolium L.] DCNA

'Golden Queen' [I. aquifolium L.] $\mathrm{NCSH}$

'Goldie' [I. opaca Ait. f. xanthocarpa Rehd.] DCNA

'Good Taste' [I. cornuta $\times$ I. pernyi] DCNA GACG
'Good Will Park' [I. opaca Ait.] DCNA

'Governor William Paca' [I. opaca Ait.] MDWP

'Gracean' [I. aquifolium L.] DCNA

'Grandpappy' [I. opaca Ait.] NCBW

'Grandview' [I. cornuta Lindl. \& Paxt.] DCNA

'Gray's Bigleaf' [I. vomitoria Ait.] $\mathrm{NCMH}$

'Gray's Little Leaf' [I. vomitoria Ait.] DCNA TXDM

'Green Dragon' [I. crenata Thunb. ex J.A.Murr.] DCNA MDLT

'Green Knight' [I. aquifolium L.] DCNA GACG

'Green Lustre' [I. crenata Thunb. ex J.A.Murr.] DCNA GACG VATA

'Green Maid' [I. aquifolium L.] DCNA GACG

'Green Screw' [I. aquifolium L.]

='Crispa'

'Green Shadow' [I. integra Thunb. ex J.A.Murr.] DCNA

'Green Thumb' [I. crenata Thunb. ex J.A.Murr.] DCNA

'Grier' [I. crenata Thunb. ex J.A.Murr.] VATA

'Griscom' [I. opaca Ait.] DCNA

'Hamlet' [I. opaca Ait.] DCNA

'Handsworthensis' [I. aquifolium L.] DCNA

'Harriet' [I. opaca Ait.] DCNA 
'Harry Gunning' [I. ciliospinosa $\times$

I. leucoclada] DCNA

'Harvest Red' [I. serrata $\times$ I. verticillata] DCNA MDBG

'Hastata' [I. aquifolium L.] GACG

'Hatfield' [I. crenata Thunb. ex J.A.Murr.] GACG

'Hazel' [I. $\times$ altaclerensis (Loud.) Dallim.] DCNA

'Hazel' [I. aquifolium L.]

= 'Hazel' [I. $\times$ altaclerensis]

'H. B. Red' [I. decidua Walt.]

$=$ 'Pocahontas'

'Hedgeholly' [I. opaca Ait.] DCNA

'Helen Makepeace' [I. opaca Ait.] DCNA

'Helleri' [I. crenata Thunb. ex J.A.Murr.] DCNA MDBG NCEG

'Hendersonii' [I. Xaltaclerensis (Loud.) Dallim.] DCNA

'Hendersonii Aurea' [I. $\times$ altaclerensis (Loud.) Dallim.] GACG

'Heterophylla Aureomarginata'

[I. aquifolium L.]

= 'Elegantissima'

'Hetzii' [I. crenata Thunb. ex J.A.Murr.] GACG

^ 'Highlight' [I. crenata Thunb. ex

J.A.Murr.]

DCNA

'Hodginsii' [I. Xaltaclerensis (Loud.)

Dallim.] DCNA GACG VAWM

'Hohman' [I. X koehneana Loesn.] MDBG

'Hollowell' [I. cornuta $\times$ I. aquifolium] DCNA - MDHA
'Hollycroft Jack' [I. × altaclerensis (Loud.) Dallim.]

= 'Hollycroft Jack' [I. aquifolium]

'Hollycroft Jack' [I. aquifolium L.] DCNA

'Homer' [I. opaca Ait.] DCNA

'Honeycomb' [I. crenata Thunb. ex J.A.Murr.] MDMN

'Hopkins' [I. opaca Ait.] DCNA

'Howard' [I. Xattenuata Ashe] DCNA GABS GAFN FLDG GACG LASL

'Howard' [I. opaca Ait.]

= 'Howard' [I. $\times$ attenuata $]$

'Huber's Compact' [I. vomitoria Ait.] LASL

'Hume' [I. cornuta Lindl. \& Paxt.] MDMN VATA

'Hume \# 1' [I. Xattenuata Ashe] DCNA

'Hume \#2' [I. Xattenuata Ashe] ALFN DCNA GACG

'Hume's Choice' [I. opaca Ait.] MDMN

'Hutchinson' [I. $\times$ attenuata Ashe] MSWB

'Indian Bayou \#3' [I. decidua Walt.] LASL

'Indian Chief' [I. cornuta $\times I$. pernyi] GACG

'Ingramii' [I. aquifolium L.] MDTN

insignis Hook.

= I. kingiana

integra Thunb. ex J.A.Murr.

Mochi $\mathrm{H}$.

$\begin{array}{lll}\text { ALBG } & \text { DCNA } & \text { GAIS } \\ \text { ALTD } & \text { FLMG } & \text { LAAL } \\ \text { DCCG } & \text { FLUF } & \text { VAWR }\end{array}$


integra $\times I$. aquifolium

(Plants of this cross from different origins are widely distributed)

DCNA MDTD

integra $\times I$. cornuta

GACG

integra $\times I$. pernyi

(Plants of this cross from different origins are widely distributed) DCNA

'Integrifolium' [I. aquifolium L.] $\mathrm{NCSH}$

'Ira Nelson' [I. cornuta Lindl. \& Paxt.] DCNA LAAL LASL VACW

'Iso' [I. opaca Ait.] DCNA

'Ivory' [I. aquifolium L.] DCNA

'Ivory Hall' [I. crenata Thunb.

ex J.A.Murr.]

DECN VATA

'Ivory Queen' [I. glabra (L.) A.Gr.

f. leucocarpa F.W.Woods]

DCNA MDMN

'Ivory Tower' [I. crenata Thunb. ex

J.A.Murr.]

DECN VATA

'Jackson' [I. verticillata (L.) A.Gr.] DCNA

$\star$ 'Jade' [I. × koehneana Loesn.] DCNA

'James G. Esson' [I. $\times$ altaclerensis (Loud.)

Dallim.]

$\begin{array}{lll}\text { DCNA } & \text { GACG } & \text { MDTD } \\ \text { DEMC } & \text { MDMN } & \end{array}$

'James Swan' [I. cornuta 'Burfordii' $\times I$.

latifolia]

TNAB TNHH

'J. C. van Tol' [I. aquifolium L.] GACG

'Jeannette Adamson' [I. opaca Ait.] MDPJ

'Jersey Pinmacle' [I. crenata Thunb. ex J.A.Murr.] DCNA
'Jersey Princess' [I. opaca Ait.] DCNA MDLT VATA

'Jinny Bruner' [I. cornuta 'Burfordii'

$\times$ I. latifolia] TNAB TNHH

'John Higgins' [I. opaca Ait.] DCNA

'John Nosal' [I. crenata Thunb. ex J.A.Murr.] DCNA

'John T. Morris' [I. cornuta $\times$ I. pernyi] DCNA GACG VATA

'Joyce' [I. opaca Ait.] DCNA

'Judge Brown' [I. opaca Ait.] DCNA NCSH

'Jungle Garden' [I. cornuta Lindl. \& Paxt.] LAAL NCDU

'Kate' [I. opaca Ait.] DCNA

kingiana Cockerell DCNA

'Kingsville Dwarf' [I. crenata Thunb. ex J.A.Murr.]

DCNA MDBG SCMG

GACG NCEG VATA

'Kingsville Special' [I. cornuta Lindl. \& Paxt.] GACG

'Kirofukurin' [I. crenata Thunb. ex J.A.Murr.]

= 'Angyo'

$\times$ kiusiana Hatusima

$[$ I. buergeri $\times I$. integra] DCNA

'Knight' [I. opaca Ait.] DCNA MDWA

$\times$ koehneana Loesn.

Koehne's H.

[I. aquifolium $\times$ I. latifolia] DCNA MDBG MDMG VATA DCWH MDLT VACW VAWR

'Kunming' [I. crenata Thunb. ex J.A.Murr.] GACG 
'La Bar' [I. opaca Ait.] DCNA NCSH

'Lacerta' [I. cornuta $\times$ I. pernyi] GACG

'Lady Baltimore' [I. aquifolium L.] DCNA

laevigata (Dum.-Cours.) A.Gr.

Smooth Winterberry

DCNA GACG NCBE

'Lagniappe' [I. longipes Chapm. ex

Trelease]

ALJG

'Lake City' [I. opaca Ait.] DCNA GACG LAHG LASL

'Lassie' [I. × koehneana Loesn.] MDMN

latifolia Thunb. ex J.A.Murr.

Luster-leaved H., Broad-leaved $\mathrm{H}$.

$\begin{array}{llll}\text { ALAU } & \text { GACG } & \text { MDLT } & \text { TXDC } \\ \text { ALBG } & \text { GADG } & \text { NCDU } & \text { VACW } \\ \text { ALTD } & \text { GAEC } & \text { NCEL } & \text { VAGS } \\ \text { DCCG } & \text { GAIS } & \text { SCMG } & \text { VASC } \\ \text { DCNA } & \text { GAWH } & \text { SCMP } & \\ \text { DCWH } & \text { LASL } & \text { TNSB } & \\ \text { FLUF } & \text { MDBG } & \text { TNUT } & \end{array}$

'Latifolia' [I. crenata Thunb. ex J.A.Murr.]

(Many cultivars of different origins)
DCNA GACG MDLT
FLMG GAFN

'Latispina' [I. aquifolium L.] VADW

'Laura' [I. opaca Ait.] DCNA NCSH

'Laurifolia' [I. $\times$ altaclarensis (Loud.) Dallim. ex Rehd.] DCNA

'Laurifolia' [I. aquifolium L.]

= 'Laurifolia' [I. Xaltaclerensis]

'Lenape Moon' [I. opaca Ait.

f. xanthocarpa Rehd.] DCNA

\section{'Lepux' [I. cornuta $\times$ I. pernyi]} GACG

'Leucocarpa' [I. serrata Thunb. ex J.A.Murr.]

Yellow-fruited Japanese Winterberry DCNA leucoclada Makino

Yellow-stemmed $\mathrm{H}$. DCNA

'Lewis' [I. aquifolium L.] MDHA

'Libby's Favorite' [I. integra $\times I$. cornuta] ALTD

'Lilliput' [I. aquifolium L.] DCNA

'Lilygold' [I. aquifolium L.] DCNA

'Lindleyana' [I. crenata Thunb. ex J.A.Murr.] GACG

'Little Bull' [I. aquifolium L.] DCNA

liukiuensis Loesn.

Liukiu $\mathrm{H}$. GACG

'Lock Raven' [I. $\times$ koehneana Loesn.] DCNA

lohfauensis Merr. DCNA

'Longfellow' [I. crenata Thunb. ex J.A.Murr.] DCNA

'Longifolia' [I. crenata Thunb. ex J.A.Murr.]

(Name applied to numerous clones of diverse origins.) DCWH

longipes Chapm. ex Trelease

Long-stalked H., Georgian H.
ALTD
LAHG
NCDU
DCNA
MDTD
TNFB
TXLL

'Longspra' [I. aquifolium L.] $\mathrm{NCSH}$

'Lord' [I. rotunda Thunb. ex J.A.Murr.] DCNA

'Louise' [I. aquifolium L.] DCNA

'Louise Holmes' [I. $\times$ attenuata Ashe] DCNA VACW

'Lowei' [I. myrtifolia Walt.] DCNA FLUF 
'Lowell' [I. opaca Ait.] DCNA

'Loyce Nelson' [I. crenata Thunb. ex J.A.Murr.]

DCNA

'Luteo-variegata' [I. crenata Thunb. ex J.A.Murr.] GACG

\section{'Lutescens' [I. aquifolium L.]} GACG

$\star$ 'Lydia Morris' [I. cornuta $\times$ I. pernyi] DCNA

'Lyra' [I. cornuta $\times$ I. pernyi] GACG

macrocarpa Oliv. DCNA

macropoda Miq. DCNA VABF

'Maderensis Variegata' [I. aquifolium L.] DCNA

'Mae' [I. opaca Ait.] DCNA

'Magna Semen' [I. opaca Ait.] DCNA

'Major' [I. crenata Thunb. ex J.A.Murr.] (Name applied to numerous clones of different origins) DCNA GACG

'Malcolm S. Whipple' [I. aquifolium L.] MDBG

'Mamie Eisenhower' [I. opaca Ait.] DCNA GACG

'Manig' [I. opaca Ait.] DCNA MDBG

'Maplehurst' [I. cornuta $\times$ I. aquifolium] DCNA

'Margaret Moran' [I. opaca Ait.

f. xanthocarpa Rehd.] DCNA

'Mariesii' [I. crenata Thunb. ex J.A.Murr.] Coin-leaved $\mathrm{H}$.
DCNA
GACG
GAEC
VAPH

'Marnockii' [I. $\times$ altaclerensis (Loud.)

Dallim.] GACG

'Marshal Tito' [I. aquifolium L.] DCNA

'Mary Nell'

[(I. cornuta 'Burfordii' $\times$ I. pernyi)

'Red Delight' $\times$ I. latifolia] ALTD

'Maryland' [I. opaca Ait.] DCNA

'Maryland Beauty' [I. verticillata (L.) A.Gr.] MDJN

'Maryland Dwarf' [I. opaca Ait.] DCNA MDBG VATA

maximowicziana Loesn. var. kanehirae (Yamamoto) Yamazaki DCNA GACG VATA

'Maxwell' [I. crenata Thunb. ex J.A.Murr.] GACG

'Medallion' [I. cornuta Lindl. \& Paxt.] DCNA

'Menantico' [I. opaca Ait.] DCNA

'Mentor Dense' [I. crenata Thunb. ex J.A.Murr.] DCNA GACG

'Mentor Glossy' [I. crenata Thunb. ex J.A.Murr.] GACG

'Merry Christmas' [I. opaca Ait.] MDAL

$\times$ meserveae S.Y.Hu

[I. rugosa $\times$ I. aquifolium]

(The "blue" hollies; see cultivar names beginning with Blue)

'Mesglog' [I. × meserveae S.Y.Hu]

$=$ 'Golden Girl'

'Microphylla' [I. crenata Thunb. ex J.A.Murr.]

(Name applied to numerous clones of different origins)
ALFN
DCNA
GACG

'Microphylla Supreme' [I. crenata Thunb. ex J.A.Murr.] GACG 
'Midas Touch' [I. crenata Thunb. ex

J.A.Murr.] DCNA

'Miss Helen' [I. opaca Ait.] DCNA MDMN

'Miss Liberty' [I. opaca Ait.] SCWI

'Miss Muffet' [I. crenata Thunb. ex J.A.Murr.] DECN VATA

'Mistigold' [I. aquifolium L.]

$=$ 'Elegantissima'

'Mobjack Supreme' [I. crenata Thunb. ex J.A.Murr.] VATA

\section{'Monstrosa' [I. aquifolium L.]} GACG

montana T.\&G. ex A.Gr.

$=$ I. monticola

monticola A.Gr.

Mountain $\mathrm{H}$. DCNA GACG SCBR

'Moonglow' [I. cornuta $\times$ I. pernyi] GACG

'Moonlight' [I. aquifolium L.]

= 'Flavescens'

'Morgan Gold' [I. opaca Ait.

f. xanthocarpa Rehd.] DCIW

'Morrell No. 1' [I. cornuta Lindl. \& Paxt.] GACG

'Morrell No. 3' [I. cornuta Lindl. \& Paxt.] GACG

'Morris Dwarf' [I. crenata Thunb. ex

J.A.Murr.] GACG

'Mount Halla' [I. crenata Thunb. ex J.A.Murr.] DCNA

'Mrs. Santa' [I. opaca Ait.] DCNA

'Mundyi' [I. $\times$ altaclerensis (Loud.) Dallim.] DCNA GACG mutchagara Makino

= I. maximowicziana var. kanahirae

myrtifolia Walt.

Myrtle-leaved $\mathrm{H}$.
DCNA
LAHG
SCJF
GACG MSFG
SCRH
GAFN
SCBR
VATA

myrtifolia Walt.

(Yellow-fruited; not equivalent to 'Lowei') GAEC

'Myrtifolia' [I. aquifolium L.] GACG

'Nakada' [I. crenata Thunb. ex J.A.Murr.] DCNA MDTD VATA

'Nakaharae' [I. sugerokii Maxim.] DCNA

'Nana' [I. glabra (L.) A.Gr.]

(Several clones of different origins) DCNA

'Nana' [I. verticillata (L.) A.Gr.]

$=$ 'Red Sprite'

'Nana' [I. vomitoria Ait.]

(Several clones of different origins)
ALAU
FLMG
GACG
LAHG

'Nanking' [I. crenata Thunb. ex J.A.Murr.] GACG

'NASA' [I. $\times$ attenuata Ashe] ALTD

'Natchez Belle' [I. longipes Chapm. ex Trelease] $\mathrm{MSBH}$

'National' [I. cornuta Lindl. \& Paxt.] ALTD

'Needle Point' [I. cornuta Lindl. \& Paxt.] LASL

'Nellie R. Stevens' [I. cornuta $\times$

I. aquifolium]

$\begin{array}{llll}\text { ALTD } & \text { DCWH } & \text { MDLT } & \text { TNUT } \\ \text { DCCG } & \text { GACG } & \text { NCTP } & \text { VATA } \\ \text { DCNA } & \text { MDBG } & \text { SCKG } & \end{array}$

'Nelson West' [I. opaca Ait.] DCNA

'N. F. Barnes' [I. aquifolium L.]

GACG 
'Nigrescens' [I. Xaltaclerensis (Loud.)

Dallim.]

DCNA

nobilis Gumbleton

= I. kingiana

'NYBG \#2' [I. aquifolium L.] DCNA

'Oconee River' [I. crenata Thunb. ex J.A.Murr.]

GACG

'Old Gold' [I. opaca Ait. f. xanthocarpa Rehd.] DCNA

'Old Heavy Berry' [I. opaca Ait.] DCNA GACG VACW

'Oleafera' [I. crenata Thunb. ex J.A.Murr.] GACG

'Olga' [I. cornuta Lindl. \& Paxt.] $\mathrm{DCCB}$

opaca Ait.

American $\mathrm{H}$.

$\begin{array}{llll}\text { ALSH } & \text { FLCG } & \text { MDLT } & \text { SCNS } \\ \text { DCCG } & \text { FLMG } & \text { MDMG } & \text { VACW } \\ \text { DCNA } & \text { GAOH } & \text { SCBR } & \\ \text { DCWH } & \text { MDBG } & \text { SCMP } & \end{array}$

opaca Ait. var. arenicola (Ashe) Ashe

= I. cumulicola

opaca Ait. f. subintegra Weatherby

DCNA MDAB MDSJ SCMP

FLPW MDJS NCBE

LASL MDMN SCBR

opaca Ait. f. xanthocarpa Rehd.

Yellow-fruited American H. DCNA GACG NCEG VACW

$\star$ 'Oriole' [(I. myrtifolia $\times$ I. opaca)

$\times$ I. myrtifolia] DCNA

'Osa' [I. opaca Ait.] DCNA

'O. Spring' [I. cornuta Lindl. \& Paxt.] DCNA

'Otis Miley' [I. vomitoria Ait.] GACG

'Painted Lady' [I. aquifolium L.] GACG
'Pale Moon' [I. aquifolium L.] GACG

'Palmetto' [I. opaca Ait.] ALTD

paraguariensis St.Hil.

Paraguay Tea, Yerba Maté DCNA FLUF

'Peconic' [I. crenata Thunb. ex J.A.Murr.] DCNA

pedunculosa Miq.

Evergreen Long-stalked $\mathrm{H}$.

$\begin{array}{llll}\text { ALTD } & \text { DEWG } & \text { MDLT } & \text { MDTD } \\ \text { DCCG } & \text { GACG } & \text { MDMG } & \text { SCWI } \\ \text { DCNA } & \text { GAEC } & \text { MDMN } & \end{array}$

pedunculosa Miq. var. continentalis Loesn.

$=$ I. pedunculosa

'Pendula' [I. aquifolium L.] DCNA GACG

perado Ait. ssp. platyphylla (P.B.Webb \& Berth.) S.Andrews DCNA

'Perkins \# 1' [I. aquifolium L.] DCNA

'Perkins-de-Wilde \# 1' [I. opaca Ait.] DCNA

'Perkins-de-Wilde \#2' [I. opaca Ait. ] DCNA

'Perkins-de-Wilde \#3' [I. opaca Ait.] DCNA

'Perle LeClair' [I. opaca Ait. f. subintegra Weatherby] DCNA

NCDU

pernyi Franch.

$\begin{array}{llll}\text { ALFN } & \text { FLMG } & \text { GALA } & \text { VABF } \\ \text { ALTD } & \text { GACG } & \text { MDMG } & \text { VAWR } \\ \text { DCNA } & \text { GAFN } & \text { TNUT } & \end{array}$

pernyi Franch. var. veitchii (J.G.Veitch) Bean $=$ I. bioritensis

'Perrine' [I. opaca Ait.]

DCNA

'Petite' [I. aquifolium L.]

DCNA GACG

'Phantom Gold' [I. aquifolium L.]

DCNA NCSH 


\section{ILEX}

'Piccolo' [I. crenata Thunb. ex J.A.Murr.] DECN

'Pin Cushion' [I. opaca Ait.] DCNA

'Pinto' [I. aquifolium L.] DCNA GACG

'Planifolia' [I. aquifolium L.] DCNA GACG

'Pocahontas' [I. decidua Walt.]

(Originally known as 'H. B. Red', an invalid name) DCNA

'Polly' [I. opaca Ait.] DCNA

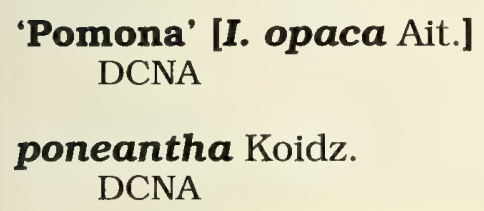

'Pride Dwarf' [I. crenata Thunb. ex J.A.Murr.] DCNA

'Pride's Tiny' [I. crenata Thunb. ex J.A.Murr.] GACG

\section{'Princess Pat' [I. aquifolium L.]} DCNA

pubescens Hook. \& Arn. ALTD DCNA FLUF

purpurea Hassk.

Peach-leaved H., Kachi H.
DCNA GAIS
TNSN
GACG
GAUG TNUT

'Pyramidalis' [I. aquifolium L.] DCNA

'Pyramidalis' [I. crenata Thunb. ex J.A.Murr.] MDAL

'Pyramidalis Compacta' [I. aquifolium L.] GACG

\section{'Recurva' [I. aquifolium L.]} DCNA GACG
'Recurva' [I. pernyi Franck] DCNA

'Red Delight' [I. cornuta 'Burfordii' $\times$ I. pernyi] DCNA GACG

'Rederly' [I. aquifolium L.] DCNA GACG

'Red Robe' [I. cornuta $\times$ I. pernyi] GACG

'Red Sprite' [I. verticillata (L.) A.Gr.] DCNA

'Repandens' [I. crenata Thunb. ex

J.A.Murr.] DCNA GACG

'Reynolds' [I. opaca Ait.] DCNA TNTV

'Richard E. Lincoln' [I. verticillata (L.) A.Gr.] MDES

'Richards' [I. opaca Ait.] MDHR

'Ricker' [I. aquifolium L.] DCNA MDHA

'Riddle Farm' [I. aquifolium L.] DCNA

'Rock Garden'

$[$ I. $\times$ aquipernyi $\times(I$. integra $\times I$. pernyi $)$ 'Accent'] DCNA

'Rocky Creek' [I. crenata Thunb. ex J.A.Murr.] DCNA

rotunda Thunb. ex J.A.Murr. Kurogane $\mathrm{H}$.
ALTD
FLFS GACH
VANB
DCNA
FLUF GAIS
FLFB
GACG LAAL

'Rotunda' [I. cornuta Lindl. \& Paxt.]

$\begin{array}{llll}\text { ALTD } & \text { FLUF } & \text { MDLT } & \text { TXHA } \\ \text { DCLC } & \text { GACG } & \text { MDTD } & \\ \text { DCNA } & \text { GAFN } & \text { SCMG } & \end{array}$

'Royal Red' [I. $\times$ altaclerensis (Loud.) Dallim.] DCNA GACG

'Royal Red' [I. aquifolium L.]

= 'Royal Red' [I. $\times$ altaclerensis $]$ 
ILEX

'Rubricaulis Aurea' [I. aquifolium L.] DCNA GACG

$\star$ 'Ruby' [I. × koehneana Loesn.] DCNA MDTD VATA

'Ruby Red' [I. opaca Ait.] TNTV

rugosa F.Schmidt

Tsuru H., Creeping H. DCNA

'Rushton' [I. opaca Ait.] DCNA

'R. V. P. Special' [I. cornuta Lindl. \& Paxt.] MDHD

'St. Ann' [I. opaca Ait.] DCNA

'St. John's' [I. opaca Ait.] MDSJ

'St. Mary' [I. opaca Ait.] DCNA GACG

'San Jose' [I. Xaquipernyi Gable] GACG MDTD

'San Jose' [I. Xkoehneana Loesn.] DCNA GACG

'Sandy Hook \#5' [I. opaca Ait.] DCNA

'Sara Higgins' [I. opaca Ait.] $\mathrm{NCSH}$

'Satyr Hill' [I. opaca Ait.] MDMN VATA

'Savannah' [I. $\times$ attenuata Ashe]

$\begin{array}{llll}\text { ALBG } & \text { GACG } & \text { LASL } & \text { SCKG } \\ \text { DCNA } & \text { GAET } & \text { SCBR } & \\ \text { FLUF } & \text { LAHG } & \text { SCFW } & \end{array}$

'Schlupp' [I. opaca Ait.] VACW

'Scotica' [I. aquifolium L.] MDBG

'Scram's Dwarf' [I. aquifolium L.] DCNA

'Secrest' [I. opaca Ait.] DCNA

'Semala' [I. integra $\times$ I. cornuta]
'Sentinel' [I. crenata Thunb. ex J.A.Murr.] VATA

* 'September Gem' [I. ciliospinosa $\times$

I. $\times$ aquipernyi] DCNA MDWP

serrata Thunb. ex J.A.Murr.

Japanese Winterberry

DCNA GACG MDPJ NCBE

DEEM MDBG MDTD

serrata $\times$ I. verticillata $\mathrm{SCCU}$

serrata Thunb. ex J.A.Murr. var. argutidens (Miq.) Rehd.

= I. serrata

serrata Thunb. ex J.A.Murr. var. sieboldii

(Miq.) Rehd.

=I. serrata

'Shanghai' [I. crenata Thunb. ex J.A.Murr.] GACG

'Shangri-La' [I. cornuta Lindl. \& Paxt.] DCNA GACG

'Shaver' [I. verticillata (L.) A.Gr.] DCNA

shennongiiaensis T.R.Dudley \& Sun DCNA

'Shilling's' [I. vomitoria Ait.] GACL NCTP

'Shilling's Dwarf' [I. vomitoria Ait.]

(This cultivar and 'Schilling's' are confused) GAJI

'Shiu-ying' [I. cornuta Lindl. \& Paxt.] MDMN

'Shortspra' [I. aquifolium L.] DCNA GACG

sikkimensis Hook.

Sikkim H. DCNA

'Silver Edge' [I. aquifolium L.]

= 'Argentea Marginata'

'Silver Milkboy' [I. aquifolium L.] GACG

'Silver Milkmaid' [I. aquifolium L.] DCNA 


\section{ILEX}

'Silver Princess' [I. aquifolium L.]

= 'Argentea Marginata'

'Sirofukurin' [I. crenata Thunb. ex J.A.Murr.]

= 'Snowflake'

'Slim Jim' [I. opaca Ait.] DCNA

'Snowflake' [I. crenata Thunb. ex J.A.Murr.] DCNA

$\star$ 'Sparkleberry' [I. serrata $\times I$. verticillata] DCNA VATA

'Sparkler' [I. aquifolium L.] GACG

spinigera (Loesn.) Loesn. DCNA

'Star' [I. opaca Ait.] DCNA

'Starker's Silver' [I. aquifolium L.] = 'Elegantissima'

'Stokes' [I. crenata Thunb. ex J.A.Murr.] DCNA GACG

'Stokes Dwarf' [I. vomitoria Ait.] LASL

sugerokii Maxim. DCNA VATA

'Sunnybrooke' [I. aquifolium L.] DCNA GACG

^ 'Sunny Foster' [I. $\times$ attenuata Ashe] DCNA MDBG

\section{'Sunnyside' [I. aquifolium L.]} DCNA

'Sunset' [I. verticillata (L.) A.Gr.] DCNA

'Taber' [I. opaca Ait.] GACG

'Taber \#2' [I. opaca Ait.] ALFN

$\star$ 'Tanager' [(I. myrtifolia $\times$ I. opaca $) \times$ I. myrtifolia] DCNA
'Tankard's Compact' [I. glebra (L.) A.Gr.] VATA

'Tennyson' [I. crenata Thunb.

ex J.A.Murr.]

DCNA GACG

'Teufel's Hybrid' [I. aquifolium L.] DCNA GACG

'Teufel's Variegated' [I. aquifolium L.] GACG

thomsonii (Hook.f.) Hara

$=I$. crenata var. thomsonii

'Thornton' [I. aquifolium L.] DCNA

'Tiny' [I. crenata Thunb. ex J.A.Murr.]

= 'Pride's Tiny'

'Tiny' [I. opaca Ait.] DCNA

'Tiny Tim' [I. crenata Thunb. ex J.A.Murr.] = 'Pride's Tiny'

'Titan' [I. cornuta $\times$ I. pernyi] DCNA GACG MDBG

'Tom Everett' [I. aquifolium L.] GACG

'T-one' [I. crenata Thunb. ex J.A.Murr.] VATA

'Toner' [I. opaca Ait.] DCSE MDHD MDKN

'Topeli' [I. $\times$ attenuata Ashe] DCNA

'Trisco' [I. opaca Ait.] DCNA

'24 Karat' [I. opaca Ait.] MDPM

'Tyke' [I. crenata Thunb. ex J.A.Murr.

var. paludosa (Nakai) Hara] SCCU

'Variegata' [I. cornuta Lindl. \& Paxt.] GACG

'Vaseyi' [I. crenata Thunb. ex J.A.Murr.] GACG

'Veitchii' [I. pernyi Franch.]

$=\mathrm{I}$, bioritensis 
'Vera' [I. opaca Ait.]

MDBG

verticillata (L.) A.Gr.

Winterberry, Black Alder

$\begin{array}{llll}\text { ALMG } & \text { MDBG } & \text { SCCU } & \text { VAPH } \\ \text { ALTD } & \text { MDLT } & \text { TNFB } & \text { VATA } \\ \text { ALUA } & \text { NCAS } & \text { VAAC } & \\ \text { DCNA } & \text { NCBE } & \text { VAGW } & \\ \text { GACG } & \text { SCBR } & \text { VAHR } & \end{array}$

verticillata (L.) A.Gr. f. chrysocarpa

Robinson

Yellow-berried Winterberry DCNA

'Virginia Dare' [I. vomitoria Ait.]

= 'Dare County'

'Virgo' $[$ I. cornuta $\times$ I. pernyi] DCNA

'Viridis' [I. glabra (L.) A.Gr.] DCNA

vomitoria Ait.

Yaupon

$\begin{array}{llll}\text { ALBG } & \text { FLMG } & \text { NCDU } & \text { SCUC } \\ \text { ARSN } & \text { FLUF } & \text { NCEG } & \text { TXDM } \\ \text { DCNA } & \text { GACG } & \text { SCBR } & \text { VACW } \\ \text { FLAL } & \text { GAJI } & \text { SCMP } & \text { VAGW } \\ \text { FLCG } & \text { GAJY } & \text { SCNS } & \end{array}$

vomitoria Ait. var. chiapiensis Sharp

Mexican Yaupon

DCNA

vomitoria Ait. f. pendula Foret \& Solym.

Pendulous Yaupon
DCNA LAHG LASL
GACG LAHP

'Walker' [I. cornuta Lindl. \& Paxt.] GACG

'Watereriana' [I. aquifolium L.]

Waterer's Gold H. DCNA

'Wayne' [I. crenata Thunb. ex J.A.Murx.] MDAL

'Wheeler \# 1' [I. opaca]

='Dick'

'Wheeler \#4' [I. aquifolium L.]

DCNA NCSH

'Wheeler \#4' [I. opaca Ait.]

(Cultivar name illegitimate) $\mathrm{NCSH}$
'Whittingtonensis' [I. aquifolium L.] DCNA

'William Cowgill' [I. ciliospinosa $\times$

I. leucoclada] MDBG

'William Hawkins' [I. opaca Ait.] ALTD

'William Jackson' [I. crenata Thunb. ex J.A.Murr.]

DCNA

'Willow Leaf' [I. crenata Thunb. ex J.A.Murr.] GACG

'Wilmat Yellow' [I. opaca Ait.

f. xanthocarpa Weatherby] GACG

wilsonii Loesn.

Wilson's $\mathrm{H}$. DCNA GACG

'Wilsonii' [I. $\times$ altaclerensis (Loud.)

Dallim.] DCNA GACG MDLT

'Wintergreen' [I. aquifolium L.] DCNA

'Winter King' [I. aquifolium L.] DCNA

'Winter Queen' [I. aquifolium L.] DCNA

'Winter Red' [I. verticillata (L.) A.Gr.] DCNA MDBG VAGS

'Wirt L. Winn' [I. $\times$ koehneana Loesn.] DCNA MDLT MDTD

'Yawkeyii' [I. vomitoria Ait.] DCNA SCBR

'Yellow Beam' [I. aquifolium L.] DCNA

'Yellow Jacquet' [I. opaca Ait.

f. xanthocarpa Rehd.] DCNA

'Yonkers' [I. aquifolium L.] DCNA

'Yule' [I. opaca Ait.] DCNA 
'Yule Glow' [I. aquifolium L.]

GACG

'Yunnan' [I. crenata Thunb. ex J.A.Murr.] GACG

yunnanensis Franch.

Yunnan $\mathrm{H}$.

DCNA

yunnanensis Franch. var. gentilis Loesn. DCNA

\section{'Zero' [I. aquifolium L.]}

DCNA GACG

zhejiangensis C.J.Tseng DCNA

\section{ILLICIUM L.}

ILLICIACEAE

Illicium Family

anisatum L.

Japanese Anise Tree
ALEH
GACG
TXLL
ALTD
GAEC
VATA

floridanum Ellis

Florida Anise

$\begin{array}{llll}\text { ALAU } & \text { FLGS } & \text { GAEC } & \text { SCWI } \\ \text { ALBG } & \text { FLMG } & \text { GAWH } & \text { TNDG } \\ \text { ALEH } & \text { FLUF } & \text { LALG } & \text { TXLL } \\ \text { ALSH } & \text { GACE } & \text { MDLT } & \text { VACW } \\ \text { DCNA } & \text { GACG } & \text { NCBE } & \text { VAPH } \\ \text { FLAL } & \text { GACH } & \text { SCMP } & \text { VAZC }\end{array}$

floridanum Ellis f. album F.G.Mey. \& Mazzeo
ALTD
DCNA SCWI

henryi Diels

Chinese Anise Tree

ALTD

mexicanum A.C.Sm.

SCWI

parviflorum Michx. ex Vent.

$\begin{array}{llll}\text { FLLE } & \text { LAGN } & \text { SCMG } & \text { VATA } \\ \text { FLMG } & \text { NCAG } & \text { SCMP } & \\ \text { FLUF } & \text { NCDU } & \text { TNFR } & \\ \text { GAJC } & \text { SCBR } & \text { VACW } & \end{array}$

'Semmes' [I. floridanum Ellis f. album

F.G.Mey. \& Mazzeo]
ALWR
DCNA

INDIGOFERA L. $-$

FABACEAE (Faboideae)

Indigo Bean Family
ITEA L.

SAXIFRAGACEAE (Iteoideae)

Saxifrage Family

ilicifolia Oliv. NCEL

virginica $\mathrm{L}$.

Virginia Willow

$\begin{array}{llll}\text { DCNA } & \text { LASL } & \text { SCBR } & \text { VACW } \\ \text { GAGB } & \text { MDBG } & \text { TNRT } & \\ \text { LALG } & \text { MDLT } & \text { TXRS } & \end{array}$

JACARANDA Juss. BIGNONIACEAE

Bignonia Family acutifolia HBK.

Jacaranda, Green Ebony

LALG 

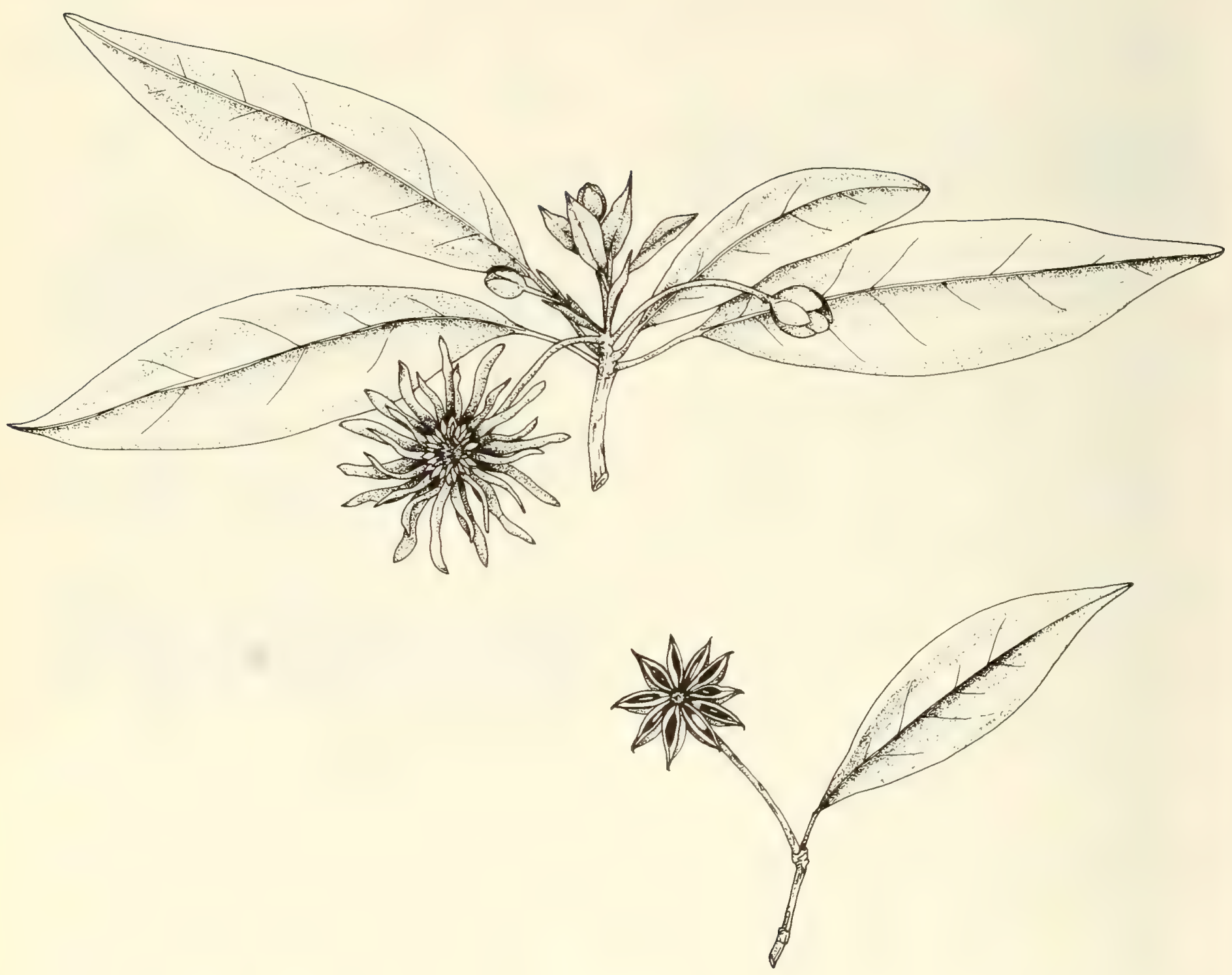

ILLICIUM floridanum Ellis

[illustrator Susan M. Johnston] 


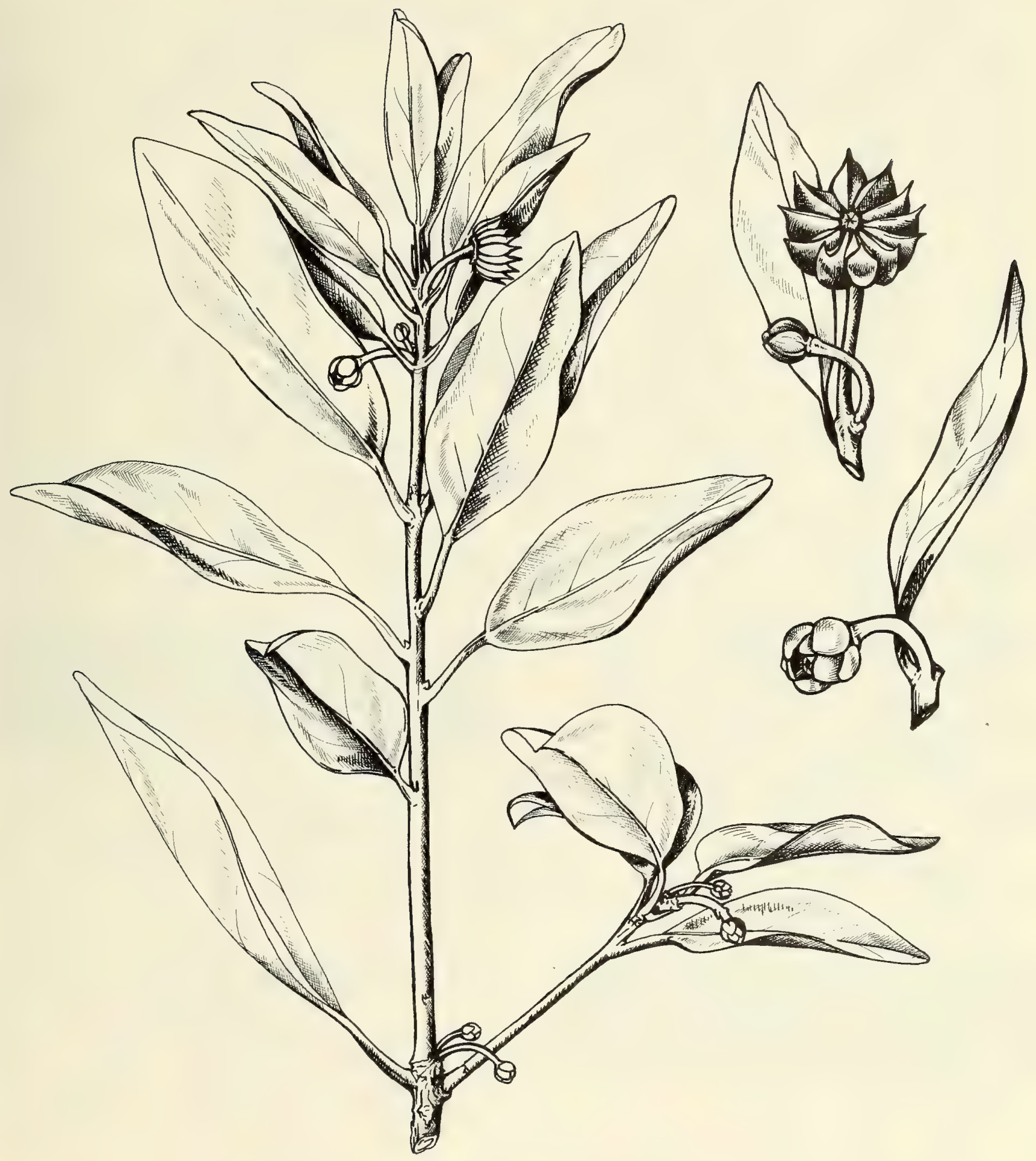

ILLICIUM parviflorum Michx. ex Vent. [illustrator Susan M. Johnston] 
JASMINUM L.

Jasmine OLEACEAE

beesianum Forrest \& Diels MDGD

floridum Bunge

$\begin{array}{llll}\text { ALBH } & \text { NCDU } & \text { TNRM } & \text { VANB } \\ \text { GABS } & \text { SCBR } & \text { TNUT } & \text { VAPH } \\ \text { LAHG } & \text { SCCU } & \text { TXLL } & \\ \text { MDGD } & \text { SCWI } & \text { VAGW } & \end{array}$

fruticans $\mathrm{L}$.

SCWI

humile $\mathrm{L}$.

LAMP LASL

mesnyi Hance

Primrose J.
ALSH
GAJI
LASL
SCUC
FLDG
LAAP
SCBR
VACW

multiflorum (Burm.f.) Andr.

Star J.

FLUF LALG

nitidum Skan

Angel-wing J.
FLUF
GACG
LALG

nudiflorum Lindl.

Winter $J$.

MDBG MDLA MDLT

parkeri S.T.Dunn

VACW

'Revolutum' [J. humile L.]

Italian $\mathrm{J}$.

SCWI

$\times$ stephanense E.Lemoine

[J. beesianum $\times J$. officinale]

SCWI

JUGLANS L. JUGLANDACEAE

ailantifolia Carr.

DCNA TNFE TNSC

DCWR TNLW

ailantifolia Carr. var. cordiformis (Maxim.)

Rehd.

Japanese W.

DCNA
'Broadview' [J. regia L.] MDGD

cinerea L.

Butternut DCCG

'Hansen' [J. regia L.] MDGD

'Laciniata' [J. nigra L.] TNNE

major (Torr.) Heller

Arizona W.

DCNA

'McDermid' [J. regia L.] MDGD

nigra $\mathrm{L}$.

Black W.

$\begin{array}{llll}\text { DCCG } & \text { GAJS } & \text { MDNA } & \text { VAWP } \\ \text { DEMC } & \text { MDCP } & \text { SCBR } & \\ \text { FLUF } & \text { MDHN } & \text { TNUT } & \\ \text { GAIS } & \text { MDLT } & \text { VACW } & \end{array}$

'Paradox' $[J$. hindsii $\times J$. regia] DCNA

regia $\mathrm{L}$

English W., Persian W. DCCG VACW

JUNIPERUS L. Juniper

CUPRESSACEAE Cypress Family

'Admirabilis' [J. horizontalis Moench] DCNA

'Alba' [J. scopulorum Sarg.] DCNA

'Arcadia' [J. sabina L.] DCNA

'Aurea' [J. chinensis L.] VAMP

'Aurea' [J. virginiana L.] DCNA

'Bar Harbor' [ $J$. horizontalis Moench] DCNA FLUF MDBG

'Berkshire' [J. communis L.] DCNA MDBG

'Blaauw' [J. chinensis L.] MDNA 


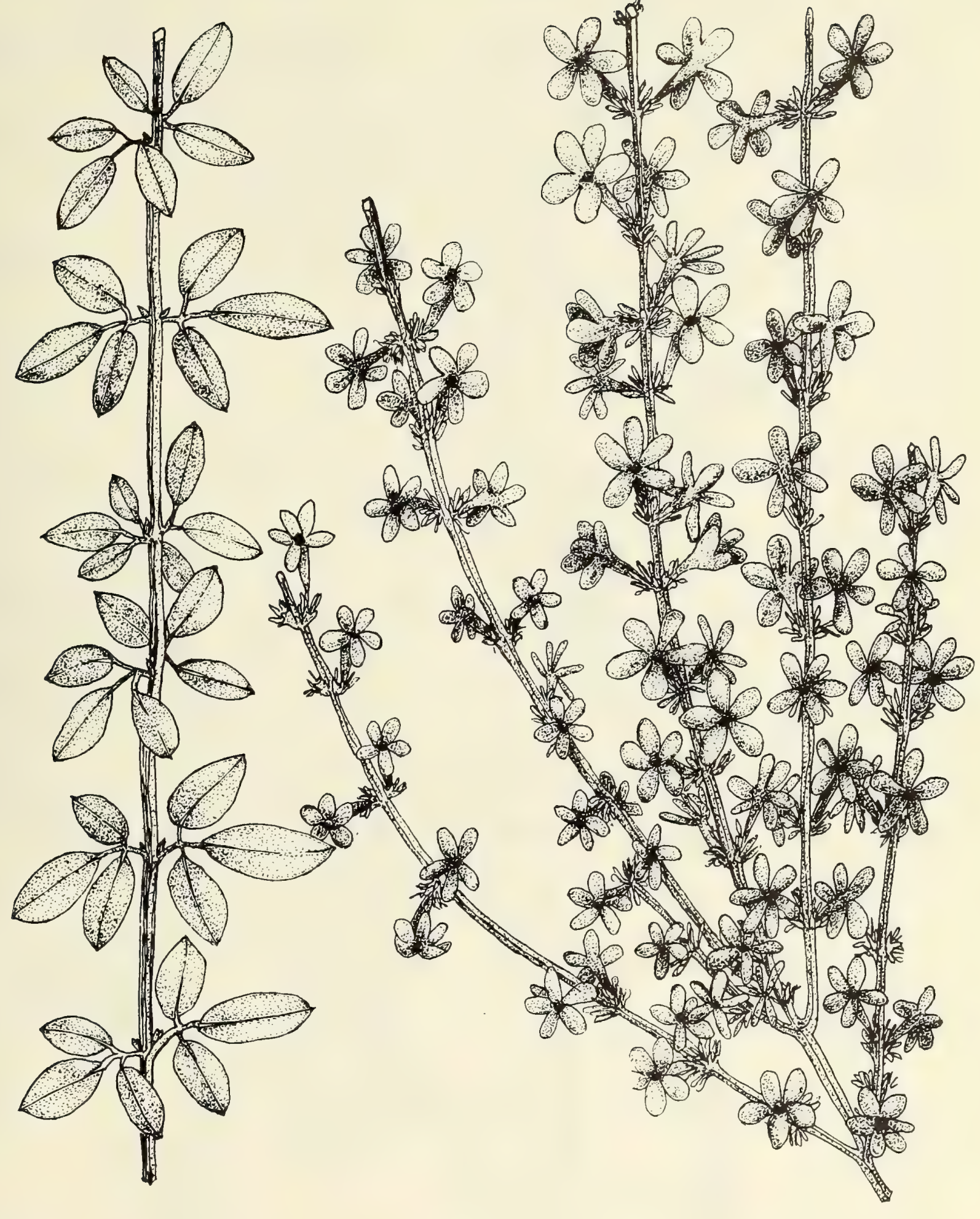

JASMINUM nudiflorum Lindl.

[illustrator Lillian Nicholson Meyer] 


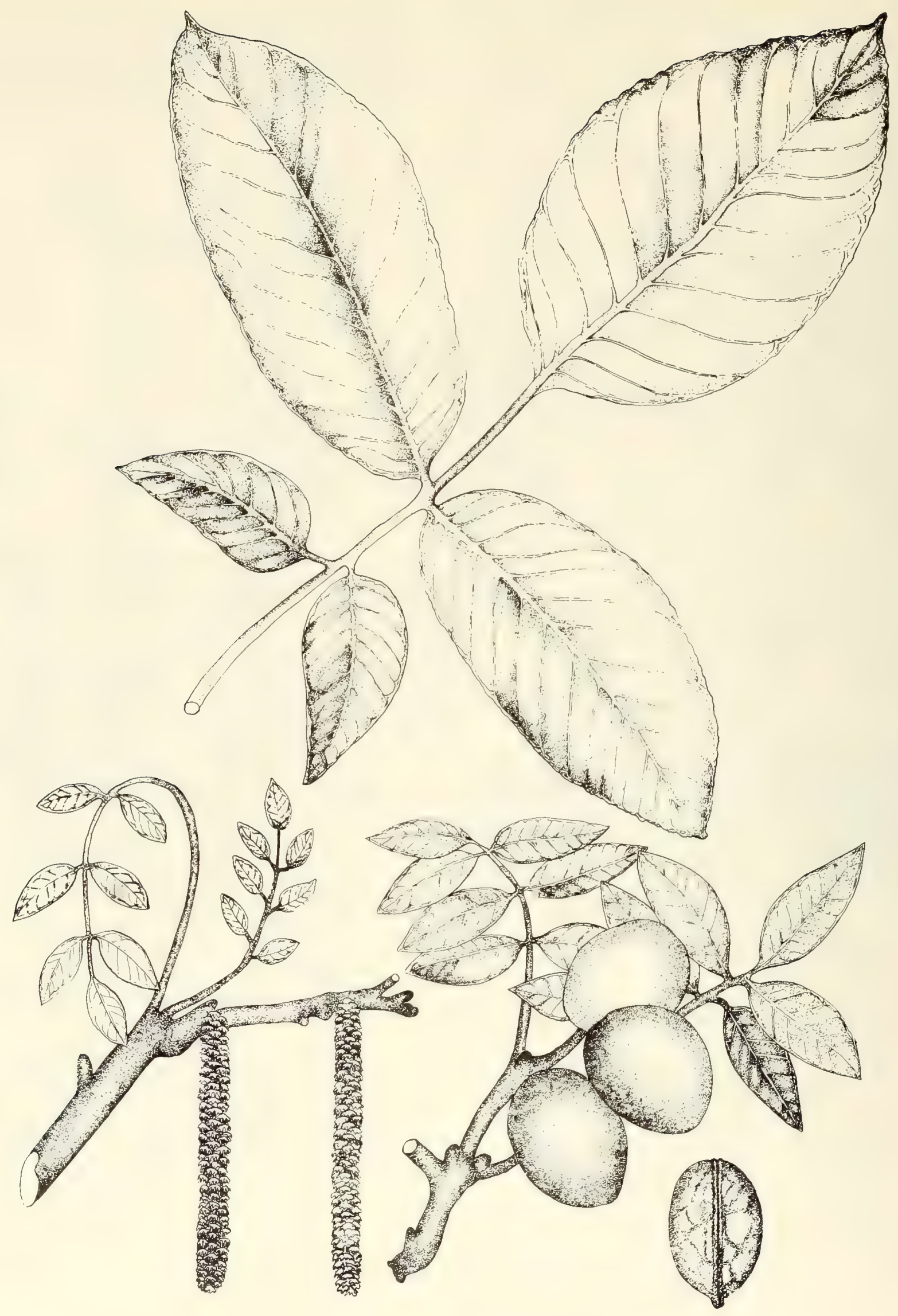

JUGLANS regia L.

[illustrator Lillian Nicholson Meyer] 
JUNIPERUS

'Blue Chip' [J. horizontalis Moench] DCNA

'Blue Cloud' [J. $\times$ media Van Melle]

[J. sabina $\times$ J. chinensis]

$$
\text { DCNA FLMG }
$$

'Blue Forest' [J. horizontalis Moench] DCNA MDBG

'Blue Heaven' [J. scopulorum Sarg.] MDBG

'Blue Horizon' [ $J$. horizontalis Moench] DCNA

'Blue Muffet' [J. horizontalis Moench] MDLT

'Blue Pacific' [J. conferta Parl.] VATA

'Blue Rug' [J. horizontalis Moench] GAEC

'Blue Star' [J. squamata Buch.-Ham. ex Lamb.] DCNA MDBG MDLT

'Blue Vase' [ $\boldsymbol{J}$. $\times$ media Van Melle] [J. sabina $\times J$. chinensis]
ALBH
DCNA
FLUF
LASL

'Broadmoor' [J. sabina L.]

DCNA

'Buffalo' [J. sabina L.]

DCNA

'Canaertii' [J. virginiana L.] LASL VACW

'Chandler Blue' [J. scopulorum Sarg.] DCNA

chinensis $\mathrm{L}$.

Chinese $\mathrm{J}$.

$\begin{array}{llll}\text { ALAU } & \text { FLMG } & \text { MDMR } & \text { VATA } \\ \text { ALBG } & \text { FLUF } & \text { SCMG } & \\ \text { ARUM } & \text { GANG } & \text { TNBC } & \end{array}$

FLLE LATU VABF

chinensis L. var. sargentii A.Henry

Sargent J.

$\begin{array}{ll}\text { DCNA } & \text { GAUG } \\ \text { GAFN } & \text { LAAL }\end{array}$

'Columnaris' [J. chinensis L.] DCNA

communis $\mathrm{L}$. DCNA GAEC GAFN VASK communis L. ssp. depressq (Pursh) Franco Ground J. DCNA

communis L. var. montana Ait. DCNA GAEC

conferta Parl.

Shore $J$.

$\begin{array}{llll}\text { ALAU } & \text { FLLE } & \text { LAAL } & \text { SCBR } \\ \text { ALBG } & \text { FLMG } & \text { LAHG } & \text { SCMG } \\ \text { ALFN } & \text { FLUF } & \text { LATU } & \text { VACW } \\ \text { DCNA } & \text { GAFN } & \text { MDLT } & \text { VANB } \\ \text { DEMC } & \text { GAJI } & \text { MSHB } & \text { VAPH } \\ \text { FLFS } & \text { GAUG } & \text { NCAS } & \end{array}$

'Den Boer' [ $\boldsymbol{J} . \times$ media Van Melle] [J. sabina $\times$ J. chinensis] DCNA

deppeana Steud. var. pachyphlaea (Torr.) Martinez

Alligator J. $\mathrm{VABF}$

'Douglasii' [J. horizontalis Moench] DCNA

'Echiniformis' [J. chinensis L.] DCNA

'Emerald Sea' [J. conferta Parl.] DCNA MDBG VATA

'Expansa' [J. davurica Pall.] DCNA FLUF

'Expansa Aureo-spicata' [J. davurica Pall.] ALFN DCNA LASL SCMG

'Expansa Variegata' [J. davurica Pall.] DCNA MDBG

'Fastigiata' [J. sabina L.] DCNA

'Fastigiata' [J. virginiana L.] DCNA

'Filicinus' [ $J$. horizontalis Moench] DCNA

'Filicinus Minimus' [J. horizontalis Moench] MDBG

formosana Hayata NCWT

'Fruitlandii' [J. $\times$ media Van Melle] [J. sabina $\times$ J. chinensis] GAFN 
JUNIPERUS

'Gareei' [J. scopulorum Sarg.] DCNA

'Glauca' [J. horizontalis Moench] ALBH

'Glauca' [J. virginiana L.] ALTD

'Glenmore' [J. horizontalis Moench] DCNA

'Globosa Cinerea' [J. chinensis L.] DCNA

'Glomerata' [ $J$. horizontalis Moench] DCNA

'Gold Star' [J. chinensis L.] MDBG

'Gray Gleam' [J. scopulorum Sarg.] DCNA

'Grey Owl' [J. virginiana L.] SCJF VATA

'Gulf Tide' [ $J$. conferta Parl.] SCMG

'Hetzii' [J. chinensis L.]

Hetz Blue J.

MDBG MDJW NCDU SCCU

'Hibernica' [ $J$. communis L.]

Irish J.

$\begin{array}{llll}\text { ALAU } & \text { DCNA } & \text { GAGM } & \text { VAMP } \\ \text { ALBH } & \text { GAEC } & \text { NCBE } & \end{array}$

'Hill's Silver' [J. scopulorum Sarg.] DCNA

horizontalis Moench

Creeping J.

$\begin{array}{lll}\text { DCCG } & \text { NCDU } & \text { VATA } \\ \text { DCNA } & \text { NCWC }\end{array}$

'Hornibrookii' [J. communis L. var. montana Ait.] DCNA

'Hughes' [J. horizontalis Moench] DCNA

'Kaizuka' [J. chinensis L.] Hollywood $J$.

$\begin{array}{lll}\text { ALBG } & \text { FLUF } & \text { SCCU } \\ \text { DCNA } & \text { LASL } & \text { VABF } \\ \text { FLFS } & \text { MDBG } & \text { VATA }\end{array}$

'Kaizuka Variegated' [J. chinensis L.] MDBG VATA

'Keteleeri' [J. virginiana L.] ALFN VACW

'Lakewood' [J. scopulorum Sarg.] DCNA

'Lakewood Globe' [J. scopulorum Sarg.] DCNA

'Livida' [J. horizontalis Moench] DCNA

'Loderi' [J. squamata Buch.-Ham. ex Lamb.] DCNA

'Marcella' [ $J$. horizontalis Moench] DCNA

'Meyeri' [J. squamata Buch.-Ham. ex Lamb.] GAEC LAAL

'Mint Julep' [J. $\times$ media Van Melle] [J. sabina $\times$ J. chinensis] FLUF

'Nana' [J. procumbens (Endl.) Miq.] DCNA MDBG

'Nova' [J. virginiana L.] DCNA

'Oblonga Pendula' [J. communis L.] MDEM NCSM VACW VAPH

'Old Gold' [J. $\times$ media Van Melle] [J. sabina $\times$ J. chinensis] DCNA

pachyphlaea Torr.

$=J$. deppeana var. pachyphlaea

'Parsonii' [J. chinensis L.] VATA

'Pathfinder' [J. scopulorum Sarg.] DCCG DCNA

'Pencil Point' [J. communis L.] DCNA

'Petraea' [J. horizontalis Moench] DCNA 
JUNIPERUS

'Pfitzeriana' [J. $\times$ media Van Melle]

Pfitzer J.

[J. sabina $\times J$. chinensis]

$\begin{array}{lll}\text { ALIT } & \text { DCNA } & \text { MDAB } \\ \text { ALSM } & \text { FLMG } & \text { NCWC } \\ \text { ARRB } & \text { FLPM } & \text { SCFW }\end{array}$

'Pfitzeriana Aurea' [J. × media Van Melle] Golden Pfitzer J.

[J. sabina $\times J$. chinensis]
GAEC
MDJS
TNMB

'Pfitzeriana Compacta' [J. × media Van

Melle]

[J. sabina $\times J$. chinensis]

DCNA

'Platinum' [J. scopulorum Sarg.] DCNA

'Plumosa' [ $J$. horizontalis Moench]

Andorra $\mathrm{J}$

GAUG SCMP

'Plumosa Aurea' [J. $\times$ media Van Melle]

[J. sabina $\times J$. chinensis]

DCNA

'Prince of Wales' [ $J$. horizontalis Moench] DCNA

procumbens (Endl.) Miq.

$\begin{array}{llll}\text { DCNA } & \text { FLUF } & \text { LAHG } & \text { VAMP } \\ \text { FLDG } & \text { GAAB } & \text { LASL } & \text { VATA } \\ \text { FLLE } & \text { GAEC } & \text { SCMG } & \end{array}$

'Procumbens' [J. horizontalis Moench] DCNA

'Pulchella' [J. horizontalis Moench] DCNA

'Reptans' [J. virginiana L.] DCNA

rigida Sieb. \& Zucc.

Needle J. DCNA GAEC LAAL

'Robusta Green' [J. chinensis L.] DCNA VATA

'San Jose' [J. chinensis L.] ARSN GAUG

scopulorum Sarg.

Rocky Mountain J. DCNA

'Seagreen' [J. chinensis L.] SCJF
'Sentinel' [J. communis L.] DCNA

'Sheppardii' [J. chinensis L.] DCNA

silicicola (Small) Bailey

Southern Red Cedar

$\begin{array}{llll}\text { ALBG } & \text { FLCG } & \text { GATS } & \text { SCMP } \\ \text { ALTR } & \text { FLMG } & \text { NCRM } & \text { TXMS } \\ \text { ALUA } & \text { FLUF } & \text { SCMG } & \end{array}$

'Skandia' [J. sabina L.] DCNA

'Skyrocket' [J. virginiana L.] MDBG VATA

'Spartan' [J. chinensis L.] DCNA

squamata Buch.-Ham. ex Lamb.

Single-seed J.

FLMG NCGM

'Stricta' [J. excelsa Bieb.] DCNA VATA

'Tolleson's Blue Weeping' [J. scopulorum Sarg.] DCNA MDMR VAMD

'Torulosa' [J. chinensis L.]

= 'Kaizuka'

'Variegata' [ $J$. chinensis L.] GAFN TNUT

virginiana $\mathrm{L}$.

Red Cedar

$\begin{array}{llll}\text { DCNA } & \text { MDHN } & \text { SCFS } & \text { VACW } \\ \text { FLCG } & \text { MDLT } & \text { SCMG } & \text { VAMP } \\ \text { FLUF } & \text { NCDU } & \text { SCWP } & \\ \text { GAUG } & \text { NCWT } & \text { TNHG } & \\ \text { MDBG } & \text { SCCU } & \text { TNSG } & \end{array}$

'Viridis' [J. chinensis L. var. sargentii A.Henry] DCNA

'Von Ehren' [J. sabina L.] LAAL

'Wichita Blue' [J. scopulorum Sarg.] DCNA

'Wiltonii' [J. horizontalis Moench] DCNA GAUG MDBG VATA 
JUSTICIA L.

ACANTHACEAE

Acanthus Family

brandegeana Wassh. \& L.B.Sm.

Shrimp Plant

FLCG FLDG FLUF

californica (Benth.) D.Gibson

Shrimp Plant

TXWE

'Yellow Queen' [J. brandegeana Wassh. \&

L.B.Sm.]

LALG

KADSURA JUSS.

SCHISANDRACEAE Schisandra Family

japonica (Thunb.) Dunal

Kadsura Vine

NCEL SCAC SCCU

KALMIA L.

ERICACEAE

Heath Family

angustifolia $\mathrm{L}$.

Sheep Laurel

DEWG MDLT VAZC

angustifolia L. var. carolina (Small) Fern. NCDU

\section{$\star$ 'Bettina' [K. latifolia L.] DCNA}

cuneata Michx.

White Wicky SCWI

'Dexter Pink' [K. latifolia L.] MDBG

'Fuscata' [K. latifolia L.] MDLT

hirsuta $\times \mathbf{K}$. latifolia GAEC SCWI

latifolia L.

Mountain Laurel
ALAU
FLMG
LALG
MDBG
ALSH
FLUF
MDLT
NCOP
VACW
DEWG
GAHC
NCBE
VAGW
VAHB

latifolia L. f. angustata Rehd. GAEC
'Ostbo Red' [K. latifolia L.] MDLT

'Sharon Rose' [K. latifolia L.] MDBG

'Shooting Star' [K. latifolia L.] GAEC

KALOPANAX Miq.

ARALIACEAE

Ginseng Family

pictus (Thunb.) Nakai

$=\boldsymbol{K}$. septemlobus

ricinifolius (Sieb. \& Zucc.) Miq.

$=K$. septemlobus

septemlobus (Thunb. ex J.A.Murr.) Koidz. DCNA MDBG MDGJ

KERRIA DC .

ROSACEAE

Rose Family

'Albescens' [K. japonica (L.) DC.] DCNA

japonica (L.) DC.

Japanese Rose
DEMC
MSMN VAPH

GAEC

SCWI

VAWR

'Picta' [K. japonica (L.) DC.] MDBG MDFM MDLT

'Pleniflora' [K. japonica (L.) DC.] ARHS SCCU VADW VAWL MDJS TNCT VAGW

KOELREUTERIA LaXm. Golden-rain Tree SAPINDACEAE Soapberry Family

bipinnata Franch

DCNA DCVK

VATA

elegans (Seemann) A.C.Sm. ssp. formosana (Hayata) F.G.Mey.

$\begin{array}{lll}\text { ALTD } & \text { FLUF } & \text { MSPR } \\ \text { FLPE } & \text { LASL } & \text { TXMA }\end{array}$

'Fastigiata' [K. paniculata Laxm.] DCNA TNTV

formosana Hayata

= $\boldsymbol{K}$. elegans ssp. formosana 
paniculata Laxm.

$\begin{array}{llll}\text { DCCG } & \text { GAUG } & \text { MDWP } & \text { TNUT } \\ \text { DCJM } & \text { LAHG } & \text { MSMN } & \text { VACP } \\ \text { DCNA } & \text { MDBG } & \text { NCBE } & \text { VACW } \\ \text { DCSE } & \text { MDJB } & \text { SCCU } & \text { VAMP } \\ \text { DCWH } & \text { MDLT } & \text { TNSC } & \text { VATA } \\ \text { DEMC } & \text { MDNA } & \text { TNSN } & \end{array}$

paniculata Laxm. var. apiculata (Rehd. \& Wils.) Rehd.

$=\mathbf{K}$. paniculata

'September' [K. paniculata Laxm.] DCNA

KOLKWITZIA Graebn.

CAPRIFOLIACEAE Honeysuckle Family (Contributed by T. R. Dudley)

\section{amabilis Graebn. \\ Beauty Bush

$\begin{array}{llll}\text { DCFP } & \text { MDBE } & \text { TNSC } & \text { VACM } \\ \text { DEWG } & \text { MDDF } & \text { TNSN } & \text { VASK } \\ \text { GABS } & \text { NCBE } & \text { TNTV } & \end{array}$ \\ 'Rosea' [K. amabilis Graebn.] DCNA \\ +LABURNOCYTISUS Schneid. \\ FABACEAE (Faboideae) Bean Family \\ [Laburnum + Cytisus]}

adamii (Poit.) Schneid.

[Laburnum anagyroides + Cytisus purpureus] MDBG

LABURNUM Medik. Golden-chain Tree FABACEAE (Faboideae) Bean Family

anagyroides Medik.

Golden-chain Tree

DCNA DEEM MDLA VACW

'Vossii' [L. × watereri (Kirchn.) Dipp.]

$=$ L. anagyroides

$\times$ watereri (Kirchn.) Dipp.

[L. anagyroides $\times$ L. alpinum]

DCNA MDLT VAGS

DEMC MDTP
LAGERSTROEMTA L. LYTHRACEAE

Crape Myrtle Loosestrife Family

$\star$ 'Acoma'

$[L$. indica 'Pink Ruffles' $\times(L$. indica $\times$

L. fauriei sdlg.)] DCNA

'Alba' [L. indica L.] DCCG GAMM VAMP FLUF VACM

$\star$ 'Apalachee'

[L. indica /Asuka dwarf hybrid/ $\times$ L. fauriei] DCNA

'Basham's Party Pink'

$[L$. indica $\times$ L. fauriei] TXSB

$\star$ 'Biloxi'

$[(\boldsymbol{L}$. indica $/$ dwarf red $/ \times$ L. fauriei $) \times$

( $L$. indica 'Low Flame' $\times$ L. fauriei)] DCNA

$\star$ 'Caddo'

[( $\mathrm{F}_{2}$ sdlg. from 'Basham's Party Pink' $\times$

'Cherokee') $\times$ unknown pollen parent] DCNA

'Carolina Beauty' [L. indica L.] SCCU

$\star$ 'Catawba'

[L. indica /lilac/ $\times$ L. indica /lavender/] DCNA SCCU

$\star$ 'Cherokee'

$[L$. indica 'Hardy Red' $\times L$. indica

'Low Flame'] DCNA MDLT

$\star$ 'Choctaw'

[(L. indica $\times$ L. fauriei $) \times$ 'Potomac'] DCNA

$\star$ 'Comanche'

$[\boldsymbol{L}$. indica $/$ dark red $/ \times(\boldsymbol{L}$. indica $\times$ L. fauriei sdlg.)] DCNA

$\star$ 'Conestoga'

[L. indica / white $/ \times L$. indica 'Low Flame'] DCNA

fauriei Koehne DCNA FLUF LASL TXPH 
$\star$ 'Hopi'

$[(L$. indica 'Pink Lace' $\times L$. fauriei $) \times L$. indica 'Alba-Nana']

DCNA

indica $\mathrm{L}$.

$\begin{array}{llll}\text { ALBG } & \text { FLUF } & \text { NCSM } & \text { VACW } \\ \text { ALCM } & \text { LARP } & \text { NCTE } & \text { VAHC } \\ \text { DCCG } & \text { LASL } & \text { SCMP } & \text { VAMP } \\ \text { FLMG } & \text { NCDU } & \text { VACM } & \end{array}$

$\star$ 'Lipan'

$[(L$. indica 'Pink Lace' $\times L$. fauriei $) \times((L$. indica $/ \mathrm{red} / \times$ 'Carolina Beauty') $\times$ 'Basham's Party Pink')]

DCNA

\section{$\star$ 'Miami'}

$[(L$. indica 'Pink Lace' $\times L$. fauriei $) \times(L$. indica 'Firebird' $\times(L$. indica $\times L$. fauriei sdlg.))] DCNA
$\star$ 'Muskogee'
$[L$. indica 'Pink Lace' $\times$ L. fauriei] DCNA

\section{$\star$ 'Natchez' \\ [L. indica 'Pink Lace' $\times$ L. fauriei] DCNA}

\section{$\star$ 'Osage'}

$[(\boldsymbol{L}$. indica $/$ dwarf red $/ \times \boldsymbol{L}$. fauriei $) \times(\boldsymbol{L}$. indica 'Pink Lace' $\times$ L. fauriei)] DCNA

\section{$\star$ 'Pecos'}

$[(\boldsymbol{L}$. indica $/$ dwarf red/ $\times \boldsymbol{L}$. fauriei $) \times(\boldsymbol{L}$. indica /dark red/ $\times$ L. fauriei)] DCNA

\section{$\star$ 'Potomac' [L. indica L.]}

(Seed was colchicine treated) DCNA SCCU

$\star$ 'Powhatan'

[L. indica /lilac/ $\times$ L. indica /lavender/] DCNA

\section{$\star$ 'Seminole'}

[L. indica $/$ pink $/ \times$ L. indica 'Low Flame'] DCNA

\section{$\star$ 'Sioux'}

[ $(L$. indica 'Tiny Fire' $\times(L$. indica $\times L$. fauriei sdlg. $)) \times((L$. indica 'Pink Lace' $\times$ L. fauriei $) \times$ 'Catawba')] DCNA

subcostata Koehne FLUF LASL
^ 'Tonto'

[( $($ L. indica 'Pink Lace' $\times$ L. fauriei $) \times$

'Catawba') $\times$ 'Tuscarora'] DCNA

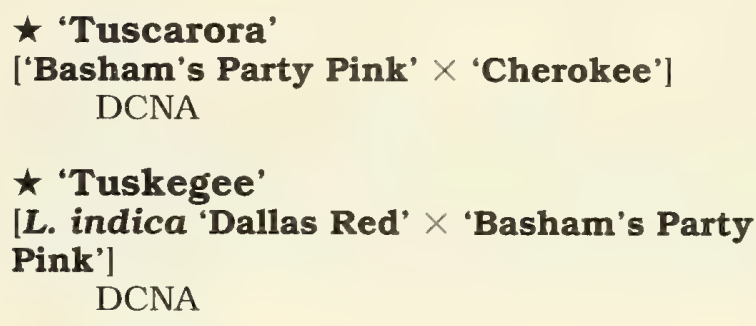
indica 'Firebird' $\times(L$. indica $\times L$. fauriei sdlg.))]

$$
\text { DCNA }
$$

\section{$\star$ 'Yuma'}

$[(L$. indica 'Pink Lace' $\times$ L. fauriei $) \times((L$. amabilis $\times \boldsymbol{L}$. indica / hardy light pink/) $\times$ L. indica /very dwarf red/)] DCNA

\section{$\star$ 'Zuni'}

$[(\boldsymbol{L}$. indica $/$ dwarf red/ $\times \boldsymbol{L}$. fauriei $) \times \boldsymbol{L}$. indica 'Low Flame'] DCNA

\section{LANTANA L. VERBENACEAE} Verbena Family

\section{camara L.

$\begin{array}{llll}\text { ALBG } & \text { GAAG } & \text { LAAL } & \text { LALG } \\ \text { FLUF } & \text { GATS } & \text { LAHG } & \text { VACW }\end{array}$

montevidensis (Spreng.) Briq.

Trailing Lantana

$$
\text { ALBG FLCG SCHW TXRS }
$$

sellowiana Link \& Otto

$=L$. montevidensis

LARIX Mill. Larch PINACEAE Pine Family

decidua Mill.

European L.

$\begin{array}{llll}\text { DCCG } & \text { MDBG } & \text { MDNA } & \text { VABF } \\ \text { DCNA } & \text { MDHN } & \text { NCBE } & \end{array}$

gmelinii (Rupr.) Rupr.

Dahurian L. MDPJ 
kaempferi (Lamb.) Carr.

Japanese L.

DCCG DCNA MDGD NCBE

leptolepis (Sieb. \& Zucc.) Gord.

= L. kaempferi

LAURUS L. LAURACEAE

Laurel Laurel Family

nobilis $\mathrm{L}$.

Grecian L.

$\begin{array}{llll}\text { ALSH } & \text { MSRN } & \text { SCHW } & \text { VACS } \\ \text { FLCG } & \text { NCTP } & \text { SCUC } & \\ \text { LARP } & \text { SCBR } & \text { TXSH } & \end{array}$

LAVANDULA L. Lavender LAMIACEAE Mint Family

angustifolia Mill

DCNA MDBG MDLT VACS

'Hidcote' [L. angustifolia Mill. ssp.

angustifolia]

DCNA MDBG VAGS

$\times$ intermedia Emeric ex Loisel.

Lavandin

[L. angustifolia $\times$ L. latifolia]

DCNA

officinalis $\mathrm{L}$.

$=L$. angustifolia

'Provence' [L. angustifolia Mill.]

DCNA

stoechas L.

French L.

SCWI

\section{LEDUM L.}

ERICACEAE

Heath Family

palustre L. var. diversipilosum Nakai

Wild Rosemary

VAGS

\section{LEIOPHYLUM R.Hedwig} ERICACEAE

Sand Myrtle Heath Family buxifolium (Bergius) Ell. var. hugeri (Small) Schneid.

DEMC GAEC

LEITNERIA Chapm.

LEITNERIACEAE

Leitneria Family

floridana Chapm.

Florida Corkwood

DCNA

\section{LEPTODERMIS Wall.}

RUBIACEAE

Madder Family

oblonga Bunge

DEWG TNSN TNTV

LEPTOSPERMUM J.R. \& J.G. Forst. MYRTACEAE Myrtle Family

'Plenum' [L. scoparium J.R. \& J.G. Forst.]

New Zealand Tea Tree LASL

LESPEDEZA Michx. Bush Clover FABACEAE (Faboideae) Bean Family

'Albiflora' [L. thunbergii (DC.) Nakai] DCNA GACG NCSM

bicolor Turcz. MDGD MDSC

bicolor Turcz. f. acutifolia Matsum.

= L. bicolor var. bicolor

cyrtobotrya Miq. MDSC

maximowiczii Schneid. MDSC

penduliflora (Oudemans) Nakai

$=$ L. thunbergii

thunbergii (DC.) Nakai

Japanese Bush C. MDSC MSHB NCWK

buxifolium (Bergius) Ell.

Box S. M.

SCWI 


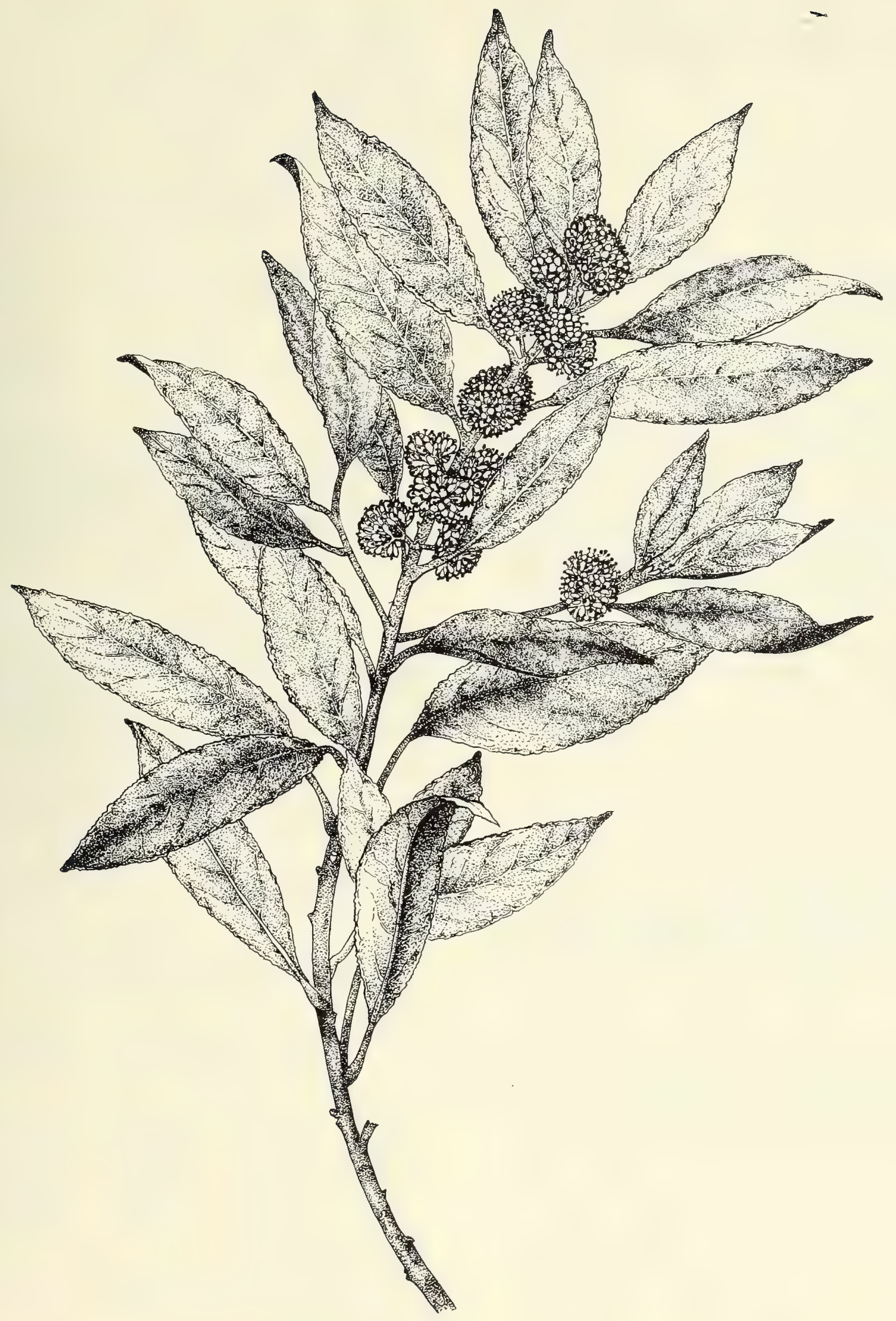

LAURUS nobilis L. (staminate) [illustrator Lillian Nicholson Meyer] 
LEUCAENA Benth.

FABACEAE (Mimosoideae) Bean Family

glauca (L.) Benth.

=L. leucocephala

leucocephala (Lam.) de Wit

White Popinac, Ipil-ipil

FLUF

\section{LEUCOPHYLLUM Humboldt \&}

Bonpland

\section{SCROPHULARIACEAE Figwort Family}

frutescens (Berl.) I.M.Johnst.

Ceniza. Barometer Bush

FLUF LASL TXLL TXSE

\section{LEUCOTHOE D.DON ERICACEAE}

Heath Family

axillaris (Lam.) D.Don

GAEC SCDB SCWI VACW

Carinella (TM) [L. fontanesiana $\times$

L. ?axillaris]

= Trademark name for 'Zebekot'

catesbaei (Walt.) A.Gr.

$=$ L. fontanesiana

editorum Fern. \& Schub.

=L. fontanesiana

fontanesiana (Steud.) Sleumer

Doghobble

$\begin{array}{llll}\text { DCNA } & \text { MDWP } & \text { TNDG } & \text { VAPO } \\ \text { DEWG } & \text { NCAS } & \text { VACW } & \text { VATA } \\ \text { GACG } & \text { NCBE } & \text { VADW } & \\ \text { GAUG } & \text { SCCU } & \text { VAGS } & \\ \text { MDLT } & \text { SCWI } & \text { VAGW } & \end{array}$

'Girard's Rainbow' [L. fontanesiana

(Steud.) Sleumer]

GACG GAJS MDBG

Lovita (TM) [L. fontanesiana $\times$

L. ?axillaris]

= Trademark name for 'Zebonard'

'Nana' [L. fontanesiana (Steud.) Sleumer] GACG MDIT

populifolia (Lam.) Dipp.

= Agarista populifolia racemosa (L.) A.Gr.

Sweetbells

$\begin{array}{llll}\text { GAEC } & \text { LALG } & \text { NCDU } & \text { VACW } \\ \text { GAJS } & \text { MDLT } & \text { SCBR } & \end{array}$

'Rollissonii' [L. fontanesiana (Steud.)

Sleumer] MDBG

'Zebekot' [L. fontanesiana $\times$ L. ?axillaris] (Plant Patent No. 5,224; patented name for Carinella(TM)) MDFM

'Zebonard' [ $L$. fontanesiana $\times$

L. ?axillaris]

(Plant Patent No. 5,229; patented name for Lovita(TM))

MDFM

\section{LIBOCEDRUS Endl.}

CUPRESSACEAE Cypress Family

decurrens Torr.

= Calocedrus decurrens

LIGUSTRUM L. Privet OLEACEAE Olive Family

'Aureo-marginatum' [L. ovalifolium

Hassk.] $\mathrm{SCCU}$

compactum Hook. ᄃ. \& Thoms. DCNA

delavayanum Hariot $\mathrm{SCCU}$

'Erecta' [L. japonicum Thunb.] FLGS

'Fraseri' [L. japonicum Thunb.] FLGS

'Fraseri' [L. sinense Lour.] ALBG FLMG GAIS TNTV ALFN FLUF NCWM ALPH GAAB SCCU FLCG GACG SCLM

ioandrum Diels

= L. delavayanum

'Iwata' [L. japonicum Thunb.] FI,MG 


\section{LIGUSTRUM}

japonicum Thunb.

Japanese $P$.

$\begin{array}{llll}\text { ALBG } & \text { DCWH } & \text { NCDU } & \text { SCUC } \\ \text { ALCW } & \text { FLMG } & \text { NCWC } & \text { TNUT } \\ \text { ALFN } & \text { GAUG } & \text { SCBR } & \text { TXPO } \\ \text { DCCG } & \text { MDJH } & \text { SCRH } & \end{array}$

'Lake Treska' [ $\boldsymbol{L}$. japonicum Thunb.] FLUF

lucidum Ait.f.

Wax-leaf $P$.
ALAU
NCDU
SCUC
VAWR
FLUF
SCCU
TXPO
LAAL
SCMP
VACM

'Nobilis' [L. japonicum Thunb.]
FLGS
SCCU

obtusifolium Sieb. \& Zucc.

Border P
ALBH
MDLT
VARC
DEWG
NCCA

ovalifolium Hassk.

California P.

DCCG LAHG MDCP VARO

'Pendulum' [L. sinense Lour.]

Weeping $\mathrm{P}$.

DCNA DCNE

quihoui Carr.

$\begin{array}{llll}\text { FLIF } & \text { MDKN } & \text { MDNA } & \text { TXMS } \\ \text { LAAP } & \text { MDKS } & \text { SCAK } & \end{array}$

'Repandens' [L. japonicum Thunb.] ALFN

'Rotundifolium' [L. japonicum Thunb.]
ALBG
FLMG
LAHG
FLDG
FLUF
SCCU
FLGS
GAHC
SCRH

sempervirens (Franch.) Mansf.

LAGN

'Silver Star' [L. japonicum Thunb.] SCCU

sinense Lour.

Chinese P.

$\begin{array}{llll}\text { ALBH } & \text { GAUG } & \text { NCBE } & \text { TNUT } \\ \text { DCRR } & \text { LAAL } & \text { NCDU } & \text { VACM } \\ \text { FLUF } & \text { MDLT } & \text { NCGP } & \text { VARO } \\ \text { GAOH } & \text { MDSJ } & \text { SCMP } & \text { VASC }\end{array}$

'Suwanee River' [L. japonicum Thunb.] ALFN SCBR SCCU tschonoskii Decne.

DCNA

'Variegatum' [L. japonicum Thunb.] LASL.

$\times$ vicaryi Rehd.

[L. ovalifolium f. aureum $\times L$. vulgare]
FLUF
SCCU

vulgare L.

Common $\mathrm{P}$

DEWG TXRS

'Wimbish' [L. sinense Lour.]

DCNA GAEC VACW

LINDERA Thunb.

Spicebush

LAURACEAE

Laurel Family

angustifolia W.C.Cheng MDBG

benzoin (L.) $\mathrm{Bl}$.
DCNA
MDBG
NCBE
DEWG MDWP VACW

benzoin (L.) Bl. var. pubescens (Palm. \& Steyerm.) Rehd.

TXLL

obtusiloba Bl.

DCNA VACW

strychnifolia (Sieb. \& Zucc.) F.Vill. DCNA

LIPPIA L.

VERBENACEAE

Verbena Family

citriodora

= Aloysia gratissima

LIQUIDAMBAR L. Sweet Gum HAMAMELIDACEAE Witch-hazel Family

formosana Hance

Formosan S.G.

$\begin{array}{llll}\text { ALTD } & \text { GAIS } & \text { MDGJ } & \text { TXMS } \\ \text { DCUR } & \text { GAUG } & \text { MDPJ } & \text { VABF } \\ \text { FLUF } & \text { LAAL } & \text { SCCU } & \end{array}$

'Gum Ball' [L. styraciflua L.]

GAEC TNSM TNSN TNTV 
'Obtusiloba'

= L. styraciflua f. rotundiloba

orientalis Mill.

DCNA GAIS

styraciflua L.

S.G.

$\begin{array}{llll}\text { ALBG } & \text { LAAL } & \text { SCBR } & \text { TNGN } \\ \text { DCCG } & \text { MDPJ } & \text { SCCU } & \text { VAHC } \\ \text { FLUF } & \text { NCBE } & \text { SCMP } & \text { VAMP }\end{array}$

styraciflua L. f. rotundiloba Rehd. NCCA

'Variegata' [L. styraciflua L.] VAGS

LIRIODENDRON L. MAGNOLIACEAE

Tulip Tree Magnolia Family

'Aureo-marginatum' [L. tulipifera L.] DCSH

chinense (Hemsl.) Sarg.

Chinese Tulip T.
NCCA VABF

tulipifera L.

Tulip T., Yellow Poplar

$\begin{array}{llll}\text { ALBH } & \text { LASL } & \text { MSEP } & \text { VACM } \\ \text { DCCG } & \text { MDBP } & \text { NCSM } & \text { VACW } \\ \text { DCWH } & \text { MDHN } & \text { SCBR } & \text { VAGW } \\ \text { DEWG } & \text { MDLT } & \text { TNSC } & \text { VATA } \\ \text { GAES } & \text { MDSJ } & \text { VABF } & \end{array}$

\section{LITHOCARPUS Bl. Tanbark Oak FAGACEAE Beech Family}

corneus (Lour.) Rehd. GAIS

edulis (Makino) Nakai GAIS

glaber (Thunb.) Nakai

Tanbark Oak

GAIS

henryi (Seemann) Rehd. \& Wils.
ALTD
DCNA
GAIS
VACW

LITSEA Lam.

LAURACEAE

Laurel Family

aestivalis (L.) Fern.

Pond-spice

SCBR

LIVISTONA R.Br.

ARECACEAE

Palm Family

chinensis (Jacq.) R.Br. ex Mart.

Chinese Fan Palm

FLUF

LONICERA L. Honeysuckle CAPRIFOLIACEAE Honeysuckle Family (Contributed by T. R. Dudley)

'Arnold Red' [L. tatarica L.] MDPJ

'Baggesen's Gold' [L. nitida Wils.] MDBG

$\times$ bella Zab.

[L. morrowii $\times$ L. tatarica] VACW

$\times$ brownii (Regel) Carr.

[L. sempervirens $\times$ L. hirsuta] GAFN LARS

chinensis P.W.Wats.

$=L$. japonica f. chinensis

'Ernest Wilson' [L. nitida Wils.] GACG

flava Sims

Yellow $\mathrm{H}$. SCWI

'Floribunda' [L. korolkowii Stapf] MDTD

fragrantissima Lindl. \& Paxt. Winter $\mathrm{H}$.

$\begin{array}{llll}\text { ALIT } & \text { FLMG } & \text { MDLT } & \text { SCUC } \\ \text { ALSH } & \text { GAOH } & \text { MDTD } & \text { TNCT } \\ \text { ARPT } & \text { GATS } & \text { MSHB } & \text { TNDG } \\ \text { DCCG } & \text { GAWP } & \text { NCBE } & \text { VACW } \\ \text { DCHU } & \text { LARP } & \text { SCBR } & \text { VADW } \\ \text { DEWG } & \text { MDDP } & \text { SCDB } & \text { VAMP }\end{array}$

gracilipes Miq. MDKN 


\section{LONICERA}

gracilipes Miq. var. glandulosa Maxim. MDAB

'Hack's Red' [L. tatarica L.] DCNA

$\times$ heckrottii Rehd.

Everblooming $\mathrm{H}$.

[L. $\times$ americana $\times$ L. sempervirens $]$
ARPT GAFN SCCU

japonica Thunb. ex J.A.Murr.

Japanese $\mathrm{H}$.
ALBG
LAAL MSHB
ALBH
MDBG TNSC

japonica Thunb. ex J.A.Murr. f. chinensis (P.W.Wats.) Hara
ALBH
VAGW

maackii Maxim.

Amur $\mathrm{H}$.

$\begin{array}{llll}\text { DCAG } & \text { LAHP } & \text { MDTD } & \text { VACW } \\ \text { DEEM } & \text { MDAB } & \text { NCDU } & \text { VAMP } \\ \text { GANA } & \text { MDBW } & \text { TNRM } & \text { VARG } \\ \text { GAUG } & \text { MDDF } & \text { VABF } & \end{array}$

maackii Maxim. var. podocarpa Franch. MDAB MDGD

'Magnifica' [L. sempervirens L.]

= 'Superba'

morrowii A.Gr.

Morrow's $\mathrm{H}$.

DEEM MDTD TNNE VAWD

MDLT NCBE TNRM

'Nana' [L. xylosteum L.] VAGS

nitida Wils. MDMG

pileata Oliv.

Privet $\mathrm{H}$.

$\begin{array}{llll}\text { DCNA } & \text { GAGB } & \text { TNCT } & \text { VANB } \\ \text { FLUF } & \text { GAUG } & \text { TNDR } & \\ \text { GACG } & \text { MDLT } & \text { VADW } & \end{array}$

'Punicea' [L. tatarica L.]

MDAB MDGD

sempervirens $\mathrm{L}$.

Trumpet $\mathrm{H}$.

$\begin{array}{llll}\text { ALEH } & \text { MDLT } & \text { TNHD } & \text { VAGW } \\ \text { GAUG } & \text { MDMG } & \text { TNRM } & \text { VAPH } \\ \text { LAHG } & \text { MSHB } & \text { TNSN } & \text { VAWP } \\ \text { LALG } & \text { SCBR } & \text { TXPS } & \text { VAZC } \\ \text { LASL } & \text { SCCU } & \text { VACW } & \\ \text { MDBG } & \text { SCWI } & \text { VADW } & \end{array}$

standishii Jacq.

Standish's H.

DEWG MDLT

subsessilis Rehd. DCNA

'Sulphurea' [L. sempervirens L.] DCNA SCWI VACW

'Superba' [L. sempervirens L.] SCWI VAGS VAPH

tatarica L.

Tatarian H.

$\begin{array}{lll}\text { MDFF } & \text { MDLA } & \text { MDMG } \\ \text { MDJH } & \text { MDMC } & \text { NCBE }\end{array}$

xylosteum L.

Fly $\mathrm{H}$. MDHN VAGS

xylosteum L. f. glabrescens Zab. VALU

LOROPETALUM R.Br. ex Reichenb. HAMAMELIDACEAE Witch-hazel Family

chinense (R.Br.) Oliv.

$\begin{array}{llll}\text { ALEH } & \text { GACE } & \text { GAUG } & \text { SCLM } \\ \text { DCNA } & \text { GACG } & \text { MDLT } & \text { SCRH } \\ \text { FLGS } & \text { GACH } & \text { NCWD } & \text { SCWI } \\ \text { FLMG } & \text { GACL } & \text { SCBR } & \text { VACW } \\ \text { FLUF } & \text { GAIS } & \text { SCCU } & \\ \text { GAAB } & \text { GAJI } & \text { SCDB } & \\ \text { GABS } & \text { GAJY } & \text { SCFW } & \end{array}$

LYCIUM L. Matrimony Vine SOLANACEAE Nightshade Family

carolinianum Walt.

Carolina M. V. SCWI

chinense Mill.

Chinese M. V. MDSC

LYONLA Nutt.

ERICACEAE Heath Family

ferruginea (Walt.) Nutt. GAEC

ligustrina (L.) DC. GAEC SCBR VAPO 
lucida (Lam.) K.Koch

Fetterbush

SCBR SCWI TXLL VAPH

mariana (L.) D.Don

Staggerbush

SCWI

MAACKIA Rupr.

FABACEAE (Faboideae) Bean Family

amurensis Rupr. \& Maxim.

MDJS SCCU VAIS

NCBE TNTV

tenuifolia (Hemsl.) Hand.-Mazz.

DCNA

\section{MACFADYENA A.DC. BIGNONIACEAE \\ Bignonia Family}

unguis-cati (L.) A.Gentry

Cat's-claw

$\begin{array}{llll}\text { ALBG } & \text { FLUF } & \text { LAHG } & \text { SCHW } \\ \text { FLCG } & \text { LAAL } & \text { SCBH } & \end{array}$

MACHILUS Nees

= PERSEA

MACLURA Nutt.

MORACEAE

Mulberry Family

pomifera (Raf.) Schneid.

Osage Orange

$\begin{array}{llll}\text { DCCG } & \text { MDLT } & \text { NCTE } & \text { VAWR } \\ \text { DCSH } & \text { MDSJ } & \text { NCWT } & \\ \text { GAEH } & \text { MSMN } & \text { TNLW } & \\ \text { MDHN } & \text { NCSM } & \text { VACW } & \end{array}$

MAGNOLIA L. MAGNOLIACEAE

acuminata $\mathrm{L}$.

Cucumber Tree

$\begin{array}{llll}\text { DCCG } & \text { GAGC } & \text { TNHG } & \text { VAMP } \\ \text { DCNA } & \text { GASC } & \text { TNMB } & \text { VAWR } \\ \text { DEEM } & \text { NCBE } & \text { VACM } & \\ \text { GACG } & \text { SCBR } & \text { VACW } & \end{array}$

'Alba' [M. $\times$ soulangeana Soul.-Bod.]

[M. denudata $\times$ M. liliiflora $]$

DCNA
'Alexandrina' [M. $\times$ soulangeana Soul.-

Bod.]

[M. denudata $\times$ M. liliiflora] DCNA

$\star$ 'Ann' [M. liliiflora 'Nigra' $\times$ M. stellata 'Rosea'] DCNA

ashei Weatherby

Florida Bigleaf M.

$\begin{array}{llll}\text { DCNA } & \text { MDFM } & \text { MSFG } & \text { TNSN } \\ \text { GAEC } & \text { MDLT } & \text { NCBE } & \text { TNTV }\end{array}$

'Baldwin' [M. grandiflora L.] DCNA

$\star$ 'Betty' [M. Liliiflora 'Nigra' $\times$ M. stellata 'Rosea'] DCNA

'Biloba' [M. officinalis Rehd. \& Wils.]

= M. officinalis var. biloba

'Charles Dickens' [M. grandiflora L.] TNSN TNTV

'C. H. Kern' [M. $\times$ soulangeana Soul.-Bod.] [M. denudata $\times$ M. liliiflora] DCNA

'Claudia Wanamaker' [M. grandiflora L.] SCSG

cordata Michx.

= M. acuminata

cylindrica Wils. MDLT

dealbata Zucc.

Mexican Bigleaf $M$. MSFG

denudata Desr.

Yulan, Lily Tree
DCNA
FLMG
MDLT

'Diva' [M. sprengeri Pamp.]

Goddess M. DCNA MDLT

'Elizabeth' [M. acuminata $\times$ M. denudata] DCNA

fraseri Walt.

Fraser M.

$$
\text { NCAS TNTV VAMP }
$$

$\star$ 'Freeman' [M. virginiana $x$ M. grandiflora] DCNA 

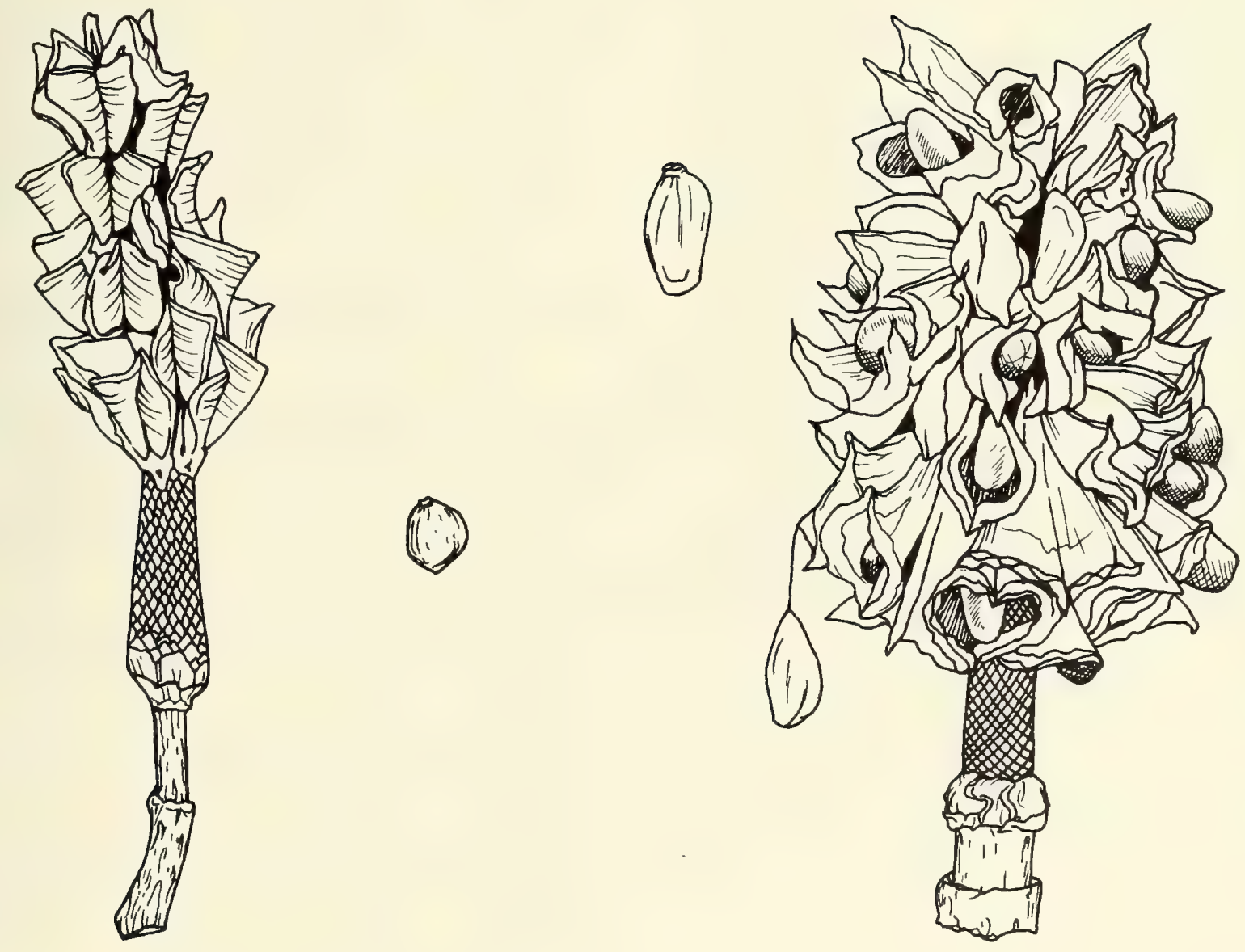
$\star$ 'Galaxy' [M. liliiflora 'Nigra' $\times$ M.

sprengeri 'Diva']

DCNA MDLT

glauca L.

$=$ M. virginiana

'Grace McDade' [M. × soulangeana Soul.Bod.]

[M. denudata $\times$ M. liliiflora] DCNA

grandiflora L.

Southern M.

$\begin{array}{llll}\text { ALBG } & \text { FLMG } & \text { MDCP } & \text { SCMP } \\ \text { DCCG } & \text { FLUF } & \text { MDFM } & \text { SCRP } \\ \text { DCPC } & \text { GAHC } & \text { MDHN } & \text { TNHG } \\ \text { DCSH } & \text { GATN } & \text { MSEP } & \text { TNUT } \\ \text { DCWA } & \text { LAAL } & \text { NCDU } & \text { VAGW } \\ \text { DCWH } & \text { LAHG } & \text { NCTE } & \text { VAMP } \\ \text { DEWG } & \text { LASL } & \text { SCBR } & \text { VATA }\end{array}$

'Hasse' [M. grandiflora L.] SCSG

'Henry Hicks' [M. virginiana L.] DCNA TNSN TNTV

heptapeta (Buc'hoz) Dandy

= M. denudata

hypoleuca Sieb. \& Zucc.

= M. obovata

* 'Jane' [M. liliiflora 'Reflorescens' $\times$

M. stellata 'Waterlily']

DCNA

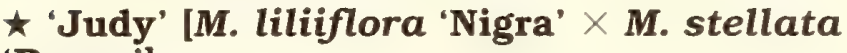
'Rosea'] DCNA

kobus DC.

Kobus M.

DCNA DCWR MDLT

'Lennei' [M. × soulangeana Soul.-Bod.]

[M. denudata $\times$ M. liliiflora]

ALON DCNA LAAL

DCCG FLMG VACG

liliiflora Desr.

Lily-flowered M.

LAAL VAGW

'Lilliputian' [M. × soulangeana Soul.-Bod.]

[M. denudata $\times M$. liliiflora]

DCNA

'Little Gem' [M. grandiflora L.] $\times$ loebneri Kache

[M. kobus $\times$ M. stellata]

DCNA DCWH

DCWR DEWG

macrophylla Michx.

Big-leaf M.

$\begin{array}{llll}\text { DCCG } & \text { GAEC } & \text { MSFG } & \text { TNSC } \\ \text { DCPC } & \text { LASL } & \text { NCBE } & \text { TNSN } \\ \text { DEEM } & \text { MDAM } & \text { NCTE } & \text { VAWH } \\ \text { DEWG } & \text { MDHC } & \text { SCBR } & \\ \text { GACB } & \text { MDPJ } & \text { SCMP } & \end{array}$

'Majestic Beauty' [M. grandiflora L.] DCNA

'Margaret Davis' [M. grandiflora L.] SCSG

$\star$ 'Maryland' [M. virginiana $\times$

M. grandiflora] DCNA

'Merrill' [M. × loebneri Kache]

[M. kobus $\times$ M. stellata] DCNA

'Mrs. Jack' [M. salicifolia (Sieb. \& Zucc.) Maxim.] MDLT

'Neil McEacharn' [M. $\times$ loebneri Kache] [M. kobus $\times$ M. stellata] DCNA

'Nigra' [M. liliiflora Desr.] DCNA

* 'Nimbus' [M. obovata $\times$ M. virginiana] DCNA

'O'Neill' [M. liliiflora Desr.] MDLT

obovata Thunb. DCNA GAFN MDLT

officinalis Rehd. \& Wils. GAEH MDJS

officinalis Rehd. \& Wils. var. biloba Rehd. \& Wils. DCNA GAWH MDJS

parviflora Sieb. \& Zucc.

$=M$. sieboldii

'Picture' [M. $\times$ soulangeana Soul.-Bod.]

[M. denudata $\times$ M. liliiflora] MDLT

'Pink Stardust' [M. stellata Sieb. \& Zucc.] MDLT 
$\star$ 'Pinkie' [M. liliiflora 'Reflorescens' $\times M$. stellata 'Rosea'] DCNA

'Pioneer' [M. grandiflora L.] DCNA

praecocissima Koidz.

= M. kobus

'Praecox' [M. grandiflora L.] DCNA

'Praecox Fastigiata' [M. grandiflora L.] DCNA

$\times$ proctoriana Rehd.

[M. kobus $\times$ M. salicifolia] GAFN

'Purpliana' [M. $\times$ soulangeana Soul.-Bod.] [M. denudata $\times$ M. liliiflora] DCNA

pyramidata Bartram ex Pursh ALBG DCNA MDLT TNTV

quinquepeta (Buc'hoz) Dandy

$=\mathbf{M}$. liliiflora

$\star$ 'Randy' [M. liliiflora 'Nigra' $\times M$. stellata'Rosea'] DCNA

'Reflorescens' [M. liliiflora Desr.] DCNA

$\star$ 'Ricki' [M. liliiflora 'Nigra' $\times$ M. stellata 'Rosea'] DCNA

'Rohrbach' [M. stellata (Sieb. \& Zucc.) Maxim.] DCNA

'Rosea' [M. stellata (Sieb. \& Zucc.) Maxim.] $\begin{array}{lll}\text { ALOS } & \text { DEWG } & \text { LAHP } \\ \text { DCNA } & \text { FLMG } & \text { MDLT }\end{array}$

'Royal Star' [M. stellata (Sieb. \& Zucc.)

Maxim.] DCNA MDLT

'Rubra' [M. × soulangeana Soul.-Bod.] [M. denudata $\times M$. liliiflora] SCBR

'Rubra' [M. stellata (Sieb. \& Zucc.) Maxim.] DCNA
'Rustica' [M. $\times$ soulangeana Soul.-Bod.]

[M. denudata $\times$ M. liliiflora ${ }^{-}$ DCNA

'St. Mary' [M. grandiflora L.]

$$
\text { DCNA FLUF }
$$

'Samuel Sommer' [M. grandiflora L.]

(Plant Patent No. 2,015) MDLT

'San Jose' [M. X soulangeana Soul.-Bod.] [M. denudata $\times M$. liliiflora] DCNA DEWG

$\star$ 'Satellite' [M. virginiana L.] DCNA

'Shady Grove No. 4' [M. grandiflora L.] SCSG

'Shady Grove No. 5' [M. grandiflora L.] SCSG

'Shady Grove No. 6' [M. grandiflora L.] SCSG

sieboldii K.Koch

Otama M.

$\begin{array}{llll}\text { DEWG } & \text { MDBG } & \text { TNSN } & \text { VAGS } \\ \text { GAFN } & \text { MDLT } & \text { TNTV } & \end{array}$

$\times$ soulangeana Soul.-Bod.

Saucer M.

[M. denudata $\times$ M. liliiflora]

$\begin{array}{llll}\text { DCCG } & \text { FLDG } & \text { LASL } & \text { MDLT } \\ \text { DCNA } & \text { GAAG } & \text { MDHN } & \text { SCBR } \\ \text { DCWH } & \text { GATS } & \text { MDJS } & \text { VAGG }\end{array}$

$\star$ 'Spectrum' [M. liliiflora 'Nigra' $\times M$. sprengeri 'Diva']

DCNA

'Spring Snow' [M. $\times$ loebneri Kache]

[M. kobus $\times$ M. stellata] MDLT

stellata (Sieb. \& Zucc.) Maxim.

Star Magnolia
DCCG
DCWH
DEWG
MDLT

'Sundance' [M. acuminata $\times$

M. ?denudata]

DCNA

'Sundew' [M. $\times$ soulangeana Soul.-Bod.] [M. denudata $\times$ M. liliiflora]

DCNA 
'Superba Rosea' [M. X soulangeana Soul.Bod.]

[M. denudata $\times$ M. liliiflora] DCNA

$\star$ 'Susan' [M. liliiflora 'Nigra' $\times M$.

stellata 'Rosea'] DCNA MDLT

tripetala L.

Umbrella M.

$\begin{array}{llll}\text { ALBG } & \text { DEWG } & \text { MDLT } & \text { NCCA } \\ \text { DCCG } & \text { GARW } & \text { MDMS } & \text { SCMP } \\ \text { DCNA } & \text { GAUG } & \text { MDVV } & \text { TNSC } \\ \text { DEEM } & \text { MDJS } & \text { NCBE } & \text { VACW }\end{array}$

'Variegata' [M. grandiflora L.] LASL

$\times$ veitchii Bean

[M. campbellii $\times$ M. denudata] DCNA NCEL

'Verbanica' [M. $\times$ soulangeana Soul.-Bod.] [M. denudata $\times M$. liliiflora] DCNA

'Victoria' [M. grandiflora L.] DCNA

virginiana $L$.

Sweet Bay

$\begin{array}{llll}\text { ALAU } & \text { FLUF } & \text { MDBG } & \text { NCCA } \\ \text { ALBG } & \text { GAEC } & \text { MDHN } & \text { SCCU } \\ \text { ALBH } & \text { GAUG } & \text { MDJH } & \text { TNPW } \\ \text { DCCG } & \text { LAAL } & \text { MDLT } & \text { TNUT } \\ \text { DCNA } & \text { LAHG } & \text { MSMN } & \text { VAGW } \\ \text { DCWA } & \text { LALG } & \text { NCBE } & \text { VAHC }\end{array}$

virginiana L. var. australis Sarg.

= M. virginiana

'Wada's Memory' [M. $\times$ kewensis Pearce] [M. kobus $\times$ M. salicifolia]
DCNA
DEW
MDLT

'Water Lily' [M. stellata Sieb. \& Zucc.] DCNA

$\times$ watsonii Hook.f.

$=$ M. $\times$ wieseneri

$\times$ wieseneri Carr.

[M. obovata $\times M$. sieboldii] DCNA VAGS

'Woodsman' [M. $\times$ brooklynensis Kalmb.] [M. acuminata $\times$ M. liliiflora]

MDBG NCAK $\times$ MAHOBERBERIS Schneid.

BERBERIDACEAE

[Berberis $\times$ Mahonia]

Barberry Family

aquisargentii Kruessm.

[Berberis sargentiana $\times$ Mahonia aquifolium] TNTV

miethkeana Melander \& Eade

[Berberis 'Renton' $\times$ Mahonia aquifolium] DCNA SCCU

MAHONIA Nutt. BERBERIDACEAE

Barberry Family

aquifolium (Pursh) Nutt.

Oregon Hollygrape

$\begin{array}{lll}\text { DCNA } & \text { GAEC } & \text { MDLT } \\ \text { DEMC } & \text { GAUG } & \text { NCDU } \\ \text { GACG } & \text { MDKN } & \text { VACW }\end{array}$

bealei (Fort.) Carr.

Leatherleaf Hollygrape

$\begin{array}{llll}\text { ALBG } & \text { FLDG } & \text { MDHN } & \text { TXGM } \\ \text { ALRH } & \text { FLMG } & \text { MDLA } & \text { VACW } \\ \text { ARSN } & \text { FLUF } & \text { MDLT } & \text { VAMP } \\ \text { DCCG } & \text { GACH } & \text { MSMN } & \\ \text { DCNA } & \text { GAEC } & \text { NCCA } & \\ \text { DCTF } & \text { GAOH } & \text { SCPP } & \end{array}$

'Charity' [M. × media Brickell]

[M. napaulensis $\times$ M. oiwakensis] DCNA

chochoca Fedde TXLL

fortunei (Lindl.) Fedde

Chinese M.

$\begin{array}{llll}\text { ALBG } & \text { FLUF } & \text { MSEP } & \text { TXHA } \\ \text { FLDG } & \text { LAAL } & \text { SCBR } & \text { TXLL } \\ \text { FLMG } & \text { LASL } & \text { SCJM } & \end{array}$

japonica (Thunb. ex J.A.Murr.) DC. Japanese M. GACG

oiwakensis Hayata ALEH FLUF LAMP VACW

pinnata (Lag.) Fedde

Cluster M.

$\begin{array}{llll}\text { ALBH } & \text { TNHS } & \text { TNPW } & \text { VAGG } \\ \text { MDBG } & \text { TNMB } & \text { TNUT } & \end{array}$

swazeyi Buckl.

Texas Hollygrape

TXGM 


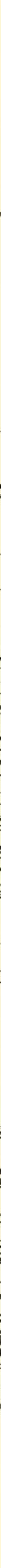

MAGNOLIA tripetala L.

[illustrator Lillian Nicholson Meyer] 
trifoliolata (Moric.) Fedle:

latredo M

WIIF TXIXC TXIL VACW

MALLOTUS LOU.

EUPHORBIACEAE

Spurge Family

japonicus (Thumb. ex L.I.) Muell.-Arg.

ditpanese: $M$

litur Gints

$\times$ MALOSORBUS Browic\%

ROSACEAE

|Malus $\times$ Sorbus|

Rose Family

florentina (Juccagni) Browle"

|Malus domestica $\times$ Sorbus torminalis|

I) ( NA

MALPIGHIA L。 MALPIGHIACEAE

Malpighia Family

glabra $\mathrm{l}$.

Barbatos Cherry

IIITH LASL.

MALUS Mill,

ROSACEAE

Rose Family

'Adirondack' [M. halliana hybrid]

I) ( $N A$

'Aldenhamensis' [M. X purpurea (Barbicr)

Rohi.]

[M. $\times$ atrosanguinea $\times M$. domestica

'Niedzwetzkeyana']

$$
\text { DCAR DCNA GININ }
$$

'Almey'

DCNA GANN MITEL

angustifolia (Ait.) Michx.

Sonthem Crabapple

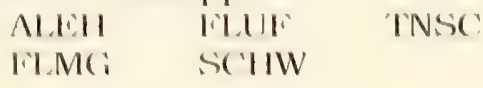

arnoldiana (Rehd.) Sarg.

[M. baccata $\times M$. floribunda]

I)CAR I)CNA

'Athabasca' [M. Xadstringens Zab.]

$\mid$ M. baccata $\times$ M. domestica|

MI)(il) atrosanguinea (Spacth) Schneid.

[M. halliana $\times$ M. sieboldii]

IXC:AR MSMN VACW

baccata (L.) Borkh.

Siberian Crabapple.

I)(NA MI)(iI)

baccata (L.) Borkh. var, mandschurica (Maxim.) Schncid.
I)C:AR
I)CNA

\section{'Centennial'}

I) $\mathrm{N} \wedge$

coronaria (L.) Mill.

Wild Sweet Crabapple

GACG NCOP VAGG

coronaria (L.) Mill. var. dasycalyx Rehd. MI)(iI)

coronaria (L.) Mill, var, elongata Rehd. I) C:NA

cralaegifolia Koehne

$=\times$ Malosorbus florentina

'Crimson Brilliant'

I) C.N $\Lambda$

'David'

1) ( N N

domestica l3orkh.

Common Apple:

(Cultigen of mixed parentage) MIIT VAC'W VATK

'Dorothea' [M. Xscheideckeri (Spaeth) 'Lab.]

|M. floribunda $\times$ M. prunifolia $\mid$ I)C:NA IOEMC

'East Malling'

I) ( N $\Lambda$

'Eleyi' [M. $\times$ purpurea (Barbier) Rehd.] $\mid M$. $\times$ atrosanguinea $\times M$. domestica 'Niedzwetzkyana'| $(\mathrm{i} \wedge \mathrm{FF}$

'Evelyn' DCNA

'Flame'

I) C:N $\Lambda$

florentina (Zuccagni) Schneid.

$=\times$ Malosorbus florentina 
floribunda Sieb.

Showy Crabapple
DCAR
DCWH
GACG
VASK
DCCG
DEMC
MDHP
DCNA
FLMG
VACW

$\star$ 'Fuji' [M. sieboldii (Regel) Rehd.]

DCNA MDGD

'Golden Anniversary'

MDGD

'Golden Hornet' [M. × robusta (Carr.)

Rehd.]

[M. baccata $\times$ M. prunifolia] DEMC

'Guiding Star' DCNA

halliana Koehne DCNA

'Henrietta Crosby' [M. $\times$ arnoldiana $\times M$. domestica 'Niedzwetzkyana'] DCNA

'Henry F. Dupont' DCNA

'Hillier' DCNA

'Hopa' [M. × adstringens Zab.]

[M. baccata $\times$ M. domestica] $\begin{array}{lll}\text { DCCC } & \text { DCNA } & \text { LATU } \\ \text { DCCG } & \text { LAGN } & \text { NCTP }\end{array}$

hupehensis (Pamp.) Rehd. DCWH DEMC SCCU

ioensis (A.Wood) Britton var. palmeri Rehd. DCNA

'Katherine' [M. $\times$ hartwigii Koehne]

[M. baccata $\times$ M. halliana] DCWH VACW VATA

lancifolia Rehd. DCNA

'Lemoinei' [M. × purpurea (Barbier) Rehd.] $[\boldsymbol{M}$. $\times$ atrosanguinea $\times \boldsymbol{M}$. domestica

'Niedzwetzkyana'] MDBG MDGD

'Marshall Oyama' DCNA

'Mary Potter' [M. $\times$ atrosanguinea $\times M$. sargentii 'Rosea'] DCNA MDPJ
'Mathews'

DCNA

$\times$ micromalus Makino

[M. baccata $\times$ M. spectabilis] DCNA

'Mrs. Bayard Thayer' DCNA

* 'Naragansett' [M. /hybrid \#28/ × $\boldsymbol{M}$.

'Wintergold'] DCNA

'Nieuwlandiana' [M. coronaria (L.) Mill.] GACG

'Normand' DCNA

'Nova' [M. ioensis (A.Wood) Britton] DCNA

'Oporto' DCNA

'Parkmanii' [M. halliana Koehne] $\begin{array}{ll}\text { DCNA } & \text { DEWG } \\ \text { DEMC } & \text { MDHN }\end{array}$

'Pink Pearl' DCNA

'Pink Spires' DCNA

$\times$ platycarpa Rehd.

[M. coronaria $\times$ M. domestica] DCNA

'Plena' [M. ioensis (A.Wood) Britton] Bechtel's Crabapple DCNA MDBG NCOP

'Prairie Rose' DCNA

'Prince George's' DCNA

'Profusion' DCNA

prunifolia (Willd.) Borkh. DCNA FLBW

pumila Mill.

$=$ M. domestica 
$\times$ purpurea (Barbier) Rehd.

[M. $\times$ atrosanguinea $\times M$. domestica

'Niedzwetzkeyana']
DCCG
DEMC FLMG

'Pygmy'

$$
\text { DCNA }
$$

'Red Jade'

DCNA TNSC

'Red Jewel'

DCNA

'Red Silver'

DCNA

'Red Tip' [M. × soulardii (Bailey) Britton]

[M. ioensis $\times M$. domestica] MDGD

$\times$ robusta (Carr.) Rehd.

[M. baccata $\times$ M. prunifolia] DCAR DCNA NCDU

'Rondo'

DCNA

'Rosea' [M. sargentii Rehd.] DCNA

sargentii Rehd.

Sargent's Crabapple
DCAR GARW
VACW
DCNA
SCCU

$\times$ scheideckeri (Spaeth) Zab.

[M. floribunda $\times$ M. prunifolia]

DCCG DCNA SCCU

'Shakespeare'

DCNA

sieboldii (Regel) Rehd.

Toringo Crabapple

DCAR DCNA NCDU VATA

sieboldii var. zumi (Matsum.) Asami

= M. zumi

'Silver Moon'

DCNA

'Sissipuk'

DCNA

spectabilis (Ait.) Borkh.

DCAR

'Thomas Roland'

DCNA
'Timiskaming'

DCNA

'Tops-in-Bloom'

DCNA

'Van Eseltine' [M. $\times$ arnoldiana $\times \boldsymbol{M}$. spectabilis] DCNA MDRP

'Veitch's Scarlet' [M. domestica L.] DCNA

'Wintergold'

DCNA

'Wynema'

DCNA MDGD

$\times$ zumi (Matsum.) Rehd.

[M. baccata var. mandshurica $\times$ M. sieboldii] DCNA DEMC

MALVAVISCUS Fabr. non Adans. MALVACEAE

Mallow Family

arboreus Cav. var. drummondii Schery

Turk's Cap

VACW

arboreus Cav. var. penduliflorus (Sesse \& Moc. ex DC.) Schery

Turk's Cap

$$
\text { ALDM FLUF }
$$

drummondii Torr. \& A.Gr.

= M. arboreus var. drummondii

MANIHOT Mill.

Cassava

EUPHORBIACEAE

Spurge Family

grahamii Hook.

FLUF LAAL MSBN

MELALEUCA L.

MYRTACEAE

Myrtle Family

leucadendron (L.) L.

$=M$. quinquenervia

quinquenervia (Cav.) S.T.Blake

Paperbark Tree, Punk Tree

FLUF 
MELIA L.

MELIACEAE

Mahogany Family

azedarach $\mathrm{L}$.

Chinaberry

$\begin{array}{llll}\text { ALCG } & \text { FLUF } & \text { NCDU } & \text { VAWR } \\ \text { ARUM } & \text { MDPJ } & \text { SCMG } & \\ \text { DCNA } & \text { NCCA } & \text { VACW } & \end{array}$

MENZIESIA Sm.

ERICACEAE

Heath Family

pilosa (Michx.) Juss.

Minniebush

VAPO

MESPILUS L.

\section{ROSACEAE}

Rose Family

germanica L.

Medlar

DCHU VABF VACW

METASEQUOIA Miki ex H.H.Hu \&

W.C.Cheng

TAXODIACEAE

Taxodium Family

glyptostroboides H.H.Hu \& W.C.Cheng

Dawn Redwood

$\begin{array}{llll}\text { ALAU } & \text { GAUG } & \text { NCDU } & \text { VATA } \\ \text { DCNA } & \text { MDBG } & \text { SCMG } & \text { VAWM } \\ \text { DEMC } & \text { MDJS } & \text { TNHD } & \\ \text { GAFN } & \text { MDPJ } & \text { VACW } & \end{array}$

$\star$ 'National' [M. glyptostroboides H.H.Hu

\& W.C.Cheng]

DCNA

MICHELIA L.

MAGNOLIACEAE Magnolia Family

compressa (Maxim.) Sarg.

NCOP figo (Lour.) Spreng.

Banana Shrub

$\begin{array}{llll}\text { ALBG } & \text { GAFF } & \text { LALG } & \text { SCMG } \\ \text { ALSH } & \text { GAHC } & \text { LASL } & \text { SCMP } \\ \text { FLDG } & \text { GAJI } & \text { MDFM } & \text { SCWI } \\ \text { FLMG } & \text { GATS } & \text { MSMN } & \text { TXHE } \\ \text { FLRM } & \text { LAAL } & \text { SCFW } & \\ \text { FLUF } & \text { LAHG } & \text { SCJM } & \end{array}$

fuscata (Andr.) Bl. ex Wall.

$=$ M. figo

MICROBIOTA Komar.

CUPRESSACEAE

Cypress Family

decussata Komar.

DCNA GAEC MDBG

MICROCITRUS Swingle

RUTACEAE

Citrus Family

australasica (F.Muell.) Swingle

Australian Finger Lime

FLUF

MILLETTIA Wight \& Arn.

FABACEAE (Faboideae)

Bean Family

reticulata Benth.

Leatherleaf $\mathrm{M}$.

FLUF TXCT

MIMOSA L.

FABACEAE (Mimosoideae) Bean Family

biuncifera Benth.

Catclaw M. LAHG LASL TXCT

pigra L. var. berlandieri (A.Gr.) B.L.Turner DCNA TXPS

\section{MITCHELLA L.}

RUBIACEAE

Madder Family

repens $\mathrm{L}$.

Partridgeberry

GAEC MDLT NCEG SCBR 


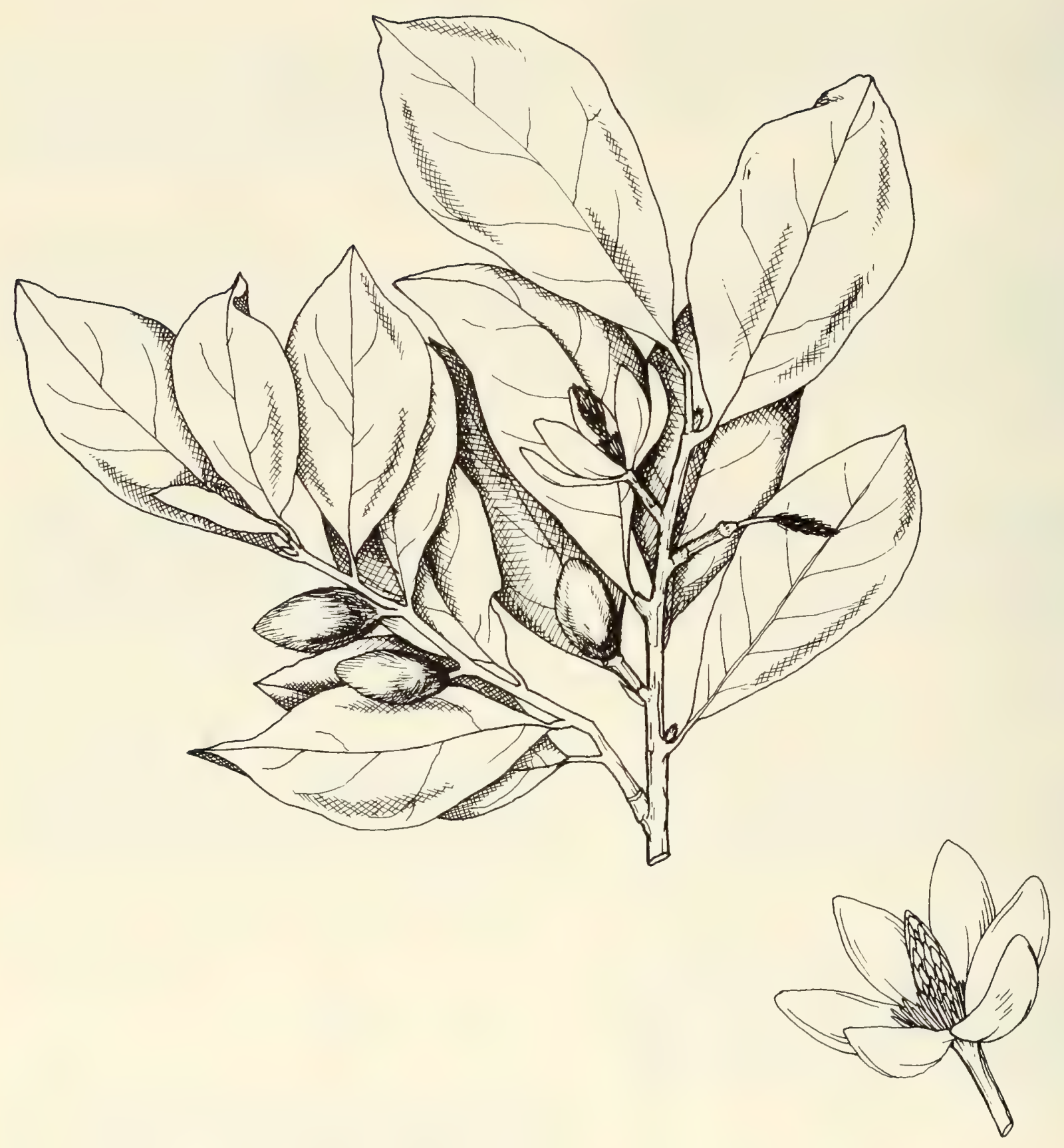

MICHELIA figo (Lour.) Spreng.

[illustrator Susan M. Johnston] 
MORUS L.

Mulberry

MORACEAE

Mulberry Family

alba L.

White M.

$\begin{array}{llll}\text { DCSH } & \text { MDHN } & \text { SCCL } & \text { VACW } \\ \text { DEMC } & \text { MDLT } & \text { TNSG } & \text { VAGG } \\ \text { FLLT } & \text { NCCA } & \text { TNTV } & \text { VAMP }\end{array}$

'Hicks' [M. alba L.]

TNNE

'Pendula' [M. alba L.]

Weeping $M$.

DCNC MSMN TNTV

$\mathrm{DCSH}$ SCUC

rubra L.

Red M.

ALHC GAUG TXDC

ALTR NCBE VACW

FLUF TNSC VAMP

'Teas Weeping' [M. alba L.] TNSN

MUEHLENBECKIA Meisn.

POLYGONACEAE

Buckwheat Family

axillaris (Hook.f.) Walp.

Matbrush Wire Vine

ALHC

MURRAYA Koenig ex L.

RUTACEAE

Citrus Family

exotica $\mathrm{L}$.

= M. paniculata

paniculata (L.) Jack

Orange Jasmine, Chinese Box FLUF
MUSA L. MUSACEAE

Banana Banana Family basjoo Sieb. \& Zucc. DCNA

rosacea Jacq.

LASL

VACW

'Sapientum' [M. $\times$ paradisiaca L.]

[M. acuminata $\times M$. balbisiana]

FLUF

velutina $\mathrm{H}$.Wendl. \& Drude GACG LASL

\section{MYRICA L.}

MYRICACEAE

Bayberry Family

cerifera L.

Wax-myrtle, Waxberry

$\begin{array}{llll}\text { ALBG } & \text { GACG } & \text { LASL } & \text { SCCU } \\ \text { FLMG } & \text { GAIS } & \text { MDJS } & \text { TXHA } \\ \text { FLPW } & \text { GAJY } & \text { MDLT } & \text { VAMP } \\ \text { FLUF } & \text { LAHG } & \text { NCTP } & \end{array}$

heterophylla Raf.

Wax-myrtle GAEC TXSE

inodora Bartram SCWI

pensylvanica Loisel.

Bayberry

DCNA MDBG TNBO

DEMC MDTD

pumila Michx.

$=$ M. pusilla

pusilla Raf.

Dwarf Wax-myrtle SCWI TXSE

rubra Sieb. \& Zucc.

FLUF GAIS VAWL

MYRTUS L.

Myrtle

MYRTACEAE

Myrtle Family

communis $\mathrm{L}$.

Common $\mathrm{M}$.

$\begin{array}{llll}\text { FLMG } & \text { GADR } & \text { SCKS } & \text { SCNR } \\ \text { FLUF } & \text { GAJI } & \text { SCMG } & \text { TXLL }\end{array}$

'Microphylla' [M. communis L.]

Dwarf Myrtle
ALFN
FLMG SCBR

ornata Roxb.

$=\mathbf{M}$. rosacea 
NANDINA Thunb.

BERBERIDACEAE

Barberry Family

'Alba' [N. domestica Thunb.] ARRB FLMG MDBG

domestica Thunb.

Heavenly Bamboo

$\begin{array}{llll}\text { ALBG } & \text { FLMG } & \text { MDBG } & \text { VACW } \\ \text { ALRH } & \text { FLUF } & \text { MDLT } & \text { VANB } \\ \text { ARRB } & \text { GAHC } & \text { MSBN } & \\ \text { DCNA } & \text { LAAL } & \text { MSEN } & \\ \text { FLDG } & \text { LASL } & \text { TNSG } & \end{array}$

'Harbor Dwarf' [N. domestica Thunb.]

$=$ 'Harbour Dwarf'

'Harbour Dwarf' [N. domestica Thunb.] GACG

'Purpurea Nana' [N. domestica Thunb.] NCNB VATA

\section{NEILLIA D.Don \\ ROSACEAE}

Rose Family

sinensis Oliv.

Chinese Neillia

NCCA TNSN

\section{NEMOPANTHUS Raf. AQUIFOLIACEAE \\ Holly Family}

mucronatus (L.) Trelease

Catberry, Northern Mountain Holly

DCNA

\section{NEOPIERIS Britton \\ ERICACEAE \\ = Lyonia mariana \\ Heath Family}

NERIUM L.

APOCYNACEAE Dogbane Family

indicum Mill.

$=N$. oleander

odorum Ait.

$=N$. oleander oleander L.

Oleander

$\begin{array}{llll}\text { ALBG } & \text { LAHG } & \text { MSBN } & \text { VANB } \\ \text { FLUF } & \text { LASL } & \text { SCWC } & \end{array}$

'Variegata' [ $N$. oleander L.]

Variegated Oleander

DCBG

\section{NEVIUSIA A.Gr.}

ROSACEAE

Rose Family

alabamensis A.Gr.

Snow-wreath

$\begin{array}{llll}\text { ALUA } & \text { NCCA } & \text { TNCT } & \text { VAPH } \\ \text { DCNA } & \text { SCBR } & \text { TNTV } & \\ \text { DEMC } & \text { SCWI } & \text { TNUT } & \\ \text { GAEC } & \text { TNAB } & \text { VACW } & \end{array}$

NICODEMIA Tenore

BUDDLEJACEAE

Buddleja Family

diversifolia Tenore

FLUF

NICOTIANA L.

SOLANACEAE

Nightshade Family

glauca R.Graham

Tree Tobacco

LASL

NIEREMBERGIA Ruiz \& Pavon

SOLANACEAE Nightshade Family

frutescens Durieu

= N. scoparia

scoparia Sendtn.

Tall Cup-flower

GACE GAUG

NYSSA L.

NYSSACEAE Sour-gum Family

ogeche Marsh.

Ogeechee Tupelo

DCNA SCBR VAWM


Sour Gum, Black Gum

sylvatica Marsh. var. biflora (Walt.) Sarg. SCBR

OCHNA L.

\section{OCHNACEAE}

Ochna Family sylvatica Marsh.
ALAU FLMG
DCCG LAHG
NCAS VAGW
DCWH
MDBP
TNFN
DEMC
MDJH
TNHG
VACW

armatus Diels

$\begin{array}{llll}\text { ALTD } & \text { GAEH } & \text { MD̃MG } & \text { VAGS } \\ \text { GACG } & \text { MDBG } & \text { NCDU } & \end{array}$

'Aurantiacus' [O. fragrans Lour.]

DCNA SCHS SCMG

$\times$ fortunei Carr.

[O. fragrans $\times$ O. heterophyllus var. heterophyllus ]

$\begin{array}{llll}\text { ALAU } & \text { FLMG } & \text { MDMG } & \text { NCTP } \\ \text { ALBG } & \text { GAUG } & \text { NCCA } & \text { VAMP } \\ \text { ALTD } & \text { LARP } & \text { NCDU } & \\ \text { DCCG } & \text { MDCO } & \text { NCGP } & \end{array}$

fragrans Lour.

Sweet Olive, Tea Olive

$\begin{array}{llll}\text { ALBG } & \text { LAAL } & \text { MDRP } & \text { SCMG } \\ \text { DCNA } & \text { LAGN } & \text { NCDU } & \text { SCMP } \\ \text { FLDG } & \text { LARP } & \text { NCEL } & \text { SCUC } \\ \text { FLMG } & \text { LASL } & \text { NCRC } & \text { TXMK } \\ \text { FLUF } & \text { LATU } & \text { SCEA } & \text { VANB } \\ \text { GAAB } & \text { MDFM } & \text { SCFW } & \end{array}$

OLEA L.

OLEACEAE Olive Family

europaea L.

Olive

FLUF LAAL TXSN

ORIGANUM L.

LAMIACEAE

Mint Family

onites $\mathrm{L}$.

Pot Marjoram

DCNA

ORIXA Thunb.

RUTACEAE

Citrus Family

japonica Thunb.

MDKN

OSMANTHUS Lour.

OLEACEAE

Olive Family

americanus (L.) A.Gr.

Devilwood

ALAU GACG NCTP VACW

ALBG GATS SCBR VAWR

ALIT GAUG SCMP

FLMG NCBE TNDG

aquifolium Sieb.

$=0$. heterophyllus var. heterophyllus
'Gulf Tide' [O. heterophyllus (G.Don)

P.S.Green var. heterophyllus]

MDGD TNSN VATA

heterophyllus (G.Don) P.S.Green var. heterophyllus

Holly Olive

$\begin{array}{llll}\text { ALTD } & \text { GATS } & \text { MDSJ } & \text { NCWC } \\ \text { DCCG } & \text { MDGD } & \text { NCCA } & \text { VAGS } \\ \text { FLMG } & \text { MDLT } & \text { NCDU } & \end{array}$

ilicifolius (Hassk.) Hort. ex Carr.

$=0$. heterophyllus var. heterophyllus

'Purpureus' [O. heterophyllus (G.Don)

P.S.Green var. heterophyllus]

VAGS

'Rotundifolius' [O. heterophyllus (G.Don)

P.S.Green var. heterophyllus]
ALTD
LALG
SCCU
GAUG
MDMG VANB

'San Jose' [O. $\times$ fortunei Carr.]

[O. fragrans $\times$ O. heterophyllus

var. heterophyllus ]

DCCG DCNA GABS NCEL

'Variegatus' [O. heterophyllus (G.Don)

P.S.Green var. heterophyllus]
ALBG
FLUF
LALG
FLMG GACG VADW 
OSTEOMELES Lindl. ROSACEAE

Rose Family

schwerinae Schneid.

Chinese Boneberry

DCNA

OSTRYA Scop.

\section{BETULACEAE}

Birch Family

virginiana (Mill.) K.Koch

Hop Hornbeam

$\begin{array}{lll}\text { ALAU } & \text { FLUF } & \text { VAMP } \\ \text { DCCG } & \text { TNFN } & \end{array}$

\section{OXYDENDRUM DC.}

ERICACEAE

Heath Family

arboreum (L.) DC.

Sourwood, Sorrel Tree

$\begin{array}{llll}\text { ALAU } & \text { MDJH } & \text { SCBR } & \text { VANB } \\ \text { DCNA } & \text { MDKN } & \text { TNSC } & \text { VAPO } \\ \text { DCWR } & \text { MDLT } & \text { VACP } & \end{array}$

PACHYSANDRA MichX.

BUXACEAE Boxwood Family

axillaris Franch.

NCWD

procumbens Michx.

Allegheny P., Allegheny Spurge

MDBG SCWI VAPH

'Silveredge' [P. terminalis Sieb. \& Zucc.] DCNA

terminalis Sieb. \& Zucc.

Japanese P., Japanese Spurge

DCNA DEWG LALG VACW

PACHYSTIMA Endl.

= PAXISTIMA

PAEONIA L. Peony

PAEONIACEAE

Peony Family

suffruticosa Andr.

Tree P.

DCWH VACW
PALAFOXIA Lag.

ASTERACEAE

Aster Family

feayi A.Gr.

SCWI

PARKINSONLA L.

FABACEAE (Caesalpinioideae)

Bean Family

aculeata $\mathrm{L}$

Jerusalem Thorn

$\begin{array}{lll}\text { FLUF } & \text { LASL } & \text { SCSB } \\ \text { LALG } & \text { MSMN } & \end{array}$

PARROTLA C.A.Mey. HAMAMELIDACEAE Witch-hazel Family

persica (DC.) C.A.Mey.

Persian Ironwood

$\begin{array}{lll}\text { DCEL } & \text { GAEH } & \text { VABF } \\ \text { DCWH } & \text { MDBG } & \text { VACW } \\ \text { DEEM } & \text { MDSJ } & \text { VAMP }\end{array}$

PARROTIOPSIS (Niedenzu) Schneid. HAMAMELIDACEAE Witch-hazel Family

jacquemontiana (Decne.) Rehd. DCNA

PARTHENOCISSUS Planch.

VITACEAE Grape Family

henryana (Hemsl.) Diels \& Gilg DCNA MDMG VAPH

quinquefolia (L.) Planch.

Virginia Creeper, Woodbine MDLT

tricuspidata (Sieb. \& Zucc.) Planch.

Boston Ivy

DCCG SCRH

PASANIA (Miq.) Oerst.

= LITHOCARPUS 


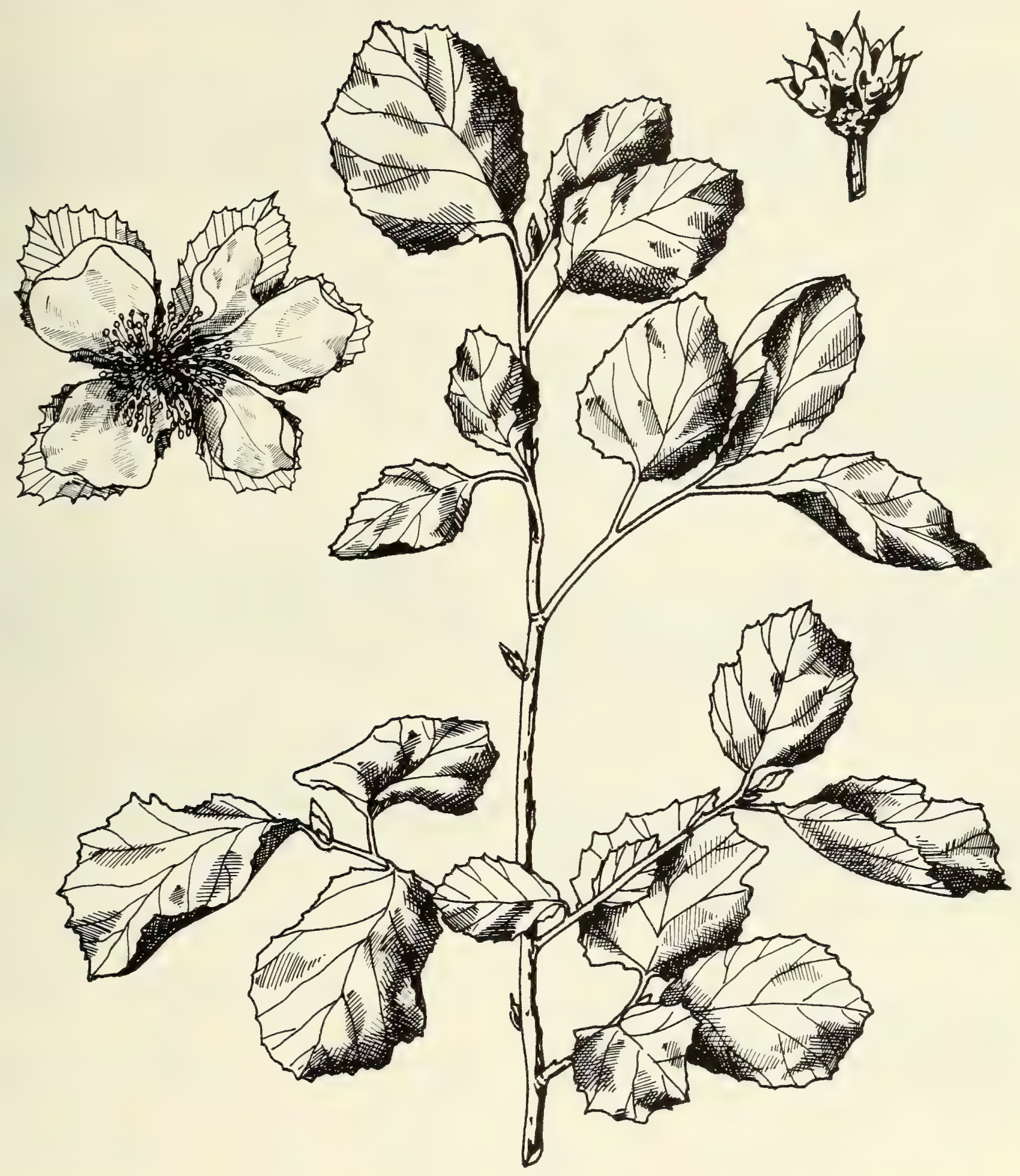

PARROTIOPSIS jacquemontiana (Decne.) Rehd.

[illustrator Susan M. Johnston] 


\section{PASSIFLORA L.}

PASSIFLORACEAE Passion-flower Family

coccinea Aubl.

Red Passionflower

GACG LASL

PAULOWNLA Sieb. \& Zucc.

SCROPHULARIACEAE Figwort Family

\section{kawakamii Ito} SCWI

tomentosa (Thunb. ex J.A.Murr.) Steud.

Princess Tree

$\begin{array}{llll}\text { DCWH } & \text { MDHN } & \text { MSRN } & \text { VAWR } \\ \text { GAAB } & \text { MDLT } & \text { TNHG } & \end{array}$

\section{PAUROTIS COOK}

\section{ARECACEAE}

Palm Family

wrightii (Griseb.) Britton

= Acoelorrhaphe wrightii

PAVONIA Cav.

MALVACEAE Mallow Family

hastata Cav.

Spearleaf P.

LASL

\section{PAXISTIMA Raf.}

CELASTRACEAE

Staff-tree Family

canbyi A.Gr.

Cliff-green

DCNA MDLT

PERIPLOCA L.

ASCLEPIADACEAE

Asclepias Family
PEROVSKIA Karelin

LAMIACEAE

Mint Family

atriplicifolia Benth.

Russian Sage

MDBG VAGS

PERSEA Mill.

LAURACEAE

Laurel Family

\section{americana Mill.}

Avocado, Alligator Pear

FLUF

borbonia (L.) Spreng.

Red Bay

$\begin{array}{llll}\text { ALAU } & \text { LAMP } & \text { SCBR } & \text { VACW } \\ \text { FLUF } & \text { NCGP } & \text { SCMP } & \text { VANB }\end{array}$

humilis Nash

SCWI

thunbergii (Sieb. \& Zucc.) Kosterm.

LASL

PETTERIA Presl

FABACEAE (Faboideae)

Bean Family

ramentacea Presl

MDGD

\section{PHELLODENDRON Rupr.}

RUTACEAE

Citrus Family

amurense Rupr.

Amur Cork-tree

$\begin{array}{llll}\text { DCTB } & \text { GARW } & \text { MDGJ } & \text { VABF } \\ \text { DCWH } & \text { MDCP } & \text { TNSN } & \text { VABP }\end{array}$

amurense Rupr. var. japonicum (Maxim.)

Ohwi

DCLC MDWG TNWF

amurense Rupr. var. lavallei (Dode)

Sprague

TNUT

graeca

TNSN 
PHILADELPHUS L. Mock Orange SAXIFRAGACEAE (Hydrangeoideae) Saxifrage Family

'Belle Etoile' [P. coronarius L.] VAPH

coronarius L.

European Mock O.

$\begin{array}{llll}\text { GAOH } & \text { MDCP } & \text { MDPJ } & \text { VAGW } \\ \text { GAVA } & \text { MDHN } & \text { TNSN } & \text { VANS } \\ \text { MDBP } & \text { MDLT } & \text { VACW } & \end{array}$

'Duplex' [P. coronarius L.] MDJS VABF

$\times$ falconeri Sarg.

[P. coronarius $\times P$. laxus] MSMN NCCA

gordonianus Lindl.

$=P$. lewisii

hirsutus Nutt. GAEC

inodorus $\mathrm{L}$.

$\begin{array}{llll}\text { FLDJ } & \text { LALG } & \text { NCCA } & \text { TXMA } \\ \text { FLMG } & \text { MSHB } & \text { SCCU } & \text { VACW } \\ \text { GAOH } & \text { NCBE } & \text { SCRH } & \text { VAGG }\end{array}$

$\times$ lemoinei V.Lemoine

[P. microphyllus $\times \boldsymbol{P}$. coronarius] VAPH

lewisii Pursh MDJS

'Minnesota Snowflake' [P. $\times$ virginalis

Rehd.]

[P. $\times$ lemoinei $\times P$. ?nivalis 'Plenus'] DEWG

'Natchez' [P. coronarius L.] DCNA

'Nymans' [P. delavayi L.Henry] DCNA

pubescens Loisel. SCBR

pubescens Loisel. var. verrucosus (Schrad. ex DC.) S.Y.Hu DCDO MDSJ VAHS

satsumanus Sieb. ex Miq. var. nikoensis Rehd.

DEWG schrenkii Rupr. DCNA

'Silberregen' DCNA

triflorus Wall. $\mathrm{VABF}$

verrucosus Schrad. ex DC.

$=\boldsymbol{P}$. pubescens var. verrucosus

'Virginal' [P. $\times$ virginalis Rehd.]

[P. $\times$ lemoinei $\times P$. ? nivalis 'Plenus']

$\begin{array}{lll}\text { ALBH } & \text { DEWG } & \text { MDCC } \\ \text { DCDO } & \text { GAPG } & \text { MDJS }\end{array}$

'Voie Lactee'

MDGD

PHILLYREA L.

OLEACEAE

Olive Family

angustifolia L.

Narrow-leaf $P$.

ALTD SCPP

latifolia L. var. media (L.) Schneid.

Tree P. LASL

media L.

$=P$. latifolia var. media

'Spinosa' [P. latifolia L. var. media (L.) Schneid.] GAUG

PHOENIX L.

ARECACEAE

Palm Family

canariensis Hort. ex Chabaud

Canary Island Date Palm

FLUF

reclinata Jacq.

Senegal Date Palm FLUF

sylvestris $\times P$. canariensis FLUF 
PHOTINLA Lindl. ROSACEAE

Rose Family

'Birmingham' [P. $\times$ fraseri W.J.Dress]

$[\boldsymbol{P}$. serratifolia $\times \boldsymbol{P}$. glabra]

$\begin{array}{llll}\text { ALBG } & \text { FLUF } & \text { SCWE } & \text { VAWR } \\ \text { ALFN } & \text { GAWH } & \text { TXSE } & \\ \text { ALTD } & \text { MSHB } & \text { VACW } & \\ \text { DCSD } & \text { SCFW } & \text { VAMP } & \end{array}$

glabra (Thunb. ex J.A.Murr.) Maxim. Japanese P.

$\begin{array}{llll}\text { FLGS } & \text { GAJY } & \text { SCCL } & \text { VAMP } \\ \text { FLMG } & \text { MSHB } & \text { SCCS } & \\ \text { GACG } & \text { NCCA } & \text { SCMG } & \end{array}$

serratifolia (Desf.) Kalk

Chinese P.

$\begin{array}{llll}\text { ALBG } & \text { GACG } & \text { NCTE } & \text { SCUC } \\ \text { ALTD } & \text { GAUG } & \text { SCBR } & \text { TNMB } \\ \text { ARRB } & \text { LASL } & \text { SCDB } & \text { VANB } \\ \text { DCCG } & \text { MSEN } & \text { SCFW } & \text { VAOD } \\ \text { FLMG } & \text { NCDU } & \text { SCMG } & \text { VAWP } \\ \text { FLUF } & \text { NCOP } & \text { SCMP } & \end{array}$

serrulata Lindl.

$=\boldsymbol{P}$. serratifolia

villosa (Thunb. ex J.A.Murr.) DC.

DCWR MDBG NCBE VAWR

DEWG MDGJ VABF

GAEH MDPJ VAGH

villosa (Thunb. ex J.A.Murr.) DC.

var. laevis (Thunb. ex J.A.Murr.) Dipp. MDGJ

villosa (Thunb. ex J.A.Murr.) DC.

var. maximowicziana (Lev.) Rehd.
DCCG
DEMC
NCBE

villosa (Thunb. ex J.A.Murr.) DC.

var. sinica Rehd. \& Wils.

VACW

PHYGELIUS E.Mey. ex Benth.

SCROPHULARIACEAE

Figwort Family

capensis E.Mey.

Cape Fuchsia

VACW
PHYLLANTHUS L.

EUPHORBIACEAE

Spurge Family

nivosa W.G.Sm.

= Breynia nivosa

PHYLLOSTACHYS Sieb. \& Zucc.

Bamboo

POACEAE

Grass Family

angusta McClure MDST

arcana McClure GAIS

aurea A.\& C.Riv.

Fishpole B., Golden B.
FLDG
GAIS
VAMP

aureosulcata McClure

Yellow-groove B.

TNFE

bambusoides Sieb. \& Zucc.

GAIS NCOP

'Castillon' [P. bambusoides Sieb. \& Zucc.] MDFM

congesta McClure GAIS

dulcis McClure

Sweetshoot B.

GAIS MDFM

elegans McClure

GAIS

flexuosa A.\& C.Riv. GAIS

'Henon' [P. nigra (Lodd.) Munro] GAIS VABB

heterocycla (Carr.) Mitf.

Giant Timber Bamboo ALBG GAIS SCCU SCWL

meyeri McClure FLUF GAIS

nidularia Munro GAIS

nigra (Loud.) Munro GAIS 
nuda McClure

MDGD MDPJ

propinqua McClure

GAIS

pubescens Mazel ex Houz.de Leh.

$=\boldsymbol{P}$. heterocycla

rubromarginata McClure DCNA

'Smoothsheath' [P. nidularia Munro] GAIS

'Solidstem' [P. purpurata McClure] GAIS

'Straightstem' [P. purpurata McClure] GAIS

viridiglaucescens A.\& C.Riv. GAIS

viridis (Young) McClure GAIS NCSL

vivax McClure

GAIS

PHYSOCARPUS (Cambess.) Maxim. Ninebark

ROSACEAE Rose Family

amurensis (Maxim.) Maxim. DCNA

intermedius (Rydb.) Schneid. TNBM

'Luteus' [P. opulifolius (L.) Maxim.] VACW

malvaceous (Greene) O.Ktze. TNUT

opulifolius (L.) Maxim. GAEC MDGJ TNFN

PICEA A.Dietr. PINACEAE

abies (L.) Karst.

Norway S.

$\begin{array}{llll}\text { DCNA } & \text { MDBG } & \text { MDJS } & \text { TNWF } \\ \text { GAHC } & \text { MDHN } & \text { NCBE } & \text { VASP }\end{array}$

alba (Ait.) Link

$=$ P. glauca

alcoquiana (J.G.Veitch ex Lindl.) Carr.

Alcock's S.

DCNA

asperata Mast.

Dragon S.

DCNA NCBE

asperata Mast. var. heterolepis (Rehd. \& Wils.) W.C.Cheng ex Rehd.

DCNA

'Aurea Compacta' [P. orientalis (L.) Link] DCNA

'Barryi' [P. abies (L.) Karst.] DCNA

'Beissneri Compacta' [P. mariana (Mill.) BSP.] DCNA

'Brevifolia' [P. abies (L.) Karst.] DCNA

'Capitata' [P. abies (L.) Karst.] DCNA

'Clanbrassiliana' [P. abies (L.) Karst.] DCNA

'Compacta' [P. pungens Engelm.] DCNA

'Compacta Asselyn' [P. abies (L.) Karst.] DCNA

'Conica' [P. glauca (Moench) Voss]

Dwarf Alberta S. $\begin{array}{lll}\text { DCNA } & \text { MDBG } & \text { MDLT } \\ \text { GAEC } & \text { MDJS } & \text { VATW }\end{array}$

'Doumetii' [P. mariana (Mill.) BSP.] Black S. NCBE

'Echiniformis' [P. glauca (Moench) Voss] MDLT

'Foxtail' [P. pungens Engelm.] DCNA

gemmata Rehd. \& Wils. DCNA

glauca (Moench) Voss

White S.

$\begin{array}{llll}\text { MDAF } & \text { MDJS } & \text { TNUT } & \text { VATA } \\ \text { MDHN } & \text { TNFE } & \text { VARG } & \end{array}$


'Glauca' [P. asperata Mast.] DCNA

'Glauca Pendula' [P. pungens Engelm.

f. glauca (Regel) Beissn.] DCNA

'Glauca Procumbens' [P. pungens Engelm.

f. glauca (Regel) Beissn.] DCNA

'Glauca Prostrata' [P. pungens Engelm.

f. glauca (Regel) Beissn.] DCNA

glehnii (F.Schmidt) Mast. DCNA NCBE

'Globosa' [P. abies (L.) Karst.] DCNA

'Globosa' [P. pungens Engelm.] DCNA

'Gracilis' [P. orientalis (L.) Link] DCNA

'Gregoryana Parsonii' [P. abies (L.) Karst.] DCNA

'Highlandia' [P. abies (L.) Karst.] DCNA

'Hoopsii' [P. pungens Engelm.] DCNA

'Howell's Dwarf [P. jezoensis (Sieb. \& Zucc.) Carr.] MDBG

'Humilis' [P. abies (L.) Karst.] DCNA

'Hunnewelliana' [P. pungens Engelm.] DCNA MDBG

'Hystrix' [P. abies (L.) Karst.] DCNA

'Inversa' [P. abies (L.) Karst.] DCNA MDBG NCBE

'Iseli Fastigiata' [P. pungens Engelm.] DCNA

'Kingsville' [P. abies (L.) Karst.] DCNA

'Koster' [P. pungens Engelm.] DCNA MDBG

'Little Gem' [P. abies (L.) Karst.] DCNA
'Maxwellii' [P. abies (L.) Karst.] DCNA MDBG

'Merhii' [P. abies (L.) Karst.] DCNA

'Microphylla' [P. abies (L.) Karst.] DCNA

'Microsperma' [P. abies (L.) Karst.] DCNA

'Montgomery' [P. pungens Engelm.] DCNA

'Montigena' [P. abies (L.) Karst.] DCNA

'Mucronata' [P. abies (L.) Karst.] DCNA MDBG

'Nidiformis' [P. abies (L.) Karst.] DCNA MDBG MDLT

obovata Ledeb. DCNA

'Ohlendorffii' [P. abies (L.) Karst.] DCNA

'Oldhamiana' [P. abies (L.) Karst.] DCNA

omorika (Pancic) Purk.

Serbian S. DCNA MDBG MDLT

orientalis (L.) Link

Oriental S.

DCNA DEWG NCBE VAAC

DCWH MDJS NCKH VABF

'Parsonii' [P. abies (L.) Karst.] DCNA

'Pendula' [P. abies (L.) Karst.] DCNA MDJS MDLT

'Pendula' [P. omorika (Pancic) Purk.] DCNA

polita (Sieb. \& Zucc.) Carr.

$=P$. torano

'Procumbens' [P. abies (L.) Karst.] DCNA

'Prostrata' [P. abies (L.) Karst.] DCNA

'Pseudoprostrata' [P. abies (L.) Karst.] DCNA 


\section{PICEA}

'Pumila' [P. abies (L.) Karst.] DCNA

'Pumila Glauca' [P. abies (L.) Karst.] DCNA

pungens Engelm.

Colorado S. DCNA MDBG VASP

pungens Engelm. f. glauca (Regel) Beissn. Colorado Blue S. DCNA

'Pygmaea' [P. abies (L.) Karst.] DCNA MDLT

'Pyramidata' [P. abies (L.) Karst.] DCNA

'Remonte' [P. abies (L.) Karst.] DCNA

'Repens' [P. abies (L.) Karst.] DCNA

'Tabuliformis' [P. abies (L.) Karst.] DCNA

torano (K.Koch) Koehne

Tiger-tail S. DCNA NCBE VAMP

'Wild Acres' [P. glauca (Moench) Voss] DCNA

\section{PIERIS D.Don}

\section{ERICACEAE}

Heath Family

'Bert Chandler' [P. japonica (Thunb. ex

J.A.Murr.) D.Don ex G.Don] MDLT MDMG

'Brower's Beauty' [P. floribunda $\times \boldsymbol{P}$. japonica] DCNA MDBG MDLT

'Compacta' [P. japonica (Thunb. ex J.A.Murr.) D.Don ex G.Don] DCNA

'Crispa' [P. japonica (Thunb. ex J.A.Murr.) D.Don ex G.Don] DCNA MDBG

'Daisen' [P. japonica (Thunb. ex J.A.Murr.)

D.Don ex G.Don] DCNA MDBG
'Dorothy Wyckoff' $[\boldsymbol{P}$. japonica (Thunb. ex J.A.Murr.) D.Don ex G.DonkDEWG MDLT VAPH

'Flamingo' [P. japonica (Thunb. ex J.A.Murr.) D.Don ex G.Don] DCNA MDBG

floribunda (Pursh) Benth. \& Hook.

Fetterbush DEWG MDLT MDWP NCDB

'Forest Flame' [P. japonica $\times P$. formosa] DEWG MDFM MDJW VAPH

formosa (Wall.) D.Don MDKN

forrestii R.Harrow ex W.W.Sm.

= P. formosa

japonica (Thunb. ex J.A.Murr.) D.Don ex

G.Don

Japanese Andromeda

$\begin{array}{llll}\text { ALBG } & \text { DEWG } & \text { MDLT } & \text { VACW } \\ \text { ALBH } & \text { GACG } & \text { TNCT } & \\ \text { DCNA } & \text { GASM } & \text { TNDG } & \end{array}$

'Mountain Fire' $[P$. formosa $\times P$. japonica] MDLT

phillyreifolia (Hook.) DC.

$\begin{array}{ll}\text { ALAU } & \text { MDKN VAPH } \\ \text { GAEC } & \text { SCWI }\end{array}$

'Purity' [P. japonica (Thunb. ex J.A.Murr.)

D.Don ex G.Don] DCNA

'Pygmaea' [P. japonica (Thunb. ex

J.A.Murr.) D.Don ex G.Don] DCNA GAEC

'Pygmy Variegata' [P. japonica (Thunb. ex J.A.Murr.) D.Don ex G.Don] MDKN

'Red Mill' [P. japonica (Thunb. ex J.A.Murr.) D.Don ex G.Don] MDBG MDLT

'Scarlet O'Hara' [P. japonica (Thunb. ex J.A.Murr.) D.Don ex G.Don] DCNA MDBG

'Shojo' [P. japonica (Thunb. ex J.A.Murr.) D.Don ex G.Don] MDLT

taiwanensis Hayata DCNA DEWG 
'Variegata' [P. japonica (Thunb. ex

J.A.Murr.) D.Don ex G.Don]

DCNA MDBG VATW

DEWG MDLT

'Wada' [P. japonica (Thunb. ex J.A.Murr.)

D.Don ex G.Don]

VAPH

'Wakehurst' [P. formosa (Wall.) D.Don] DCNA

'Whitecaps' [P. japonica (Thunb. ex

J.A.Murr.) D.Don ex G.Don] DCNA

'White Cascade' [P. japonica (Thunb. ex J.A.Murr.) D.Don ex G.Don] DCNA MDBG

PINCKNEYA MichX.

RUBIACEAE Madder Family

pubens Michx.

VAPH

PINUS L.

Pine

PINACEAE

Pine Family

'Adcock's Dwarf' [P. parviflora Sieb. \& Zucc.]

DCNA

'Albyns' [P. sylvestris L.]

DCNA

'Amelia Dwarf' [P. strobus L.]

DCNA

'Argentea Compacta' [P. sylvestris L.] DCNA

armandii Franch. DCNA

'Arnold Dwarf' [P. peuce Griseb.] DCNA

attenuata J.G.Lemmon

Knob-cone P. VABF

'Aurea' [P. sylvestris L.]

DCNA

ayacahuite K.Ehrenb. GAEC
'Baldwin' [P. parviflora Sieb. \& Zucc.] DCNA

banksiana Lamb.

Jack P. TNMB TNUT

'Beuvronensis' [P. sylvestris L.] DCNA

brutia Tenore DCNA

bungeana Zucc. ex Endl.

Lacebark P.

DCNA MDBA VABF

GAUG TNRM

cembra L.

Swiss Stone P

DCNA MDBG MDPJ

cembra L. var. sibirica Loud. DCNA

cembroides Zucc.

Mexican Stone P.
DCNA
VABF

clausa (Chapm. ex Engelm.) Vasey ex Sarg.

Sand P.

ALAU FLFB FLPL FLUF

'Compact Gem' [P. leucodermis Ant.] DCNA

'Compacta Glauca' [P. cembra L.] DCNA

'Contorta' [P. strobus L.] MDBG

'Corticosa' [P. thunbergiana Franco] DCNA

densiflora Sieb. \& Zucc.

Japanese Red P.

$\begin{array}{lll}\text { ALTD } & \text { FLHJ } & \text { GAFN } \\ \text { DCNA } & \text { FLUF } & \text { VANB }\end{array}$

'Dwarf' [P. strobus L.]

GAJS

'Dwarf Blue' [P. pumila (Pall.) Regel] DCNA

echinata Mill.

Shortleaf P.

$\begin{array}{llll}\text { DCCG } & \text { FLMG } & \text { GAIS } & \text { SCFW } \\ \text { DCNC } & \text { GACE } & \text { NCBM } & \text { TNFL }\end{array}$

'Elf' [P. strobus L.]

DCNA 
elliottii Engelm.

Slash P.

$$
\text { ALAU FLFB }
$$

excelsa Wall. ex D.Don

$=P$. wallichiana

'Fastigiata' [P. strobus L.] GAEC MDBG MDJS VABF

'Fastigiata' [P. sylvestris L.] DCNA MDBG

flexilis James

Limber P.

$$
\text { DCCG DCNA MDBG }
$$

glabra Walt.

Spruce P.
ALEH
LAMP
SCFW
FLMG
LASL
SCMP
FLUF
NCCA
TXPS

'Glauca' [P. parviflora Sieb. \& Zucc.] DCNA

'Glauca Nana' [P. parviflora Sieb. \& Zucc.] DCNA

'Glenmore' [P. flexilis James] DCNA

'Globosa' [P. nigra Arn.] DCNA

'Globosa Viridis' [P. sylvestris L.] DCNA

'Gnom' [P. mugo Turra var. mughus (Scop.)

Zenari] DCNA

greggii Engelm. \& Parl. TXMA

griffithii McClelland

$=P$. wallichiana

halepensis Mill.

Aleppo P. TXAO

heldreichii Christ var. leucodermis (Ant.)

Markgr. ex Fitschen

$=P$. leucodermis

'Hornibrookiana' [P. nigra Arn.] DCNA

'Kokonoe' [P. parviflora Sieb. \& Zucc.] DCNA koraiensis Sieb. \& Zucc.

Korean P.

DCNA MDDF MDJS

leucodermis Ant.

DCNA VABF

longifolia Roxb.

$=$ P. roxburghii

'Merrimack' [P. strobus L.] DCNA

'Monstrosa' [P. nigra Arn.] GAEC

'Mops' [P. mugo Turra var. mughus (Scop.) Zenari]

DCNA

'Moseri' [P. sylvestris L.] DCNA

mugo Turra var. mugo

Swiss Mountain P.

$\begin{array}{lll}\text { ALTD } & \text { DEWG } & \text { MDLT } \\ \text { DCLC } & \text { GAFN } & \text { TNHD } \\ \text { DCNA } & \text { MDBG } & \text { VABF }\end{array}$

mugo Turra var. pumilio (Haenke) Zenari MDBG

'Nana' [P. nigra Arn.]

(Nana group)

DCNA GAEC MDBG MDLT

'Nana' [P. strobus L.]

DCNA MDBG MDLT

nepalensis Chambr.

$=\boldsymbol{P}$. wallichiana

nigra Arn. ssp. nigra

Austrian P.

$\begin{array}{llll}\text { DCLC } & \text { MDHN } & \text { SCCU } & \text { VAMP } \\ \text { MDAF } & \text { MDLA } & \text { VABF } & \text { VATW } \\ \text { MDCP } & \text { MDSJ } & \text { VAHN } & \text { VAWR }\end{array}$

'Oculus-draconis' [P. densiflora Sieb. \& Zucc.]

$$
\begin{array}{lll}
\text { DCNA } & \text { MDBG } & \text { TNTV } \\
\text { GAJS } & \text { MDTD } &
\end{array}
$$

palustris Mill.

Longleaf $\mathrm{P}$.

$$
\begin{array}{lll}
\text { ALSM } & \text { NCGP } & \text { TNBM } \\
\text { NCBE } & \text { NCWK } &
\end{array}
$$

parviflora Sieb. \& Zucc.

Japanese White P.
DCNA
MDJS
DEWG MDLT SCMG VAMP
MDTD
TNTV 
patula Schiede \& Deppe

Jelecote P.

$\mathrm{SCCU}$

'Pendula' [P. densiflora Sieb. \& Zucc.]

Weeping Japanese Red P.
DCNA MDJS
MDBG MDLT

'Pendula' [P. ponderosa Dougl. ex P.\& C.Laws.] DCNA

'Pendula' [P. strobus L.] DCNA MDBG MDLT

pinea L.

Italian Stone P., Umbrella P. LAMP

ponderosa Dougl. ex P.\& C.Laws Western Yellow P., Ponderosa P. $\mathrm{VABF}$

pumila (Pall.) Regel DCNA $\underset{\text { DCNA }}{\text { 'Pumila' [P. strobus L.] }}$

pungens Lamb.

Table-mountain $\mathrm{P}$. DCNA SCCU VABF

'Radiata' [P. strobus L.] DCNA

'Repens' [P. sylvestris L.] DCNA

resinosa Ait.

Red P. DCCG DCNA TNSC VATG

rigida Mill.

Pitch P. MDDF

roxburghii Sarg.

Long-leaved Indian P., Chir P. TXHJ

sabiniana Dougl. ex D.Don

Digger $\mathrm{P}$. $\mathrm{VABF}$

'Seacrest' [P. strobus L.] DCNA

'Sentinel' [P. sylvestris L.] DCNA serotina Michx.

Pond $\mathrm{P}$

ALAU GACL

'Spaan's Dwarf [P. contorta Dougl. ex Loud.]

DCNA

strobus L.

Eastern White P.

$\begin{array}{llll}\text { ALIT } & \text { MDHN } & \text { NCOP } & \text { VAGW } \\ \text { DCNA } & \text { MDJS } & \text { SCAK } & \text { VAMP } \\ \text { GAOH } & \text { MDLT } & \text { SCCU } & \\ \text { GAUG } & \text { NCCA } & \text { TNBM } & \\ \text { MDBG } & \text { NCKH } & \text { TNHG } & \end{array}$

strobus $\times P$. wallichiana

DCNA

sylvestris $\mathrm{L}$.

Scotch P.

$\begin{array}{lll}\text { DCNA } & \text { NCCM } & \text { TNSC } \\ \text { GAUG } & \text { SCCU } & \text { VABF }\end{array}$

taeda L.

Loblolly P.

$\begin{array}{llll}\text { DCWH } & \text { GAIS } & \text { MDBG } & \text { SCMG } \\ \text { FLMG } & \text { LAAL } & \text { NCGP } & \text { TNRM }\end{array}$

'Tanoyosho' [P. densiflora Sieb. \& Zucc.] $=P$. densiflora 'Umbraculifera'

thunbergiana Franco

Japanese Black P.

$\begin{array}{llll}\text { ALTD } & \text { DCNA } & \text { MDKN } & \text { TNCT } \\ \text { DCCG } & \text { LAMP } & \text { MDLT } & \text { VABF } \\ \text { DCFP } & \text { MDBG } & \text { NCBE } & \text { VATA }\end{array}$

thunbergii Parl.

$=P$. thunbergiana

\section{'Torulosa' [P. strobus L.] DCNA}

'Umbraculifera' [P. densiflora Sieb. \& Zucc.]

Japanese Umbrella P.

$\begin{array}{llll}\text { ALOS } & \text { DCNA } & \text { MDJS } & \text { TNSC } \\ \text { DCCG } & \text { MDBG } & \text { MDLT } & \text { VATS }\end{array}$

'Venus' [P. parviflora Sieb. \& Zucc.] DCNA

virginiana Mill.

Virginia P., Scrub P.

$\begin{array}{llll}\text { ALAU } & \text { GAJS } & \text { NCWK } & \text { VAGW } \\ \text { DCNA } & \text { GAUG } & \text { SCCU } & \\ \text { GACE } & \text { MDBG } & \text { TNSC } & \\ \text { GAEC } & \text { NCCA } & \text { VABF } & \end{array}$

wallichiana A.B.Jacks.

Himalayan P.

MDBG VAMP 
'Watnong' [P. parviflora Sieb. \& Zucc.] DCNA

'Yatsubusa' [P. thunbergiana Franco] DCNA

'Zebrina' [P. wallichiana A.B.Jacks.] DCNA

PISTACIA L. Pistache ANACARDIACEAE Cashew Family

chinensis Bunge

Chinese P.

$\begin{array}{llll}\text { ALBT } & \text { GAIS } & \text { SCEM } & \text { VAHM } \\ \text { ALTD } & \text { LAAL } & \text { SCRP } & \text { VAMP } \\ \text { DCNA } & \text { LASL } & \text { TNSG } & \\ \text { FLH } & \text { MDGD } & \text { TXMS } & \\ \text { FLUF } & \text { MSMN } & \text { VABF } & \end{array}$

texana Swingle

Texas $\mathrm{P}$.

TXLL TXMS

PITHECELLOBIUM Mart.

FABACEAE (Mimosoideae) Bean Family

ebano (Berl.) Muller

Texas Ebony

TXRE

flexicaule (Benth.) Coult.

$=P$. ebano

PITHECOLOBIUM Benth.

\section{= PITHECELLOBIUM}

PITTOSPORUM Banks ex Sol.

\section{PITTOSPORACEAE Pittosporum Family}

glabratum Lindl. LAAL

tobira (Willd.) Ait

Japanese P.

$\begin{array}{llll}\text { DCNA } & \text { LAAL } & \text { SCHW } & \text { VANB } \\ \text { FLMG } & \text { LARP } & \text { SCMP } & \text { VARR } \\ \text { FLUF } & \text { LASL } & \text { SCUC } & \\ \text { GAHC } & \text { NCGP } & \text { TXSH } & \\ \text { GAJI } & \text { SCBR } & \text { VAMF } & \end{array}$

undulatum Vent.

Victorian Box

FLUF _ LAAL
'Variegata' [P. tobira (Willd.) Ait.]

FLMG LALG SCBR SCJM

GACH MSEN SCCU TXSH

'Wheeler's Dwarf' [P. tobira (Willd.) Ait.] FLMG FLUF

PLANERA J.F.Gmel.

ULMACEAE

Elm Family

aquatica (Walt.) J.F.Gmel.

Water Elm

SCMP

PLATANUS L.

Sycamore

PLATANACEAE

Plane-tree Family

$\times$ acerifolia (Ait.) Willd.

[P. occidentalis $\times \boldsymbol{P}$. orientalis]

London Plane

$\begin{array}{llll}\text { DCCG } & \text { MDHN } & \text { NCBE } & \text { VASC }\end{array}$

DEMC MDJH TNUT

$\star$ 'Columbia' [P. $\times$ acerifolia (Ait.) Willd.]

[P. occidentalis $\times P$. orientalis] DCNA

$\times$ hispanica Muenchh.

= $\boldsymbol{P}$. $\times$ acerifolia

$\star$ 'Liberty' [P. $\times$ acerifolia (Ait.) Willd.]

$[P$. occidentalis $\times P$. orientalis $]$

DCNA FLLT

occidentalis $\mathrm{L}$.

American S.

DCCG FLUF MDHN TNUT

orientalis $\mathrm{L}$.

Oriental Plane

DCNA FLUF

PLATYCARYA Sieb. \& Zucc.

JUGLANDACEAE

Walnut Family

strobilacea Sieb. \& Zucc. TNLW VABF

PLATYCLADUS Spach

CUPRESSACEAE Cypress Family

'Aurea Nana' [P. orientalis (L.) Franco]

ALFN FLCG GABC LASL

DCNA FLEM GAFN SCMG 
'Bakeri' [P. orientalis (L.) Franco] ALAU ALFN SCMG

'Berckman's Golden' [P. orientalis (L.)

Franco] ALAU

'Beverleyensis' [P. orientalis (L.) Franco] DCNA LAAL

'Conspicua' [P. orientalis (L.) Franco] DCNA SCCU

'Flagelliformis' [P. orientalis (L.) Franco] GAGC

'Fruitlandii' [P. orientalis (L.) Franco] ALFN

'Hohman' [P. orientalis (L.) Franco] ALBH

'Juniperoides' [P. orientalis (L.) Franco] DCNA

orientalis (L.) Franco

Oriental Arborvitae

$\begin{array}{llll}\text { ALSH } & \text { FLUF } & \text { MDJS } & \text { SCRH } \\ \text { ALUA } & \text { GAJI } & \text { MDLA } & \text { TNUT } \\ \text { DCFP } & \text { GASU } & \text { NCCA } & \\ \text { FLDG } & \text { LAAL } & \text { SCBS } & \\ \text { FLMG } & \text { MDCP } & \text { SCMG } & \end{array}$

'Rosedalis' [P. orientalis (L.) Franco] MDBG

'Semperaurea' [P. orientalis (L.) Franco] GAJI

PLUMBAGO L.

PLUMBAGINACEAE Leadwort Family

auriculata Lam.

Cape Leadwort

$\begin{array}{lll}\text { ALBG } & \text { GACG } & \text { LALG } \\ \text { FLUF } & \text { LAAL } & \text { SCHW }\end{array}$

capensis Thunb.

= P. auriculata

PODOCARPUS L'Her. ex Pers.

PODOCARPACEAE Podocarpus Family

gracilior Pilger

African Fern Pine

FLUF macrophyllus (Thunb. ex J.A.Murr.) Sweet Yew P.

$\begin{array}{lll}\text { ALBG } & \text { LAAL } & \text { MSMN } \\ \text { FLMG } & \text { LASL } & \text { NCCA } \\ \text { FLUF } & \text { MSHB } & \text { SCMG }\end{array}$

macrophyllus (Thunb. ex J.A.Murr.) Sweet var. maki Sieb.

$\begin{array}{llll}\text { ALBG } & \text { FLUF } & \text { LALG } & \text { NCDU } \\ \text { ALON } & \text { GAHC } & \text { LARP } & \text { SCBR } \\ \text { FLDC } & \text { GAIS } & \text { LASL } & \text { SCMG } \\ \text { FLDG } & \text { GAJI } & \text { MSMN } & \text { SCWI } \\ \text { FLMG } & \text { GATS } & \text { NCCA } & \end{array}$

nagi (Thunb. ex J.A.Murr.) Makino

Broadleaf P.

$\begin{array}{lll}\text { FLRP } & \text { GACH } & \text { LAGN } \\ \text { FLUF } & \text { LAAL } & \text { TXHA }\end{array}$

totara D.Don

Totara Pine

ALEH

POINCIANA L.

= CAESALPINIA

POLIOTHYRSIS Oliv.

FLACOURTIACEAE

Flacourtia Family

sinensis Oliv.

DCNA VAGS

POLYGONELLA MichX.

POLYGONACEAE Buckwheat Family

americana (Fischer \& C.A.Mey.) Small SCBR SCWI

myriophylla (Small) Horton SCWI

polygama (Vent.) Engelm. \& A.Gr. SCWI

PONCIRUS Raf.

RUTACEAE

Citrus Family

trifoliata (L.) Raf.

Hardy Orange

$\begin{array}{llll}\text { ALTD } & \text { FLUF } & \text { MDLA } & \text { VAMP } \\ \text { DCCG } & \text { GAHC } & \text { SCCU } & \text { VAPH } \\ \text { DCWH } & \text { GAIS } & \text { VACW } & \text { VATA } \\ \text { FLMG } & \text { MDHN } & \text { VAGW } & \end{array}$


POPULUS L.

Poplar

SALICACEAE

Willow Family

alba L.

White P

$\begin{array}{lll}\text { ALTC } & \text { NCDU } & \text { VACW } \\ \text { MDHN } & \text { TNFR } & \text { VASP }\end{array}$

alba L. var. bolleana (Lauche) Otto

$=$ 'Pyramidalis'

balsamifera L. var. candicans (Ait.) A.Gr.

$=P$. candicans

balsamifera L. var. subcordata Hylander

$=\boldsymbol{P}$. candicans

bolleana Lauche

= 'Pyramidalis'

$\times$ canadensis Moench

Carolina P.

[P. deltoides $\times P$. nigra]

VACP

candicans Ait.

Balm-of-Gilead NCCA VAGW

$\times$ canescens (Ait.) Sm.

Gray P.

[P. alba $\times$ P. tremula]

MDPJ VAGW VASP

MDWP VARB

deltoides Marsh.

Cottonwood

$\begin{array}{lll}\text { DCCG } & \text { GAUG } & \text { TXWH } \\ \text { FLUF } & \text { MSMN } & \text { VASC }\end{array}$

'Fastigiata' [P. simonii Carr.] SCCU

$\times$ gileadensis Rouleau

$=P$. candicans

grandidentata Michx.

Large-toothed Aspen

VAWP

heterophylla L.

Swamp Cottonwood SCBR VACW

'Italica' [P. nigra L.]

Lombardy P.
ALBH
GAVI
TNBO
VASP

maximowiczii A.Henry

Japanese P. VAGG

maximowiczii $\times P$. trichocarpa VATA

'Pyramidalis' [P. alba L.]

GABS MDNA TNSC

GAUG NCWT

tremuloides Michx.

Quaking Aspen

DCNA VASP

PORLIERIA Ruiz \& Pavon

ZYGOPHYLLACEAE

Caltrop Family

angustifolia (Engelm.) A.Gr.

Texas $\mathrm{P}$

LASL

POTENTILLA L.

Cinquefoil

ROSACEAE

Rose Family

'Abbotswood' [P. fruticosa L.]

VAGS

'Everest' [P. fruticosa L.] MDWP

fruticosa L.

Shrubby C.

MDBG MDJS VAGS

'Grandiflora' [P. fruticosa L.] MDKN

'Jackman's' [P. fruticosa L. VATA

'Katherine Dykes' [P. fruticosa L.] MDKN

'Maanelys' [P. fruticosa L.] MDKN VATA

'Moonlight' [P. fruticosa L.]

$=$ 'Maanelys'

'Primrose Beauty' [P. fruticosa L.] MDKN

'Purdomii' [P. fruticosa L.]

= 'William Purdom'

'Sophie Blush'

DCNA 
tridentata Ait.

DCNA

'William Purdom' [P. fruticosa L.]

DEMC MDKN

\section{PRINSEPIA Royle}

ROSACEAE

Rose Family

sinensis (Oliv.) Oliv.

DEWG

PROSOPIS L.

FABACEAE (Mimosoideae) Bean Family

glandulosa Torr. var. torreyana (L.Benson)

M.C.Johnst.

Western Honey Mesquite

TXGS

PRUNUS L.

ROSACEAE

Rose Family

'Accolade' [P. sargentii $\times$ P. $\times$ subhirtella] DEWG

'Akebono' [P. $\times$ yedoensis Matsum.]

[Parentage unknown]
DCNA
DCTB
DCWH
DCWM

'Alba' [P. mume Sieb. \& Zucc.] MDBG

'Alba' [P. persica (L.) Batsch] NCOP

'Albo-plena' [P. glandulosa Thunb.] ALIT NCEG TNDG VAPH

'Albo-plena' [P. persica (L.) Batsch] DCNA NCEG VACW LAAP SCLM

'Amanogawa' [P. serrulata Lindl.] DCNA

americana Marsh.

Wild Plum VASK

amygdalus Batsch

= P. dulcis angustifolia Marsh.

Chickasaw Plum

LATU NCDF

apetala (Sieb. \& Zucc.) Franch. \& Sav. DCNA

'Ariake'

(Sato zakura group) DCPP

armeniaca L.

Apricot

MDPD NCBE VACW VAJL

'Asagi'

(Sato zakura group)

MDGD

'Atropurpurea' [P. cerasifera Ehrh.]

Purple-leaf Plum

$\begin{array}{lll}\text { ALBG } & \text { FLHP } & \text { SCUC } \\ \text { ALSH } & \text { LAMP } & \text { VACW }\end{array}$

'Autumnalis' [P. $\times$ subhirtella Lindl.]

[Parentage unknown]

DCCG GAEC MDLT

DCNA MDBG NCEL

DCWH MDKN VANB

avium L.

Sweet Cherry

$\begin{array}{lll}\text { DCCC } & \text { MDHN } & \text { VACW } \\ \text { MDHC } & \text { MDJH } & \text { VASP }\end{array}$

'Benden'

(Sato zakura group)

MDGD

'Beni fugen'

= 'Fugenzo'

$\times$ blireiana Andre

[P. cerasifera 'Atropurpurea' $\times \boldsymbol{P}$. mume] DCDO LATU

'Botan zakura'

(Sato zakura group) MDKN

'Camelliifolia' [P. laurocerasus L.] ALFN

'Camelliifolia' [P. persica (L.) Batsch] DCCG

campanulata Maxim.

Taiwan Cherry

ALCW ALEH FLMG GATS


caroliniana Ait.

Carolina Cherry Laurel

$\begin{array}{llll}\text { ALAU } & \text { FLLE } & \text { MSBN } & \text { TNDR } \\ \text { ALBG } & \text { FLPM } & \text { NCDU } & \text { TNRM } \\ \text { ALEH } & \text { FLUF } & \text { NCTP } & \text { TNUT } \\ \text { ALIT } & \text { GAHC } & \text { SCCU } & \text { VACW } \\ \text { ARRB } & \text { GARW } & \text { SCMP } & \text { VASC } \\ \text { DCWH } & \text { LAHG } & \text { SCUC } & \text { VAWR } \\ \text { FLCG } & \text { LASL } & \text { SCWI } & \end{array}$

cerasifera Ehrh. MDBG MDGD

cerasoides D.Don var. campanulata (Maxim.) Koidz.

= $P$. campanulata

$\times$ cistena N.E.Hansen

$[P$. cerasifera 'Atropurpurea' $\times P$. pumilal MDBG

conradinae Koehne MDGD

cyclamina Koehne DCNA

'Daikaku' [P. serrulata Lindl.] DCNA

'Dianthiflora' [P. persica (L.) Batsch] FLHP GADG SCHW

domestica L. ssp. insititia (L.) Schneid. Damson Plum VACW

'Dr. S. Edwin Mueller' [P. sargentii Rehd.] MDKN

dulcis (Mill.) D.A.Webb

Almond VACW

'Duplex' [P. persica (L.) Batsch] NCOP

'Elizabeth' [P. $\times$ subhirtella Miq.]

[Parentage unknown] MDKN

'Fastigiata' [P. persica (L.) Batsch] VACW

'February Pink' [P. incisa Thunb. ex

J.A.Murr.] MDGD

'Flore-plena' [P. $\times$ subhirtella Miq. var. ascendens (Makino) Wils.]

[Parentage unknown] MDGD
'Forest Green' [P. laurocerasus L.] DCNA

'Fudan sakura' [P. serrulata Lindl.] DCNA

'Fugenzo'

(Sato zakura group) MDGD MDKN

'Fuku rokuju' [P. serrulata Lindl.] MDKN

glandulosa Thunb. ex J.A.Murr.

Flowering Almond LAHP LASL MSMV SCCU

'Gozanoma nioi' [P. serrulata Lindl.] MDKN

'Grandiflorus' [P. padus L.] DCNA

'Gyoiko'

(Sato zakura group) MDGD

'Hally Jolivette' [ $(P . \times$ subhirtella $\times$

P. $\times$ yedoensis) $\times$ P. $\times$ subhirtella] DCNA DEWG VAPH DCWH MDKN

'Hatazakura'

(Sato zakura group) MDGD MDKN

$\times$ hillieri (listed name with no standing)

= 'Spire'

\section{'Hizakura'}

(Sato zakura group) MDKN

'Hosokawa' [P. serrulata Lindl.] DCTB MDGD

humilis Bunge NCEL

'Imose' [P. serrulata Lindl.] MDGD

incisa Thunb. ex J.A.Murr. DCNA VAPH

incisa Thunb. ex J.A.Murr. f. serrata Koidz. ex Wils. MDGD

incisa Thunb. ex J.A.Murr. var. tomentosa Koidz. DCNA 
japonica Thunb. ex J.A.Murr.

Oriental Bushcherry

ALEH

'Jugatsu zakura' [P. × subhirtella Miq.]

= 'Autumnalis'

'Kiku shidare' [P. serrulata Lindl.] MDKN

'Kursar'

(P. nipponica var. kurilensis (Miyabe) Wils. $\times$ unknown pollen parent) MDGD

'Kwanzan' [P. serrulata Lindl.]

Kwanzan Cherry

$\begin{array}{llll}\text { DCCG } & \text { GACG } & \text { MDBG } & \text { TNCT } \\ \text { FLMG } & \text { GARW } & \text { NCTP } & \end{array}$

laurocerasus L.

Cherry Laurel, English Laurel

$\begin{array}{llll}\text { DCCG } & \text { GAUG } & \text { NCWC } & \text { VACM } \\ \text { FLMG } & \text { MDWP } & \text { SCDB } & \text { VAHC } \\ \text { GACC } & \text { NCBM } & \text { SCFW } & \text { VAMP } \\ \text { GAHC } & \text { NCOP } & \text { TNAB } & \end{array}$

laurocerasus L. var. schipkaensis Spaeth Carpathian Cherry Laurel

$\begin{array}{llll}\text { DCCG } & \text { GAUG } & \text { NCWT } & \text { VAGS } \\ \text { DEEM } & \text { MDMM } & \text { TNCT } & \text { VATA } \\ \text { GACG } & \text { MDWP } & \text { TNUT } & \text { VATW }\end{array}$

lusitanica L.

Portuguese Cherry Laurel DCNA GARW

mahaleb L.

Mahaleb Cherry ALBH

maritima Marsh.

Beach Plum MDGD VAGW

'Matsubara Red' [P. mume Sieb. \& Zucc.] MDBG

'Meigetsu'

(Sato zakura group) MDKN

'Methley' [P. maritima Marsh.] ALEH

mexicana S.Wats.

Mexican Plum

FLUF LAHG MSEN TXMA

'Montmorency' [P. cerasus L.]

Pie Cherry

MDWP
'Moseri' [P. cerasifera Ehrh.] GAFN

'Mt. Fuji' [P. serrulata Lindl.] DCPP MDLT

'Multiplex' [P. triloba Lindl.]

Double-flowered Flowering Almond DEWG NCGM VAGW

mume Sieb. \& Zucc.

Japanese Apricot

$\begin{array}{llll}\text { DCNA } & \text { GAIS } & \text { MDMW } & \text { NCOP } \\ \text { GAHC } & \text { MDGD } & \text { NCDU } & \text { SCDB }\end{array}$

munsoniana W.F.Wight \& Hedr.

Wild Goose Plum FLUF NCCA TNBO

'Nishiki ume' [P. mume Sieb. \& Zucc.] MDLT

'Ojochin'

(Sato zakura group) MDKN

'Okami' [P. $\times$ incam Fletcher]

$[P$. incisa $\times P$. campanulata] DCNA

'Oshima zakura' [P. serrulata Lindl.] MDGD

'Otto Luyken' [P. laurocerasus L.] GACG MDBG MDLT

padus $\mathrm{L}$.

Bird Cherry

GARW

'Pandora' [P. $\times$ subhirtella $\times$

$P$. $\times$ yedoensis] MDGD

'Peggy Clarke' [P. mume Sieb. \& Zucc.] DCNA

'Pendula' [P. X subhirtella Miq.]

= 'Shidare higan'

persica (L.) Batsch

Peach DCCG DCNA GAGM VACW

persica (L.) Batsch var. nectarina (Ait.) Maxim.

$=P$. persica var. nucipersica

'Pink Perfection' [P. serrulata Lindl.] MDGD

pissardii Carr.

= 'Atropurpurea' [P. cerasifera] 
PRUNUS

'Plena' [P. avium L.]

DCNA

'Purpusii' [P. cerasifera Ehrh.] MDGD

'Rancho' [P. sargentii Rehd.] DCNA

rivularis Scheele

Creek Plum TXLL

'Rosea' [P. serrulata Lindl.] MDGD

'Rosea' [P. $\times$ subhirtella Miq.]

[Parentage unknown] MDGD

'Rosea Plena' [P. glandulosa]

$=$ 'Sinensis'

'Rosemary Clarke' [P. mume Sieb. \& Zucc.] DCNA

'Rubro-plena' [P. persica (L.) Batsch] NCOP

sargentii Rehd.

Sargent Cherry DCNA MDKN MDLT NCDU

'Schubert' [P. virginiana L.] DCNA

'Sekiyama' [P. serrulata Lindl.]

= 'Kwanzan'

'Senriko'

(Sato zakura group) MDGD MDKN

serotina Ehrh.

Black Cherry

$\begin{array}{llll}\text { ALON } & \text { LAAL } & \text { MSBN } & \text { TNMB } \\ \text { DCNA } & \text { MDCP } & \text { TNHG } & \text { VAPO } \\ \text { FLLE } & \text { MDLT } & \text { TNHS } & \text { VASJ }\end{array}$

serrula Franch.

MDKA

serrulata Lindl.

Japanese Flowering Cherry
FLMG
MDGD

serrulata Lindl. var. spontanea (Maxim.)

Wils.

DCNA
'Shidare higan' [P. $\times$ subhirtella Lindl.]

Weeping Higan Cherry

[Parentage unknown]

$\begin{array}{llll}\text { DCCG } & \text { DEMC } & \text { MDGD } & \text { MDPJ } \\ \text { DCNA } & \text { MDBG } & \text { MDKN } & \text { NCWC } \\ \text { DCPP } & \text { MDDF } & \text { MDLT } & \end{array}$

'Shidare yoshino' [P. $\times$ yedoensis Matsum.]

[Parentage unknown] MDGD

'Shirofugen' [P. serrulata Lindl.]

(Sato zakura group)

DCNA MDGD NCOP

'Shirotae' [P. serrulata Lindl.]

(Sato zakura group) DCMA MDKN NCRW

'Shogetsu'

(Sato zakura group) MDGD

$\times$ sieboldii (Carr.) Wittmack

Takasago Cherry

$[\boldsymbol{P}$. apetala $\times \boldsymbol{P}$. serrulata $]$ DCNA

'Sinensis' [P. glandulosa Thunb.] ALSM DEWG NCOP VAPH

'Snow Fountain' DCNA

'Somei yoshino' [P. $\times$ yedoensis Matsum.] Yoshino Cherry

[Parentage unknown]

$$
\begin{array}{lll}
\text { DCCG } & \text { MDDF } & \text { MDLT } \\
\text { DCTB } & \text { MDGD } &
\end{array}
$$

'Spire' $[\boldsymbol{P}$. sargentii $\times \boldsymbol{P}$. ?incisa] MDGD

$\times$ subhirtella Miq.

Higan Cherry

(Alleged hybrid of unknown origin) DCCG DCNA SCCU

syodoi Nakai

DCNA

'Tai haku' [P. serrulata Lindl.] MDGD

'Takasago'

$=P$. sieboldii

'Taki nioi'

(Sato zakura group) DCPP 
'Thundercloud' [P. cerasifera Ehrh.] MDBG SCCU VATA

tomentosa Thunb. ex J.A.Murr.

Nanking Cherry

DEMC MDLT VACW

DEWG MDOF

triloba Lindl.

Flowering Almond DEWG

umbellata Ell.

Black-sloe Plum FLMG GAGM

'Umineko' [P. incisa $\times \boldsymbol{P}$. serrulata] MDGD

verecunda (Koidz.) Koehne DCNA

'Versicolor' [P. persica (L.) Batsch] NCOP

'Wase mikayo' [P. serrulata Lindl.] MDKN

'W. B. Clarke' [P. mume Sieb. \& Zucc.] MDBG

'White Glory' [P. persica (L.) Batsch var. nucipersica (Borkh.) Schneid.]

Nectarine NCNS

'Yae akebono'

(Sato zakura group) MDKN

'Yae murasaki zakura'

(Yato zakura group) MDKN

'Yae shidare higan' [P. $\times$ subhirtella Lindl.] [Parentage unknown] DCCC MDGD MDLT MDNH

'Yama zakura' [P. serrulata Lindl.] MDGD

$\times$ yedoensis Matsum.

Tokyo Cherry

(Alleged hybrid of unknown origin) ALON DCPP GAIH

'Yoshino'

= 'Somei yoshino'

'Zabeliana' [P. laurocerasus L.] DCNA
PSEUDOCYDONIA (Schneid.) Schneid. ROSACEAE Rose Family

sinensis (Dum.-Cours.) Thouin

Chinese Quince

$\begin{array}{lll}\text { DCNA } & \text { LASL } & \text { NCEL } \\ \text { FLDG } & \text { MDBG } & \text { VAGG } \\ \text { LAAL } & \text { MDJH } & \text { VATA }\end{array}$

PSEUDOLARIX Gord. PINACEAE

Pine Family

amabilis (J.Nelson) Rehd.

Golden Larch

DCNA MDPJ VAMP

PSEUDOSASA Makino ex Nakai

POACEAE

Grass Family

japonica Sieb. \& Zucc. ex Steud.

Metake
ALON
DCMC
LALG
MDFM

PSEUDOTSUGA Carr. Douglas Fir PINACEAE Pine Family

'Densa' [P. menziesii (Mirb.) Franco

var. glauca (Beissn.) Franco] DCNA

'Fastigiata' [P. menziesii (Mirb.) Franco var. glauca (Beissn.) Franco] DCNA

'Glauca Pendula' [P. menziesii (Mirb.) Franco var. glauca (Beissn.) Franco]
DCNA
VAAC

menziesii (Mirb.) Franco var. glauca (Beissn.) Franco

Rocky Mountain Douglas Fir
DCCG
GAEC
MDBG
SCUC
DCNA
GATN
MDNA
VAAC
DEMC
GAUG
$\mathrm{NCKH}$
VAMP

'Oudemansii' [P. menziesii (Mirb.) Franco var. glauca (Beissn.) Franco]

DCNA 


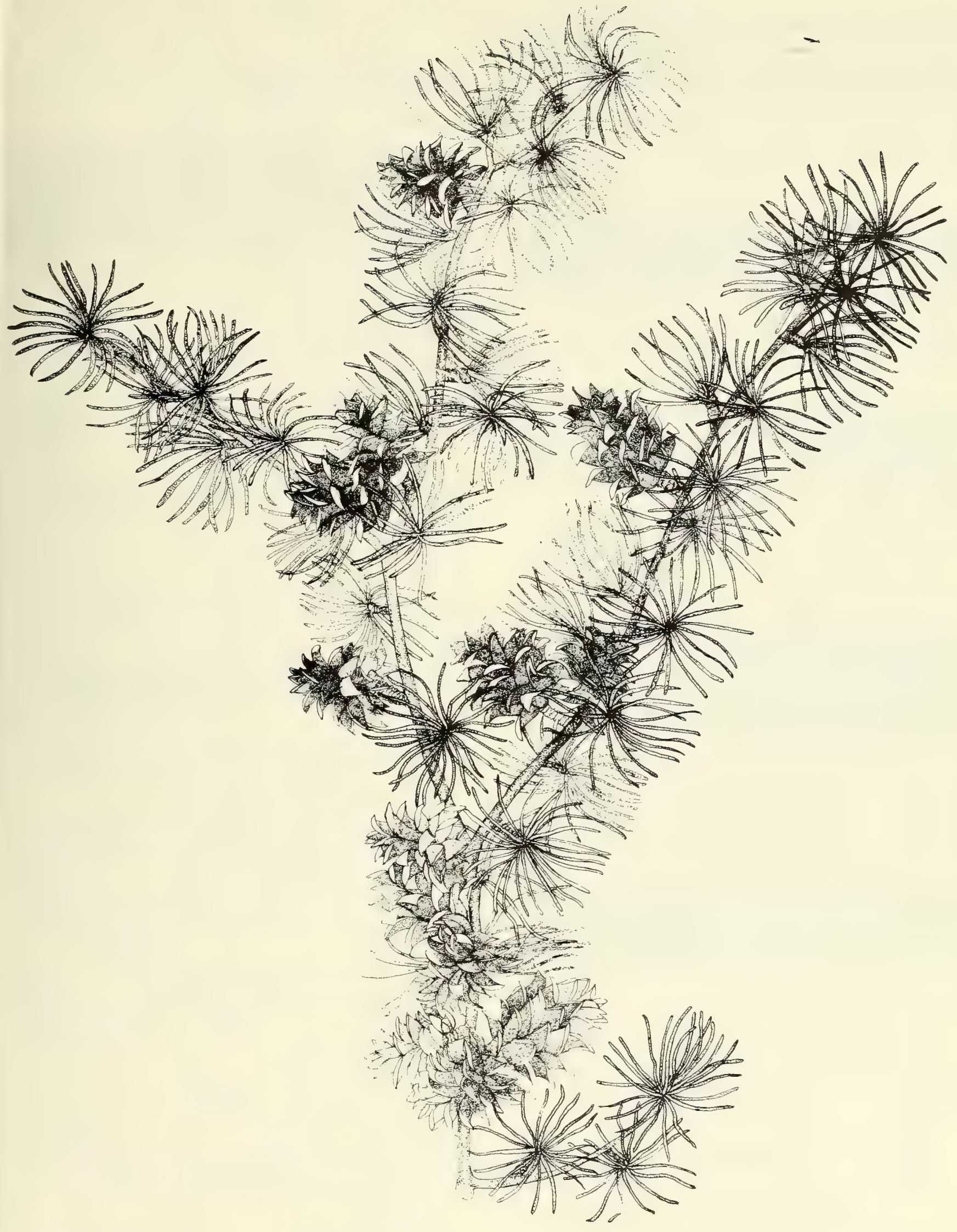

PSEUDOLARUX amabilis (J. Nelson) Rehd. [illustrator Lillian Nicholson Meyer] 
PSIDIUM L.

Purple Guava MTRTACEAE Myrtle Family

littorale Raddi var. longipes (O. Berg)

Fosberg

Purple Guava FLUF LAAL

PTELEA L. RUTACEAE

trifoliata L.

Wafer Ash DCWH NCBE VACW VAGG

PTEROCARYA Kunth JUGLANDACEAE Walnut Family

stenoptera DC.

Chinese W. $\begin{array}{llll}\text { DCNA } & \text { GANG } & \text { TNSG } & \text { VACW } \\ \text { GAIS } & \text { NCDS } & \text { TXMA } & \end{array}$

PTEROSTYRAX Sieb. \& Zucc.

STYRACACEAE Epaulette Tree Storax Family

corymbosum Sieb. \& Zucc. MDGJ

Hop Tree Citrus Family

hispidum Sieb. \& Zucc.

DCNA DEWG TNTV VAGS

PUNICA L.

PUNICACEAE Pomegranate Family

granatum L.

Pomegranate

$\begin{array}{lll}\text { DCWA } & \text { LAMP } & \text { SCKG } \\ \text { FLCG } & \text { MSMN } & \text { VAGS }\end{array}$

granatum L. var. nana (L.) Pers.

$=$ 'Nana'

'Legrellei' [P. granatum L.] $\begin{array}{lll}\text { LAHG } & \text { SCWC VAFN } \\ \text { MSEP } & \text { VABR }\end{array}$

'Mme. Legrelle' [P. granatum L.]

= 'Legrellei'
'Nana' [P. granatum L.]

Dwarf Pomegranate GAUG LASL SCCU VABF

'Plena' [P. granatum L.] GAUG LAMP VAGS

\section{PYRACANTHA M.J.Roem. Firethorn ROSACEAE Rose Family}

DCNA GAIS N NCWK
NChneid.

$\star$ 'Apache'

$[P$. koidzumii 'Victory' $\times(P$. koidzumii 'Rosedale' $\times$ P. fortuneana 'Orange Glow')] DCNA

atalantioides (Hance) Stapf DCNA DCSH GAIS

'Aurea' [P. atalantioides (Hance) Stapf] VATA

'Bad Zwischenahn' DCNA

'Baker'

DCNA

'Belli' [P. koidzumii (Hayata) Rehd.] DCNA

'Bloss'

DCNA

'Bound' [P. coccinea Roem.] DCNA

'Brilliant'

DCNA

'Cal Poly' DCNA

'Chadwick' [P. coccinea Roem.] DCNA

'Chinese Brocade' DCNA

coccinea Roem. SCCU VAGW

'Cole's Erect' [P. coccinea Roem.] DCNA

'Coplen's Royal' DCNA 
crenatoserrata (Hance) Rehd.

$=\boldsymbol{P}$. fortuneana

crenulata (D.Don) Roem.

DCNA GAIS NCBE SCMP

FLUF MSHB SCMG

crenulata (D.Don) Roem. f. flava

Meunissier

DCNA

crenulata (D.Don) Roem. var. kansuensis

Rehd. DCNA

crenulata (D.Don) Roem. var. rogersiana

A.B.Jacks.

DCNA

'Crimson Tide' [P. koidzumii (Hayata)

Rehd.]

DCNA

'Dauerbrand' [P. coccinea Roem.] DCNA

'Dr. Hook'

DCNA

'Early Red'

DCNA

'Eddie's Coral' $[\boldsymbol{P}$. coccinea $\times \boldsymbol{P}$. crenulata

var. rogersiana]

DCNA

'Flava' [P. crenulata var. rogersiana] DCNA

fortuneana (Maxim.) $\mathrm{Li}$

DCNA GAIS SCMG VACW

FLJU MSHB SCMP

gibbsii Rehd. non A.B.Jacks.

var. yunnanensis Osborn

$=\boldsymbol{P}$. fortuneana

'Gnome' [P. angustifolia (Franch.)

Schneid.]

(Plant Patent No. 1,816)

DCNA

'Gnozam' [P. angustifolia (Franch.)

Schneid.]

= 'Gnome'

'Gold Nugget' [P. coccinea Roem.] DCNA

'Golden Charmer' DCNA
'Golden Queen'

DCNA

'Government Red' [P. koidzumii (Hayata)

Rehd.]

ALBH

'Graberi' [P. fortuneana (Maxim.) Li] DCNA VANB

'Heyden's Bright Yellow' DCNA

'Heyden's Hi Yellow' DCNA

'Ingleside Crimson' DCNA

'Kasan' [P. coccinea Roem.] DCNA

'Keessen' [P. coccinea Roem.] DCNA

'Knap Hill Buttercup' DCNA

koidzumii (Hayata) Rehd.

$\begin{array}{llll}\text { DCNA } & \text { FLUF } & \text { NCOP } & \text { SCKG } \\ \text { FLCG } & \text { GATS } & \text { SCBR } & \\ \text { FLMA } & \text { NCEL } & \text { SCFW } & \end{array}$

'Lalandei' [P. coccinea Roem.] DCNA

'Low Boy' [P. coccinea Roem.] DCNA GACG

'Low Dense' [P. koidzumii (Hayata) Rehd.] DCNA FLUF

'Miller' [P. koidzumii (Hayata) Rehd.] DCNA

'Minute Man' [P. coccinea Roem.] DCNA

'Mioun' DCNA

$\star$ 'Mohave' [P. koidzumii $\times$ P. coccinea 'Wyattii'] DCNA NCDU

'Monon' [P. angustifolia (Franch.) Schneid.] $=$ 'Gnome'

'Monrovia' [P. coccinea Roem.] DCNA 


\section{PYRACANTHA}

'Moonbeam'

DCNA

FLUF VANB

$\star$ 'Navaho' [P. angustifolia $\times$

$P$. 'Watereri']

DCNA

'Orange Giant' [P. coccinea Roem.] DCNA

'Orange Glow' [P. fortuneana (Maxim.) Li] DCNA VATA

\section{'Orange King'}

DCNA

'Pauciflora' [P. coccinea Roem.] DCNA

'Pine Cone' DCNA

'Pinkie' DCNA

'Praecox' [P. coccinea Roem.] DCNA

'Pride of Portsmouth' DCNA

$\star$ 'Pueblo' [P. koidzumii 'Belli' $\times P$. coccinea var. pauciflora] DCNA

'Pyrabox' DCNA

\section{'Red Berry'} DCNA

rogersiana (A.B.Jacks.) Chittenden

$=P$. crenulata var. rogersiana

'Rosedale' [P. koidzumii (Hayata) Rehd.] DCNA

'Runyan' [P. coccinea Roem.] DCNA

'San Jose' [P. koidzumii $\times$ P. fortuneana] DCNA

'Santa Cruz' DCNA

'Schwartz' DCNA

'Select Yellow' DCNA
'Sensation'

DCNA

'Sepers' [P. coccinea Roem.] DCNA

$\star$ 'Shawnee' [P. koidzumii $\times$

$P$. fortuneana] DCNA

'Spring Hill' DCNA

'Stribling' DCNA

'Taliensis' DCNA

$\star$ 'Teton' [P. 'Orangeglow' $\times$ P. 'Flava'] DCNA

'Thornless' [P. coccinea Roem.] DCNA

'Tiny Tim' DCNA

'True Yellow' DCNA

'Variegated' GACG LALG LASL

'Vincent' [P. coccinea Roem.] DCNA

'Walder' DCNA

'Walder Prostrate' DCNA

'Watereri' DCNA

'Waterer's Dwarf' DCNA

'Waterer's Orange' DCNA

'Waterer's Yellow' DCNA

'Wayside's Compact' [P. coccinea Roem.] DCNA

'Weaver's Superb' DCNA

'Wheeler' DCNA 
'Wight Early' DCNA

'Wonderberry' DCNA

'Wyattii' [P. coccinea Roem.] DCNA

'Yella Berry' DCNA

'Yokohama' DCNA

'Yukon Belle' [P. angustifolia (Franch.)

Schneid.]

= 'Gnome'

'Yukon Jack' [P. angustifolia (Franch.) Schneid.]

$=$ 'Gnome'

yunnanensis (M.L.Vilm. ex Mottet)

Chittenden

$=\boldsymbol{P}$. fortuneana

XPYRACOMELES Rehd. ex Guill.

ROSACEAE
[Osteomeles $\times$ Pyracantha]

vilmorinii Rehd. ex Guill.

[Pyracantha fortuneana $\times$ Osteomeles subrotunda] DCNA FLUF

PYRUS L. Pear

\section{ROSACEAE} Rose Family

amygdaliformis Villars MDAB

'Aristocrat' [P. calleryana Decne.] DCNA VATA

betulifolia Bunge

Birchleaf P. ALCW MDAB

'Bradford' [P. calleryana Decne.]

Bradford $P$.
ALEH
DCNA
MDAB
VACW
DCCG
GAAB
MDLT
VATA

calleryana Decne.

Callery P.

$\begin{array}{llll}\text { ALCW } & \text { ARPT } & \text { LATU } & \text { SCEM } \\ \text { ALTD } & \text { LASL } & \text { MDAB } & \text { TNUT }\end{array}$

calleryana Decne. var. dimorphophylla (Makino) Koidz. $\mathrm{MDAB}$

$\times$ canescens Spach

[P. nivalis $\times P$. salicifolia] MDAB

$\star$ ‘Capital' [P. calleryana Decne.] DCNA

'Chanticleer' [P. calleryana Decne.] DCNA

'Chojure' [P. pyrifolia (Burm.) Nakai] MDKN

communis $\mathrm{L}$.

Common $\mathrm{P}$. MDAB VACW VAMP VASK

elaeagrifolia Pall. $\mathrm{MDAB}$

fauriei Schneid. $\mathrm{MDAB}$

kawakamii Hayata

Evergreen $\mathrm{P}$. DCNA FLMG TXSH

$\times$ lecontei Rehd.

Leconte $\mathrm{P}$.

[P. communis $\times \boldsymbol{P}$. pyrifolia] $\begin{array}{lll}\text { ALSM } & \text { LAAP } & \text { SCMG } \\ \text { FLDG } & \text { MDWP } & \text { SCMP }\end{array}$

$\times$ michauxii Bosc ex Poir.

[P. ?amygdaliformis $\times P$. nivalis] MDAB

nivalis Jacq.

Snow P. MDAB MDGD MDHN

pashia Buch.-Ham. ex D.Don $\mathrm{MDCH}$

'Pendula' [P. salicifolia Pall.] DCNA MDAB

phaeocarpa Rehd. MDAB

bretschneideri Rehd. MDAB 
pyrifolia (Burm.f.) Nakai

Sand P., Asian P.

$\begin{array}{lll}\text { LATU } & \text { MDCH } & \text { TXMS } \\ \text { MDAB } & \text { MDGD } & \text { VATG }\end{array}$

pyrifolia (Burm.f.) Nakai var. culta (Makino) Nakai

Nashi P. MDAB MDPJ

regelii Rehd. MDAB

salicifolia Pall. MDAB VAPH

ussuriensis Maxim. DCCI MDAB

ussuriensis Maxim. var. hondoensis (Kikuchi \& Nakai) Rehd.
DCCI
MDAB

ussuriensis Maxim. var. ovoidea Rehd. MDAB

'Whitehouse' [P. calleryana Decne.] DCNA VATA

QUERCUS L. FAGACEAE

acutissima Carruthers

Oriental Sawtooth O.

$\begin{array}{llll}\text { ARUM } & \text { GAFN } & \text { MDTH } & \text { TXMS } \\ \text { DCAE } & \text { GAIS } & \text { NCBE } & \text { VABP } \\ \text { DCNA } & \text { LAAL } & \text { NCGR } & \\ \text { FLUF } & \text { MDGD } & \text { TNSG } & \\ \text { GACG } & \text { MDLA } & \text { TXHG } & \end{array}$

alba L.

White O.

$\begin{array}{lll}\text { ALAU } & \text { DEMC } & \text { MDWM } \\ \text { ARUM } & \text { GAUG } & \text { SCBR } \\ \text { DCCG } & \text { MDHN } & \text { SCMP }\end{array}$

alba $\times$ Q. virginiana SCRH

arkansana Sarg.

Arkansas Oak ARCA

$\times$ asheana Little

[Q. incana $\times$ Q. laevis] FLUF

austrina Small FLUF bicolor Willd.

Swamp White O. DCCG SCMG

$\times$ bimundorum Palm.

[Q. alba $\times$ 9. robur] $\mathrm{DCCH}$

borealis Michx.f.

= Q. rubra

catesbaei Michx.

$=Q$. laevis

cerris L.

Turkey O. NCTP TNWF

chenii Nakai DCNA

coccinea Muenchh.

Scarlet O. DCNG DEMC MDAF MDHN

'Columna' [Q. robur 'Fastigiata' $\times \boldsymbol{Q}$. petraea 'Muscaviensis'] DCNA GAUG

$\times$ comptonae Sarg.

Compton $\mathrm{O}$.

[Q. lyrata $\times$ Q. virginiana]
DCHP
LASL
VACW
LAMB
VACG

'Cristata' [Q. robur L.]

DCNA

'Cucullata' [Q. robur L.] DCNA

dentata Thunb. ex J.A.Murr.

Daimyo $\mathrm{O}$.

$$
\text { DCNA GAIS GAUG LAAL }
$$

fabri Hance DCNA

falcata Michx.

Southern Red O., Spanish O. ALBG GAHC SCBR

falcata Michx. var. pagodifolia Ell. SCMP

'Falkenbergensis' [Q. petraea (Mattusch.) Liebl.] DCNA

farnetto Tenore

$=\mathbf{Q}$. frainetto 
frainetto Tenore

Italian Oak

DCNA

'Geisleri' [Q. petraea (Mattusch.) Liebl.] DCNA

georgiana M.A.Curtis

Georgia O.

GAWD

gilva $\mathrm{Bl}$.

LAAL

glandulifera $\mathrm{Bl}$.

Konara Oak DCNA

glauca Thunb. ex J.A.Murray Ring-cupped $\mathrm{O}$.

$\begin{array}{llll}\text { ALAU } & \text { GACD } & \text { NCOP } & \text { TXHG } \\ \text { ALON } & \text { GAHC } & \text { SCBR } & \text { VANB } \\ \text { ALTD } & \text { GAIS } & \text { SCKG } & \text { VAWR } \\ \text { DCNA } & \text { GAJI } & \text { SCRP } & \\ \text { FLMG } & \text { LAAL } & \text { TNMB } & \\ \text { FLUF } & \text { MSMN } & \text { TNUT } & \end{array}$

haas Kotschy DCNA

hemisphaerica Bartram ex Willd.

Darlington $\mathrm{O}$. DCNA GAWM SCSG SCWP

$\times$ heterophylla Michx.f.

Bartram O.

[Q. phellos $\times$ Q. rubra]

DCCG

imbricaria Michx.

Shingle O.

DCNA MDBG

incana Bartram

Bluejack O.

FLUF

laevis Walt.

Turkey $\mathrm{O}$.

FLUF NCGP

laurifolia Michx.

Laurel $\mathrm{O}$.

$\begin{array}{llll}\text { ALAU } & \text { ALSH } & \text { MDKB } & \text { SCBR } \\ \text { ALBB } & \text { DCNA } & \text { NCAG } & \text { SCKG } \\ \text { ALBG } & \text { FLUF } & \text { NCTP } & \text { SCSG }\end{array}$

lyrata Walt.

Overcup O.

DCNG FLUF SCBR macrocarpa Michx.

Bur O.

$\begin{array}{lll}\text { DCCG } & \text { DCNA } & \text { GAR̄C } \\ \text { DCMS } & \text { GACD } & \text { NCWM }\end{array}$

margaretta Ashe

Sand Post O.

GAGR

maxima Marsh.

= Q. rubra

michawxii Nutt.

Basket O.

DCCG FLMG SCBR

minima (Sarg.) Small

SCWI

mongolica Fischer var. grosseserrata (B1.) Rehd. \& Wils.

DCNA

'Muscaviensis' [Q. petraea (Mattusch.) Liebl.] DCNA

myrsinifolia $\mathrm{Bl}$.

Japanese Evergreen O.

$\begin{array}{lll}\text { ALON } & \text { FLMG } & \text { LAAL } \\ \text { ALTD } & \text { FLUF } & \text { MSHB } \\ \text { DCNA } & \text { GAUG } & \text { NCEL }\end{array}$

nigra $\mathrm{L}$.

Water $\mathrm{O}$.

$\begin{array}{lll}\text { ALBG } & \text { FLUF } & \text { SCBR } \\ \text { DCHP } & \text { GAJS } & \text { SCDH } \\ \text { FLLE } & \text { NCTE } & \text { VAMP }\end{array}$

nuttallii Palm.

Nuttall O.

LAAP

pagoda Raf.

= Q. falcata var. pagodifolia

palustris Muenchh.

Pin $\mathrm{O}$.

$$
\text { DCCG DCNA SCBR }
$$

petraea (Mattusch.) Liebl.

Durmast Oak

DCNA

phellos L.

Willow $\mathrm{O}$.

DCCG SCBR SCWP VAGG

NCDU SCMG VACM VALC

phillyraeoides A.Gr.

Ubame O.

DCNA GAIS SCCU TXPS 
prinus L.

Chestnut O.

DCCG MDHN VAGF

pubescens Willd.

DCCG DCLC

robur $\mathrm{L}$.

English O.

DCCG DCNA MDCP NCTP

robur L. f. fastigiata (Lam.) O.Schwarz Columnar English O.
DCNA MDAB
VAMP
GAUG TNSN

$\times$ rosacea Bechst.

[Q. petraea $\times$ Q. robur]

DCNA

rubra L.

Red O.
DCCG
DEMC
GAUG
MDHN

'Salicifolia' [Q. robur L.] DCNA

$\times$ sargentii Rehd.

Sargent $\mathrm{O}$.

[Q. prinus $\times$ Q. robur]

DCHP

serrata Thunb. ex J.A.Murr.

= Q. glandulifera

shumardii Buckl.

Shumard O.

$$
\text { ALAU FLUF SCMP }
$$

stellata Wang.

Post O.

$$
\text { DCCG GAJS }
$$

stellata Wang. var. margaretta (Ashe) Sarg.

$=\mathbf{Q}$. margaretta

suber $\mathrm{L}$.

Cork O.

$$
\text { ALUA NCTE SCRP }
$$

variabilis $B 1$.

Chinese Cork O.

$$
\text { DCDO DCNA FLGS MDDF }
$$

velutina Lam.

Black O.

MDHN SCBR virginiana Mill.

Southern Live O.

$\begin{array}{llll}\text { ALBB } & \text { GAAB } & \text { SCKG } & \text { VARO } \\ \text { ALCM } & \text { LAAP } & \text { SCMG } & \text { VASC } \\ \text { ALEC } & \text { NCAG } & \text { SCMP } & \text { VATA } \\ \text { ALTD } & \text { SCBP } & \text { VACM } & \text { VAWR } \\ \text { FLCG } & \text { SCBR } & \text { VAGW } & \\ \text { FLUF } & \text { SCDH } & \text { VANB } & \end{array}$

RAPHIOLEPIS Lindl.

= RHAPHIOLEPIS

REHSONIA Stritch

= WISTERIA

RHAMNUS L.

Buckthorn

RHAMNACEAE

Buckthorn Family

alaternus $\mathrm{L}$.

Italian B.

$\mathrm{SCCU}$

carolinianus L.

Carolina B.

GAUG MSMN TNSC

catharticus $\mathrm{L}$.

Common B.

$\begin{array}{ll}\text { DEEM } & \text { MDGJ TNRG } \\ \text { MDBP } & \text { TNLW }\end{array}$

davuricus Pall.

$\begin{array}{lll}\text { MDGJ } & \text { TNLW } & \text { VACW } \\ \text { TNDR } & \text { TNUT } & \end{array}$

frangula $\mathrm{L}$.

= Frangula alnus

japonicus Maxim.

DCNA

utilis Decne.

LAAL MDBP

RHAPHIOLEPIS Lindl.

ROSACEAE

Rose Family

'Enchantress' [R. indica (L.) Lindl.] FLRH VACW 
indica (L.) Lindl.

Indian Hawthorn

$\begin{array}{llll}\text { ALTD } & \text { GAUG } & \text { SCBA } & \text { SCPR } \\ \text { FLMG } & \text { GAWH } & \text { SCBH } & \text { SCRH } \\ \text { FLRH } & \text { MSHB } & \text { SCCU } & \\ \text { GACL } & \text { NCEG } & \text { SCFW } & \\ \text { GATS } & \text { NCOP } & \text { SCMP } & \end{array}$

indica var. integerrima (Hook.) Kitam.

$=R$. umbellata var. integerrima

'Majestic Beauty'

TXSN

'Pink Cloud'

TXSN

'Snow' [R. indica (L.) Lindl.] TXSE

'Springtime'

TXSN

umbellata (Thunb. ex J.A.Murr.) Makino

Yeddo Hawthorn
ALBG GAIS VATA
GACH NCEG

umbellata (Thunb. ex J.A.Murr.) Makino

var. integerrima (Hook. \& Arn.) Rehd.

$\begin{array}{lll}\text { DCNA } & \text { LAHG } & \text { SCLM } \\ \text { FLUF } & \text { NCAG } & \text { VANB } \\ \text { GATS } & \text { SCBR } & \text { VATA }\end{array}$

\section{RHAPIDOPHYLLUM H.Wendl. \& Drude ARECACEAE \\ Palm Family}

hystrix (Pursh) H.Wendl. \& Drude

Needle Palm

$\begin{array}{llll}\text { ALAU } & \text { FLUF } & \text { LAMP } & \text { SCWI } \\ \text { FLDG } & \text { GAHC } & \text { NCOP } & \text { TXSE }\end{array}$

RHAPIS L.f. ex Ait.

ARECACEAE

Palm Family

excelsa (Thunb. ex J.A.Murr.) A.Henry

FLUF

\section{RHODODENDRON L.}

\section{ERICACEAE}

Heath Family

(The plants recognized by landscapers and horticulturists as "rhododendrons" fall into two main categories: lepidote (scaly) and elepidote (nonscaly). Botanically, the lepidotes are found in subgenus Rhododendron and the elepidotes in subgenus Hymenanthes. The leaves of the lepidotes have minute surface scales and are generally smaller than those of the elepidotes. The lepidotes include, among others, species native to the southeastern United States (for example, $\boldsymbol{R}$. minus), to subarctic regions (for example, $\boldsymbol{R}$. lapponicum), and to the Alps and Pyrenees (for example, R. ferrugineum). Many of the subarctic and alpine species and their hybrids do not thrive in the summer heat of the southeastern United States.

The elepidotes ( $\boldsymbol{R}$. maximum and $\boldsymbol{R}$.

catawbiense, for example) are species generally recognized by the layperson as rhododendrons.

Some of the elepidotes have leaves that are glabrous (without hairs), while others have leaves with indumentum (matted branched hairs) on the lower surface. Azaleas are also members of the genus Rhododendron. Approximately 15 deciduous species are native in the eastern United States, and a number of these have been crossed with Japanese, Chinese, and eastEuropean species to develop showy hybrids (for example, the Ghent, Knap Hill, Exbury, and Ilam hybrids). The evergreen azaleas are of east-Asian origin. Their degree of leaf retention may vary with severity of the climate.)

'A. Bedford'

(Elepidote rhododenron) VAZC

'Addy Wery'

(Evergreen azalea) DEMC

alabamense Rehd.

Alabama Azalea

(Deciduous azalea)

$\begin{array}{llll}\text { DCNA } & \text { FLMG } & \text { MDLT } & \text { SCBR } \\ \text { DEWG } & \text { GACG } & \text { NCBE } & \end{array}$

'Albiflorum' [R. mucronulatum Turcz.]

(Lepidote rhododendron) DCNA

'Album' [R. carolinianum Rehd.]

(Lepidote rhododendron) DEWG MDLT

'Album Elegans' [R. catawbiense hybrid] (Elepidote rhododendron) DCWH

amagianum Makino

Mt. Amagi Azalea

(Deciduous azalea) MDMG

\section{'Amethystinum'}

(Evergreen azalea; Mucronatum group) DEMC 


\section{'Amoenum'}

(Evergreen azalea; Obtusum group) DEWG NCBE

'Anah Kruschke' [R. ponticum seedling] (Elepidote rhododendron) VATA

\section{'Annie E. Endtz'}

(Elepidote rhododendron) VANB

\section{'April Rose'}

(Evergreen azalea) SCMG

arborescens (Pursh) Torr.

Sweet Azalea

(Deciduous azalea)

$$
\text { DEMC DEWG NCBE TNFN }
$$

atlanticum (Ashe) Rehd.

Coastal Azalea

(Deciduous azalea)

$$
\begin{array}{lll}
\text { DCNA } & \text { MDLT } & \text { SCBR } \\
\text { DEWG } & \text { NCBE } &
\end{array}
$$

augustinii Hemsl.

(Lepidote rhododendron)

MDMG VANB

austrinum (Small) Rehd.

Florida Azalea

(Deciduous azalea)

$\begin{array}{llll}\text { ALEH } & \text { FLAL } & \text { MDLT } & \text { TXLL } \\ \text { ALFS } & \text { FLMG } & \text { NCBE } & \text { VAGS } \\ \text { ARHS } & \text { GACG } & \text { SCBR } & \text { VATW } \\ \text { DCNA } & \text { GATS } & \text { SCMG } & \\ \text { DEWG } & \text { MDKN } & \text { SCWI } & \end{array}$

\section{'Autumn Glory'}

(Elepidote rhododendron) VAZC

\section{'Azma'}

(Elepidote rhododendron)

$$
\text { VANB }
$$

\section{'Azor'}

(Elepidote rhododendron)

$$
\text { VANB }
$$

bakeri (W.P.Lemmon \& McKay) Hume

Cumberland Azalea

(Deciduous azalea)
DEWG
MDLT
GAEC MDMG
TNAB

\section{'Ballerina'}

(Deciduous azalea)

$$
\text { DEMC }
$$

'Beauty of Littleworth'

(Elepidote rhododendron) DCNA

'Beni kirishima'

(Evergreen azalea) MDMG

\section{'Ben Morrison'}

(Evergreen azalea)

$$
\text { MDMG }
$$

\section{'Betty Wormald'}

(Elepidote rhododendron) DCNA

\section{'Bibiani'}

(Elepidote rhododendron)

$$
\text { ALEH }
$$

\section{'Blaauw's Pink'}

(Evergreen azalea) DCWH

\section{'Blue Tit'}

(Lepidote rhododendron)

$$
\text { DCNA }
$$

\section{'Bosley 1020'}

(Elepidote rhododendron) VATA

$\star$ 'Bowie' [R. chapmanii $\times$ R. minus]

(Lepidote rhododendron) DCNA

brachycarpum D.Don ex G.Don Fujiama R.

(Elepidote rhododendron) VANB

\section{'Brandywine'}

(Lepidote rhododendron)

$$
\text { DCNA }
$$

\section{'Brick-dust Red'}

(Evergreen azalea) SCMG

\section{'Cadis'}

(Elepidote rhododendron)

$$
\text { DEMC }
$$

calendulaceum (Michx.) Torr.

Flame Azalea

(Deciduous azalea)

$\begin{array}{llll}\text { DEWG } & \text { MDMG } & \text { VADF } & \text { VAZC } \\ \text { FLMG } & \text { NCBE } & \text { VAGW } & \\ \text { GAEC } & \text { SCBR } & \text { VASP } & \end{array}$


$\star$ 'Camp's Red' [R. bakeri (W.P.Lemmon \& McKay) Hume]

(Deciduous azalea)

DCNA MDKN

canescens (Michx.) Sweet

Piedmont Azalea

(Deciduous azalea)

$\begin{array}{llll}\text { DEWG } & \text { GASM } & \text { NCBE } & \text { SCMG } \\ \text { FLMG } & \text { LAAL } & \text { NCOP } & \text { SCWI } \\ \text { FLUF } & \text { LASL } & \text { SCBR } & \text { TNDG } \\ \text { GACG } & \text { MDLT } & \text { SCFW } & \text { TXLL }\end{array}$

'Carmen'

(Evergreen azalea) DEMC

'Carminata Splendens'

(Evergreen azalea; Amoenum hybrid) DCNA

carolinianum Rehd.

Carolina R.

(Lepidote rhododendron) $\begin{array}{lll}\text { DCWH } & \text { MDKN } & \text { VANB } \\ \text { DEWG } & \text { MDLA } & \text { VATW }\end{array}$

carolinianum $\times \boldsymbol{R}$. mucronulatum

(Lepidote rhododendron) DCNA

carolinianum $\times$ R. racemosum

(Lepidote rhododendron) DCNA

catawbiense Michx.

Mountain Rosebay

(Elepidote rhododendron)

DCCG MDOM VAHB VAPO

'Cerise'

(Evergreen azalea) SCMG

chapmanii A.Gr.

Chapman R.

(Lepidote rhododendron) $\begin{array}{llll}\text { DCNA } & \text { FLUF } & \text { NCBE } & \text { TXLL } \\ \text { FLMG } & \text { GAEC } & \text { SCWI } & \text { VANB }\end{array}$

'Cherry Red'

(Elepidote rhododendron) MDLT

'Christmas Cheer'

(Evergreen azalea) ALEH ARPT FLMG

'Christopher Wren'

(Deciduous azalea) DEMC MDBG
'Coccinea Major'

(Evergreen azalea)

FLMG

SCMG

'Coccinea Speciosa'

(Deciduous azalea) DEMC

'Comte de Newport'

(Evergreen azalea) SCMG

'Conemaugh' [R. racemosum $\times$

R. mucronulatum]

(Lepidote rhododendron) DEWG

'Conestoga' [R. carolinianum $\times$

$\boldsymbol{R}$. racemosum]

(Lepidote rhododendron) DCNA DEWG

'Conewago' [R. carolinianum $\times$

R. mucronulatum]

(Lepidote rhododendron) DEWG

'Coral Bells'

(Evergreen azalea) ALEH ARPT

'Coral Cluster'

(Evergreen azalea) DCNA

'Corneille'

(Deciduous azalea) MDLT

'Cornell Pink' [R. mucronulatum Turcz.]

(Lepidote rhododendron) DCNA DEWG MDBG

'Crater Lake' [R. augustinii Hemsl.]

(Lepidote rhododendron) MDBG

cumberlandense E.Braun

$=\boldsymbol{R}$. bakeri

'Cunningham's White' [R. caucasicum $\times$ R. ponticum var. album]

(Elepidote rhododendron) VANB

'Cynthia' [R. catawbiense $\times$

R. griffithianum]

(Elepidote rhododendron) VANB

dauricum L.

(Lepidote rhododendron) DCNA 
'David Gable' [R. catawbiense

'Atrosanguineum' $\times \boldsymbol{R}$. fortunei]

(Elepidote rhododendron)

DEWG MDLT

decorum Franch.

(Elepidote rhododendron)

MDFM VANB

degronianum Carr. ssp. heptamerum

(Maxim.) Hara

(Elepidote rhododendron)

$\begin{array}{llll}\text { DCNA } & \text { MDKN } & \text { MDMG } & \text { VANB } \\ \text { DEWG } & \text { MDLT } & \text { SCWI } & \end{array}$

\section{'Dexter Purple'}

(Elepidote rhododendron)

MDLT

'Dexter's Champagne'

(Elepidote rhododendron) MDLT

discolor Franch.

$=\boldsymbol{R}$. fortunei subsp. discolor

\section{'Dora Amateis' [R. carolinianum $\times$}

R. ciliatum]

(Lepidote rhododendron) DEMC GAEC SCWI

'Dorsett' [R. kaempferi Planch.]

(Evergreen azalea) DCNA

\section{'Duc de Rohan'}

(Evergreen azalea)

$$
\text { FLMG SCMG }
$$

\section{'Early Lavender'}

(Evergreen azalea) SCMG

\section{'Early Salmon'}

(Evergreen azalea)

$$
\text { SCMG }
$$

\section{'Evening Glow'}

(Elepidote rhododendron) MDLT

'Everestianum' [R. catawbiense hybrid] (Elepidote rhododendron)

$$
\text { VANB }
$$

fargesii Franch.

$=\boldsymbol{R}$. oreodoxa var. fargesii

'Fawley'

(Deciduous azalea)

DEMC
'Fedora'

(Evergreen azalea) DEMC

\section{'Flamingo'}

(Evergreen azalea) VATA

flammeum (Michx.) Sarg.

Oconee Azalea

(Deciduous azalea)

$\begin{array}{lll}\text { DCNA } & \text { FLMG } & \text { MDLT } \\ \text { DEWG } & \text { GACG } & \text { NCBE }\end{array}$

flavum (Hoffmgg.) G.Don

= R. luteum

'Flowerdale Pink'

(Evergreen azalea) SCMG

\section{'Flowerdale Red'}

(Evergreen azalea) SCMG

\section{'Formosa'}

(Evergreen azalea)

$$
\text { ALBG FLUF SCMG }
$$

'Forsterianum' [R. veitchianum $\times$ R. edgeworthii]

(Lepidote rhododendron) DCNA

fortunei Lindl.

(Elepidote rhododendron)
DCWH
NCBE
VATW
MDMG VANB

fortunei Lindl. ssp. discolor (Franch.)

Chamb.

(Elepidote rhododendron) MDKN MDMG VATW

\section{'George Lindley Taber'}

(Evergreen azalea; sport of 'Phoeniceum') FLGS FLMG MDLG

'Georgiana Maclay'

(Evergreen azalea)

FLMG

'Giant Elegans'

(Evergreen azalea)

SCMG

'Giant White'

(Evergreen azalea)

SCMG

'Gibraltar'

(Deciduous azalea) MDLT 


\section{Glenn Dale Azaleas}

This series of 454 named cultivars was created at the U.S. Plant Introduction Garden, Glenn Dale, MD, by B.Y. Morrison, who became the first director of the U.S. National Arboretum. The project began around 1929, with the objective of creating azaleas with flowers as large as those of the Southern Indian hybrids, but hardy in the Washington, DC, area. Many of the selections met this criterion, and others equally hardy were selected for different ornamental values (see B.Y. Morrison. 1953. The Glenn Dale azaleas. U.S. Department of Agriculture, Agriculture Monograph No. 20, Washington, DC.) A list of the Glenn Dale azalea cultivars documented in the U.S. National Arboretum herbarium appears in Appendix C.

\section{'Glory of Sunninghill' \\ (Evergreen azalea) FLMG}

\section{'Golden Dream' \\ (Deciduous azalea) MDLT}

'Gomer Waterer' [R. catawbiense hybrid] (Elepidote rhododendron) SCWI

'Gumpo' [R. eriocarpum (Hayata) Nakai] (Evergreen azalea) LAHG

\section{'Helen'}

(Evergreen azalea) VATA

'Hinode giri' (often 'Hinodegiri')

(Evergreen azalea) ARPT

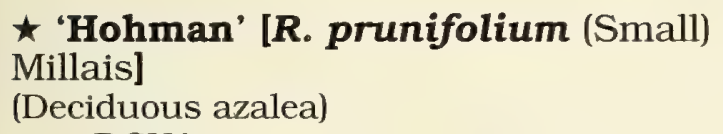

\section{'Hugh Koster'}

(Elepidote rhododendron) VANB

'Ima shojo'

\section{= 'Christmas Cheer'}

indicum (L.) Sweet

Indica Azalea

(Evergreen azalea)

DCNA LAAL LAHG TNSG

indicum (L.) Sweet var. tamurae Makino

$=\boldsymbol{R}$. tamurae

'Iveryana'

(Evergreen azalea) FLMG SCMG

japonicum (A.Gr.) Suring. DCNA

'Jean Marie de Montague'

= 'The Hon. Jean Marie de Montague'

'John Walter' [R. catawbiense $\times$

$\boldsymbol{R}$. arboreum ssp. arboreum]

(Elepidote rhododendron) VANB

kaempferi Planch.

Kaempfer Azalea

(Evergreen azalea) DEWG MDGD

kaempferi $\times \boldsymbol{R}$. komiyamae

(Evergreen azalea; natural hybrid) MDGD

kanahirae Wils.

Taibei Azalea

(Evergreen azalea) MDGD

keiskei Miq.

(Lepidote rhododendron) DCNA DEMC

keiskei $\times \boldsymbol{R}$. racemosum

(Lepidote rhododendron) DEWG MDKN

'Ken Janeck' [R. yakushimanum Nakai ssp. yakushimanum]

(Elepidote rhododendron) MDLT

kiusianum Makino

Kyushu Azalea

(Evergreen azalea) DEWG MDGD VANB

'Koran yuki'

(Evergreen azalea) DCNA

'Koromo shikibu'

(Evergreen azalea) DCNA

'Kristin'

(Elepidote rhododendron) MDLT 


\section{'La Roche' \\ (Evergreen azalea) TNCT \\ 'Lady Mulberry' \\ (Evergreen azalea) SCMG \\ lasiostylum Hayata \\ (Evergreen azalea) MDGD \\ 'Late Orchid' \\ (Evergreen azalea) SCMG \\ 'Lawsal' \\ (Evergreen azalea) SCMG \\ ledifolium (Hook.) G.Don \\ = 'Mucronatum'}

'Linearifolium' [R. macrosepalum Maxim.]

Spider Azalea

(Evergreen azalea) MDGD

'Lizette'

(Evergreen azalea) DCNA

\section{'Lucinda' \\ (Evergreen azalea) DCNA}

\section{luteum Sweet}

Pontic Azalea

(Deciduous azalea)

(The early synonymy of this plant is confused. It is possible that $\boldsymbol{R}$. flavum may have priority over $\boldsymbol{R}$. luteum under the rules governing botanical nomenclature. The International Registrar for Rhododendron, however, lists the subject plant as $\boldsymbol{R}$. luteum, and that name is almost universally used in horticulture and the nursery trade.)

$$
\text { DCNA DEWG MDGD MDKN }
$$

\section{'Luteum' [R. carolinianum Rehd.]}

(Lepidote rhododendron) DCNA

macranthum (Bunge) G.Don

$=R$. indicum

macrosepalum Maxim.

Large-sepal Azalea

(Evergreen azalea)

$$
\text { VAPH }
$$

'Madame de Bruin'

(Elepidote rhododendron) VANB
'Magnolia Alba'

(Evergreen azalea) SCMG

makinoi Tagg

$=R$. yakushimanum ssp. makinoi

'Maria Derby'

(Evergreen azalea) MDMG

'Mary Fleming' [(R. keiskei $\times$ $\boldsymbol{R}$. racemosum) $\times \boldsymbol{R}$. keiskei] (Lepidote rhododendron) MDLT

\section{maximum L.}

Rosebay

(Elepidote rhododendron)

$\begin{array}{llll}\text { DCNA } & \text { MDET } & \text { MDLT } & \text { NCDR } \\ \text { MDBG } & \text { MDHN } & \text { MDMG } & \text { TNHD } \\ \text { MDDF } & \text { MDJH } & \text { NCBE } & \text { VASP }\end{array}$

maximum L.

(Elepidote rhododendron; red-flowered form) VATW

metternichii Sieb. \& Zucc.

$=\boldsymbol{R}$. degronianum ssp. heptamerum

micranthum Turcz.

(Lepidote rhododendron) MDLT SCWI VATW

minus Michx.

Piedmont R.

(Lepidote rhododendron)

$\begin{array}{lll}\text { DCSH } & \text { MDCH } & \text { SCWI } \\ \text { DEWG } & \text { NCBE } & \text { TNAB } \\ \text { GAEC } & \text { SCBR } & \text { VAPO }\end{array}$

'Mrs. Betty Robertson'

(Elepidote rhododendron) VANB

'Mrs. Charles S. Sargent' [R. catawbiense hybrid]

(Elepidote rhododendron) VANB

'Mrs. E. C. Stirling' [R. griffithianum hybrid]

(Elepidote rhododendron) VANB

'Mrs. G. G. Gerbing'

(Evergreen azalea) FLMG

'Mrs. LBJ'

(Evergreen azalea) MDGD 
'Mrs. R. S. Holford'

(Elepidote rhododendron) VATA

mucronatum (Bl.) G.Don

$=$ Mucronatum group

\section{'Mucronatum'}

Snow Azalea

(Evergreen azalea; Mucronatum group)

(Although Chamberlain and Rae (1990) retain $R$. mucronatum (Bl.) G. Don var. mucronatum as a botanical taxon, they note that the plant is "only known in cultivation." They also note that var. mucronatum "may occur in the wild as the albino form of $[R$. mucronatum] var. ripense." Because of the amibiguity surrounding its origin, the Snow Azalea is accorded cultivar status here as 'Mucronatum', and its closely related variants are identified as being in the "Mucronatum group.")

$\begin{array}{lll}\text { ARPT } & \text { DEWG } & \text { MDGD } \\ \text { DCDO } & \text { LASL } & \text { MSHB }\end{array}$

mucronulatum Turcz.

(Lepidote rhododendron)
DEMC
DEWG
MDBG MDLT

'Myrtifolium' [R. minus $\times$ R. hirsutum]

(Lepidote rhododendron; this cultivar name sometimes leads to confusion with the quite different $\boldsymbol{R}$. myrtifolium)

MDLT MDMG

'Nadine'

(Evergreen azalea) DCNA

'Narcissiflora'

(Deciduous azalea) MDLT

\section{'Nodding Bells'}

(Elepidote rhododendron) VADF

nudiflorum (L.) Torr.

$=R$. periclymenoides

oblongifolium (Small) Millais

Texas Azalea

(Deciduous azalea) MDLT NCBE SCWI

obtusum (Lindl.) Planch.

= Obtusum group

\section{'Obtusum'}

Kirishima Azalea

(Evergreen azalea; Obtusum group) FLMG
'Obtusum Album'

(Evergreen azalea; Obtusum group) DCHU

occidentale (Torr. \& A.Gr.) A. Gr.

Western Azalea

(Deciduous azalea) DCNA

oldhamii Maxim.

Oldham Azalea

(Evergreen azalea)

$\begin{array}{ll}\text { DCNA } & \text { MDGD } \\ \text { GADG } & \text { SCWI }\end{array}$

oreodoxa Franch. var. fargesii (Franch.)

Chamb.

(Elepidote rhododendron) DEWG

'Oritani'

(Lepidote rhododendron) MDLT

ovatum (Lindl.) Planch. ex Maxim.

(Azaleastrum) DCNA GACG

'Palestrina'

(Evergreen azalea) DEMC

periclymenoides (Michx.) Shinners

Pinxterbloom

(Deciduous azalea) $\begin{array}{llll}\text { DCNA } & \text { MDLT } & \text { VAAC } & \text { VAGW } \\ \text { DEWG } & \text { NCBE } & \text { VADW } & \text { VATW }\end{array}$

phoeniceum (Sweet) G.Don

= 'Phoeniceum'

'Phoeniceum' [R. scabrum selection or hybrid]

(Evergreen azalea; Phoeniceum group) ARGD MDGD SCMG

'Pink Diamond' [R. griffithianum $\times$ $\boldsymbol{R}$. fortunei ssp. fortunei]

(Elepidote rhododendron; Loderi group) VANB

'Pioneer'

(Lepidote rhododendron; Gable hybrid) DCNA MDBG MDLT

'P. J. M.' [R. carolinianum $\times$

R. dauricum]

(Lepidote rhododendron; 'P.J.M.' originally applied to various seedlings from the cross; other cultivar names are being given to clonal selections, e.g. 'P.J. Mezitt'
DEMC
MDBG 
'P. J. Mezitt' [R. carolinianum $\times$

R. dauricum]

(Lepidote rhododendron; P.J.M. group) GACG MDLT

\section{'Plenum'}

(Evergreen azalea; Mucronatum group) MDMG

'Ponticum Roseum' [R. ponticum $\times$ R. maximum]

(Elepidote rhododendron) VATA

\section{'Ponticum Variegatum' [R. ponticum sport] \\ (Elepidote rhododendron) DCNA}

poukhanense Lev.

$=\boldsymbol{R}$. yedoense var. poukhanense

\section{'President Claeys' \\ (Evergreen azalea) SCMG}

'Pride of Dorking'

(Evergreen azalea) FLMG

'Pride of Mobile'

(Evergreen azalea)

$$
\text { ALCW SCMG }
$$

'Pride of Summerville'

= 'Lawsal'

prinophyllum (Small) Millais

Roseshell Azalea

(Deciduous azalea)

$$
\begin{array}{lll}
\text { DEWG } & \text { MDTD } & \text { SCBR } \\
\text { MDLT } & \text { NCBE } &
\end{array}
$$

prunifolium (Small) Millais

Plumleaf Azalea

(Deciduous azalea)
DCNA
MDLT
NCBE

punctatum Andr.

(Lepidote rhododendron) SCBR

'Purple Splendor'

(Evergreen azalea) DCNA

\section{'Queen of Orange' \\ (Evergreen azalea) SCMG}

'Ramapo' [R. fastigiatum $\times$

$\boldsymbol{R}$. carolinianum]

(Lepidote rhododendron) NCAS

'Red Flare' [R. keiskei Miq.]

(Lepidote rhododendron) MDLT

'Red Formosa'

(Evergreen azalea) FLMG

'Red Head'

(Elepidote rhododendron) VANB

reticulatum D.Don ex G.Don

Rose Azalea

(Deciduous azalea) DCNA MDGD

'Robert Allison'

(Elepidote rhododendron) DEWG

roseum (Loisel.) Rehd.

$=R$. prinophyllum

'Roseum Elegans' [R. catawbiense hybrid]

(Elepidote rhododendron) DCCG DCWH VAMP

'Roseum Magnificum'

(Evergreen azalea) SCMG

'Royal Red'

(Evergreen azalea) FLMG

\section{'Royal Splendor'}

(Evergreen azalea) SCMG

rubropilosum Hayata

(Evergreen azalea) MDGD MSBA

'Salmon King'

(Evergreen azalea) SCMG

schlippenbachii Maxim.

Royal Azalea

(Deciduous azalea)
DCNA
DEWG
MDPB
DEMC
TNDG

racemosum $\times \boldsymbol{R}$. keiskei

(Lepidote rhododendron)

DCNA 


\section{RHODODENDRON}

schlippenbachii Maxim.

Royal Azalea

(Deciduous azalea; white-flowered form)
DCNA
DEWG
MDBG
MDKN

serpyllifolium (A.Gr.) Miq.

Wild-thyme Azalea

(Evergreen azalea)

MDJW

serrulatum (Small) Millais

Hammock Sweet Azalea

(Deciduous azalea)

LASL NCBE

\section{'Shrimp Pink'}

(Lepidote rhododendron)

MDBG

simiarum Hance

(Elepidote rhododendron)

DCNA MDGD

simsii Planch.

Sims Azalea

(Evergreen azalea)

VANB

simsii Planch. var. tamurae (Makino) Kaneh.

\& Hatusima

$=R$. tamurae

\section{'Small Elegans'}

(Evergreen azalea) SCMG

smirnowii Traut.

(Elepidote rhododendron)

DEWG MDKN MDLT

speciosum (Willd.) Sweet

= R. flammeum

\section{'Spring Glory'}

(Elepidote rhododendron) DCNA

\section{'Stewartstonian'}

(Evergreen azalea) DEMC

\section{'Suetsuma'}

(Evergreen azalea) DCNA

\section{'Sunset'}

(Evergreen azalea) DCNA

\section{'Susugonoito'}

(Evergreen azalea) DCNA

\section{RHODODENDRON}

tamurae (Makino) Masam.

Dwarf Indica Azalea

(Evergreen azalea; Chamberlaiñ \& Rae (1990) believe that, contrary to Ohwi's interpretation, $\boldsymbol{R}$. tamurae is a synonym of $\boldsymbol{R}$. eriocarpum) DCNA

'The Hon. Jean Marie de Montague'

[R. griffithianum hybrid]

(Elepidote rhododendron) VANB

'Tiffany'

(Lepidote rhododendron) MDLT

tosaense Makino

(Evergreen azalea) DCNA

'Trilby'

(Elepidote rhododendron) VAZC

vaseyi A.Gr.

Pinkshell Azalea

(Deciduous azalea)
DCNA
GACG
MDLT
DEMC MDKN MDWJ
NCBE

vaseyi A.Gr. f. album (Bean) Rehd.

(Deciduous azalea)

GAEC MDLT

vernicosum Franch.

(Elepidote rhododendron) VANB VATW

'Viscosepala'

(Deciduous azalea) MDLT

viscosum (L.) Torr.

Swamp Azalea

(Deciduous azalea)

$\begin{array}{lll}\text { DCNA } & \text { MDBG } & \text { MDTD } \\ \text { DEWG } & \text { MDLT } & \text { NCBE } \\ \text { GAEC } & \text { MDMG } & \text { VAPH }\end{array}$

weyrichii Maxim.

(Deciduous azalea) MDGD

'Wheatley'

(Elepidote rhododendron) MDLT

'Wheeldon's Pink' [R. mucronulatum Turcz.]

(Lepidote rhododendron)

DEWG 
'William Bull'

(Evergreen azalea) FLMG

williamsianum Rehd. \& Wils.

(Elepidote rhododendron) DCNA

'Windbeam'

(Lepidote rhododendron)
DEMC
GAEC
MDLT

'Winterthur'

(Evergreen azalea; Mucronatum group) DEWG

\section{'Wissahickon'}

(Elepidote rhododendron) MDLT

'Wyanokie'

(Lepidote rhododendron) DCNA

'Yae shojo'

(Evergreen azalea) DCNA

yakushimanum Nakai ssp. makinoi (Tagg) Chamb.

(Elepidote rhododendron) VAZC

yakushimanum Nakai ssp. yakushimanum

(Elepidote rhododendron) DEWG MDBG MDLT

yedoense Maxim. ex Regel

var. poukhanense (Lev.) Nakai

Korean Azalea

(Evergreen azalea)
DEWG
GAEC
NCBE

yedoense Maxim. ex Regel

var. poukhanense (Lev.) Nakai

(Evergreen azalea; white-flowered form) DCNA

RHODOLEIA Champ. ex Hook. HAMAMELIDACEAE Witch-hazel Family

championii Hook. FLUF
RHODOTYPOS Sieb. \& Zucc. Jetbead ROSACEAE Rose Family

scandens (Thunb.) Makino

DEMC MDGD NCBE VACW

MDBG MDJH NCCA VAGG

RHUS L. Sumac

ANACARDIACEAE Cashew Family

aromatica Ait.

Fragrant S.
DCNA
DEMC
VACW

chinensis Mill. $\mathrm{VABF}$

copallina L.

Shining S., Winged S.
ALBG
SCCU VAPO

glabra L.

Smooth S.

DEMC

'Laciniata' [R. typhina L.]

MDBG MDLT TNSC

michauxii Sarg.

GAWD

punjabensis J.L.Stewart var. sinica (Diels)

Rehd. \& Wils.

DCNA

radicans $\mathrm{L}$.

= Toxicodendron radicans

typhina $\mathrm{L}$.

Staghorn S. DCNA VACW

verniciflua Stokes

= Toxicodendron vernicifluum

RIBES L. Currant, Gooseberry SAXIFRAGACEAE (Ribesioideae) Saxifrage Family

alpinum $\mathrm{L}$.

Mountain C. MDWP

curvatum Small

Granite G.

TXLL 
echinellum (Cov.) Rehd.

Florida G.

MDJW SCWI

grossularia L.

$=$ R. uva-crispa

odoratum H.Wendl.

Clove C.

$$
\begin{array}{lll}
\text { ARWS } & \text { MDBG } & \text { TNSM } \\
\text { GAOH } & \text { MDGL } &
\end{array}
$$

'Pixwell' [R. uva-crispa L.] MDFM

'Pulborough Scarlet' [R. sanguineum

Pursh]

MDBG

uva-crispa $\mathrm{L}$.

English G.

MDFM

ROBINIA L. Locust

FABACEAE (Faboideae) Bean Family

$\times$ ambigua Poir.

[R. pseudoacacia $\times$ R. viscosa] DCNA

'Bicolor' [R. pseudoacacia L.] DCNA

boyntonii Ashe NCCA

'Burgundy' [R. pseudoacacia L.] DCNA

'Decaisneana' [R. $\times$ ambigua Poir.]

[R. pseudoacacia $\times \boldsymbol{R}$. viscosa] DCNA

'Fastigiata' [R. pseudoacacia L.]

= 'Pyramidalis'

'Frisia' [R. pseudoacacia L.] DCNA

hispida L.

Rose Acacia, Bristly Locust NCCA VACW VAGC

pseudoacacia L.

Black L.

$\begin{array}{llll}\text { DCNA } & \text { GAJA } & \text { MDHN } & \text { SCMP } \\ \text { DCWR } & \text { MDBP } & \text { MDLT } & \text { TNFR } \\ \text { FLWJ } & \text { MDCP } & \text { MSBN } & \text { TXHA } \\ \text { GAES } & \text { MDGJ } & \text { SCFW } & \text { VACW }\end{array}$

pseudoacacia L. var. rectissima Raber

Ship-mast Locust

DCNA

'Purple Rose' [R. pseudoacacia L.] DCNA

'Pyramidalis' [R. pseudoacacia L.] MDGJ VAJL

'Rectissima' [R. pseudoacacia L.]

= R. pseudoacacia var. rectissima

'Sandraudiga' [R. pseudoacacia L.] DCNA

'Tortuosa' [R. pseudoacacia L.] DCNA

'Unifoliola' [R. pseudoacacia L.] DCNA

viscosa Vent.

Clammy L.

NCCA

ROSA L.

Rose

ROSACEAE

Rose Family

(The list includes cultivars introduced mainly before 1900; dates indicate year of introduction to gardens.)

acicularis Lindl.

Prickly R. VABF

'Agathe Incarnata'

(Damask; 1866) DCNA NCCB

'Aglaia' [R. multiflora $\times$ R. 'Reve d'Or'] (Yellow Rambler; 1896) VACW

$\times$ alba L.

White Rose-of-York

[R. ?canina $\times \boldsymbol{R} . \times$ damascena]

(Alba; before 1597)

NCCB VAGW

'Alba' [R. rugosa Thunb. ex J.A.Murr.] DCNA NCCB VANB VAPH

'Alba Plena' [R. banksiae Ait.f. var. banksiae]

Banks R. (double white)

(1807)

$\begin{array}{lll}\text { GATS } & \text { SCMG } & \text { SCNS } \\ \text { LAGN } & \text { SCMP } & \text { VAMW }\end{array}$


'Alberic Barbier' [R. wichuraiana $\times$

'Shirley Hibberd']

(Rambler; 1900)

$\mathrm{NCCB}$

'Alfred de Dalmas'

(Moss; 1855)

DCNA

'Alika'

(Gallica Grandiflora; 1906)

NCCB

'America' [R. wichuraiana $\times$

R. multiflora]

(Multiflora Hybrid; rambler; 1915) $\mathrm{NCCB}$

'Anais Segalas'

(Gallica; 1837)

DCNA NCCB

'Angelica Minor'

(Alba)

$\mathrm{NCCB}$

'Arielle'

(Damask Perpetual)

$\mathrm{NCCB}$

'Aurora'

[Parentage unknown]

$\mathrm{NCCB}$

'Baltimore Belle' $[\boldsymbol{R}$. setigera $\times \boldsymbol{R}$. gallica hybrid]

(1843)

DCNA

banksiae Ait.

(See cultivars 'Alba Plena', 'Lutea', and

'Lutescens')

'Baronne Prevost'

(Hybrid Perpetual; 1842) NCCB

'Beauty of Glazenwood'

= 'Fortune's Double Yellow'

'Belinda'

(Hybrid Musk; 1936)

$\mathrm{NCCB}$

'Belle Amour'

(Alba; before 1950) $\mathrm{NCCB}$

'Belle de Crecy'

(Gallica; before 1848) DCNA NCCB
'Belle Isador'

(China)

NCCB

'Belle Vichysoise'

(Noisette; 1897) NCCB

'Bicolor' [R. foetida J.Herrm.]

(Before 1590) DCNA

$\times$ bifera Pers.

$=\boldsymbol{R}$. damascena var. semperflorens

'Blanc Double de Coubert'

(Hybrid Rugosa; 1892) MDJS NCCB

'Blanchefleur'

(Centifolia; 1835)

NCCB

'Blanche Moreau'

(Moss; 1880)

DCNA

blanda Ait.

Meadow R.

(1773)

NCCB

'Blush Boursault'

(Shrub)

NCCB

bracteata J.C.Wendl.

$\mathrm{M}^{\mathrm{C}}$ Cartney $\mathrm{R}$.

(1793)

$\begin{array}{lll}\text { ALTR } & \text { GACG } & \text { NCCB } \\ \text { DCNA } & \text { MSEP } & \text { SCBR }\end{array}$

brunonii Lindl.

Himalayan Musk R.

(1822)

$$
\text { DCNA NCCB }
$$

'Bullata' [R. centifolia L.]

(Before 1815)

DCNA

'Camaieux'

(Gallica; 1830)

DCNA NCCB

canina $\mathrm{L}$.

Dog R.

(Before 1737)

DCNA NCCB VASK

'Captain Christy'

(Hybrid Tea; 1881)

NCCB 
'Cardinal de Richelieu'

(Gallica; 1840)

DCNA

'Carnea' [R. multiflora Thunb.]

(1804) NCCB

carolina L.

Pasture R.

(1826)

TNKS

'Catherine Mermet'

(Tea; 1869)

DCNA

'Celeste' [R. $\times$ alba L.]

[R. ?canina $\times \boldsymbol{R}$. $\times$ damascena]

(Alba; before 1759)

DCNA NCCB

'Celestial' [R. $\times$ alba L.]

$=$ 'Celeste'

'Celsiana'

(Damask; before 1750)

NCCB

centifolia L.

Cabbage R., Provence R.

(1596)

$$
\text { DCNA NCCB VAGW }
$$

'Champney's Pink Cluster'

Champney R.

(Noisette; 1811 )

DCNA

'Charles de Mills'

(Gallica)

$\mathrm{NCCB}$

chinensis Jacq.

China R.

(1759)

$$
\text { MSHB VAGW }
$$

chinensis Jacq. f. mutabilis (Corr.) Rehd.

= 'Mutabilis'

'Commandant Beaurepaire'

(Bourbon; 1874)

NCCB NCMM

'Comte de Chambord'

(Portland; 1860)

DCNA NCCB

'Conditorum' [R. gallica L.]

Tidbit R., Hungarian R.

DESC
'Conrad Ferdinand Meyer' [R. rugosa

hybrid $\times$ 'Gloire de Dijon']

(Hybrid Rugosa; 1899)

$\mathrm{NCCB}$

'Cramoisi Superieur'

(China; 1832) NCMM VACW

'Crested Jewel'

(Moss; 1971) NCCB

'Cristata' [R. centifolia L.]

Crested Moss R.

(Crested Moss; 1827)

$$
\text { NCCA NCMM }
$$

$\times$ damascena Mill.

Damask R.

[R. gallica $\times R$. moschata]

(16th century; before Miller's use of the name

$\boldsymbol{R}$. $\times$ damascena, J. Herrmann applied the epithet damascena, apparently to a different rose. Miller's name thus may be a later homonym and therefore illegitimate, but there is no generally accepted replacement.)

$$
\text { DCNA LASL NCCB VAGW }
$$

$\times$ damascena Mill. var. semperflorens

(Loisel.) Rowley

Four Seasons R., Monthly R.

[R. gallica $\times \boldsymbol{R}$. moschata]

(Ancient)

$$
\text { DCNA GASH NCCB }
$$

'De la Grifferaie'

(Shrub; 1845)

$$
\text { NCCB }
$$

'Delicata'

(Hybrid Rugosa; 1898) $\mathrm{NCCB}$

'Duc de Fitzjames'

(Gallica) NCCB

'Duc de Guiche'

(Gallica; before 1829) NCCB

'Duchesse de Brabant'

(Tea; 1857)

DCNA SCHW

'Duchesse de Montebello'

(Gallica; before 1829)

DCNA NCCB 
$\times$ dupontii Deseglise

[R. ?gallica $\times \boldsymbol{R}$. moschata]

(Before 1817)

DCNA NCMM

eglanteria L.

$=\boldsymbol{R}$. rubiginosa

'Empress Josephine' [R. $\times$ francofurtana Muenchh.]

[R. gallica $\times \boldsymbol{R}$. cinnamomea]

(Gallica)

DCNA

'Ernst Calvat'

(Bourbon)

NCMM

'Fantin Latour'

(Centifolia)

DCNA NCCB

'Felicite et Perpetue' [R. sempervirens hybrid]

(1827)

$\mathrm{NCCB}$

'Felicite Parmentier'

(Alba; 1834)

NCMM

'F. J. Grootendorst'

(Hybrid Rugosa; 1918) MSMN

foetida J.Herrm.

Austrian Brier

(Before 1542)

DCNA

forrestiana Boulenger

(1918)

DCNA

'Fortune's Double Yellow' [R, Xodorata (Andr.) Sweet var. pseudindica (Lindl.) Rehd.]

$[\boldsymbol{R}$. chinensis $\times \boldsymbol{R}$. gigantea]

(1845)

VACW

$\times$ fortuneana Lindl.

$[\boldsymbol{R}$. banksiae $\times \boldsymbol{R}$. ?laevigata]

(1850)

$\begin{array}{lll}\text { DCNA } & \text { GAIS } & \text { TXBA } \\ \text { FLUF } & \text { GATS } & \text { VACW }\end{array}$

'Frasers Pink Musk'

(Noisette; 1818)

$\mathrm{NCCB}$
'Frau Dagmar Hartopp'

(Hybrid Rugosa)

NCCB

'Frau Dagmar Hastrup'

= 'Frau Dagmar Hartopp'

'Frau Karl Druschki'

(Hybrid Perpetual; 1906) $\mathrm{NCCB}$

'Fun Jwan Lo' [R. Xodorata (Andr.) Sweet]

[R. chinensis $\times \boldsymbol{R}$. gigantea] SCMG

'Gardenia' [R. wichuraiana $\times$ 'Perle des Jardins']

(Rambler; 1899) $\mathrm{NCCB}$

'General Jacqueminot'

(Hybrid Perpetual; 1853)

DCNA

'General Kleber'

(Moss; 1856)

$\mathrm{NCCB}$

glauca Pourr.

(Before 1830)

DCNA

'Gloire de Dijon'

[? Tea $\times$ 'Souvenir de la Malmaison']

(1853)

DCNA

'Gloire des Mosseux'

(Moss; 1853)

DCNA GASH NCCB

'Gold of Ophir'

= 'Fortune's Double Yellow'

'Gracilis' [R. × Iheritieranea Thory]

Boursault R.

[R. chinensis $\times \boldsymbol{R}$. ?pendulina] $\mathrm{NCCB}$

'Hanosa' [R. rugosa Thunb.] DCDO

$\times$ harisonii Rivers

Yellow Rose-of-Texas

[R. foetida $\times R$. pimpinellifolia]

(ca. 1830)

$\begin{array}{lll}\text { DCNA } & \text { MDHN } & \text { VABF } \\ \text { MDFM } & \text { NCMM } & \text { VAGW }\end{array}$

'Henri Martin'

(Moss; 1863)

NCMM 
'Hermosa'

(China; 1840)

DCNA NCMM

'Honorine de Brabant'

(Bourbon)

DCNA NCCB

hugonis Hemsl.

$=R$. xanthina f. hugonis

'Ipsilante'

(Gallica; 1821)

$\mathrm{NCCB}$

'Isabella Sprunt'

(Tea; 1865)

DCNA

\section{'Jacques Cartier'}

(Portland; 1868)

GASH NCCB

'Jeanne d'Arc'

(Alba; 1818)

$\mathrm{NCCB}$

'Jeanne de Montfort'

(Moss; 1851 or 1854) MDHN

'Juno'

(Centifolia; before 1832)

DCNA

'Koenigin von Daenemark'

[R. ? $\times$ alba $\times$ Damask Hybrid] (Alba; 1826)

DCNA MDJS NCMM

laevigata Michx.

Cherokee R.

(1759)

$\begin{array}{lll}\text { FLMG } & \text { SCBR } & \text { SCMP } \\ \text { GAIS } & \text { SCCU } & \text { SCWI } \\ \text { GAJI } & \text { SCMG } & \text { VACW }\end{array}$

'La France'

(Hybrid Tea; 1867) DCNA

'Leda'

(Damask; before 1827)

DCNA NCCB

'Louis Philippe'

(China; 1834)

FLMS

'Louise Odier'

(Bourbon; 1851)

DCNA NCCB
'Lutea' [R. banksiae Ait.f. var. banksiae] Banks R. (double yellow), LadysBanks' R.

(1824)

$\begin{array}{llll}\text { DCNA } & \text { LAGN } & \text { SCHW } & \text { SCWI } \\ \text { GACG } & \text { MSMN } & \text { SCMG } & \text { TXMK } \\ \text { GATS } & \text { NCOP } & \text { SCUC } & \end{array}$

'Lutescens' [R. banksiae Ait.f. var.

normalis Regel]

Banks R. (single yellow)

(ca. 1870)

FLCG

'Mabel Morrison'

(Hybrid Perpetual; 1878) NCCB

'Macrantha'

[Hort., not Desp.] DCNA

'Macrantha' [R. $\times$ waitziana Tratt.]

(Early 18th Century)

[R. canina $\times R$. gallica] NCCB

'Maiden's Blush' [R. $\times$ alba $\times$

R. centifolia]

(1797)

DCNA GASH MDHN NCMM

'Marcel Bourgouin'

(Gallica; 1899)

$\mathrm{NCCB}$

'Marie Louise'

(Damask; 1813)

NCMM

'May Queen' [R. wichuraiana $\times$ 'Champion of the World']

(Rambler; 1898)

$\mathrm{NCCB}$

'Minima' [R. chinensis Jacq.]

Fairy Rose

(1815)

DCNA

'Mme. Alfred Carriere'

(Noisette; 1879) DCNA

'Mme. Hardy'

(Damask; 1832)

DCNA NCCB

'Mme. Isaac Pereire'

(Bourbon; 1881)

NCMM 
'Mme. Legras de Saint Germain'

(Hybrid Alba; 1846) NCCB

'Mme. Louis Leveque'

(Moss; 1898)

NCMM

'Mme. Pierre Oger'

(Bourbon; 1878) NCMM

'Mme. Plantier'

(Hybrid Alba; 1835) DCNA NCCB

moschata J.Herrm.

Musk R.

(1540)

DCNA

moschata var. nepalensis Lindl.

$=\boldsymbol{R}$. brunonii

moyesii Hemsl. \& Wils.

(1894)

DCNA

multibracteata Hemsl. \& Wils. (1910)

DCNA

multiflora Thunb. ex J.A.Murr.

Japanese R., Baby R.

(Before 1868)

$\begin{array}{lll}\text { ALBH } & \text { NCCA } & \text { VACW } \\ \text { DEMC } & \text { NCWC } & \text { VAHI } \\ \text { MSHB } & \text { SCBR } & \text { VASK }\end{array}$

'Muscosa' [R. centifolia L.]

Moss R.

(Before 1750)

DCNA NCCB VAGW

mutabilis Corr.

= 'Mutabilis'

'Mutabilis' [R. chinensis Jacq.]

(Cult. 1932 or earlier)

$$
\text { GAUG LASL NCCB }
$$

'Niphetos'

(Tea; 1889)

DCNA

$\times$ noisettiana Thory

Noisette R.

[R. chinensis $\times \boldsymbol{R}$. moschata]

(ca. 1812)

$$
\text { NCCB VABR VAGW }
$$

'Nuits de Young'

(Moss; 1845) $\mathrm{NCCB}$

$\times$ odorata (Andr.) Sweet

Tea R.

[R. chinensis $\times \boldsymbol{R}$. gigantea]

(Cult. 1752) NCCB

'Oeillet de Saint Arquey'

= 'Serratipetala'

'Oeillet Panachee'

Striped Moss

(Moss; 1888)

DCNA NCMM

'Officinalis'

Apothecary R.

(Gallica; 13th Century)

DCNA NCCB

'Old Blush' [R. ×odorata (Andr.) Sweet]

Monthly R.

$[R$. chinensis $\times R$. gigantea]

(1752)

DCNA NCCB VAGW

'Omar Khayyam'

(Damask; 1893)

$\mathrm{NCCB}$

palustris Marsh.

Swamp R.

(1726)

FLUF SCBR

$\times$ paulii Rehd.

[R. arvensis $\times \boldsymbol{R}$. rugosa]

(Before 1903)

DEMC

'Paul Neyron'

(Hybrid Perpetual; 1869)

DCNA NCCB

'Perle d'Or'

(Polyantha; 1884) $\mathrm{NCCB}$

'Perseana' [R. foetida J.Herrm.]

Persian Yellow R.

(1837)

$$
\text { DCNA VAGW }
$$

'Petite de Hollande'

(Centifolia; before 1802)

DCNA NCCB 
pimpinellifolia $\mathrm{L}$.

Scotch R.

(Before 1600)

$$
\text { VAGW }
$$

pimpinellifolia L. var. altaica (Willd.)

Thory

(Ca. 1820)

DCDO

'Platyphylla' [R. multiflora Thunb. ex J.A.Murr.]

Seven Sisters R.

(1817) $\mathrm{NCCB}$

'Plena' [R. moschata J.Herrm.]

(Before 1596) DCNA

\section{'Pompon Elegant'}

(Hybrid China)

$\mathrm{NCCB}$

'President de Seze'

(Gallica; 1836)

NCCB

'Prince Camille de Rohan'

(Hybrid Perpetual; 1861)

$$
\text { DCNA }
$$

'Ramona' [R. $\times$ anemonoides Rehd.]

Red Cherokee R.

[R. laevigata $\times \mathbf{R}$. ?odorata]

(1913)

$$
\text { SCLS VACW }
$$

'Rampant' [R. sempervirens L.]

(1830) $\mathrm{NCCB}$

'Reine des Violettes'

(Hybrid Perpetual; 1860) DCNA NCCB

\section{'Reine Victoria'}

(Bourbon; 1872) DCNA MDJS

'Reve d'Or'

(Noisette; 1869) FLCG

'Rivers' George IV'

(Hybrid China; 1820) DCNA NCCB

'Rose de Meaux'

(Centifolia; 1789)

NCMM
'Rose des Peintres' [R. centifolia L.]

(Centifolia)

DCNA NCMM

roxburghii Tratt. f. normalis Rehd. \& Wils.

Chestnut R., Bur R.

(Flowers single; 1908)
DCNA
GASH
MDHN MSMN

'Roxburghii' [R. roxburghii Tratt.]

(Flowers double; before 1814)

$\begin{array}{llll}\text { GAJS } & \text { MDHN } & \text { NCCB } & \text { VAWP } \\ \text { LARP } & \text { MSMV } & \text { SCBR } & \end{array}$

rubiginosa $\mathrm{L}$.

(Before 1551)

$\begin{array}{lll}\text { DCNA } & \text { MDHN } & \text { NCCB } \\ \text { GASH } & \text { MDJS } & \text { VAGW }\end{array}$

rubrifolia Villars

$=\boldsymbol{R}$. glauca

rugosa Thunb. ex J.A.Murr.

Rugosa R.

(Ca. 1845)

DCNA VACW VANB

'Safrano'

(Tea; 1839)

DCNA

'Salet'

(Moss; 1854)

DCNA

'Schneezwerg'

(Hybrid Rugosa)

MDJS

'Semiplena' [R. $\times$ alba L.]

(Alba)

[R. canina $\times \boldsymbol{R} . \times$ damascena] DCNA

'Serratipetala'

(China; 1912) NCCB

setigera Michx.

Prairie R

(1810)

$\begin{array}{lll}\text { DCNA } & \text { SCBR } & \text { VABF } \\ \text { NCCB } & \text { SCMP } & \end{array}$

'Shailer's Provence'

(Centifolia)

$\mathrm{NCCB}$

'Sombreuil'

(Tea; 1850)

DCNA 
spinosissima L.

$=\boldsymbol{R}$. pimpinellifolia

'Stanwell Perpetual' [R. $\times$ damascena var. semperflorens $\times \boldsymbol{R}$. pimpinellifolia]

(Hybrid Pimpinellifolia; 1838) DCNA MDHN

'Suzanne' [R. laxa $\times$ R. pimpinellifolia] (Shrub; 1950) MDJS NCCB

'The Fairy'

(Polyantha; 1932) VANB

'Tour de Malakoff'

(Centifolia; 1856) NCCB

'Trigintipetala' [R. $\times$ damascena Mill.] Kazanlik Rose

[R. gallica $\times$ R. moschata]

(Before 1850)

DCNA

'Tuscany'

(Gallica; 1596) DCNA

'Tuscany Superb'

(Gallica) DCNA NCCB

'Variegata' [R. centifolia L.] (1845) NCMM

'Variegata di Bologna'

(Bourbon; 1909) NCMM

'Versicolor' [R. $\times$ damascena Mill.]

York and Lancaster R.

[R. gallica $\times \boldsymbol{R}$. moschata]

(Before 1700) DCNA NCCB

'Versicolor' [R. gallica L.]

(Before 1581) DCNA MDFM VAGW

DESC NCCB

virginiana Mill.

Virginia R.

(Before 1807)

$\begin{array}{llll}\text { DCNA } & \text { NCCB } & \text { VACW } & \text { VAPO } \\ \text { MDRL } & \text { NCDU } & \text { VAGS } & \end{array}$

'Viridiflora' [R. chinensis Jacq.]

Green R.

(1855)

LASL wichuraiana Crepin

Memorial R.

(1891)

NCWC TNBO VAWE

wichuraiana Crepin var. poteriifolia

Koidz.

DCNA

woodsii Lindl.

(1820)

$\mathrm{VABF}$

xanthina Lindl. f. hugonis

(Hemsl.) Roberts

Father Hugo R.

(1899)

$\begin{array}{ll}\text { DCDO } & \text { MDJS NCBE } \\ \text { DEWG } & \text { MDLA }\end{array}$

ROSMARINUS L.

LAMIACEAE

Mint Family

officinalis $\mathrm{L}$.

Rosemary

FLUF SCCL VANB

LAHG VACW VATA

'Prostratus' [R. officinalis L.] GAJI

RUBUS L.

ROSACEAE

Rose Family

calycinoides Hayata \& Koidz.

DCNA VAGS VAPH

cockburnianus Hemsl.

MDBG

'Coronarius' [R. rosifolius Sm.]

Blackberry Rose
GAIH
MDFM SCWI

odoratus L.

Thimbleberry

MDJH

RUSCUS L.

LILIACEAE

Lily Family

aculeatus $\mathrm{L}$.

Butcher's Broom

$\begin{array}{llll}\text { DCNA } & \text { LAAL } & \text { NCEL } & \text { SCBR } \\ \text { GACC } & \text { LASL } & \text { NCTP } & \text { TXHN } \\ \text { GAUG } & \text { MSEN } & \text { NCWM } & \end{array}$


hypoglossum L.

TXDM

RUSSELIA Jacq.

SCROPHULARIACEAE

Figwort Family

equisetiformis Schlechtend. \& Cham.

Coral Plant

ALBG LAAL LASL

SABAL Adans.

ARECACEAE

Palm Family

minor (Jacq.f.) Pers.

Dwarf Palmetto

LASL NCTE SCBR SCWI

palmetto (Walt.) Lodd. ex J.A. \& J.H.

Schultes

Cabbage Palm

ALBG FLUF SCBR

SAGERETIA Brongn.

RHAMNACEAE

Buckthorn Family

thea (Osbeck) M.C.Johnst.

LAAL TXMS

theezans (L.) Brongn.

$=\mathrm{S}$. thea

SALIX L.

Willow

SALICACEAE

Willow Family

aegyptiaca L. DCNA MDSC

alba L.

White W.

$\begin{array}{lll}\text { NCDU } & \text { TNLW } & \text { VAHI } \\ \text { TNBM } & \text { VAGG }\end{array}$

alba $\times$ S. fragilis

DCSH DEMC MDSC

babylonica L.

Weeping $\mathrm{W}$.

$\begin{array}{llll}\text { ALEH } & \text { GANG } & \text { NCTP } & \text { VACW } \\ \text { ARRR } & \text { GAUG } & \text { NCWD } & \text { VALC } \\ \text { DCDO } & \text { LAHG } & \text { TNCT } & \text { VARG } \\ \text { DCNA } & \text { MDGD } & \text { TXWW } & \\ \text { GAAB } & \text { MDSJ } & \text { VACM } & \end{array}$

$\times$ bicolor Ehrh.

[S. arbuscula $\times$ S. nigricans] MDSC

$\times$ blanda Anderss.

Niobe W.

[S. babylonica $\times$ S. fragilis]

$$
\text { DCNA }
$$

caprea L.

Goat W.

DCNA DCRP

caprea $\times$ S. elaeagnos

DCNA

caprea $\times$ S. purpurea

DCNA

chaenomeles Kimura

DCNA

'Chrysocoma'

[S. alba var. vitellina $\times \mathbf{S}$. babylonica] ALBG DCNA VACW VAHI

cinerea L.

Gray W.

$$
\begin{array}{lll}
\text { DCNA } & \text { MDPJ } & \text { SCCU } \\
\text { GACG } & \text { MDSC } & \text { TNTV }
\end{array}
$$

cordata Michx. MDSC

'Crispa' [S. babylonica L.] TNTV

$\times$ dasyclados Wimmer

[S. caprea $\times$ S. cinerea $\times$ S. viminalis] MDSC

discolor Muhl. DCNA

elaeagnos Scop. DEMC MDSC

'Eugenei' [S. purpurea L.] DCNA

fragilis $\mathrm{L}$. DCAG

'Ginme' [S. $\times$ tsugaluensis Koidz.]

[S. integra $\times$ S.vulpina] MDSC

'Ginryu' [S. subopposita Miq.] MDBG

gracilistyla Miq. DCNA SCBR VANB 
hookeriana Barratt var. tomentosa

J.K.Henry

DCNA

humilis Marsh.

DCNA

integra Thunb. ex J.A.Murr. DCNA

interior Rowlee MDSC

irrorata Anderss. MDSC

japonica Thunb. ex J.A.Murr. DCNA

$\times$ laestadiana Hartm.

[S. cineria $\times$ S. lapponum] MDSC

'Melanostachys' [S. gracilistyla Miq.] DCNA

miyabeana Seemen DCNA

muscina Dode DCNA MDSC

$\times$ myricoides Muhl.

[S. rigida $\times$ S. sericea] MDSC

myrsinifolia Salisb. DCNA MDSC

nigra $\mathrm{L}$.

Black W.

$\begin{array}{llll}\text { ALAU } & \text { DEWG } & \text { LAAL } & \text { MSMV } \\ \text { ALTD } & \text { GAFN } & \text { MSBN } & \text { NCDU }\end{array}$

'Pendula' [S. alba L. var. vitellina (L.)

Stokes] DEWG

'Pendula' [S. purpurea L.] MDSC

pentandra $\mathrm{L}$. DCNA

$\times$ pontederana Willd.

[S. cineria $\times$ S. purpurea] MDSC

purpurea L.

Basket W. DCNA MDSC purpurea L. var. amplexicaulis (Bory \& Chaub.) Boiss.

GACG

purpurea L. f. gracilis (Gren. \& Godr.) Schneid. DCNA MDSC

repens L. ssp. argentea (Sm.) A.A. \& E.G. Camus DCNA MDSC

rigida Muhl. MDSC

$\times$ rubra Huds.

[S. purpurea $\times$ S. viminalis] MDSC

'Sekka' [S. sachalinensis F.Schmidt] DCNA MDBG VANB GACG MDGD

$\times$ sepulcralis Simonk.

[S. alba $\times \mathbf{S}$. babylonica] DCNA DEMC MDKN MDSJ

sericans Tausch ex A. Kerner MDSC

sericea Marsh. DCNA

$\times$ seringeana Gaudin

[S. caprea $\times$ S. elaeagnos] MDSC

$\times$ smithiana Willd.

[S. caprea $\times$ S. viminalis] MDSC

syrticola Fern. MDSC

'Tortuosa' [S. babylonica L.] Contorted Hankow W.

$\begin{array}{llll}\text { ALBH } & \text { GACD } & \text { MDLA } & \text { VAWR } \\ \text { DEWG } & \text { GAUG } & \text { VACW } & \\ \text { FLUF } & \text { MDBG } & \text { VATA } & \end{array}$

'Tortuosa' [S. matsudana Koidz.]

= 'Tortuosa' [S. babylonica]

udensis Traut. DCNA

variegata Franch. MDSC

vulpina Anderss. DCNA 
SALVIA L. Sage LAMIACEAE Mint Family

greggii A.Gr.

Autumn S. LAHG SCWI VACW VAPH

leucantha Cav.

Mexican Bush S.

SCWI

SAMBUCUS L. Elder CAPRIFOLLACEAE Honeysuckle Family (Contributed by T. R. Dudley)

'Aurea' [S. nigra L.]

MDBG

canadensis $\mathrm{L}$.

American E.

$\begin{array}{llll}\text { ALBG } & \text { LAAL } & \text { TNSC } & \text { VAHR } \\ \text { DCNA } & \text { MDTD } & \text { VAGW } & \end{array}$

ebulus L.

Dwarf E.

DCNA

racemosa L. ssp. pubens (Michx.) H.House American Red E. MDWP

racemosa ssp. sieboldiana (Miq.) Hara Asiatic Red E.

DCNA

sieboldiana B1. ex Graebn.

= S. racemosa $\mathrm{ssp}$. sieboldiana

SANTOLINA L. ASTERACEAE Aster Family

chamaecyparissus $\mathrm{L}$.

Lavender Cotton MDBG MDLT TNUT
SAPINDUS L. SAPINDACEAE
Soapberry Soapberry Family drummondii Hook. \& Arn.

Texas S.

$\begin{array}{llll}\text { ALUA } & \text { GATG } & \text { MSMN } & \text { VABF } \\ \text { DCNA } & \text { LAAL } & \text { TNSC } & \\ \text { GAMP } & \text { LAHP } & \text { TXZH } & \end{array}$

marginatus Willd.

Florida S.

GACB

mukorossi Gaertn.

Chinese S.

$\begin{array}{ll}\text { ALCS } & \text { GACR } \\ \text { FLUF } & \text { GAIS }\end{array}$

SAPIUM P.Br.

Tallow Tree

EUPHORBLACEAE

Spurge Family

japonicum (Sieb. \& Zucc.) Pax \& K.Hoffm. MDBG

sebiferum (L.) Roxb.

Chinese Tallow T.

$\begin{array}{llll}\text { ALAU } & \text { GAIS } & \text { SCCL } & \text { VACW } \\ \text { ALBG } & \text { LAAL } & \text { SCJC } & \\ \text { ALCG } & \text { LASL } & \text { SCMG } & \\ \text { FLWG } & \text { LATU } & \text { TXGS } & \end{array}$

SARCOCOCCA Lindl. BUXACEAE

Sweet Box Boxwood Family

hookeriana Baill. var. digyna Franch. GACG

hookeriana Baill. var. humilis Rehd. \& Wils.

$\begin{array}{lll}\text { DCNA } & \text { GAWH } & \text { MDSJ } \\ \text { DEMC } & \text { MDBG } & \text { NCGC } \\ \text { DEWG } & \text { MDLT } & \text { SCWI }\end{array}$

ruscifolia Stapf

Fragrant Sweet B.

NCWM VACW VANB

SASA Makino \& Shibata

POACEAE

Grass Family

veitchii (Carr.) Rehd.

Kuma Bamboo

LAAL TXLL

SASSAFRAS T.Nees \& Eberm.

LAURACEAE

Laurel Family

albidum (Nutt.) Nees

Sassafras

MDJS VACW VAWR

MDLA VAPH 


SATUREJA L.
LAMLACEAE
montana L.
Winter S.
DCNA

SCHINUS

Peppertree

ANACARDIACEAE

Cashew Family

terebinthifolius Raddi

Brazilian P.

FLUF

SCHIZOPHRAGMA Sieb. \& Zucc. SAXIFRAGACEAE (Hydrangeoideae)

Saxifrage Family

hydrangeoides Sieb. \& Zucc.

Japanese Hydrangea Vine
ALUA
MDGD
MDMG
DCNA
MDLT

\section{SCIADOPITYS Sieb. \& Zucc. \\ TAXODIACEAE Taxodium Family}

verticillata (Thunb. ex J.A.Murr.) Sieb. \& Zucc.

Umbrella Pine

$\begin{array}{llll}\text { DCNA } & \text { MDBG } & \text { MDLT } & \text { VANP } \\ \text { DEWG } & \text { MDJS } & \text { VABF } & \end{array}$

SEBASTIANA Spreng.

EUPHORBIACEAE

Spurge Family

ligustrina (Michx.) Muell.-Arg. GAEC

SEMIARUINDINARIA Makino ex Nakai POACEAE Grass Family

fastuosa (Latour-Marl. ex Mitf.) Makino ex Nakai SCJC

SENECIO L.

ASTERACEAE

Aster Family

salignus DC.

FLUF

SEQUOIA Endl.

TAXODIACEAE

'Adpressa' [S. sempervirens (D.Don) Endl.] DCNA

sempervirens (D.Don) Endl.

Coast Redwood

$\begin{array}{llll}\text { ALAU } & \text { SCMG } & \text { VACW } & \text { VAOD } \\ \text { MDPL } & \text { TNMB } & \text { VAMR } & \text { VAWM } \\ \text { SCHA } & \text { VABR } & \text { VANB } & \end{array}$

SEQUOLADENDRON J.Buchh.

TAXODIACEAE

Giant Sequoia Taxodium Family

giganteum (Lindl.) J.Buchh.

G. S., Big Tree

DCCG NCBR

SERENOA Hook.f.

ARECACEAE

Palm Family

repens (Bartram) Small

Saw Palmetto

FLUF

SERISSA Comm. ex Juss.

RUBIACEAE

Madder Family

foetida (L.f.) Lam.

= S. japonica

japonica (Thunb.) Thunb.

$\begin{array}{llll}\text { ALFS } & \text { FLUF } & \text { GAUG } & \text { VANB } \\ \text { DCNA } & \text { GAJI } & \text { LALG } & \\ \text { FLCG } & \text { GAJY } & \text { SCCU } & \\ \text { FLLE } & \text { GATS } & \text { VACW } & \end{array}$




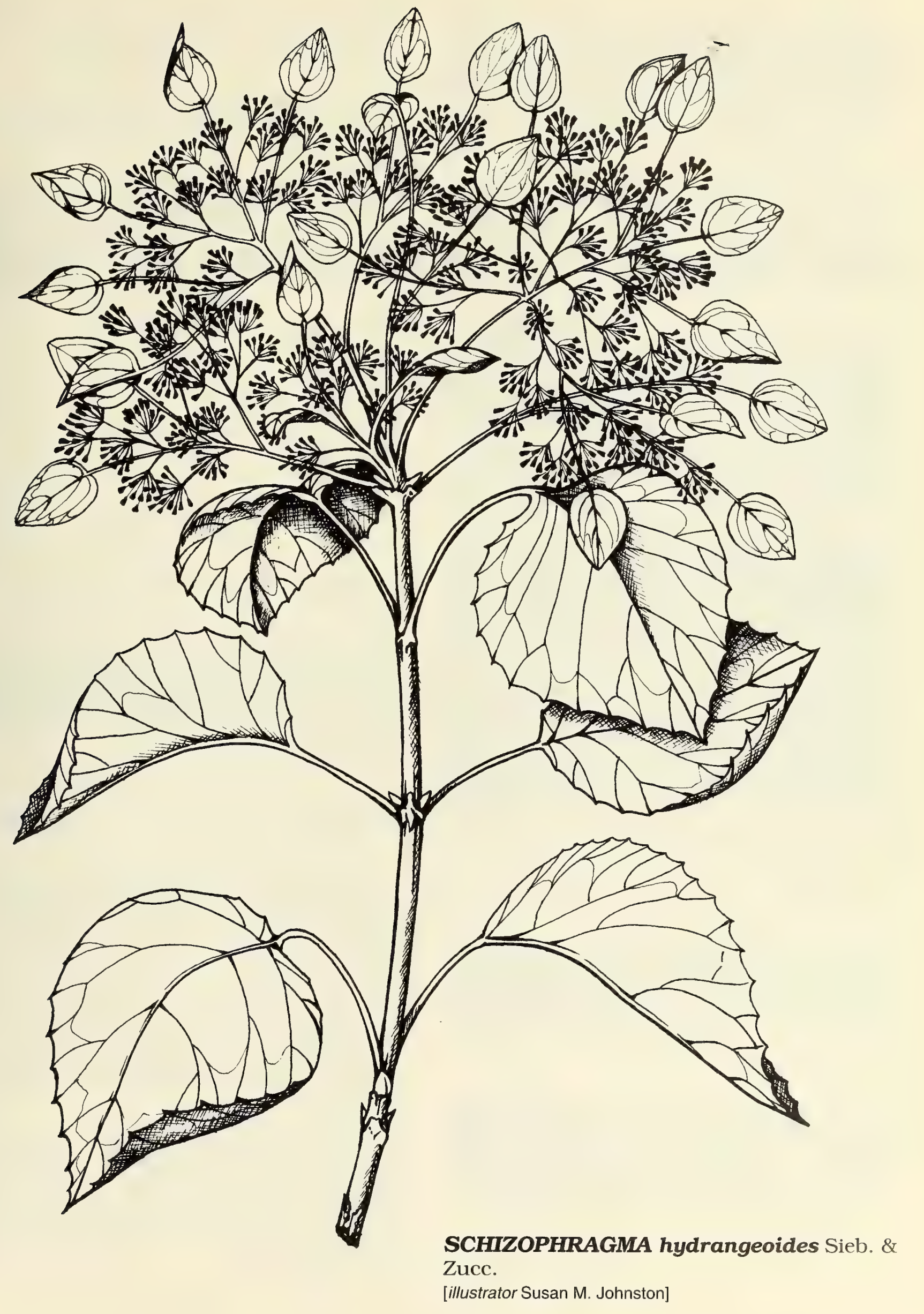


SESBANIA Scop.

FABACEAE (Faboideae)

Bean Family

drummondii (Rydb.) Cory

SCWI

punicea (Cav.) Benth.

LAAL NCEG SCMP

LASL SCBR SCWI

tripetii (Poit.) F.T.Hubb.

= S. punicea

SEVERINIA Tenore ex Endl.

RUTACEAE

Citrus Family

buxifolia (Poir.) Tenore

Chinese Box Orange
FLCG
FLUF
LAAL
FLMG
GAJI
VACW

SHIBATAEA Makino ex Nakai

POACEAE

Grass Family

kumasaca (Zoll.) Makino

NCBE

\section{SINARUNDINARIA Nakai POACEAE \\ Grass Family}

murielae (Gamble) Nakai

= Thamnocalamus spathaceus

nitida (Mitf.) Nakai

DCNA

\section{SIPHONOSMANTHUS Stapf \\ OLEACEAE \\ Olive Family}

delavayi (Franch.) Stapf
ALTD MDJW

SKIMIMA Thunb.

Skimmia

RUTACEAE

Citrus Family

'Fisheri' [S. japonica Thunb.] DCNA

$\times$ foremanii H.Knight

[S. japonica $\times$ S. reevesiana] VAGS

'Fructo-albo' [S. japonica Thunb.]

White-fruited S. DCNA

japonica Thunb.

DCNA GACG MDLT

DEMC MDBG VAPH

japonica Thunb. var. repens (Nakai) Ohwi DCNA

laureola Sieb. \& Zucc.

DCNA

'Nana' [S. japonica Thunb.]

DEMC MDBG

reevesiana Fort. DCNA VAPH

'Veitchii' [S. japonica Thunb.] DCNA

'Wisley Red' [S. japonica Thunb.] DCNA

SMILAX L. SMILACACEAE

Greenbrier Cat-brier Family

biflora Sieb. ex Miq SCWI

\section{laurifolia L.} SCWI

pumila Walt. SCBR SCWI

smallii Morong ALON GAUG TNHD

SOLANUM L. SOLANACEAE Nightshade Family

diphyllum $\mathrm{L}$.

FLUF TXGS

jasminoides Paxt.

Potato Vine

GACL MSMN TXGM

rantonnetii Carr.

Blue Potato Bush

TXPS TXRS 
seaforthianum Andr.

Brazilian Nightshade

ALBG

wendlandii Hook.f.

Paradise Flower

LALG

SOPHORA L.

FABACEAE (Faboideae) Bean Family

affinis $\mathrm{T} . \& \mathrm{G}$.

LASL TXDM

davidii (Franch.) Skeels

SCWI TNLW VABF

'Dot' [S. japonica L.]

ALTD

japonica $\mathrm{L}$.

Pagoda Tree, Chinese Scholar Tree

$\begin{array}{lll}\text { DCCG } & \text { DEMC } & \text { VAMP } \\ \text { DCNA } & \text { TNLW } & \text { VASC }\end{array}$

DCNA TNLW VASC

'Pendula' [S. japonica L.] MDBG MDCP MDHN VAMP

'Regent' [S. japonica L.] DCNA

secundiflora (Ort.) Lag. ex DC.

Mescal Bean

$\begin{array}{llll}\text { FLJH } & \text { LAHG } & \text { TXJS } & \text { TXSH } \\ \text { FLMG } & \text { MSMN } & \text { TXPS } & \text { VACW }\end{array}$

viciifolia Hance

$=\mathbf{S}$. davidii

SORBARIA (Ser. ex DC.) A.Braun

False Spirea

ROSACEAE

Rose Family

kirilowii (Regel) Maxim. VAGO

tomentosa (Lindl.) Rehd. var. tomentosa DCBG DCNA NCBE

$\times$ SORBARONLA Schneid. ROSACEAE

[Aronia $\times$ Sorbus]

Rose Family

'Brilliantissima'

DCNA fallax Schneid.

[Aronia melanocarpa $\times$ Sorbus aucuparia] MDAB

sorbifolia (Poir.) Schneid.

[Aronia melanocarpa $\times$ Sorbus americana] DCNA

XSORBOCOTONEASTER Pojark.

ROSACEAE

Rose Family

[Cotoneaster $\times$ Sorbus]

pozdnjakovii Pojark.

[Cotoneaster niger $\times$ Sorbus sibirica] DCNA

$\times$ SORBOPYRUS Schneid.

ROSACEAE

Rose Family

[Pyrus $\times$ Sorbus]

'Bulbiformis' [ $\times$ S. auricularis (Knoop)

Schneid.]

[Pyrus communis $\times$ Sorbus aria] MDSC

SORBUS L.

Mountain Ash

ROSACEAE

Rose Family

alnifolia (Sieb. \& Zucc.) K.Koch DCNA MDGD VABF

americana Marsh.

American Mountain A. DCWH DEWG NCAS

'Apricot Queen' DCNA

aucuparia L.

Rowan

$\begin{array}{lll}\text { DCMS } & \text { DEMC } & \text { TNBO } \\ \text { DCNA } & \text { NCAS } & \end{array}$

'Cardinal' [S. aucuparia L.] DCNA

domestica L.

Service Tree NCMS

'Fastigiata' [S. aucuparia L.] MDKN

hybrida L. VASK 
'Magnifica' [S. aria (L.) Crantz] DCNA

'Pendula' [S. aucuparia L.] MDKN

pohuashanensis (Hance) Hedl. MDKN

'Red Copper Glow' DCNA

torminalis Crantz DCNA

SPARTIUM L.

FABACEAE (Faboideae)

Bean Family

junceum L.

Spanish Broom

SCWI VACW

SPIRAEA L. Spirea ROSACEAE Rose Family

alba Du Roi DCNA

albiflora (Miq.) Zab. DCSH

'Alpestris' [S. $\times$ lemoinei Zab.]

[S. bullata $\times$ S. $\times$ bumalda] DEWG

'Alpina' [S. japonica L.f.]

$\begin{array}{llll}\text { DCNA } & \text { GAEC } & \text { VAGS } & \text { VATA } \\ \text { GAAM } & \text { SCCU } & \text { VAPH } & \end{array}$

'Anthony Waterer' [S. $\times$ bumalda Burv.]

[S. albiflora $\times \mathbf{S}$. japonica]

MDBN MDJS NCAS SCBR

arcuata Hook.f.

DCNA MDDF

$\times$ arguta Zab.

[S. multiflora $\times$ S. thunbergii]

DEWG

betulifolia Pall.

DCNA

$\times$ billiardii Herincq

[S. douglasii $\times$ S. salicifolia]

DEWG VACW blumei G.Don DCNA

bullata Maxim. DEWG MDJW

$\times$ bumalda Burv.

[S. albiflora $\times$ S. japonica] TNBM TNFE VAPH

cantoniensis Lour. Reeves S.

$\begin{array}{llll}\text { DCWR } & \text { FLUF } & \text { MDPJ } & \text { SCPP } \\ \text { DEWG } & \text { GACG } & \text { NCTE } & \text { TNDG } \\ \text { FLJU } & \text { LAAP } & \text { SCMG } & \\ \text { FLMG } & \text { LATU } & \text { SCMM } & \end{array}$

chinensis Maxim. GAIS SCPP

'Compacta' [S. thunbergii Sieb. ex. Bl.] DCNA

'Crispa' [S. × bumalda Burv.]

[S. albiflora $\times$ S. japonica] ALBH VAPH

'Goldflame' [S. $\times$ bumalda Burv.]

[S. albiflora $\times$ S. japonica] DCNA

japonica L.f.

Japanese $\mathrm{S}$.

$\begin{array}{llll}\text { DCNA } & \text { LAHG } & \text { NCBE } & \text { VATA } \\ \text { DEWG } & \text { MDBG } & \text { SCCU } & \end{array}$

'Lanceata' [S. cantoniensis Lour.]

$\begin{array}{llll}\text { ALBG } & \text { FLLT } & \text { LAAP } & \text { NCSM } \\ \text { ALBH } & \text { FLMG } & \text { LALG } & \text { NCTE } \\ \text { ALEH } & \text { FLUF } & \text { LASL } & \text { SCLM } \\ \text { DEWG } & \text { GAJI } & \text { MDGJ } & \text { TNDG } \\ \text { FLJU } & \text { GAVI } & \text { MDLA } & \end{array}$

$\times$ margaritae Zab.

[S. japonica $\times$ S. $\times$ superba] DEWG MDJH

miyabei Koidz. DCNA

nipponica Maxim. var. tosaensis (Yatabe) Makino DCWH VATA

prunifolia Sieb. \& Zucc.

Bridal Wreath

ALIT FLDG MDMM VAPH

ARRB FLMG SCMP VATA

DCDO GACG TNCT VAWR 
reevesiana Lindl.

$=\mathbf{S}$. cantoniensis

salicifolia L.

Willowleaf S.

$\mathrm{SCCU}$

$\times$ sanssouciana K.Koch

[S. douglasii $\times$ S. japonica]

DCNA DEWG

'Snowmound' [S. nipponica Maxim.

var. tosaensis (Yatabe) Makino]

MDBG SCCU TNTV

MDBN TNSN

'Swan Lake' [S. trilobata L.] DEWG

thunbergii Sieb. ex Bl.

$\begin{array}{llll}\text { ALBG } & \text { FLUF } & \text { SCLM } & \text { TNDG } \\ \text { DEWG } & \text { GAGM } & \text { SCMP } & \\ \text { FLMG } & \text { NCBE } & \text { SCUC } & \end{array}$

tomentosa $\mathrm{L}$.

Hardhack

$\mathrm{NCDB}$

$\times$ vanhouttei (C. Briot) Zab.

Vanhoutte S., Bridal Wreath

[S. cantoniensis $\times$ S. trilobata]

$\begin{array}{llll}\text { ARRB } & \text { GACG } & \text { NCGR } & \text { VAGJ } \\ \text { DCDO } & \text { MDHF } & \text { SCLM } & \text { VAMP } \\ \text { DCWR } & \text { MDLT } & \text { SCMP } & \\ \text { DEWG } & \text { NCBE } & \text { VACW } & \end{array}$

STACHYURUS Sieb. \& Zucc.

\section{STACHYURACEAE \\ Stachyurus Family}

chinensis Franch.

DCNA

'Issai' [S. praecox Sieb. \& Zucc.]

MDMG

praecox Sieb. \& Zucc.

DCNA MDLT VAGS

STAPHYLEA L.

STAPHYLEACEAE $\quad$ Bladdernut Family

colchica Steven

DCNA DEWG

pinnata $\mathrm{L}$.

NCBE trifolia L.

ALAU DCNA VACW VAGW

STEPHANANDRA Sieb. \& Zucc.

ROSACEAE

Rose Family

'Crispa' [S. incisa (Thunb. ex J.A.Murr.)

Zab.]

MDBG

incisa (Thunb. ex J.A.Murr.) Zab.

Lace Shrub

DEMC MDLN NCBE

DEWG MDLT

tanakae (Franch. \& Sav.) Franch. \& Sav. DCNA

\section{STEWARTIA L.}

THEACEAE

Tea Family

malacodendron $\mathrm{L}$.

Silky Camellia

GAEC MDKN MDLT MDMG

monadelpha Sieb. \& Zucc.

$\begin{array}{lll}\text { ALBG } & \text { MDBG } & \text { SCWI } \\ \text { DCNA } & \text { MDLT } & \text { TNAB } \\ \text { GARW } & \text { NCBE } & \text { VAGS }\end{array}$

ovata (Cav.) Weatherby

Mountain Camellia

DCNA TNPW

pentagyna L'Her.

= S. ovata

pseudocamellia Maxim.

Japanese S.

$\begin{array}{llll}\text { DCCG } & \text { MDFA } & \text { NCDB } & \text { TNAB } \\ \text { DCNA } & \text { MDLT } & \text { NCDU } & \text { VAGS } \\ \text { DEMC } & \text { MDMG } & \text { NCEL } & \text { VATA } \\ \text { MDBG } & \text { NCBE } & \text { SCBR } & \end{array}$

rostrata Spongberg

DEWG VAGS

serrata Maxim.

VAGS

sinensis Rehd. \& Wils.

DCNA GAEC TNSN 
STIGMAPHYLLON JUSS. MALPIGHIACEAE

Malpighia Family

ledifolium (HBK.) Small TXCT

STRANVAESIA Lindl. ROSACEAE Rose Family

davidiana Decne.

Chinese S.

$\begin{array}{llll}\text { DCCG } & \text { DCWA } & \text { MDLT } & \text { TNUT } \\ \text { DCNA } & \text { MDBG } & \text { TNLW } & \end{array}$

'Lutea' [S. davidiana Decne.]

Yellow-fruited Chinese S. DCCG

niitakayamensis (Hayata) Hayata VAPH

STYRAX L.

STYRACACEAE

americanus Lam.

American Snowbell TNSN TXMA

'Carillon' [S. japonica Sieb. \& Zucc.] DCNA

dasyanthus Perk. MDKN

grandifolius Ait.

Bigleaf Snowbell

$\begin{array}{lll}\text { LAHG } & \text { NCDU } & \text { VACW } \\ \text { NCAG } & \text { SCBR } & \end{array}$

japonicus Sieb. \& Zucc.

Japanese Snowbell

$\begin{array}{llll}\text { DCSH } & \text { MDGJ } & \text { TNSG } & \text { VACW } \\ \text { DEWG } & \text { MDLT } & \text { TNSN } & \text { VAGS } \\ \text { MDBG } & \text { TNSC } & \text { TNUT } & \end{array}$

obassia Sieb. \& Zucc.

Fragrant Snowbell

$\begin{array}{llll}\text { DCDO } & \text { MDBG } & \text { MDPJ } & \text { VANB } \\ \text { DEEM } & \text { MDGJ } & \text { TNTV } & \text { VATA } \\ \text { MDAB } & \text { MDLT } & \text { VAGS } & \end{array}$

'Pink Chimes' [S. japonica Sieb. \& Zucc.] DCNA MDBG

platanifolius Engelm.

Texas Snowbell MDFM
SYAGRUS Mart.

ARECACEAE

Palm Family

romanzoffianum (Cham.) Glassman

Queen Palm

FLUF

romanzoffianum $\times$ Butia capitata FLUF

$\times$ SYCOPARROTIA P.Endress \&

J.Anliker

HAMAMELIDACEAE Witch-hazel Family

[Parrotia $\times$ Sycopsis]

semidecidua P.Endress \& J.Anliker

[Parrotia persica $\times$ Sycopsis sinensis] DCNA

SYCOPSIS Oliv.

HAMAMELIDACEAE Witch-hazel Family

sinensis Oliv.

DCNA MDBG

SYMPHORICARPOS Duham. Snowberry CAPRIFOLIACEAE Honeysuckle Family

(Contributed by T. R. Dudley)

albus (L.) S.F.Blake

MDLG MDPJ

albus (L.) S.F.Blake var. laevigatus (Fern.) S.F.Blake MDJS

$\times$ chenaultii Rehd.

[S. microphyllus $\times \mathbf{S}$. orbiculatus] VARE

orbiculatus Moench

Coralberry, Indian Currant

$\begin{array}{llll}\text { ALAU } & \text { NCBE } & \text { VAHB } & \text { VASP } \\ \text { DCCG } & \text { TXDC } & \text { VAPO } & \end{array}$

SYMPLOCOS Jacq. SYMPLOCACEAE

Sweetleaf Family

chinensis (Lour.) Druce f. pilosa (Nakai) Ohwi = S. paniculata 

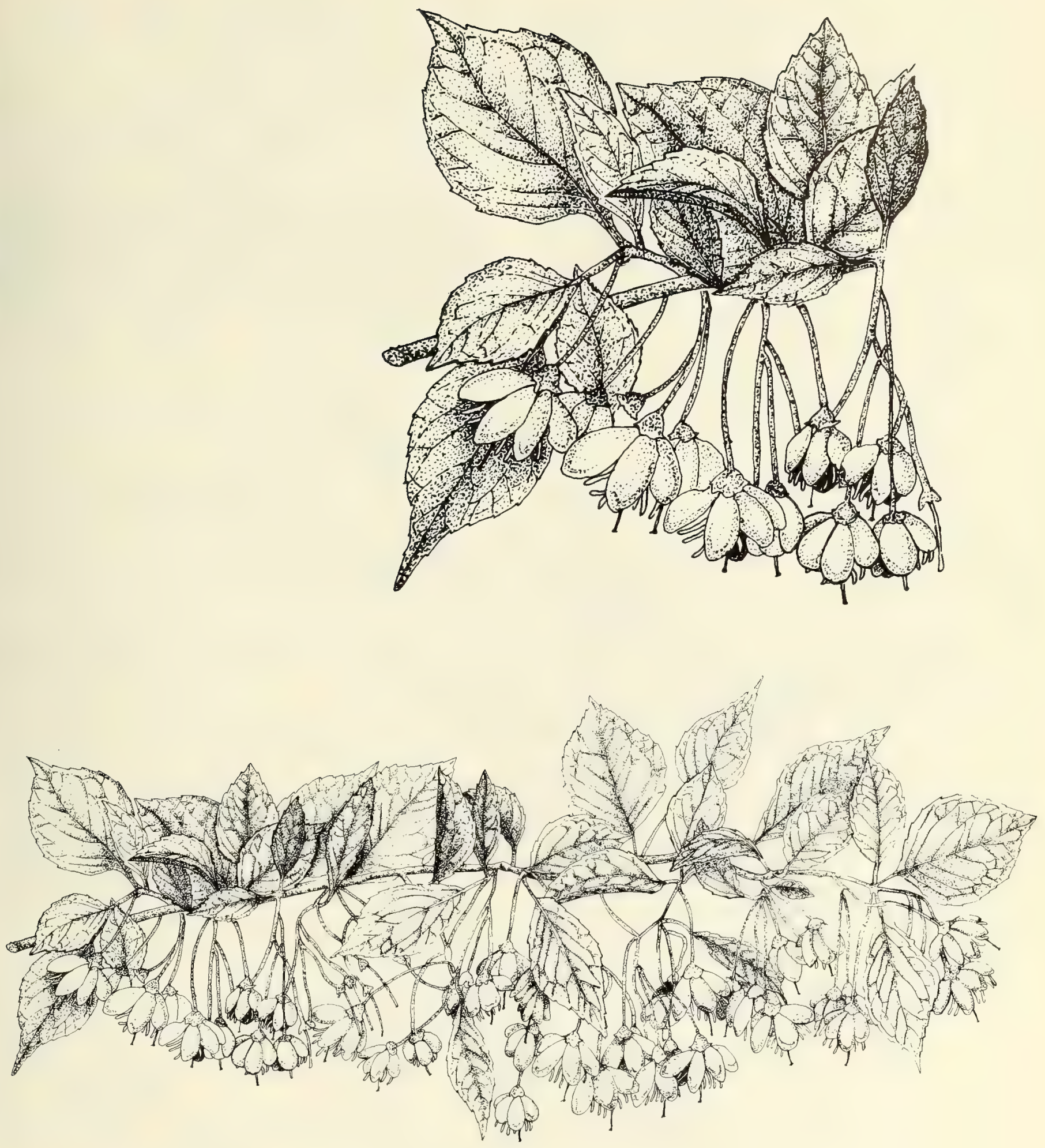

STYRAX japonicus Sieb. \& Zucc.

[illustrator Lillian Nicholson Meyer] 

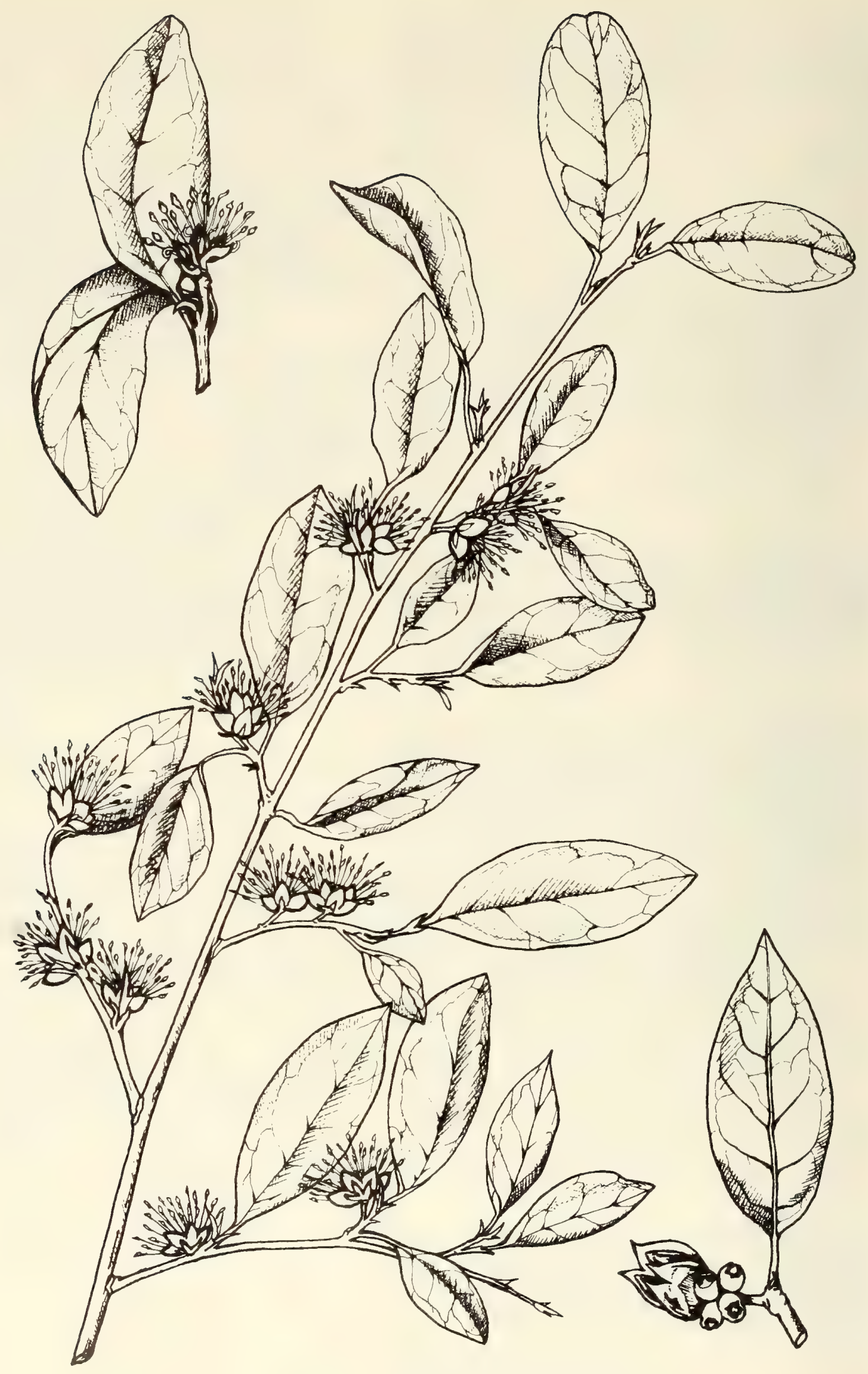

SYCOPSIS sinensis Oliv.

[illustrator Susan M. Johnston] 
lucida Sieb. \& Zucc. ALTD

paniculata (Thunb. ex J.A.Murr.) Miq. DCNA MDBG MDPJ

DEEM MDKN TNDR

tinctoria (L.) L'Her.

Horse Sugar, Yellow-wood
ALBG
GAEC
NCOP
FLMG
LARP SCBR

SYRINGA L. OLEACEAE

Lilac Olive Family

'Alba' [S. vulgaris L.] $\begin{array}{llll}\text { ARRB } & \text { MDMM } & \text { VAGW } & \text { VAWR } \\ \text { MDLA } & \text { VACW } & \text { VASP } & \end{array}$

'Alba Plena' [S. vulgaris L.]

Double White L.

MDLA

\section{'Alexander's Perfection'} DCNA

amurensis var. japonica (Maxim.) Franch. \& Sav.

$=\mathrm{S}$. reticulata

'Annabelle' [S. oblata Lindl. var. dilatata (Nakai) Rehd.] DCNA

'Buffon' [S. $\times$ hyacinthiflora (V.Lemoine) Rehd.]

[S. oblata $\times$ S. vulgaris]

DCNA

'Charles Giant' [S. $\times$ hyacinthiflora

(V.Lemoine) Rehd.]

[S. oblata $\times$ S. vulgaris]

DEWG

'Charles Joly' [S. vulgaris L.] DCNA

'Charles Nordine' [S. vulgaris L.] DCNA

$\times$ chinensis Willd.

Chinese L.

[S. $\times$ persica $\times$ S. vulgaris] DCNA

'Crayton' [S. villosa Vahl]

Late Lilac

DEWG
'Decaisne' [S. vulgaris L.] DCNA

'Esther Staley' [S. vulgaris L.] DCNA

'Ethel M. Webster' [S. $\times$ prestoniae

McKelvey]

[S. reflexa $\times$ S. villosa] DEWG

'Henri Robert' [S. vulgaris L.] DCNA

$\times$ henryi Schneid.

[S. josikaea $\times$ S. villosa] DEWG

'Jessica'

DCNA

josikaea Jacq.f. ex Reichenb.

Hungarian L. NCBE

'Jules Ferry' [S. vulgaris L.] DCNA

'Katherine Havemeyer' [S. vulgaris L.] DCNA

laciniata Mill.

Cutleaf L.

$\begin{array}{llll}\text { DCNA } & \text { GAFN } & \text { MDWP } & \text { SCCU } \\ \text { DEWG } & \text { GAJS } & \text { NCBE } & \end{array}$

'Laciniata' [S. persica L.] MDWP NCBE SCCU

'Lamartine' [S. $\times$ hyacinthiflora (Lem.) Rehd.]

[S. oblata $\times$ S. vulgaris]

DCNA DEWG

'Laurentian' [S. oblata Lindl. var. dilatata (Nakai) Rehd.]

DCNA

'Leon Gambetta' [S. vulgaris L.] DCNA

'Marechal Lannes' [S. vulgaris L.] DCNA

'Maurice Barres' [S. vulgaris L.] DCNA

meyeri Schneid.

DCNA DEWG VAGS

microphylla Diels

DEWG 
'Miss Ellen Willmott' [S. vulgaris L.] DCNA

'Miss Kim' [S. patula (Palib.) Nakai] MDJS

'Mme. Charles Souchet' [S. vulgaris L.] DCNA

'Monge' [S. vulgaris L.] DCNA

'Mrs. Edward Harding' [S. vulgaris L.] DCNA

oblata Lindl. var. dilatata (Nakai) Rehd. VATA

patula (Palib.) Nakai DCNA VACW

persica $\mathrm{L}$.

Persian L. $\begin{array}{llll}\text { DEWG } & \text { MDWP } & \text { VACW } & \text { VAWR } \\ \text { MDPJ } & \text { SCCU } & \text { VAGW } & \end{array}$

'Pocohantas' [S. $\times$ hyacinthiflora (Lem.) Rehd.]

[S. oblata $\times$ S. vulgaris]

DEWG

'President Grevy' [S. vulgaris L.] DCNA

'Primrose' [S. vulgaris L.] DEWG

'Priscella' [S. vulgaris L.] DCNA

pubescens Turcz. DCNA DEWG

'Purpurea' [S. vulgaris L.] Common Lilac

$\begin{array}{lll}\text { ARRB } & \text { MDLA } & \text { TNCT } \\ \text { DCWH } & \text { MDMM } & \text { VAGW } \\ \text { GAAM } & \text { MDSM } & \text { VASP }\end{array}$

'Rene Jarry-Desloges' [S. vulgaris L.] DCNA

reticulata (Bl.) Hara

Japanese Tree L.

$\begin{array}{llll}\text { DCNA } & \text { DEWG } & \text { MDHC } & \text { VAMP } \\ \text { DCSH } & \text { GAOH } & \text { TNRW } & \text { VATA } \\ \text { DCWH } & \text { MDBR } & \text { TNTV } & \end{array}$

'Sarah Sands' [S. vulgaris L.] DCNA
'Superba' [S. microphylla Diels] DEWG

'Vaubon' [S. $\times$ hyacinthiflora (Lem.) Rehd.] [S. oblata $\times$ S. vulgaris] DCNA

velutina Komar.

= S. patula

villosa Vahl

Late L.

MDBG

vulgaris L. var. alba Weston

= 'Alba'

vulgaris L. var. purpurea Weston

= 'Purpurea'

yunnanensis Franch. DCNA

'Zulu' [S. vulgaris L.] DCNA

TABERNAEMONTANA L. APOCYNACEAE Dogbane Family

coronaria (Jacq.) Willd.

= $\mathrm{T}$. divaricata

divaricata (L.) R.Br. ex Roem. \&

J.A.Schultes

Crape Jasmine

LALG

TATWANIA Hayata

TAXODIACEAE

Taxodium Family

cryptomerioides Hayata

ALTD DCNA GACG

TAMARIX L.

TAMARICACEAE

Tamarisk Family

parviflora $\mathrm{DC}$.

DEWG VACW

pentandra Pall.

$=T$. ramosissima

ramosissima Ledeb.

DCNA TXHN VAGS 
'Rubra' [T. ramosissima Ledeb.] DCNA

'Summer Glow' [T. ramosissima Ledeb.] DCNA

TAXODIUM L.C.Rich. TAXODIACEAE

Bald Cypress Taxodium Family

ascendens Brongn.

$=T$. distichum var. nutans

distichum (L.) L.C.Rich. var. distichum

Bald Cypress

$\begin{array}{llll}\text { ALAU } & \text { DCWH } & \text { NCCA } & \text { VAMC } \\ \text { ALBG } & \text { DEMC } & \text { SCBR } & \text { VAMP } \\ \text { ARUM } & \text { GAMW } & \text { SCMG } & \\ \text { DCCG } & \text { MDBG } & \text { TNFE } & \\ \text { DCNA } & \text { MSEP } & \text { TNSC } & \end{array}$

distichum (L.) L.C.Rich. var. imbricarium (Nutt.) Croom

= $\mathrm{T}$. distichum var. nutans

distichum (L.) L.C.Rich. var. nutans (Ait.)

Sweet

Pond C.

$\begin{array}{llll}\text { ALAU } & \text { DEMC } & \text { MDCP } & \text { SCBR } \\ \text { ALBG } & \text { FLMG } & \text { NCCA } & \text { VAMP } \\ \text { DCNA } & \text { MDBG } & \text { NCOP } & \end{array}$

mucronatum Tenore

Montezuma Cypress
LASL
TXDM
TXPS

'Nutans' [T. distichum (L.) L.C.Rich.]

$=T$. distichum var. nutans

'Pendens' [T. distichum (L.) L.C.Rich.] DCNA

TAXUS L.

TAXACEAE

'Adpressa' [T. baccata L.] NCBE

'Adpressa Fowle' [T. baccata L.] DCNA

\section{'Amersfoort' [T. baccata L.]} DCNA MDTD

\section{'Aurea' [T. baccata L.]}

Golden Y. DCSH

\section{'Aurea Marginata' [T. baccata L.] VABR VAMP}

baccata $\mathrm{L}$.

English Y.
DCCG
MDFM NCTE
VASJ
DCNA
MDRT
VACS
VAWR

baccata L. f. fastigiata (Lindl.) Pilger $\begin{array}{llll}\text { FLMG } & \text { MDHN } & \text { VABA } & \text { VAMP } \\ \text { GABG } & \text { MDJH } & \text { VAGS }\end{array}$

canadensis Marsh.

Canadian Y., Ground Hemlock SCBR VADW

'Citation' [T. $\times$ media Rehd.]

[T. baccata $\times T$. cuspidata] DCNA

cuspidata Sieb. \& Zucc.

Japanese Y.
ALTD
DCCG MDDF
VASP

'Elegantissima' [T. baccata L.] VAFN

'Expansa' [T. cuspidata Sieb. \& Zucc.] MDLT

'Fastigiata Robusta' [T. baccata L.] DCNA

floridana Nutt.

Florida Y.

$$
\text { FLUF TNAB }
$$

'Flushing' [T. $\times$ media Rehd.]

[T. baccata $\times T$. cuspidata] DCNA

'Fowle' [T. baccata L.]

= 'Adpressa Fowle'

'Hicksii' [T. $\times$ media Rehd.]

[T. baccata $\times T$. cuspidata] DCNA FLMG NCDU

'Luteobaccata' [T. cuspidata Sieb. \& Zucc.] DCNA

$\times$ media Rehd.

[T. baccata $\times T$. cuspidata] DCFP DCNA DCSI MDAB

'Minima' [T. cuspidata Sieb. \& Zucc.] DCNA

'Nana' [T. cuspidata Sieb. \& Zucc.] MDTD

'Nana Variegata' [T. cuspidata Sieb. \& Zucc.] DCNA 
'Overeynderi' [T. baccata L.] DCCG

'Repandens' [T. baccata L.] DCNA MDBG MDHN

'Richard Horsey' [T. $\times$ hunnewelliana

Rehd.]

[T . canadensis $\times T$. cuspidata] DCNA

'Stricta' $[\boldsymbol{T}$. baccata $]$

$=T$. baccata f. fastigiata

'Thompson' [T. cuspidata Sieb. \& Zucc.] DCNA

TECOMA JusS.

BIGNONIACEAE Bignonia Family

stans (L.) HBK. var. angustata Rehd. TXLL

TECOMARIA (Endl.) Spach

BIGNONIACEAE Bignonia Family

capensis (Thunb.) Spach

Cape Honeysuckle

FLUF

TERNSTROEMIA Mutis ex L.f.

THEACEAE

Tea Family

gymnanthera (Wight \& Arn.) Sprague

(Distinct from Cleyera japonica, this plant is often grown under that name because of its confused nomenclatural history; referral to

Cleyera japonica Thunb., pro parte, merely continues the chances for misidentification of the plant.)

$\begin{array}{llll}\text { ALAU } & \text { FLMG } & \text { LAMP } & \text { SCKG } \\ \text { ALBG } & \text { FLUF } & \text { LASL } & \text { VAMP } \\ \text { ALTD } & \text { GAHC } & \text { MSHB } & \text { VANB } \\ \text { DCCG } & \text { GAMM } & \text { NCAG } & \text { VAWM } \\ \text { DCDO } & \text { GAWP } & \text { NCDU } & \\ \text { DCNA } & \text { LAHG } & \text { NCOP } & \\ \text { FLCG } & \text { LALG } & \text { NCTE } & \end{array}$

japonica Thunb.

$=T \cdot$ gymnanthera
TETRAPANAX (K.Koch) K.Koch

ARALIACEAE

Ginseng Family

papyriferus (Hook.) K.Koch

Rice-paper Plant

MSHB

$\begin{array}{llr}\text { TEUCRIUM } & \text { L. } & \text { Germander } \\ \text { LAMIACEAE } & \text { Mint Family }\end{array}$

chamaedrys $\mathrm{L}$.

European Germander

DCNA

$\times$ lucidrys Boom

[T. chamaedrys $\times$ T. lucidum] DCNA

lucidum $\mathrm{L}$.

MDHN

THAMNOCALAMUS MunRO

POACEAE Grass Family

spathaceus (Franch.) Soderstrom

Green Fountain Bamboo

DCNA MDFM

THRYALLIS Mart.

MALPIGHIACEAE Malpighia Family

glauca (Cav.) O.Ktze.

= Galphimia glauca

THUJA L.

Arborvitae

CUPRESSACEAE

Cypress Family

'Alba' [T. occidentalis L.] DCNA

'Aurea' [T. occidentalis L.] VAMP

'Beaufort' [T. occidentalis L.] DCNA

'Buchananii' [T. occidentalis L.] DCNA

'Columna' [T. occidentalis L.] MDJS 
THUJA

'Compacta' [T. occidentalis L.] DCNA

'Cuprea' [T. plicata D.Don] DCNA

'Endean' [T. occidentalis L.] DCNA

'Fastigiata' [T. occidentalis L.] NCBE

'Filifera' [T. occidentalis L.] NCBE

'Filiformis' [T. occidentalis L.] DCNA

'Froebelii' [T. occidentalis L.] DCNA

'Globosa' [T. occidentalis L.] DCNA SCCU

'Globosa Rheindiana' [T. occidentalis L.] DCNA

'Hetz Midget' [T. occidentalis L.] DCNA

'Holmstrup' [T. occidentalis L.] DCNA

'Hudsonica' [T. occidentalis L.] DCNA

'Little Gem' [T. occidentalis L.] VATA

'Lutea' [T. occidentalis L.] VABF

'Malonyana' [T. occidentalis L.] DCNA

occidentalis $\mathrm{L}$.

American A.

$\begin{array}{llll}\text { ALSH } & \text { MDHN } & \text { NCCA } & \text { TNBV } \\ \text { DCNA } & \text { MDJH } & \text { NCSM } & \end{array}$

'Ohlendorfii' [T. occidentalis L.] MDBG

orientalis $\mathrm{L}$.

= Platycladus orientalis

'Pendula' [T. occidentalis L.] TNTV

plicata J.Donn ex G.Don

Western Red Cedar

GAEC TNUT
'Pygmaea' [T. occidentalis L.] DCNA MDBG =

'Pyramidalis' [T. occidentalis L.] MDBG MDHN

'Pyramidalis Nigra' [T. occidentalis L.] VATA

'Recurva Nana' [T. occidentalis L.] DCNA

'Rheingold' [T. occidentalis L.] DCNA

'Rogersii' [T. plicata D.Don] DCNA

'Semperaurea' [T. occidentalis L.] DCNA

'Spiralis' [T. occidentalis L.] NCBE

standishii (Gord.) Carr. DCNA

'Stricta' [T. occidentalis L.] DCNA

'Sunkist' [T. occidentalis L.] DCNA MDBG

'Techny' [T. occidentalis L.] GACG

'Umbraculifera' [T. occidentalis L.] DCNA

'Wareana' [T. occidentalis L.] DCNA

'Wareana Lutescens' [T. occidentalis L.] DCNA

'Woodwardii' [T. occidentalis L.] DCNA

'Zebrina' [T. plicata D.Don] DCNA

THUJOPSIS Sieb. \& Zucc. ex Endl.

False Arborvitae

CUPRESSACEAE

Cypress Family

dolabrata (Thunb. ex L.f.) Sieb. \& Zucc. Hiba Arborvitae DCNA MDBG MDLT NCKN 
dolabrata (Thunb. ex L.f.) Sieb. \& Zucc.

var. hondae Makino

DCNA MDGD

'Nana' [T. dolabrata (Thunb. ex L.f.) Sieb. \& Zucc.]

LASL

THUNBERGIA Retz.

\section{ACANTHACEAE Acanthus Family}

grandiflora (Roxb. ex Rottl.) Roxb.

Blue Trumpet Vine

TXAB

THYMUS L.

\section{LAMIACEAE}

Mint Family

vulgaris $\mathrm{L}$.

Common Thyme

DCNA MDBG

\section{TIBOUCHINA Aubl.}

MELASTOMATACEAE Melastoma Family

semidecandra Hort. non (DC.) Cogn.

= $T$. urvilleana

urvilleana (DC.) Cogn.

Glory Bush

ALBG FLUF

TILIA L.

\section{TILLACEAE}

Basswood, Linden Linden Family

americana L.

American L.

$\begin{array}{llll}\text { ALUA } & \text { DCMS } & \text { MDHN } & \text { VABR } \\ \text { DCBG } & \text { DCNA } & \text { MDNA } & \text { VACM } \\ \text { DCCC } & \text { DCSI } & \text { NCBE } & \text { VACW } \\ \text { DCCG } & \text { DCTB } & \text { SCBR } & \text { VAMP } \\ \text { DCJJ } & \text { FLUF } & \text { TNFE } & \text { VARO } \\ \text { DCMH } & \text { MDAB } & \text { TNSG } & \end{array}$

caroliniana Mill.

$=T$. americana

cordata Mill.

Littleleaf L.

DCCG

DCJM

DCMH

DCMN

DCNA

DCNY

DCSH

DCWH

DESR

GARW
MDBG

MDHN

MDJH

MDLT

NCDU
SCCU

TNSC

TNUT

VACM $\times$ euchlora K.Koch

[T. ? cordata $\times T$. dasystyla]

$\begin{array}{ll}\text { DCBG } & \text { DCML MDNA } \\ \text { DCJM } & \text { DCNA }\end{array}$

$\times$ europaea L.

[T. cordata $\times T$. platyphyllos]

$\begin{array}{llll}\text { DCGP } & \text { GAAC } & \text { MDSJ } & \text { VASC } \\ \text { DCJJ } & \text { GALE } & \text { NCBE } & \text { VAWR } \\ \text { DCML } & \text { MDHC } & \text { NCCP } & \\ \text { DCMN } & \text { MDHN } & \text { VACM } & \end{array}$

heterophylla Vent.

= $\mathrm{T}$. americana

'Laciniata' [T. platyphyllos Scop.]

DCBG MDCP VALC

DCML TNTV

$\times$ moltkei Spaeth

[T. americana $\times$ T. tomentosa 'Pendula'] DCML MDNA

mongolica Maxim.

Mongolian L.

DCNA

'Pendula' [T. tomentosa Moench] DCGP DCPA DCWH

petiolaris $\mathrm{DC}$

= $T$. tomentosa 'Pendula'

platyphyllos Scop.

Bigleaf L.

$\begin{array}{llll}\text { DCBG } & \text { MDDF } & \text { NCBE } & \text { VAMP } \\ \text { DCCG } & \text { MDEC } & \text { TNFE } & \text { VARB } \\ \text { DCML } & \text { MDHN } & \text { TNHG } & \\ \text { DCWH } & \text { MDJH } & \text { TNSG } & \\ \text { MDCP } & \text { MDPJ } & \text { VAMF } & \end{array}$

tomentosa Moench

Silver L.

$\begin{array}{llll}\text { DCAC } & \text { DCWH } & \text { MDCP } & \text { TNUT } \\ \text { DCCG } & \text { GAEH } & \text { MDJH } & \text { VALC } \\ \text { DCGP } & \text { MDCL } & \text { NCBE } & \end{array}$

'Vitifolia' [T. platyphyllos Scop.]

DCSI

$\times$ vulgaris Hayne

$=$ T. $\times$ europaea

TIPUANA (Benth.) Benth.

FABACEAE (Faboideae)

Bean Family

tipu (Benth.) O.Ktze.

Tipu Tree, Rosewood FLUF 
TOONA (Endl.) M.J.Roem.

MELIACEAE

Mahogany Family

sinensis (Juss.) M.J.Roem.

Chinese $\mathrm{T}$.

$\begin{array}{llll}\text { DCCG } & \text { DCNA } & \text { MDBG } & \text { VABF } \\ \text { DCGD } & \text { DCTN } & \text { TNRT } & \end{array}$

TORREYA Arn.

TAXACEAE

Yew Family

'Gold Strike' [T. nucifera (L.) Sieb. \& Zucc.] DCNA

nucifera (L.) Sieb. \& Zucc.

Japanese $\mathrm{T}$.

DCDO DCNA TNTV

taxifolia Arn.

Stinking Cedar, Florida T.

$\begin{array}{llll}\text { ALEH } & \text { FLDG } & \text { FLUF } & \text { SCBR } \\ \text { DCNA } & \text { FLMG } & \text { NCBE } & \text { SCMG } \\ \text { FLAL } & \text { FLTP } & \text { NCHS } & \end{array}$

TOXICODENDRON Mill.

\section{ANACARDIACEAE Cashew Family}

radicans (L.) O.Ktze.

Poison Ivy

MDBG

vernicifluum (Stokes) F.A.Barkley

Varnish Tree

DCNA

\section{TRACHELOSPERMUM LEM. \\ APOCYNACEAE Dogbane Family}

asiaticum (Sieb. \& Zucc.) Nakai GAEC GAWH

difforme (Walt.) A.Gr. MSEP SCBR

jasminoides (Lindl.) Lem.

Confederate Jasmine, Star Jasmine

$\begin{array}{llll}\text { FLPM } & \text { GAUG } & \text { MSEN } & \text { SCCM } \\ \text { FLUF } & \text { LALG } & \text { NCDU } & \text { SCWI } \\ \text { GAOH } & \text { LASL } & \text { NCWM } & \end{array}$

jasminoides (Lindl.) Lem. var. pubescens Makino LAHG MSMN
'Variegatum' [T. jasminoides (Lindl.) Lem.] ALBG LASL SCWC

TRACHYCARPUS H.Wendl.

ARECACEAE

Palm Family

fortunei (Hook.) H.Wendl.

Windmill Palm

$\begin{array}{lll}\text { ALBG } & \text { SCFW } \\ \text { FLMG } & \text { TXDM }\end{array}$

TRIPETALEIA Sieb. \& Zucc.

$$
\text { = ELLIOTLA }
$$

TRIPTERYGIUM Hook.f.

\section{CELASTRACEAE Staff-tree Family}

regelii Sprague $\&$ Takeda

Three-wing Wingnut DCNA TNTV

TRITHRINAX Mart.

ARECACEAE

Palm Family

acanthocoma Drude

FLUF

TROCHODENDRON Sieb. \& Zucc. TROCHODENDRACEAE

Trochodendron Family

aralioides Sieb. \& Zucc.

MDLT

TSUGA Carr.

Hemlock

PINACEAE

Pine Family

'Abbott's Dwarf' [T. canadensis (L.) Carr.] MDMG

'Angustifolia' [T. canadensis (L.) Carr.] DCNA

'Armistice' [T. canadensis (L.) Carr.] DCNA

'Beaujean' [T. canadensis (L.) Carr.] DCNA 
'Bennett' [T. canadensis (L.) Carr.] DCNA

'Boulevard' [T. canadensis (L.) Carr.] DCNA

'Brandley' [T. canadensis (L.) Carr.] DCNA

\section{canadensis (L.) Carr.}

Canadian $\mathrm{H}$.

$\begin{array}{llll}\text { ALEH } & \text { GAAB } & \text { NCBE } & \text { TNWF } \\ \text { DCCG } & \text { MDHN } & \text { NCTE } & \text { VAMP } \\ \text { DCFP } & \text { MDJS } & \text { SCCU } & \text { VAPO } \\ \text { DCNA } & \text { MDLA } & \text { SCPP } & \text { VAWP } \\ \text { DCWH } & \text { MDLT } & \text { TNDG } & \text { VAWR } \\ \text { FLUF } & \text { MDWP } & \text { TNSM } & \end{array}$

caroliniana Engelm.

Carolina $\mathrm{H}$.

$\begin{array}{llll}\text { DCWH } & \text { MDLT } & \text { NCBE } & \text { VAPO } \\ \text { MDBG } & \text { MDNA } & \text { NCCA } & \\ \text { MDDF } & \text { MDWP } & \text { NCDU } & \\ \text { MDLA } & \text { NCAS } & \text { VAML } & \end{array}$

chinensis (Franch.) Pritz. DCNA

'Cinnamomea' [T. canadensis (L.) Carr.] DCNA

'Curtis Ideal' [T. canadensis (L.) Carr.] DCNA

'Curtis Spreader' [T. canadensis (L.) Carr.] DCNA

diversifolia (Maxim.) Mast. DCNA GAEC

'Doc's Choice' [T. canadensis (L.) Carr.] DCNA

'Doran' [T. canadensis (L.) Carr.] DCNA

'Fastigiata' [T. canadensis (L.) Carr.] DCNA

'Gensch White' [T. canadensis (L.) Carr.] MDLT

'Globosa' [T. canadensis (L.) Carr.] DCNA

'Hawkersmith Weeping' [T. canadensis

(L.) Carr.] TNSN

'Henry Hohman' [T. canadensis (L.) Carr.] DCNA
'Jacqueline Verkade' [ $T$. canadensis (L.)

Carr.] DCNA

'Jervis' [T. canadensis (L.) Carr.] DCNA

'Kelsey's Weeping' [T. canadensis (L.) Carr.] DCNA

'Macrophylla' [T. canadensis (L.) Carr.] DCNA

'Minima' [T. canadensis (L.) Carr.] DCNA

'Minuta' [T. canadensis (L.) Carr.] DCNA

'Pendula' [T. canadensis (L.) Carr.]

Weeping $\mathrm{H}$. DCNA MDLT MDNA NCBE

'Sargentii' [T. canadensis (L.) Carr.]

Sargent's Weeping H. DCNA MDBG

sieboldii Carr. MDKN MDLT

'Verkade Recurved' [T. canadensis (L.) Carr.] DCNA

'Von Helms' [T. canadensis (L.) Carr.] DCNA

'Youngcone' [T. canadensis (L.) Carr.] DCNA

ULMUS L. ULMACEAE

Elm Elm Family

alata Michx.

Winged $\mathrm{E}$.

$\begin{array}{llll}\text { ARSN } & \text { GAUG } & \text { SCCU } & \text { VAMP } \\ \text { FLUF } & \text { LAAP } & \text { SCMG } & \text { VASC } \\ \text { GAAB } & \text { NCDU } & \text { VACW } & \text { VAWR } \\ \text { GATN } & \text { NCWT } & \text { VAGG } & \end{array}$

americana L.

American E.

$\begin{array}{llll}\text { DCCG } & \text { MDCP } & \text { SCUC } & \text { TNSC } \\ \text { DCNA } & \text { MDHN } & \text { TNDR } & \text { VACW } \\ \text { DCWH } & \text { SCCU } & \text { TNLW } & \text { VASC }\end{array}$

'Augustine Ascending' [U. americana L.] DCWH NCDU VASJ 
'Belgica' [U. $\times$ hollandica Mill.]

[U. minor $\times U$. glabra] DCCG DCWP

'Camperdownii' [U. glabra Huds.] DCWH TNDR VACW VAWP

carpinifolia Gleditsch

$=U$. minor

'Christine Buisman' [U. minor Mill.] DCPP

crassifolia Nutt.

Cedar E. TXGS

'Dauvessei' [U. $\times$ hollandica Mill.]

[U. minor $\times$ U. glabra] DCWP

'Drake' [U. parvifolia Jacq.] SCCU SCSG VATA

'Dynasty' [U. parvifolia Jacq.] DCNA

elliptica K.Koch $\mathrm{VABF}$

'Frosty' [U. parvifolia Jacq.] GAFG

fulva Michx.

= U. rubra

glabra Huds.

Wych E., Scotch E. DCCG

'Hokkaido' [U. parvifolia Jacq.] GAFG

$\times$ hollandica Mill.

[U. minor $\times U$. glabra] VAMW

$\star$ 'Homestead'

[U. pumila $\times((U . \times$ hollandica 'Vegeta' $\times$

$U$. minor $) \times(U$. pumila var, arborea $\times$

$U$. minor 'Hoersholm'))] DCNA

japonica (Rehd.) Sarg. DCNA

japonica $\times U$. wilsoniana DCTB

laevis Pall.

European White E. $\begin{array}{ll}\text { DCCG } & \text { DCSE } \\ \text { DCDO } & \text { MDDF }\end{array}$ macrocarpa Hance DCNA MDGD

'Major' [U. $\times$ hollandica Mill.]

[U. minor $\times U$. glabra] DCCG

'Marginata' [U. procera Salisb.] DCSE

minor Mill. DCCG SCCU TNRM

'Moline' [U. americana L.]

Moline Elm MDGD

parvifolia Jacq.

Chinese E.

$\begin{array}{llll}\text { ALAU } & \text { DCLC } & \text { GAJY } & \text { SCCU } \\ \text { ALFN } & \text { FLMN } & \text { MDAF } & \\ \text { DCAC } & \text { FLUF } & \text { NCEL } & \end{array}$

$\star$ 'Pioneer' [U. $\times$ hollandica]

[U. minor $\times$ U. glabra]

DCNA

procera Salisb.

English E.
DCCG
DCWH
MDHN
DCSE
MDCP

pumila L.

Siberian E.

$\begin{array}{llll}\text { ALBH } & \text { DCWR } & \text { MDGG } & \text { SCNS } \\ \text { ARPT } & \text { GACL } & \text { MDMM } & \text { VAWD } \\ \text { DCCG } & \text { LATU } & \text { NCCC } & \end{array}$

rubra Muhl.

Slippery E.
DCCG
NCKH VACW
MDHN
SCMP VAMP

'Sarniensis' [U. minor Mill.]

Jersey E.

DCLP

serotina Sarg.

September E.

NCBE VASC

'Superba' [U. $\times$ hollandica Mill.]

[U. minor $\times U$. glabra]

DCGP

thomasii Sarg.

Rock E.

$\mathrm{SCCU}$

'Umbraculifera' [U. minor Mill.]

Globe E.

NCBE 


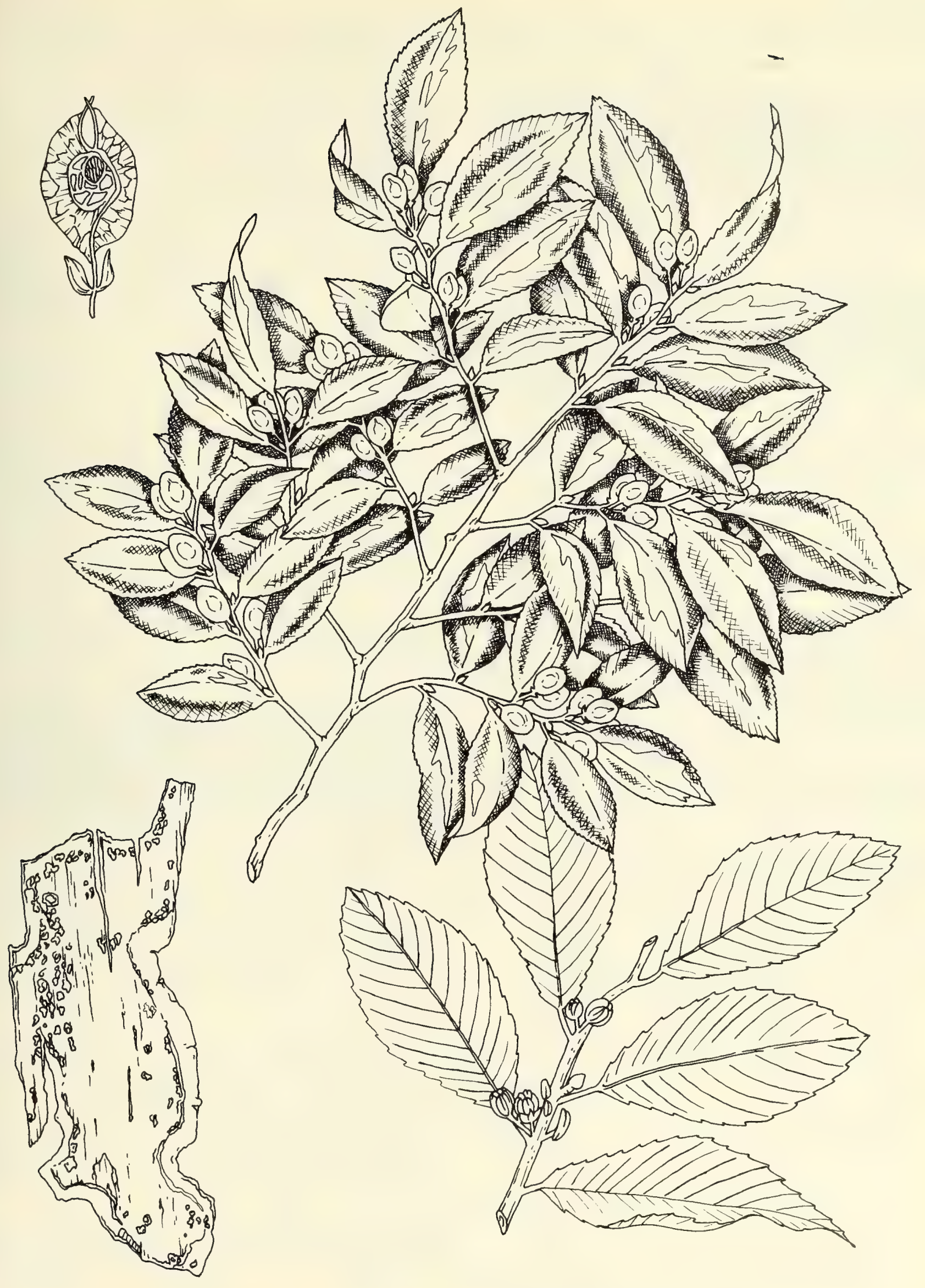

ULMUS parvifolia Jacq.

[illustrator Susan M. Johnston] 
'Wredei' [U. minor Mill.]

DCPL

UNGNADIA Endl.

\section{SAPINDACEAE}

Soapberry Family

speciosa Endl.

Mexican Buckeye

LASL SCWI TXDC TXLL

VACCINIUM L. ERICACEAE

L. Blueberry Heath Family

amoenum Ait.

GASM

arboreum Marsh.

Farkleberry, Sparkleberry $\begin{array}{llll}\text { ALBG } & \text { FLUF } & \text { LALG } & \text { SCHS } \\ \text { FLMG } & \text { LAHG } & \text { MSMN } & \end{array}$

ashei Reade

$=\boldsymbol{V}$. corymbosum, in part

atrococcum (A.Gr.) Heller

$=\mathrm{V}$. corymbosum, in part

'Bloodstone' [V. crassifolium Andr. ssp. sempervirens (Rayner \& Henderson)

Kirkman \& Bal.]

NCNS

bracteatum Thunb. ex J.A.Murr. DCNA

constablaei A.Gr.

$=\mathbf{V}$. corymbosum, in part

corymbosum L.

Highbush B.

$\begin{array}{llll}\text { ALON } & \text { LAHG } & \text { SCCU } & \text { VACW } \\ \text { DCNA } & \text { LALG } & \text { SCKG } & \text { VAPH } \\ \text { FLDG } & \text { MDWJ } & \text { SCWI } & \text { VAPO } \\ \text { GACG } & \text { SCBR } & \text { TNMB } & \text { VATW }\end{array}$

crassifolium Andr.

Creeping B.

SCWI

cylindraceum Sm.

DCNA

elliottii Chapm.

$=\mathrm{V}$. corymbosum, in part

floridanum (Nutt.) Sleumer

$=V$. stamineum, in part fuscatum Ait.

$=\mathrm{V}$. corymbosum, in part

myrsinites Lam.

Ground B.

$\begin{array}{ll}\text { ALTD } & \text { GAJI } \\ \text { FLUF } & \text { SCWI }\end{array}$

simulatum Small VACW

stamineum L.

Deerberry

ALBG GACG MDLT VACW

tenellum Ait.

SCBR

vacillans Torr.

MDLT

virgatum Ait.

= V. corymbosum, in part

vitis-idaea $\mathrm{L}$.

Cowberry, Mountain Cranberry DCNA DEMC

'Wells Delight' [V. crassifolium Andr. ssp. crassifolium] NCNS

\section{VIBURNUM L.}

CAPRIFOLIACEAE Honeysuckle Family (Contributed by T. R. Dudley)

acerifolium L.

Maple-leaved V.

GAUG MDLT VAAC

$\star$ 'Alleghany' [V. $\times$ rhytidophylloides

Suring.]

[V. lantana $\times$ V. rhytidophyllum]

DCNA MDLT

alnifolium Marsh.

$=V$. lantanoides

'Aurantiacum' [V. setigerum Hance] DCNA MDBG MDGD

awabuki K.Koch

$\begin{array}{llll}\text { FLJU } & \text { FLUF } & \text { LASL } & \text { SCCU } \\ \text { FLMG } & \text { LAAL } & \text { NCDU } & \text { SCMG }\end{array}$

'Ben Blackburn' [V. rhytidophyllum Hemsl.]

MDKN 


\section{VIBURNUM}

betulifolium Batal.

Birch-leaved V. GAUG

bitchiuense Makino

Yeddo V.

DCWH GAEC VAPH VARG

brachybotryum Hemsl.

GACG

buddleifolium Wright

Buddleja-leaved V.

DEWG MDKN

burejaeticum Regel \& Herd.

Manchurian V.

$$
\text { MDLT MDTD TNUT }
$$

$\times$ burkwoodii Burkw. \& Skipwith

Burkwood's V.

[V. carlesii $\times$ V. utile]

$\begin{array}{llll}\text { DCNA } & \text { MDDF } & \text { TNMB } & \text { VAPH } \\ \text { DEWG } & \text { SCCU } & \text { TNUT } & \\ \text { GAFN } & \text { TNDG } & \text { VACW } & \end{array}$

calvum Rehd.

LASL

$\times$ carlcephalum Burkw. ex Pike

[V. carlesii $\times V$. macrocephalum]

$\begin{array}{llll}\text { ARWS } & \text { MDLT } & \text { TNCT } & \text { VACW } \\ \text { DEWG } & \text { MDME } & \text { TNMB } & \text { VAWM }\end{array}$

carlesii Hemsl.

Korean-spice V.

$\begin{array}{lll}\text { DCNA } & \text { MDMM } & \text { VANB } \\ \text { GAWH } & \text { TNCT } & \text { VAPH } \\ \text { MDBG } & \text { TNDG } & \text { VAWM }\end{array}$

carlesii Hemsl. var. bitchiuense (Makino)

Nakai

$=V$. bitchiuense

cassinoides $\mathrm{L}$.

Withe-rod V. NCBE TXHN

$\star$ 'Catskill' [V. dilatatum Thunb. ex

J.A.Murr.]

DCNA MDBG

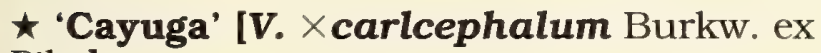

Pike]

[V. carlesii $\times$ V. macrocephalum]

DCNA

'Chenault' [V. $\times$ burkwoodii Burkw. \&

Skipwith]

[V. carlesii $\times$ V. utile]

MDBG MDLT $\star$ 'Chesapeake'

[V. $\times$ carlcephalum 'Cayuga' $\measuredangle$ V. utile] DCNA GAEC

$\star$ 'Chippewa'

[V. japonicum $\times$ V. dilatatum] DCNA

cinnamomifolium Rehd.

Cinnamon-leaved V. LASL

'Compacta' [V. carlesii Hemsl.] DEWG

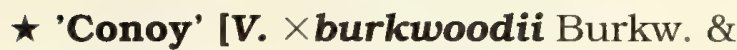

Skipwith]

[V. carlesii $\times$ V. utile] DCNA

cylindricum D.Don GAIS

dasyanthum Rehd. MDKN

'Dawn' [V. $\times$ bodnantense Aberc.]

[V. farreri $\times$ V. grandiflorum]

DEWG MDLT

'Deben' [V. $\times$ bodnantense Aberc.]

[V. farreri $\times$ V. grandiflorum] DEWG

dentatum $\mathrm{L}$.

Arrow-wood $\begin{array}{lll}\text { MDBG } & \text { NCBE } & \text { SCWI } \\ \text { MDLT } & \text { SCBR } & \text { TNSN }\end{array}$

dilatatum Thunb. ex J.A.Murr.

Linden $\mathrm{V}$.

$\begin{array}{llll}\text { DEWG } & \text { MDGD } & \text { SCCU } & \text { VANB } \\ \text { GAEC } & \text { MDLT } & \text { VABF } & \text { VATA } \\ \text { MDBG } & \text { NCBE } & \text { VAFC } & \end{array}$

$\star$ 'Erie' [V. dilatatum Thunb. ex J.A.Murr.] DCNA MDBG

erosum Thunb. ex J.A.Murr. GACG MDGD NCSW

GAEC MDPJ

$\star$ 'Eskimo' [V. $\times$ carlcephalum 'Cayuga' $\times$ V. utile] DCNA

farreri Stearn DEWG

foetidum Wall. var. rectangulatum (Graebn.) Rehd. DCNA 
fordiae Hemsl. TNTV

fragrans Bunge

= V. farreri

$\times$ hillieri Stearn

[V. erubescens $\times V$. henryi] MDBG

hirtulum Rehd. $\mathrm{SCCU}$

hupehense Rehd. MDGD TNTV

$\star$ 'Huron' [V. lobophyllum $\times$

V. japonicum] DCNA

ichangense (Hemsl.) Rehd. MDKN

* 'Iroquois' [V. dilatatum Thunb. ex J.A.Murr.] DCNA MDBG

japonicum (Thunb. ex J.A.Murr.) Spreng. Japanese V.

$\begin{array}{lll}\text { DCNA } & \text { GAJI } & \text { MDGD } \\ \text { FLCG } & \text { LALG } & \text { MDLT } \\ \text { FLUF } & \text { MDBG } & \text { SCBH }\end{array}$

$\times$ juddii Rehd.

[V. bitchiuense $\times$ V. carlesii] DEWG MDBG VAPH

lantana $\mathrm{L}$.

Wayfaring Tree MDJS MDLT NCBE

lantanoides Michx.

Hobblebush

$\mathrm{NCDB}$

lentago $\mathrm{L}$.

Nannyberry, Sheepberry VACW VAGW

lentago L. f. sphaerocarpum (Fern.) Rehd. MDBG

lobophyllum Graebn. DEWG MDBG

luzonicum Rolfe

Philippine V.

TXLL TXSE

macrocephalum Fort. f. keteleeri (Carr.) Rehd. VAAC macrocephalum Fort. f. macrocephalum Chinese Snowball
DCWH
GACG
MDKN
DEWG
MDBG
VAGS

'Mariesii' [V. plicatum Thunb.

f. tomentosum (Thunb. ex J.A.Murr.)

Rehd.]

$\begin{array}{llll}\text { DCWR } & \text { MDLA } & \text { TNDG } & \text { VATW } \\ \text { MDJS } & \text { MDLT } & \text { VANB } & \end{array}$

$\star$ 'Mohawk' [V. $\times$ burkwoodii $\times$ V. carlesii] DCNA MDBG

$\star$ 'Mohican' [V. Lantana L.] DCNA

molle Michx.

Poison Haw

DEWG

'Mt. Fuji' [V. plicatum Fort. f. tomentosum (Thunb. ex J.A.Murr.) Rehd.] DCNA

mullaha Buch.-Ham. ex D.Don DCNA

'Newport' [V. plicatum Thunb. f. plicatum] GACG MDBG

nudum L.

Nannyberry Haw, Possum Haw
ALBG
LAHG
SCBR
VACW
ALUA
LALG
TNUT

obovatum Walt.

Walter's V.
ALEH
DCNA
TXPS
ALTD SCBR

odoratissimum Ker-Gawl.

Sweet V.

$$
\begin{array}{llll}
\text { FLEM } & \text { LAAL } & \text { MSBN } & \text { MSMN } \\
\text { FLMN } & \text { LAHG } & \text { MSEP } & \text { TXJS }
\end{array}
$$

odoratissimum Ker-Gawl. var. awabuki

(K.Koch) K.Koch ex Ruempler

$=$ V. awabuki

$\star$ 'Oneida' [V. dilatatum $\times$

V. lobophyllum]

DCNA

'Onondaga' [V. sargentii Koehne] DCNA

opulus L.

European Cranberry-bush MDTD VAAC VASK 


\section{VIBURNUM}

ovatifolium Rehd.

DEWG

plicatum Thunb. f. lanceolatum (Rehd.)

Rehd.

\section{TNUT}

plicatum Thunb. f. parvifolium (Miq.)

Rehd.

$$
\text { MDKN VATW }
$$

plicatum Thunb. f. plicatum

Japanese Snowball

$\begin{array}{llll}\text { ALBH } & \text { MDJS } & \text { NCCA } & \text { VAMP } \\ \text { DCDO } & \text { MDKN } & \text { NCSM } & \text { VASK } \\ \text { GACG } & \text { MDLA } & \text { VAAC } & \\ \text { MDBG } & \text { NCBE } & \text { VACW } & \end{array}$

plicatum Thunb. var. rotundifolium Rehd. Round-leaved double-file V.
ARPT
MDDF

plicatum Thunb. f. tomentosum (Thunb. ex J.A.Murr.) Rehd.

Double-file V.

$\begin{array}{llll}\text { ALBH } & \text { GACG } & \text { MDKN } & \text { VACW } \\ \text { DCWH } & \text { GAFN } & \text { NCGC } & \text { VAPH } \\ \text { DEWG } & \text { MDBG } & \text { VAAC } & \text { VASK }\end{array}$

'Pragense' [V. rhytidophyllum $\times$ V. utile] TNTV VAGS VATA

prunifolium $\mathrm{L}$.

Black Haw, Sheepberry

$\begin{array}{llll}\text { DCNA } & \text { MDLA } & \text { TNMB } & \text { VAMP } \\ \text { GARW } & \text { MDLT } & \text { TNRG } & \text { VAPO } \\ \text { MDBA } & \text { NCBE } & \text { VACW } & \text { VATA } \\ \text { MDBG } & \text { SCCU } & \text { VAGS } & \\ \text { MDKN } & \text { TNDG } & \text { VAGW } & \end{array}$

recognitum Fern

Northern Arrow-wood

DCCG LASL VACW VAGW

'Reticulatum' [V. sieboldii Miq.] MDPJ VADW

$\times$ rhytidocarpum E.Lemoine

[V. buddleifolium $\times V$. rhytidophyllum] GACG MDBG TNPW VATW

$\times$ rhytidophylloides Suring.

[V. lantana $\times$ V. rhytidophyllum]

GACG MDPJ VATA

MDKN VAMP

rhytidophyllum Hemsl.

Leatherleaf $\mathrm{V}$.

$\begin{array}{llll}\text { ALTD } & \text { MDLA } & \text { NCBE } & \text { TNMB } \\ \text { GAOH } & \text { MDLT } & \text { NCDU } & \text { TNPW } \\ \text { GAUG } & \text { MDMG } & \text { NCWC } & \text { VACW } \\ \text { GAWH } & \text { MDPJ } & \text { TNCT } & \text { VAMP }\end{array}$

'Roseum' [V. opulus L.]

Common Snowball, European Snowball $\begin{array}{lll}\text { GAOH } & \text { VACW } & \text { VAGW } \\ \text { MDTD } & \text { VADW } & \text { VASP }\end{array}$

'Roseum' [V. rhytidophyllum Hemsl.] MDKN

rufidulum Raf.

Rusty Black Haw, Southern Black Haw

$\begin{array}{lll}\text { ALTD } & \text { LARP } & \text { TNSG } \\ \text { GACG } & \text { MDGJ } & \text { TXMK } \\ \text { LAAL } & \text { SCBR } & \text { TXRS }\end{array}$

'St. Keverne' [V. plicatum Thunb.

f. tomentosum (Thunb. ex J.A.Murr.) Rehd.] TNTV

sargentii Koehne MDBG MDGD VACW

sargentii Koehne var. calvescens Rehd. SCCU

'Seneca' [V. sieboldii Miq.] DCNA MDBG

setigerum Hance

Tea-leaved V.

$\begin{array}{llll}\text { GAAB } & \text { MDBG } & \text { MDPJ } & \text { VABF } \\ \text { GACG } & \text { MDDF } & \text { NCBE } & \text { VATW } \\ \text { GAEC } & \text { MDJS } & \text { SCCU } & \text { VAWR } \\ \text { GAEH } & \text { MDKN } & \text { TNMB } & \\ \text { GAIS } & \text { MDLT } & \text { TNSN } & \end{array}$

$\star$ 'Shasta' [V. plicatum f. tomentosum $\times$

V. plicatum f. tomentosum 'Mariesii'] DCNA

$\star$ 'Shoshoni' [V. plicatum Fort.

f. tomentosum (Thunb. ex J.A.Murr.) Rehd.] DCNA

sieboldii Miq.

Tree V.

$\begin{array}{llll}\text { DCCG } & \text { MDJH } & \text { MDLT } & \text { VATA } \\ \text { DEMC } & \text { MDKN } & \text { MDWS } & \\ \text { DEWG } & \text { MDLA } & \text { TNUT } & \end{array}$

stellulatum Wall.

= V. mullaha

suspensum Lindl.

$\begin{array}{lll}\text { ALEH } & \text { GAFN } & \text { LAGN } \\ \text { FLMG } & \text { GAJI } & \text { SCBR } \\ \text { FLUF } & \text { LAAL } & \text { TXSH }\end{array}$

$\star$ 'Susquehanna' [V. sargentii Koehne] DCNA

theiferum Rehd.

= V. setigerum 
tinus L.

Laurustinus

$\begin{array}{llll}\text { FLUF } & \text { MSMN } & \text { SCFW } & \text { VACW } \\ \text { GACG } & \text { NCEG } & \text { SCHW } & \\ \text { LALG } & \text { NCTP } & \text { SCMG } & \\ \text { LASL } & \text { SCBR } & \text { SCMP } & \end{array}$

trilobum Marsh.

Highbush Cranberry

$\begin{array}{lll}\text { DCDO } & \text { NCAS } & \text { VARG } \\ \text { MDBG } & \text { NCCA } & \end{array}$

urceolatum Sieb. \& Zucc.

DCNA

utile Hemsl.

GAEC MDBG NCEL SCWI

'Variegatum' [V. rhytidophyllum Hemsl.] MDBG

\footnotetext{
'Willow Leaf' [V. × rhytidophylloides

Suring.]

[V. lantana $\times$ V. rhytidophyllum]

DEWG GACG
}

'Willowwood' [V. × rhytidophylloides

Suring.]

[V. Lantana $\times \mathrm{V}$. rhytidophyllum]

TNPW

wrightii Miq. MDGD MDJS MDKN VABF

VINCA L. APOCYNACEAE

'Alba' [V. minor L.] MDLT MDSM

'Atropurpurea' [V. minor L.] VAPH

major L.

Greater P. FLDG FLMG MDWP VAWR

minor $\mathrm{L}$.

Common P. MDLT VACW

'Multiplex' [V. minor L.]

Double-flowered P. DEWG

'Oxyloba' [V. major L.] MDFM

'Variegata' [V. major L.] VAPH
VITEX L.

VERBENACEAE

Verbena Family

agnus-castus L.

Chaste Tree

$\begin{array}{llll}\text { ALAU } & \text { LAMP } & \text { NCBE } & \text { VACP } \\ \text { ALBG } & \text { MDBG } & \text { NCSM } & \text { VACW } \\ \text { DCCG } & \text { MDBR } & \text { SCBR } & \\ \text { GAAB } & \text { MDFD } & \text { SCCU } & \\ \text { LAAL } & \text { MDWP } & \text { SCMP } & \end{array}$

'Incisa' [V. negundo L.]

FLUF VACW

negundo $\mathrm{L}$.

FLUF GAIS

rotundifolia L.f.

MDFM

'Silver Spire' [V. agnus-castus L.]

DCNA

'Variegata' [V. trifolia L.]

FLUF

VITIS L.

Grape

VITACEAE

Grape Family

candicans Engelm.

$=V$. mustangensis

labrusca L.

Fox G.

NCDU

mustangensis Buckl.

Mustang $\mathrm{G}$.

DCNA

rotundifolia Michx.

Muscadine G., Scuppernong

NCMO SCPL

WASHINGTONIA H.Wendl.

ARECACEAE

Washington Palm

Palm Family

robusta $\mathrm{H}$.Wendl.

LAAL 
WEIGELA Thunb.

CAPRIFOLIACEAE

Honeysuckle Family

(Contributed by T. R. Dudley)

'Bristol Ruby' [W. florida $\times$

W. 'Eva Rathke']

VAMP

decora (Nakai) Nakai

DCDO NCBE

'Eva Rathke' [W. floribunda $\times$

W. coraeensis]

DEWG

'Feerie'

(Hybrid of unknown parentage)

DEWG

florida (Bunge) A.DC.

$\begin{array}{lll}\text { ALBH } & \text { MDLA } \\ \text { LAHG } & \text { SCMG }\end{array}$

hortensis (Sieb. \& Zucc.) K.Koch

FLMG NCBE SCCU VALU

$\times$ hybrida Jaeger

= Untenable name for unassigned hybrids

'Mont Blanc'

(Hybrid of unknown parentage)

DEWG

'Newport Red'

(Hybrid of unknown parentage)

DEWG

'Rosea' [W. praecox (V.Lemoine) Bailey] DCNA

subsessilis (Nakai) Bailey DCNA

'Vanicek'

= 'Newport Red'

'Variegata' [W. florida (Bunge) A.DC.] MDPJ VAGS

WESTRINGIA Sm.

LAMIACEAE Mint Family

fruticosa (Willd.) Druce

$=W$. rosmariniformis

rosmariniformis $\mathrm{Sm}$.

FLUF
WIKSTROEMIA Endl. -

THYMELAEACEAE

Mezereum Family

trichotoma (Thunb.) Makino

DCNA

WISTERIA Nutt.

Wisteria

FABACEAE (Faboideae)

Bean Family

'Alba' [W. brachybotrys Sieb. \& Zucc.] DCNA

'Alba' [W. floribunda (Willd.) DC.]

DCDO MDBG NCDU VAGS

DEEM MDPJ TNCT VANB

'Alba' [W. sinensis (Sims) Sweet]

FLMN SCLO SCWI VAGS

GASM SCMG TXLL

brachybotrys Sieb. \& Zucc.

DCNA

floribunda (Willd.) DC.

Japanese W.

$\begin{array}{llll}\text { ALBG } & \text { DEEM } & \text { MDBG } & \text { SCMG } \\ \text { DCCC } & \text { FLUF } & \text { MDJH } & \text { TNSC } \\ \text { DCDO } & \text { GAHC } & \text { MSHB } & \text { VAGS } \\ \text { DCNA } & \text { GANG } & \text { NCCB } & \text { VANB } \\ \text { DCWH } & \text { GASS } & \text { SCDB } & \end{array}$

$\times$ formosa Rehd.

[W. floribunda $\times W$. sinensis]

$\begin{array}{llll}\text { ALEH } & \text { FLUF } & \text { MSEN } & \text { SCFW } \\ \text { DCCC } & \text { GAJI } & \text { MSHB } & \text { VATW } \\ \text { FLCG } & \text { LAAL } & \text { SCBR } & \end{array}$

frutescens (L.) Poir.

American W.

$\begin{array}{llll}\text { DCNA } & \text { GALF } & \text { SCWI } & \text { VACW } \\ \text { GAFN } & \text { SCBR } & \text { TXWE } & \end{array}$

'Honbeni' [W. floribunda (Willd.) DC.] DCNA

'Itoe kokuryu' [W. floribunda (Willd.) DC.] DCNA

'Jabo' [W. floribunda (Willd.) DC.] DCNA

'Koshigaya' [W. floribunda (Willd.) DC.] MDGD

'Macrobotrys' [W. floribunda (Willd.) DC.] MDBG

macrostachya Nutt.

$=\boldsymbol{W}$. frutescens 
'Murasaki kapitan' [W. brachybotrys Sieb. \& Zucc.] DCNA

'Nivea' [W. frutescens (L.) Poir.] SCWI

'Noda' [W. floribunda (Willd.) DC.] DCNA

'Ossai' [W. floribunda (Willd.) DC.] DCNA

'Rosea' [W. floribunda (Willd.) DC.] DCNA GASM VAGS DEEM MDBG

'Shino kapitan' [W. floribunda (Willd.) DC.] DCNA

sinensis (Sims) Sweet

Chinese W.

$\begin{array}{llll}\text { DCDO } & \text { FLMG } & \text { MSHB } & \text { SCWI } \\ \text { DEWG } & \text { GAVI } & \text { NCGP } & \text { VAGS } \\ \text { FLDG } & \text { LASL } & \text { SCPP } & \end{array}$

'Siro kapitan' [W. venusta Rehd. \& Wils.] DCNA

villosa Rehd.

ARRB DCDO DCNA

'Violacea Plena' [W. floribunda (Willd.) DC.]

$\begin{array}{lll}\text { DCNA } & \text { GAWP } & \text { NCOP } \\ \text { GASM } & \text { MDBG } & \text { SCMP }\end{array}$

\section{XANTHOCERAS Bunge}

SAPINDACEAE

Soapberry Family

sorbifolium Bunge

Yellowhorn

DEWG VAGS VAPH

XANTHORHIZA Marsh.

RANUNCULACEAE Buttercup Family

simplicissima Marsh.

Shrub Yellowroot

SCWI VAPO

XOLISMA Raf.

$=$ LYONLA
XYLOSMA J.G.Forst.

FLACOURTLACEAE

Flacourtia Family

congestum (Lour.) Merr.

FLUF LASL TXSH

LAAL NCOP

flexuosum (HBK.) Hemsl.

FLUF

senticosum Hance

$=\mathrm{X}$. congestum

YUCCA L. Adam's Needle, Spanish Dagger AGAVACEAE Agave Family

'Bright Edge' [Y. filamentosa L.] VAGS

filamentosa $\mathrm{L}$.

Adam's Needle

TNHS

filamentosa L. var. smalliana (Fern.) Ahles

$=$ Y. smalliana

gloriosa L.

Spanish Dagger $\begin{array}{lll}\text { DCCG } & \text { FLMG } & \text { NCRM } \\ \text { DCNA } & \text { LAAL } & \end{array}$

'Golden Sword' [Y. filamentosa L.] VAGS

'Marginata' [Y. aloifolia L.]

Spanish Bayonet

ALBG LAAL

recurvifolia Salisb.

VAGS VATA

smalliana Fern.

VATA

whipplei Torr.

Our-Lord's-Candle ALBG

ZAMIA L.

CYCADACEAE

Cycad Family

floridana A.DC.

= Z. pumila

integrifolia Ait.

= Z. pumila 
pumila L.

Coontie $\begin{array}{llll}\text { FLCG } & \text { FLMG } & \text { GACG } & \text { GASM } \\ \text { FLDG } & \text { FLUF } & \text { GAJI } & \end{array}$

ZANTHOXYLUM L. Prickly Ash RUTACEAE Citrus Family

bungei Planch.

= Z . simulans

clava-herculis $\mathrm{L}$.

Southern P. A. $\begin{array}{ll}\text { FLUF } & \text { LAAL } \\ \text { FLWC } & \text { MSMN }\end{array}$

fagara (L.) Sarg.

Wild Lime TXPH

piperitum $\mathrm{DC}$. DCNA

simulans Hance

$\begin{array}{lll}\text { GAEC } & \text { GAIS } & \text { TNSG } \\ \text { GAEH } & \text { MDPL } & \text { VABF }\end{array}$

ZELKOVA Spach

ULMACEAE

Elm Family
ZENOBIA D.Don

ERICACEAE

$-$

Heath Family

pulverulenta (Bartram) Pollard MDLT SCWI VAPH

pulverulenta (Bartram) Pollard f. nuda (Vent.) Fern. SCBR

ZIZIPHUS Mill.

RHAMNACEAE

Buckthorn Family

'Contorta' [Z. jujuba Mill.]

TNSN

jujuba Mill.

Jujube

$\begin{array}{llll}\text { DCCG } & \text { GAIS } & \text { MSEP } & \text { TNNE } \\ \text { DCNA } & \text { GALE } & \text { MSMN } & \text { TNRM } \\ \text { GAHC } & \text { LAAL } & \text { SCWI } & \text { VABF }\end{array}$

acuminata (Lindl.) Planch.

$=\mathbf{Z}$. serrata

carpinifolia (Pall.) K.Koch

Caucasian Z.

DCLC

DCUS

crenata Spach

$=\mathbf{Z}$. carpinifolia

schneideriana Hand.-Mazz.

DCAC DCCG MDBG MDGD

serrata (Thunb.) Makino

Japanese Z., Keaki

$\begin{array}{llll}\text { ALIT } & \text { DCGC } & \text { MDBG } & \text { MDJH } \\ \text { DCCG } & \text { DCGP } & \text { MDGG } & \text { TNDR } \\ \text { DCEL } & \text { GAIS } & \text { MDIH } & \end{array}$

$\times$ verschaffeltii Nichols.

[Z. carpinifolia $\times$ Z. serrata] MDGD 
Appendix A: Authors Cited

Abel: Clarke Abel,

1789-1826, United Kingdom

Aberc.: Henry Duncan McLaren,

2nd Baron Aberconway,

1879-1953, United Kingdom

Adams: William Preston Adams,

1930- , United Kingdom (Hypericum)

Adans.: Michel Adanson,

1727-1806, France

Ahles: Harry E. Ahles,

1924-1981, United States

Ahrendt: Leslie Walter Allen Ahrendt,

1903-1969, United Kingdom (Berberis, Mahonia)

Airy-Shaw: Herbert Kenneth Airy-Shaw,

1902-1985, United Kingdom

Ait.: William Aiton,

1731-1793, United Kingdom (cultivated plants)

Ait.f.: William Townsend Aiton,

1766-1849, United Kingdom (cultivated plants)

Alex.: Edward Johnston Alexander,

1901-1985, United States

Ames, L.M.: Lawrence M. Ames,

1900-1966, United States

Anderss.: Nils Johan Andersson.

1821-1880, Sweden (Salix)

Andr.: Henry Charles Andrews,

1752?-1830, United Kingdom

Andre: Édouard-François André,

1840-1911, France

Andrews, S.: Susyn Andrews,

1953- , United Kingdom (Ilex)

Anliker, J.: Johann Anliker,

fl. 1931, Switzerland
Ant.: Franz Antoine,

1815-1886, Austria (Coniferae)

Arn.: George Arnott (Arnold) Walker Arnott,

1799-1868, United Kingdom

Asami: Yoshichi Asami,

1894- , Japan (Malus)

Ashe: William Willard Ashe,

1872-1932, United States

Aubl.: Jean Baptiste Christophore Fusée Aublet, 1720-1778, France

Audubon: John James Audubon,

1785-1851, United States

BSP.: See Britton; Sterns; Poggenburg

Bailey: Liberty Hyde Bailey, 1858-1954, United States (cultivated plants)

Baill.: Henri Ernest Baillon,

1827-1895, France

Baker: John Gilbert Baker, 1834-1920, United Kingdom

Bal.: Benedict Balansa,

1825-1892, France

Balf.f.: Isaac Bayley Balfour, 1853-1922, United Kingdom

Ballington: James R. Ballington, contemporary, United States (horticultural plants)

Banks: Joseph Banks,

1743-1820, United Kingdom

Barbier: Barbier,

fl. 1911, France (nurseryman)

Barkley, F.A.: Fred Alexander Barkley, 1908-1989, United States

Barratt: Joseph Barratt, 1796-1882, United States (Salix)

Bartling: Friedrich Gottlieb Bartling, 1798-1875, Germany

Bartram: William Bartram, 1739-1823, United States 
Batal.: Alexander Theodorowicz Batalin,

1847-1896, Russia

Batsch: August Johann Georg Karl Batsch, 1761-1802, Germany (horticulture)

Beadle: Chauncey Delos Beadle, 1866-1950, United States

Bean: William Jackson Bean, 1863-1947, United Kingdom (dendrology)

Becc.: Odoardo Beccari, 1843-1920, Italy (palms)

Bechst.: Johann Matthaeus Bechstein, 1757-1822, Germany

Beissn.: Ludwig Beissner, 1843-1927, Germany (Coniferae)

Benn.: John Joseph Bennett, 1801-1876, United Kingdom

Benson, L.: Lyman David Benson, 1909-1993, United States

Benth.: George Bentham, 1800-1884, United Kingdom

Bercht.: Friedrich von Berchtold, 1781-1876, Czechoslovakia

Berg, O.: Otto Karl Berg, 1815-1866, Germany

Bergius: Peter Jonas Bergius, 1730-1790, Sweden

Berl.: Jean Louis Berlandier, 1805-1851, France

Berth.: Sabin Berthelot, 1794-1880, France

Bieb.: Friedrich August Marschall von Bieberstein, 1768-1826, Germany, Russia

Bl.: Karl Ludwig von Blume, 1796-1862, Netherlands

Blake, S.F.: Sydney Fay Blake,

1892-1959, United States

Blake, S.T.: Stanley Thatcher Blake, 1910-1973, Australia
Blanco: Francisco Manuel Blanco, 1780-1845, Spain, Philippines.

Bois: Désiré Georges Jean Marie Bois, 1856-1946, France

Boiss.: Pierre Edmond Boissier, 1810-1885, Switzerland

Bonpland: Aimé Jacques Alexandre Bonpland, 1773-1858, France, South America

Boom: Boudewijn Karel Boom, 1903-1980, Netherlands (cultivated plants)

Booth: J.B. Booth, 1836-1908, Germany (nurseryman)

Bord.-Rey: O. Bordères-Rey, fl. 1939-68, France (Coniferae)

Borkh.: Moritz Balthasar Borkhausen, 1760-1806, Germany

Bory: Jean Baptiste Geneviève Marcellin Bory de Saint-Vincent, 1778-1846, France

Bosc: Louis Augustin Guillaume Bosc, 1759-1828, France

Boulenger: George Albert Boulenger, 1858-1937, Belgium (Rosa)

Br., N.E.: Nicholas Edward Brown, 1849-1934, United Kingdom

Br., P.: Patrick Browne, 1720-1790, Ireland

Br., R.: Robert Brown, 1773-1858, United Kingdom

Brandegee: Townshend Stith Brandegee, 1843-1925, United States

Braun, A.: Alexander Karl Heinrich Braun, 1805-1877, Germany

Braun, E.: Emma Lucy Braun, 1889-1971, United States

Brickell: Christopher David Brickell, 1932- , United Kingdom (cultivated plants) 
Briot, C.: Pierre Louis (Charles, in error) Briot, 1804-1888, France

Briq.: John Isaac Briquet,

1870-1931, Switzerland

Britton: Nathaniel Lord Britton,

1859-1934, United States

Brongn.: Adolphe Théodore Brongniart, 1801-1876, France

Browicz: Kazimierz Browicz,

1925- , Poland (dendrology)

Buch.-Ham.: Francis Buchanan-Hamilton,

1762-1829, United Kingdom

Buchh., J.: John Theodore Buchholz,

1888-1951, United States

Buc'hoz: Pierre Joseph Buc'hoz,

1731-1807, France

Buckl.: Samuel Botsford Buckley,

1809-1884, United States

Buerger: Heinrich Bürger,

1806-1858, Germany, Netherlands

Bunge: Alexander Andrejewitsch von Bunge,

1803-1890, Russia

Bur.: Louis Édouard Bureau,

1830-1918, France

Burkw.: Arthur Burkwood,

1888-1951, United Kingdom (nurseryman)

Burm.: Johannes Burman,

1707-1779, Netherlands

Burm.f.: Nikolaas Laurens Burman,

1734-1793, Netherlands

Burret: (Maximilian) Karl Ewald Burret,

1883-1964, Germany

Burv.: Fréderic Burvenich,

1857-1917, Belgium

Cambess.: Jacques Cambessèdes,

1799-1863, France

Camus, A.A.: Aimée Antoinette Camus,

1879-1965, France
Camus, E.G.: Edmond Gustav Camus,

1852-1915, France

Candolle: See DC.

Carr.: Elie Abel Carrière,

1818-1896, France (dendrology)

Carruthers: William Carruthers, 1830-1922, United Kingdom

Cav.: Antonio José Cavanilles, 1745-1804, Spain

Chabaud: J. Benjamin Chabaud, 1833-1915, France

Cham.: Ludolf Karl Adalbert von Chamisso, 1781-1838, Germany

Chamb.: Charles Joseph Chamberlain, 1863-1943, United States

Chambr.: Georges de Chambray, 1783-1849, France

Champ.: John George Champion, 1815-1854, United Kingdom

Chao: Jew Ming Chao,

fl. 1977, China

Chapm.: Alvan Wentworth Chapman, 1809-1899, United States

Chaub.: Louis Athanase (Anastase) Chaubard, 1785-1854, France

Cheng, M.: Mien Cheng,

fl. 1956-1980, China

Cheng, W.C.: Wan Chun Cheng,

1904-1983, China (dendrology)

Chev., A.: Auguste Jean Baptiste Chevalier, 1873-1956, France

Chittenden: Frederick James Chittenden, 1873-1950, United Kingdom (cultivated plants)

Christ: Konrad Hermann Heinrich Christ, 1833-1933, Switzerland

Clarke: Charles Baron Clarke, 1832-1906, United Kingdom 
Cochet: Pierre Charles Marie Cochet,

1866-1936, France

Cockerell: Theodore Dru Alison Cockerell, 1866-1948, United States

Cogn.: Célestin Alfred Cogniaux,

1841-1916, Belgium

Cohen-Stuart: Combertus Pieter Cohen-Stuart, 1889-1945, Netherlands, Java

Coll.: Henry Collett,

1836-1901, United Kingdom

Comber: Harold Frederick Comber,

1897-1969, United Kingdom

Comm.: Philibert Commerson,

1727-1773, France

Cook: Orator Fuller Cook,

1867-1949, United States

Corr.: Louis Henry Correvon,

1854-1939, Switzerland

Correa: José Francisco Corrêa da Serra,

1751-1823, Portugal

Correll: Donovan Stewart Correll,

1908-1983, United States

Cory: Victor Louis Cory,

1880-1964, United States

Coult.: John Merle Coulter,

1851-1928, United States

Cov.: Frederick Vernon Coville,

1867-1937, United States

Cowan: John Macqueen Cowan,

1891-1960, United Kingdom

Craib: William Grant Craib,

1882-1933, United Kingdom

Crantz: Heinrich Johann Nepomuk von Crantz, 1722-1797, Austria

Crepin: François Crépin,

1830-1903, Belgium

Croom: Hardy Bryan Croom,

1797-1837, United States
Curtis: William Curtis,

1746-1799, United Kingdom =-

Curtis, M.A.: Moses Ashley Curtis,

1808-1872, United States

DC.: Augustin Pyramus de Candolle,

1778-1841, Switzerland

DC., A.: Alphonse Louis Pierre

Pyramus de Candolle,

1806-1893, Switzerland

Dallim.: William Dallimore,

1871-1959, United Kingdom (horticulture, dendrology)

Dandy: James Edgar Dandy,

1903-1976, United Kingdom (Magnolia)

Davis: Peter Hadland Davis,

1918-1992, United Kingdom

Decne.: Joseph Decaisne,

1807-1882, France

Dehnh.: Friedrich Dehnhardt,

1787-1870, Germany

de Lannoy: de Lannoy,

fl. 1863, France

Deppe: Ferdinand Deppe,

1794-1861, Germany

Deseglise: Pierre Alfred Déséglise,

1823-1883, France

Desf.: René Louiche Desfontaines,

1750-1833, France

Desm.: Yves Desmarais,

1918- , Canada

Desmoul.: Charles Robert des Moulins, 1798-1875, France

Desp.: Narcisse Henri François Desportes,

1776-1856, France

Desr.: Louis Auguste Joseph Desrousseaux, 1753-1838, France

de Wit: Hendrik Cornelius Dirk de Wit,

1909- 
Dicks.: George Frederick Dickson,

fl. 1839, United Kingdom

Dieck: Georg Dieck,

1847-1925, Germany

Diels: Friedrich Ludwig Emil Diels,

1874-1945, Germany

Dietr., A.: Albert Gottfried Dietrich,

1795-1856, Germany

Dietr., D.: David Nathaniel Friedrich Dietrich,

1799-1888, Germany

Dipp.: Leopold Dippel,

1827-1914, Germany (dendrology)

Dode: Louis-Albert Dode,

1875-1943, France

Don, D.: David Don,

1799-1841, United Kingdom

Don, G.: George Don,

1798-1856, United Kingdom

Donn, J.: James Donn,

1758-1813, United Kingdom

Doorenbos: J. Doorenbos,

1921- , Netherlands (dendrology)

Dougl.: David Douglas,

1798-1834, United Kingdom

Drake: Emmanuel Drake del Castillo,

1855-1904, France

Dress, W.J.: William John Dress,

1918- , United States (cultivated plants)

Druce: George Claridge Druce,

1850 1932, United Kingdom

Drude: Carl Georg Oscar Drude,

1852-1933, Germany

Dudley, T.R.: Theodore Robert Dudley,

1936- , United States (Ilex, Viburnum, cultivated plants)

Duham.: Henri Louis Duhamel du Monceau,

1700-1782, France
Dum.-Cours.: George Louis Marie Dumont de

Courset,

1746-1824, France

Dunal: Michel Félix Dunal,

1789-1856, France

Dunn, S.T.: Stephen Troyte Dunn,

1868-1938, United Kingdom

Durande: Jean François Durande,

1732-1794, France

Durazz.: Antonio Durazzini,

fl. 1772- , Italy

Durieu: Michel Charles Durieu de Maisonneuve, 1796-1878, France

Du Roi: Johann Philipp Du Roi,

1741-1785, Germany

Eade: George William Eade,

1905- , United States

Eberm.: Carl Heinrich Ebermaier, 1802-1870, Germany

Eckl.: Christian Friedrich Ecklon, 1795-1868, Germany

Edgew.: Michael Pakenham Edgeworth,

1812-1881, United Kingdom

Egglest.: Willard Webster Eggleston,

1863-1935, United States

Ehrenb., K.: Karl August Ehrenberg,

1801-1849, Germany

Ehrh.: Jakob Friedrich Ehrhart,

1742-1795, Germany

El1.: Stephen Elliot,

1771-1830, United States

Ellis: John Ellis,

1710-1776, United Kingdom

Emeric: D. Emeric,

fl. ca. 1828 , France

End1.: Stephan Friedrich Ladislaus Endlicher, 1804-1849, Austria

Endress, P.: Peter Karl Endress,

1942- , Switzerland (Hamamelidaceae) 
Engelm.: George Engelmann, 1809-1884, United States

Engl.: Heinrich Gustav Adolf Engler, 1844-1930, Germany

Exell: Arthur Wallis Exell, 1901- , United Kingdom

Fabr.: Philipp Conrad Fabricius, 1714-1774, Germany

Fedde: Friedrich Karl Georg Fedde, 1873-1942, Germany

Ferguson, A.R.: Allan Ross Ferguson, 1943- , New Zealand (Actinidia)

Fern.: Merritt Lyndon Fernald, 1873-1950, United States

Fischer: Friedrich Ernst Ludwig von Fischer, 1782-1854, Russia

Fitschen: Jost Fitschen, 1869-1947, Germany (Coniferae)

Fletcher: Fletcher \& Sons Nursery,

fl. 1913, United Kingdom

Flinck: Karl Evert Flinck, 1915- , Sweden

Florin: Carl Rudolph Florin, 1894-1965, Sweden

Forbes: James Forbes, 1773-1861, United Kingdom

Foret: James Aloysius Foret, 1921 - , United States

Forrest: George Forrest, 1873-1932, United Kingdom (collector in China)

Forssk.: Pehr Forsskål, 1732-1763, Finland

Forst., J.G.: Johann Georg Adam Forster, 1754-1794, Germany

Forst., J.R.: Johann Reinhold Forster, 1729-1798, Germany

Fort.: Robert Fortune, 1812-1880, United Kingdom (collector in China)
Fosberg: Francis Raymond Fosberg, 1908- , United States

Frahm: G. Frahm,

fl. 1898- , Germany

Franch.: Adrien René Franchet, 1834-1900, France

Franco: João Manuel Antonio Paes do Amaral Franco, 1921- , Portugal

Gable: Joseph Benson Gable, 1886-1972, United States (nurseryman)

Gaertn.: Joseph Gaertner, 1732-1791, Germany

Gaertn., C.F.: Carl Friedrich von Gaertner, 1772-1850, Germany

Gagnep.: François Gagnepain, 1866-1952, France

Gamble: James Sykes Gamble, 1847-1925, United Kingdom

Gard.: Alexander Garden, 1730-1792, United Kingdom, United States

Gatt.: Augustin Gattinger, 1825-1903, United States

Gaudin: Jean François Aimée Gottlieb Philippe Gaudin,

1766-1833, Switzerland

Gaussen: Henri Marcel Gaussen, 1891-1981, France

Gentry, A.: Alwyn Howard Gentry, 1945-1993, United States

Gibson, D.: Dorothy Nash Gibson, 1921- . United States

Gilg: Ernst Friedrich Gilg, 1867-1933, Germany

Gillies: John Gillies, 1792-1834, United Kingdom

Glassman: Sidney Frederick Glassman, 1919- , United States 
Gleditsch: Johann Gottlieb Gleditsch,

1714-1786, Germany

Glend.: Robert Glendinning,

1805-1862, United Kingdom

Gmel., J.F.: Johann Friedrich Gmelin,

1748-1804, Germany

Godfrey: Robert Kenneth Godfrey,

$1911-$, United States

Godr:: Dominique Alexandre Godron,

1807-1880, France

Gord.: George Gordon,

1806-1879, Ireland (Coniferae)

Gr., A.: Asa Gray,

1810-1888, United States

Graebn.: Karl Otto Robert Peter Paul Graebner, 1871-1933, Germany

Graham, R.: Robert C. Graham,

1786-1845, United Kingdom

Green, P.S.: Peter Shaw Green,

1920- , United Kingdom

Greene: Edward Lee Greene,

1843-1915, United States

Gren.: Jean Charles Marie Grenier,

1808-1875, France

Griseb.: August Heinrich Rudolph Grisebach,

1814-1879, Germany

Grootend.: Herman Johannes Grootendorst,

1911- , Netherlands (dendrology, Rhododendron)

Guill.: André Guillaumin,

1885-1974, France

Gumbleton: William Edward Gumbleton,

1840-1911, Ireland

HBK.: See Humboldt; Bonpland; Kunth

Haenke: Thaddäus Peregrinus Xaverius Haenke,

1761-1817, Czechoslovakia

Hance: Henry Fletcher Hance,

1827-1886, United Kingdom, China
Hand.-Mazz.: Heinrich R. E. Handel-Mazzetti, 1882-1940, Austria

Hansen, N.E.: Niels Ebbesen Hansen, 1866-1950, Denmark

Hara: Hiroshi Hara, 1911-1986, Japan

Hariot: Paul Auguste Hariot, 1854-1917, France

Harrow, R.: Robert Lewis Harrow, 1867-1954, United Kingdom

Hartm.: Carl Johan Hartman, 1790-1849, Sweden

Hartw.: Carl Theodor Hartweg, 1812-1871, Germany

Harv.: William Henry Harvey, 1811-1866, Ireland

Hassk.: Justus Karl Hasskarl, 1811-1894, Germany, Java

Hatusima: Sumihiko Hatusima, 1906- , Japan (dendrology)

Hayata: Bunzō Hayata,

1874-1934, Japan, Taiwan

Hayne: Friedrich Gottlob Hayne, 1763-1832, Germany

Hedl.: Johan Theodor Hedlund, 1861-1953, Sweden

Hedr.: Ulysses Prentiss Hedrick, 1870-1951, United States

Hedwig, R.: Romanus Adolf Hedwig, 1772-1806, Germany

Heller: Amos Arthur Heller, 1867-1944, United States

Hemsl.: William Botting Hemsley, 1843-1924, United Kingdom

Henderson: James Henderson, fl. 1980, United States

Henry, A.: Augustine Henry, 1857-1930, Ireland, China 
Henry, J.K.: Joseph Kaye Henry, 1866-1930, Canada

Henry, L.: Louis Henry, 1853-1903, France (horticulture)

Herd.: Ferdinand Gottfried Maximilian Theobold von Herder,

1828-1896, Germany, Russia

Herincq: Francois Hérincq,

1820-1891, France

Herrm., J.: J. Herrmann,

1738-1800, France

Hesse: Hermann Albrecht Hesse,

1852-1937, Germany (dendrology)

Hibb.: James Shirley Hibberd,

1825-1890, United Kingdom (horticulture)

Hiern: William Philip Hiern,

1839-1925, United Kingdom

Hildebr.: F.H.G. Hildebrand,

1835-1915, Germany

Hill, J.: John Hill,

1716-1775, United Kingdom

Hochst.: Christian Ferdinand Friedrich

Hochstetter,

1787-1860, Germany

Hoffm., K.: Käthe Hoffmann,

1883-ca.1931, Germany

Hoffmgg.: Johann Centurius von Hoffmannsegg,

1766-1849, Germany

Holtt.: Richard Eric Holttum,

1895-1990, United Kingdom

Hook.: William Jackson Hooker,

1785-1865, United Kingdom

Hook.f.: Joseph Dalton Hooker,

1817-1911, United Kingdom

Hoppe: David Heinrich Hoppe,

1760-1846, Germany

Hort.: Hortorum (of gardens) or hortulanorum (of gardeners)
Horton: James H. Horton,

1931- , United States

Horvath: Michael Henry Horvath,

fl. 1934, United States (Berberis)

House, H.: Homer Doliver House,

1878-1949, United States

Houtt.: Maarten Houttuyn,

1720-1798, Netherlands

Houz. de Leh.: Jean Auguste-Hippolyte Houzeau de Lehaie,

1867-1959, Belgium (bamboo)

Hu, H.H.: Hsen Hsu Hu (Xian Su Hu),

1894-1968, China (dendrology)

Hu, S.Y.: Shiu-Ying Hu,

1910- , United States, China

Hubb., F.T.: Frederic Tracy Hubbard,

1875-1962, United States

Huds.: William Hudson,

1730-1793, United Kingdom

Hull: John Hull,

1761-1843, United Kingdom

Humboldt: Friedrich Wilhelm Heinrich Alexander von Humboldt,

1769-1859, Germany, South America

Hume: Hardrada Harold Hume,

1875-1965, United States, Canada (Ilex,

Camellia)

Hutch.: John Hutchinson,

1884-1972, United Kingdom

Hylander: Nils Hylander,

1904-1970, Sweden

Hylmo: Bertil Hylmö,

1915- , Sweden

Ingram, J .: John William Ingram,

1924- , United States (cultivated plants)

Ito: Tokutarō Itō,

1868-1941, Japan

Jack: William Jack,

1795-1822, United Kingdom 
Jacks., A.B.: Albert Bruce Jackson, 1876-1947, United Kingdom (Coniferae)

Jacq.: Nicolaus Joseph von Jacquin, 1727-1817, Austria

Jacq.f.: Joseph Franz von Jacquin, 1766-1839, Austria

Jacques: Henri Antoine Jacques,

1782-1866, France

Jaeger: Hermann Jaeger,

1815-1890, Germany (horticulture)

James: Edwin James,

1797-1861, United States

Jennison: Harry Milliken Jennison,

1885-1940, United States

Johnst., I.M.: Ivan Murray Johnston,

1898-1960, United States

Johnst., M.C.: Marshall Corning Johnston,

1930- , United States

Jong: Pieter Cornelius DeJong,

1938- , Netherlands (Acer)

Judd: Walter Stephen Judd,

1951- , United States

Juss.: Antoine Laurent de Jussieu,

1748-1836, France

Kache: Paul Kache,

1882-1945, Germany (horticulture)

Kalk.: Cornelis Kalkman,

1928- , Netherlands

Kalmb.: George Anthony Kalmbacher,

1897-1977, United States (horticulture)

Kaneh.: Ryōzō Kanehira,

1882-1947, Japan, Taiwan (dendrology)

Kanitz: August Kanitz,

1843-1896, Hungary

Karelin: Gregor Silitsch Karelin,

1801-1872, Russia

Karst.: Gustav Karl Wilhelm Hermann Karsten, 1817-1908, Germany
Ker-Gawl.: John Bellenden Ker Gawler, 1764-1842, United Kingdom

Kerner, A.: Anton Joseph Kerner von Marilaun, 1831-1898, Austria

Kikuchi: Akio Kikuchi,

1883-1951, Japan

Kimura: Arika Kimura,

1900- , Japan

Kirchn.: George Kirchner,

1837-1885, Germany

Kirkman: W. Benson Kirkman, contemporary, United States

Kitam.: Sirō Kitamura,

1906- , Japan

Klotz.: Johann Friedrich Klotzsch,

1805-1860, Germany

Knight, H.: Henry Knight,

1834-1896, United Kingdom (nurseryman)

Knight, J.: Joseph Knight,

1777?-1855, United Kingdom (nurseryman)

Knoop: Johann Hermann Knoop,

ca.1700-1769, Germany (horticulture)

Koch, K.: Karl Heinrich Emil Koch,

1809-1879, Germany (dendrology)

Kochs: Julius Kochs,

1900- , Germany

Koehne: Bernhard Adalbert Emil Koehne, 1848-1918, Germany (dendrology)

Koenig: Johann Gerhard König, 1728-1785, Germany

Koidz.: Gen'ichi Koidzumi,

1883-1953, Japan

Komar.: Vladimir Leontjevich Komarov, 1869-1945, Russia

Kosterm.: André Joseph Guillaume Henri Kostermans,

1907- , Netherlands, Indonesia

Kotschy: Carl Georg Theodor Kotschy, 1813-1866, Austria 
Kruessm.: Gerd Krüssmann, 1910-1980, Germany (dendrology)

Ktze., O.: Karl Ernst Otto Kuntze, 1843-1907, Germany

Kudo: Yūshun Kudō, 1887-1932, Japan

Kunth: Carl Sigismund Kunth, 1788-1850, Germany

Kurz: Wilhelm Sulpiz Kurz, 1834-1878, Germany

L'Her.: Charles Louis L'Héritier de Brutelle, 1746-1800, France

L.: Carl Linnaeus, 1707-1778, Sweden

L.f.: Carl Linnaeus, filius, 1741-1783, Sweden

Labill.: Jacques Julien Houtton de Labillardière, 1755-1834, France

Lag.: Mariano Lagasca y Segura,

1776-1839, Spain

Lam.: Jean Baptiste Antoine Pierre de Monnet de Lamarck, 1744-1829, France

Lamb.: Alymer Bourke Lambert, 1761-1842, United Kingdom

Lange, J.: Johan Martin Christian Lange, 1818-1898, Denmark

Latour-Marl.: Joseph (Bory) Latour-Marliac, 1830-1911, France

Lauche: Wilhelm Lauche, 1827-1883, Germany (dendrology)

Lauener: Lucien André Lauener, 1918-1991, United Kingdom

Lav.: Pierre Alphonse Martin Lavallée, 1836-1884, France

Laws., C.: Charles Lawson, 1794-1873, United Kingdom (Coniferae)

Laws., P.: Peter Lawson,

fl. ca.1870, United Kingdom (Coniferae)
Laxm.: Erich Laxmann, 1737-1796, Finland, Russia =

Ledeb.: Karl Friedrich von Ledebour, 1785-1851, Germany

Lem.: (Antoine) Charles Lemaire, 1801-1871, France

Lemmon, J.G.: John Gill Lemmon, 1832-1908, United States

Lemmon, W.P.: W.P. Lemmon,

fl. 1938, United States (Rhododendron)

Lemoine, E.: Émile Lemoine,

1862-1943, France

Lemoine, V.: Pierre Louis Victor Lemoine, 1823-1911, France

Lev.: Augustin Abel Hector Léveillé, 1863-1918, France

Lex.: Juan José Martinez de Lexarza, 1785-1824, Mexico

Li: Hui Lin Li,

$1911-$, United States

Liang, C.F.: Chou Fen Liang, 1921- , China (Actinidia)

Liebl.: Franz Kaspar Lieblein, 1744-1810, Germany

Lindl.: John Lindley, 1799-1865, United Kingdom

Link: Johann Heinrich Friedrich Link, 1767-1851, Germany

Little: Elbert Luther Little, Jr., 1907- , United States

Litvin.: Dmitri Ivanovich Litvinov, 1854-1929, Russia

Liu: Tang Shui Liu, fl. 1966-1982, Taiwan (Coniferae, Abies)

Llave: Pablo de La Llave, 1773-1833, Mexico

Lodd.: Conrad Loddiges, 1738-1826, Netherlands, United Kingdom 
Loesn.: Ludwig Eduard Theodor Loesener, 1865-1941, Germany

Loisel.: Jean Louis Auguste Loiseleur-

Deslongchamps,

1774-1849, France

Lotsy: Johannes Paulus Lotsy,

1867-1931, Netherlands

Loud.: John Claudius Loudon,

1783-1843, United Kingdom

Lour.: João de Loureiro,

1717-1791, Portugal

Lundell: Cyrus Longworth Lundell,

1907- , United States

Macf.: James Macfadyen,

1798-1850, United Kingdom

Maiden: Joseph Henry Maiden,

1859-1925, United Kingdom, Australia

Maire: Réne Charles Joseph Ernest Maire,

1878-1949, France, Algeria

Makino: Tomitarō Makino,

1862-1957, Japan

Manetti: Guiseppe Manetti,

1831-1858, Italy

Mansf.: Rudolph Mansfeld,

1901-1960, Germany

Markgr.: Friedrich Markgraf,

1897-1987, Germany, Switzerland

Marq.: Cecil Victor Boley Marquand,

1897-1943, United Kingdom

Marsh.: Humphrey Marshall,

1722-1801, United States

Marsili: Giovanni Marsili,

1727-1794, Italy

Mart.: Carl Friedrich Philipp von Martius,

1794-1868, Germany

Martinez: Maximino Martinez,

1888-1964. Mexico

Masam.: Genkei Masamune,

1899- , Japan
Mast.: Maxwell Tylden Masters,

1833-1907, United Kingdom

Matsum.: Jinzō Matsumura,

1856-1928, Japan

Mattusch.: Heinrich Gottfried von Mattuschka, 1734-1779, Germany

Maxim.: Carl Johann Maximowicz, 1827-1891, Russia

Mayr: Heinrich Mayr,

1856-1911, Germany (dendrology)

Mazel: Eugène Mazel,

fl. 1872, France (cultivated plants)

Mazzeo: Peter M. Mazzeo,

1940- , United States (dendrology)

McClelland: John McClelland,

1805-1883, United Kingdom

McClint.: Elizabeth May McClintock, 1912- , United States (cultivated plants, Hydrangea)

McClure: Floyd Alonso McClure, 1897-1970, United States (bamboo)

McKay: John A. McKay,

fl. 1938, United States (Rhododendron)

McKelvey: Susan Adams (nee Delano) McKelvey, 1888-1964, United States

Medik.: Friedrich Kasimir Medikus,

1736-1808, Germany

Meikle: Robert Desmond Meikle,

1923- , United Kingdom

Meisn.: Carl Daniel Friedrich Meisner,

1800-1874, Switzerland

Melander: Leonard William Melander.

1893- , United States

Merr.: Elmer Drew Merrill,

1876-1956, United States

Meunissier: Auguste Alexandre Meunissier, 1876-1947, France

Mey., C.A.: Carl Anton von Meyer,

1795-1855, Russia 
Mey., E.: Ernst Heinrich Friedrich Meyer, 1791-1858, Germany

Mey., F.G.: Frederick Gustav Meyer, 1917- , United States

Michx.: André Michaux, 1746-1803, France, United States

Michx.f.: François André Michaux, 1770-1855, France, United States

Miers: John Miers, 1789-1879, United Kingdom

Miki: Shigeru Miki, 1901-1974, Japan

Mill.: Philip Miller, 1691-1771, United Kingdom

Millais: John Guille Millais, 1865-1931, United Kingdom, (Magnolia, Rhododendron)

Miq.: Friedrich Anton Wilhelm Miquel, 1811-1871, Netherlands

Mirb.: Charles François Brisseau de Mirbel, 1776-1854, France

Mitf.: Algernon Bertram Freeman Mitford, 1837-1916, United Kingdom (bamboo)

Miyabe: Kingo Miyabe,

1860-1951, Japan

Moc.: José Mariano Moçiño,

1757-1820, Mexico

Moench: Conrad Moench, 1744-1805, Germany

Mol.: Juan Ignacio Molina, 1737-1829, Chile

Momiy.: Yasuichi Momiyama,

1904- , Japan

Moore, H.E.: Harold Emery Moore, Jr., 1917-1980, United States

Moore, R.J.: Raymond John Moore, 1918- , Canada

Moore, T.: Thomas Moore, 1821-1887, United Kingdom
Moric.: Moïse Étienne (Stefano) Moricand, 1779-1854, Switzerland

Morley: Brian D. Morley, 1943- , Australia

Morong: Thomas Morong, 1827-1894, United States

Mottet: Séraphin Joseph Mottet, 1861-1930, France (horticulture)

Muell., F.: Ferdinand Jacob Heinrich von Mueller,

1825-1896, Germany, Australia

Muell.-Arg.: Johannes Müller Argoviensis [of Aargau],

1828-1896, Switzerland

Muenchh.: Otto von Münchhausen,

1716-1774, Germany

Muhl.: Gotthilf Henry Ernest Muhlenberg, 1753-1815, United States

Muller: Cornelius Herman Muller, 1909- , United States

Munro: William Munro, 1818-1880, United Kingdom (bamboo)

Murata: Gen Murata (Gen Nakai), 1927- , Japan

Murr., A.: Andrew Murray, 1812-1878, United Kingdom (Coniferae)

Murr., E.: Albert Edward Murray, 1935- , United States

Murr., J.A.: Johan Andreas Murray, 1740-1791, Sweden

Mutis: José Celestino Bruno Mutis, 1732-1808, Spain, Colombia

Nakai: Takenoshin Nakai, 1882-1952, Japan

Nash: George Valentine Nash, 1864-1921, United States

Necker: Noel Martin Joseph de Necker, 1730-1793, France 
Nees: Christian Gottfried Nees von Esenbeck, 1776-1858, Germany

Nees, T.: Theodor Friedrich Ludwig Nees von Esenbeck,

1787-1837, Germany

Nelson, J.: John Nelson (pseudonym "Johannes Senilis"),

fl. 1860's, United Kingdom

Nemoto: Kwanji Nemoto,

1860-1936, Japan

Nichols.: George Nicholson,

1847-1908, United Kingdom (horticulture)

Niedenzu: Franz Joseph Niedenzu, 1857-1937, Germany

Nutt.: Thomas Nuttall,

1786-1859, United Kingdom, United States

Oerst.: Anders Sandoe Oersted,

1816-1872, Denmark, Central America

Ohwi: Jisaburo Ohwi,

1905-1977, Japan

Oliv.: Daniel Oliver,

1830-1916, United Kingdom

Opiz: Philipp Maximilian Opiz,

1787-1858, Czechoslovakia

Ort.: Casimiro Gómez Ortega,

1740-1818, Spain

Osbeck: Pehr Osbeck,

1723-1805, Sweden (collector in China)

Osborn: Arthur Osborn,

1878-1964, United Kingdom

Otto: Christoph Friedrich Otto,

1783-1856, Germany

Ottol.: Kornelius Johannes Willem Ottolander,

1822-1887, Netherlands

Oudemans: Cornelius Anton Jan Abraham

Oudemans.

1825-1906, Netherlands (dendrology)

Palib.: Ivan Vladimirovich Palibin,

1872-1949, Russia
Pall.: Peter Simon Pallas, 1741-1811, Germany, Russia

Palm.: Ernest Jesse Palmer, 1875-1962, United Kingdom, United States

Pamp.: Renato Pampanini, 1875-1949, Italy

Pancic.: Josef Pančić,

1814-1888, Yugoslavia

Parl.: Filippo Parlatore,

1816-1877, Italy (Coniferae)

Pavon: José Antonio Pavón, 1754-1844, Spain, Peru, Chile

Pax: Ferdinand Albin Pax, 1858-1942, Germany

Paxt.: Joseph Paxton,

1803-1865, United Kingdom

Pearce: Sydney Albert Pearce,

1906-1972, United Kingdom (cultivated plants)

Perk.: Janet Russell Perkins,

1853-1933, United States

Pers.: Christiaan Hendrik Persoon,

1761-1836, So. Africa, Germany, France

Phipps: James Bird Phipps,

1934- , Canada (Crataegus)

Pierre: Jean Baptiste Louis Pierre, 1833-1905, France

Pike: Arnold Pike,

fl. 1890's, United Kingdom

Pilger: Robert Knud Friedrich Pilger, 1876-1953, Germany

Planch.: Jules Émile Planchon,

1823-1888, France

Poggenburg: Justus Ferdinand Poggenburg, 1840-1893, Germany, United States

Poir.: Jean Louis Marie Poiret,

1755-1834, France

Poit.: Pierre Antoine Poiteau,

1766-1854, France (cultivated plants) 
Pojark.: Antonina Ivanovna Pojarkova, 1897-1980, Russia

Pollard: Charles Louis Pollard, 1872-1945, United States

Pourr.: Pierre André Pourret, 1754-1818, France

Prain: David Prain, 1857-1944, United Kingdom

Presl: Karel Boriwag Presl, 1794-1852, Czechoslovakia

Presl, J.: Jan Svatopluk Presl, 1791-1849, Czechoslovakia

Pritz.: Ernst Georg Pritzel, 1875-1946, Germany

Purk.: Emanuel von Purkyně, 1832-1882, Czechoslovakia

Pursh: Frederick Traugott Pursh, 1774-1820, Germany, United States

Raber: Oran Lee Raber, 1893-1940, United States

Raddi: Giuseppe Raddi, 1770-1829, Italy

Raeusch.: Ernst Adolf Raeuschel, fl. 1772-1797, Germany

Raf.: Constantine Samuel Rafinesque, 1783-1840, United States

Rayner: Douglas A. Rayner, 1949- , United States

Reade: John Moore Reade, 1876-1937, Canada, United States

Regel: Eduard August von Regel, 1815-1892, Germany, Russia

Rehd.: Alfred Rehder, 1863-1949, Germany, United States (dendrology)

Reichenb.: Heinrich Gottlieb Ludwig Reichenbach, 1793-1879, Germany

Retz.: Anders Jahan Retzius, 1742-1821, Sweden
Reveal: James Lauritz Reveal,

1941- , United States

Rich., A.: Achille Richard,

1794-1852, France

Rich., L.C.: Louis Claude Marie Richard,

1754-1821, France

Riv., A.: Marie Auguste Rivière,

1821-1877, France (bamboo)

Riv., C.: Charles Marie Rivière,

1845-?, France (bamboo)

Rivers: Thomas Rivers,

1798-1877, United Kingdom (nurseryman)

Robinson: Benjamin Lincoln Robinson,

1864-1935, United States

Robs., N.: Norman K. B. Robson,

1928- , United Kingdom (Hypericum)

Roberts: A.V. Roberts,

United Kingdom

Roem.: Johann Jacob Roemer,

1763-1819, Germany

Roem., M.J.: Max J. Roemer,

1791-1849, Germany

Rolfe: Robert Allen Rolfe,

1855-1921, United Kingdom

Rose: Joseph Nelson Rose,

1862-1928, United States

Roth: Albrecht Wilhelm Roth, 1757-1834, Germany

Rottl:: Johann Peter Rottler, 1749-1836, France, India

Rouleau: Joseph Albert Ernest Rouleau, 1916-1991, Canada

Rowlee: Willard Winfield Rowlee, 1861-1923, United States

Rowley: Gordon Douglas Rowley, 1921- , United Kingdom

Roxb.: William Roxburgh, 1751-1815, United Kingdom, India 
Royle: John Forbes Royle, 1798-1858, United Kingdom

Rudd: Velva Elaine Rudd, 1910- , United States

Ruempler: Karl Theodor Rümpler, 1817-1891, Germany

Ruiz: Hipólito Ruiz López,

1754-1815, Spain, Peru, Chile

Rupr.: Franz Joseph Ruprecht, 1814-1870, Czechoslovakia, Russia

Rushforth: K.D. Rushforth,

fl. 1983, United Kingdom (Coniferae)

Rydb.: Per Axel Rydberg,

1860-1931, United States

Salisb.: Richard Anthony Salisbury,

1761-1829, United Kingdom

Sarg.: Charles Sprague Sargent,

1841-1927, United States (dendrology)

Sav.: Paul Amedée Ludovic Savatier,

1830-1891, France (flora of Japan)

Schaeffer: Jacob Christian Schaeffer,

1718-1790, Germany

Schauer: Johannes Conrad Schauer.

1813-1848, Germany

Scheele: Georg Heinrich Adolph Scheele,

1808-1864, Germany

Schelle: Ernst Schelle,

1864-1945, Germany (horticulture)

Schery: Robert Walter Schery,

1917-1987, United States

Schiede: Christian Julius Wilhelm Schiede,

1798-1836, Germany

Schlechtend.: Diederich Franz Leonhard von

Schlechtendal,

1794-1866, Germany

Schmidt, F.: Friedrich Karl Schmidt,

1832-1908, Russia

Schneid.: Camillo Karl Schneider,

1876-1951, Germany, United States (dendrology)
Schottky: Ernst Max Schottky,

1888-1915, Germany

Schrad.: Heinrich Adolph Schrader, 1767-1836, Germany

Schreber: Johann Christian Daniel von Schreber, 1739-1810, Germany

Schub.: Bernice Giduz Schubert, 1913- , United States

Schultes, J.A.: Josef August Schultes, 1773-1831, Austria

Schultes, J.H.: Julius Hermann Schultes, 1804-1840, Austria

Schultz, H.: Elmer H. Schultz,

fl. 1934, United States (Berberis)

Schum.: Karl Moritz Schumann, 1851-1904, Germany

Schwarz, O.: Otto Karl Anton Schwarz, 1900-1983, Germany

Schwerin: Fritz Kurt Alexander von Schwerin, 1856-1934, Germany (dendrology)

Scop.: Giovanni Antonio Scopoli, 1723-1788, Austria, Italy

Sealy: Joseph Robert Sealy, 1907- , United Kingdom

Seemann: Berthold Carl Seemann, 1825-1871, Germany

Seemen: Karl Otto von Seemen, 1838-1910, Germany

Seldin: Margaret J. Seldin,

fl. 1976, United States

Sendtn.: Otto Sendtner, 1813-1859, Germany

Ser.: Nicolas Charles Seringe, 1776-1858, France

Sesse: Martin de Sessé y Lacasta, 1751-1808, Spain, Mexico

Sharp: Aaron John Sharp, 1904- , United States 
Sharp, W.: Ward McClintic Sharp, 1904- , United States

Shibata: Keita Shibata, 1877-1949, Japan

Shinners: Lloyd Herbert Shinners, 1918-1971, Canada, United States

Sieb.: Philipp Franz von Siebold, 1796-1866, Germany

Simmonds: Arthur Simmonds, 1892-1968, United Kingdom (horticulture)

Simonk.: Lájos Simonkai, 1851-1910, Hungary

Sims: John Sims, 1749-1831, United Kingdom

Skan: Sidney Alfred Skan, 1870-1939, United Kingdom (horticulture)

Skeels: Homer Collar Skeels, 1873-1934, United States

Skipwith: Geoffrey Skipwith, fl. 1924-29, United Kingdom (nurseryman)

Skvortz.: Boris Vassilievich Skvortzov, 1890-1980, Poland, Brazil

Sleumer: Hermann Otto Sleumer, 1906- , Germany, Netherlands

Sm.: James Edward Smith, 1759-1828, United Kingdom

Sm., A.C.: Albert Charles Smith, 1906- , United States

Sm., A.R.: Alan Reed Smith, 1938- , United Kingdom

Sm., E.A.: Eugene Allen Smith, 1841-1927, United States

Sm., L.B.: Lyman Bradford Smith, 1904- . United States

Sm., W.G.: William Gardner Smith, 1866-1928, United Kingdom

Sm., W.W.: William Wright Smith, 1875-1956, United Kingdom
Small: John Kunkel Small, 1869-1938, United States

Soderstrom: Thomas Robert Soderstrom, 1936-1987, United States

Sol.: Daniel Carl Solander, 1733-1782, Sweden, United Kingdom

Solym.: Sigmond L. Solymosy, 1906-1974, United States

Soul.-Bod.: Étienne Soulange-Bodin, 1774-1846, France (horticulture)

Spach: Édouard Spach, 1801-1879, France

Spaeth: Franz Ludwig Späth, 1838-1913, Germany (horticulture)

Spongberg: Stephen A. Spongberg, 1942- , United States

Sprague: Thomas Archibald Sprague, 1877-1958, United Kingdom

Spreng.: Kurt Polykarp Joachim Sprengel, 1766-1833, Germany

St. Hil.: Auguste François César Prouvencal de Saint-Hilaire, 1779-1853, France

Stapf: Otto Stapf, 1857-1933, Austria, United Kingdom

Staunt.: George Leonard Staunton, 1737-1801, United Kingdom

Stearn: William Thomas Stearn,

1911- , United Kingdom

Sterns: Emerson Ellick Sterns,

1846-1926, United States

Steud.: Ernst Gottlieb von Steudel, 1783-1856, Germany

Steven: Christian von Steven, 1781-1863, France, United States

Stewart, J.L.: John Lindsay Stewart, 1832-1873, United Kingdom, India

Steyerm.: Julian Alfred Steyermark, 1909-1988, United States 
Stokes: Jonathan Stokes,

1755-1831, United Kingdom

Stritch: Lawrence R. Stritch,

1953- , United States

Sukachev: Vladimir Nikolaevich Sukachev,

1880-1967, Russia

Sun: Siang Chung Sun,

1908- , China

Suring.: Willem Frederik Reinier Suringar, 1832-1898, Netherlands

Svenson: Henry Knute Svenson, 1897-1986, United States

Swartz, O.: Olof Peter Swartz,

1760-1818, Sweden

Sweet: Robert Sweet,

1783-1835, United Kingdom (cultivated plants)

Swingle: Walter Tennyson Swingle,

1871-1952, United States (Citrus)

Szysz.: Ignaz von Szyszylowicz,

1857-1910, Poland

T. \& G.: See Torr.; A. Gr.

Tagg: Henry Frank Tagg,

1874-1933, United Kingdom (Rhododendron)

Takeda: Hisayoshi Takeda,

1883-1972, Japan

Tarbox: Gurdon Lucius Tarbox, Jr.,

1927- , United States (horticulture)

Tausch: Ignaz Friedrich Tausch,

1793-1848, Czechoslovakia

Taylor, T.M.C.: Thomas Mayne

Cunninghame Taylor,

1904-1983, Canada, South Africa

Temple: F.L. Temple,

fl. 1885-1893, United States (nurseryman)

Tenore: Michele Tenore,

1780-1861, Italy

Teran: Manuel de Mier y Terán,

1789?-1852, Mexico
Thoms.: Thomas Thomson,

1817-1878, United Kingdom

Thory: Claude Antoine Thory, 1759-1827, France

Thouin: André Thouin, 1747-1824, France

Thunb.: Carl Peter Thunberg, 1743-1828, Sweden, Japan, South Africa

Tieghem: Philippe Édouard Léon van Tieghem, 1839-1914, France

Tobl.: Friedrich Tobler,

1879-1957, Switzerland (Hedera)

Torr.: John Torrey,

1796-1873, United States

Tratt.: Leopold Trattinnick,

1764-1849, Austria

Traut.: Ernst Rudolph von Trautvetter, 1809-1889, Russia

Trecul: Auguste Adolfe Lucien Trécul, 1818-1896, France

Trelease: William Trelease,

1857-1945, United States

Trew: Christoph Jakob Trew,

1695-1769, Germany

Troncoso: Nélida Sara Troncoso,

1914-1988, Argentina

Tseng, C.J.: Chang Jiang Tseng,

1896-1980, China (Ilex)

Turcz.: Nikolai Stepanovich Turczaninow, 1796-1863, Russia

Turner, B.L.: Billie Lee Turner,

1925- , United States

Turra: Antonio Turra, 1730-1796, Italy

Turrill: William Bertram Turrill, 1890-1961, United Kingdom

Tutch.: William James Tutcher, 1867-1920, United Kingdom 
Uyeki: Homiki Uyeki,

1882- , Japan

Vahl: Martin Henrichsen Vahl,

1749-1804, Denmark

Van Melle: Peter Jacobus van Melle, 1891-1953, Netherlands, United States (Juniperus)

Van.: Eugène Vaniot, ?-1913, France

Vasey: George Vasey, 1822-1893, United States

Veitch, J.G.: John Gould Veitch, 1839-1870, United Kingdom

Vent.: Étienne Pierre Ventenat, 1757-1808, France

Vill.: Celestino Fernández-Villar, 1838-1907, Spain, Philippines

Villars: Dominique Villars, 1745-1814, France

Vilm., M.L.: Auguste Louis Maurice Levêque de Vilmorin,

1849-1918, France

Vis.: Roberto de Visiani,

1800-1878, Italy

Voss: Andreas Voss,

1857-1924, Germany (cultivated plants)

Vrughtman, F.: Freek Vrughtman,

1927- , Canada (horticulture)

Wall.: Nathaniel Wallich (Nathan Wolff),

1786-1854, Denmark, India, Nepal

Wallroth: Carl Friedrich Wilhelm Wallroth, 1792-1857, Germany

Walp.: Wilhelm Gerhard Walpers,

1816-1853, Germany

Walt.: Thomas Walter,

1740-1789, United States

Wang.: Friedrich Adam Julius von Wangenheim, 1749-1800, Germany
Warder: John Aston Warder, 1812-1883, United States (horticulture)

Wassh.: Dieter Carl Wasshausen, 1938- , United States (Acanthaceae)

Wats., P.W.: Peter William Watson, 1761-1830, United Kingdom

Wats., S.: Sereno Watson, 1826-1892, United States

Weatherby: Charles Alfred Weatherby, 1875-1949, United States

Webb, D.A.: David Allardice Webb, 1912- , Ireland

Webb, P.B.: Philip Barker Webb, 1793-1854, United Kingdom

Weber: Georg Heinrich Weber, 1752-1828, Germany

Wendl., H.: Hermann Wendland, 1825-1903, Germany

Wendl., J.C.: Johann Christoph Wendland, 1755-1828, Germany

Wesmael: Alfred Wesmael, 1832-1905, Belgium (dendrology)

Weston: Richard Weston, 1733-1806, United Kingdom

Weyer: W. van de Weyer,

fl. 1914-20, United Kingdom

Wieg.: Karl McKay Wiegand, 1873-1942, United States

Wight: Robert Wight,

1796-1872, United Kingdom, India

Wight, W.F.: William Franklin Wight,

1874-1954, United States

Willd.: Karl Ludwig von Willdenow,

1765-1812, Germany

Wils.: Ernest Henry Wilson,

1876-1930, United Kingdom, United States

Wilson, P.: Percy Wilson,

1879-1944, United States 
Wimmer: Christian Friedrich Heinrich Wimmer,

1803-1868, Germany

Wittmack: Max Carl Ludwig Wittmack,

1839-1929, Germany

Wolf, E.: Egbert Ludwigowitsch Wolf,

1860-1931, Germany (dendrology)

Wood, A.: Alphonso Wood, 1810-1881, United States

Woods, F.W.: Frank W. Woods, contemporary, United States

Wright: Charles Henry Wright, 1864-1941, United Kingdom

Wright, J.: John Wright, 1811-1846, United States

Yamamoto: Yoshimatsu Yamamoto, 1893-1947, Japan, Taiwan

Yamazaki: Takasi Yamazaki, 1921- , Japan

Yatabe: Ryōkichi Yatabe, 1851-1899, Japan

Young: Robert Armstrong Young, 1876-1963, United States

Zab.: Hermann Zabel, 1832-1912, Germany (dendrology)

Zenari: Silvia Zenari, 1896-1956, Italy

Zeyh.: Carl Ludwig Philipp Zeyher, 1799-1858, Germany, So. Africa

Zoll.: Heinrich Zollinger,

1818-1859, Switzerland, Java

Zucc.: Joseph Gerhard Zuccarini, 1797-1848, Germany

Zuccagni: Attilio Zuccagni, 1754-1807, Italy 


\section{Appendix B: Plant Collection Sites, Cited by Acronym}

The acronyms cited here identify the 677 sites where plant specimens were collected. Arranged alphabetically by state, the acronyms consist of two elements: (1) the first two letters are the postal code for the state or the District of Columbia, and (2) the second two letters identify a precise locale in the state where the plant was collected. ALAU thus stands for Alabama, Auburn University; and DCNA, for the District of Columbia, the U.S. National Arboretum.

ALAU Auburn University, Auburn, Lee Co., AL

ALBB Bank in downtown area, Birmingham, Jefferson Co., AL

ALBG Bellingrath Gardens, Theodore, Mobile Co., AL ALBH Byers Nursery, Huntsville, Madison Co., AL

ALBT Bryce State Mental Hospital, Tuscaloosa, Tuscaloosa Co., AL

ALCG Vacant corner lot on Church Street near Government Street, Mobile, Mobile Co., AL

ALCM Church Street Graveyard, Mobile, Mobile Co., AL

ALCS Chamberlain Street, Mobile, Mobile Co., AL

ALCW Nursery of Clarence H. Welch, US Highway 98, Wilmer, Mobile Co., AL

ALDM House on Dauphin Street, Mobile, Mobile Co., AL

ALEC Elmwood Cemetery, Birmingham, Jefferson Co., AL

ALEH Private garden, 4558 Brookmoor Drive, Mobile, Mobile Co., AL

ALFN Fraser Nursery, Birmingham, Jefferson Co., AL

ALFS Horticulture Field Station Mobile (Spring Hill School), Mobile, Mobile Co., AL

ALHC Private garden, 168 Peachtree Circle, Birmingham, Jefferson Co., AL

ALIT Milepost 36.5 on Interstate 85 near Tuskegee exit, Macon Co., AL

ALJG Private garden, Eight Mile, Mobile Co., AL

ALMC Beech Grove United Methodist Church,

Francisco, Jackson Co., AL

ALMG Magnolia Gardens, Chunchulla,

Mobile Co., AL

ALON Overlook Nurseries, Route 98, Mobile, Mobile Co., AL

ALOS Overlook Nursery, Schillinger Road, West Mobile, Mobile Co., AL

ALPH Albert Pick Motel, US Route 72, Huntsville, Madison Co., AL

ALRH Private garden, 4058 Old Shell Road, Spring Hill, Mobile, Mobile Co., AL

ALSH Spring Hill College, Spring Hill, Mobile, Mobile Co., AL
ALSM Scenic Motel, Highway 98, Mobile, Mobile Co., AL

ALTC Roadside, US Route 11, $3 \mathrm{mi}$. south of Tuscaloosa, Tuscaloosa Co.. AL

ALTD Tom Dodd Nursery, Semmes, Mobile Co., AL

ALTM Travelodge Motel, Government Street, Mobile, Mobile Co., AL

ALTR Roadside, US Route 11 at Tombigbee River (west side), Sumter Co., AL

ALUA University of Alabama, Tuscaloosa, Tuscaloosa Co., AL

ALWR Private garden, Wulff Road, Semmes, Mobile Co., AL

ARCA Private garden, Calion, Union Co., AR

ARGD Private garden, Glendale, Lincoln Co., AR

ARHS Private garden, 34 Edgehill Road, Little Rock, Pulaski Co., AR

ARPT Private garden, 4 Longfellow Lane, Little Rock, Pulaski Co., AR

ARRB Private garden, 36 River Ridge Road, Little Rock, Pulaski Co., AR

ARRR Roadside, Route 130, Scott, Pulaski Co., AR

ARSN Private garden, 12 Sherrill Road, Little Rock, Pulaski Co., AR

ARUM University of Arkansas, Monticello, Drew Co., AR

ARWS Private garden, 8 Longfellow Place, Little Rock, Pulaski Co., AR

DCAC National Academy of Sciences, Wisconsin Avenue NW, Washington, DC

DCAD Vacant lot at corner of Anacostia Avenue and Douglas Street NE, Washington, DC

DCAE Arnold Estate Garden (now abandoned), 4000 block, Massachusetts Avenue NW. Washington, DC

DCAG Kenilworth Aquatic Gardens, Douglas Street NE (off Kenilworth Avenue), Washington, DC

DCAR Anacostia Park, near Sousa Bridge (Pennsylvania Avenue SE), Washington, DC

DCBG US Botanic Garden grounds, 3d Street SW, Washington, DC

DCCA Center for the Aging, 2601 18th Street NE, Washington, DC

DCCB Private garden, 6010 31st Street NW, Washington, DC

DCCC Cosmos Club, 2121 Massachusetts Avenue NW, Washington, DC

DCCG US Capitol grounds, Washington DC

DCCH Sidewalk along Delaware Avenue NE, by Senate Office Building, Washington, DC

DCCI Columbia Island, Washington, DC

DCCK Small park at Columbia and Kalorama Roads NW, Washington, DC

DCCL Private garden, 3815 Alton Place NW, Washington, DC

DCCT Connecticut Avenue NW, between Kanawha and Military Roads, Washington, DC

DCDG Private garden, Dahlia Street and Georgia Avenue NW, Washington, DC

DCDO Dumbarton Oaks Garden, 31st and R Streets NW, Washington, DC

DCEL Vacant lot near Eastern Avenue and Laurel Street NW, Washington, DC 
DCFM Vacant lot across from Franciscan Monastery, 18th Street NE, Washington, DC

DCFP Folger Park, 2d and D Streets SE, Washington, DC

DCGC Near greenhouse, Independence Avenue at 1st Street SW, Washington, DC

DCGD Private garden at 1617 29th Street (Georgetown), Washington, DC

DCGP Garfield Park, F Street between 1st and 3rd Streets SE, Washington, DC

DCGW George Washington University campus, Washington, DC

DCHM Hillwood Museum, 4115 Linnean Avenue NW, Washington, DC

DCHP East Potomac Golf Course, Hains Point, Washington, DC

DCHU Howard University campus, Washington, DC

DCIW Private garden, 7433 Berkeley Terrace NW. Washington, DC

DCJJ Near John Paul Jones statue, 17th Street and Independence Avenue SW, Washington, DC

DCJM Jefferson Memorial grounds, Washington, DC

DCJS Private garden, corner of Jackson Street and 14th Street NE, Washington, DC

DCLC Library of Congress, Washington, DC

DCLP Lafayette Park, Washington, DC

DCMA Private garden, MacArthur Boulevard near Arizona Avenue NW, Washington, DC

DCMC Private garden, 161631 st Street NW (Georgetown), Washington, DC

DCMH Meridian Hill Park, 16th Street NW, Washington, DC

DCML The Mall, Washington, DC

DCMN Fort McNair, Washington, DC

DCMS US Navy, Bureau of Medicine and Surgery, 23d and E Streets NW, Washington, DC

DCNA US National Arboretum, Washington, DC

DCNC National Cathedral, Washington, DC

DCNE Private garden, 3910 18th Street NE, Washington, DC

DCNG National Gallery of Art, Washington, DC

DCNY New York Avenue and 7th Street NW, Washington, DC

DCPA Pan American Building, 17th and C Streets NW, Washington, DC

DCPC Rock Creek Park Cemetery, Rock Creek Church Road NW, Washington, DC

DCPG National Portrait Gallery, F and 7th Streets NW, Washington, DC

DCPL Old District of Columbia Public Library (now UDC), New York Avenue and 7th Street NW, Washington, DC

DCPP East Potomac Park, Washington, DC

DCRC Rock Creek Park, Washington, DC

DCRP Private garden, 2122 Rand Place NE, Washington, DC

DCRR Private garden, 3051 Porter Street NW, Washington, DC

DCRS Private garden, 3224 R Street NW, Washington, DC

DCSD Department of State, C and 22d Streets NW, Washington, DC
DCSE Saint Elizabeth's Hospital grounds, Martin Luther King Jr Avenue SE, Washington, DC

DCSG Edge of woods at corner of Sargent Road and Galloway Road NE, Washington, DC

DCSH Soldiers Home (near police station), Washington, DC

DCSI Old Smithsonian Institution building (the "Castle"), Washington, DC

DCTB Tidal Basin, Washington, DC

DCTF Private garden, 1700 24th Street NE, Washington, DC

DCTN Private garden, 4701 Fessenden Street NW, Washington, DC

DCUR Mount Alto, site for new Russian Federation Embassy, Wisconsin Avenue NW, Washington, DC

DCUS Union Square, 1st Street and The Mall, Washington, DC

DCVK Private garden, 152 North Carolina Avenue SE, Washington, DC

DCWA Westchester Apartments, 4000 Cathedral Avenue NW, Washington, DC

DCWH The White House, 1600 Pennsylvania Avenue NW, Washington, DC

DCWM Washington Monument grounds, Washington, DC

DCWP West Potomac Park, Washington, DC

DCWR Grounds of Walter Reed Hospital, 16th Street NW, Washington, DC

DECN Cannon Nursery, Greenwood, Sussex Co., DE

DEEM Eleutherian Mills, Greenville,

New Castle Co., DE

DEFC Frorer Collection, Wilmington,

New Castle Co., DE

DEFL Private garden, Bridgeville, Sussex Co., DE

DEMC Mount Cuba Botanical Park, Greenville, New Castle Co., DE

DESC Delaware State College, Dover, Kent Co., DE

DESR Along Route 52, 1/2 mi. south of state line, near Snuffmill Road, Centerville, New Castle Co., DE

DEWG Winterthur Gardens, Winterthur, New Castle Co., DE

FLAL Private garden, Northwest 45th Avenue, Gainesville, Alachua Co., FL

FLBF Briar Farm, 15th Street NW, Gainesville, Alachua Co., FL

FLBW Private garden, Lakeview Drive, DeFuniak Springs, Walton Co., FL

FLCG Cummer Gallery of Art, 829 Riverside Avenue, Jacksonville, Duval Co., FL

FLCM Cemetery adjacent to Monticello Nursery, Monticello, Jefferson Co., FL

FLCS Apartment building, Charles Street, Tallahassee, Leon Co., FL

FLDC Doyle Conner Building, 2010 Southwest 34th Street, Gainesville, Alachua Co., FL

FLDG Dickinson's Glen Arden, Chipley, Washington Co., FL

FLDH Private garden, 1313 Southwest 23d Drive, Gainesville, Alachua Co., FL 
FLDJ Private garden, 335 Cypress Street,

DeFuniak Springs, Walton Co., FL

FLEM Lake Ella Motel, Tallahassee, Leon Co., FL

FLFB Private garden, 2356 West University Avenue, Gainesville, Alachua Co., FL

FLFS Florida State University, Tallahassee, Leon Co., FL

FLGA Apartment house, 13th Street and 13th Avenue SW, Gainesville, Alachua Co., FL

FLGS Glen Saint Mary Nurseries, Glen Street, Glen Saint Mary, Baker Co., FL

FLHJ Howard Johnson Restaurant, 13th Street NW and 29th Road, Gainesville, Alachua Co., FL

FLHP Private garden, Highland Park area, Jacksonville, Duval Co., FL

FLIF IFAS Horticultural Unit (University of Florida), Millhopper Road, Gainesville, Alachua Co., FL

FLJH Private garden, 2 Northwest 29th Street, Gainesville, Alachua Co., FL

FLJU Jacksonville University, Jacksonville, Duval Co., FL

FLLE American Legion Building, Lake Ella, Tallahassee, Leon Co., FL

FLLT Building along Route 27 near Lake Ella, Tallahassee, Leon Co., FL

FLMA Marcos de Apalache Museum, Saint Marks, Wakulla Co., FL

FLMG Alfred B. Maclay Gardens State Park, US Route 319, Leon Co., FL

FLMM Mason Manor, 1827 Stockton Street, Jacksonville, Duval Co., FL

FLMN Monticello Nursery, Monticello, Jefferson Co., FL

FLMS Along Highway 441, south side of Gainesville, Alachua Co., FL

FLMT Vacant lot on Monroe Street, Tallahassee, Leon Co. FL

FLPE Private garden, 4859 Empire Avenue, Jacksonville, Duval Co. FL

FLPL Park Street and Laclede (western section), Jacksonville, Duval Co., FL

FLPM Prince Murat Motel, Monroe Street, Tallahassee, Leon Co., FL

FLPS Private garden, Park Street extension, Riverside area (western section), Jacksonville, Duval Co., FL

FLPU Private garden, Providence, Union Co., FL

FLPW Welcome station, Florida state line, Route 1, Nassau Co., FL

FLRD Private garden, Randall and Dancy Streets, Jacksonville, Duval Co., FL

FLRH North Florida Landscaping Co., 1310 Glen Laura Road, Jacksonville, Duval Co., FL

FLRI Riverside Motel, Route 17, near Florida state line, Nassau Co., FL

FLRM Private garden, San Jose Boulevard, Jacksonville, Duval Co., FL

FLRP Private garden, 4806 River Basin Drive and River Point Road, Jacksonville, Duval Co., FL

FLTP Torreya State Park, Liberty Co., FL

FLUF University of Florida, Gainesville, Alachua Co., FL
FLWC Women's Club of Jacksonville, Park Street. Jacksonville, Duval Co., FL

FLWG West Gate Shopping Centre, University Avenue, Gainesville, Alachua Co., FL

FLWJ Private garden, 2243 University Boulevard. North Jacksonville, Duval Co., FL

GAAB Atlanta Botanical Garden, Piedmont Park, Prado Street S, Atlanta, Fulton Co., GA

GAAC Camak House, Finley and Meigs Streets, Athens, Clarke Co., GA

GAAG Private garden, Augusta, Richmond Co., GA

GAAM Carr's Hill, Athens, Clarke Co., GA

GAAT Private garden, Athens, Clarke Co., GA

GABC Bonaventure Cemetery, Savannah, Chatham Co., GA

GABG Barnsley Gardens, Route 3, Adairsville, Bartow Co., GA

GABP Buttner Park, Savannah, Chatham Co., GA

GABQ Private garden, 209 Sherwood Road, Rome, Floyd Co., GA

GABS Berry School, Rome, Floyd Co., GA

GABW Benjamin Wilson House, Calhoun Square, Savannah, Chatham Co., GA

GACB Along Canton bypass, Route 5, Cherokee Co., GA

GACC Christ Church, near Fort Frederica, Saint Simon's Island, Glynn Co., GA

GACD Private garden, 5 Club Drive, Rome, Floyd Co., GA

GACE Private garden, 4130 Amsterdam Circle, Savannah, Chatham Co., GA

GACG Callaway Gardens, Pine Mountain, Harris Co., GA

GACH Cloisters Hotel, Sea Island, Saint Simon's Island, Glynn Co., GA

GACL Private garden, 308 East 5th Street, Savannah, Chatham Co., GA

GACM Maxwelton Plantation, Colonel's Island, Liberty Co., GA

GACR Old Knight Plantation, Quitman, Brooks Co., GA

GACW Clark and Waddell Streets, Athens, Clarke Co., GA

GADG Diamond Garden, South Rockwell Avenue, Vernonburg, Chatham Co., GA

GADR Private garden, corner of Drayton and Gaston Streets, Savannah, Chatham Co., GA

GAEC Private garden, Highway 140, Canton, Cherokee Co., GA

GAEH Mimosa Hall, 127 Bullock Avenue, Roswell, Fulton Co., GA

GAES Private garden, 316 55th Street, East Savannah, Chatham Co., GA

GAET Private garden, 2021 East 37th Street, Savannah, Chatham Co., GA

GAFF Private garden, front of 318 55th Street E, Savannah, Chatham Co., GA

GAFG Private garden, Hamilton, Harris Co., GA

GAFN Fruitland Nursery, Augusta, Richmond Co., GA

GAGB University of Georgia Botanical Garden, Athens, Clarke Co., GA 
GAGC Gould's Corner, Walton Way and Milledge Road, Augusta, Richmond Co., GA

GAGM Abandoned garden along Route 27 Alt., $10 \mathrm{mi}$. north of Greenville, Meriwether Co., GA

GAGN Private garden, front of 415 Green Street, Augusta, Richmond Co., GA

GAGR Private garden, 2111 Gardner Street, Augusta, Richmond Co., GA

GAGS Private garden, adjacent to 2111 Gardner Street, Augusta, Richmond Co., GA

GAHC Private garden, 933 Milledge Road, Augusta, Richmond Co., GA

GAHS Private garden, 869 Hill Street, Athens, Clark Co., GA

GAIH Private garden, Isle of Hope, La Roche Avenue, Savannah, Chatham Co., GA

GAIS Plant Introduction Station, Route 17, $10 \mathrm{mi}$. south of Savannah, Chatham Co., GA

GAJA Edge of southbound Route 301, ca. 2 mi. south of Altamaha River bridge, near Jesup, Wayne Co., GA

GAJC Private garden, 3820 Northside Drive, Atlanta, Fulton Co., GA

GAJI Private garden, Jekyll Island, Glynn Co., GA

GAJS Cedar Lane Farms Nursery, Madison,

Morgan Co., GA

GAJY Private garden, 881 Conway Drive W, Atlanta, Fulton Co., GA

GAKN Private garden, 357 Academy Street, Madison, Morgan Co., GA

GALA Private garden, 3700 Northside Drive NW, Atlanta, Fulton Co., GA

GALE Lawton B. Evans School grounds, Walton Way, Augusta, Richmond Co., GA

GALF Private garden, 1 Beech Knoll Drive, Canton, Cherokee Co., GA

GALH Private garden, Thomson, McDuffie Co., GA

GALS Private garden, Lumpkin Street, Athens, Clarke Co., GA

GAMC Private garden, 720 Milledge Circle, Athens, Clarke Co., GA

GAMD Private garden, Hallowcreek Lane, Athens, Clarke Co., GA

GAME Private garden, 708 Milledge Road, Augusta, Richmond Co., GA

GAMM City Hall, Madison, Morgan Co., GA

GAMP Georgia May Park, 4th Avenue at Fenwick, Augusta, Richmond Co., GA

GAMW Private garden, 126 Dearing Street, Athens, Clark Co., GA

GANA Private garden, 530 Academy Street, Madison, Morgan Co., GA

GANG Augusta National Golf Course, Augusta, Richmond Co., GA

GAOH Private garden, Oak Hill, Rome, Floyd Co., GA

GAPG Private garden, Athens, Clarke Co., GA

GAPM Corner of State and Main Streets,

Pine Mountain, Harris Co., GA

GAPN Private garden, 3036 Pine Needle Street, Augusta, Richmond Co., GA

GARC Private garden, 188 South Milledge Avenue, Athens, Clarke Co. GA
GARW Reinhardt College, Waleska, Cherokee Co., GA

GASC Sadis Church, Route 140, Cherokee Co., GA

GASH Tullie Smith House Restoration, 3099 Andrews Drive NW, Atlanta, Fulton Co., GA

GASM Private garden, 4637 Oakview Drive, Savannah, Chatham Co., GA

GASS Queens Court Motel, Saint Simon's Island, Glynn Co., GA

GASU Summerville Cemetery, Cuming and Johns Roads, Augusta, Richmond Co., GA

GATC Theta Chi Fraternity House, 645 Milledge Avenue, Athens, Clarke Co., GA

GATG Taylor-Grady House, Prince Avenue, Athens, Clarke Co., GA

GATN Bonar Hall, Dixy Avenue, Madison, Morgan Co., GA

GATS Private garden, Route 6, Turner's Rock, Savannah, Chatham Co., GA

GAUG University of Georgia, Athens, Clark Co., GA

GAVA DeSoto Hotel, Kingston, Floyd Co., GA

GAVI Valley Inn Motel, Route 27, Harris Co., GA

GAWD Private garden, 2600 Lexington Road, Athens, Clarke Co., GA

GAWH Private garden, Harden Bridge Road, Banks Co., GA

GAWJ Private garden along Route 83, near West Jefferson and Washington Streets, Madison, Morgan Co., GA

GAWM Westover Memorial Park, Wheeler Road and Monte Sano Avenue, Augusta,

Richmond Co., GA

GAWP Wormsloe Plantation, RR 6 (Skidaway Road), Savannah, Chatham Co., GA

GAWS Private garden, 575 Waddell Street, Athens, Clarke Co., GA

LAAL Jungle Gardens, Avery Island, Iberia Parish, LA

LAAP Audubon Park, near Tulane University campus, New Orleans, Orleans Parish, LA

LAAU Private garden, 120 Amelia Street and University Avenue W, Lafayette,

Lafayette Parish, LA

LAGN Grandview Nursery, Youngsville, Lafayette Parish, LA

LAHG Hodges Gardens, Sabine Parish, LA

LAHP Haphazard Plantation, Concordia Parish, LA

LAHS Private garden, 117 Rio Vista Avenue, Jefferson Parish, LA

LAIS International Salt Co., Avery Island, Iberia Parish, LA

LALG Longue Vue Gardens, 11 Garden Lane, New Orleans, Orleans Parish, LA

LAMB Private garden, corner of Johnston and E Streets, Lafayette, Lafayette Parish, LA

LAMP Mary Plantation, Braithwaite, Plaquemines Parish, LA

LANR Private garden at house near Main Road (Louisiana Route 1), New Roads, Pointe Coupee Parish, LA

LAPA Private garden, 456 Audubon Street, New Orleans, Orleans Parish, LA 
LARP Rosedown Plantation, Saint Francisville, West Feliciana Parish, LA

LARS Private garden, Jonesville, Concordia Parish, LA

LASL Southwestern Louisiana University, Lafayette, Lafayette Parish, LA

LATU Tulane University, New Orleans, Orleans Parish, LA

MDAB USDA Agricultural Research Center (formerly Plant Industry Sta.), Beltsville, Prince George's Co., MD

MDAC Adventist Church, Laurel and Eastern Avenues, Takoma Park, Montgomery Co., MD

MDAF Society of American Foresters, 5400 Grosvenor Lane, Bethesda, Montgomery Co., MD

MDAG Private garden, Bethesda, Montgomery Co., MD

MDAL Private garden, Lutherville, Baltimore Co., MD

MDAM Private garden at house across from US Post Office, Ashton, Montgomery Co., MD

MDAN Angelica Nurseries, RR 1, Kennedyville, Kent Co., MD

MDBA Bond Arboretum, Easton, Talbot Co., MD

MDBD Brighton Dam, Brighton Dam Road, Ashton, Montgomery Co., MD

MDBE Private garden, 1600 Bishop Road, Edgewater, Anne Arundel Co., MD

MDBG Brookside Gardens, 1500 Glenallen Road, Wheaton, Montgomery Co., MD

MDBM Private garden, 6704 Glenn Avenue, Glenn Dale, Prince George's Co., MD

MDBN J. H. Burton Nursery, 5950 Ager Road, Hyattsville, Prince George's Co., MD

MDBO Field along B \& O Railroad, Jackson, Cecil Co., MD

MDBP Jesup Blair Park, Silver Spring, Montgomery Co., MD

MDBR Bordley-Randall House, Randall Court, Annapolis, Anne Arundel Co., MD

MDBW Blackwater Wildlife Refuge, Dorcester Co., MD

MDCA Private garden across from St. John's College, College Avenue, Annapolis,

Anne Arundel Co., MD

MDCC Along Route 4 at Calvert County line, Calvert Co., MD

MDCD Crescent Drive, Greenbelt, Prince George's Co., MD

MDCG Private garden, 4327 Kentbury Drive, Bethesda, Montgomery Co., MD

MDCH Private garden, 6510 River Road, Bethesda, Montgomery Co., MD

MDCL Carson Circle and LaSalle Drive, Avondale, Prince George's Co., MD

MDCO Corner of Colesville Road and Dale Drive, Silver Spring, Montgomery Co., MD

MDCP Mount Clare, Carroll Park, Baltimore, MD

MDCR Private garden, 1634 Chesapeake Road, Edgewater, Anne Arundel Co., MD

MDDF Private garden, "In the Woods," 8922 Spring Valley Road, Chevy Chase, Montgomery Co., MD
MDDP Private garden, corner of Dale and Pershing Drives, Silver Spring, Moutgomery Co., MD

MDDS Private garden, 727 Dartmouth Street, Silver Spring, Montgomery Co., MD

MDEC Episcopal Church, Prince Frederick, Calvert Co., MD

MDEF Private garden, near Elm Avenue, Frederick, Frederick Co., MD

MDEM Private garden, 400 East Main Street, Middletown, Frederick Co., MD

MDES Private garden, Haller Vista Avenue, Frederick, Frederick Co., MD

MDET Private garden, 7100 Armat Drive, Bethesda, Montgomery Co., MD

MDFA Private garden, Camden Street, Salisbury, Wicomico Co., MD

MDFD Private garden, 730 Dartmouth Avenue, Silver Spring, Montgomery Co., MD

MDFF Along Route 15 at Frederick, Frederick Co., MD

MDFM Private garden, 7417 Buffalo Avenue, Takoma Park, Montgomery Co., MD

MDGB Small park near shopping area, Greenbelt, Prince George's Co., MD

MDGC Goucher College (Science Building), Towson, Baltimore Co., MD

MDGD USDA Plant Introduction Station (now National Plant Quarantine Center), Glenn Dale, Prince George's Co., MD

MDGG Private garden, 11002 Kenilworth Avenue, Garrett Park, Montgomery Co., MD

MDGJ Private garden, Bryans Road, Charles Co., MD

MDGL Private garden, 7112 Cedar Avenue, Takoma Park, Montgomery Co., MD

MDGR Private garden, 22 Orchard Way, North Rockville, Montgomery Co., MD

MDHA Henry Allanson Memorial Garden, Scientists' Cliffs, Port Republic, Calvert Co., MD

MDHC Hood College, Frederick, Frederick Co., MD

MDHD Private garden, 3108 Lancer Drive, Hyattsville, Prince George's Co., MD

MDHF Memorial Hospital grounds, Frederick, Frederick Co., MD

MDHH Hardy Farm, $7 \mathrm{mi}$. N of Potomac River bridge, Faulkner, Charles Co., MD

MDHN Hampton National Historic Site, 535 Hampton Lane, Towson, Baltimore Co., MD

MDHP Hilltop and Park Valley Roads, near Piney Branch Road, Takoma Park, Montgomery Co., MD

MDHS Private garden, 24 Acacia, Scientists' Cliffs, Port Republic, Calvert Co., MD

MDHW Private garden, 7001 Glenn Brook, Bethesda, Montgomery Co., MD

MDIH National Institutes of Health, Bethesda, Montgomery Co., MD

MDJB Jones Mill and Brookville Roads, adjacent to Route 410 at Rock Creek, Montgomery Co., MD

MDJC Private garden, New Carrollton, Prince George's Co., MD

MDJH Johns Hopkins University campus, Baltimore, MD 
MDJN Jenkins Nursery, Mitchelville,

Prince George's Co., MD

MDJR Private garden, 6200 Kennedy Drive, Kenwood, Chevy Chase, Montgomery Co., MD

MDJS Private garden, 1304 West Seminary Road, Lutherville, Baltimore Co., MD

MDJW Private garden, 4400 Samar Street, Beltsville, Prince George's Co., MD

MDKA Private garden, 1138 Kenilworth Avenue, Garrett Park, Montgomery Co., MD

MDKB Private garden, 6102 Kirby Street, Bethesda, Montgomery Co., MD

MDKN Kingsville Nursery, Kingsville, Baltimore Co., MD

MDKS Private garden, Kennett Street, Silver Spring, Montgomery Co., MD

MDLA Tulip Hill, Harwood, Anne Arundel Co., MD

MDLG Landon School Gardens, Bethesda, Montgomery Co., MD

MDLM Across from National Library of Medicine, Bethesda, Montgomery Co., MD

MDLN Lyon Nursery, 13520 New Hampshire Avenue, Silver Spring, Montgomery Co., MD

MDLS Private garden, 415 Ethan Allen, Takoma Park, Prince George's Co., MD

MDLT London Town Publik House and Gardens, 839 Londontown Road, Edgewater, Anne Arundel Co., MD

MDMC Montgomery College, Fenton Street, Takoma Park, Montgomery Co., MD

MDME METRO Station, Colesville Road, Silver Spring, Montgomery Co., MD

MDMG McCrillis Gardens and Gallery, 6910 Greentree Road, Bethesda. Montgomery Co., MD

MDMM Montpelier Mansion, 12828 Laurel-Bowie Road, Laurel, Prince George's Co., MD

MDMN McLean Nursery, Satyr Hill Road, Towson, Baltimore Co., MD

MDMR Restaurant on Mayo Road (near Londontown Road), Edgewater, Anne Arundel Co., MD

MDMS Private garden, 1 Valley View, Takoma Park, Montgomery Co., MD

MDMW Private garden, 2212 Merzerott Road, Adelphi. Prince George's Co., MD

MDNA US Naval Academy, Annapolis, Anne Arundel Co., MD

MDNH Bethesda Naval Hospital, Bethesda, Montgomery Co., MD

MDOF Olallie Farm, Glenn Dale, Prince George's Co., MD

MDOM Chatolanee Hill, Owings Mills, Baltimore Co., MD

MDPB Private garden, 7432 Piney Branch Road, Takoma Park, Montgomery Co., MD

MDPD Private garden, 420 Pershing Drive, Silver Spring, Montgomery Co., MD

MDPF Private garden, Fox Chapel Drive, Lutherville, Baltimore Co., MD

MDPJ Private garden, Scientists' Cliffs, Prince Frederick, Calvert Co., MD
MDPL Private garden, 7114 Eversfield Drive, College Heights Estates, Hyattsville, Prince George's Co., MD

MDPM Private garden, South Glen Road, Potomac, Montgomery Co., MD

MDRL Private garden, 11954 Simpson Road, Clarksville, Howard Co., MD

MDRP Private garden, 4502 Brandon Lane, Beltsville, Prince George's Co., MD

MDRT Rideout Tenements, 114 Duke of Gloucester Street, Annapolis, Anne Arundel Co., MD

MDSC Plant Materials Center, Soil Conservation Service, Beltsville, Prince George's Co., MD

MDSG Private garden, 4102 Chelmont Lane, Bowie, Prince George's Co., MD

MDSH Satyr Hill Nursery, Baltimore, MD

MDSJ Saint John's College, Annapolis, Anne Arundel Co., MD

MDSM Private garden, 10926 Montrose Street, Garrett Park, Montgomery Co., MD

MDSS Private garden at corner of Georgia Avenue and Highland Drive, Silver Spring, Montgomery Co., MD

MDST Private garden, 5520 Spruce Tree Avenue, Bethesda, Montgomery Co., MD

MDTD Private garden, 10041 Worrell Avenue, Glenn Dale, Prince George's Co., MD

MDTH Tyler Heights Elementary School, Janwal Street, Annapolis, Anne Arundel Co., MD

MDTN Tingle Nursery, Pittsville, Wicomico Co., MD

MDTP Gymnasium, Chicago Avenue near Philadelphia Avenue, Takoma Park, Montgomery Co., MD

MDTW Towson Nursery, Towson, Baltimore Co., MD

MDUM North Administration Building, University of Maryland, College Park, Prince George's Co., MD

MDVV Corner of Valley View and Maple Avenues, Takoma Park, Montgomery Co., MD

MDWA Ten Oaks Nursery, Clarkesville, Howard Co., MD

MDWG Private garden, Burnt Mills, 10705 Blossom Lane, Silver Spring, Montgomery Co., MD

MDWJ Roadside at intersection of Wisconsin Avenue and Jones Bridge Road, Montgomery Co., MD

MDWK Private garden, 415 Pershing Drive, Silver Spring, Montgomery Co., MD

MDWM Wye Oak State Park, Wye Mills, Talbot Co., MD

MDWP The William Paca Garden, 1 Martin Street, Annapolis, Anne Arundel Co., MD

MDWS Private garden, 40 Cornhill Street, Annapolis, Anne Arundel Co., MD

MSBA Private garden, Pass Christian, Harrison Co., MS

MSBH Private garden, Crosby, Wilkinson Co., MS

MSBN Bellemont Motel, US Route 61 near southern boundary of Natchez, Adams Co., MS

MSEN Elmscourt garden, Natchez, Adams Co., MS 
MSEP D'Evereux Plantation, Natchez, Adams Co., MS

MSFG Gloster Arboretum, Highway 33, Gloster,

Amite Co., MS

MSHB Private garden, Hattiesburg, Forrest Co., MS

MSKR Private garden, Perry Co., MS

MSMN Mistletoe House, Airport Road, Natchez, Adams Co., MS

MSMV Magnolia Vale, Natchez, Adams Co., MS

MSPR Private garden, Poplarville, Pearl River Co., MS

MSRN Rosalie House garden, Natchez,

Adams Co., MS

MSWB Private garden, Port Gibson, Claiborne Co., MS

NCAG Cemetery of Airlie Gardens, Wrightsville Beach

Hwy., near Wilmington, New Hanover Co., NC

NCAK Private garden, 240 Tranquility Place,

Hendersonville, Henderson Co., NC

NCAS Appalachian State University, Boone,

Watauga Co., NC

NCBE Biltmore Estate Gardens, Biltmore,

Buncombe Co., NC

NCBM Private garden, 410 Buchanan and Monmouth Avenues, Durham, Durham Co., NC

NCBR YMCA Blue Ridge Assembly, near Black Mountain, Buncombe Co., NC

NCBW Private garden, Willard, Pender Co., NC

NCCA Coker Arboretum, University of North Carolina, Chapel Hill, Orange Co., NC

NCCB Chatwood, Faucette Mill Road, Hillsborough, Orange Co., NC

NCCC Calico Cottage Restaurant, Saluda, Polk Co., NC

NCCG Clarendon Gardens, Pinehurst, Moore Co., NC

NCCM Carleton Motel, Rocky Mount,

Edgecombe Co., NC

NCCP Cameron Park School, Hillsborough,

Orange Co., NC

NCCS Church Street, Rocky Mount,

Edgecombe Co., NC

NCCW Private garden, Weaver Dairy Road,

Chapel Hill, Orange Co., NC

NCDB Daniel Boone Native Gardens, Boone,

Watauga Co., NC

NCDF Private garden, Dixie Lane, Hendersonville, Henderson Co., NC

NCDR Bluff on Dan River, below Leaksville,

Rockingham Co., NC

NCDS Private garden, 2706 Spencer Street, Durham, Durham Co., NC

NCDU Duke University, Durham, Durham Co., NC

NCEG Elizabethan Gardens, Manteo, Dare Co., NC

NCEL Private garden, 384 Ridgewood Road,

Charlotte, Mecklenburg Co., NC

NCFR Fred Rees Nursery, Lake Hosea, Saluda,

Henderson Co., NC

NCGC Private garden, Chisholm Street, Saluda, Polk Co., NC

NCGM Gra-Mar Lodge, Box 122, Fayetteville,

Cumberland Co., NC

NCGP Greenfield Park, Wilmington,

New Hanover Co., NC

NCGR Park near Guess Road and Interstate 85 ,

Durham, Durham Co., NC
NCHO Private garden, Hillsborough, Orange Co., NC

NCHS Private garden, Norlina, Larren Co., NC

NCJP Private garden, 2260 Crawford, Durham,

Durham Co., NC

NCKH Private garden, West King and South Hasel Streets, Hillsborough, Orange Co., NC

NCKK Private garden, North Rugby Road, RR 8 Box 48, Hendersonville,

Henderson Co., NC

NCKN Kitty's Nursery, 106 Airlie Road, Wilmington, New Hanover Co., NC

NCMH Private garden, Manns Harbor, Dare Co., NC

NCMM Private garden, Elon College,

Alamance Co., NC

NCMO Private garden (Mother Vine), Manteo, Dare Co., NC

NCMS Private garden, Ravenswood Road, Flatrock, Henderson Co., NC

NCNB Private garden, Pollock Street, New Bern, Craven Co., NC

NCNS North Carolina State University Arboretum, Raleigh, Wake Co., NC

NCOP Orton Plantation, off Route 133, $13 \mathrm{mi}$. south of Wilmington, Brunswick Co., NC

NCPL Planters National Bank, Rocky Mount, Edgecombe Co., NC

NCPR Prudent National Bank, Rocky Mount, Edgecombe Co., NC

NCRC Private garden, 2800 Saint Andrews Lane, Charlotte, Mecklenburg Co., NC

NCRM Riverside Motel, Southport, Brunswick Co., NC

NCRW Private garden, 342 Ridgewood Avenue, Charlotte, Mecklenburg Co., NC

NCSH Ebersole Holly Garden, Sand Hills Community College, Southern Pines, Moore Co., NC

NCSL Near house of Sidney Lanier, along stream 300 yards from Route 176, Tryon, Polk Co., NC

NCSM Saint Matthew's Episcopal Church, Hillsborough, Orange Co., NC

NCSW Parking strip on South Wake Street, Hillsborough, Orange Co., NC

NCTE Calvary Episcopal Church, Church Street, Tarboro, Edgecombe Co., NC

NCTP Tryon Palace, New Bern, Craven Co., NC

NCTR Intersection of Towerview Road and Edwin Avenue, Durham, Durham Co., NC

NCWC Western Carolina University, Cullowhee, Jackson Co., NC

NCWD We-Do Nursery, RR 5 Box 724, Marion, McDowell Co., NC

NCWK Private garden, Norlina, Warren Co., NC

NCWM Private garden, 2828 Saint Andrews Lane, Charlotte, Mecklenburg Co., NC

NCWT Episcopal Cemetery, West Tryon Street, Hillsborough, Orange Co., NC

SCAB Private garden, 933 Abbeville Street, Aiken, Aiken Co., SC

SCAC Private garden, 127 Greenville Street, Aiken, Aiken Co., SC

SCAK Private garden, Aiken, Aiken Co., SC

SCBA Private garden, Barre and Calhoun Streets, Charleston, Charleston Co., SC 
SCBG Bailston Garden, Richland Street, Columbia, Richland Co., SC

SCBH Private garden, 68 South Battery, Charleston, Charleston Co., SC

SCBP Boone Hall Plantation, Route 17, Charleston Co., SC

SCBR Brookgreen Gardens, Route 17, Georgetown Co., SC

SCBS Burt-Stark Mansion, North Main and Ellis Streets, Abbeville, Abbeville Co., SC

SCBY Private garden, 531 Berrie Road, Aiken, Aiken Co., SC

SCCC Private garden, 7 Church Street, Charleston, Charleston Co., SC

SCCF Private garden, Carolina and Forest Avenues, Aiken, Aiken Co., SC

SCCG Church of Prince George, Winyah, Georgetown, Georgetown Co., SC

SCCL Charlestowne Landing, Charleston, Charleston Co., SC

SCCM Charleston Motor Hotel, Meeting Street, Charleston, Charleston Co., SC

SCCS Private garden at house across from 7 Church Street, Charleston, Charleston Co., SC

SCCU Clemson University, Clemson, Pickens Co., SC

SCCY Cypress Gardens, off US Route 52,

Berkeley Co., SC

SCDB Private garden, Dibble and Bissel Roads, Aiken, Aiken Co., SC

SCDH Drayton Hall, north of Charleston, Charleston Co., SC

SCEA Private garden, 120 Edwards Avenue, Darlington, Darlington Co., SC

SCEG Edisto Memorial Garden, Orangeburg, Orangeburg Co., SC

SCEI Private garden (formerly Baynard's Bluff), Bluffton, Beaufort Co.. SC

SCEM Private garden, 27 King Street, Charleston, Charleston Co., SC

SCFR Private garden, 100 Tradd Street, Charleston, Charleston Co., SC

SCFS Graveyard of First Scots Presbyterian Church, Meeting Street, Charleston,

Charleston Co., SC

SCFW Private garden, 1500 Old Town Road, Charleston, Charleston Co., SC

SCGB Corner of Greenville and Barnwell Streets, Aiken, Aiken Co., SC

SCHA Private garden, 419 North Main Street, Abbeville, Abbeville Co., SC

SCHG Hopelands Garden, behind Hall of Fame Building, Whiskey and Dupree Place, Aiken, Aiken Co., SC

SCHS Private garden, Summerville, Dorchester Co., SC

SCHW Heywood-Washington House, 87 Church Street, Charleston. Charleston Co., SC

SCJA Private garden, 718 South Boundary, Aiken, Aiken Co.. SC

SCJC Joyce Cottage, between Chesterfield and Newberry Streets, Aiken, Aiken Co., SC

SCJF John L. Frierson Nursery, Norway, Orangeburg Co., SC
SCJM Private garden, 42 Society Street, Charleston, Charleston Co., SC

SCKG Kalmia Gardens, Coker College, Hartsville, Darlington Co., SC

SCKS Private garden, King Street, Charleston, Charleston Co., SC

SCLM Private garden, 74 South Battery, Charleston, Charleston Co., SC

SCLO Live Oak Memorial Gardens, Charleston, Charleston Co., SC

SCLS Garden by entrance gate, 22 Lagare Street, Charleston, Charleston Co., SC

SCMA Private garden, 206 South Main Street, Abbeville, Abbeville Co., SC

SCMG Magnolia Gardens, north of Charleston, Charleston Co., SC

SCMI Private garden, 204 South Main Street, Abbeville, Abbeville Co., SC

SCMM Motel near southern boundary of Manning, Clarendon Co., SC

SCMP Middleton Place Gardens, Dorcester Co., SC

SCNR Nathaniel Russell House, Meeting Street, Charleston, Charleston Co., SC

SCNS Private garden, 14 Legare Street, Charleston, Charleston Co., SC

SCPL Palmetto Landscaping and Nursery Co., 247 Springville Road, Darlington,

Darlington Co., SC

SCPP Pomaria Plantation, US Route 176, Pomaria, Newberry Co., SC

SCPR Median strip, Pendleton and Richland Avenues, Aiken, Aiken Co., SC

SCRH Rosehill, 200 block of Greenville Street, Aiken, Aiken Co., SC

SCRL Median strip, Richland and Lancaster Avenues, Aiken, Aiken Co., SC

SCRP Redcliffe State Park, Beech Island, Route 125, Aiken Co., SC

SCRS Roadside, Route 17 Alt. north of Summerville, Dorchester Co., SC

SCSB Private garden, along South Battery Street, Charleston, Charleston Co., SC

SCSG Shady Grove Nursery, 3030 Charleston Road SW, Orangeburg, Orangeburg Co., SC

SCSR Private garden, 1115 Glen Oaks Road, Columbia, Richland Co., SC

SCTR Private garden, near Table Rock State Park, Highway 11, Greenville Co., SC

SCUC University of South Carolina Campus, South Caroliniana Library, Columbia, Richland Co., SC

SCWC Private garden, 30 King Street, Charleston, Charleston Co., SC

SCWE Wendy's restaurant, US Route 1, Aiken, Aiken Co., SC

SCWF Private garden, 4032 Rosewood Drive, Columbia, Richland Co., SC

SCWI Woodlanders, Inc. (nursery), 1128 Colleton Avenue, Aiken, Aiken Co., SC

SCWL Woodland, southwest corner of Colleton and Laurens Streets, Aiken, Aiken Co., SC 
SCWP Private garden, Mechaniesville Highway,

Darlington Co., SC

SCWR Williamsburg and Richland Streets, Aiken, Aiken Co., SC

TNAB Private garden, 709 Forest Hills Boulevard, Knoxville, Knox Co., TN

TNBC Bay View Motel, US Route 27 (north of tunnel), Chattanooga, Hamilton Co., TN

TNBM Private garden, 3359 Lakeview Drive (formerly Sanford Arboretum), Knoxville, Knox Co., TN

TNBO Boyd Nursery, McMinnville, Warren Co., TN

TNBV Private garden, Route 261, 2 mi. north of Bakersville, Mitchell Co., TN

TNCG Chickasaw Golf Course, Central Avenue and Lafayette Street, Memphis, Shelby Co., TN

TNCT Cheekwood, Tennessee Botanical Gardens, Cheek Road, Nashville, Davidson Co., TN

TNDG Dixon Gallery and Gardens, 4339 Park Avenue, Memphis, Shelby Co., TN

TNDR Private garden, 3417 Lakeview Drive (formerly Sanford Arboretum),

Knoxville, Knox Co., TN

TNFB Private garden, Alcoa, Blount Co., TN

TNFE Private garden, 645 Cheowa Circle (formerly Sanford Arboretum), Knoxville, Knox Co., TN

TNFL Fort Loudoun Dam (near powerhouse), Loudoun Co., TN

TNFN Forest Nursery Company, McMinnville, Warren Co., TN

TNFR Private garden, 3400 Lakeview Drive (formerly Sanford Arboretum), Knoxville, Knox Co., TN

TNGN Private garden, 3410 Lakeview Drive (formerly Sanford Arboretum), Knoxville, Knox Co., TN

TNHD Private garden, 1079 Scenic Drive, Knoxville, Knox Co., TN

TNHG The Hermitage (home of Andrew Jackson), Hermitage, Davidson Co,, TN

TNHH Holly Haven Nursery, Knoxville, Knox Co., TN

TNHS Private garden, 3350 Lakeview Drive (formerly Sanford Arboretum), Knoxville, Knox Co., TN

TNHT Private garden, 600 High Street, Winchester, Franklin Co., TN

TNKS Keith Spring Mountain (1,200 ft. alt.), Franklin Co., TN

TNLW Private garden, 3428 Lakeview Drive (formerly Sanford Arboretum), Knoxville, Knox Co., TN

TNMB Memphis Botanic Garden, Audubon Park, 750 Cherry Street, Memphis, Shelby Co., TN

TNML Private garden, Montreal Lane, Oak Ridge, Anderson Co., TN

TNNE Norris Experimental Area, Tennessee Valley Authority, Norris, Anderson Co., TN

TNPC Corner of Parkway E and Central Avenue, Memphis, Shelby Co., TN

TNPW Phytotektor, Inc., Winchester, Franklin Co., TN
TNRG Private garden, 3351 Lakeview Drive (formerly Sanford Arboretum), Knoxville, Knox Co., TN

TNRM Private garden, 3403 Lakeview Drive (formerly Sanford Arboretum), Knoxville, Knox Co., TN

TNRT Private garden, 4805 East Summit, Knoxville, Knox Co., TN

TNRU Gravesite of Ferdinand Rugel (botanist), Old Westminster Cemetery, ca. $2 \mathrm{mi}$. from White Pine, Jefferson Co., TN

TNRW Private garden, 3358 Lakeview Drive (formerly Sanford Arboretum), Knoxville, Knox Co., TN

TNSB Swan Bakery, 1801 Magnolia Avenue NE, Knoxville, Knox Co., TN

TNSC Private garden, Holt Road, Nashville, Davidson Co., TN

TNSG Private garden, 3418 Lakeview Drive (formerly Sanford Arboretum), Knoxville, Knox Co., TN

TNSM Private garden, McMinnville, Warren Co., TN

TNSN Shadow Nursery, Route 64, Winchester, Franklin Co., TN

TNTV Tennessee Valley Nursery, Winchester, Franklin Co., TN

TNUT University of Tennessee, Agriculture Campus, Knoxville, Knox Co., TN

TNWF Private garden, 3425 Lakeview Drive (formerly Sanford Arboretum), Knoxville, Knox Co., TN

TXAB Private garden, 2 Tealway, River Oaks, Houston, Harris Co., TX

TXAO Private garden, 4019 Avenue O, Galveston, Galveston Co., TX

TXAV Private garden, 4318 Avenue O, Galveston, Galveston Co., TX

TXBA Private garden, 2030 Banks Avenue, Houston, Harris Co., TX

TXCC Private garden, 3325 Chevy Chase Drive, Houston, Harris Co., TX

TXCT Private garden, Spring Branch, Holidan Street, Houston, Harris Co., TX

TXDC Private garden, 3408 Ella Lee Lane, Houston, Harris Co., TX

TXDM Private garden, 3453 Inwood Street, Houston, Harris Co., TX

TXEN Eagleson Nursery, Port Arthur, Jefferson Co., TX

TXGM Private garden, 2515 Reba Street, Houston, Harris Co., TX

TXGS Private garden, Bel Air Boulevard, Sharpstown, Houston, Harris Co., TX

TXHA Houston Arboretum and Botanical Gardens, Woodway Lane, Houston, Harris Co., TX

TXHE Hogg Estate, Bayou Bend, 2940 Lazy Lane Boulevard, Houston, Harris Co., TX

TXHG Private garden, 406 Hedwig Green Street, Houston, Harris Co., TX

TXHJ Howard Johnson Motel, Interstate 10, west of Houston, Harris Co., TX

TXHL Old nursery of Lynn Lowrey, 1404 Upland, Houston, Harris Co., TX 
TXHN Hester Nursery, 3029 Todville Road, PO Box 413, Seabrook, Harris Co., TX

TXJS Private garden, 1702 Broadway Street, Galveston, Galveston Co., TX

TXLL Nursery of Lynn Lowrey, 2323 Sleepy Hollow Road, Conroe, Montgomery Co., TX

TXMA Private garden, 3376 Inwood Street, Houston, Harris Co., TX

TXMK Private garden, 2 Longbow Lane, Houston, Harris Co., TX

TXMS Museum of Natural Sciences, Houston, Harris Co., TX

TXPH Prudential Building, 1100 Halcombe Boulevard, Houston, Harris Co., TX

TXPO Private garden, 10656 Memorial Drive, Houston, Harris Co., TX

TXPS Private garden, 6810 Hendon, Sharpstown, Houston, Harris Co., TX

TXRE Private garden, 11108 Meadowick, Houston, Harris Co., TX

TXRS Private garden, Woodland Garden, Houston, Harris Co., TX

TXSB Private garden, Spring Branch area, Houston, Harris Co., TX

TXSC Private garden, 7107 Sharp Crest, Sharpstown, Houston, Harris Co., TX

TXSE Private garden, 700 Cavalcade Street W, Houston, Harris Co., TX

TXSH Private garden, Avenue N 1/2, Galveston, Galveston Co., TX

TXSN Seaside Nursery, 2315 45th Street, Galveston. Galveston Co., TX

TXVO Private garden, $3 \mathrm{mi}$. north of Vidor (1 mi. north of Interstate 10), Orange Co., TX

TXWE West End Market, 2203 45th Street,

Galveston. Galveston Co., TX

TXWH Whataburgers' Hamburger Place, 3639 Westheimer Road, Houston,

Harris Co., TX

TXWW Private garden, 4 Woods Edge Lane, Houston, Harris Co., TX

TXZH Zoo grounds, Hermann Park, Houston, Harris Co., TX

VAAC Arlington National Cemetery, Arlington, Arlington Co., VA

VAAH American Horticultural Society Headquarters, River Farm. Mount Vernon, Fairfax Co., VA

VAAL Private garden, Aldie, Loudon Co., VA

VABA Private garden, Front Street, Accomac, Accomack Co., VA

VABB Bamboo grove along Richmond Road, about 2 mi. west of Williamsburg, James City Co., VA

VABF Blandy Experimental Farm (O. E. White Arboretum), Boyce, Clarke Co., VA

VABK Private garden, Henrico Co., VA

VABP Bryan Park, Richmond, VA

VABR Private garden, Roseland, Route 605, Accomac, Accomack Co., VA

VACG Carter's Grove Plantation, James City Co., VA VACM Claremont Manor, Claremont, Surry Co., VA
VACP Chippokes Plantation State Park,

Surry Co., VA

VACR Private garden, 1306 Claremont Avenue, Richmond, VA

VACS Private garden, Front Street, Accomac, Accomack Co., VA

VACW Colonial Williamsburg, Williamsburg, James City Co., VA

VADC Cultivated area along Dickey Creek, Dickey Knob, Highway 16, Sugar Grove, Smythe Co., VA

VADF Private garden, 116 Woodland Drive, Newport News, VA

VADW Private garden, 8709 Cherokee Road, Richmond, VA

VAFB Along Interstate 95 near Fredericksburg, Spotsylvania Co., VA

VAFC Falls Church City Hall, Falls Church, Fairfax Co.,VA

VAFN Private garden, Back Street, Accomac Accomack Co., VA

VAFR Old house on Flat Ridge Road, Sugar Grove, Smythe Co., VA

VAGC Roadbank along Interstate $81,5 \mathrm{mi}$. north of Groseclose, Wythe Co., VA

VAGF Greenbrier Farms Nursery, 201 Hickory Road, Chesapeake, VA

VAGG The Lewis Ginter Botanical Garden, Lakeside Avenue, Henrico Co., VA

VAGH Glass-blower house, Jamestown, James City Co., VA

VAGJ House at junction of Routes 16 and 741, Grayson Co., VA

VAGM Private garden, 8008 Park Street N, Dunn Loring, Fairfax Co., VA

VAGO 4602 17th Street N, Arlington, Arlington Co., VA

VAGS Gulf Stream Nursery (Jacques Legendre), Wachapreague, Accomack Co., VA

VAGU Gunston Hall, Fairfax Co., VA

VAGW George Washington Estate, Mount Vernon, Fairfax Co., VA

VAHB Harry F. Byrd, Sr., Visitors' Center, Shenandoah National Park, Madison Co., VA

VAHC Hollywood Cemetery, Richmond, VA

VAHF Private garden, Route 601, Flat Ridge Road, Sugar Grove, Smythe Co., VA

VAHI Holiday Inn Motel, Marion, Smythe Co., VA

VAHM Hermitage Methodist Home, Hermitage Avenue, Richmond, VA

VAHN Hill's Nursery and Camellia Gardens, Arlington, Arlington Co., VA

VAHR Humpback Rocks Visitors' Center, Blue Ridge Parkway, Augusta Co., VA

VAHS Private garden, across from store in downtown area, Sugar Grove, Smythe Co., VA

VAIG Glenwood Garden Center, Richmond, VA

VAIS Private garden, Idlewood Street, Richmond, VA

VAJL Private garden, 2822 Avenham Avenue SW, Roanoke, Roanoke Co., VA

VAKH Garden at Kenmore House, Fredericksburg, Spotsylvania Co., VA 
VALC Jefferson Lakeside Country Club, Lakeside Avenue, Henrico Co., VA

VALU Private garden, Main Street, Luray, Page Co., VA

VAMC Medical College of Virginia, College and Marshall Streets, Richmond, VA

VAMD McDonald's Garden Center, Hampton, VA

VAMF Private garden, near East and Market Streets, Onancock, Accomack Co., VA

VAML Private garden, Mountain Lake, Giles Co., VA

VAMP Maymont Park, Richmond, VA

VAMR Private garden, 1027 Magnolia Road (along street), Norfolk, VA

VAMW Mews across from Saint John's Church, off 24th Street, Richmond, VA

VANB Norfolk Botanical Gardens, Airport Way, Norfolk, VA

VANP Heronwood, Route 623 near Upperville, Loudon Co., VA

VANS Roadside near settlement along Route 16 near Marion, Smythe Co., VA

VAOD Old Dominion University, Norfolk, VA

VAPC Private garden, corner of Pope Avenue and Crestwood Road, Richmond, VA

VAPH Private garden, 219 Robanna Shores, Seaford, York Co., VA

VAPO Peaks of Otter Lodge, Blue Ridge Parkway, Bedford Co., VA

VAPS Private garden, Pitt Street, Fredericksburg, Spotsylvania Co., VA

VARB Anly Farm, Onancock, Accomack Co,, VA

VARC Near milepost 155, Interstate 81, Roanoke Co., VA

VARD Private garden, Richmond, VA

VARE Private garden, 6611 Brauwner St, McLean, Fairfax Co, VA

VARG Russell's Grocery, near stream, along Route 16, Sugar Grove, Smythe Co., VA

VARH Private garden, Nock's Pasture, Back Street, Accomac, Accomack Co., VA

VARO Hotel Roanoke grounds, Roanoke, Roanoke Co., VA

VARR Private garden, Quinby, Accomack Co., VA

VASC State Capitol Building grounds, Richmond, VA

VASG Abandoned house, Route 601, Sugar Grove, Smythe Co., VA

VASJ Saint John's Churchyard, Richmond, VA

VASK Skyland, Shenandoah National Park, Page Co., VA

VASP Shenandoah National Park, VA

VASS Stony Point School, Richmond, VA

VATA Virginia Truck and Ornamental Research Station (Tidewater Arboretum), 1444 Diamond Springs Road, Virginia Beach, VA

VATG Thornton Gap, Shenandoah National Park, VA

VATS Todd Shopping Center, 220 Mercury

Boulevard, Hampton, VA

VATW Private garden, 6311 Three Chopt Road, Richmond, VA

VAWD Private garden, Wassona Drive, Marion, Smythe Co., VA
VAWE Wytheville exit, Interstate 81, Wythe Co., VA

VAWH Westbrook Psychiatric Hespital, 1500 Westbrook Avenue, Richmond, VA

VAWI Private garden, 610 North Cameron Street, Winchester, VA

VAWL White Lion Motel, Williamsburg, James City Co., VA

VAWM College of William and Mary, Williamsburg, James City Co., VA

VAWP Woodlawn Plantation gardens, Fairfax Co., VA

VAWR Private garden, Highway 6, 3 mi. west of Manakin, Goochland Co., VA

VAZC Private garden, 5236 Shenstone Circle, Virginia Beach, VA 
Appendix C: Glenn Dale Azalea Cultivars Documented in the U.S. National Arboretum Herbarium

\begin{tabular}{|c|c|}
\hline Abbott & Cocktail \\
\hline Acme & Colleen \\
\hline Acrobat & Commando \\
\hline Adorable & Commodore \\
\hline Advance & Con Amore \\
\hline Alight & Concordia \\
\hline Allegory & Consuela \\
\hline Allure & Content \\
\hline Altair & Copperman \\
\hline Ambrosia & Coquette \\
\hline Anchorite & Cordial \\
\hline Andros & Corsair \\
\hline Angela Place & Crinoline \\
\hline Anthem & Cygnet \\
\hline Antique & Cytherea \\
\hline Aphrodite & Damaris \\
\hline Arcadia & Damask \\
\hline Argosy & Damozel \\
\hline Astarte & Daphnis \\
\hline Ave Maria & Darkness \\
\hline Aviator & Dawning \\
\hline Bacchante & Dayspring \\
\hline Bagatelle & Dazzler \\
\hline Bagdad & Delight \\
\hline Beacon & Delos \\
\hline Bettina & Demure \\
\hline Bishop & Desire \\
\hline Blushing Maid & Dimity \\
\hline Bohemian & Dowager \\
\hline Boldface & Dragon \\
\hline Bolivar & Dream \\
\hline Bopeep & Driven Snow \\
\hline Bountiful & Duenna \\
\hline Bowman & Echo \\
\hline Brangaene & Egoist \\
\hline Bravo & Ember \\
\hline Buccaneer & Enchantment \\
\hline Burgundy & Eros \\
\hline Cadenza & Eucharis \\
\hline Camelot & Evangeline \\
\hline Cantabile & Evensong \\
\hline Capella & Everest \\
\hline Captivation & F. C. Bradford \\
\hline Carbineer & Fairy Bells \\
\hline Carnival & Fakir \\
\hline Carrara & Fandango \\
\hline Cascade & Fantasy \\
\hline Cavalier & Fashion \\
\hline Cavatina & Fawn \\
\hline Challenger & Felicity \\
\hline Chameleon & Folly \\
\hline Chanticleer & Frivolity \\
\hline Cherry Spot & Furbelow \\
\hline Chloe & Futurity \\
\hline Chum & Galathea \\
\hline Cinnabar & Galaxy \\
\hline
\end{tabular}

Ganymede

Gawain

Geisha

Glacier

Gladiator

Glamour

Glee

Gracious

Granat

Grandam

Grandee

Greeting

Grenadier

Guerdon

Harbinger

Harlequin

Helen Fox

Helen Gunning

Hopeful

Illusion

Isolde

Jamboree

Janet Noyes

Jessica

Jubilant

Juneglow

Kashmir

Kathleen

Killarney

Kobold

Lacquer

Leonore

Lillie Maude

Limerick

Litany

Louise Dowdle

Lucette

Luminary

Lustre

Madcap

Madeira

Madrigal

Mandarin

Manhattan

Marionette

Marjorie

Marmora

Martha Hitchcock

Mary Margaret

Mascot

Masquerade

Masterpiece

Matins

Mavis

Mavourneen

Mayflower

Medea

Melanie

Memento

Merlin

Meteor

Minstrel

Modesty
Moira

Morgana

Mother of Pearl

Motley

Muscadine

Nerissa

Niagara

Niphetos

Nocturne

Noreen

Novelty

Oriflamme

Orison

Paladin

Parade

Pastel

Patriot

Peerless

Peter Pan

Phoebe

Pied Piper

Pilgrim

Pinkie

Pinocchio

Pippin

Pirate

Pixie

Polonaise

Portent

Prelate

Presto

Progress

Prosperity

Prudence

Puck

Punchinello

Quakeress

Ranger

Red Bird

Red Hussar

Refrain

Remembrance

Revery

Reward

Robinhood

Rogue

Rosalie

Roselight

Rosette

Roundelay

Safrano

Saga

Sambo

Samite

Samson

Sarabande

Satrap

Satyr

Scherzo

Scholar

Scout

Seafoam

Sebastian
Seneca

Serenade

Serenity

Signal

Silver Cup

Silver Lace

Silver Mist

Silver Moon

Simplicity

Sligo

Snowclad

Snowscape

Sonata

Souvenir

Spangles

Sprite

Stampede

Stardust

Sterling

Surprise

Suwanee

Swansong

Swashbuckler

Taffeta

Tanager

Tango

Templar

Temptation

Thisbe

Touchstone

Treasure

Trilby

Trinket

Tristan

Trophy

Valentine

Vanity

Vespers

Vestal

Viking

Violetta

Vision

Volcan

Warrior

Wavelet

Welcome

Whimsical

Whirlwind

Wildfire

Winedrop

Winner

Wisdom

Witchery

Zephyr

Zulu 
Appendix D: Plants Listed by

Botanical Names and Cultivar Names

This appendix provides a cross-reference to the legitimate botanical names and, where applicable, the cultivar names for the plants included in the catalog. Genera are arranged alphabetically. The catalog presents the specific epithets and cultivar names in alphabetical order following each genus. This list is organized as follows:

Genus name and family name

Specific epithet or hybrid specific epithet Cultivar name for selected species material Forma (minor variant of species)

Infraspecific epithet for subspecies or varietas Cultivar name at infraspecific rank

Hybrid without cultivar name

Hybrid with cultivar name

Cultivar name not otherwise identified.

\section{ABELIA R.Br.}

CAPRIFOLIACEAE Honeysuckle Family

chinensis $\mathrm{R}$. Br.

$\times$ grandiflora (Andre) Rehd.

$\times$ grandiflora (Andre) Rehd. 'Prostrata'

$\times$ grandiflora (Andre) Rehd. 'Sherwoodii'

$(\times$ grandiflora $\times$ A. schumannii)

'Edward Goucher'

\section{ABELIOPHYLLUM Nakai OLEACEAE}

Olive Family

distichum Nakai

\section{ABIES Mill.} PINACEAE

Fir

Pine Family

alba Mill.

balsamea (L.) Mill.

cephalonica Loud.

chensiensis Tieghem ssp. salouenensis

(Bord.-Rey. \& Gaussen) Rushforth

cilicica (Ant. \& Kotschy) Carr.

concolor (Gord. \& Glend.) Lindl.

ex Hildebr.

firma Sieb. \& Zucc.

fraseri (Pursh) Poir.

holophylla Maxim.

homolepis Sieb. \& Zucc.

koreana Wils. nordmanniana (Steven) Spach

numidica de Lannoy ex Carr.

pinsapo Boiss.

pinsapo Boiss. 'Glauca'

procera Rehd.

$\times$ umbellata (Mayr) Liu

$\times$ vilmorinii Mast.
ABUTILON Mill. MALVACEAE
Flowering Maple
Mallow Family

pictum (Gillies ex Hook. \& Arn.) Walp. pictum (Gillies ex Hook. \& Arn.) Walp.

'Thompsonii'

ACACIA Mill. FABACEAE (Mimosoideae) Bean Family

berlandieri Benth.

farnesiana (L.) Willd.

rigidula Benth.

roemeriana Scheele

wrightii Benth.

ACALYPHA L.

EUPHORBLACEAE Spurge Family

hispida Burm.f.

ACCA O.Berg

MYRTACEAE

Myrtle Family

sellowiana (O.Berg) Burret

ACER L.

ACERACEAE

Maple

Maple Family

buergerianum Miq.

buergerianum Miq. 'Mino yatsufusa'

buergerianum Miq. 'Nauto kaede'

buergerianum Miq. ssp. formosanum

(Hayata) E.Murr. \& Lauener

'Miyasama'

campbellii Hiern ssp. flabellatum

(Rehd.) E.Murr.

campestre $\mathrm{L}$.

campestre L. 'Nanum'

campestre L. var. leiocarpum (Opiz)

Wallroth

capillipes Maxim.

cappadocicum Gleditsch

cappadocicum Gleditsch 'Aureum'

carpinifolium Sieb. \& Zucc.

cissifolium (Sieb. \& Zucc.) K.Koch

davidii Franch.

diabolicum $\mathrm{Bl}$. ex K.Koch

$\times$ freemanii E.Murr. 
ginnala Maxim.

griseum (Franch.) Pax

japonicum Thunb. ex J.A.Murr.

japonicum Thunb. ex J.A.Murr.

'Aconitifolium'

japonicum Thunb. ex J.A.Murr.

'Aureum'

japonicum Thunb. ex J.A.Murr.

'Itaya'

japonicum Thunb. ex J.A.Murr.

'Kinugasayama'

japonicum Thunb. ex J.A.Murr.

'O isami'

japonicum Thunb. ex J.A.Murr.

'O take'

japonicum Thunb. ex J.A.Murr.

'Vitifolium'

japonicum Thunb. ex J.A.Murr.

'Yayoigasa'

longipes Franch. ssp. amplum

(Rehd.) Jong

mandshuricum Maxim.

maximowiczianum Miq.

miyabei Maxim.

monspessulanum $\mathrm{L}$.

negundo $\mathrm{L}$.

negundo L. 'Variegatum'

negundo L. ssp. mexicanum (DC.)

Wesmael

negundo L. ssp. negundo

var. texanum Pax

oblongum Wall. ex DC.

oliverianum Pax

palmatum Thunb. ex J.A.Murr.

'Akaji nishiki'

palmatum Thunb. ex J.A.Murr.

'Aoyagi'

palmatum Thunb. ex J.A.Murr.

'Asahi zuru'

palmatum Thunb. ex J.A.Murr.

'Atropurpureum'

palmatum Thunb. ex J.A.Murr.

'Atropurpureum Superbum'

palmatum Thunb. ex J.A.Murr.

'Aureo-variegatum'

palmatum Thunb. ex J.A.Murr.

'Aureum'

palmatum Thunb. ex J.A.Murr.

'Autumn Glory'

palmatum Thunb. ex J.A.Murr.

'Bloodgood'

palmatum Thunb. ex J.A.Murr.

'Burgundy Lace'

palmatum Thunb. ex J.A.Murr.

'Butterfly'

palmatum Thunb. ex J.A.Murr.

'Chirimen nishiki' palmatum Thunb. ex J.A.Murr. 'Chitoseyama'

palmatum Thunb. ex J.A.Murr. 'Corallinum'

palmatum Thunb. ex J.A.Murr. 'Crimson Queen'

palmatum Thunb. ex J.A.Murr.

'Dissectum'

palmatum Thunb. ex J.A.Murr.

'Dissectum Flavescens'

palmatum Thunb. ex J.A.Murr.

'Dissectum Palmatifidum'

palmatum Thunb. ex J.A.Murr.

'Dissectum Rubrifolium'

palmatum Thunb. ex J.A.Murr.

'Dissectum Variegatum'

palmatum Thunb. ex J.A.Murr. 'Filigree'

palmatum Thunb. ex J.A.Murr. 'Garnet'

palmatum Thunb. ex J.A.Murr.

'Hagoromo'

palmatum Thunb. ex J.A.Murr. 'Hessei'

palmatum Thunb. ex J.A.Murr.

'Higasayama'

palmatum Thunb. ex J.A.Murr.

'Hogyoku'

palmatum Thunb. ex J.A.Murr.

'Ichigyo ji'

palmatum Thunb. ex J.A.Murr.

'Iijima sunago'

palmatum Thunb. ex J.A.Murr.

'Inaba shidare'

palmatum Thunb. ex J.A.Murr.

'Jiro shidare'

palmatum Thunb. ex J.A.Murr.

'Kagiri nishiki'

palmatum Thunb. ex J.A.Murr.

'Killarney'

palmatum Thunb. ex J.A.Murr.

'Kingsville Red'

palmatum Thunb. ex J.A.Murr.

'Koshimino'

palmatum Thunb. ex J.A.Murr.

'Kurabeyama'

palmatum Thunb. ex J.A.Murr.

'Linearilobum'

palmatum Thunb. ex J.A.Murr.

'Lutescens'

palmatum Thunb. ex J.A.Murr. 'Maiko'

palmatum Thunb. ex J.A.Murr.

'Masukagami'

palmatum Thunb. ex J.A.Murr.

'Matsukaze'

palmatum Thunb. ex J.A.Murr. 'Mioun'

palmatum Thunb. ex J.A.Murr.

'Mizu kuguri'

palmatum Thunb. ex J.A.Murr.

'Monzukushi' 
palmatum Thunb. ex J.A.Murr.

'Moonfire'

palmatum Thunb. ex J.A.Murr.

'Mure hibari'

palmatum Thunb. ex J.A.Murr.

'Naruo nishiki'

palmatum Thunb. ex J.A.Murr. 'Nomura'

palmatum Thunb. ex J.A.Murr. 'Nomura

nishiki'

palmatum Thunb. ex J.A.Murr. 'Ogino nagare'

palmatum Thunb. ex J.A.Murr. 'Ogon sarasa'

palmatum Thunb. ex J.A.Murr.

'O kagami'

palmatum Thunb. ex J.A.Murr.

'Okushimo'

palmatum Thunb. ex J.A.Murr. 'Omato'

palmatum Thunb. ex J.A.Murr.

'Oo shi rini'

palmatum Thunb. ex J.A.Murr. 'Oregon Sunset'

palmatum Thunb. ex J.A.Murr. 'Orido nishiki'

palmatum Thunb. ex J.A.Murr.

'Ornatum'

palmatum Thunb. ex J.A.Murr.

'Osakazuki'

palmatum Thunb. ex J.A.Murr.

'Oshio beni'

palmatum Thunb. ex J.A.Murr.

'Oshu shidare'

palmatum Thunb. ex J.A.Murr. 'Pixie'

palmatum Thunb. ex J.A.Murr. 'Ribbon Leaf'

palmatum Thunb. ex J.A.Murr. 'Sagara nishiki'

palmatum Thunb. ex J.A.Murr. 'Sango kaku'

palmatum Thunb. ex J.A.Murr.

'Sazanami'

palmatum Thunb. ex J.A.Murr. 'Seigen'

palmatum Thunb. ex J.A.Murr. 'Seiryu'

palmatum Thunb. ex J.A.Murr.

'Sherwood Flame'

palmatum Thunb. ex J.A.Murr.

'Shigitatsu sawa'

palmatum Thunb. ex J.A.Murr.

'Shigurezome'

palmatum Thunb. ex J.A.Murr. 'Shino buga oka'

palmatum Thunb. ex J.A.Murr.

'Shirigosan'

palmatum Thunb. ex J.A.Murr.

'Shishigashira'

palmatum Thunb. ex J.A.Murr. 'Shojo' palmatum Thunb. ex J.A.Murr.

'Tamukeyama'

palmatum Thunb. ex J.Ä.Murr. 'Tatsuta'

palmatum Thunb. ex J.A.Murr. 'The

Bishop'

palmatum Thunb. ex J.A.Murr. 'Toyama

nishiki'

palmatum Thunb. ex J.A.Murr.

'Trompenburg'

palmatum Thunb. ex J.A.Murr.

'Tsuchi uno'

palmatum Thunb. ex J.A.Murr.

'Tsuku bane'

palmatum Thunb. ex J.A.Murr.

'Tsukushi gata'

palmatum Thunb. ex J.A.Murr.

'Tsuri nishiki'

palmatum Thunb. ex J.A.Murr.

'Utsu semi'

palmatum Thunb. ex J.A.Murr.

'Versicolor'

palmatum Thunb. ex J.A.Murr.

'Waka momiji'

palmatum Thunb. ex J.A.Murr.

'Waterfall'

palmatum Thunb. ex J.A.Murr.

'Yatsubusa'

palmatum Thunb. ex J.A.Murr.

'Yezo nishiki'

palmatum Thunb. ex J.A.Murr.

'Yukigumi'

palmatum Thunb. ex J.A.Murr.

var. amoenum (Carr.) Ohwi

palmatum Thunb. ex J.A.Murr.

var. matsumurae (Koidz.) Makino

palmatum Thunb. ex J.A.Murr.

var. palmatum

pensylvanicum L.

platanoides L.

platanoides L. 'Almira'

platanoides L. 'Chas. F. Irish'

platanoides L. 'Crimson King'

platanoides L. 'Drummondii'

platanoides L. 'Faassen's Black'

platanoides L. 'Oekonomierat Stoll'

platanoides L. 'Schwedleri'

pseudoplatanus L.

pseudoplatanus L. 'Atropurpureum'

pseudoplatanus L. 'Brilliantissimum'

pseudosieboldianum (Pax) Komar.

rubrum L.

rubrum L. 'Gerling'

rubrum L. 'October Glory'

rubrum L. 'Pyramidale'

rubrum L. 'Schlesingeri'

rubrum L. 'Tilford'

rubrum L. var. drummondii (Nutt.) Sarg. 
rubrum L. var. trilobum T. \& G.

ex K.Koch

rufinerve Sieb. \& Zucc.

rufinerve Sieb. \& Zucc. 'Hatsuyuki'

rufinerve Sieb. \& Zucc. 'Variegatum'

saccharinum L.

saccharinum L. 'Palmatum'

saccharum Marsh. 'Newton Sentry'

saccharum Marsh. 'Sweet Shadow Cut-

Leaf'

saccharum Marsh. 'Temple's Upright'

saccharum Marsh. ssp. floridanum

(Chapm.) Desm.

saccharum Marsh. ssp. leucoderme

(Small) Desm.

saccharum Marsh. ssp. nigrum (Michx.f.)

Desm.

saccharum Marsh. var. rugelii (Pax)

Rehd.

saccharum Marsh. ssp. saccharum

shirasawanum Koidz.

sieboldianum Miq.

sieboldianum Miq. 'Kohauchina kaido'

sieboldianum Miq. 'Mikasayama'

spicatum Lam.

tataricum L.

tegmentosum Maxim.

truncatum Bunge

truncatum Bunge 'Akikaze nishiki'

truncatum Bunge 'Tokiwa nishiki'

truncatum Bunge 'Usugumo'

truncatum Bunge f. dissectum Wesmael

velutinum Boiss. var. vanvolxemii

(Mast.) Rehd.

wilsonii Rehd.

$\times$ zoeschense Pax

ACOELORRHAPHE H.Wendl.

\section{ARECACEAE}

Palm Family

wrightii (Griseb. \& H.Wendl.) H.Wendl. ex Becc.

\section{ACTINIDIA Lindl.} ACTINIDIACEAE Actinidia Family

arguta (Sieb. \& Zucc.) Planch. ex Miq. deliciosa (A.Chev.) C.F.Liang \&

A.R.Ferguson var. deliciosa deliciosa (A.Chev.) C.F.Liang \&

A.R.Ferguson var, deliciosa 'Chico' $\times$ fairchildii Rehd.

kolomikta (Maxim. \& Rupr.) Maxim.

polygama (Sieb. \& Zucc.) Maxim.

purpurea Rehd.
ADINA Salisb.

RUBLACEAE

Madder Family

rubella Hance

AESCULUS L. Horsechestnut, Buckeye HIPPOCASTANACEAE Buckeye Family

$\times$ bushii Schneid.

californica (Spach) Nutt.

$\times$ carnea Hayne

$\times$ carnea Hayne 'Briotii'

$\times$ dupontii Sarg.

flava Sol.

glabra Willd.

$\times$ glaucescens Sarg.

hippocastanum L.

hippocastanum L. 'Baumanii'

hippocastanum L. 'Memmingeri'

$\times$ hybrida DC.

$\times$ marilandica Booth ex Kirchn.

$\times$ mutabilis (Spach) Scheele

parviflora Walt.

parviflora Walt. var. serotina Rehd.

pavia L.

pavia L. var. flavescens (Sarg.) Correll

$\times$ plantierensis Andre

sylvatica Bartram

$\times$ woerlitzensis Koehne

AGARISTA D.Don ex G.Don

ERICACEAE Heath Family

populifolia (Lam.) D.Don ex Judd

AGAVE L.

AGAVACEAE

Century Plant

Agave Family

americana $\mathrm{L}$.

americana L. 'Marginata'

AILANTHUS Desf.

SIMAROUBACEAE Quassia Family

altissima (Mill.) Swingle

AKEBIA Decne.

Akebia

LARDIZABALACEAE Lardizabala Family

quinata (Thunb. ex Houtt.) Decne.

quinata (Thunb. ex Houtt.) Decne.

'Shirobana' 
ALBIZIA Durazz.

FABACEAE (Mimosoideae)

Bean Family

julibrissin Durazz.

kalkora (Roxb.) Prain

ALEURITES J.R. \& J.G. Forst.

EUPHORBIACEAE

Spurge Family

fordii Hemsl.

montana (Lour.) Wils.

ALLAMANDA L.

APOCYNACEAE

Dogbane Family

cathartica L.

ALNUS Mill.

BETULACEAE

Alder

Birch Family

formosana (Burkw.) Makino

glutinosa (L.) Gaertn.

hirsuta (Spach) Rupr. var. sibirica

(Spach) Schneid.

japonica (Thunb.) Steud.

maritima (Marsh.) Nutt.

serrulata (Ait.) Willd.

ALOYSIA Juss.

VERBENACEAE

Verbena Family

gratissima (Gillies \& Hook.) Troncoso

$\begin{array}{llr}\text { AMELANCHIER Medik. } & \begin{array}{r}\text { Shadbush } \\ \text { Rose Family }\end{array} \\ \text { ROSACEAE } & \end{array}$

alnifolia (Nutt.) Nutt.

arborea (Michx.f.) Fern.

asiatica (Sieb. \& Zucc.) Endl.

canadensis (L.) Medik.

laevis Wieg.

laevis Wieg. 'Rosea'

sanguinea (Pursh) DC.

spicata (Lam.) K.Koch

'Success'

AMORPHA L.

FABACEAE (Faboideae)

Bean Family

fruticosa L.

glabra Desf. ex Poir.
AMPELOPSIS Michx.

VITACEAE

- Grape Family

arborea (L.) Koehne

glandulosa var. brevipedunculata

(Maxim.) Momiy.

glandulosa var. brevipedunculata

(Maxim.) Momiy. 'Elegans'

AMTRIS P.Br.

RUTACEAE

Citrus Family

texana (Buckl.) P.Wilson

ANDROMEDA L.

ERICACEAE

Heath Family

polifolia L.

polifolia L. 'Nana'

ANISACANTHUS Nees

ACANTHACEAE Acanthus Family

thurberi (Torr.) A.Gr.

ANTIGONON Endl.

POLYGONACEAE Buckwheat Family

leptopus Hook. \& Arn.

APHANANTHE Planch.

ULMACEAE

Elm Family

aspera (Thunb. ex J.A.Murr.) Planch.

ARALIA L.

ARALIACEAE Ginseng Family

elata (Miq.) Seemann

spinosa $\mathrm{L}$.

ARAUCARIA Juss.

ARAUCARIACEAE Araucaria Family

araucana (Mol.) K.Koch

bidwillii Hook.

ARBUTUS L.

ERICACEAE

Heath Family

unedo L.

ARCTOSTAPHYLOS Adans. ERICACEAE Heath Family

uva-ursi (L.) Spreng. 
ARDISIA O.Swartz MYRSINACEAE

Myrsine Family

crenata Sims

crenata Sims 'Alba'

crispa (Thunb. ex J.A.Murr.) A.DC.

japonica (Thunb.) Bl.

ARISTOLOCHLA L.

ARISTOLOCHLACEAE Aristolochia Family

elegans Mast.

ARONLA Medik.

ROSACEAE

Chokeberry

Rose Family

arbutifolia (L.) Ell.

melanocarpa (Michx.) Ell.

prunifolia (Marsh.) Rehd.

ARTEMISIA L.

ASTERACEAE

Aster Family

abrotanum L.

ARUNDINARIA Michx.

POACEAE

Grass Family

pygmaea (Miq.) Mitf. 'Variegata'

ASCYRUM L.

HYPERICACEAE St. John's-wort Family

hypericoides L.

ASIMINA Adans.

ANNONACEAE

Custard-apple Family

triloba (L.) Dunal

ASTER L.

ASTERACEAE

Aster Family

carolinianus Walt.

AUCUBA Thunb.

CORNACEAE

Dogwood Family

chinensis Benth.

japonica Thunb.

japonica Thunb. 'Goldieana'

japonica Thunb. 'Limbata'

japonica Thunb. 'Longifolia'

japonica Thunb. 'Meigetsu'

japonica Thunb. 'Nana'

japonica Thunb. 'Salicifolia'

japonica Thunb. 'Variegata'

japonica Thunb. var. borealis Miyabe \&

Kudo
BACCHARIS L.

Groundsel

ASTERACEAE

Aster Family

glomeruliflora Pers.

halimifolia L.

halimifolia L. 'Dauphin Island'

BAMBUSA Schreber POACEAE

Bamboo

Grass Family

multiplex (Lour.) Raeusch.

multiplex (Lour.) Raeusch. 'Alphonse

Karr'

multiplex (Lour.) Raeusch. 'Fernleaf'

multiplex (Lour.) Raeusch. 'Silverstripe'

multiplex (Lour.) Raeusch. 'Variegata'

tuldoides Munro

BAUHINIA L.

FABACEAE (Caesalpinioideae)

Bean Family

divaricata $\mathrm{L}$.

lunarioides A.Gr. ex S.Wats.

yunnanensis Franch.

BEFARIA Mutis ex L.

ERICACEAE

Heath Family

racemosa Vent.

BERBERIS L.

BERBERIDACEAE

Barberry

Barberry Family

$\times$ gladwynensis Li 'William Penn'

hookeri Lem.

hookeri Lem. var. viridis Schneid.

$\times$ hybrido-gagnepainii Suring.

'Chenault'

julianae Schneid.

julianae Schneid. 'Byers'

koreana Palib. 'Atropurpurea'

$\times$ media Grootend. 'Parkjuweel'

$\times$ mentorensis H.Schultz \& Horvath

ex L.M.Ames

mouillacana Schneid.

sargentiana Schneid.

soulieana Schneid.

thunbergii DC.

thunbergii DC. 'Atropurpurea'

thunbergii DC. 'Atropurpurea Nana'

thunbergii DC. 'Aurea'

thunbergii DC. 'Crimson Pygmy'

thunbergii DC. 'Kobold'

thunbergii DC. 'Sparkle'

thunbergii DC. 'Special'

verruculosa Hemsl. \& Wils.

$\times$ wisleyensis Ahrendt 
BERCHEMIA Necker ex DC.

RHAMNACEAE Buckthorn Family

racemosa Sieb. \& Zucc.

racemosa Sieb. \& Zucc. 'Issai'

scandens (J.Hill) K.Koch

BETULA L.

Birch

BETULACEAE

Birch Family

alleghaniensis Britton

costata Traut.

davurica Pall.

jaquemontii Spach

lenta $\mathrm{L}$.

maximowicziana Regel

nigra $\mathrm{L}$.

occidentalis Hook.

papyrifera Marsh.

papyrifera $\times$ B. maximowicziana

pendula Roth

pendula $\times$ B. nigra

pendula Roth 'Dalecarlica'

pendula Roth 'Purpurea'

pendula Roth 'Youngii'

platyphylla Sukachev var. japonica

(Miq.) Hara

populifolia Marsh.

potaninii Batal.

pubescens Ehrh.

uber (Ashe) Fern.

BIGNONIA L.

BIGNONIACEAE Bignonia Family

capreolata L.

BREYNLA J.R. \& J.G. Forst. EUPHORBIACEAE

Spurge Family

disticha J.R. \& J.G.Forst.

BROUSSONETIA L'Her. ex Vent.

MORACEAE

Mulberry Family

papyrifera (L.) Vent.

papyrifera (L.) Vent. 'Variegata'

BRUCKENTHALIA Reichenb.

ERICACEAE

Heath Family

spiculifolia (Salisb.) Reichenb.

BRUGMANSIA Pers.

SOLANACEAE Nightshade Family

suaveolens (Humboldt \& Bonpland

ex Willd.) Bercht. \& J.Presl
BRUNFELSIA L.

SOLANACEAE Nightshade Family

australis Benth.

BUCKLEYA Torr.

SANTALACEAE Sandalwood Family

distichophylla (Nutt.) Torr.

BUDDLEJA L.

Butterfly Bush

BUDDLEJACEAE

Buddleja Family

alternifolia Maxim.

crispa Benth. var. farreri (Balf.f. \&

W.W.Sm.)Hand.-Mazz.

davidii Franch.

davidii Franch. 'Alba'

fallowiana Balf.f. \& W.W.Sm.

farreri Balf.f. \& W.W.Sm.

japonica Hemsl.

lindleyana Fort. ex Lindl.

venenifera Makino

$\times$ weyeriana Weyer

$\times$ weyeriana Weyer 'Sungold'

$\times$ whiteana R.J.Moore

BUMELIA O.Swartz

SAPOTACEAE Sapodilla Family

lanuginosa (Michx.) Pers.

lycioides (L.) Pers.

tenax (L.) Willd.

BUTIA (Becc.) Becc.

ARECACEAE Palm Family

capitata (Mart.) Becc.

BUXUS L. Box

BUXACEAE Boxwood Family

balearica Lam.

harlandii Hance

harlandii Hance 'Richard'

microphylla Sieb. \& Zucc.

microphylla Sieb. \& Zucc. 'Compacta'

microphylla Sieb. \& Zucc. 'Curly Locks'

microphylla Sieb. \& Zucc. 'Grace

Hendrick Phillips'

microphylla Sieb. \& Zucc. 'Green Pillow'

microphylla Sieb. \& Zucc. 'Helen

Whiting'

microphylla Sieb. \& Zucc. 'Henry

Hohman'

microphylla Sieb. \& Zucc. 'John

Baldwin 
microphylla Sieb. \& Zucc. 'Sunnyside' microphylla Sieb. \& Zucc. var. japonica

(Muell.-Arg.) Rehd. \& Wils.

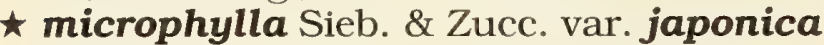

(Muell.-Arg.) Rehd. \& Wils. 'Morris

Dwarf

$\star$ microphylla Sieb. \& Zucc. var. japonica

(Muell.-Arg.) Rehd. \& Wils. 'Morris

Midget'

$\star$ microphylla Sieb. \& Zucc. var. japonica

(Muell.-Arg.) Rehd. \& Wils. 'National' sempervirens L.

sempervirens L. 'Abilene'

sempervirens L. 'Agram'

sempervirens L. 'Angustifolia'

sempervirens L. 'Arborescens'

sempervirens L. 'Arborescens

Decussata'

sempervirens L. 'Argenteo-variegata'

sempervirens L. 'Aristocrat'

sempervirens L. 'Aurea Pendula'

sempervirens L. 'Aureo-variegata'

sempervirens L. 'Belleville'

sempervirens L. 'Bullata'

sempervirens L. 'Butterworth'

sempervirens L. 'Denmark'

sempervirens L. 'Edgar Anderson'

sempervirens L. 'Elegantissima'

sempervirens L. 'Fastigiata'

sempervirens L. 'Glauca'

sempervirens L. 'Graham Blandy'

sempervirens L. 'Handsworthiensis'

sempervirens L. 'Hardwickensis'

sempervirens L. 'Heinrich Bruns'

sempervirens L. 'Henry Shaw'

sempervirens L. 'Inglis'

sempervirens L. 'Ipek'

sempervirens L. 'Joe Gable'

sempervirens L. 'Joy'

sempervirens L. 'Latifolia

Macrophylla'

sempervirens L. 'Latifolia Nova'

sempervirens L. 'Lynnhaven'

sempervirens L. 'Macrophylla'

sempervirens L. 'Marginata'

sempervirens L. 'Memorial'

sempervirens L. 'Myrtifolia'

sempervirens L. 'Nish'

sempervirens L. 'Northern Find'

sempervirens L. 'Northern New York'

sempervirens L. 'Northland'

sempervirens L. 'Pendula'

sempervirens L. 'Ponteyi'

sempervirens L. 'Prostrata'

sempervirens L. 'Pyramidalis'

sempervirens L. 'Rotundifolia'

sempervirens L. 'Ste. Genevieve' sempervirens L. 'Salicifolia'

sempervirens L. 'Salicifolia Elata'

sempervirens L. 'Suffruticosa'

sempervirens L. 'Vardar Valley'

sempervirens L. 'Varifolia'

sempervirens L. 'Welleri'

sinica (Rehd. \& Wils.) M.Cheng

var. insularis (Nakai) M.Cheng

sinica (Rehd. \& Wils.) M.Cheng

var. insularis (Nakai) M.Cheng

'Pincushion'

sinica (Rehd. \& Wils.) M.Cheng

var. insularis (Nakai) M.Cheng

'Tall Boy'

sinica (Rehd. \& Wils.) M.Cheng

var. insularis (Nakai) M.Cheng

'Tide Hill'

sinica (Rehd. \& Wils.) M.Cheng

var. insularis (Nakai) M.Cheng

'Winter Beauty'

sinica (Rehd. \& Wils.) M.Cheng

var. insularis (Nakai) M.Cheng

'Wintergreen'

stenophylla Hance

wallichiana Baill.

'Green Gem'

'Green Mountain'

'Green Velvet'

CAESALPINIA L.

FABACEAE (Caesalpinioideae) Bean Family

gilliesii Hook.

CALLIANDRA Benth.

FABACEAE (Mimosoideae) Bean Family

tweediei Benth.

CALLICARPA L

VERBENACEAE

Beautyberry

Verbena Family

americana $\mathrm{L}$.

americana L. 'Lactea'

bodinieri Lev.

bodinieri Lev. var. giraldii Rehd.

dichotoma (Lour.) K.Koch

dichotoma (Lour.) K.Koch 'Leucocarpa'

japonica Thunb.

macrophylla Vahl

tosaensis Makino

CALLISTEMON R.Br. MYRTACEAE

Bottlebrush Myrtle Family

citrinus (Curtis) Skeels

linearis (Schrad. \& J.C.Wendl.) DC.

rigidus $\mathrm{R}$. Br. 
rugulosus Miq.

salignus (Sm.) DC.

viminalis (Sol. ex Gaertn.) G.Don

CALLITRIS Vent. CUPRESSACEAE

Cypress Family

columellaris F.Muell.

CALLUNA Salisb.

Heather

ERICACEAE Heath Family

vulgaris (L.) Hull

vulgaris (L.) Hull 'August Beauty'

vulgaris (L.) Hull 'Aureafolia'

vulgaris (L.) Hull 'H. E. Beale'

vulgaris (L.) Hull 'Juno'

vulgaris (L.) Hull 'Plena'

vulgaris (L.) Hull 'Silver Queen'

vulgaris (L.) Hull 'Tib'

\section{CALOCEDRUS KurZ}

CUPRESSACEAE Cypress Family

decurrens (Torr.) Florin

CALYCANTHUS L.

CALYCANTHACEAE Calycanthus Family

floridus $\mathrm{L}$.

floridus L. 'Athens'

floridus L. 'Margarita'

CAMELLIA L.

THEACEAE

Tea Family

assimilis Champ. ex Benth.

crapnelliana Tutch.

cuspidata (Kochs) J.G.Veitch

drupifera (Lour.) Pierre

fraterna Hance

(fraterna $\times$ C. japonica)

'Little Princess'

granthamiana Sealy

$\star$ (hiemalis 'Billy Wylam' $\times$ C. oleifera)

'Frost Princess'

$\star$ ('Frost Princess' $\times$ C. oleifera)

'Polar Ice'

$\star$ ('Frost Princess' $\times$ C. oleifera)

'Snow Flurry'

$\star$ ('Frost Princess' $\times$ C. oleifera)

'Winter's Hope'

$\star$ (hiemalis 'Otome' $\times$ C. oleifera)

'Winter's Rose'

$\star$ (hiemalis 'Shishigashira' $\times$ C. oleifera)

'Frost Prince' $\star$ ('Frost Prince' $\times$ C. hiemalis)

'Winter's Star'

$\star$ (hongkongensis $\times$ C. rusticana)

'Sunworshiper'

japonica L.

japonica L. 'Alice Morrison'

japonica L. 'Anemoniflora'

japonica L. 'Aunt Jetty'

japonica L. 'Barbara Morgan'

japonica L. 'Chandleri Elegans'

japonica L. 'Daitairin'

japonica L. 'Doctor Tinsley'

$\star$ japonica L. 'Frost Queen'

japonica L. 'Gov. Mouton Variegated'

japonica L. 'Guilio Nuccio'

japonica L. 'Kumasaka'

japonica L. 'Leucantha'

japonica L. 'Marie Wood'

japonica L. 'Professor Sargent'

japonica L. 'Reverend John G. Drayton'

japonica L. 'Sarah Frost'

japonica L. 'Sieboldii'

japonica L. 'Tama no ura'

japonica L. 'Ville de Nantes'

$\star$ (japonica 'Arabella' $\times$ C. 'Fragrant

Pink') 'Ack-Scent Red'

$\star$ (japonica 'Fragrant Star' $\times$ C. 'Fragrant

Pink') 'Ack-Scent Pink'

$\star$ (japonica 'Fragrant Star' $\times$ C. 'Fragrant

Pink') 'Ack-Scent Spice'

$\star$ (japonica 'Fragrant Star' $\times$ C. 'Fragrant

Pink') 'Ack-Scent Star'

$\star$ (japonica 'Frost Queen' $\times$ C. 'Fragrant

Pink') 'Ack-Scent Sno'

$\star$ (japonica 'Frost Queen' $\times$ C. 'Fragrant

Pink') 'Ack-Scent White'

$\star$ (japonica 'Kenyo-Tai' $\times$ C. lutchuensis)

'Cinnamon Cindy'

$\star$ (japonica 'Kramer's Supreme' $\times$

C. 'Fragrant Pink Improved')

'Ack-Scent'

kissii Wall.

lutchuensis Ito

oleifera Abel

$\star$ (oleifera $\times$ C. sasanqua

'Takara-awase') 'Winter's Charm'

reticulata Lindl. 'Capt. Rawes'

(reticulata $\times$ C. saluenensis)

'Inspiration'

$\star$ (rusticana 'Yoshida' $\times$ C. lutchuensis)

'Fragrant Pink'

$\star$ (rusticana $\times$ C. lutchuensis)

'Fragrant Joy'

saluenensis Stapf

sasanqua Thunb. ex J.A.Murr.

sasanqua Thunb. ex J.A.Murr. 'Annette'

sasanqua Thunb. ex J.A.Murr.

'Cherokee' 
sasanqua Thunb. ex J.A.Murr.

'Cleopatra'

sasanqua Thunb. ex J.A.Murr.

'Crimson Bride'

sasanqua Thunb. ex J.A.Murr.

'Sharon Elizabeth'

sasanqua Thunb. ex J.A.Murr.

'Texas Star'

(sasanqua 'Maiden's Blush' +

C. japonica) +'Daisy Eagleson'

$\star$ (sasanqua 'Onishiki' $\times$ C. kissii)

'Two Marthas'

sinensis (L.) O.Ktze.

tenuifolia (Hayata) Cohen-Stuart

tsaii H.H.Hu

vernalis (Makino) Makino 'Dawn'

vernalis (Makino) Makino 'Sayehime'

$\times$ williamsii W.W.Sm. 'Donation'

$\times$ williamsii W.W.Sm. 'Donation

Sport'

$\times$ williamsii W.W.Sm. 'November

Pink'

$\star$ 'Fragrant Pink Improved' (Polyploid

'Fragrant Pink') 'Tiny Princess'

CAMPSIS Lour.

BIGNONIACEAE

Bignonia Family

grandiflora (Thunb. ex J.A.Murr.)

Schum.

radicans (L.) Seemann ex Bur.

radicans (L.) Seemann ex Bur.

'Yellow Trumpet'

$\times$ tagliabuana (Vis.) Rehd. 'Mme. Galen'

CAMPYLOTROPIS Bunge

FABACEAE (Papilionoideae) Bean Family

macrocarpa Bunge

CARAGANA Fabr.

FABACEAE (Faboideae)

Pea Shrub

Bean Family

arborescens Lam.

arborescens Lam. 'Lorbergii'

arborescens Lam. 'Pendula'

frutex (L.) K.Koch 'Sylvatica'

sinica (Buc'hoz) Rehd.

CARISSA L.

APOCYNACEAE Dogbane Family

macrocarpa (Eckl. \& Zeyh.) A.DC.
CARPINUS L.

BETULACEAE

Hornbeam

Birch Family

betulus $\mathrm{L}$.

betulus L. 'Fastigiata'

betulus L. 'Purpurea'

caroliniana Walt.

caroliniana Walt. 'Ascendens'

cordata Bl.

japonica $\mathrm{Bl}$.

orientalis Mill.

CARYA Nutt.

JUGLANDACEAE

Hickory

Walnut Family

aquatica (Michx.f.) Nutt.

cordiformis (Wang.) K.Koch

glabra (Mill.) Sweet

illinoinensis (Wang.) K.Koch

illinoinensis (Wang.) K.Koch 'Mehan'

illinoinensis (Wang.) K.Koch 'Stuart'

illinoinensis $\times$ C. $\mathrm{sp}$.

(illinoinensis $\times$ C. sp.) 'Koon'

laciniosa (Michx.f.) Loud.

ovalis (Wang.) Sarg.

ovata (Mill.) K. Koch

ovata (Mill.) K.Koch 'Glover'

ovata (Mill.) K.Koch 'Hines'

ovata (Mill.) K.Koch 'Lake'

ovata (Mill.) K.Koch 'Lingenfelter'

ovata (Mill.) K.Koch 'Romig'

ovata (Mill.) K.Koch 'Schaul'

ovata (Mill.) K.Koch 'Schinnerling'

ovata (Mill.) K.Koch 'Vest'

ovata (Mill.) K.Koch var. pubescens Sarg.

pallida (Ashe) Engl. \& Graebn.

tomentosa (Lam.) Nutt.

\section{CARYOPTERIS Bunge}

VERBENACEAE

Verbena Family

$\times$ clandonensis Simmonds

incana (Thunb. ex Houtt.) Miq.

CASIMIROA Llave \& LeX.

RUTACEAE

Citrus Family

edulis Llave \& Lex.

pringlei (S.Wats.) Engelm.

CASSIA L.

Senna

FABACEAE (Caesalpinioideae) Bean Family

alata L.

coluteoides Coll.

corymbosa Lam. 
CASTANEA Mill.

FAGACEAE

Chestnut Beech Family

crenata Sieb. \& Zucc.

mollissima $\mathrm{Bl}$.

mollissima Bl. 'Crane'

mollissima Bl. 'Meiling'

mollissima Bl. 'Nanking'

mollissima $\mathrm{Bl}$. 'Orrin'

sativa Mill.

sativa L. 'Argenteo-variegata'

CASTANOPSIS (D.Don) Spach

\section{FAGACEAE}

Chinquapin Beech Family

cuspidata (Thunb. ex J.A.Murray)

Schottky

cuspidata (Thunb.) Schottky

var. sieboldii (Makino) Nakai

sclerophylla Schottky

CASUARINA Adans.

CASUARINACEAE Casuarina Family

cunninghamiana Miq.

CATALPA Scop.

BIGNONLACEAE

Bignonia Family

bignonioides Walt.

bungei C.A.Mey.

ovata G.Don

speciosa Warder ex Engelm.

CEANOTHUS L.

RHAMNACEAE

Buckthorn Family

americanus $\mathrm{L}$.

$\times$ delilianus Spach

$\times$ delilianus Spach 'Gloire de Versailles'

microphyllus Michx.

$\times$ pallidus Lindl. 'Marie Simon'

velutinus Dougl.

CEDRUS Trew

PINACEAE

atlantica (Endl.) Manetti ex Carr.

atlantica (Endl.) Manetti ex Carr. 'Aurea'

atlantica (Endl.) Manetti ex Carr.

'Fastigiata'

atlantica (Endl.) Manetti ex Carr.

'Glauca'

atlantica (Endl.) Manetti ex Carr.

'Glauca Pendula' atlantica (Endl.) Manetti ex Carr.

'Pendula'

deodara (Roxb.) G.Don

deodara (Roxb.) G.Don 'Aurea'

deodara (Roxb.) G.Don 'Fastigiata'

deodara (Roxb.) G.Don 'Limelight'

deodara (Roxb.) G.Don 'Pendula'

deodara (Roxb.) G.Don 'Pygmy'

deodara (Roxb.) G.Don 'Repandens'

libani A.Rich.

libani A.Rich. 'Nana'

libani A.Rich. ssp. brevifolia (Hook.f.)

Meikle

libani A.Rich. var. stenocoma

(O.Schwarz) Davis

CELASTRUS L. CELASTRACEAE

Bittersweet Staff-tree Family

angulatus Maxim.

orbiculatus Thunb. ex J.A.Murr.

rosthornianus Loesn.

CELTIS L.

ULMACEAE

Hackberry

Elm Family

australis $\mathrm{L}$.

caucasica Willd.

laevigata Willd.

occidentalis $\mathrm{L}$.

pallida Torr.

sinensis Pers.

sinensis Pers. var. japonica (Planch.)

Nakai

tala Gillies ex Planch.

CEPHALANTHUS L.

RUBLACEAE

Madder Family

occidentalis L.

CEPHALOTAXUS Sieb. \& Zucc. ex Endl. CEPHALOTAXACEAE Plum-yew Family

harringtonia (J.Knight ex Forbes) K.Koch harringtonia (J.Knight ex Forbes) K.Koch 'Fastigiata'

harringtonia (J.Knight ex Forbes) K.Koch 'Nana'

CERATIOLA MichX. EMPETRACEAE Crowberry Family ericoides Michx. 
CERATOSTIGMA Bunge

PLUMBAGINACEAE Leadwort Family

plumbaginoides Bunge

willmottianum Stapf

CERCIDIPHYLLUM Sieb. \& ZuCc.

CERCIDIPHYLLACEAE Katsura-tree Family

japonicum Sieb. \& Zucc.

japonicum Sieb. \& Zucc. 'Pendula'

\section{CERCIS L.}

FABACEAE (Caesalpinioideae) Bean Family

canadensis $\mathrm{L}$.

canadensis L. 'Alba'

canadensis L. 'Forest Pansy'

canadensis L. 'Plena'

canadensis L. 'Ruby Atkinson'

canadensis L. 'Wither's Pink Charm'

canadensis L. ssp. texensis (S.Wats.)

E.Murr. 'Alba'

canadensis L. ssp. texensis (S.Wats.)

E.Murr. 'Oklahoma'

chinensis Bunge

chinensis Bunge 'Alba'

siliquastrum L. 'Rubrum'

\section{CESTRUM L.}

SOLANACEAE

Nightshade Family

nocturnum $\mathrm{L}$.

\section{CHAENOMELES Lindl. ROSACEAE}

Rose Family

$\times$ californica Clarke \& Weber

'Rosy Morn'

japonica (Thunb.) Lindl. ex Spach

speciosa (Sweet) Nakai

speciosa (Sweet) Nakai 'Apple Blossom'

speciosa (Sweet) Nakai 'Candida'

speciosa (Sweet) Nakai 'Cardinalis'

speciosa (Sweet) Nakai 'Hanazono'

speciosa (Sweet) Nakai 'Marmorata'

speciosa (Sweet) Nakai 'Nivalis'

speciosa (Sweet) Nakai 'Phyllis Moore'

speciosa (Sweet) Nakai 'Rubra'

speciosa (Sweet) Nakai 'Simonii'

speciosa (Sweet) Nakai 'Snow'

speciosa (Sweet) Nakai 'Toyo nishiki'

speciosa (Sweet) Nakai 'Umbilicata'

superba (Frahm) Rehd.

superba (Frahm) Rehd. 'Corallina'

superba (Frahm) Rehd. 'Crimson and Gold ×superba (Frahm) Rehd. 'Knap Hill Scarlet'

$\times$ superba (Frahm) Rehd. 'Mandarin'

$\times$ superba (Frahm) Rehd. 'Perfecta'

$\times$ superba (Frahm) Rehd. 'Pink Lady'

$\times$ superba (Frahm) Rehd. 'Roxana Foster'

$\times$ superba (Frahm) Rehd. 'Ruby Glow'

$\times$ superba (Frahm) Rehd. 'Stanford Red'

$\times$ vilmoriniana Weber 'Mt. Everest'

CHAMAECYPARIS Spach False Cypress CUPRESSACEAE

Cypress Family

funebris (Endl.) Franco

lawsoniana (A.Murr.) Parl.

lawsoniana (A.Murr.) Parl. 'Allumii'

lawsoniana (A.Murr.) Parl. 'Blom'

lawsoniana (A.Murr.) Parl. 'Filiformis'

lawsoniana (A.Murr.) Parl. 'Filiformis Compacta'

lawsoniana (A.Murr.) Parl. 'Lutea'

lawsoniana (A.Murr.) Parl.

'Lycopodioides'

lawsoniana (A.Murr.) Parl.

'Pembury Blue'

lawsoniana (A.Murr.) Parl.

'Pendula Vera'

nootkatensis (D.Don) Spach

obtusa (Sieb. \& Zucc.) Endl.

obtusa (Sieb. \& Zucc.) Endl. 'Albospica'

obtusa (Sieb. \& Zucc.) Endl. 'Breviramea' obtusa (Sieb. \& Zucc.) Endl. 'Contorta'

obtusa (Sieb. \& Zucc.) Endl.

'Coralliformis'

obtusa (Sieb. \& Zucc.) Endl. 'Crippsii'

obtusa (Sieb. \& Zucc.) Endl. 'Filicoides'

obtusa (Sieb. \& Zucc.) Endl. 'Filiformis

Aurea'

obtusa (Sieb. \& Zucc.) Endl. 'Gold Drop'

obtusa (Sieb. \& Zucc.) Endl. 'Gracilis'

obtusa (Sieb. \& Zucc.) Endl. 'Intermedia'

obtusa (Sieb. \& Zucc.) Endl. 'Kosteri'

obtusa (Sieb. \& Zucc.) Endl.

'Lycopodioides'

obtusa (Sieb. \& Zucc.) Endl. 'Mariesii'

obtusa (Sieb. \& Zucc.) Endl. 'Nana'

obtusa (Sieb. \& Zucc.) Endl. 'Nana

Argentea'

obtusa (Sieb. \& Zucc.) Endl. 'Nana Aurea'

obtusa (Sieb. \& Zucc.) Endl. 'Nana

Gracilis'

obtusa (Sieb. \& Zucc.) Endl. 'Nana

Pyramidalis'

obtusa (Sieb. \& Zucc.) Endl. 'Pygmaea'

obtusa (Sieb. \& Zucc.) Endl. 'Pygmaea

Aurescens'

obtusa (Sieb. \& Zucc.) Endl. 'Reis Dwarf' 
obtusa (Sieb. \& Zucc.) Endl. 'Repens' obtusa (Sieb. \& Zucc.) Endl. 'Rigid Dwarf' obtusa (Sieb. \& Zucc.) Endl. 'Sanderi' obtusa (Sieb. \& Zucc.) Endl. 'Spiralis' obtusa (Sieb. \& Zucc.) Endl. 'Stoneham' obtusa (Sieb. \& Zucc.) Endl. 'Tempelhof' obtusa (Sieb. \& Zucc.) Endl. 'Tetragona Aurea'

obtusa (Sieb. \& Zucc.) Endl. 'Tonia' obtusa (Sieb. \& Zucc.) Endl. 'Verdonii' pisifera (Sieb. \& Zucc.) Endl.

pisifera (Sieb. \& Zucc.) Endl. 'Aurea Nana'

pisifera (Sieb. \& Zucc.) Endl. 'Boulevard' pisifera (Sieb. \& Zucc.) Endl. 'Compacta' pisifera (Sieb. \& Zucc.) Endl. 'Filifera' pisifera (Sieb. \& Zucc.) Endl. 'Filifera Aurea'

pisifera (Sieb. \& Zucc.) Endl. 'Gold Spangle'

pisifera (Sieb. \& Zucc.) Endl. 'Golden Mop'

pisifera (Sieb. \& Zucc.) Endl.

'Juniperoides Aurea'

pisifera (Sieb. \& Zucc.) Endl. 'Monstrosa' pisifera (Sieb. \& Zucc.) Endl. 'Plumosa' pisifera (Sieb. \& Zucc.) Endl. 'Plumosa Aurea' pisifera (Sieb. \& Zucc.) Endl. 'Plumosa Aurea Nana' pisifera (Sieb. \& Zucc.) Endl. 'Plumosa Compacta' pisifera (Sieb. \& Zucc.) Endl. 'Plumosa Compressa' pisifera (Sieb. \& Zucc.) Endl. 'Pygmaea' pisifera (Sieb. \& Zucc.) Endl. 'Snow' pisifera (Sieb. \& Zucc.) Endl. 'Squarrosa' pisifera (Sieb. \& Zucc.) Endl. 'Squarrosa Intermedia' pisifera (Sieb. \& Zucc.) Endl. 'Squarrosa Minima'

thyoides (L.) BSP.

thyoides (L.) BSP. 'Andelyensis'

thyoides (L.) BSP. 'Ericoides'

CHAMAECYTISUS Link FABACEAE (Faboideae) Bean Family

supinus (L.) Link

CHAMAEDAPHNE Moench ERICACEAE Heath Family

calyculata (L.) Moench
CHAMAEROPS L.

ARECACEAE - Palm Family

humilis L.

CHILOPSIS D.Don

BIGNONIACEAE

Bignonia Family

linearis (Cav.) Sweet

CHIMONANTHUS Lindl.

CALYCANTHACEAE Calycanthus Family

nitens Oliv.

praecox (L.) Link

praecox (L.) Link 'Luteus'

praecox (L.) Link 'Mangetsu'

\section{CHIONANTHUS L. Fringe Tree OLEACEAE Olive Family}

retusus Lindl. \& Paxt.

retusus Lindl. \& Paxt. var. serrulatus

(Hayata) Koidz.

virginicus $\mathrm{L}$.

CHOSENIA Nakai

SALICACEAE Willow Family

arbutifolia (Pall.) Skvortz.

CHRYSANTHEMUM L.

ASTERACEAE

Aster Family

nipponicum (Franch. ex Maxim.) Matsum.

CINNAMOMUM Schaeffer

LAURACEAE

Laurel Family

camphora (L.) T. Nees \& Eberm.

daphnoides Sieb. \& Zucc.

CISSUS L.

VITACEAE

Grape Family

incisa (Nutt.) Desmoul.

CISTUS L.

CISTACEAE

Rock-rose Family

$\times$ hybridus Pourr.

ladanifer $\mathrm{L}$.

$\times$ purpureus Lam. 
CITHAREXYLUM L. VERBENACEAE

Verbena Family

berlandieri Robinson

$\times$ CITROFORTUNELLA J.Ingram \& H.E.Moore

RUTACEAE

Citrus Family

mitis (Blanco) J.Ingram \& H.E.Moore

$\times$ CITRONCIRUS J.Ingram \& H.E.Moore RUTACEAE

Citrus Family

webberi J.Ingram \& H.E.Moore

CITRUS L.

RUTACEAE

Citrus Family

aurantium $\mathrm{L}$.

hystrix DC.

medica L. 'Etrog'

$\times$ paradisi Macf.

reticulata Blanco

'Thomasville Citrangequat'

CLADRASTIS Raf.

FABACEAE (Faboideae) Bean Family

kentukea (Dum.-Cours.) Rudd

\section{CLEMATIS L.}

RANUNCULACEAE Buttercup Family

armandii Franch.

armandii Franch. 'Farquhariana'

chrysocoma Franch. var. sericea

(Franch.) Schneid.

crispa $\mathrm{L}$.

$\times$ jackmanii T.Moore

$\times$ jackmanii T.Moore 'Superba'

montana Buch.-Ham. 'Grandiflora'

pitcheri T. \& G.

stans Sieb. \& Zucc.

terniflora DC.

texensis Buckl.

$\times$ triternata DC. 'Rubro-marginata'

versicolor Britton

viorna $\mathrm{L}$.

$\star$ viticella L. 'Betty Corning'
CLERODENDRUM L.

\section{VERBENACEAE}

Verbena Family

bungei Steud.

indicum (L.) O.Ktze.

philippinum Schauer

thomsonae Balf.f.

trichotomum Thunb.

CLETHRA L.

CLETHRACEAE

White Alder

White-alder Family

acuminata Michx.

alnifolia $\mathrm{L}$.

alnifolia L. 'Rosea'

barbinervis Sieb. \& Zucc.

fargesii Franch.

tomentosa Lam.

CLEYERA Thunb.

THEACEAE

Tea Family

japonica Thunb.

japonica Thunb. 'Tricolor'

CLIFTONIA Banks ex C.F.Gaertn.

CYRILLACEAE

Cyrilla Family

monophylla (Lam.) Britton ex Sarg.

\section{CLINOPODIUM L.}

LAMIACEAE Mint Family

ashei (Weatherby) Small

coccineum (Nutt.) O.Ktze.

dentatum (Chapm.) O.Ktze.

CLYTOSTOMA Miers ex Bur.

BIGNONIACEAE Bignonia Family

callistegioides (Cham.) Bur.

CNEORUM L.

CNEORACEAE

Spurge-olive Family

tricoccon $\mathrm{L}$.

COCCULUS DC.

MENISPERMACEAE Moonseed Family

laurifolius DC.

trilobus (Thunb.) DC.

COLUTEA L.

FABACEAE (Faboideae) Bean Family

arborescens L. 
COMPTONLA L'Her. ex Ait.

MYRICACEAE Wax-myrtle Family

peregrina (L.) Coult.

CONDALIA Cav.

RHAMNACEAE Buckthorn Family

hookeri M.C.Johnst.

CONRADINA A.Gr.

LAMIACEAE Mint Family

canescens (T. \& G.) A.Gr.

glabra Shinners

grandiflora Small

verticillata Jennison

CORDIA L.

BORAGINACEAE

Borage Family

boissieri A.DC.

CORIARIA L.

CORIARIACEAE Coriaria Family

japonica A.Gr.

CORNUS L.

Dogwood

CORNACEAE

Dogwood Family

alba L.

alba L. 'Argenteo-marginata'

alba L. 'Kesselringii'

alba L. 'Sibirica'

alternifolia L.f.

amomum Mill.

asperifolia Michx.

australis C.A.Mey. var. koenigii

(Schneid.) Wang.

controversa Hemsl.

coreana Wang.

drummondii C.A.Mey.

florida L.

florida L. 'Bay Beauty'

florida L. 'Cherokee Chief'

florida L. 'Cherokee Princess'

florida L. 'Cherokee Sunset'

florida L. 'Cloud Nine'

florida L. 'Dwarf'

florida L. 'First Lady'

florida L. 'Fragrant Cloud'

florida L. 'Prosser Red'

florida L. 'Rainbow'

florida L. 'Royal Red'

florida L. 'Salicifolia'

florida L. 'Springtime'

florida L. 'Steele's Fastigiata'

florida L. 'Stokes' Pink'

florida L. 'Sweetwater'

florida L. 'Welch's Junior Miss'

florida L. 'Welchii'

florida L. f. pluribracteata Rehd.

florida L. f. rubra (Weston) Schelle

florida L. f. xanthocarpa Rehd.

foemina Mill.

kousa (Buerger ex Miq.) Hance

kousa (Buerger ex Miq.) Hance

'Gold Star'

kousa (Buerger ex Miq.) Hance

'Lustgarten Weeping'

kousa (Buerger ex Miq.) Hance

'Milky Way'

kousa (Buerger ex Miq.) Hance 'Rubra'

kousa (Buerger ex Miq.) Hance

'Variegata'

mas $\mathrm{L}$.

mas L. 'Aurea'

mas L. 'Macrocarpa'

mas L. 'Nana'

mas L. 'Variegata'

nuttallii Audubon

obliqua Raf.

officinalis Sieb. \& Zucc.

paucinervis Hance

racemosa Lam.

sanguinea $\mathrm{L}$.

sericea $\mathrm{L}$.

sericea L. 'Flaviramea'

walteri Wang.

CORYLOPSIS Sieb. \& Zucc. Winter Hazel HAMAMELIDACEAE Witch-hazel Family

glabrescens Franch. \& Sav.

pauciflora Sieb. \& Zucc.

sinensis Hemsl. 'Winterthur'

sinensis Hemsl. var. calvescens

Rehd. \& Wils.

sinensis Hemsl. var. calvescens

Rehd. \& Wils. f. veitchiana

(Bean) Morley \& Chao

sinensis Hemsl. var. sinensis

sinensis Hemsl. var. sinensis

'Spring Purple'

spicata Sieb. \& Zucc.
CORYLUS L.

BETULACEAE

Hazelnut, Filbert Birch Family americana Marsh.

americana Walt. 'Rush'

(americana $\times$ C. avellana) 'Potomac'

(americana $\times$ C. avellana) 'Reed' 
avellana $\mathrm{L}$.

avellana L. 'Contorta'

avellana L. 'Cosford'

avellana L. 'Fusco-rubra'

chinensis Franch.

colurna L.

cornuta Marsh.

maxima Mill. 'Purpurea'

sieboldiana $\mathrm{Bl}$.

sieboldiana $\mathrm{Bl}$. var. mandschurica

(Maxim. \& Rupr.) Schneid.

COTINUS Mill. ANACARDIACEAE

coggygria Scop.

coggygria Scop. 'Baby Doll'

coggygria Scop. 'Flame'

coggygria Scop. 'Nordine Red'

coggygria Scop. 'Purpureus'

coggygria Scop. 'Royal Purple'

coggygria Scop. 'Rubrifolius'

coggygria Scop. 'Velvet Cloak'

obovatus Raf.

COTONEASTER Medik. ROSACEAE

Rose Family

acutifolius Turcz.

adpressus Bois

adpressus Bois 'Hessei'

ambiguus Rehd. \& Wils.

apiculatus Rehd. \& Wils.

congestus Baker

conspicuus Marq.

conspicuus Marq. 'Decorus'

dammeri Schneid.

dammeri Schneid. 'Lowfast'

dammeri Schneid. 'Royal Beauty'

dammeri Schneid. 'Skogsholmen'

dielsianus Pritz. ex Diels

divaricatus Rehd. \& Wils.

floccosus (Rehd. \& Wils.) Flinck \& Hylmo

foveolatus Rehd. \& Wils.

franchetii Bois

horizontalis Decne.

horizontalis Decne. 'Variegata'

horizontalis Decne. var. perpusillus

Schneid.

ignavus $\mathrm{E}$.Wolf

integerrimus Medik.

lacteus W.W.Sm.

lucidus Schlechtend.

microphyllus Wall. ex Lindl.

microphyllus Wall. ex Lindl.

f. thymifolius (Lindl.) Koehne microphyllus Wall. ex Lindl.

var. cochleatus (Franch.) Rehd. \& Wils.

nanshan Mottet

nitens Rehd. \& Wils.

nummularius Fischer \& C.A.Mey.

obscurus Rehd. \& Wils.

racemiflorus (Desf.) K.Koch

racemiflorus (Desf.) K.Koch

var. soongoricus (Regel \& Herd.)

Schneid.

roseus Edgew.

salicifolius Franch.

salicifolius Franch. 'Parkteppich'

salicifolius Franch. 'Scarlet Leader'

silvestrii Pamp.

splendens Flinck \& Hylmo

sternianus (Turrill) Boom

$\times$ watereri Exell

$\times$ watereri Exell 'Cornubia'

wilsonii Nakai

zabelii Schneid.

CRATAEGOSORBUS Makino ex

Pojark.

ROSACEAE

Rose Family

miczurinii Pojark.

CRATAEGUS L. ROSACEAE

Hawthorn

Rose Family

aestivalis (Walt.) T. \& G.

arnoldiana Sarg.

brachyacantha Sarg. \& Engelm.

calpodendron (Ehrh.) Medik.

coccinioides Ashe

compta Sarg.

concinna Beadle

crus-galli L.

flava Ait.

floridana Sarg.

fulleriana Sarg.

intricata J.Lange

lacrimata Small

laevigata (Poir.) DC.

laevigata (Poir.) DC. 'Autumn Glory'

laevigata (Poir.) DC. 'Paul's Scarlet'

laevigata (Poir.) DC. 'Plena'

laevigata (Poir.) DC. 'Superba'

$\times$ lavallei Herincq ex Lav.

$\times$ lavallei Herincq ex Lav. 'Carrierei'

macrosperma Ashe

marshallii Egglest.

mollis (T. \& G.) Scheele

monogyna Jacq.

monogyna Jacq. 'Inermis'

monogyna Jacq. 'Praecox' 
monogyna Jacq. 'Stricta'

$\times$ mordenensis Boom 'Toba'

opaca Hook. \& Arn.

pennsylvanica Ashe

phaenopyrum (L.f.) Medik.

pinnatifida Bunge

populifolia Walt.

pringlei Sarg.

pruinosa (H.Wendl.) K.Koch

var. leiophylla (Sarg.) Phipps

punctata Jacq.

punctata Jacq. 'Inermis'

schuettei Ashe

spathulata Michx.

uniflora Moench

vaileae Britton

viridis $\mathrm{L}$.

viridis $\mathrm{L}$. 'Winter King'

CROTON L.

EUPHORBIACEAE Spurge Family

alabamensis E.A.Sm.

CRYPTOMERIA
TAXODIACEAE

japonica (Thunb. ex L.f.) D.Don

japonica (Thunb. ex L.f.) D.Don

'Bandai sugi'

japonica (Thunb. ex L.f.) D.Don 'Cristata'

japonica (Thunb. ex L.f.) D.Don

'Dacrydioides'

japonica (Thunb. ex L.f.) D.Don 'Elegans'

japonica (Thunb. ex L.f.) D.Don

'Enko sugi'

japonica (Thunb. ex L.f.) D.Don 'Globosa'

japonica (Thunb. ex L.f.) D.Don

'Globosa Nana'

japonica (Thunb. ex L.f.) D.Don

'Jindai sugi'

japonica (Thunb. ex L.f.) D.Don

'Knaptonensis'

japonica (Thunb. ex L.f.) D.Don 'Lobbii'

japonica (Thunb. ex L.f.) D.Don

'Lycopodioides'

japonica (Thunb. ex L.f.) D.Don

'Pygmaea'

japonica (Thunb. ex L.f.) D.Don

'Vilmoriniana'

japonica (Thunb. ex L.f.) D.Don 'Yoshino'

CUDRANLA Trecul

MORACEAE Mulberry Family

tricuspidata (Carr.) Bur. ex Lav.
CUNIINGHAMIA R.Br.

TAXODIACEAE

China Fir

Jaxodium Family

lanceolata (Lamb.) Hook.f.

lanceolata (Lamb.) Hook.f. 'Glauca'

CUPHEA P.Br.

LYTHRACEAE

Loosestrife Family

hyssopifolia HBK.

micropetala HBK.

$\times$ CUPRESSOCYPARIS Dallim.
CUPRESSACEAE

leylandii (Dallim. \& A.B.Jacks.) Dallim.

leylandii (Dallim. \& A.B.Jacks.) Dallim.

'Leighton Green'

$\star$ leylandii (Dallim. \& A.B.Jacks.) Dallim.

'Silver Dust'

$\begin{array}{lr}\text { CUPRESSUS L. } & \text { Cypress } \\ \text { CUPRESSACEAE } & \text { Cypress Family }\end{array}$

Cypress Family

arizonica Greene

arizonica Greene 'Gareei'

lusitanica Mill.

macrocarpa Hartw.

sempervirens L.

CYCAS L.

CYCADACEAE

Cycad Family

circinalis L.

revoluta Thunb.

CYDONLA Mill.

ROSACEAE

Quince

Rose Family

oblonga Mill.

CYRILLA Gard. ex L.

CYRILLACEAE

Cyrilla Family

racemiflora $\mathrm{L}$.

CYTISUS Desf.

FABACEAE (Faboideae)

Broom

Bean Family

battandieri Maire

commutatus (Willd.) Briq.

decumbens (Durande) Spach

$\times$ praecox Bean

$\times$ praecox Bean 'Hollandia'

$\times$ praecox Bean 'Moonlight'

scoparius (L.) Link

scoparius (L.) Link 'Andreanus' 
DALBERGIA L.f. FABACEAE (Faboideae)

Bean Family

hupeana Hance

sissoo Roxb. ex DC.

DANAE Medik.

LILIACEAE

Lily Family

racemosa (L.) Moench

DAPHNE L.

THYMELAEACEAE Mezereum Family

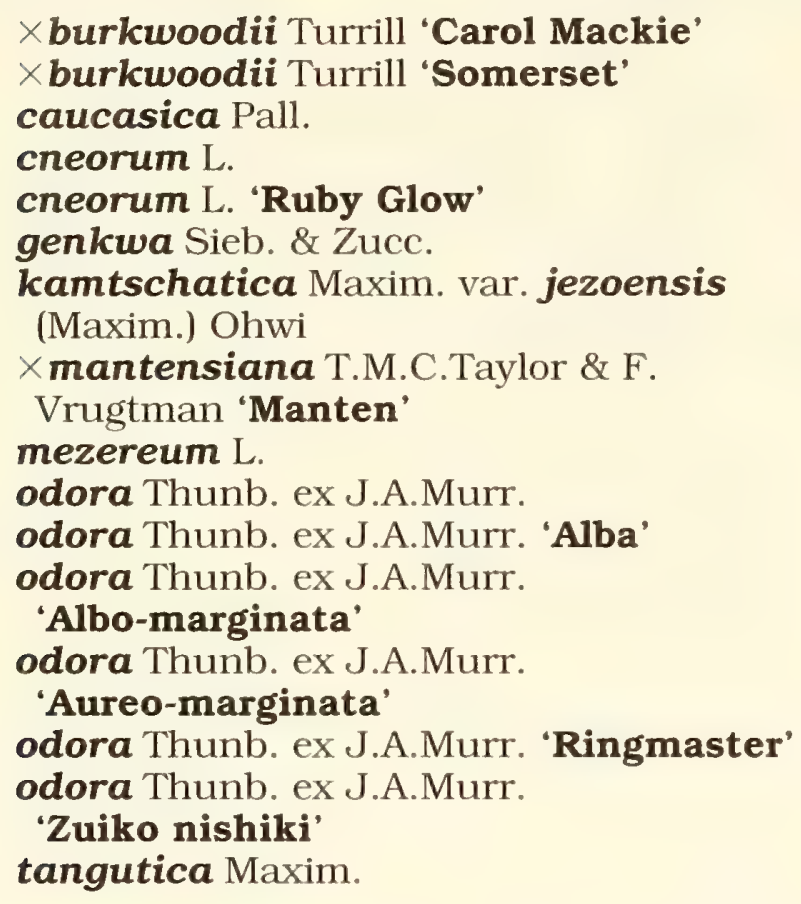

\section{DAPHNIPHYLLUM Bl.} EUPHORBIACEAE

macropodum Miq.

DAVIDIA Baill. NYSSACEAE Sour-gum Family

involucrata Baill. var. vilmoriniana (Dode) Wang.

DECUMARIA L.

SAXIFRAGACEAE (Hydrangeoideae) Saxifrage Family

barbara L.
DENDROPANAX Decne. \& Planch. ARALIACEAE Ginseng Family

trifidus (Thunb. ex J.A.Murr.) Makino ex Hara

DEUTZIA Thunb.

SAXIFRAGACEAE (Hydrangeoideae)

Saxifrage Family

$\times$ candelabrum (Lem.) Rehd.

$\times$ candida (Lem.) Rehd.

chunii H.H.Hu

discolor Hemsl.

$\times$ elegantissima (Lem.) Rehd.

$\times$ elegantissima (Lem.) Rehd.

'Conspicua'

glabrata Komar.

gracilis Sieb. \& Zucc.

gracilis Sieb. \& Zucc. 'Mohican'

gracilis Sieb. \& Zucc. 'Nikko'

$\times$ hybrida E.Lemoine 'Contraste'

$\times$ hybrida E.Lemoine 'Magicien'

$\times$ lemoinei E.Lemoine ex Bois

$\times$ magnifica (Lem.) Rehd.

$\times$ magnifica (Lem.) Rehd. 'Eburnea'

$\times$ magnifica (Lem.) Rehd. 'Eminens'

$\times$ magnifica (Lem.) Rehd. 'Erecta'

$\times$ magnifica (Lem.) Rehd. 'Formosa'

$\times$ magnifica (Lem.) Rehd. 'Latiflora'

$\times$ rosea (Lem.) Rehd.

$\times$ rosea (Lem.) Rehd. 'Carminea' scabra Thunb.

scabra Thunb. 'Candidissima'

scabra Thunb. 'Plena'

scabra Thunb. 'Pride of Rochester'

scabra Thunb. 'Summer Snow'

scabra Thunb. 'Suspensa'

scabra Thunb. 'Watereri'

schneideriana Rehd.

sieboldiana Maxim.

DIERVILLA Mill. CAPRIFOLIACEAE

Bush Honeysuckle Honeysuckle Family

lonicera Mill.

rivularis Gatt.

$\times$ splendens (Carr.) Kirchn.

DIOON Lindl.

CYCADACEAE

Cycad Family

edule Lindl. 
DIOSPYROS

EBENACEAE

Persimmon Ebony Family

kaki L.f.

kaki L.f. 'Hagabushi'

kaki L.f. 'Kyungsun bansi'

sinensis Hemsl.

texana Scheele

virginiana $\mathrm{L}$.

DIPELTA Maxim.

CAPRIFOLIACEAE Honeysuckle Family

floribunda Maxim.

yunnanensis Franch.

DIRCA L.

THYMELAEACEAE Mezereum Family

palustris $\mathrm{L}$.

DISANTHUS MaXim.

HAMAMELIDACEAE Witch-hazel Family

cercidifolius Maxim.

DISTYLIUM Sieb. \& Zucc.

HAMAMELIDACEAE Witch-hazel Family

racemosum Sieb. \& Zucc.

racemosum Sieb. \& Zucc. 'Akebono'

DURANTA L.

VERBENACEAE Verbena Family

repens $\mathrm{L}$.

EDGEWORTHIA Meisn.

THYMELAEACEAE Mezereum Family

chrysantha Lindl.

EHRETIA P.Br.

BORAGINACEAE Borage Family

acuminata R.Br. var. serrata (Roxb.)

I.M.Johnst.

anacua (Teran \& Berl.) I.M.Johnst.

ELAEAGNUS L.

ELAEAGNACEAE

Oleaster Family

angustifolia L.

$\times$ ebbingei Doorenbos

glabra Thunb. ex J.A.Murr.

macrophylla Thunb.

multiflora Thunb. ex J.A.Murr. pungens Thunb. ex J.A.Murr.

pungens Thunb. ex J.A.Murr. 'Aurea'

pungens Thunb. ex J.A.Murr.

'Fruitlandii'

pungens Thunb. ex J.A.Murr. 'Maculata' pungens Thunb. ex J.A.Murr. 'Variegata' umbellata Thunb. ex J.A.Murr.

ELEUTHEROCOCCUS MaXim.

ARALIACEAE Ginseng Family

gracilistylus (W.W.Sm.) S.Y.Hu

sieboldianus (Makino) Koidz.

trifoliatus (L.) S.Y.Hu

ELLIOTTIA Muhl. ex Ell.

ERICACEAE

Heath Family

bracteata (Maxim.) Hook.f.

racemosa Muhl. ex Ell.

ENKIANTHUS Lour.

ERICACEAE

Heath Family

campanulatus (Miq.) Nichols.

campanulatus (Miq.) Nichols.

'Ambassador'

perulatus (Miq.) Schneid.

ERICA L.

Heath

ERICACEAE

Heath Family

carnea L.

carnea L. 'Springwood Pink'

carnea L. 'Springwood White'

$\times$ darleyensis Bean 'Silberschmelze'

vagans $\mathrm{L}$.

ERIOBOTRYA Lindl. ROSACEAE

Loquat

Rose Family

deflexa (Hemsl.) Nakai

japonica (Thunb.) Lindl.

ERYTHRINA L.

FABACEAE (Faboideae) Bean Family

$\times$ bidwillii Lindl.

crista-galli L.

herbacea L.

ESCALLONIA Mutis ex L.f.

SAXIFRAGACEAE (Escallonioideae)

Saxifrage Family

bifida Link \& Otto

rosea Griseb.

rubra (Ruiz \& Pavon) Pers. 
EUCALYPTUS L'Her.

MYRTACEAE

camaldulensis Dehnh.

coccifera Hook.f.

dalrympleana Maiden

gunnii Hook.f.

robusta $\mathrm{Sm}$.

viminalis Labill.

EUCOMMIA Oliv.

EUCOMMIACEAE

Eucommia Family

ulmoides Oliv.

EUODIA J.R. \& J.G. Forst.

RUTACEAE

Citrus Family

daniellii (Benn.) Hemsl.

\section{EUONYMUS L. \\ Spindle Tree \\ CELASTRACEAE \\ Staff-tree Family}

alatus (Thunb. ex J.A.Murr.) Sieb.

alatus (Thunb. ex J.A.Murr.) Sieb.

'Compactus'

alatus (Thunb. ex J.A.Murr.) Sieb.

f. apterus (Regel) Rehd.

americanus $\mathrm{L}$.

bungeanus Maxim.

bungeanus Maxim. var. semipersistans

(Rehd.) Schneid.

europaeus L.

europaeus L. 'Aldenhamensis'

fortunei (Turcz.) Hand.-Mazz.

fortunei (Turcz.) Hand.-Mazz.

'Emerald Gaiety'

fortunei (Turcz.) Hand.-Mazz. 'Gracilis'

fortunei (Turcz.) Hand.-Mazz. 'Harlequin'

fortunei (Turcz.) Hand.-Mazz. 'Longwood'

fortunei (Turcz.) Hand.-Mazz. 'Minima'

fortunei (Turcz.) Hand.-Mazz. 'Sarcoxie'

fortunei (Turcz.) Hand.-Mazz. 'Silver

Gueen'

fortunei (Turcz.) Hand.-Mazz.

'Variegatus'

fortunei (Turcz.) Hand.-Mazz.

var. radicans (Miq.) Rehd.

japonicus Thunb.

japonicus Thunb. 'Albo-marginatus'

japonicus Thunb. 'Argenteo-variegatus'

japonicus Thunb. 'Aureo-marginatus'

japonicus Thunb. 'Aureus'

japonicus Thunb. 'Duc d'Anjou'

japonicus Thunb. 'Macrophyllus'

japonicus Thunb. 'Microphyllus'

japonicus Thunb. 'Silver King' kiautschovicus Loesn.

kiautschovicus Loesn. 'Manhattan'

lanceolatus Yatabe

maackii Rupr.

nanus Bieb.

sieboldianus $\mathrm{Bl}$.

wilsonii Sprague

EUPHORBIA L. EUPHORBIACEAE

Spurge

Spurge Family

characias L. ssp. wulfenii (Hoppe ex

K.Koch) A.R.Sm.

leucocephala Lotsy

pulcherrima Willd.

EUPTELEA Sieb. \& Zucc.

EUPTELEACEAE Euptelea Family

polyandra Sieb. \& Zucc.

EURYA Thunb.

THEACEAE

Tea Family

acuminata DC.

emarginata (Thunb. ex J.A.Murr.)

Makino

japonica Thunb.

japonica Thunb. 'Confetti'

japonica Thunb. 'Harmony'

$\star$ japonica Thunb. 'Winter Wine'

EUSCAPHIS Sieb. \& Zucc.

STAPHYLEACEAE Bladdernut Family

japonica (Thunb. ex J.A.Murr.) Kanitz

EXOCHORDA Lindl.

ROSACEAE

Rose Family

$\times$ macrantha (V.Lemoine) Schneid.

'The Bride'

racemosa (Lindl.) Rehd.

FAGUS L.

Beech

FAGACEAE

Beech Family

grandifolia Ehrh.

sylvatica $\mathrm{L}$.

sylvatica L. 'Asplenifolia'

sylvatica L. 'Cristata'

sylvatica L. 'Dawyck'

sylvatica L. 'Laciniata'

sylvatica L. 'Pendula'

sylvatica L. 'Purpurea Tricolor'

sylvatica L. 'Quercifolia'

sylvatica L. 'Rohanii' 
sylvatica L. 'Rotundifolia'

sylvatica $\mathrm{L}$. 'Zlatia'

sylvatica L. f. purpurea (Ait.) Schneid.

FALLUGIA Endl.

ROSACEAE Rose Family

paradoxa (D.Don) Endl.

$\times$ FATSHEDERA Guill.

ARALIACEAE Ginseng Family

lizei (Cochet) Guill.

FATSIA Decne. \& Planch.

ARALIACEAE Ginseng Family

japonica (Thunb.) Decne. \& Planch.

FICUS L.

MORACEAE

carica L.

carica $\times$ F. pumila

palmata Forssk.

pumila L.

pumila L. 'Minima'

tikoua Bur.

FIRMIANA Marsili

STERCULIACEAE

Sterculia Family

simplex (L.) W.F.Wight

FONTANESIA Labill.

OLEACEAE

Olive Family

fortunei Carr.

FORESTIERA Poir.

OLEACEAE

Olive Family

acuminata (Michx.) Poir.

FORSYTHIA Vahl OLEACEAE

Golden Bells Olive Family

$\times$ intermedia Zab.

$\times$ intermedia Zab. 'Karl Sax'

$\times$ intermedia Zab. 'Lynwood'

$\times$ intermedia Zab. 'New Hampshire Gold'

$\times$ intermedia Zab. 'Spectabilis'

$\times$ intermedia Zab. 'Spring Glory'

$\times$ intermedia Zab. 'Variegata'

koreana Nakai 'Ilgwang'

mandshurica Nakai 'Vermont Sun'

ovata Nakai ovata (?hybrid) 'Ottawa'

(ovata $\times$ F. $\times$ intermedia 'Spring

Glory') 'Winterthur'

suspensa (Thunb.) Vahl

suspensa (Thunb.) Vahl 'Pallida'

suspensa (Thunb.) Vahl var. fortunei

(Lindl.) Rehd.

viridissima Lindl.

viridissima Lindl. 'Bronxensis'

FORTUNEARIA Rehd. \& Wils.

HAMAMELIDACEAE Witch-hazel Family

sinensis Rehd. \& Wils.

FORTUNELLA Swingle

RUTACEAE

Kumquat

Citrus Family

$\times$ crassifolia Swingle

japonica (Thunb.) Swingle

FOTHERGILLA L.

HAMAMELIDACEAE Witch-hazel Family

gardenii J.A.Murr.

major (Sims) Lodd.

major $\times$ F. gardenii

FRANGULA Mill.

RHAMNACEAE Buckthorn Family

alnus Mill.

FRANKLINIA Marsh.

THEACEAE

Tea Family

alatamaha Marsh.

FRAXINUS L.

Ash

OLEACEAE

Olive Family

americana L.

americana L. var. biltmoreana (Beadle)

J.Wright

berlandieriana A.DC.

chinensis Roxb. var. rhynchophylla

(Hance) Hemsl.

excelsior $\mathrm{L}$.

excelsior L. 'Aurea'

excelsior L. 'Doorenbos \#5'

holotricha Koehne

longicuspis Sieb. \& Zucc.

nigra Marsh.

ornus L.

ornus L. $\times$ F. excelsior L.

pennsylvanica Marsh. 
pennsylvanica Marsh.

var. subintegerrima (Vahl) Fern.

sieboldiana $\mathrm{Bl}$.

velutina Torr. var. coriacea (S.Wats.)

Rehd.

velutina Torr. var. glabra Rehd.

GALPHIMIA Cav. MALPIGHIACEAE

Malpighia Family

glauca Cav.

GARDENIA Ellis RUBIACEAE

Madder Family

jasminoides Ellis

jasminoides Ellis 'Radicans'

GAULTHERIA L.

ERICACEAE

Heath Family

procumbens $\mathrm{L}$.

GAYLUSSACIA HBK. $\begin{gathered}\text { Huckleberry } \\ \text { ERICACEAE }\end{gathered}$
Heath Family

baccata (Wang.) K.Koch

brachycera (Michx.) A.Gr.

GELSEMIUM JusS.

LOGANIACEAE Logania Family

rankinii Small

sempervirens (L.) Ait.f.

sempervirens (L.) Ait.f. 'Pride of Augusta'

GENISTA L.

Broom

FABACEAE (Faboideae)

Bean Family

germanica L. 'Prostrata'

lydia Boiss.

GINKGO L.

GINKGOACEAE

Ginkgo Family

biloba L.

biloba L. 'Fastigiata'

biloba L. 'Lakeview'

biloba L. 'Mayfield'

GLEDITSIA L.

Honeylocust

FABACEAE (Caesalpinioideae) Bean Family triacanthos L. f. inermis (Pursh)

Schneid.

triacanthos L. f. inermis (Pursh)

Schneid. 'Shademaster'

triacanthos L. f. inermis (Pursh)

Schneid. 'Sunburst'

GLOCHIDION J.R. \& J.G.Forst. EUPHORBIACEAE

Spurge Family

puberum (L.) Hutch.

GLYCOSMIS Correa

RUTACEAE

Citrus Family

citrifolia (Willd.) Lindl.

GLYPTOSTROBUS Endl.

TAXODIACEAE Taxodium Family

lineatus (Poir.) Druce

GORDONIA Ellis

THEACEAE

Tea Family

axillaris (Roxb. ex Ker-Gawl.) D.Dietr.

chrysandra Cowan

lasianthus (L.) Ellis

GREWIA L.

TILIACEAE Linden Family

biloba G.Don

GYMNOCLADUS Lam.

FABACEAE (Caesalpinioideae) Bean Family

dioica (L.) K.Koch

HALESIA Ellis ex L. Silverbell Tree STYRACACEAE

Storax Family

carolina L.

carolina L. 'Rosea'

diptera Ellis

diptera Ellis var. magniflora Godfrey parviflora Michx.

HALIMODENDRON Fischer ex DC. FABACEAE (Faboideae) Bean Family

halodendron (L.) Voss

aquatica Marsh.

japonica Miq.

triacanthos $\mathrm{L}$. 
HAMAMELIS L.

HAMAMELIDACEAE

Witch Hazel

Witch-hazel Family

$\times$ intermedia Rehd.

$\times$ intermedia Rehd. 'Arnold Promise'

$\times$ intermedia Rehd. 'Feuerzauber'

$\times$ intermedia Rehd. 'Jelena'

$\times$ intermedia Rehd. 'Luna'

$\times$ intermedia Rehd. 'Orange Beauty'

$\times$ intermedia Rehd. 'Pallida'

$\times$ intermedia Rehd. 'Primavera'

$\times$ intermedia Rehd. 'Rubra'

$\times$ intermedia Rehd. 'Ruby Glow'

$\times$ intermedia Rehd. 'Vesna'

japonica Sieb. \& Zucc.

japonica Sieb. \& Zucc. 'Zuccariniana'

japonica Sieb. \& Zucc.

f. flavopurpurascens (Makino) Rehd. mollis Oliv.

mollis Oliv. 'Brevipetala'

vernalis Sarg.

vernalis Sarg. 'Carnea'

vernalis Sarg. 'Christmas Cheer'

vernalis Sarg. 'Copper'

vernalis Sarg. 'Lombart's Weeping'

vernalis Sarg. 'Sandra'

virginiana $\mathrm{L}$.

HAMELIA Jacq.

RUBIACEAE

Madder Family

patens Jacq.

HEDERA L.

Ivy

ARALIACEAE

Ginseng Family

canariensis Willd.

canariensis Willd. 'Canary Cream'

canariensis Willd. 'Variegata'

colchica K.Koch

colchica K.Koch var. dentata Hibb.

'Sulfur Heart'

helix $\mathrm{L}$.

helix L. 'Alpha'

helix L. 'Anchor'

helix L. 'Angularis Aurea'

helix L. Arborescens group

helix L. 'Arrowhead'

helix L. 'Aurea Spectabilis'

helix L. 'Baby Merion'

helix L. 'Baccifer'

helix L. 'Big Deal'

helix L. 'Boskoop'

helix L. 'Bulgaria'

helix L. 'California'

helix L. 'California Gold'

helix L. 'Carolina Crinkle' helix L. 'Cascade'

helix L. 'Cathedral Wa11'

helix L. 'Cavendishii'

helix L. 'Christian'

helix L. 'Chrysantha'

helix L. 'Cockle Shell'

helix L. 'Conglomerata'

helix L. 'Crenata'

helix L. 'Deltoidea'

helix L. 'Dentata'

helix L. 'Denticulata'

helix L. 'Digitata'

helix L. 'Discolor'

helix L. 'Dragon Claw'

helix L. 'Edison'

helix L. 'Emerald Beauty'

helix L. 'Emerald Gem'

helix L. 'Emerald Jewel'

helix L. 'Erecta'

helix L. 'Erin'

helix L. 'Eva'

helix L. 'Fan'

helix L. 'Fantasia'

helix L. 'Ferney'

helix L. 'Fleur'

helix L. 'Fleur de Lis'

helix L. 'Four Square'

helix L. 'Garland'

helix L. 'Gavotte'

helix L. 'Ginkgo'

helix L. 'Glacier'

helix L. 'Gladiator'

helix L. 'Glymii'

helix L. 'Goldcraft'

helix L. 'Gold Dust'

helix L. 'Gold Heart'

helix L. 'Goods Selfbranching'

helix L. 'Gracilis'

helix L. 'Green Crown'

helix L. 'Green Finger'

helix L. 'Green Quartz'

helix L. 'Green Ripples'

helix L. 'Green Spear'

helix L. 'Green Velvet'

helix L. 'Hahn Selfbranching'

helix L. 'Hahn Variegated'

helix L. 'Harold'

helix L. 'Harrison'

helix L. 'Hebron'

helix L. 'Helvetica'

helix L. 'Heterophylla'

helix L. 'Hibernica'

helix L. 'Hite Miniature'

helix L. 'Holly'

helix L. 'Ideal'

helix L. 'Imp'

helix L. 'Itsy Bitsy' 
helix L. 'Iva Lace'

helix L. 'Jack Frost'

helix L. 'La Platta'

helix L. 'Lady Kay'

helix L. 'Lobata Major'

helix L. 'Lolla Rookh'

helix L. 'Long Point'

helix L. 'Lucida Aurea'

helix L. 'Luzzi'

helix L. 'Manda Crested'

helix L. 'Manda Fringette'

helix L. 'Manda's Star'

helix L. 'Maple Queen'

helix L. 'Marbled Dragon'

helix L. 'Marginata'

helix L. 'Meagheri'

helix L. 'Merion Beauty'

helix L. 'Merrie's Albany'

helix L. 'Microphylla Variegata'

helix L. 'Midget'

helix L. 'Miniature Needlepoint'

helix L. 'Minima'

helix L. 'Minor Marmorata'

helix L. 'Mount Vernon'

helix L. 'My Variegated'

helix L. 'Needlepoint'

helix L. 'Obscura'

helix L. 'Paper Doll'

helix L. 'Parsley Crested'

helix L. 'Pedata'

helix L. 'Perfection'

helix L. 'Permanent Wave'

helix L. 'Pin Oak'

helix L. 'Pin Oak Improved'

helix L. 'Pittsburgh'

helix L. 'Pittsburgh Variegated'

helix L. 'Pixie'

helix L. 'Plume de Or'

helix L. 'Preston Tiny'

helix L. 'Purpurea'

helix L. 'Ralf'

helix L. 'Rambler'

helix L. 'Ray's Supreme'

helix L. 'Ripples'

helix L. 'Rochester'

helix L. 'Roehr's Minor'

helix L. 'Rubaiyat'

helix L. 'Russell Gold'

helix L. 'Ruth'

helix L. 'Sagittaefolia'

helix L. 'Sea Foam'

helix L. 'Shannon'

helix L. 'Silver Queen'

helix L. 'Sinclair Silverleaf'

helix L. 'Small Deal'

helix L. 'Spearpoint'

helix L. 'Springtime Snow' helix L. 'Staghorn'

helix L. 'Stardust'

helix L. 'Stare'

helix L. 'Sulphurea'

helix L. 'Susan Gibles'

helix L. 'Suzanne'

helix L. 'Sylvanian'

helix L. 'Teardrop'

helix L. 'Teena'

helix L. 'Telecurl'

helix L. 'Tesselata'

helix L. 'Thorndale'

helix L. 'Tidal Wave'

helix L. 'Tribairn'

helix L. 'Triloba'

helix L. 'Triton'

helix L. 'Trustee'

helix L. '238th Street'

helix L. 'Ustlers'

helix L. 'Vanderhof'

helix L. 'Walthamensis'

helix L. 'Weber's California'

helix L. 'Wilson'

helix L. 'Woodsii'

helix L. var. poetica Weston

helix L. var. taurica Rehd. 'Yalta'

nepalensis K.Koch var. sinensis Tobl.

rhombea (Miq.) Bean

\section{HELIANTHEMUM Mill.}

CISTACEAE Rock-rose Family

apenninum (L.) Mill.

apenninum (L.) Mill. var. roseum

(Jacq.) Schneid.

nummularium (L.) Mill.

nummularium (L.) Mill. 'Buttercup'

nummularium (L.) Mill. 'Fireball'

HELICHRYSUM Mill. Strawflower ASTERACEAE Aster Family

italicum (Roth) G.Don

HEMIPTELEA Planch.

ULMACEAE

Elm Family

davidii (Hance) Planch.

HEPTACODIUM Rehd.

CAPRIFOLIACEAE Honeysuckle Family

miconioides Rehd. 
HETEROPTERIS

MALPIGHIACEAE

HBK.

Malpighia Family

angustifolia Griseb.

syringifolia Griseb.

\section{HIBISCUS L.}

\section{MALVACEAE}

Mallow Family

mutabilis $\mathrm{L}$.

paramutabilis Bailey

rosa-sinensis L. 'Albo-laciniata'

rosa-sinensis L. 'Brilliant'

syriacus L.

syriacus L. 'Admiral Dewey'

syriacus L. 'Aka-Yae'

syriacus L. 'Albus Plenus'

syriacus L. 'Amarantus'

$\star$ syriacus L. 'Aphrodite'

syriacus L. 'Ardens'

syriacus L. 'Ardens Plena'

syriacus L. 'Bicolor'

syriacus L. 'Blue Bird'

syriacus L. 'Blue Rouge'

syriacus L. 'Boule de Feu'

syriacus L. 'Caeruleus Plenus'

syriacus L. 'Campanha'

syriacus L. 'Celestial Blue'

syriacus L. 'Colie Mullins'

syriacus L. 'Comte de Flandre'

syriacus L. 'Comte de Haimout'

syriacus L. 'Dela Vaux'

syriacus L. 'De La Veuve'

$\star$ syriacus L. 'Diana'

syriacus L. 'Double Light Pink'

syriacus L. 'Duc de Brabant'

syriacus L. 'Effie Riegel'

syriacus L. 'Elegantissimus'

syriacus L. 'Gion mamori'

syriacus L. 'Grandiflorus Superbus'

syriacus L. 'Hagan Hybrid \#2'

syriacus L. 'Hamabo'

$\star$ syriacus L. 'Helene'

syriacus L. 'Hinomaru'

syriacus L. 'Hitoe'

syriacus L. 'Jeanne d'Arc'

syriacus L. 'Koki yae'

syriacus L. 'Kreider Blue'

syriacus L. 'La Fleur'

syriacus L. 'Lady Stanley'

syriacus L. 'Leopoldii'

syriacus L. 'Leopoldii Plenus'

syriacus L. 'Lovely Pink'

syriacus L. 'Lucy'

syriacus L. 'Luteus Plenus'

syriacus L. 'Martha Jane'

syriacus L. 'Mauve Queen' syriacus L. 'Meehanii'

syriacus L. 'Mimihara' -

$\star$ syriacus L. 'Minerva'

syriacus L. 'Monstrosus'

syriacus L. 'Monstrosus Plenus'

syriacus L. 'Monstrosus Simple'

syriacus L. 'Oiseau Bleu'

syriacus L. 'Perry's Purple'

syriacus L. 'Pheasant Eye'

syriacus L. 'Pink Delight'

syriacus L. 'Plume'

syriacus L. 'Pom Pom Rouge'

syriacus L. 'Pompon'

syriacus L. 'Pulcherrimus'

syriacus L. 'Puniceus Plenus'

syriacus L. 'Purpurea Semiplena'

syriacus L. 'Purpureus Plenus'

syriacus L. 'Ranunculiflorus'

syriacus L. 'Ranunculiflorus Plenus'

syriacus L. 'Rosalinda'

syriacus L. 'Roseus Plenus'

syriacus L. 'Roxanus'

syriacus L. 'Rubis'

syriacus L. 'Rubra Grandiflora'

syriacus L. 'Rubra Plena'

syriacus L. 'Shiro hanagasa'

syriacus L. 'Shiro midare'

syriacus L. 'Sir de Charles Breton'

syriacus L. 'Snowdrift'

syriacus L. 'Soft Pink'

syriacus L. 'Sokobeni yae'

syriacus L. 'Sonde'

syriacus L. 'Souvenir de Charles Breton'

syriacus L. 'Speciosus'

syriacus L. 'Spectabilis Plena'

syriacus L. 'Suminokura'

syriacus L. 'Suminokura hanagasa'

syriacus L. 'Suminokura yae'

syriacus L. 'Tamausagi'

syriacus L. 'Totus Albus'

syriacus L. 'Usu hitoe'

syriacus L. 'Variegatus'

syriacus L. 'Violaceus Plenus'

syriacus L. 'Violet Clair'

syriacus L. 'White Red Eye'

syriacus L. 'White Supreme'

syriacus L. 'William P. Smith'

syriacus L. 'Woodbridge'

syriacus L. 'Zulauf'

HOVENIA Thunb.

RHAMNACEAE

Buckthorn Family

dulcis Thunb. 
HTDRANGEA L.

SAXIFRAGACEAE (Hydrangeoideae)

Saxifrage Family

anomala D.Don ssp. petiolaris

(Sieb. \& Zucc.) McClint.

arborescens L. 'Annabelle'

macrophylla (Thunb. ex J.A.Murr.) Ser.

macrophylla (Thunb. ex J.A.Murr.) Ser.

'Grayswood'

macrophylla (Thunb. ex J.A.Murr.) Ser.

'Maculata'

macrophylla (Thunb. ex J.A.Murr.) Ser.

ssp. serrata (Thunb. ex J.A.Murr.)

Makino

macrophylla (Thunb. ex J.A.Murr.) Ser.

ssp. serrata (Thunb. ex J.A.Murr.)

Makino 'Prolifera'

paniculata Sieb.

paniculata Sieb. 'Grandiflora'

paniculata Sieb. 'Tardiva'

quercifolia Bartram

quercifolia Bartram 'Harmony'

quercifolia Bartram 'Snowflake'

scandens (L.f.) Ser. ssp. liukiuensis

(Nakai) McClint.

HYPERICUM L.

St. John's-Wort

HYPERICACEAE

St. John's-wort Family

brachyphyllum (Spach) Steud.

buckleyi M.A.Curtis

calycinum $\mathrm{L}$.

(?calycinum $\times$ H. forrestii) 'Hidcote'

fasciculatum Lam.

frondosum Michx.

frondosum Michx. 'Sunburst'

galioides Lam.

hircinum $\mathrm{L}$.

hookerianum Wight \& Arn.

kalmianum L.

lissophloeus Adams

lloydii (Svenson) Adams

microsepalum (T. \& G.) A.Gr.

patulum Thunb. ex J.A.Murr.

prolificum L.

reductum (Svenson) Adams

stans (Michx.) Adams \& N.Robs.

'Van Fleetii'

HYSSOPUS L.

LAMIACEAE

Mint Family

officinalis L.
IDESIA MaXim.

FLACOURTIACEAE

Flacourtia Family

polycarpa Maxim.

ILEX L.

AQUIFOLIACEAE

Holly

Holly Family

$\times$ altaclerensis (Loud.) Dallim.

$\times$ altaclerensis (Loud.) Dallim. 'Alice'

$\times$ altaclerensis (Loud.) Dallim.

'Balearica'

$\times$ altaclerensis (Loud.) Dallim. 'Belgica'

$\times$ altaclerensis (Loud.) Dallim. 'Belin's

Weeping'

$\times$ altaclerensis (Loud.) Dallim.

'Camelliifolia'

$\times$ altaclerensis (Loud.) Dallim. 'Cherry

Berry'

$\times$ altaclerensis (Loud.) Dallim. 'Colburn'

$\times$ altaclerensis (Loud.) Dallim. 'Early

Cluster'

$\times$ altaclerensis (Loud.) Dallim. 'Eldridge'

$\times$ altaclerensis (Loud.) Dallim. 'Father

Charles'

$\times$ altaclerensis (Loud.) Dallim. 'Firelight'

$\times$ altaclerensis (Loud.) Dallim. 'Hazel'

$\times$ altaclerensis (Loud.) Dallim.

'Hendersonii'

$\times$ altaclerensis (Loud.) Dallim.

'Hendersonii Aurea'

$\times$ altaclerensis (Loud.) Dallim.

'Hodginsii'

$\times$ altaclerensis (Loud.) Dallim. 'James G.

Esson'

$\times$ altaclerensis (Loud.) Dallim.

'Laurifolia'

$\times$ altaclerensis (Loud.) Dallim.

'Marnockii'

$\times$ altaclerensis (Loud.) Dallim. 'Mundyi'

$\times$ altaclerensis (Loud.) Dallim.

'Nigrescens'

$\times$ altaclerensis (Loud.) Dallim.

'Royal Red'

$\times$ altaclerensis (Loud.) Dallim. 'Wilsonii'

ambigua (Michx.) Torr.

amelanchier M.A.Curtis

aquifolium L.

aquifolium L. 'Angustifolium'

aquifolium L. 'Apricot'

aquifolium L. 'Argentea Marginata'

aquifolium L. 'Atlas'

aquifolium L. 'Aureo-marginata'

aquifolium L. 'Aurifodina'

aquifolium L. 'Bacciflava'

aquifolium L. 'Balkans'

aquifolium L. 'Barnes' 
aquifolium L. 'Beacon'

aquifolium L. 'Beauty Spra'

aquifolium L. 'Beauty Spra Espalier'

aquifolium L. 'Berigold'

aquifolium L. 'Big Bull'

aquifolium L. 'Bleeg'

aquifolium L. 'Bodley's Bleeg'

aquifolium L. 'Bonanza'

aquifolium L. 'Bronze'

aquifolium L. 'Brownell'

aquifolium L. 'Butler'

aquifolium L. 'Campus Variegated'

aquifolium L. 'Captain Bonneville'

aquifolium L. 'Chief'

aquifolium L. 'Clouded Gold'

aquifolium L. 'Coleman'

aquifolium L. 'Cover Girl'

aquifolium L. 'Crinkle Variegated'

aquifolium L. 'Crispa'

aquifolium L. 'Crispa Aureo-picta'

aquifolium L. 'Daddyo'

aquifolium L. 'Deluxe'

aquifolium L. 'Dude'

aquifolium L. 'Dumbarton Oaks'

aquifolium L. 'Elegantissima'

aquifolium L. 'Escort'

aquifolium L. 'Favorite'

aquifolium L. 'Ferox'

aquifolium L. 'Ferox Argentea'

aquifolium L. 'Ferox Aurea Marginata'

aquifolium L. 'Firecracker'

aquifolium L. 'Flavescens'

aquifolium L. 'Foxii'

aquifolium L. 'Fructo-lutea'

aquifolium L. 'Globe'

aquifolium L. 'Golden Butterfly'

aquifolium L. 'Golden Milkboy'

aquifolium L. 'Golden Milkmaid'

aquifolium L. 'Golden Queen'

aquifolium L. 'Gracean'

aquifolium L. 'Green Knight'

aquifolium L. 'Green Maid'

aquifolium L. 'Handsworthensis'

aquifolium L. 'Hastata'

aquifolium L. 'Hollycroft Jack'

aquifolium L. 'Ingramii'

aquifolium L. 'Integrifolium'

aquifolium L. 'Ivory'

aquifolium L. 'J. C. van Tol'

aquifolium L. 'Lady Baltimore'

aquifolium L. 'Latispina'

aquifolium L. 'Lewis'

aquifolium L. 'Lilliput'

aquifolium L. 'Lilygold'

aquifolium L. 'Little Bull'

aquifolium L. 'Longspra'

aquifolium L. 'Louise' aquifolium L. 'Lutescens'

aquifolium L. 'Maderensis Variegata'

aquifolium L. 'Malcolm S. Whipple'

aquifolium L. 'Marshal Tito'

aquifolium L. 'Monstrosa'

aquifolium L. 'Myrtifolia'

aquifolium L. 'N. F. Barnes'

aquifolium L. 'NYBG \#2'

aquifolium L. 'Painted Lady'

aquifolium L. 'Pale Moon'

aquifolium L. 'Pendula'

aquifolium L. 'Perkins \# 1'

aquifolium L. 'Petite'

aquifolium L. 'Phantom Gold'

aquifolium L. 'Pinto'

aquifolium L. 'Planifolia'

aquifolium L. 'Pot-O-Gold'

aquifolium L. 'Princess Pat'

aquifolium L. 'Pyramidalis'

aquifolium L. 'Pyramidalis Compacta'

aquifolium L. 'Recurva'

aquifolium L. 'Rederly'

aquifolium L. 'Ricker'

aquifolium L. 'Riddle Farm'

aquifolium L. 'Rubricaulis Aurea'

aquifolium L. 'Scotia'

aquifolium L. 'Scram's Dwarf'

aquifolium L. 'Shortspra'

aquifolium L. 'Silver Milkboy'

aquifolium L. 'Silver Milkmaid'

aquifolium L. 'Sparkler'

aquifolium L. 'Sunnybrooke'

aquifolium L. 'Sunnyside'

aquifolium L. 'Teufel's Hybrid'

aquifolium L. 'Teufel's Variegated'

aquifolium L. 'Thornton'

aquifolium L. 'Tom Everett'

aquifolium L. 'Watereriana'

aquifolium L. 'Wheeler \#4'

aquifolium L. 'Whittingtonensis'

aquifolium L. 'Winter King'

aquifolium L. 'Winter Queen'

aquifolium L. 'Wintergreen'

aquifolium L. 'Yellow Beam'

aquifolium L. 'Yonkers'

aquifolium L. 'Yule Glow'

aquifolium L. 'Zero'

(aquifolium $\times I$. ciliospinosa) 'Brilliant'

aquifolium $\times$ I. spinigera

$\times$ aquipernyi Gable

$\times$ aquipernyi Gable 'Aquipern'

× aquipernyi Gable 'Dragon Lady'

$\times$ aquipernyi Gable 'Gable'

$\times$ aquipernyi Gable 'San Jose'

( $\times$ aquipernyi $\times(I$. integra $\times I$. pernyi $)$

'Accent') 'Rock Garden'

asprella (Hook. \& Arn.) Champ. ex Benth. 
$\times$ attenuata Ashe
$\times$ attenuata Ashe 'Alagold'
$\times$ attenuata Ashe 'Eagleson'
$\times$ attenuata Ashe 'East Palatka'
$\times$ attenuata Ashe 'Edna Jean'
$\times$ attenuata Ashe 'Erma Byrd'
$\times$ attenuata Ashe 'Foster \#1'
$\times$ attenuata Ashe 'Foster \#2'
$\times$ attenuata Ashe 'Foster \#3'
$\times$ attenuata Ashe 'Foster \#4'
$\times$ attenuata Ashe 'Howard'
$\times$ attenuata Ashe 'Hume \#1'
$\times$ attenuata Ashe 'Hume \#2'
$\times$ attenuata Ashe 'Hutchinson'
$\times$ attenuata Ashe 'Louise Holmes'
$\times$ attenuata Ashe 'NASA'
$\times$ attenuata Ashe 'Savannah'
$\times$ attenuata Ashe 'Sunny Foster'
$\times$ attenuata Ashe 'Topeli'
beadlei Ashe
$\times$ beanii Rehd.
bioritensis Hayata
buergeri Miq.
buswellii Small
canariensis Poir.
cassine L.
cassine L. 'Baldwin'
cassine L. var. angustifolia Ait.
cassine L. var. angustifolia
a aurea-baccata Taabox

f. aurea-baccata Tarbox ex S.F.Blake cassine L. var. bryanii Tarbox

ex S.F.Blake

ciliospinosa Loesn.

$\star$ (ciliospinosa $\times$ I. $\times$ aquipernyi)

'September Gem'

ciliospinosa $\times$ I. fargesi

(ciliospinosa $\times$ I. leucoclada)

'Harry Gunning'

(ciliospinosa $\times$ I. leucoclada)

'William Cowgill'

cinerea Champ.

colchica Pojark.

collina Alex.

corallina Franch.

coriacea (Pursh) Chapm.

cornuta Lindl. \& Paxt.

cornuta Lindl. \& Paxt. 'Aglo'

cornuta Lindl. \& Paxt.

'Anicet Delcambrie'

cornuta Lindl. \& Paxt. 'Anna Mae'

cornuta Lindl. \& Paxt. 'Avery Island'

cornuta Lindl. \& Paxt. 'Bostic'

cornuta Lindl. \& Paxt. 'Brawley'

cornuta Lindl. \& Paxt. 'Burfordii'

cornuta Lindl. \& Paxt. 'Cajun Gold'

cornuta Lindl. \& Paxt. 'Carissa'

cornuta Lind1. \& Paxt. 'Casey's Dwarf' cornuta Lindl. \& Paxt.

'Clarendon Batwing'

cornuta Lindl. \& Paxt.

'Clarendon Small Leaf'

cornuta Lindl. \& Paxt. 'Dodd Special'

cornuta Lindl. \& Paxt. 'D'Or'

cornuta Lindl. \& Paxt. 'Dr. James Foret'

cornuta Lindl. \& Paxt. 'Dr. John Creech'

cornuta Lindl. \& Paxt. 'Dwarf Burford'

cornuta Lindl. \& Paxt. 'E. A. McIlhenny'

cornuta Lindl. \& Paxt. 'Fine Line'

cornuta Lindl. \& Paxt. 'Glenwood'

cornuta Lindl. \& Paxt. 'Grandview'

cornuta Lindl. \& Paxt. 'Hume'

cornuta Lindl. \& Paxt. 'Ira Nelson'

cornuta Lindl. \& Paxt. 'Jungle Garden'

cornuta Lindl. \& Paxt.

'Kingsville Special'

cornuta Lindl. \& Paxt. 'Medallion'

cornuta Lindl. \& Paxt. 'Morrell No. 1'

cornuta Lindl. \& Paxt. 'Morrell No. 3'

cornuta Lindl. \& Paxt. 'National'

cornuta Lindl. \& Paxt. 'Needle Point'

cornuta Lindl. \& Paxt. 'Olga'

cornuta Lindl. \& Paxt. 'O. Spring'

cornuta Lindl. \& Paxt. 'Rotunda'

cornuta Lindl. \& Paxt. 'R. V. P. Special'

cornuta Lindl. \& Paxt. 'Shangri-La'

cornuta Lindl. \& Paxt. 'Shiu-Ying'

cornuta Lindl. \& Paxt. 'Variegata'

cornuta Lindl. \& Paxt. 'Walker'

(cornuta $\times$ I. aquifolium) 'Callina'

(cornuta $\times$ I. aquifolium)

'Edward J. Stevens'

(cornuta $\times$ I. aquifolium) 'Hollowell'

(cornuta $\times$ I. aquifolium) 'Maplehurst'

(cornuta $\times$ I. aquifolium)

'Nellie R. Stevens'

$\star$ (lcornuta $\times$ I. aquifolium) 'Nellie R.

Stevens' $\times$ I. leucoclada) 'Clusterberry'

cornuta $\times I$. pernyi

(cornuta $\times$ I. pernyi) 'Atlas'

(cornuta $\times$ I. pernyi) 'Audry'

(cornuta $\times$ I. pernyi) 'Brighter Shine'

(cornuta $\times$ I. pernyi) 'Cetus'

(cornuta $\times$ I. pernyi) 'Doctor Kassab'

(cornuta $\times$ I. pernyi) 'Drace'

(cornuta $\times$ I. pernyi) 'Formax'

(cornuta $\times$ I. pernyi) 'Good Taste'

(cornuta $\times$ I. pernyi) 'Indian Chief'

$\star$ (cornuta $\times$ I. pernyi) 'John T. Morris'

(cornuta $\times$ I. pernyi) 'Lacerta'

(cornuta $\times$ I. pernyi) 'Lepux'

$\star$ (cornuta $\times$ I. pernyi) 'Lydia Morris'

(cornuta $\times$ I. pernyi) 'Lyra'

(cornuta $\times$ I. pernyi) 'Moonglow'

(cornuta $\times$ I. pernyi) 'Red Robe' 
(cornuta $\times$ I. pernyi) 'Titan'

(cornuta $\times I$. pernyi) 'Virgo'

(cornuta $\times$ I. rugosa) 'China Boy'

(cornuta $\times$ I. rugosa) 'China Girl'

(cornuta 'Burfordii' $\times$ I. latifolia)

'Arthur Bruner'

(cornuta 'Burfordii' $\times$ I. latifolia)

'Bob Bruner'

(cornuta 'Burfordii' $\times$ I. latifolia)

'Emily Bruner'

(cornuta 'Burfordii' $\times$ I. latifolia)

'James Swan'

(cornuta 'Burfordii' $\times$ I. Latifolia)

'Jinny Bruner'

(cornuta 'Burfordii' $\times$ I. pernyi)

'Red Delight'

((cornuta 'Burfordii' $\times$ I. pernyi)

'Red Delight' $\times$ I. latifolia)

'Mary Nell'

crenata Thunb. ex J.A.Murr.

crenata Thunb. ex J.A.Murr. 'Angyo'

crenata Thunb. ex J.A.Murr.

'Braddock Heights'

crenata Thunb. ex J.A.Murr. 'Buxifolia'

crenata Thunb. ex J.A.Murr. 'Changsha'

crenata Thunb. ex J.A.Murr. 'Compacta'

crenata Thunb. ex J.A.Murr. 'Conners'

crenata Thunb. ex J.A.Murr. 'Convexa'

crenata Thunb. ex J.A.Murr.

'Delaware Diamond'

crenata Thunb. ex J.A.Murr. 'Divaricata'

crenata Thunb. ex J.A.Murr.

'Dwarf Cone'

crenata Thunb. ex J.A.Murr.

'Dwarf Pagoda'

crenata Thunb. ex J.A.Murr.

'Edwin Dozier'

crenata Thunb. ex J.A.Murr. 'Firefly'

crenata Thunb. ex J.A.Murr. 'Flushing'

crenata Thunb. ex J.A.Murr.

'Foster No. 1'

crenata Thunb. ex J.A.Murr.

'Foster No. 2'

crenata Thunb. ex J.A.Murr. 'Frierson'

crenata Thunb. ex J.A.Murr. 'Glass'

crenata Thunb. ex J.A.Murr. 'Glory'

crenata Thunb. ex J.A.Murr.

'Golden Gem'

crenata Thunb. ex J.A.Murr.

'Golden Heller'

crenata Thunb. ex J.A.Murr.

'Green Dragon'

crenata Thunb. ex J.A.Murr. 'Green

Lustre'

crenata Thunb. ex J.A.Murr. 'Green

Thumb'

crenata Thunb. ex J.A.Murr. 'Grier' crenata Thunb. ex J.A.Murr. 'Hatfield'

crenata Thunb. ex J.A.Aurr. 'Helleri'

crenata Thunb. ex J.A.Murr. 'Hetzii'

$\star$ crenata Thunb. ex J.A.Murr.

'Highlight'

crenata Thunb. ex J.A.Murr.

'Honeycomb'

crenata Thunb. ex J.A.Murr. 'Ivory Hall'

crenata Thunb. ex J.A.Murr.

'Ivory Tower'

crenata Thunb. ex J.A.Murr. 'Jersey

Pinnacle'

crenata Thunb. ex J.A.Murr.

'John Nosal'

crenata Thunb. ex J.A.Murr.

'Kingsville Dwarf'

crenata Thunb. ex J.A.Murr. 'Kunming'

crenata Thunb. ex J.A.Murr. 'Latifolia'

crenata Thunb. ex J.A.Murr.

'Lindleyana'

crenata Thunb. ex J.A.Murr. 'Longfellow'

crenata Thunb. ex J.A.Murr. 'Longifolia'

crenata Thunb. ex J.A.Murr.

'Loyce Nelson'

crenata Thunb. ex J.A.Murr.

'Luteo-variegata'

crenata Thunb. ex J.A.Murr. 'Major'

crenata Thunb. ex J.A.Murr. 'Mariesii'

crenata Thunb. ex J.A.Murr. 'Maxwell'

crenata Thunb. ex J.A.Murr.

'Mentor Dense'

crenata Thunb. ex J.A.Murr.

'Mentor Glossy'

crenata Thunb. ex J.A.Murr.

'Microphylla'

crenata Thunb. ex J.A.Murr.

'Microphylla Supreme'

crenata Thunb. ex J.A.Murr.

'Midas Touch'

crenata Thunb. ex J.A.Murr.

'Miss Muffet'

crenata Thunb. ex J.A.Murr.

'Mobjack Supreme'

crenata Thunb. ex J.A.Murr.

'Morris Dwarf'

crenata Thunb. ex J.A.Murr.

'Mount Halla'

crenata Thunb. ex J.A.Murr. 'Nakada'

crenata Thunb. ex J.A.Murr. 'Nanking'

crenata Thunb. ex J.A.Murr.

'Oconee River'

crenata Thunb. ex J.A.Murr. 'Oleafera' crenata Thunb. ex J.A.Murr. 'Peconic' crenata Thunb. ex J.A.Murr. 'Piccolo' crenata Thunb. ex J.A.Murr.

'Pride Dwarf' 
crenata Thunb. ex J.A.Murr.

'Pride's Tiny'

crenata Thunb. ex J.A.Murr.

'Pyramidalis'

crenata Thunb. ex J.A.Murr.

'Repandens'

crenata Thunb. ex J.A.Murr.

'Rocky Creek'

crenata Thunb. ex J.A.Murr. 'Sentinel' crenata Thunb. ex J.A.Murr. 'Shanghai' crenata Thunb. ex J.A.Murr. 'Snowflake' crenata Thunb. ex J.A.Murr. 'Stokes' crenata Thunb. ex J.A.Murr. 'Tennyson' crenata Thunb. ex J.A.Murr. 'T-one' crenata Thunb. ex J.A.Murr. 'Vaseyi' crenata Thunb. ex J.A.Murr. 'Wayne' crenata Thunb. ex J.A.Murr.

'William Jackson'

crenata Thunb. ex J.A.Murr.

'Willow Leaf'

crenata Thunb. ex J.A.Murr. 'Yunnan' crenata Thunb. ex J.A.Murr.

f. watanabeana Makino crenata Thunb. ex J.A.Murr.

ssp. fukasawana (Makino) Murata crenata Thunb. ex J.A.Murr.

var. paludosa (Nakai) Hara crenata Thunb. ex J.A.Murr.

var. paludosa (Nakai) Hara 'Carefree' crenata Thunb. ex J.A.Murr.

var. paludosa (Nakai) Hara 'Crescent' crenata Thunb. ex J.A.Murr.

var. paludosa (Nakai) Hara 'Gayle' crenata Thunb. ex J.A.Murr.

var. paludosa (Nakai) Hara 'Tyke' crenata Thunb. ex J.A.Murr.

var. thomsonii (Hook.f.) Loesn. cumulicola Small

cumulicola Small 'Fort McCoy'

curtissii (Fern.) Small

cyrtura Merr.

decidua Walt.

decidua Walt. 'Byers Golden'

decidua Walt. 'Indian Bayou \#3'

decidua Walt. 'Pocahontas'

dimorphophylla Koidz.

dipyrena Wall.

fargesii Franch.

fargesii Franch. ssp. melanotricha

(Merr.) S.Andrews

ficoidea Hemsl.

geniculata Maxim.

georgei Comber

glabra (L.) A.Gr.

glabra (L.) A.Gr. 'Nana'

glabra (L.) A.Gr. 'Tankard's Compact'

glabra (L.) A.Gr. 'Viridis' glabra (L.) A.Gr. f. leucocarpa

F.W.Woods

glabra (L.) A.Gr. f. leucocarpa

F.W.Woods 'Ivory Queen'

integra Thunb. ex J.A.Murr.

integra Thunb. ex J.A.Murr.

'Green Shadow'

integra $\times$ I. aquifolium

integra $\times I$. cornuta

(integra $\times$ I. cornuta) 'Libby's Favorite'

(integra $\times I$. cornuta) 'Semala'

integra $\times I$. pernyi

$\star$ (integra $\times I$. pernyi) 'Accent'

$\star$ (integra $\times$ I. pernyi) 'Elegance'

kingiana Cockerell

$\times$ kiusiana Hatusima

$\times$ koehneana Loesn.

$\times$ koehneana Loesn. 'Chieftan'

$\times$ koehneana Loesn. 'Hohman'

$\star \times$ koehneana Loesn. 'Jade'

$\times$ koehneana Loesn. 'Lassie'

$\times$ koehneana Loesn. 'Lock Raven'

$\star \times$ koehneana Loesn. 'Ruby'

$\times$ koehneana Loesn. 'San Jose'

$\times$ koehneana Loesn. 'Wirt L. Winn'

laevigata (Dum.-Cours.) A.Gr.

latifolia Thunb. ex J.A.Murr.

leucoclada Makino

liukiuensis Loesn.

lohfauensis Merr.

longipes Chapm. ex Trelease

longipes Chapm. ex Trelease 'Lagniappe'

longipes Chapm. ex Trelease

'Natchez Belle'

macrocarpa Oliv.

macropoda Miq.

maximowicziana Loesn. var. kanehirae

(Yamamoto) Yamazaki

$\times$ meserveae S.Y.Hu 'Blue Angel'

$\times$ meserveae S.Y.Hu 'Blue Boy'

$\times$ meserveae S.Y.Hu 'Blue Girl'

$\times$ meserveae S.Y.Hu 'Blue Maid'

$\times$ meserveae S.Y.Hu 'Blue Prince'

$\times$ meserveae S.Y.Hu 'Blue Princess'

$\times$ meserveae S.Y.Hu 'Blue Stallion'

$\times$ meserveae S.Y.Hu 'Golden Girl'

monticola A. Gr.

myrtifolia Walt.

myrtifolia Walt. 'Lowei'

$\star(($ myrtifolia $\times$ I. opaca $) \times I$. myrtifolia $)$

'Oriole'

$\star(($ myrtifolia $\times$ I. opaca $) \times I$. myrtifolia $)$

'Tanager'

opaca Ait.

opaca Ait. 'Aalto'

opaca Ait. 'Aalto \#5' 
opaca Ait. 'Aalto \#5A'

opaca Ait. 'Andorra'

opaca Ait. 'Anne Arundel'

opaca Ait. 'Arden'

opaca Ait. 'B. \& O.'

opaca Ait. 'Barclay'

opaca Ait. 'Beulah'

opaca Ait. 'Boyce Thompson'

opaca Ait. 'Boyce Thompson \#3'

opaca Ait. 'Brown \#5'

opaca Ait. 'Brown \# 16'

opaca Ait. 'Cape Christmas'

opaca Ait. 'Charles'

opaca Ait. 'Christmas Carol'

opaca Ait. 'Christmas Hedge'

opaca Ait. 'Clarendon'

opaca Ait. 'Clarendon Spreading'

opaca Ait. 'Clarissa'

opaca Ait. 'Clark'

opaca Ait. 'Croonenberg'

opaca Ait. 'Cumberland'

opaca Ait. 'Dan Fenton'

opaca Ait. 'Dick'

opaca Ait. 'Dorsey'

opaca Ait. 'Dr. T. B. Symons'

opaca Ait. 'Elizabeth'

opaca Ait. 'Emily'

opaca Ait. 'Farage'

opaca Ait. 'Faulkner'

opaca Ait. 'Felten's Selection'

opaca Ait. 'Fire Chief'

opaca Ait. 'Formal'

opaca Ait. 'Francis Lewis'

opaca Ait. 'Freeman'

opaca Ait. 'Gee'

opaca Ait. 'Golden Fleece'

opaca Ait. 'Good Will Park'

opaca Ait. 'Governor William Paca'

opaca Ait. 'Grandpappy'

opaca Ait. 'Griscom'

opaca Ait. 'Hamlet'

opaca Ait. 'Harriet'

opaca Ait. 'Hedgeholly'

opaca Ait. 'Helen Makepeace'

opaca Ait. 'Homer'

opaca Ait. 'Hopkins'

opaca Ait. 'Hume's Choice'

opaca Ait. 'Iso'

opaca Ait. 'Jeannette Adamson'

opaca Ait. 'Jersey Princess'

opaca Ait. 'John Higgins'

opaca Ait. 'Joyce'

opaca Ait. 'Judge Brown'

opaca Ait. 'Kate'

opaca Ait. 'Knight'

opaca Ait. 'La Bar'

opaca Ait. 'Lake City' opaca Ait. 'Laura'

opaca Ait. 'Lowell'

opaca Ait. 'Mae'

opaca Ait. 'Magna Semen'

opaca Ait. 'Mamie Eisenhower'

opaca Ait. 'Manig'

opaca Ait. 'Maryland'

opaca Ait. 'Maryland Dwarf'

opaca Ait. 'Menantico'

opaca Ait. 'Merry Christmas'

opaca Ait. 'Miss Helen'

opaca Ait. 'Miss Liberty'

opaca Ait. 'Mrs. Santa'

opaca Ait. 'Nelson West'

opaca Ait. 'Old Heavy Berry'

opaca Ait. 'Osa'

opaca Ait. 'Palmetto'

opaca Ait. 'Perkins-de-Wilde \# 1'

opaca Ait. 'Perkins-de-Wilde \#2'

opaca Ait. 'Perkins-de-Wilde \#3'

opaca Ait. 'Perrine'

opaca Ait. 'Pin Cushion'

opaca Ait. 'Polly'

opaca Ait. 'Pomona'

opaca Ait. 'Reynolds'

opaca Ait. 'Richards'

opaca Ait. 'Ruby Red'

opaca Ait. 'Rushton'

opaca Ait. 'St. Ann'

opaca Ait. 'St. John's'

opaca Ait. 'St. Mary'

opaca Ait. 'Sandy Hook \#5'

opaca Ait. 'Sara Higgins'

opaca Ait. 'Satyr Hill'

opaca Ait. 'Schlupp'

opaca Ait. 'Secrest'

opaca Ait. 'Slim Jim'

opaca Ait. 'Star'

opaca Ait. 'Taber'

opaca Ait. 'Taber \#2'

opaca Ait. 'Tiny'

opaca Ait. 'Toner'

opaca Ait. 'Trisco'

opaca Ait. '24 Karat'

opaca Ait. 'Vera'

opaca Ait. 'Wheeler \#4'

opaca Ait. 'William Hawkins'

opaca Ait. 'Yule'

opaca Ait. f. subintegra Weatherby

opaca Ait. f. subintegra Weatherby

'Perle LeClair'

opaca Ait. f. xanthocarpa Rehd.

opaca Ait. f. xanthocarpa Rehd.

'Boyce Thompson Xanthocarpa'

opaca Ait. f. xanthocarpa Rehd.

'Calloway' 
opaca Ait. f. xanthocarpa Rehd.

'Canary'

opaca Ait. f. xanthocarpa Rehd.

'Corpening \# 1'

opaca Ait. f. xanthocarpa Rehd.

'Dengle Belles'

opaca Ait. f. xanthocarpa Rehd.

'Fruitland Nursery'

opaca Ait. f. xanthocarpa Rehd. 'Goldie'

opaca Ait. f. xanthocarpa Rehd.

'Lenape Moon'

opaca Ait. f. xanthocarpa Rehd.

'Margaret Moran'

opaca Ait. f. xanthocarpa Rehd.

'Morgan Gold'

opaca Ait. f. xanthocarpa Rehd.

'Old Gold'

opaca Ait. f. xanthocarpa Rehd.

'Wilmat Yellow'

opaca Ait. f. xanthocarpa Rehd.

'Yellow Jacquet'

paraguariensis St.Hil.

pedunculosa Miq.

perado Ait. ssp. platyphylla (P.B.Webb

\& Berth.) S.Andrews

pernyi Franch.

pernyi Franch. 'Recurva'

poneantha Koidz.

pubescens Hook. \& Arn.

purpurea Hassk.

rotunda Thunb. ex J.A.Murr.

rotunda Thunb. ex J.A.Murr. 'Lord'

rugosa F.Schmidt

serrata Thunb. ex J.A.Murr.

serrata Thunb. ex J.A.Murr.

'Leucocarpa'

serrata $\times I$. verticillata

$\star$ (serrata $\times$ I. verticillata) 'Apollo'

(serrata $\times$ I. verticillata) 'Autumn Glow'

(serrata $\times$ I. verticillata) 'Harvest Red'

$\star$ (serrata $\times$ I. verticillata) 'Sparkleberry'

shennongjiaensis T.R.Dudley \& Sun

sikkimensis Hook.

spinigera (Loesn.) Loesn.

sugerokii Maxim.

sugerokii Maxim. 'Aka tsuge'

sugerokii Maxim. 'Nakaharae'

verticillata (L.) A.Gr.

verticillata (L.) A.Gr. 'Afterglow'

verticillata (L.) A.Gr. 'Aurantiaca'

verticillata (L.) A.Gr. 'Bright Horizon'

verticillata (L.) A.Gr. 'Cacapon'

verticillata (L.) A.Gr. 'Christmas Cheer'

verticillata (L.) A.Gr. 'Christmas Gem'

verticillata (L.) A.Gr. 'Earlibright'

verticillata (L.) A.Gr. 'Fairfax'

verticillata (L.) A.Gr. 'Jackson'

verticillata (L.) A.Gr. 'Maryland Beauty' verticillata (L.) A.Gr. 'Red Sprite' verticillata (L.) A.Gr. 'Richard E.

Lincoln'

verticillata (L.) A.Gr. 'Shaver'

verticillata (L.) A.Gr. 'Sunset'

verticillata (L.) A.Gr. 'Winter Red'

verticillata (L.) A.Gr. f. chrysocarpa

Robinson

vomitoria Ait.

vomitoria Ait. 'Compacta'

vomitoria Ait. 'Dare County'

vomitoria Ait. 'Dewerth'

vomitoria Ait. 'Fort McCoy'

vomitoria Ait. 'Gray's Bigleaf

vomitoria Ait. 'Gray's Little Leaf'

vomitoria Ait. 'Huber's Compact'

vomitoria Ait. 'Nana'

vomitoria Ait. 'Otis Miley'

vomitoria Ait. 'Shilling's'

vomitoria Ait. 'Shilling's Dwarf'

vomitoria Ait. 'Stokes Dwarf'

vomitoria Ait. 'Yawkeyii'

vomitoria Ait. f. pendula Foret \& Solym.

vomitoria Ait. f. pendula Foret \& Solym.

'Folsom's Weeping'

vomitoria Ait. var. chiapiensis Sharp

wilsonii Loesn.

yunnanensis Franch.

yunnanensis Franch. var. gentilis Loesn. zhejiangensis C.J.Tseng

\section{ILLICIUM L.}

ILLICIACEAE

Illicium Family

anisatum $\mathrm{L}$.

floridanum Ellis

floridanum Ellis f. album F.G.Mey. \&

Mazzeo

floridanum Ellis f. album F.G.Mey. \&

Mazzeo 'Semmes'

henryi Diels

mexicanum A.C.Sm.

parviflorum Michx. ex Vent.

INDIGOFERA L.

FABACEAE (Faboideae)

Indigo

Bean Family

decora Lindl

decora Lindl. 'Alba'

kirilowii Maxim.

potaninii Craib

suffruticosa Mill. 
ITEA L.

SAXIFRAGACEAE (Iteoideae)

Saxifrage Family

ilicifolia Oliv. virginica $L$.

JACARANDA Juss. BIGNONIACEAE Bignonia Family acutifolia HBK.
JASMINUM L. OLEACEAE
Jasmine Olive Family

beesianum Forrest \& Diels

floridum Bunge

fruticans $\mathrm{L}$.

humile $\mathrm{L}$.

humile L. 'Revolutum'

mesnyi Hance

multiflorum (Burm.f.) Andr.

nitidum Skan

nudiflorum Lindl.

parkeri S.T.Dunn

$\times$ stephanense E.Lemoine
JUGLANS L.

JUGLANDACEAE

ailantifolia Carr.

ailantifolia Carr. var. cordiformis

(Maxim.) Rehd.

cinerea $\mathrm{L}$.

(hindsii $\times J$. regia) 'Paradox'

major (Torr.) Heller

nigra $\mathrm{L}$.

nigra L. 'Laciniata'

regia $\mathrm{L}$.

regia $\mathrm{L}$. 'Broadview'

regia L. 'Hansen'

regia $\mathrm{L}$. 'McDermid'

JUNIPERUS L. CUPRESSACEAE

Juniper

Cypress Family chinensis $\mathrm{L}$.

chinensis L. 'Aurea'

chinensis L. 'Blaauw'

chinensis L. 'Columnaris'

chinensis L. 'Echiniformis'

chinensis L. 'Globosa Cinerea'

chinensis L. 'Gold Star'

chinensis L. 'Hetzii'

chinensis L. 'Kaizuka'

chinensis L. 'Kaizuka Variegated'

chinensis L. 'Parsonii' chinensis L. 'Robusta Green'

chinensis L. 'San Jose'-

chinensis L. 'Seagreen'

chinensis L. 'Sheppardii'

chinensis L. 'Spartan'

chinensis L. 'Variegata'

chinensis L. var. sargentii A.Henry

chinensis L. var. sargentii A.Henry

'Viridis'

communis $\mathrm{L}$.

communis L. 'Berkshire'

communis L. 'Hibernica'

communis L. 'Oblonga Pendula'

communis L. 'Pencil Point'

communis L. 'Sentinel'

communis L. ssp. depressa

(Pursh) Franco

communis $\mathrm{L}$. var. montana Ait.

communis L. var. montana Ait.

'Hornibrookii'

conferta Parl.

conferta Parl. 'Blue Pacific'

conferta Parl. 'Emerald Sea'

conferta Parl. 'Gulf Tide'

davurica Pall. 'Expansa'

davurica Pall. 'Expansa Aureo-spicata'

davurica Pall. 'Expansa Variegata'

deppeana Steud. var. pachyphlaea

(Torr.) Martinez

excelsa Bieb. 'Stricta'

formosana Hayata

horizontalis Moench

horizontalis Moench 'Admirabilis'

horizontalis Moench 'Bar Harbor'

horizontalis Moench 'Blue Chip'

horizontalis Moench 'Blue Forest'

horizontalis Moench 'Blue Horizon'

horizontalis Moench 'Blue Muffet'

horizontalis Moench 'Blue Rug'

horizontalis Moench 'Douglasii'

horizontalis Moench 'Filicinus'

horizontalis Moench 'Filicinus Minimus'

horizontalis Moench 'Glauca'

horizontalis Moench 'Glenmore'

horizontalis Moench 'Glomerata'

horizontalis Moench 'Hughes'

horizontalis Moench 'Livida'

horizontalis Moench 'Marcella'

horizontalis Moench 'Petraea'

horizontalis Moench 'Plumosa'

horizontalis Moench 'Prince of Wales'

horizontalis Moench 'Procumbens'

horizontalis Moench 'Pulchella'

horizontalis Moench 'Wiltonii'

$\times$ media Van Melle 'Blue Cloud'

$\times$ media Van Melle 'Blue Vase'

$\times$ media Van Melle 'Den Boer' 
$\times$ media Van Melle 'Fruitlandii'

$\times$ media Van Melle 'Mint Julep'

$\times$ media Van Melle 'Old Gold'

$\times$ media Van Melle 'Pfitzeriana'

$\times$ media Van Melle 'Pfitzeriana Aurea'

$\times$ media Van Melle 'Pfitzeriana

Compacta'

$\times$ media Van Melle 'Plumosa Aurea'

procumbens (Endl.) Miq.

procumbens (Endl.) Miq. 'Nana'

rigida Sieb. \& Zucc.

sabina L. 'Arcadia'

sabina L. 'Broadmoor'

sabina L. 'Buffalo'

sabina L. 'Fastigiata'

sabina L. 'Skandia'

sabina L. 'Von Ehren'

scopulorum Sarg.

scopulorum Sarg. 'Alba'

scopulorum Sarg. 'Blue Heaven'

scopulorum Sarg. 'Chandler Blue'

scopulorum Sarg. 'Gareei'

scopulorum Sarg. 'Gray Gleam'

scopulorum Sarg. 'Hill's Silver'

scopulorum Sarg. 'Lakewood'

scopulorum Sarg. 'Lakewood Globe'

scopulorum Sarg. 'Pathfinder'

scopulorum Sarg. 'Platinum'

scopulorum Sarg. 'Tolleson's Blue

Weeping'

scopulorum Sarg. 'Wichita Blue'

silicicola (Small) Bailey

squamata Buch.-Ham. ex Lamb.

squamata Buch.-Ham. ex Lamb.

'Blue Star'

squamata Buch.-Ham. ex Lamb.

'Loderi'

squamata Buch.-Ham. ex Lamb.

'Meyeri'

virginiana $L$.

virginiana L. 'Aurea'

virginiana $\mathrm{L}$. 'Canaertii'

virginiana L. 'Fastigiata'

virginiana L. 'Glauca'

virginiana L. 'Grey Owl'

virginiana L. 'Keteleeri'

virginiana L. 'Nova'

virginiana $\mathrm{L}$. 'Reptans'

virginiana L. 'Skyrocket'

JUSTICIA L.

ACANTHACEAE Acanthus Family

brandegeana Wassh. \& L.B.Sm.

brandegeana Wassh. \& L.B.Sm.

'Yellow Queen'

californica (Benth.) D.Gibson
KADSURA JusS.

SCHISANDRACEAE

Schisandra Family

japonica (Thunb.) Dunal

KALMIA L.

ERICACEAE

Heath Family

angustifolia L.

angustifolia L. var. carolina (Small)

Fern.

cuneata Michx.

hirsuta $\times \mathbf{K}$. latifolia

latifolia L.

$\star$ latifolia L. 'Bettina'

latifolia L. 'Dexter Pink'

latifolia L. 'Fuscata'

latifolia L. 'Ostbo Red'

latifolia L. 'Sharon Rose'

latifolia L. 'Shooting Star'

latifolia L. f. angustata Rehd.

RALOPANAX Miq.

ARALIACEAE Ginseng Family

septemlobus (Thunb. ex J.A.Murr.) Koidz.

KERRIA DC

ROSACEAE

Rose Family

japonica (L.) DC.

japonica (L.) DC. 'Albescens'

japonica (L.) DC. 'Picta'

japonica (L.) DC. 'Pleniflora'

KOELREUTERIA LaXm.

SAPINDACEAE

Golden-rain Tree

Soapberry Family

bipinnata Franch.

elegans (Seemann) A.C.Sm.

ssp. formosana (Hayata) F.G.Mey.

paniculata Laxm.

paniculata Laxm. 'Fastigiata'

paniculata Laxm. 'September'

KOLKWITZIA Graebn.

CAPRIFOLIACEAE Honeysuckle Family

amabilis Graebn.

amabilis Graebn. 'Rosea'

+LABURNOCYTISUS Schneid.

FABACEAE (Faboideae) Bean Family

adamii (Poit.) Schneid. 
LABURNUM Medik. Golden-chain Tree FABACEAE (Faboideae) Bean Family

anagyroides Medik.

$\times$ watereri (Kirchn.) Dipp.

LAGERSTROEMIA L. LYTHRACEAE

Crape Myrtle Loosestrife Family

fauriei Koehne indica $\mathrm{L}$.

indica L. 'Alba'

indica L. 'Carolina Beauty'

$\star$ indica L. 'Potomac'

subcostata Koehne

Note: The parentage of the following cultivars is complex; see catalog entries for details.

$\star$ 'Acoma'

$\star$ 'Apalachee'

'Basham's Party Pink'

$\star$ 'Biloxi'

^ 'Caddo'

^ 'Catawba'

$\star$ 'Cherokee'

$\star$ 'Choctaw'

$\star$ 'Comanche'

$\star$ 'Conestoga'

$\star$ 'Hopi'

$\star$ 'Lipan'

$\star$ 'Miami'

$\star$ 'Muskogee'

$\star$ 'Natchez'

^ 'Osage'

$\star$ 'Pecos'

$\star$ 'Powhatan'

^ 'Seminole'

$\star$ 'Sioux'

$\star$ 'Tonto'

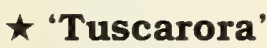

$\star$ 'Tuskegee'

$\star$ 'Wichita'

Ł'Yuma'

'Zuni'

LANTANA L.

VERBENACEAE Verbena Family

camara L.

montevidensis (Spreng.) Briq.

LARIX Mill. PINACEAE

Larch Pine Family
LAURUS L.

Laurel

LAURACEAE

- Laurel Family

nobilis $\mathrm{L}$.

LAVANDULA L.

LAMLACEAE

Lavender Mint Family

angustifolia Mill.

angustifolia Mill. 'Provence'

angustifolia Mill. ssp. angustifolia

'Hidcote'

$\times$ intermedia Emeric ex Loisel.

stoechas L.

LEDUM L.

ERICACEAE

Heath Family

palustre L. var. diversipilosum Nakai

LEIOPHYLLUM R.Hedwig Sand Myrtle ERICACEAE Heath Family

buxifolium (Bergius) Ell.

buxifolium (Bergius) Ell. var. hugeri

(Small) Schneid.

LEITNERIA Chapm.

LEITNERIACEAE

Leitneria Family

floridana Chapm.

LEPTODERMIS Wall.

RUBIACEAE Madder Family

oblonga Bunge

LEPTOSPERMUM J.R. \& J.G. Forst. MYRTACEAE Myrtle Family

scoparium J.R. \& J.G. Forst. 'Plenum'

LESPEDEZA Michx. Bush Clover FABACEAE (Faboideae) Bean Family

bicolor Turcz.

cyrtobotrya Miq.

maximowiczii Schneid.

thunbergii (DC.) Nakai

thunbergii (DC.) Nakai 'Albiflora'

LEUCAENA Benth.

FABACEAE (Mimosoideae) Bean Family

leucocephala (Lam.) de Wit

decidua Mill.

gmelinii (Rupr.) Rupr.

kaempferi (Lamb.) Carr. 
LEUCOPHYLLUM Humboldt \&

Bonpland

SCROPHULARIACEAE Figwort Family

frutescens (Berl.) I.M.Johnst.

LEUCOTHOE D.Don
ERICACEAE
axillaris (Lam.) D.Don
fontanesiana (Steud.) Sleumer
fontanesiana (Steud.) Sleumer
'Girard's Rainbow'
fontanesiana (Steud.) Sleumer 'Nana'
fontanesiana (Steud.) Sleumer
'Rollissonii'
fontanesiana (Steud.) Sleumer 'Zebekot'
fontanesiana (Steud.) Sleumer
'Zebonard'
racemosa (L.) A.Gr.

LIGUSTRUM L. Privet OLEACEAE Olive Family

compactum Hook.f. \& Thoms. delavayanum Hariot japonicum Thunb. japonicum Thunb. 'Erecta' japonicum Thunb. 'Fraseri' japonicum Thunb. 'Iwata' japonicum Thunb. 'Lake Treska' japonicum Thunb. 'Nobilis' japonicum Thunb. 'Repandens' japonicum Thunb. 'Rotundifolium' japonicum Thunb. 'Silver Star' japonicum Thunb. 'Suwanee River' japonicum Thunb. 'Variegatum' lucidum Ait.f. obtusifolium Sieb. \& Zucc. ovalifolium Hassk. ovalifolium Hassk. 'Aureo-marginatum' quihoui Carr. sempervirens (Franch.) Mansf.

sinense Lour.

sinense Lour. 'Fraseri'

sinense Lour. 'Pendulum'

sinense Lour. 'Wimbish'

tschonoskii Decne.

$\times$ vicaryi Rehd.

vulgare $\mathrm{L}$.

\section{LINDERA Thunb.}

LAURACEAE

Spicebush Laurel Family

angustifolia W.C.Cheng benzoin (L.) $\mathrm{Bl}$.

\section{LIRIODENDRON L. MAGNOLIACEAE \\ chinense (Hemsl.) Sarg. \\ tulipifera L. \\ tulipifera L. 'Aureo-marginatum'} Magnolia Family

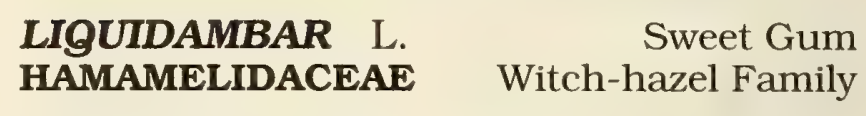

formosana Hance

orientalis Mill.

styraciflua $\mathrm{L}$.

styraciflua L. 'Gum Ball'

styraciflua L. 'Variegata'

styraciflua L. f. rotundiloba Rehd.

LITHOCARPUS Bl.

FAGACEAE

Tanbark Oak Beech Family

corneus (Lour.) Rehd.

edulis (Makino) Nakai

glaber (Thunb.) Nakai

henryi (Seemann) Rehd. \& Wils.

LITSEA Lam.

LAURACEAE Laurel Family

aestivalis (L.) Fern.

LIVISTONA R.Br.

ARECACEAE Palm Family

chinensis (Jacq.) R.Br. ex Mart.

LONICERA L.

Honeysuckle

CAPRIFOLIACEAE Honeysuckle Family

$\times$ bella Zab.

$\times$ brownii (Regel) Carr.

flava Sims

fragrantissima Lindl. \& Paxt.

gracilipes Miq.

gracilipes Miq. var. glandulosa Maxim.

$\times$ heckrottii Rehd.

japonica Thunb. ex J.A.Murr.

japonica Thunb. ex J.A.Murr.

f. chinensis (P.W.Wats.) Hara

korolkowii Stapf 'Floribunda'

maackii Maxim.

maackii Maxim. var. podocarpa Franch. 
morrowii A.Gr.

nitida Wils.

nitida Wils. 'Baggesen's Gold'

nitida Wils. 'Ernest Wilson'

pileata Oliv.

sempervirens $\mathrm{L}$.

sempervirens L. 'Sulphurea'

sempervirens L. 'Superba'

standishii Jacq.

subsessilis Rehd.

tatarica L.

tatarica L. 'Arnold Red'

tatarica L. 'Hack's Red'

tatarica L. 'Punicea'

xylosteum L.

xylosteum L. 'Nana'

xylosteum L. f. glabrescens Zab.

LOROPETALUM R.Br. ex Reichenb.

HAMAMELIDACEAE Witch-hazel Family

chinense (R.Br.) Oliv.

\section{LYCIUM L.}

SOLANACEAE

Matrimony Vine Nightshade Family

carolinianum Walt.

chinense Mill.

LYONIA Nutt.

\section{ERICACEAE}

Heath Family

ferruginea (Walt.) Nutt.

ligustrina (L.) DC.

lucida (Lam.) K.Koch

mariana (L.) D.Don

MAACKIA Rupr.

FABACEAE (Faboideae) Bean Family

amurensis Rupr. \& Maxim.

tenuifolia (Hemsl.) Hand.-Mazz.

\section{MACFADYENA A.DC.}

BIGNONIACEAE Bignonia Family

unguis-cati (L.) A.Gentry

MACLURA Nutt.

MORACEAE

Mulberry Family

pomifera (Raf.) Schneid.
MAGNOLIA L. MAGNOLIACEAE

Magnolia

Magnolia Family acuminata $\mathrm{L}$.

(acuminata $\times$ M. denudata) 'Elizabeth' (?acuminata $\times$ M. denudata)

'Sundance'

ashei Weatherby

$\times$ brooklynensis Kalmb. 'Woodsman'

cylindrica Wils.

dealbata Zucc.

denudata Desr.

fraseri Walt.

grandiflora L.

grandiflora L. 'Baldwin'

grandiflora L. 'Charles Dickens'

grandiflora L. 'Claudia Wanamaker'

grandiflora L. 'Hasse'

grandiflora L. 'Little Gem'

grandiflora L. 'Majestic Beauty'

grandiflora L. 'Margaret Davis'

grandiflora L. 'Pioneer'

grandiflora L. 'Praecox'

grandiflora L. 'Praecox Fastigiata'

grandiflora L. 'St. Mary'

grandiflora L. 'Samuel Sommer'

grandiflora L. 'Shady Grove No. 4'

grandiflora L. 'Shady Grove No. 5'

grandiflora L. 'Shady Grove No. 6'

grandiflora L. 'Variegata'

grandiflora L. 'Victoria'

$\times$ kewensis Pearce 'Wada's Memory'

kobus DC.

liliiflora Desr.

liliiflora Desr. 'Nigra'

liliiflora Desr. 'O'Neill'

liliiflora Desr. 'Reflorescens'

$\star$ (liliiflora 'Nigra' $\times$ M. sprengeri 'Diva')

'Galaxy'

$\star$ (liliiflora 'Nigra' $\times$ M. sprengeri 'Diva')

'Spectrum'

$\star$ (liliiflora 'Nigra' $\times$ M. stellata 'Rosea')

'Ann'

$\star$ (liliiflora 'Nigra' $\times$ M. stellata 'Rosea')

'Betty'

$\star$ (liliiflora 'Nigra' $\times$ M. stellata 'Rosea')

'Judy'

$\star$ (liliiflora 'Nigra' $\times$ M. stellata 'Rosea')

'Randy'

$\star$ (liliiflora 'Nigra' $\times$ M. stellata 'Rosea')

'Ricki'

$\star$ (liliiflora 'Nigra' $\times$ M. stellata 'Rosea')

'Susan'

$\star$ (liliiflora 'Reflorescens' $\times$ M. stellata

'Rosea') 'Pinkie'

$\star$ (liliiflora 'Reflorescens' $\times$ M. stellata

'Waterlily') 'Jane'

$\times$ loebneri Kache

$\times$ loebneri Kache 'Merrill'

$\times$ loebneri Kache 'Neil McEacharn'

$\times$ loebneri Kache 'Spring Snow'

macrophylla Michx. 
obovata Thunb.

$\star$ (obovata $\times M$. virginiana) 'Nimbus'

officinalis Rehd. \& Wils.

officinalis Rehd. \& Wils. var. biloba

Rehd. \& Wils.

$\times$ proctoriana Rehd.

pyramidata Bartram ex Pursh

salicifolia (Sieb. \& Zucc.) Maxim.

'Mrs. Jack'

sieboldii K.Koch

$\times$ soulangeana Soul.-Bod.

$\times$ soulangeana Soul.-Bod. 'Alba'

$\times$ soulangeana Soul.-Bod. 'Alexandrina'

$\times$ soulangeana Soul.-Bod. 'C. H. Kern'

$\times$ soulangeana Soul.-Bod.

'Grace McDade'

$\times$ soulangeana Soul.-Bod. 'Lennei'

$\times$ soulangeana Soul.-Bod. 'Lilliputian'

$\times$ soulangeana Soul.-Bod. 'Picture'

$\times$ soulangeana Soul.-Bod. 'Purpliana'

$\times$ soulangeana Soul.-Bod. 'Rubra'

$\times$ soulangeana Soul.-Bod. 'Rustica'

$\times$ soulangeana Soul.-Bod. 'San Jose'

$\times$ soulangeana Soul.-Bod. 'Sundew'

$\times$ soulangeana Soul.-Bod.

'Superba Rosea'

$\times$ soulangeana Soul.-Bod. 'Verbanica'

sprengeri Pamp. 'Diva'

stellata (Sieb. \& Zucc.) Maxim.

stellata (Sieb. \& Zucc.) Maxim.

'Pink Stardust'

stellata (Sieb. \& Zucc.) Maxim.

'Rohrbach'

stellata (Sieb. \& Zucc.) Maxim. 'Rosea'

stellata (Sieb. \& Zucc.) Maxim.

'Royal Star'

stellata (Sieb. \& Zucc.) Maxim. 'Rubra'

stellata (Sieb. \& Zucc.) Maxim.

'Water Lily'

tripetala $\mathrm{L}$.

$\times$ veitchii Bean

virginiana $L$.

virginiana L. 'Henry Hicks'

$\star$ virginiana L. 'Satellite'

$\star$ (virginiana $\times$ M. grandiflora)

'Freeman'

$\star$ (virginiana $\times$ M. grandiflora)

'Maryland'

$\times$ wieseneri Carr.

\section{$\times$ MAHOBERBERIS Schneid. BERBERIDACEAE Barberry Family}

aquisargentii Kruessm.

miethkeana Melander \& Eade
MAHONLA Nutt. BERBERIDACEAE

Barberry Family

aquifolium (Pursh) Nutt.

bealei (Fort.) Carr.

chochoca Fedde

fortunei (Lindl.) Fedde

japonica (Thunb. ex J.A.Murr.) DC.

oiwakensis Hayata

$\times$ media Brickell 'Charity'

pinnata (Lag.) Fedde

swazeyi Buckl.

trifoliolata (Moric.) Fedde

MALLOTUS LOUT.

EUPHORBIACEAE

Spurge Family

japonicus (Thunb. ex L.f.) Muell.-Arg.

$\times$ MALOSORBUS Browicz ROSACEAE

Rose Family

florentina (Zuccagni) Browicz

MALPIGHIA L.

MALPIGHIACEAE

Malpighia Family

glabra L.

MALUS Mill.

ROSACEAE

Rose Family

$\times$ adstringens Zab. 'Athabasca'

$\times$ adstringens Zab. 'Hopa'

angustifolia (Ait.) Michx.

$\times$ arnoldiana (Rehd.) Sarg.

$(\times$ arnoldiana $\times M$. domestica

'Niedzwetzkyana') 'Henrietta Crosby'

( $\times$ arnoldiana $\times$ M. spectabilis)

'Van Eseltine'

$\times$ atrosanguinea (Spaeth) Schneid.

( $\times$ atrosanguinea $\times$ M. sargentii 'Rosea')

'Mary Potter'

baccata (L.) Borkh.

baccata (L.) Borkh. var. mandschurica

(Maxim.) Schneid.

coronaria (L.) Mill.

coronaria (L.) Mill. 'Nieuwlandiana'

coronaria (L.) Mill. var. dasycalyx Rehd.

coronaria (L.) Mill. var. elongata Rehd.

domestica Borkh.

domestica Borkh. 'Veitch's Scarlet'

floribunda Sieb.

halliana Koehne

halliana Koehne 'Parkmanii'

\ halliana Koehne hybrid 'Adirondack'

$\times$ hartwigii Koehne 'Katherine' 
hupehensis (Pamp.) Rehd. ioensis (A.Wood) Britton 'Nova' ioensis (A.Wood) Britton 'Plena' ioensis (A.Wood) Britton var. palmeri Rehd.

lancifolia Rehd.

$\times$ micromalus Makino

$\times$ platycarpa Rehd.

prunifolia (Willd.) Borkh.

$\times$ purpurea (Barbier) Rehd.

$\times$ purpurea (Barbier) Rehd.

'Aldenhamensis'

$\times$ purpurea (Barbier) Rehd. 'Eleyi'

$\times$ purpurea (Barbier) Rehd. 'Lemoinei'

$\times$ robusta (Carr.) Rehd.

$\times$ robusta (Carr.) Rehd. 'Golden

Hornet'

sargentii Rehd.

sargentii Rehd. 'Rosea'

$\times$ scheideckeri (Spaeth) Zab.

Xscheideckeri (Spaeth) Zab.

'Dorothea'

sieboldii (Regel) Rehd.

$\star$ sieboldii (Regel) Rehd. 'Fuji'

$\times$ soulardii (Bailey) Britton 'Red Tip'

spectabilis (Ait.) Borkh.

$\times$ zumi (Matsum.) Rehd.

$\star$ (/hybrid \#28/ × M. 'Wintergold')

'Naragansett'

'Almey'

'Centennial'

'Crimson Brilliant'

'David'

'East Malling'

'Evelyn'

'Flame'

'Golden Anniversary'

'Guiding Star'

'Henry F. Dupont'

'Hillier'

'Marshall Oyama'

'Mathews'

'Mrs. Bayard Thayer'

'Normand'

'Oporto'

'Pink Pearl'

'Pink Spires'

'Prairie Rose'

'Prince George's'

'Profusion'

'Pygmy'

'Red Jade'

'Red Jewel'

'Red Silver'

'Rondo'

'Shakespeare'

'Silver Moon'
'Sissipuk'

'Thomas Roland'

'Timiskaming'

'Tops-in-Bloom'

'Wintergold'

'Wynema'

MALVAVISCUS Fabr, non Adans.

MALVACEAE Mallow Family

arboreus Cav. var. drummondii Schery arboreus Cav. var. penduliflorus (Sesse \& Moc. ex DC.) Schery

MANIHOT Mill. EUPHORBIACEAE

Cassava Spurge Family

grahamii Hook.

MELALEUCA L. MYRTACEAE Myrtle Family

quinquenervia (Cav.) S.T.Blake

MELIA L.

MELIACEAE Mahogany Family

azedarach $\mathrm{L}$.

MENZIESIA Sm.

ERICACEAE

Heath Family

pilosa (Michx.) Juss.

MESPILUS L.

ROSACEAE

Rose Family

germanica L.

METASEQUOLA Miki ex H.H.Hu \& W.C.Cheng

TAXODIACEAE

Taxodium Family

glyptostroboides H.H.Hu \& W.C.Cheng

$\star$ glyptostroboides H.H.Hu \& W.C.Cheng 'National'

MICHELIA L.

MAGNOLIACEAE Magnolia Family

compressa (Maxim.) Sarg.

figo (Lour.) Spreng.

MICROBIOTA Komar.

CUPRESSACEAE

Cypress Family

decussata Komar. 
MICROCITRUS Swingle

RUTACEAE

Citrus Family

australasica (F.Muell.) Swingle

MILLETTIA Wight \& Arn.

FABACEAE (Faboideae)

Bean Family

reticulata Benth.

MIMOSA L.

FABACEAE (Mimosoideae) Bean Family

biuncifera Benth.

pigra L. var. berlandieri (A.Gr.)

B.L.Turner

\section{MITCHELLA L.}

RUBLACEAE

Madder Family

repens $\mathrm{L}$.

MORUS L.

MORACEAE

Mulberry Mulberry Family

alba L.

alba L. 'Hicks'

alba L. 'Pendula'

alba L. 'Teas Weeping'

rubra $\mathrm{L}$.

MUEHLENBECKIA Meisn.

POLYGONACEAE Buckwheat Family

axillaris (Hook.f.) Walp.

MURRAYA Koenig ex L.

RUTACEAE

Citrus Family

paniculata (L.) Jack

MUSA L.

Banana

MUSACEAE

Banana Family

basjoo Sieb. \& Zucc.

$\times$ paradisiaca L. 'Sapientum'

rosacea Jacq.

velutina $\mathrm{H}$.Wendl. \& Drude

MYRICA L. MYRICACEAE

Bayberry Family

cerifera L.

heterophylla Raf. inodora Bartram

pensylvanica Loisel.

pusilla Raf.

rubra Sieb. \& Zucc.

MYRTUS L.

Myrtle

MYRTACEAE

Myrtle Family

communis $\mathrm{L}$.

communis L. 'Microphylla'

NANDINA Thunb.

BERBERIDACEAE Barberry Family

domestica Thunb.

domestica Thunb. 'Alba'

domestica Thunb. 'Harbour Dwarf'

domestica Thunb. 'Purpurea Nana'

NEILLIA D.Don

ROSACEAE

Rose Family

sinensis Oliv.

NEMOPANTHUS Raf.

AQUIFOLIACEAE

Holly Family

mucronatus (L.) Trelease

NERIUM L.

APOCYNACEAE Dogbane Family

oleander L.

oleander L. 'Variegata'

NEVTUSIA A.Gr.

ROSACEAE

Rose Family

alabamensis A.Gr.

NICODEMIA Tenore

BUDDLEJACEAE

Buddleja Family

diversifolia Tenore

NICOTLANA L.

SOLANACEAE Nightshade Family

glauca R.Graham

NIEREMBERGLA Ruiz \& Pavon

SOLANACEAE Nightshade Family

scoparia Sendtn. 
NYSSA L.

NYSSACEAE

Sour-gum Family

ogeche Marsh.

sylvatica Marsh.

sylvatica Marsh. var. biflora (Walt.) Sarg.

OCHNA L.

OCHNACEAE

Ochna Family

atropurpurea DC.

OLEA L.

OLEACEAE

europaea L.

ORIGANUM L.

LAMIACEAE

Mint Family

onites $\mathrm{L}$.

ORIXA Thunb.

RUTACEAE

Citrus Family

japonica Thunb.

OSMANTHUS Lour.

OLEACEAE

Olive Family

americanus (L.) A.Gr.

armatus Diels

$\times$ fortunei Carr.

$\times$ fortunei Carr. 'San Jose'

fragrans Lour.

fragrans Lour. 'Aurantiacus'

heterophyllus (G.Don) P.S.Green

var. heterophyllus

heterophyllus (G.Don) P.S.Green

var. heterophyllus 'Gulf Tide'

heterophyllus (G.Don) P.S.Green var. heterophyllus 'Purpureus'

heterophyllus (G.Don) P.S.Green var. heterophyllus 'Rotundifolius'

heterophyllus (G.Don) P.S.Green var. heterophyllus 'Variegatus'

OSTEOMELES Lindl. ROSACEAE

Rose Family

schwerinae Schneid.

OSTRYA Scop.

BETULACEAE

Birch Family

virginiana (Mill.) K.Koch
OXYDENDRUM DC.

ERICACEAE

$-$

Heath Family

arboreum (L.) DC.

PACHYSANDRA Michx.

BUXACEAE

Boxwood Family

axillaris Franch.

procumbens Michx.

terminalis Sieb. \& Zucc.

terminalis Sieb. \& Zucc. 'Silveredge'

PAEONIA L.

PAEONIACEAE

Peony

Peony Family

suffruticosa Andr.

PALAFOXIA Lag. ASTERACEAE

Aster Family

feayi A.Gr.

PARKINSONLA L.

FABACEAE (Caesalpinioideae) Bean Family

aculeata L.

PARROTIA C.A.Mey.

HAMAMELIDACEAE Witch-hazel Family

persica (DC.) C.A.Mey.

PARROTIOPSIS (Niedenzu) Schneid.

HAMAMELIDACEAE Witch-hazel Family

jacquemontiana (Decne.) Rehd.

PARTHENOCISSUS Planch.

VITACEAE Grape Family

henryana (Hemsl.) Diels \& Gilg

quinquefolia (L.) Planch.

tricuspidata (Sieb. \& Zucc.) Planch.

PASSIFLORA L.

PASSIFLORACEAE Passion-flower Family

coccinea Aubl.

PAULOWINIA Sieb. \& Zucc.

SCROPHULARIACEAE Figwort Family

kawakamii Ito

tomentosa (Thunb. ex J.A.Murr.) Steud. 
PAVONLA Cav.

MALVACEAE

Mallow Family

hastata Cav.

PAXISTIMA Raf.

CELASTRACEAE

Staff-tree Family

canbyi A.Gr.

PERIPLOCA L.

ASCLEPIADACEAE

Asclepias Family

graeca L.

PEROVSKIA Karelin

LAMIACEAE

Mint Family

atriplicifolia Benth.

PERSEA Mill.

LAURACEAE

Laurel Family

americana Mill.

borbonia (L.) Spreng.

humilis Nash

thunbergii (Sieb. \& Zucc.) Kosterm.

PETTERIA Presl

FABACEAE (Faboideae) Bean Family

ramentacea Presl

PHELLODENDRON Rupr. RUTACEAE

Citrus Family

amurense Rupr.

amurense Rupr. var. japonicum (Maxim.)

Ohwi

amurense Rupr. var. lavallei (Dode)

Sprague

PHILADELPHUS L. Mock Orange

SAXIFRAGACEAE (Hydrangeoideae)

Saxifrage Family

coronarius $\mathrm{L}$.

coronarius L. 'Belle Etoile'

coronarius L. 'Duplex'

coronarius L. 'Natchez'

delavayi L.Henry 'Nymans'

$\times$ falconeri Sarg.

hirsutus Nutt.

inodorus $\mathrm{L}$.

$\times$ lemoinei V.Lemoine

lewisii Pursh

pubescens Loisel. pubescens Loisel. var. verrucosus

(Schrad. ex DC.) S.Y.Hu

satsumanus Sieb. ex Miq. var. nikoensis

Rehd.

schrenkii Rupr.

triflorus Wall.

$\times$ virginalis Rehd. 'Minnesota

Snowflake'

$\times$ virginalis Rehd. 'Virginal'

'Silberregen'

'Voie Lactee'

PHILLYREA L.

OLEACEAE

Olive Family

angustifolia $\mathrm{L}$.

latifolia L. var. media (L.) Schneid. latifolia L. var. media (L.) Schneid.

'Spinosa'

\section{PHOENIX L.}

ARECACEAE Palm Family

canariensis Hort. ex Chabaud

reclinata Jacq.

sylvestris $\times \boldsymbol{P}$. canariensis

PHOTINLA Lindl.

ROSACEAE Rose Family

$\times$ fraseri W.J.Dress 'Birmingham'

glabra (Thunb. ex J.A.Murr.) Maxim. serratifolia (Desf.) Kalk.

villosa (Thunb. ex J.A.Murr.) DC.

villosa (Thunb. ex J.A.Murr.) DC.

var. laevis (Thunb. ex J.A.Murr.) Dipp.

villosa (Thunb. ex J.A.Murr.) DC.

var. maximowicziana (Lev.) Rehd.

villosa (Thunb. ex J.A.Murr.) DC.

var. sinica Rehd. \& Wils.

PHYGELIUS E.Mey. ex Benth.

SCROPHULARIACEAE Figwort Family

capensis E.Mey.

PHYLLOSTACHYS Sieb. \& Zucc.

POACEAE

Bamboo

Grass Family

angusta McClure

arcana McClure

aurea A.\& C.Riv.

aureosulcata McClure

bambusoides Sieb. \& Zucc.

bambusoides Sieb. \& Zucc. 'Castillon'

congesta McClure 
dulcis McClure

elegans McClure

flexuosa A.\& C.Riv.

heterocycla (Carr.) Mitf.

meyeri McClure

nidularia Munro

nidularia Munro 'Smoothsheath'

nigra (Loud.) Munro

nigra (Lodd.) Munro 'Henon'

nuda McClure

propinqua McClure

purpurata McClure 'Solidstem'

purpurata McClure 'Straightstem'

rubromarginata McClure

viridiglaucescens A.\& C.Riv.

viridis (Young) McClure

vivax McClure

\section{PHYSOCARPUS (Cambess.) Maxim.}

Ninebark

ROSACEAE Rose Family

amurensis (Maxim.) Maxim.

intermedius (Rydb.) Schneid.

malvaceous (Greene) O.Ktze.

opulifolius (L.) Maxim.

opulifolius (L.) Maxim. 'Luteus'
PICEA A.Dietr. PINACEAE

Spruce

Pine Family abies (L.) Karst.

abies (L.) Karst. 'Barryi'

abies (L.) Karst. 'Brevifolia'

abies (L.) Karst. 'Capitata'

abies (L.) Karst. 'Clanbrassiliana'

abies (L.) Karst. 'Compacta Asselyn'

abies (L.) Karst. 'Globosa'

abies (L.) Karst. 'Gregoryana Parsonii'

abies (L.) Karst. 'Highlandia'

abies (L.) Karst. 'Humilis'

abies (L.) Karst. 'Hystrix'

abies (L.) Karst. 'Inversa'

abies (L.) Karst. 'Kingsville'

abies (L.) Karst. 'Little Gem'

abies (L.) Karst. 'Maxwellii'

abies (L.) Karst. 'Merhii'

abies (L.) Karst. 'Microphylla'

abies (L.) Karst. 'Microsperma'

abies (L.) Karst. 'Montigena'

abies (L.) Karst. 'Mucronata'

abies (L.) Karst. 'Nidiformis'

abies (L.) Karst. 'Ohlendorffii'

abies (L.) Karst. 'Oldhamiana'

abies (L.) Karst. 'Parsonii'

abies (L.) Karst. 'Pendula'

abies (L.) Karst. 'Procumbens' abies (L.) Karst. 'Prostrata'

abies (L.) Karst. 'Pseuđoprostrata'

abies (L.) Karst. 'Pumila'

abies (L.) Karst. 'Pumila Glauca'

abies (L.) Karst. 'Pygmaea'

abies (L.) Karst. 'Pyramidata'

abies (L.) Karst. 'Remonte'

abies (L.) Karst. 'Repens'

abies (L.) Karst. 'Tabuliformis'

alcoquiana (J.G.Veitch ex Lindl.) Carr.

asperata Mast.

asperata Mast. 'Glauca'

asperata Mast. var. heterolepis (Rehd. \&

Wils.) W.C.Cheng ex Rehd.

gemmata Rehd. \& Wils.

glauca (Moench) Voss

glauca (Moench) Voss 'Conica'

glauca (Moench) Voss 'Echiniformis'

glauca (Moench) Voss 'Wild Acres'

glehnii (F.Schmidt) Mast.

jezoensis (Sieb. \& Zucc.) Carr.

'Howell's Dwarf'

mariana (Mill.) BSP.

'Beissneri Compacta'

mariana (Mill.) BSP. 'Doumetii'

obovata Ledeb.

omorika (Pancic) Purk.

omorika (Pancic) Purk. 'Pendula'

orientalis (L.) Link

orientalis (L.) Link 'Aurea Compacta'

orientalis (L.) Link 'Gracilis'

pungens Engelm.

pungens Engelm. 'Compacta'

pungens Engelm. 'Foxtail'

pungens Engelm. f. glauca (Regel)

Beissn. 'Glauca Pendula'

pungens Engelm. f. glauca (Regel)

Beissn. 'Glauca Procumbens'

pungens Engelm. f. glauca (Regel)

Beissn. 'Glauca Prostrata'

pungens Engelm. 'Globosa'

pungens Engelm. 'Hoopsii'

pungens Engelm. 'Hunnewelliana'

pungens Engelm. 'Iseli Fastigiata'

pungens Engelm. 'Koster'

pungens Engelm. 'Montgomery'

pungens Engelm. f. glauca (Regel)

Beissn.

torano (K.Koch) Koehne

\section{PIERIS D.Don}

ERICACEAE

Heath Family

floribunda (Pursh) Benth. \& Hook.

(floribunda $\times P$. japonica)

'Brower's Beauty'

formosa (Wall.) D.Don 
formosa (Wall.) D.Don 'Wakehurst'

(formosa $\times P$. japonica) 'Mountain Fire'

japonica (Thunb. ex J.A.Murr.) D.Don ex G.Don

japonica (Thunb. ex J.A.Murr.) D.Don ex G.Don 'Bert Chandler'

japonica (Thunb. ex J.A.Murr.) D.Don ex G.Don 'Compacta'

japonica (Thunb. ex J.A.Murr.) D.Don ex G.Don 'Crispa'

japonica (Thunb. ex J.A.Murr.) D.Don ex G.Don 'Daisen'

japonica (Thunb. ex J.A.Murr.) D.Don ex G.Don 'Dorothy Wyckoff'

japonica (Thunb. ex J.A.Murr.) D.Don ex G.Don 'Flamingo'

japonica (Thunb. ex J.A.Murr.) D.Don ex G.Don 'Purity'

japonica (Thunb. ex J.A.Murr.) D.Don ex G.Don 'Pygmaea'

japonica (Thunb. ex J.A.Murr.) D.Don ex G.Don 'Pygmy Variegata'

japonica (Thunb. ex J.A.Murr.) D.Don ex G.Don 'Red Mill'

japonica (Thunb. ex J.A.Murr.) D.Don ex G.Don 'Scarlet O'Hara'

japonica (Thunb. ex J.A.Murr.) D.Don ex G.Don 'Shojo'

japonica (Thunb. ex J.A.Murr.) D.Don ex G.Don 'Variegata'

japonica (Thunb. ex J.A.Murr.) D.Don ex G.Don 'Wada'

japonica (Thunb. ex J.A.Murr.) D.Don ex G.Don 'White Cascade'

japonica (Thunb. ex J.A.Murr.) D.Don ex G.Don 'Whitecaps'

(japonica $\times$ P. formosa) 'Forest Flame' phillyreifolia (Hook.) DC.

taiwanensis Hayata

PINCKNEYA MichX. RUBIACEAE Madder Family

pubens Michx.

PINUS L. Pine PINACEAE Pine Family

armandii Franch. attenuata J.G.Lemmon ayacahuite K.Ehrenb.

banksiana Lamb.

brutia Tenore bungeana Zucc. ex Endl. cembra L.

cembra L. 'Compacta Glauca'

cembra L. var. sibirica Loud.

cembroides Zucc.

clausa (Chapm. ex Engelm.) Vasey

ex Sarg.

contorta Dougl. ex Loud. 'Spaan's Dwarf'

densiflora Sieb. \& Zucc.

densiflora Sieb. \& Zucc.

'Oculus-draconis'

densiflora Sieb. \& Zucc. 'Pendula'

densiflora Sieb. \& Zucc. 'Umbraculifera'

echinata Mill.

elliottii Engelm.

flexilis James

flexilis James 'Glenmore'

glabra Walt.

greggii Engelm. \& Parl.

halepensis Mill.

koraiensis Sieb. \& Zucc.

leucodermis Ant.

leucodermis Ant. 'Compact Gem'

mugo Turra var. mugo

mugo Turra var. mughus (Scop.) Zenari

'Gnom'

mugo Turra var. mughus (Scop.) Zenari

'Mops'

mugo Turra var. pumilio (Haenke) Zenari

nigra Arn. 'Globosa'

nigra Arn. 'Hornibrookiana'

nigra Arn. 'Monstrosa'

nigra Arn. 'Nana'

nigra Arn. ssp. nigra

palustris Mill.

parviflora Sieb. \& Zucc.

parviflora Sieb. \& Zucc.

'Adcock's Dwarf'

parviflora Sieb. \& Zucc. 'Baldwin'

parviflora Sieb. \& Zucc. 'Glauca'

parviflora Sieb. \& Zucc. 'Glauca Nana'

parviflora Sieb. \& Zucc. 'Kokonoe'

parviflora Sieb. \& Zucc. 'Venus'

parviflora Sieb. \& Zucc. 'Watnong'

patula Schiede \& Deppe

peuce Griseb. 'Arnold Dwarf'

pinea $\mathrm{L}$.

ponderosa Dougl. ex P.\& C.Laws

ponderosa Dougl. ex P.\& C.Laws.

'Pendula'

pumila (Pall.) Regel

pumila (Pall.) Regel 'Dwarf Blue'

pungens Lamb.

resinosa Ait.

rigida Mill.

roxburghii Sarg.

sabiniana Dougl. ex D.Don 
serotina Michx.

strobus $\mathrm{L}$.

strobus L. 'Amelia Dwarf'

strobus L. 'Contorta'

strobus L. 'Dwarf'

strobus L. 'Elf'

strobus L. 'Fastigiata'

strobus L. 'Merrimack'

strobus L. 'Nana'

strobus L. 'Pendula'

strobus L. 'Pumila'

strobus L. 'Radiata'

strobus L. 'Seacrest'

strobus L. 'Torulosa'

strobus $\times P$. wallichiana

sylvestris $\mathrm{L}$.

sylvestris L. 'Albyns'

sylvestris L. 'Argentea Compacta'

sylvestris L. 'Aurea'

sylvestris L. 'Beuvronensis'

sylvestris L. 'Fastigiata'

sylvestris L. 'Globosa Viridis'

sylvestris L. 'Moseri'

sylvestris L. 'Repens'

sylvestris L. 'Sentinel'

taeda L.

thunbergiana Franco

thunbergiana Franco 'Corticosa'

thunbergiana Franco 'Yatsubusa'

virginiana Mill.

wallichiana A.B.Jacks.

wallichiana A.B.Jacks. 'Zebrina'

PISTACIA L. Pistache

ANACARDIACEAE Cashew Family

chinensis Bunge

texana Swingle

PITHECELLOBIUM Mart.

FABACEAE (Mimosoideae) Bean Family

ebano (Berl.) Muller

PITTOSPORUM Banks ex Sol.

PITTOSPORACEAE Pittosporum Family

glabratum Lindl.

tobira (Willd.) Ait

tobira (Willd.) Ait. 'Variegata'

tobira (Willd.) Ait. 'Wheeler's Dwarf'

undulatum Vent.
PLANERA J.F.Gmel.

ULMACEAE - Elm Family

aquatica (Walt.) J.F.Gmel.

PLATANUS L.

PLATANACEAE

Sycamore

Plane-tree Family

$\times$ acerifolia (Ait.) Willd.

$\star \times$ acerifolia (Ait.) Willd. 'Columbia'

$\star \times$ acerifolia (Ait.) Willd. 'Liberty'

occidentalis L.

orientalis $\mathrm{L}$.

PLATYCARYA Sieb. \& Zucc.

JUGLANDACEAE Walnut Family

strobilacea Sieb. \& Zucc.

PLATYCLADUS Spach

CUPRESSACEAE Cypress Family

orientalis (L.) Franco

orientalis (L.) Franco 'Aurea Nana'

orientalis (L.) Franco 'Bakeri'

orientalis (L.) Franco

'Berckman's Golden'

orientalis (L.) Franco 'Beverleyensis'

orientalis (L.) Franco 'Conspicua'

orientalis (L.) Franco 'Flagelliformis'

orientalis (L.) Franco 'Fruitlandii'

orientalis (L.) Franco 'Hohman'

orientalis (L.) Franco 'Juniperoides'

orientalis (L.) Franco 'Rosedalis'

orientalis (L.) Franco 'Semperaurea'

PLUMBAGO L.

PLUMBAGINACEAE Leadwort Family

auriculata Lam.

PODOCARPUS L'Her. ex Pers.

PODOCARPACEAE Podocarpus Family

gracilior Pilger

macrophyllus (Thunb. ex J.A.Murr.)

Sweet

macrophyllus (Thunb. ex J.A.Murr.)

Sweet var. maki Sieb.

nagi (Thunb. ex J.A.Murr.) Makino

totara D.Don

POLIOTHYRSIS Oliv.

FLACOURTIACEAE Flacourtia Family

sinensis Oliv. 
POLYGONELLA MichX.

POLYGONACEAE Buckwheat Family

americana (Fischer \& C.A.Mey.) Small

myriophylla (Small) Horton

polygama (Vent.) Engelm. \& A.Gr.

PONCIRUS Raf.

RUTACEAE Citrus Family

trifoliata (L.) Raf.

POPULUS L. Poplar

SALICACEAE

Willow Family

alba L.

alba L. 'Pyramidalis'

$\times$ canadensis Moench

candicans Ait.

$\times$ canescens (Ait.) Sm.

deltoides Marsh.

grandidentata Michx.

heterophylla L.

maximowiczii A.Henry

maximowiczii $\times P$. trichocarpa

nigra L. 'Italica'

simonii Carr. 'Fastigiata'

tremuloides Michx.

PORLIERIA Ruiz \& Pavon

ZYGOPHYLLACEAE Caltrop Family

angustifolia (Engelm.) A.Gr.

POTENTILLA L.

Cinquefoil

ROSACEAE

Rose Family

fruticosa $\mathrm{L}$.

fruticosa L. 'Abbotswood'

fruticosa L. 'Everest'

fruticosa L. 'Grandiflora'

fruticosa L. 'Jackman's'

fruticosa L. 'Katherine Dykes'

fruticosa L. 'Maanelys'

fruticosa L. 'Primrose Beauty'

fruticosa L. 'William Purdom'

tridentata Ait.

'Sophie Blush'

PRINSEPIA Royle

ROSACEAE

Rose Family

sinensis (Oliv.) Oliv.
PROSOPIS L.

FABACEAE (Mimosoideae) Bean Family

glandulosa Torr. var. torreyana

(L.Benson) M.C.Johnst.

PRUNUS L.

ROSACEAE

Rose Family

americana Marsh.

angustifolia Marsh.

apetala (Sieb. \& Zucc.) Franch. \& Sav.

armeniaca $\mathrm{L}$.

avium $\mathrm{L}$.

avium L. 'Plena'

$\times$ blireiana Andre

campanulata Maxim.

caroliniana Ait.

cerasifera Ehrh.

cerasifera Ehrh. 'Atropurpurea'

cerasifera Ehrh. 'Moseri'

cerasifera Ehrh. 'Purpusii'

cerasifera Ehrh. 'Thundercloud'

cerasus L. 'Montmorency'

$\times$ cistena N.E.Hansen

conradinae Koehne

cyclamina Koehne

domestica L. Ssp. insititia (L.) Schneid.

dulcis (Mill.) D.A.Webb

glandulosa Thunb. ex J.A.Murr.

glandulosa Thunb. ex J.A.Murr.

'Albo-plena'

glandulosa Thunb. ex J.A.Murr.

'Sinensis'

humilis Bunge

$\times$ incam Fletcher 'Okami'

incisa Thunb. ex J.A.Murr.

incisa Thunb. ex J.A.Murr.

'February Pink'

incisa Thunb. ex J.A.Murr. f. serrata

Koidz. ex Wils.

incisa Thunb. ex J.A.Murr.

var. tomentosa Koidz.

(incisa $\times P$. serrulata) 'Umineko'

japonica Thunb. ex J.A.Murr.

laurocerasus $\mathrm{L}$.

laurocerasus L. 'Camelliifolia'

laurocerasus L. 'Forest Green'

laurocerasus L. 'Otto Luyken'

laurocerasus L. 'Zabeliana'

laurocerasus L. var. schipkaensis

Spaeth

lusitanica $\mathrm{L}$.

mahaleb L.

maritima Marsh.

maritima Marsh. 'Methley'

mexicana S.Wats. 
mume Sieb. \& Zucc.

mume Sieb. \& Zucc. 'Alba'

mume Sieb. \& Zucc. 'Matsubara Red'

mume Sieb. \& Zucc. 'Nishiki ume'

mume Sieb. \& Zucc. 'Peggy Clarke'

mume Sieb. \& Zucc. 'Rosemary Clarke'

mume Sieb. \& Zucc. 'W. B. Clarke'

munsoniana W.F.Wight \& Hedr.

(nipponica Matsum. var. kurilensis

(Miyabe) Wils. $\times$ unknown pollen parent)

'Kursar'

padus L.

padus L. 'Grandiflorus'

persica (L.) Batsch

persica (L.) Batsch 'Alba'

persica (L.) Batsch 'Albo-plena'

persica (L.) Batsch 'Camelliifolia'

persica (L.) Batsch 'Dianthiflora'

persica (L.) Batsch 'Duplex'

persica (L.) Batsch 'Fastigiata'

persica (L.) Batsch 'Rubro-plena'

persica (L.) Batsch 'Versicolor'

persica (L.) Batsch var. nucipersica

(Borkh.) Schneid. 'White Glory'

rivularis Scheele

sargentii Rehd.

sargentii Rehd. 'Dr. S. Edwin Mueller'

sargentii Rehd. 'Rancho'

(sargentii $\times P$. ? incisa) 'Spire'

(sargentii $\times$ P. subhirtella) 'Accolade'

serotina Ehrh.

serrula Franch.

serrulata Lindl.

serrulata Lindl. 'Amanogawa'

serrulata Lindl. 'Daikaku'

serrulata Lindl. 'Fudan sakura'

serrulata Lindl. 'Fuku rokuju'

serrulata Lindl. 'Gozanoma nioi'

serrulata Lindl. 'Hosokawa'

serrulata Lindl. 'Imose'

serrulata Lindl. 'Kiku shidare'

serrulata Lindl. 'Kwanzan'

serrulata Lindl. 'Mt. Fuji'

serrulata Lindl. 'Oshima zakura'

serrulata Lindl. 'Pink Perfection'

serrulata Lindl. 'Rosea'

serrulata Lindl. 'Shirofugen'

serrulata Lindl. 'Shirotae'

serrulata Lindl. 'Tai haku'

serrulata Lindl. 'Wase mikayo'

serrulata Lindl. 'Yama zakura'

serrulata Lindl. var. spontanea

(Maxim.) Wils.

$\times$ sieboldii (Carr.) Wittmack

$\times$ subhirtella Miq.

$\times$ subhirtella Miq. 'Autumnalis' $\times$ subhirtella Miq. 'Elizabeth'

$\times$ subhirtella Miq. 'Ros̄ea'

$\times$ subhirtella Miq. 'Shidare higan'

$\times$ subhirtella Miq. 'Yae shidare higan'

$\times$ subhirtella Miq. var. ascendens

(Makino) Wils. 'Flore-plena'

( $\times$ subhirtella $\times \boldsymbol{P}, \times$ yedoensis)

'Pandora'

$((\times$ subhirtella $\times$ P. $\times$ yedoensis $) \times$

P. $\times$ subhirtella) 'Hally Jolivette'

syodoi Nakai

tomentosa Thunb. ex J.A.Murr.

triloba Lindl.

triloba Lindl. 'Multiplex'

umbellata Ell.

verecunda (Koidz.) Koehne

virginiana L. 'Schubert'

$\times$ yedoensis Matsum.

$\times$ yedoensis Matsum. 'Akebono'

$\times$ yedoensis Matsum. 'Shidare yoshino'

$\times$ yedoensis Matsum. 'Somei yoshino'

'Ariake'

'Asagi'

'Benden'

'Botan zakura'

'Fugenzo'

'Gyoiko'

'Hatazakura'

'Hizakura'

'Meigetsu'

'Ojochin'

'Senriko'

'Shogetsu'

'Snow Fountain'

'Taki nioi'

'Yae akebono'

'Yae murasaki zakura'

PSEUDOCYDONIA (Schneid.) Schneid. ROSACEAE

Rose Family

sinensis (Dum.-Cours.) Thouin

PSEUDOLARIX Gord. PINACEAE

Pine Family

amabilis (J.Nelson) Rehd.

PSEUDOSASA Makino ex Nakai

POACEAE Grass Family

japonica Sieb. \& Zucc. ex Steud. 
$\begin{array}{lll}\text { PSEUDOTSUGA } & \text { Carr. } & \text { Douglas Fir } \\ \text { PINACEAE } & & \text { Pine Family }\end{array}$

menziesii (Mirb.) Franco var. glauca

(Beissn.) Franco

menziesii (Mirb.) Franco var. glauca

(Beissn.) Franco 'Densa'

menziesii (Mirb.) Franco var. glauca

(Beissn.) Franco 'Fastigiata'

menziesii (Mirb.) Franco var. glauca

(Beissn.) Franco 'Glauca Pendula'

menziesii (Mirb.) Franco var. glauca

(Beissn.) Franco 'Oudemansii'

PSIDIUM L. MYRTACEAE

Purple Guava Myrtle Family

littorale Raddi var. longipes (O. Berg)

Fosberg

PTELEA L. RUTACEAE

trifoliata $\mathrm{L}$.

PTEROCARYA Kunth JUGLANDACEAE

Wingnut Walnut Family

stenoptera DC.

PTEROSTYRAX Sieb. \& Zucc.

Epaulette Tree

STYRACACEAE Storax Family

corymbosum Sieb. \& Zucc.

hispidum Sieb. \& Zucc.

PUNICA L.

PUNICACEAE Pomegranate Family

granatum L.

granatum L. 'Legrellei'

granatum L. 'Nana'

granatum L. 'Plena'

PYRACANTHA M.J.Roem. r Firethorn
ROSACEAE

angustifolia (Franch.) Schneid.

angustifolia (Franch.) Schneid. 'Gnome'

$\star$ (angustifolia $\times$ P. 'Watereri') 'Navaho'

atalantioides (Hance) Stapf

atalantioides (Hance) Stapf 'Aurea'

coccinea Roem.

coccinea Roem. 'Bound'

coccinea Roem. 'Chadwick'

coccinea Roem. 'Cole's Erect' coccinea Roem. 'Dauerbrand'

coccinea Roem. 'Gold Nugget'

coccinea Roem. 'Kasan'

coccinea Roem. 'Keessen'

coccinea Roem. 'Lalandei'

coccinea Roem. 'Low Boy'

coccinea Roem. 'Minute Man'

coccinea Roem. 'Monrovia'

coccinea Roem. 'Orange Giant'

coccinea Roem. 'Pauciflora'

coccinea Roem. 'Praecox'

coccinea Roem. 'Runyan'

coccinea Roem. 'Sepers'

coccinea Roem. 'Thornless'

coccinea Roem. 'Vincent'

coccinea Roem. 'Wayside's Compact'

coccinea Roem. 'Wyattii'

(coccinea $\times P$. crenulata

var. rogersiana) 'Eddie's Coral'

crenulata (D.Don) Roem.

crenulata (D.Don) Roem. f. flava

Meunissier

crenulata (D.Don) Roem.

var. kansuensis Rehd.

crenulata (D.Don) Roem.

var. rogersiana A.B.Jacks.

crenulata (D.Don) Roem.

var. rogersiana A.B.Jacks. 'Flava'

fortuneana (Maxim.) Li

fortuneana (Maxim.) Li 'Graberi'

fortuneana (Maxim.) Li 'Orange Glow'

$\star$ fortuneana 'Orange Glow' $x$

$P$. crenulata var. rogersiana 'Flava')

'Teton'

koidzumii (Hayata) Rehd.

koidzumii (Hayata) Rehd. 'Belli'

koidzumii (Hayata) Rehd. 'Crimson Tide'

koidzumii (Hayata) Rehd. 'Government

Red'

koidzumii (Hayata) Rehd. 'Low Dense'

koidzumii (Hayata) Rehd. 'Miller'

koidzumii (Hayata) Rehd. 'Rosedale'

$\star$ (koidzumii $\times$ P. coccinea 'Wyattii')

'Mohave'

(koidzumii $\times$ P. fortuneana) 'San Jose'

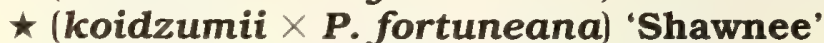

$\star$ (koidzumii 'Belli' $\times$ P. coccinea var. pauciflora) 'Pueblo'

$\star$ (koidzumii 'Victory' $\times$ (P. koidzumii

'Rosedale' $\times \boldsymbol{P}$. fortuneana

'Orange Glow')) 'Apache'

'Bad Zwischenahn'

'Baker'

'Bloss'

'Brilliant'

'Cal Poly'

'Chinese Brocade' 
'Coplen's Royal'

'Dr. Hook'

'Early Red'

'Golden Charmer'

'Golden Queen'

'Heyden's Bright Yellow'

'Heyden's Hi Yellow'

'Ingleside Crimson'

'Knap Hill Buttercup'

'Mioun'

'Moonbeam'

'Orange King'

'Pine Cone'

'Pinkie'

'Pride of Portsmouth'

'Pyrabox'

'Red Berry'

'Santa Cruz'

'Schwartz'

'Select Yellow'

'Sensation'

'Spring Hill'

'Stribling'

'Taliensis'

'Tiny Tim'

'True Yellow'

'Variegated'

'Walder'

'Walder Prostrate'

'Waterer's Dwarf'

'Waterer's Orange'

'Waterer's Yellow'

'Watereri'

'Weaver's Superb'

'Wheeler'

'Wight Early'

'Wonderberry'

'Yella Berry'

'Yokohama'

$\times$ PYRACOMELES Rehd. ex Guill.

ROSACEAE

Rose Family

vilmorinii Rehd. ex Guill.

PYRUS L.

ROSACEAE

Pear

Rose Family

amygdaliformis Villars

betulifolia Bunge

bretschneideri Rehd.

calleryana Decne.

calleryana Decne. 'Aristocrat'

calleryana Decne. 'Bradford'

* calleryana Decne. 'Capital'

calleryana Decne. 'Chanticleer'

$\star$ calleryana Decne. 'Whitehouse' calleryana Decne. var. dimorphophylla

(Makino) Koidz.

$\times$ canescens Spach

communis $\mathrm{L}$.

elaeagrifolia Pall.

fauriei Schneid.

kawakamii Hayata

$\times$ lecontei Rehd.

$\times$ michauxii Bosc ex Poir.

nivalis Jacq.

pashia Buch.-Ham. ex D.Don

phaeocarpa Rehd.

pyrifolia (Burm.f.) Nakai

pyrifolia (Burm.) Nakai 'Chojure'

pyrifolia (Burm.f.) Nakai var. culta

(Makino) Nakai

regelii Rehd.

salicifolia Pall.

salicifolia Pall. 'Pendula'

ussuriensis Maxim.

ussuriensis Maxim. var. hondoensis

(Kikuchi \& Nakai) Rehd.

ussuriensis Maxim. var. ovoidea Rehd.

QUERCUS L.

FAGACEAE

Oak

Beech Family

acutissima Carruthers

alba L.

alba $\times$ Q. virginiana

arkansana Sarg.

asheana Little

austrina Small

bicolor Willd.

$\times$ bimundorum Palm.

cerris L.

chenii Nakai

coccinea Muenchh.

$\times$ comptonae Sarg.

dentata Thunb. ex J.A.Murr.

fabri Hance

falcata Michx.

falcata Michx. var. pagodifolia Ell.

frainetto Tenore

georgiana M.A.Curtis

gilva B1.

glandulifera $\mathrm{Bl}$.

glauca Thunb. ex J.A.Murray

haas Kotschy

hemisphaerica Bartram ex Willd.

$\times$ heterophylla Michx.f.

imbricaria Michx.

incana Bartram

laevis Walt.

laurifolia Michx.

lyrata Walt.

macrocarpa Michx. 
margaretta Ashe

michauxii Nutt.

minima (Sarg.) Small

mongolica Fischer var. grosseserrata

(Bl.) Rehd. \& Wils.

myrsinifolia Bl.

nigra $\mathrm{L}$.

nuttallii Palm.

palustris Muenchh.

petraea (Mattusch.) Liebl.

petraea (Mattusch.) Liebl.

'Falkenbergensis'

petraea (Mattusch.) Liebl. 'Geisleri'

petraea (Mattusch.) Liebl. 'Muscaviensis'

phellos L.

phillyraeoides A.Gr.

prinus L.

pubescens Willd.

robur $\mathrm{L}$.

robur L. 'Cristata'

robur L. 'Cucullata'

robur L. 'Salicifolia'

robur L. f. fastigiata (Lam.) O.Schwarz

(robur f. fastigiata $\times$ Q. petraea

'Muscaviensis') 'Columna'

$\times$ rosacea Bechst.

rubra L.

$\times$ sargentii Rehd.

shumardii Buckl.

stellata Wang.

suber L.

variabilis $B 1$.

velutina Lam.

virginiana Mill.

RHAMNUS L.

RHAMNACEAE

alaternus L.

carolinianus L.

catharticus L.

davuricus Pall.

japonicus Maxim.

utilis Decne.

\section{RHAPHIOLEPIS Lindl.}

ROSACEAE

Rose Family

indica (L.) Lindl.

indica (L.) Lindl. 'Enchantress'

indica (L.) Lindl. 'Snow'

umbellata (Thunb. ex J.A.Murr.) Makino

umbellata (Thunb. ex J.A.Murr.) Makino

var. integerrima (Hook. \& Arn.) Rehd.

'Majestic Beauty'

'Pink Cloud'

'Springtime’
RHAPIDOPHYLLUM H.Wendl. \& Drude

ARECACEAE

Palm Family

hystrix (Pursh) H.Wendl. \& Drude

RHAPIS L.f. ex Ait.

ARECACEAE Palm Family

excelsa (Thunb. ex J.A.Murr.) A.Henry

\section{RHODODENDRON L.} ERICACEAE

Heath Family

alabamense Rehd.

amagianum Makino

arborescens (Pursh) Torr.

atlanticum (Ashe) Rehd.

augustinii Hemsl.

augustinii Hemsl. 'Crater Lake'

austrinum (Small) Rehd.

bakeri (W.P.Lemmon \& McKay) Hume

$\star$ bakeri (W.P.Lemmon \& McKay) Hume

'Camp's Red'

brachycarpum D.Don ex G.Don

calendulaceum (Michx.) Torr.

canescens (Michx.) Sweet

carolinianum Rehd.

carolinianum Rehd. 'Album'

carolinianum Rehd. 'Luteum'

(carolinianum $\times R$. ciliatum)

'Dora Amateis'

(carolinianum $\times \boldsymbol{R}$. dauricum)

'P. J. M.'

(carolinianum $\times \boldsymbol{R}$. dauricum)

'P. J. Mezitt'

carolinianum $\times \boldsymbol{R}$. mucronulatum

carolinianum $\times \boldsymbol{R}$. mucronulatum

'Conewago'

carolinianum $\times \boldsymbol{R}$. racemosum

(carolinianum $\times \boldsymbol{R}$. racemosum)

'Conestoga'

catawbiense Michx.

(catawbiense $\times \boldsymbol{R}$. arboreum

ssp. arboreum) 'John Walter'

(catawbiense $\times \boldsymbol{R}$. griffithianum)

'Cynthia'

(catawbiense 'Atrosanguineum' $\times$

$\boldsymbol{R}$. fortunei) 'David Gable'

catawbiense hybrid 'Album Elegans'

catawbiense hybrid 'Everestianum'

catawbiense hybrid 'Gomer Waterer'

catawbiense hybrid

'Mrs. Charles S. Sargent'

catawbiense hybrid 'Roseum Elegans'

(caucasicum $\times \boldsymbol{R}$. ponticum

var. album) 'Cunningham's White'

chapmanii A.Gr. 
$\star$ (chapmanii $\times R$. minus) 'Bowie' dauricum $\mathrm{L}$.

decorum Franch.

degronianum Carr. ssp. heptamerum

(Maxim.) Hara

eriocarpum (Hayata) Nakai 'Gumpo'

(fastigiatum $\times \boldsymbol{R}$. carolinianum)

'Ramapo'

flammeum (Michx.) Sarg.

fortunei Lindl.

fortunei Lindl. ssp. discolor (Franch.)

Chamb.

(griffithianum $\times \boldsymbol{R}$. fortunei

ssp. fortunei) 'Pink Diamond'

griffithianum hybrid

'Mrs. E. C. Stirling'

griffithianum hybrid

'The Hon. Jean Marie de Montague'

indicum (L.) Sweet

japonicum (A.Gr.) Suring.

kaempferi Planch.

kaempferi Planch. 'Dorsett'

kaempferi $\times$ R. komiyamae

kanahirae Wils.

keiskei Miq.

keiskei Miq. 'Red Flare'

keiskei $\times R$. racemosum

$(($ keiskei $\times R$. racemosum) $\times R$. keiskei)

'Mary Fleming'

kiusianum Makino

lasiostylum Hayata

luteum Sweet

macrosepalum Maxim.

macrosepalum Maxim. 'Linearifolium'

maximum $\mathrm{L}$.

micranthum Turcz.

minus Michx.

(minus $\times \boldsymbol{R}$. hirsutum) 'Myrtifolium'

mucronulatum Turcz.

mucronulatum Turcz. 'Albiflorum'

mucronulatum Turcz. 'Cornell Pink'

mucronulatum Turcz. 'Wheeldon's Pink'

oblongifolium (Small) Millais

occidentale (Torr. \& A.Gr.) A. Gr.

oldhamii Maxim.

oreodoxa Franch. var. fargesii (Franch.)

Chamb.

ovatum (Lindl.) Planch. ex Maxim.

periclymenoides (Michx.) Shinners

ponticum sport 'Ponticum Variegatum'

(ponticum $\times R$. maximum)

'Ponticum Roseum'

ponticum seedling 'Anah Kruschke'

prinophyllum (Small) Millais

prunifolium (Small) Millais

$\star$ prunifolium (Small) Millais 'Hohman'

punctatum Andr. racemosum $\times \boldsymbol{R}$. keiskei

(racemosum $\times \boldsymbol{R}$. muctonulatum)

'Conemaugh'

reticulatum D.Don ex G.Don

rubropilosum Hayata

scabrum (selection or hybrid)

'Phoeniceum'

schlippenbachii Maxim.

serpyllifolium (A.Gr.) Miq.

serrulatum (Small) Millais

simiarum Hance

simsii Planch.

smirnowii Traut.

tamurae (Makino) Masam.

tosaense Makino

vaseyi A.Gr.

vaseyi A.Gr. f. album (Bean) Rehd.

(veitchianum $\times R$. edgeworthii)

'Forsterianum'

vernicosum Franch.

viscosum (L.) Torr.

weyrichii Maxim.

williamsianum Rehd. \& Wils.

yakushimanum Nakai ssp. makinoi

(Tagg) Chamb.

yakushimanum Nakai

ssp. yakushimanum

yakushimanum Nakai

ssp. yakushimanum 'Ken Janeck'

yedoense Maxim. ex Regel

var. poukhanense (Lev.) Nakai

'A. Bedford'

'Addy Wery'

'Amethystinum'

'Amoenum'

'Annie E. Endtz'

'April Rose'

'Autumn Glory'

'Azma'

'Azor'

'Ballerina'

'Beauty of Littleworth'

'Ben Morrison'

'Beni kirishima'

'Betty Wormald'

'Bibiani'

'Blaauw's Pink'

'Blue Tit'

'Bosley 1020'

'Brandywine'

'Brick-dust Red'

'Cadis'

'Carmen'

'Carminata Splendens'

'Cerise'

'Cherry Red'

'Christmas Cheer' 
'Christopher Wren'

'Coccinea Major'

'Coccinea Speciosa'

'Comte de Newport'

'Coral Bells'

'Coral Cluster'

'Corneille'

'Dexter Purple'

'Dexter's Champagne'

'Duc de Rohan'

'Early Lavender'

'Early Salmon'

'Evening Glow'

'Fawley'

'Fedora'

'Flamingo'

'Flowerdale Pink'

'Flowerdale Red'

'Formosa'

'George Lindley Taber'

'Georgiana Maclay'

'Giant Elegans'

'Giant White'

'Gibraltar'

Glenn Dale Azaleas (see App. C)

'Glory of Sunninghill'

'Golden Dream'

'Helen'

'Hinode giri'

'Hugh Koster'

'Iveryana'

'Koran yuki'

'Koromo shikibu'

'Kristin'

'La Roche'

'Lady Mulberry'

'Late Orchid'

'Lawsal'

'Lizette'

'Lucinda'

'Madame de Bruin'

'Magnolia Alba'

'Maria Derby'

'Mrs. Betty Robertson'

'Mrs. G. G. Gerbing'

'Mrs. LBJ'

'Mrs. R. S. Holford'

'Mucronatum'

'Nadine'

'Narcissiflora'

'Nodding Bells'

'Obtusum'

'Obtusum Album'

'Oritani'

'Palestrina'

'Pioneer'

'Plenum'
'President Claeys'

'Pride of Dorking'

'Pride of Mobile'

'Purple Splendor'

'Queen of Orange'

'Red Formosa'

'Red Head'

'Robert Allison'

'Roseum Magnificum'

'Royal Red'

'Royal Splendor'

'Salmon King'

'Shrimp Pink'

'Small Elegans'

'Spring Glory'

'Stewartstonian'

'Suetsuma'

'Sunset'

'Susugonoito'

'Tiffany'

'Trilby'

'Viscosepala'

'Wheatley'

'William Bull'

'Windbeam'

'Winterthur'

'Wissahickon'

'Wyanokie'

'Yae shojo'

RHODOLEIA Champ. ex Hook.

HAMAMELIDACEAE Witch-hazel Family

championii Hook.

RHODOTYPOS Sieb. \& Zucc. Jetbead ROSACEAE Rose Family

scandens (Thunb.) Makino

RHUS L.

Sumac

ANACARDIACEAE

Cashew Family

aromatica Ait.

chinensis Mill.

copallina $\mathrm{L}$.

glabra L.

michauxii Sarg.

punjabensis J.L.Stewart var. sinica

(Diels) Rehd. \& Wils.

typhina $\mathrm{L}$.

typhina L. 'Laciniata' 
RIBES L. Currant, Gooseberry SAXIFRAGACEAE (Ribesioideae) Saxifrage Family

alpinum L.

curvatum Small

echinellum (Cov.) Rehd.

odoratum H.Wendl.

sanguineum Pursh 'Pulborough Scarlet'

uva-crispa $\mathrm{L}$.

uva-crispa L. 'Pixwell'

ROBINIA L.

FABACEAE (Faboideae)

Locust Bean Family

$\times$ ambigua Poir.

$\times$ ambigua Poir. 'Decaisneana'

boyntonii Ashe

hispida $\mathrm{L}$.

pseudoacacia L.

pseudoacacia L. 'Bicolor'

pseudoacacia L. 'Burgundy'

pseudoacacia L. 'Frisia'

pseudoacacia L. 'Purple Rose'

pseudoacacia L. 'Pyramidalis'

pseudoacacia L. 'Sandraudiga'

pseudoacacia L. 'Tortuosa'

pseudoacacia L. 'Unifoliola'

pseudoacacia L. var. rectissima Raber

viscosa Vent.

ROSA L.

ROSACEAE

acicularis Lindl.

$\times$ alba L.

$\times$ alba L. 'Celeste'

$\times$ alba L. 'Semiplena'

$(\times a l b a \times R$. centifolia)

'Maiden's Blush'

(? $\times$ alba $\times$ Damask Hybrid) 'Koenigin

von Daenemark'

$\times$ anemonoides Rehd. 'Ramona'

banksiae Ait.f. var. banksiae

'Alba Plena'

banksiae Ait.f. var. banksiae 'Lutea'

banksiae Ait.f. var. normalis Regel

'Lutescens'

blanda Ait.

bracteata J.C.Wendl.

brunonii Lindl.

canina $\mathrm{L}$.

carolina $\mathrm{L}$.

centifolia $\mathrm{L}$.

centifolia L. 'Bullata'

centifolia L. 'Cristata' centifolia L. 'Muscosa'

centifolia L. 'Rose des_Peintres'

centifolia L. 'Variegata'

chinensis Jacq.

chinensis Jacq. 'Minima'

chinensis Jacq. 'Mutabilis'

chinensis Jacq. 'Viridiflora'

(chinensis $\times \boldsymbol{R}$. gigantea) 'Old Blush'

$\times$ damascena Mill.

$\times$ damascena Mill. var. semperflorens

(Loisel.) Rowley

$\times$ damascena Mill. 'Trigintipetala'

$\times$ damascena Mill. 'Versicolor'

$(\times$ damascena var. semperflorens $\times$

R. pimpinellifolia) 'Stanwell Perpetual'

$\times$ dupontii Deseglise

foetida J.Herrm.

foetida J.Herrm. 'Bicolor'

foetida J.Herrm. 'Perseana'

forrestiana Boulenger

$\times$ fortuneana Lindl.

$\times$ francofurtana Muenchh. 'Empress

Josephine'

gallica L. 'Conditorum'

gallica L. 'Versicolor'

glauca Pourr.

$\times$ harisonii Rivers

laevigata Michx.

(laxa $\times$ R. pimpinellifolia) 'Suzanne'

$\times$ lheritieranea Thory 'Gracilis'

moschata J.Herrm.

moschata J.Herrm. 'Plena'

moyesii Hemsl. \& Wils.

multibracteata Hemsl. \& Wils.

multiflora Thunb. ex J.A.Murr

multiflora Thunb. ex J.A.Murr. 'Carnea'

multiflora Thunb. ex J.A.Murr.

'Platyphylla'

(multiflora $\times$ R. 'Reve d'Or') 'Aglaia'

$\times$ noisettiana Thory

odorata (Andr.) Sweet

odorata (Andr.) Sweet 'Fun Jwan Lo'

odorata (Andr.) Sweet var. pseudindica

(Lindl.) Rehd. 'Fortune's Double Yellow'

palustris Marsh.

$\times$ paulii Rehd.

pimpinellifolia L.

pimpinellifolia L. var. altaica (Willd.)

Thory

roxburghii Tratt. 'Roxburghii'

roxburghii Tratt. f. normalis Rehd.

$\&$ Wils.

rubiginosa $\mathrm{L}$.

rugosa Thunb. ex J.A.Murr.

rugosa Thunb. ex J.A.Murr. 'Alba'

rugosa Thunb. ex J.A.Murr. 'Hanosa'

(rugosa hybrid $\times$ 'Gloire de Dijon')

'Conrad Ferdinand Meyer' 
sempervirens L. 'Rampant'

sempervirens hybrid 'Felicite et

Perpetue'

setigera Michx.

(setigera $\times \boldsymbol{R}$. gallica hybrid)

'Baltimore Belle'

virginiana Mill.

$\times$ waitziana Tratt. 'Macrantha'

wichuraiana Crepin

wichuraiana Crepin var. poteriifolia

Koidz.

(wichuraiana $\times$ R. multiflora)

'America'

(wichuraiana $\times$ 'Champion of the

World') 'May Gueen'

(wichuraiana $\times$ 'Perle des Jardins')

'Gardenia'

(wichuraiana $\times$ 'Shirley Hibberd')

'Alberic Barbier'

woodsii Lindl.

xanthina f. hugonis (Hemsl.) Roberts

'Agathe Incarnata'

'Alfred de Dalmas'

'Alika'

'Anais Segalas'

'Angelica Minor'

'Arielle'

'Aurora'

'Baronne Prevost'

'Belinda'

'Belle Amour'

'Belle de Crecy'

'Belle Isador'

'Belle Vichysoise'

'Blanc Double de Coubert'

'Blanche Moreau'

'Blanchefleur'

'Blush Boursault'

'Camaieux'

'Captain Christy'

'Cardinal de Richelieu'

'Catherine Mermet'

'Celsiana'

'Champney's Pink Cluster'

'Charles de Mills'

'Commandant Beaurepaire'

'Comte de Chambord'

'Cramoisi Superieur'

'Crested Jewel'

'De la Grifferaie'

'Delicata'

'Duc de Fitzjames'

'Duc de Guiche'

'Duchesse de Brabant'

'Duchesse de Montebello'

'Ernst Calvat'

'Fantin Latour'

'Felicite Parmentier'
'F. J. Grootendorst'

'Fraser's Pink Musk'

'Frau Dagmar Hartopp'

'Frau Karl Druschki'

'General Jacqueminot'

'General Kleber'

'Gloire de Dijon'

'Gloire des Mosseux'

'Henri Martin'

'Hermosa'

'Honorine de Brabant'

'Ipsilante'

'Isabella Sprunt'

'Jacques Cartier'

'Jeanne d'Arc'

'Jeanne de Montfort'

'Juno'

'La France'

'Leda'

'Louis Philippe'

'Louise Odier'

'Mabel Morrison'

'Macrantha'

'Marcel Bourgouin'

'Marie Louise'

'Mme. Alfred Carriere'

'Mme. Hardy'

'Mme. Isaac Pereire'

'Mme. Legras de Saint Germain'

'Mme. Louis Leveque'

'Mme. Pierre Oger'

'Mme. Plantier'

'Niphetos'

'Nuits de Young'

'Oeillet Panachee'

'Officinalis'

'Omar Khayyam'

'Paul Neyron'

'Perle d'Or'

'Petite de Hollande'

'Pompon Elegant'

'President de Seze'

'Prince Camille de Rohan'

'Reine des Violettes'

'Reine Victoria'

'Reve d'Or'

'Rivers' George IV'

'Rose de Meaux'

'Safrano'

'Salet'

'Schneezwerg'

'Serratipetala'

'Shailer's Provence'

'Sombreuil'

'Tour de Malakoff'

'Tuscany'

'Tuscany Superb'

'Variegata di Bologna' 
ROSMARINUS L.

LAMIACEAE

Mint Family

officinalis L.

officinalis L. 'Prostratus'

RUBUS L.

ROSACEAE

Rose Family

calycinoides Hayata \& Koidz.

cockburnianus Hemsl.

odoratus L.

rosifolius Sm. 'Coronarius'

RUSCUS L.

LILIACEAE

Lily Family

aculeatus L.

hypoglossum $\mathrm{L}$.

RUSSELIA JaCq.

SCROPHULARIACEAE Figwort Family

equisetiformis Schlechtend. \& Cham.

SABAL Adans.

ARECACEAE Palm Family

minor (Jacq.f.) Pers.

palmetto (Walt.) Lodd. ex J.A. \& J.H.

Schultes

SAGERETIA Brongn.

RHAMNACEAE Buckthorn Family

thea (Osbeck) M.C.Johnst.

SALIX L.

SALICACEAE

Willow

Willow Family

aegyptiaca $\mathrm{L}$.

alba L.

alba L. var. vitellina (L.) Stokes

'Pendula'

alba $\times$ S. fragilis

(alba var. vitellina $\times \mathbf{S}$. babylonica)

'Chrysocoma'

babylonica L.

babylonica L. 'Crispa'

babylonica L. 'Tortuosa'

$\times$ bicolor Ehrh.

$\times$ blanda Anderss.

caprea L.

caprea $\times \mathbf{S}$. elaeagnos

caprea $\times$ S. purpurea

chaenomeles Kimura

cinerea L. cordata Michx.

$\times$ dasyclados Wimmer-

discolor Muhl.

elaeagnos Scop.

fragilis L.

gracilistyla Miq.

gracilistyla Miq. 'Melanostachys'

hookeriana Barratt var. tomentosa

J.K.Henry

humilis Marsh.

integra Thunb. ex J.A.Murr.

interior Rowlee

irrorata Anderss.

japonica Thunb. ex J.A.Murr.

$\times$ laestadiana Hartm.

miyabeana Seemen

muscina Dode

$\times$ myricoides Muhl.

myrsinifolia Salisb.

nigra $\mathrm{L}$.

pentandra $\mathrm{L}$.

$\times$ pontederana Willd.

purpurea L.

purpurea L. 'Eugenei'

purpurea L. 'Pendula'

purpurea L. f. gracilis (Gren. \&

Godr.) Schneid.

purpurea L. var. amplexicaulis (Bory

\& Chaub.) Boiss.

repens L. ssp. argentea (Sm.) A.A. \&

E.G. Camus

rigida Muhl.

$\times$ rubra Huds.

sachalinensis F.Schmidt 'Sekka'

$\times$ sepulcralis Simonk.

sericans Tausch ex A. Kerner

sericea Marsh.

$\times$ seringeana Gaudin

$\times$ smithiana Willd.

subopposita Miq. 'Ginryu'

syrticola Fern.

$\times$ tsugaluensis Koidz. 'Ginme'

udensis Traut.

variegata Franch.

vulpina Anderss.

SALVIA L.

LAMIACEAE

Mint Family

greggii A.Gr.

leucantha Cav. 
SAMBUCUS L.

Elder CAPRIFOLIACEAE Honeysuckle Family

canadensis L.

ebulus L.

nigra L. 'Aurea'

racemosa L. ssp. pubens (Michx.)

H.House

racemosa L. ssp. sieboldiana (Miq.) Hara

SANTOLINA L.

ASTERACEAE

Aster Family

chamaecyparissus L.

SAPINDUS L. SAPINDACEAE

Soapberry Soapberry Family

drummondii Hook. \& Arn.

marginatus Willd.

mukorossi Gaertn.

SAPIUM P.Br.

EUPHORBIACEAE

Tallow Tree

Spurge Family

japonicum (Sieb. \& Zucc.) Pax \&

K.Hoffm.

sebiferum (L.) Roxb.

SARCOCOCCA Lindl. BUXACEAE

Sweet Box Boxwood Family

hookeriana Baill. var. digyna Franch.

hookeriana Baill. var. humilis Rehd. \&

Wils.

ruscifolia Stapf

SASA Makino \& Shibata

POACEAE

Grass Family

veitchii (Carr.) Rehd.

SASSAFRAS T. Nees \& Eberm.

LAURACEAE Laurel Family

albidum (Nutt.) Nees

SATUREJA L.

LAMIACEAE Mint Family

montana L.

SCHINUS L.

ANACARDIACEAE

Peppertree

Cashew Family
SCHIZOPHRAGMA Sieb. \& Zucc.

SAXIFRAGACEAE (Hydrangeoideae)

Saxifrage Family

hydrangeoides Sieb. \& Zucc.

SCLADOPITYS Sieb. \& Zucc.

TAXODIACEAE Taxodium Family

verticillata (Thunb. ex J.A.Murr.) Sieb. \& Zucc.

SEBASTIANA Spreng.

EUPHORBLACEAE

Spurge Family

ligustrina (Michx.) Muell.-Arg.

SEMIARUNDINARIA Makino ex Nakai POACEAE

Grass Family

fastuosa (Latour-Marl. ex Mitf.) Makino ex Nakai

SENECIO L.

ASTERACEAE

Aster Family

salignus $\mathrm{DC}$.

SEQUOLA Endl. California Redwood

TAXODIACEAE Taxodium Family

sempervirens (D.Don) Endl.

sempervirens (D.Don) Endl. 'Adpressa'

SEQUOLADENDRON J. Buchh.

TAXODIACEAE

Taxodium Family

giganteum (Lindl.) J.Buchh.

SERENOA Hook.f.

ARECACEAE Palm Family

repens (Bartram) Small

SERISSA Comm. ex Juss.

RUBIACEAE Madder Family

japonica (Thunb.) Thunb.

SESBANIA Scop.

FABACEAE (Faboideae) Bean Family

drummondii (Rydb.) Cory

punicea (Cav.) Benth. 
SEVERINIA Tenore ex Endl. RUTACEAE

Citrus Family

buxifolia (Poir.) Tenore

SHIBATAEA Makino ex Nakai

POACEAE

Grass Family

kumasaca (Zoll.) Makino

SINARUNDINARIA Nakai POACEAE Grass Family

nitida (Mitf.) Nakai

SIPHONOSMANTHUS Stapf OLEACEAE Olive Family

delavayi (Franch.) Stapf

SKIMMIA Thunb. Skimmia RUTACEAE Citrus Family

$\times$ foremanii H.Knight

japonica Thunb.

japonica Thunb. 'Fisheri'

japonica Thunb. 'Fructo-albo'

japonica Thunb. 'Nana'

japonica Thunb. 'Veitchii'

japonica Thunb. 'Wisley Red'

japonica Thunb. var. repens (Nakai)

Ohwi

laureola Sieb. \& Zucc.

reevesiana Fort.

SMILAX L.

SMILACACEAE

Greenbrier Greenbrier Family

biflora Sieb. ex Miq.

laurifolia L.

pumila Walt.

smallii Morong

SOLANUM L.

SOLANACEAE

Nightshade Family

diphyllum $\mathrm{L}$.

jasminoides Paxt.

rantonnetii Carr.

seaforthianum Andr.

wendlandii Hook.f.

SOPHORA L.

FABACEAE (Faboideae)

Bean Family

affinis T. \& $\mathrm{G}$.

davidii (Franch.) Skeels

japonica L. japonica L. 'Dot'

japonica L. 'Pendula' -

japonica L. 'Regent'

secundiflora (Ort.) Lag. ex DC.

SORBARIA (Ser. ex DC.) A.Braun

ROSACEAE

False Spirea

Rose Family

kirilowii (Regel) Maxim.

tomentosa (Lindl.) Rehd. var. tomentosa

$\times$ SORBARONIA Schneid.

ROSACEAE

Rose Family

fallax Schneid.

sorbifolia (Poir.) Schneid.

'Brilliantissima'

$\times$ SORBOCOTONEASTER Pojark.

ROSACEAE

Rose Family

pozdnjakovii Pojark.

$\times$ SORBOPYRUS Schneid.

ROSACEAE

Rose Family

auricularis (Knoop) Schneid.

'Bulbiformis'

SORBUS L.

ROSACEAE

Mountain Ash

Rose Family

alnifolia (Sieb. \& Zucc.) K.Koch

americana Marsh.

aria (L.) Crantz 'Magnifica'

aucuparia L.

aucuparia L. 'Cardinal'

aucuparia L. 'Fastigiata'

aucuparia L. 'Pendula'

domestica $\mathrm{L}$.

hybrida L.

pohuashanensis (Hance) Hedl.

torminalis Crantz

'Apricot Queen'

'Red Copper Glow'

SPARTIUM L.

FABACEAE (Faboideae) Bean Family

junceum $\mathrm{L}$.

SPIRAEA L.

ROSACEAE

Spirea

Rose Family

alba Du Roi

albiflora (Miq.) Zab.

arcuata Hook.f. 
$\times$ arguta Zab.

betulifolia Pall.

$\times$ billiardii Herincq

blumei G.Don

bullata Maxim.

$\times$ bumalda Burv.

$\times$ bumalda Burv. 'Anthony Waterer'

$\times$ bumalda Burv. 'Crispa'

$\times$ bumalda Burv. 'Goldflame'

cantoniensis Lour.

cantoniensis Lour. 'Lanceata'

chinensis Maxim.

japonica L.f.

japonica L.f. 'Alpina'

$\times$ lemoinei Zab. 'Alpestris'

$\times$ margaritae Zab.

miyabei Koidz.

nipponica Maxim. var. tosaensis (Yatabe) Makino

nipponica Maxim. var. tosaensis (Yatabe) Makino 'Snowmound'

prunifolia Sieb. \& Zucc.

salicifolia L.

$\times$ sanssouciana K.Koch

thunbergii Sieb. ex Bl.

thunbergii Sieb. ex Bl. 'Compacta'

tomentosa $\mathrm{L}$.

trilobata L. 'Swan Lake'

$\times$ vanhouttei (C. Briot) Zab.

STACHYURUS Sieb. \& Zucc.

STACHYURACEAE Stachyurus Family

chinensis Franch.

praecox Sieb. \& Zucc.

praecox Sieb. \& Zucc. 'Issai'

\section{STAPHYLEA L.}

STAPHYLEACEAE Bladdernut Family

colchica Steven

pinnata L.

trifolia L.

STEPHANANDRA Sieb. \& Zucc. ROSACEAE Rose Family

incisa (Thunb. ex J.A.Murr.) Zab.

incisa (Thunb. ex J.A.Murr.) Zab. 'Crispa' tanakae (Franch. \& Sav.) Franch. \& Sav.

STEWARTIA L.

THEACEAE Tea Family

malacodendron $\mathrm{L}$.

monadelpha Sieb. \& Zucc.

ovata (Cav.) Weatherby

pseudocamellia Maxim.

rostrata Spongberg

serrata Maxim.

sinensis Rehd. \& Wils.

STIGMAPHYLLON JuSS.

MALPIGHIACEAE Malpighia Family

ledifolium (HBK.) Small

STRANVAESIA Lindl.

ROSACEAE

Rose Family

davidiana Decne.

davidiana Decne. 'Lutea'

niitakayamensis (Hayata) Hayata

STYRAX L.

STYRACACEAE

americanus Lam.

dasyanthus Perk.

grandifolius Ait.

japonicus Sieb. \& Zucc.

japonicus Sieb. \& Zucc. 'Carillon'

japonicus Sieb. \& Zucc. 'Pink Chimes'

obassia Sieb. \& Zucc.

platanifolius Engelm.

SYAGRUS Mart.

ARECACEAE

Palm Family

romanzoffianum (Cham.) Glassman romanzoffianum $\times$ Butia capitata

$\times$ SYCOPARROTIA P.Endress \& J.Anliker

HAMAMELIDACEAE Witch-hazel Family semidecidua P.Endress \& J.Anliker

SYCOPSIS Oliv.

HAMAMELIDACEAE Witch-hazel Family

sinensis Oliv.

SYMPHORICARPOS Duham.

CAPRIFOLIACEAE Snowberry

albus (L.) S.F.Blake

albus (L.) S.F.Blake var. laevigatus

(Fern.) S.F.Blake

$\times$ chenaultii Rehd.

orbiculatus Moench 
SYMPLOCOS Jacq.

SYMPLOCACEAE

Sweetleaf Family

lucida Sieb. \& Zucc.

paniculata (Thunb. ex J.A.Murr.) Miq.

tinctoria (L.) L'Her.

SYRINGA L.

OLEACEAE

Olive Family

$\times$ chinensis Willd.

$\times$ henryi Schneid.

$\times$ hyacinthiflora (V.Lemoine) Rehd.

'Buffon'

$\times$ hyacinthiflora (V.Lemoine) Rehd.

'Charles Giant'

$\times$ hyacinthiflora (V.Lemoine) Rehd.

'Lamartine'

$\times$ hyacinthiflora (V.Lemoine) Rehd.

'Pocohantas'

$\times$ hyacinthiflora (V.Lemoine) Rehd. 'Vaubon'

josikaea Jacq.f. ex Reichenb.

laciniata Mill.

meyeri Schneid.

microphylla Diels

microphylla Diels 'Superba'

oblata Lindl. var. dilatata (Nakai) Rehd.

oblata Lindl. var. dilatata (Nakai) Rehd.

'Annabelle'

oblata Lindl. var. dilatata (Nakai) Rehd.

'Laurentian'

patula (Palib.) Nakai

patula (Palib.) Nakai 'Miss Kim'

persica L.

persica L. 'Laciniata'

$\times$ prestoniae McKelvey

'Ethel M. Webster'

pubescens Turcz.

reticulata (Bl.) Hara

villosa Vahl

villosa Vahl 'Crayton'

vulgaris $\mathrm{L}$. 'Alba'

vulgaris L. 'Alba Plena'

vulgaris L. 'Charles Joly'

vulgaris $\mathrm{L}$. 'Charles Nordine'

vulgaris L. 'Decaisne'

vulgaris L. 'Esther Staley'

vulgaris $\mathrm{L}$. 'Henri Robert'

vulgaris L. 'Jules Ferry'

vulgaris L. 'Katherine Havemeyer'

vulgaris L. 'Leon Gambetta'

vulgaris L. 'Marechal Lannes'

vulgaris L. 'Maurice Barres'

vulgaris L. 'Miss Ellen Willmott'

vulgaris L. 'Mme. Charles Souchet'

vulgaris L. 'Monge' vulgaris L. 'Mrs. Edward Harding'

vulgaris L. 'President-Grevy'

vulgaris $\mathrm{L}$. 'Primrose'

vulgaris $\mathrm{L}$. 'Priscella'

vulgaris L. 'Purpurea'

vulgaris L. 'Rene Jarry-Desloges'

vulgaris L. 'Sarah Sands'

vulgaris $\mathrm{L}$. 'Zulu'

yunnanensis Franch.

'Alexander's Perfection'

'Jessica'

TABERNAEMONTANA L.

APOCYNACEAE Dogbane Family

divaricata (L.) R.Br. ex Roem. \& J.A.

Schultes

TATWANLA Hayata

TAXODIACEAE

Taxodium Family

cryptomerioides Hayata

TAMARIX L.

TAMARICACEAE Tamarisk Family

parviflora $\mathrm{DC}$.

ramosissima Ledeb.

ramosissima Ledeb. 'Rubra'

ramosissima Ledeb. 'Summer Glow'

TAXODIUM L.C.Rich. TAXODIACEAE

Bald Cypress Taxodium Family

distichum (L.) L.C.Rich. 'Pendens'

distichum (L.) L.C.Rich. var. distichum

distichum (L.) L.C.Rich. var. nutans (Ait.)

Sweet

mucronatum Tenore

TAXUS L.

TAXACEAE

Yew

Yew Family

baccata $\mathrm{L}$.

baccata L. 'Adpressa'

baccata L. 'Adpressa Fowle'

baccata L. 'Amersfoort'

baccata L. 'Aurea'

baccata L. 'Aurea Marginata'

baccata L. 'Elegantissima'

baccata L. 'Fastigiata Robusta'

baccata L. 'Overeynderi'

baccata L. 'Repandens'

baccata L. f. fastigiata (Lindl.) Pilger

canadensis Marsh.

cuspidata Sieb. \& Zucc.

cuspidata Sieb. \& Zucc. 'Expansa' 
cuspidata Sieb. \& Zucc. 'Luteobaccata' cuspidata Sieb. \& Zucc. 'Minima' cuspidata Sieb. \& Zucc. 'Nana' cuspidata Sieb. \& Zucc. 'Nana Variegata' cuspidata Sieb. \& Zucc. 'Thompson' floridana Nutt.

$\times$ hunnewelliana Rehd. 'Richard Horsey'

$\times$ media Rehd.

$\times$ media Rehd. 'Citation'

$\times$ media Rehd. 'Flushing'

$\times$ media Rehd. 'Hicksii'

TECOMA Juss.

BIGNONIACEAE

Bignonia Family

stans (L.) HBK. var. angustata Rehd.

TECOMARIA (Endl.) Spach

BIGNONIACEAE

Bignonia Family

capensis (Thunb.) Spach

TERNSTROEMIA Mutis ex L.f.

THEACEAE Tea Family

gymnanthera (Wight \& Arn.) Sprague

TETRAPANAX (K.Koch) K.Koch

ARALIACEAE Ginseng Family

papyriferus (Hook.) K.Koch

$\begin{array}{llr}\text { TEUCRIUM } & \text { L. } & \text { Germander } \\ \text { LAMIACEAE } & & \text { Mint Family }\end{array}$

chamaedrys L.

$\times$ lucidrys Boom

lucidum L.

THAMNOCALAMUS Munro

POACEAE

Grass Family

spathaceus (Franch.) Soderstrom
THUJA L. CUPRESSACEAE
Arborvitae Cypress Family occidentalis $\mathrm{L}$.

occidentalis L. 'Alba'

occidentalis L. 'Aurea'

occidentalis L. 'Beaufort'

occidentalis L. 'Buchananii'

occidentalis L. 'Columna'

occidentalis L. 'Compacta'

occidentalis L. 'Endean'

occidentalis L. 'Fastigiata'

occidentalis L. 'Filifera' occidentalis L. 'Filiformis'

occidentalis L. 'Froebelii'

occidentalis L. 'Globosa'

occidentalis L. 'Globosa Rheindiana'

occidentalis L. 'Hetz Midget'

occidentalis L. 'Holmstrup'

occidentalis L. 'Hudsonica'

occidentalis L. 'Little Gem'

occidentalis L. 'Lutea'

occidentalis L. 'Malonyana'

occidentalis L. 'Ohlendorfii'

occidentalis L. 'Pendula'

occidentalis L. 'Pygmaea'

occidentalis L. 'Pyramidalis'

occidentalis L. 'Pyramidalis Nigra'

occidentalis L. 'Recurva Nana'

occidentalis L. 'Rheingold'

occidentalis L. 'Semperaurea'

occidentalis L. 'Spiralis'

occidentalis L. 'Stricta'

occidentalis L. 'Sunkist'

occidentalis L. 'Techny'

occidentalis L. 'Umbraculifera'

occidentalis L. 'Wareana'

occidentalis L. 'Wareana Lutescens'

occidentalis L. 'Woodwardii'

plicata J.Donn ex D.Don

plicata J.Donn ex D.Don 'Cuprea'

plicata J.Donn ex D.Don 'Rogersii'

plicata J.Donn ex D.Don 'Zebrina'

standishii (Gord.) Carr.

THUJOPSIS Sieb. \& Zucc. ex Endl. False or Hiba Arborvitae

\section{CUPRESSACEAE Cypress Family}

dolabrata (Thunb. ex L.f.) Sieb. \& Zucc. dolabrata (Thunb. ex L.f.) Sieb. \& Zucc.

'Nana'

dolabrata (Thunb. ex L.f.) Sieb. \& Zucc.

var. hondae Makino

THUNBERGIA Retz.

ACANTHACEAE Acanthus Family

grandiflora (Roxb. ex Rottl.) Roxb.

THYMUS L.

LAMIACEAE Mint Family

vulgaris $\mathrm{L}$.

TIBOUCHINA Aubl.

MELASTOMATACEAE Melastoma Family

urvilleana (DC.) Cogn. 
TILIA L.

TILIACEAE

Linden, Basswood Linden Family

americana $\mathrm{L}$.

cordata Mill.

$\times$ euchlora K.Koch

$\times$ europaea $\mathrm{L}$.

$\times$ moltkei Spaeth

mongolica Maxim.

platyphyllos Scop.

platyphyllos Scop. 'Laciniata'

platyphyllos Scop. 'Vitifolia'

tomentosa Moench

tomentosa Moench 'Pendula'

TIPUANA (Benth.) Benth.

FABACEAE (Faboideae)

Bean Family

tipu (Benth.) O.Ktze.

TOONA (Endl.) M.J.Roem.

MELIACEAE Mahogany Family

sinensis (Juss.) M.J.Roem.

TORREYA Arn.

TAXACEAE Yew Family

nucifera (L.) Sieb. \& Zucc.

nucifera (L.) Sieb. \& Zucc. 'Gold Strike' taxifolia Arn.

\section{TOXICODENDRON Mill.}

ANACARDIACEAE Cashew Family

radicans (L.) O.Ktze.

vernicifluum (Stokes) F.A.Barkley

\section{TRACHELOSPERMUM LEM. \\ APOCYNACEAE Dogbane Family}

asiaticum (Sieb. \& Zucc.) Nakai

difforme (Walt.) A.Gr.

jasminoides (Lindl.) Lem.

jasminoides (Lindl.) Lem. 'Variegatum'

jasminoides (Lindl.) Lem. var. pubescens Makino

TRACHYCARPUS H.Wendl. ARECACEAE Palm Family

fortunei (Hook.) H.Wendl.

TRIPTERYGIUM Hook.f.

CELASTRACEAE Staff-tree Family

regelii Sprague \& Takeda
TRITHRINAX Mart.

ARECACEAE - Palm Family

acanthocoma Drude

TROCHODENDRON Sieb. \& Zucc. TROCHODENDRACEAE

Trochodendron Family

aralioides Sieb. \& Zucc.

TSUGA Carr.

PINACEAE

Hemlock

Pine Family

canadensis (L.) Carr.

canadensis (L.) Carr. 'Abbott's Dwarf'

canadensis (L.) Carr. 'Angustifolia'

canadensis (L.) Carr. 'Armistice'

canadensis (L.) Carr. 'Beaujean'

canadensis (L.) Carr. 'Bennett'

canadensis (L.) Carr. 'Boulevard'

canadensis (L.) Carr. 'Brandley'

canadensis (L.) Carr. 'Cinnamomea'

canadensis (L.) Carr. 'Curtis Ideal'

canadensis (L.) Carr. 'Curtis Spreader'

canadensis (L.) Carr. 'Doc's Choice'

canadensis (L.) Carr. 'Doran'

canadensis (L.) Carr. 'Fastigiata'

canadensis (L.) Carr. 'Gensch White'

canadensis (L.) Carr. 'Globosa'

canadensis (L.) Carr. 'Hawkersmith

Weeping'

canadensis (L.) Carr. 'Henry Hohman'

canadensis (L.) Carr. 'Jacqueline

Verkade'

canadensis (L.) Carr. 'Jervis'

canadensis (L.) Carr. 'Kelsey's Weeping'

canadensis (L.) Carr. 'Macrophylla'

canadensis (L.) Carr. 'Minima'

canadensis (L.) Carr. 'Minuta'

canadensis (L.) Carr. 'Pendula'

canadensis (L.) Carr. 'Sargentii'

canadensis (L.) Carr. 'Verkade

Recurved'

canadensis (L.) Carr. 'Von Helms'

canadensis (L.) Carr. 'Youngcone'

caroliniana Engelm.

chinensis (Franch.) Pritz.

diversifolia (Maxim.) Mast.

sieboldii Carr.

ULMUS L.

ULMACEAE

Elm

Elm Family

alata Michx.

americana $\mathrm{L}$.

americana L. 'Augustine Ascending'

americana L. 'Moline' 
crassifolia Nutt.

elliptica K. Koch

glabra Huds.

glabra Huds. 'Camperdownii'

$\times$ hollandica Mill.

$\times$ hollandica Mill. 'Belgica'

$\times$ hollandica Mill. 'Dauvessei'

$\times$ hollandica Mill. 'Major'

$\star \times$ hollandica Mill. 'Pioneer'

$\times$ hollandica Mill. 'Superba'

japonica (Rehd.) Sarg.

japonica $\times U$. wilsoniana

laevis Pall.

macrocarpa Hance

minor Mill.

minor Mill. 'Christine Buisman'

minor Mill. 'Sarniensis'

minor Mill. 'Umbraculifera'

minor Mill. 'Wredei'

parvifolia Jacq.

parvifolia Jacq. 'Drake'

$\star$ parvifolia Jacq. 'Dynasty'

parvifolia Jacq. 'Frosty'

parvifolia Jacq. 'Hokkaido'

procera Salisb.

procera Salisb. 'Marginata'

pumila L.

$\star$ (pumila $\times\left(\left(U_{.} \times\right.\right.$hollandica 'Vegeta' $\times$

$U$. minor $) \times(U$. pumila var. arborea $\times$

U. minor 'Hoersholm'))) 'Homestead'

rubra Muhl.

serotina Sarg.

thomasii Sarg.

UNGNADIA Endl.

SAPINDACEAE

Soapberry Family

speciosa Endl.

VACCINIUM L.

ERICACEAE

Blueberry

Heath Family

amoenum Ait.

arboreum Marsh.

bracteatum Thunb. ex J.A.Murr.

corymbosum L.

crassifolium Andr.

crassifolium Andr. ssp. crassifolium

'Wells Delight'

crassifolium Andr. ssp. sempervirens

(Rayner\&Henderson) Kirkman\&Bal.

'Bloodstone'

cylindraceum Sm.

myrsinites Lam.

simulatum Small

stamineum L.

tenellum Ait. vacillans Torr.

vitis-idaea $\mathrm{L}$.

\section{VIBURNUM L.}

CAPRIFOLIACEAE Honeysuckle Family

acerifolium L.

awabuki K.Koch

betulifolium Batal.

bitchiuense Makino

$\times$ bodnantense Aberc. 'Dawn'

$\times$ bodnantense Aberc. 'Deben'

brachybotryum Hemsl.

buddleifolium Wright

burejaeticum Regel \& Herd.

$\times$ burkwoodii Burkw. \& Skipwith

$\times$ burkwoodii Burkw. \& Skipwith

'Chenault'

$\star \times$ burkwoodii Burkw. \& Skipwith 'Conoy'

$\star(\times$ burkwoodii $\times$ V. carlesii) 'Mohawk'

calvum Rehd.

$\times$ carlcephalum Burkw. ex Pike

$\star \times$ carlcephalum Burkw. ex Pike 'Cayuga'

$\star$ ( $\times$ carlcephalum 'Cayuga' $\times$ V. utile $)$

'Chesapeake'

$\star$ ( $\times$ carlcephalum 'Cayuga' $\times$ V. utile $)$

'Eskimo'

carlesii Hemsl.

carlesii Hemsl. 'Compacta'

cassinoides L.

cinnamomifolium Rehd.

cylindricum D.Don

dasyanthum Rehd.

dentatum $\mathrm{L}$

dilatatum Thunb. ex J.A.Murr.

$\star$ dilatatum Thunb. ex J.A.Murr. 'Catskill'

$\star$ dilatatum Thunb. ex J.A.Murr. 'Erie'

* dilatatum Thunb. ex J.A.Murr. 'Iroquois'

$\star$ (dilatatum $\times$ V. lobophyllum) 'Oneida'

erosum Thunb. ex J.A.Murr.

farreri Stearn

foetidum Wall. var. rectangulatum

(Graebn.) Rehd.

fordiae Hemsl.

$\times$ hillieri Stearn

hirtulum Rehd.

hupehense Rehd.

ichangense (Hemsl.) Rehd.

japonicum (Thunb. ex J.A.Murr.) Spreng.

$\star$ (japonicum $\times$ V. dilatatum) 'Chippewa' $\times$ juddii Rehd.

lantana L.

* lantana L. 'Mohican'

lantanoides Michx.

lentago L.

lentago L. f. sphaerocarpum (Fern.)

Rehd. 
lobophyllum Graebn.

$\star$ (lobophyllum $\times$ V. japonicum) 'Huron'

luzonicum Rolfe

macrocephalum Fort. f. keteleeri (Carr.)

Rehd.

macrocephalum Fort. f. macrocephalum molle Michx.

mullaha Buch.-Ham. ex D.Don

nudum L.

obovatum Walt.

odoratissimum Ker-Gawl.

opulus L.

opulus L. 'Roseum'

ovatifolium Rehd.

plicatum Thunb. f. lanceolatum (Rehd.)

Rehd.

plicatum Thunb. f. parvifolium (Miq.)

Rehd.

plicatum Thunb. f. plicatum

plicatum Thunb. f. plicatum 'Newport'

plicatum Thunb. f. tomentosum (Thunb.

ex J.A.Murr.) Rehd.

plicatum Thunb. f. tomentosum (Thunb.

ex J.A.Murr.) Rehd. 'Mariesii'

plicatum Thunb. f. tomentosum (Thunb.

ex J.A.Murr.) Rehd. 'Mt. Fuji'

plicatum Thunb. f. tomentosum (Thunb.

ex J.A.Murr.) Rehd. 'St. Keverne'

$\star$ plicatum Thunb. f. tomentosum (Thunb.

ex J.A.Murr.) Rehd. 'Shoshoni'

$\star$ (plicatum Thunb. f. tomentosum

(Thunb. ex J.A. Murr.) Rehd. $\times$

V. plicatum f. tomentosum 'Mariesii')

'Shasta'

plicatum Thunb. var. rotundifolium

Rehd.

prunifolium L.

recognitum Fern.

$\times$ rhytidocarpum E.Lemoine

$\times$ rhytidophylloides Suring.

$\star$ ×rhytidophylloides Suring. 'Alleghany'

$\times$ rhytidophylloides Suring. 'Willow

Leaf'

$\times$ rhytidophylloides Suring.

'Willowwood'

rhytidophyllum Hemsl.

rhytidophyllum Hemsl. 'Ben Blackburn'

rhytidophyllum Hemsl. 'Roseum'

rhytidophyllum Hemsl. 'Variegatum'

(rhytidophyllum $\times$ V. utile) 'Pragense'

rufidulum Raf.

sargentii Koehne

$\star$ sargentii Koehne 'Onondaga'

* sargentii Koehne 'Susquehanna'

sargentii Koehne var. calvescens Rehd.

scabrellum Chapm. setigerum Hance

setigerum Hance 'Aurantiacum'

sieboldii Miq.

sieboldii Miq. 'Reticulatum'

$\star$ sieboldii Miq. 'Seneca'

suspensum Lindl.

tinus L.

trilobum Marsh.

urceolatum Sieb. \& Zucc.

utile Hemsl.

wrightii Miq.

VINCA L.

APOCYNACEAE

Periwinkle

Dogbane Family

major L.

major L. 'Oxyloba'

major L. 'Variegata'

minor $\mathrm{L}$.

minor L. 'Alba'

minor L. 'Atropurpurea'

minor L. 'Multiplex'

VTTEX L.

VERBENACEAE

Verbena Family

agnus-castus L.

agnus-castus L. 'Silver Spire'

negundo $\mathrm{L}$.

negundo L. 'Incisa'

rotundifolia L.f.

trifolia L. 'Variegata'

VITIS L.

VITACEAE

Grape

Grape Family

labrusca L.

mustangensis Buckl.

rotundifolia Michx.

WASHINGTONIA H.Wendl.

ARECACEAE

Washington Palm Palm Family

robusta $\mathrm{H}$.Wendl.

WEIGELA Thunb.

CAPRIFOLIACEAE Honeysuckle Family

decora (Nakai) Nakai

(floribunda $\times W$. coraeensis)

'Eva Rathke'

florida (Bunge) A.DC.

florida (Bunge) A.DC. 'Variegata'

(florida $\times$ W. 'Eva Rathke')

'Bristol Ruby' 
hortensis (Sieb. \& Zucc.) K.Koch

praecox (V.Lemoine) Bailey 'Rosea'

subsessilis (Nakai) Bailey

'Feerie'

'Mont Blanc'

'Newport Red'

WESTRINGLA Sm.

LAMIACEAE

Mint Family

rosmariniformis $\mathrm{Sm}$.

WIKSTROEMIA Endl.

THYMELAEACEAE Mezereum Family

trichotoma (Thunb.) Makino

WISTERIA Nutt.

FABACEAE (Faboideae)

Wisteria Bean Family

brachybotrys Sieb. \& Zucc.

brachybotrys Sieb. \& Zucc. 'Alba'

brachybotrys Sieb. \& Zucc. 'Murasaki

kapitan'

floribunda (Willd.) DC.

floribunda (Willd.) DC. 'Alba'

floribunda (Willd.) DC. 'Honbeni'

floribunda (Willd.) DC. 'Itoe kokuryu'

floribunda (Willd.) DC. 'Jabo'

floribunda (Willd.) DC. 'Koshigaya'

floribunda (Willd.) DC. 'Macrobotrys'

floribunda (Willd.) DC. 'Noda'

floribunda (Willd.) DC. 'Ossai'

floribunda (Willd.) DC. 'Rosea'

floribunda (Willd.) DC. 'Shino kapitan'

floribunda (Willd.) DC. 'Violacea Plena'

$\times$ formosa Rehd.

frutescens (L.) Poir.

frutescens (L.) Poir. 'Nivea'

sinensis (Sims) Sweet

sinensis (Sims) Sweet 'Alba'

venusta Rehd. \& Wils. 'Siro kapitan'

villosa Rehd.

XANTHOCERAS Bunge

SAPINDACEAE Soapberry Family

sorbifolium Bunge

XANTHORHIZA Marsh.

RANUNCULACEAE Buttercup Family
XYLOSMA J.G.Forst.

FLACOURTIACEAE

Flacourtia Family

congestum (Lour.) Merr.

flexuosum (HBK.) Hemsl.

YUCCA L. Adam's Needle, Spanish Dagger AGAVACEAE

Agave Family

aloifolia L. 'Marginata'

filamentosa $\mathrm{L}$.

filamentosa L. 'Bright Edge'

filamentosa L. 'Golden Sword'

gloriosa L.

recurvifolia Salisb.

smalliana Fern.

whipplei Torr.

ZAMLA L.

CYCADACEAE

Cycad Family

pumila L.

ZANTHOXYLUM L. Prickly Ash

RUTACEAE

Citrus Family

clava-herculis L.

fagara (L.) Sarg.

piperitum DC.

simulans Hance

ZELKOVA Spach

ULMACEAE

Elm Family

carpinifolia (Pall.) K.Koch

schneideriana Hand.-Mazz.

serrata (Thunb.) Makino

$\times$ verschaffeltii Nichols.

ZENOBLA D.DON

ERICACEAE

Heath Family

pulverulenta (Bartram) Pollard

pulverulenta (Bartram) Pollard f. nuda

(Vent.) Fern.

ZIZIPHUS Mill.

RHAMNACEAE Buckthorn Family

jujuba Mill.

jujuba Mill. 'Contorta'

simplicissima Marsh. 


\section{Appendix E: Vernacular Names}

This alphabetical list of the vernacular (or common) names of plants in the catalog has two sections: one that identifies the genera and another that identifies the species and cultivars. The list provides common names for about 40 percent of the catalog entries. Only a few common-name synonyms are given. In the list of species and cultivars, the common names are alphabetized under group names, such as alder, box, cypress, gum.

\section{Genera}

Adam's Needle: YUCCA

Akebia: AKEBIA

Alder: ALNUS

Arborvitae: THUJA

Ash: FRAXINUS

Bald Cypress: TAXODIUM

Bamboo: BAMBUSA, PHYLLOSTACHYS

Banana: MUSA

Barberry: BERBERIS

Basswood: TILIA

Beautyberry: CALLICARPA

Beech: FAGUS

Birch: BETULA

Bittersweet: CELASTRUS

Blueberry: VACCINIUM

Bottlebrush: CALLISTEMON

Box: BUXUS

Broom: CYTISUS, GENISTA

Buckeye: AESCULUS

Buckthorn: RHAMNUS

Bush Clover: LESPEDEZA

Bush Honeysuckle: DIERVILLA

Butterfly Bush: BUDDLEJA

California Redwood: SEGUOIA

Cassava: MANIHOT

Cedar: CEDRUS

Century Plant: AGAVE

Chestnut: CASTANEA

China Fir: CUNNINGHAMIA

Chinquapin: CASTANOPSIS

Chokeberry: ARONIA

Cinquefoil: POTENTILLA

Coral Tree: ERYTHRINA

Crape Myrtle: LAGERSTROEMIA

Currant: RIBES

Cypress: CUPRESSUS

Dogwood: CORNUS

Douglas Fir: PSEUDOTSUGA

Elder: SAMBUCUS

Elm: ULMUS

Epaulette Tree: PTEROSTYRAX

False Cypress: CHAMAECYPARIS
False Spirea: SORBARIA

False Arborvitae: THUJOPSIS.

Fig: FICUS

Filbert: CORYLUS

Fir: ABIES

Firethorn: PYRACANTHA

Flowering Maple: ABUTILON

Fringe Tree: CHIONANTHUS

Germander: TEUCRIUM

Giant Sequoia: SEQUOLADENDRON

Golden Bells: FORSYTHIA

Golden-rain Tree: KOELREUTERIA

Golden-chain Tree: LABURNUM

Gooseberry: RIBES

Grape: VITIS

Greenbrier: SMILAX

Groundsel: BACCHARIS

Hackberry: CELTIS

Hawthorn: CRATAEGUS

Hazelnut: CORYLUS

Heath: ERICA

Heather: CALLUNA

Hemlock: TSUGA

Hickory: CARYA

Holly: ILEX

Honeylocust: GLEDITSIA

Honeysuckle: LONICERA

Hop Tree: PTELEA

Hornbeam: CARPINUS

Horsechestnut: AESCULUS

Huckleberry: GAYLUSSACIA

Indigo: INDIGOFERA

Ivy: HEDERA

Jasmine: JASMINUM

Jessamine: GELSEMIUM

Jetbead: RHODOTYPOS

Juniper: JUNIPERUS

Kumquat: FORTUNELLA

Larch: LARIX

Laurel: LAURUS

Lavender: LAVANDULA

Lilac: SYRINGA

Linden: TILIA

Locust: ROBINIA

Loquat: ERIOBOTRYA

Magnolia: MAGNOLIA

Maple: ACER

Matrimony Vine: LYCIUM

Mimosa: ACACIA

Mock Orange: PHLADELPHUS

Mountain Ash: SORBUS

Mulberry: MORUS

Myrtle: MYRTUS

Ninebark: PHYSOCARPUS

Oak: QUERCUS

Pea Shrub: CARAGANA

Pear: PYRUS

Peony: PAEONLA

Peppertree: SCHINUS

Periwinkle: VINCA

Persimmon: DIOSPYROS

Pine: PINUS

Pistache: PISTACIA 
Poplar: POPULUS

Prickly Ash: ZANTHOXYLUM

Privet: LIGUSTRUM

Purple Guava: PSIDIUM

Quince: CYDONLA

Rose: ROSA

Sage: SALVIA

St. John's-wort: HYPERICUM

Sand Myrtle: LEIOPHYLLUM

Senna: CASSIA

Shadbush: AMELANCHIER

Silverbell Tree: HALESIA

Skimmia: SKIMMIA

Smoke Tree: COTINUS

Snowbell: STYRAX

Snowberry: SYMPHORICARPOS

Soapberry: SAPINDUS

Spanish Dagger: YUCCA

Spicebush: LINDERA

Spindle Tree: EUONYMUS

Spirea: SPIRAEA

Spruce: PICEA

Spurge: EUPHORBIA

Storax: STYRAX

Strawflower: HELICHRYSUM

Sumac: RHUS

Sweet Box: SARCOCOCCA

Sweet Gum: LIGUIDAMBAR

Sycamore: PLATANUS

Tallow Tree: SAPIUM

Tanbark Oak: LITHOCARPUS

Tulip Tree: LIRIODENDRON

Walnut: JUGLANS

Washington Palm: WASHINGTONLA

Wattle: ACACIA

White Alder: CLETHRA

Willow: SALIX

Wingnut: PTEROCARYA

Winter Hazel: CORYLOPSIS

Wisteria: WISTERIA

Witch Hazel: HAMAMELIS

Yew: TAXUS

\section{Species and Cultivars}

Aaron's Beard: HYPERICUM calycinum

Abelia, Chinese: ABELIA chinensis

Glossy: ABELIA $\times$ grandiflora

Acacia, Rose: ROBINLA hispida

Sweet: ACACIA farnesiana

Adam's Needle: YUCCA filamentosa

Alder, Black: ALNUS glutinosa,

ILEX verticillata

European: ALNUS glutinosa

Seaside: ALNUS maritima

Smooth: ALNUS serrulata

Witch: FOTHERGILLA gardenii

Allspice, Carolina: CALYCANTHUS floridus

Almond: PRUNUS dulcis

Double-flowered Flowering: PRUNUS triloba 'Multiplex'
Flowering: PRUNUS glandulosa, $\boldsymbol{P}$. triloba, Althea, Shrub: HIBISCUS syriacus

Anacahuita: CORDIA boisseri

Andromeda, Japanese: PIERIS japonica

Angel's Trumpet: BRUGMANSLA suaveolens

Anise, Florida: ILLICIUM floridanum

Anise Tree, Chinese: ILLICIUM henryi

Japanese: ILLICIUM anisatum

Apache Plume: FALLUGIA paradoxa

Apple, Common: MALUS domestica

Apricot: PRUNUS armeniaca

Japanese: PRUNUS mume

Arborvitae, American: THUJA occidentalis

Hiba: THUJOPSIS dolabrata

Oriental: PLATYCLADUS orientalis

Ardisia, Coral: ARDISLA crispa

Japanese: ARDISIA japonica

Arrow-wood: VBBURNUM dentatum

Northern: VIBURNUM recognitum

Ash. American: FRAXINUS americana

American Mountain: SORBUS americana

Berlandier: FRAXINUS berlandieriana

Biltmore: FRAXINUS americana var. biltmoreana

Black: FRAXINUS nigra

European: FRAXINUS excelsior

Flowering: FRAXINUS ornus

Green: FRAXINUS pennsylanica

var. subintegerrima

Leatherleaf: FRAXINUS velutina var. coriacea

Modesto: FRAXINUS velutina var. glabra

Red: FRAXINUS pennsylvanica

Wafer: PTELEA trifoliata

White: FRAXINUS americana

Aspen, Large-toothed: POPULUS grandidentata

Quaking: POPULUS tremuloides

Aster, Carolina: ASTER carolinianus

Aucuba, Japanese: AUCUBA japonica

Avocado: PERSEA americana

Azalea, Alabama: RHODODENDRON alabamense

Coastal: RHODODENDRON atlanticum

Cumberland: RHODODENDRON bakeri

Dwarf Indica: RHODODENDRON tamurae

Flame: RHODODENDRON calendulaceum

Florida: RHODODENDRON austrinum

Hammock Sweet: RHODODENDRON serrulatum

Indica: RHODODENDRON indicum

Kaempfer: RHODODENDRON kaempferi

Kirishima: RHODODENDRON 'Obtusum'

Korean: RHODODENDRON yedoense var. poukhanense

Kyushu: RHODODENDRON kiusianum

Large-sepal: RHODODENDRON macrosepalum

Mt. Amagi: RHODODENDRON amagianum

Oconee: RHODODENDRON flammeum

Oldham: RHODODENDRON oldhamii

Piedmont: RHODODENDRON canescens

Pinkshell: RHODODENDRON vaseyi

Plumleaf: RHODODENDRON prunifolium

Pontic: RHODODENDRON luteum

Rose: RHODODENDRON reticulatum

Roseshell: RHODODENDRON prinophyllum 
Royal: RHODODENDRON schlippenbachii

Sims: RHODODENDRON simsii

Snow: RHODODENDRON 'Mucronatum'

Spider: RHODODENDRON macrosepalum

'Linearifolium'

Swamp: RHODODENDRON viscosum

Sweet: RHODODENDRON arborescens

Taibei: RHODODENDRON kanahirae

Texas: RHODODENDRON oblongifolium

Western: RHODODENDRON occidentale

Wild-thyme: RHODODENDRON serpyllifolium

Baccharis, Southern: BACCHARIS glomerulifera

Balm-of-Gilead: POPULUS candicans

Bamboo, Fishpole: PHYLLOSTACHYS aurea

Giant Timber: PHYLLOSTACHYS heterocycla

Golden: PHYLLOSTACHYS aurea

Green Fountain: THAMNOCALAMUS spathaceus

Heavenly: NANDINA domestica

Hedge: BAMBUSA multiplex

Kuma: SASA veitchii

Sweetshoot: PHYLLOSTACHYS dulcis

Yellow-groove: PHYLLOSTACHYS aureosulcata

Banana Shrub: MICHELIA figo

Barberry, Byers Wintergreen: BERBERIS julianae

'Byers'

Dwarf Redleaf Japanese: BERBERIS thunbergii 'Atropurpurea Nana'

Hooker: BERBERIS hookeri, BERBERIS hookeri var. viridis

Japanese: BERBERIS thunbergii

Mentor: BERBERIS $\times$ mentorensis

Redleaf Japanese: BERBERIS thunbergii

'Atropurpurea'

Redleaf Korean: BERBERIS koreana

'Atropurpurea'

Sargent: BERBERIS sargentiana

Soulie: BERBERIS soulieana

Warty: BERBERIS verruculosa

Wintergreen: BERBERIS julianae

Yellowleaf Japanese: BERBERIS thunbergii 'Aurea'

Barometer Bush: LEUCOPHYLLUM frutescens

Bauhinia, Texas: BAUHINIA lunarioides

Bay, Loblolly: GORDONLA lasianthus

Red: PERSEA borbonia

Sweet: MAGNOLIA virginiana

Bayberry: MYRICA pensylvanica

Bean, Coral: ERYTHRINA herbacea

Indian: CATALPA bignonioides

Mescal: SOPHORA secundiflora

Bearberry: ARCTOSTAPHYLOS uva-ursi

Beauty Bush: KOLKWITZLA amabilis

Beautyberry, American: CALLICARPA americana

Bodinier: CALLICARPA bodinieri

Japanese: CALLICARPA japonica

Purple: CALLICARPA dichotoma

White-fruited: CALLICARPA americana 'Lactea'

Beech, American: FAGUS grandifolia

Columnar: FAGUS sylvatica 'Dawyck'

European: FAGUS sylvatica

Fernleaf: FAGUS sylvatica 'Laciniata'

Purple: FAGUS sylvatica f. purpurea

Weeping: FAGUS sylvatica 'Pendula'
Beefwood: CASUARINA cunninghamiana

Big Tree: SEGUOLADENDRON_giganteum

Birch, European White: BETULA pendula

Gray: BETULA populifolia

Hairy: BETULA pubescens

Japanese White: BETULA platyphylla

var. japonica

Paper: BETULA papyrifera

River: BETULA nigra

Sweet: BETULA lenta

Virginia Roundleaf: BETULA uber

Yellow: BETULA alleghaniensis

Bitternut: CARYA cordiformis

Bittersweet, Oriental: CELASTRUS orbiculatus

Blueberry, Creeping: VACCINIUM crassifolium

Ground: VACCINIUM myrsinites

Highbush: VACCINIUM corymbosum

Boneberry, Chinese: OSTEOMELES schwerinae

Bottlebrush, Narrowleaf: CALLISTEMON linearis

Stiff: CALLISTEMON rigidus

Willow: CALLISTEMON salignus

Box, Balearic: BUXUS balearica

Chinese: MURRAYA paniculata

Columnar: BUXUS sempervirens 'Fastigiata'

Common: BUXUS sempervirens

Dwarf English: BUXUS sempervirens 'Suffruticosa'

Handsworth: BUXUS sempervirens

'Handsworthiensis'

Harland: BUXUS harlandii

Japanese: BUXUS microphylla, B. microphylla

var. japonica

Korean: BUXUS sinica var. insularis

Tree: BUXUS sempervirens 'Arborescens'

Victorian: PITTOSPORUM undulatum

Weeping: BUXUS sempervirens 'Pendula'

Willow-leaved: BUXUS sempervirens 'Angustifolia'

Yugoslavian: BUXUS sempervirens 'Vardar Valley'

Boxelder: ACER negundo

Bridal Wreath: SPIRAEA prunifolia, S. $\times$ vanhouttei

Brier, Austrian: ROSA foetida

Broom, Butcher's: RUSCUS aculeatus

Scotch: CYTISUS scoparius

Spanish: SPARTIUM junceum

Warminster: CYTISUS $\times$ praecox

Buckeye, Bottlebrush: AESCULUS parviflora

California: AESCULUS californica

Mexican: UNGNADIA speciosa

Ohio: AESCULUS glabra

Red: AESCULUS pavia

Yellow: AESCULUS flava

Buckthorn, Carolina: RHAMNUS carolinianus

Common: RHAMNUS catharticus

Italian: RHAMNUS alaternus

Southern: BUMELIA lycioides

Buckwheat Tree: CLIFTONIA monophylla

Buddleja, Fountain: BUDDLEJA alternifolia

Orange-eye: BUDDLEJA davidii

Bunya-Bunya Tree: ARAUCARIA bidwillii

Bushcherry, Oriental: PRUNUS japonica

Bush Clover, Japanese: LESPEDEZA thunbergii

Bush Honeysuckle, Dwarf: DIERVILLA lonicera

Georgia: DIERVILLA rivularis 
Butternut: JUGLANS cinerea

Buttonbush: CEPHALANTHUS occidentalis Chinese: ADINA rubella

Calamondin: $\times$ CITROFORTUNELLA mitis

Camellia, Common: CAMELLIA japonica

Mountain: STEWARTIA ovata

Oil: CAMELLIA oleifera

Sasanqua: CAMELLIA sasanqua

Silky: STEWARTIA malacodendron

Warratah: CAMELLIA japonica 'Anemoniflora'

Camphor Tree: CINNAMOMUM camphora

Cassine: ILEX cassine

Cat's-claw: MACFADYENA unguis-cati

Catalpa, Chinese: CATALPA ovata

Common: CATALPA bignonioides

Manchurian: CATALPA bungei

Western: CATALPA speciosa

Catberry: NEMOPANTHUS mucronatus

Ceanothus, Snowbrush: CEANOTHUS velutinus

Cedar, Alaska: CHAMAECYPARIS nootkatensis

Atlantic White: CHAMAECYPARIS thyoides

Atlas: CEDRUS atlantica

Blue Atlas: CEDRUS atlantica 'Glauca'

Cyprus: CEDRUS libani ssp. brevifolia

Deodar: CEDRUS deodara

Incense: CALOCEDRUS decurrens

Japanese: CRYPTOMERIA japonica

-of-Lebanon: CEDRUS libani

Port Orford: CHAMAECYPARIS lawsoniana

Red: JUNIPERUS virginiana

Southern Red: JUNIPERUS silicicola

Stinking: TORREYA taxifolia

Western Red: THUJA plicata

Ceniza: LEUCOPHYLUM frutescens

Century Plant, Variegated: AGAVE americana 'Marginata'

Chaste Tree: VITEX agnus-castus

Chenille Plant: ACALYPHA hispida

Cherry, Barbados: MALPIGHIA glabra

Bird: PRUNUS padus

Black: PRUNUS serotina

Cornelian: CORNUS mas

Higan: PRUNUS $\times$ subhirtella

Japanese Cornelian: CORNUS officinalis

Japanese Flowering: PRUNUS serrulata

Kwanzan: PRUNUS serrulata 'Kwanzan'

Mahaleb: PRUNUS mahaleb

Nanking: PRUNUS tomentosa

Sargent: PRUNUS sargentii

Sour: PRUNUS cerasus

Sweet: PRUNUS avium

Taiwan: PRUNUS campanulata

Takasago: PRUNUS $\times$ sieboldii

Tokyo: PRUNUS $\times$ yedoensis

Weeping Higan: PRUNUS $\times$ subhirtella

'Shidare Higan'

Yoshino: PRUNUS $\times$ yedoensis 'Somei Yoshino'

Chestnut, Chinese: CASTANEA mollissima

Common: CASTANEA sativa

European: CASTANEA sativa

Japanese: CASTANEA crenata

Chinaberry: MELIA azedarach

Chinquapin, Japanese: CASTANOPSIS cuspidata
Chittamwood: BUMELIA lanuginosa

Chocolate Vine: AKEBIA quinata

Chokeberry, Black: ARONLA melanocarpa

Purplefruit: ARONLA prunifolia

Red: ARONIA arbutifolia

Cinquefoil, Shrubby: POTENTILLA fruticosa

Citrange: $\times$ CITRONCIRUS webberi

Citron: CITRUS medica 'Etrog'

Clematis, Evergreen: CLEMATIS armandii

Goldwool: CLEMATIS chrysocoma var. sericea

Pink-flowered Evergreen: CLEMATIS armandii 'Farquhariana'

Scarlet: CLEMATIS texensis

Cleyera, Japanese: CLEYERA japonica

Variegated: CLEYERA japonica 'Tricolor'

Cliff-green: PAXISTIMA canbyi

Coffee-tree, Kentucky: GYMNOCLADUS dioica

Conradina, Bluesage: CONRADINA canescens

Whorled: CONRADINA verticillata

Coontie: ZAMIA pumila

Coralbeads: COCCULUS triloba

Coralberry: ARDISIA crenata, SYMPHORICARPOS orbiculatus

Coral Plant: RUSSELIA equisetiformis

Coral Tree, Cockspur: ERYTHRINA crista-galli

Coriaria, Japanese: CORIARLA japonica

Cork-tree, Amur: PHELLODENDRON amurense

Corkwood, Florida: LEITNERIA floridana

Cotoneaster, Bloodberry: COTONEASTER obscurus

Cranberry: COTONEASTER apiculatus

Creeping: COTONEASTER adpressus

Diels: COTONEASTER dielsianus

European: COTONEASTER integerrimus

Rock: COTONEASTER horizontalis

Spreading: COTONEASTER divaricatus

Wintergreen: COTONEASTER conspicuus

Cottonwood: POPULUS deltoides

Swamp: POPULUS heterophylla

Cowberry: VACCINIUM vitis-idaea

Crabapple, Bechtel's: MALUS ioensis 'Plena'

Sargent's: MALUS sargentii

Showy: MALUS floribunda

Siberian: MALUS baccata

Southern: MALUS angustifolia

Toringo: MALUS sieboldii

Wild Sweet: MALUS coronaria

Cranberry, Highbush: VIBURNUM trilobum

Mountain: VACCINIUM vitis-idaea

Cranberry-bush, European: VBURNUM opulus

Creeper, Mexican: ANTIGONON leptopus

Virginia: PARTHENOCISSUS quinquefolia

Cross Vine: BIGNONLA capreolata

Croton, Alabama: CROTON alabamensis

Cucumber Tree: MAGNOLIA acuminata

Cup-flower, Tall: NIEREMBERGIA scoparia

Currant, Clove: RIBES odoratum

Indian: SYMPHORICARPOS orbiculatus

Mountain: RIBES alpinum

Cypress, Bald: TAXODIUM distichum var. distichum

Italian: CUPRESSUS sempervirens

Lawson: CHAMAECYPARIS lawsoniana

Leyland: $\times$ CUPRESSOCYPARIS leylandii 
Monterey: CUPRESSUS macrocarpa Montezuma: TAXODIUM mucronatum Mourning: CHAMAECYPARIS funebris Pond: TAXODIUM distichum var. nutans Portuguese: CUPRESSUS lusitanica

Dahoon: ILEX cassine

Narrow-leaved: ILEX cassine var, angustifolia

Yellow-berried: ILEX cassine var. angustifolia

f. aurea-baccata

Daisy, Nippon: CHRYSANTHEMUM nipponicum

Daphne, February: DAPHNE mezereum

Lilac: DAPHNE genkwa

Rose: DAPHIE cneorum

Winter: DAPHIE odora

Deerberry: VACCINIUM stamineum

Deutzia, Rough-leaved: DEUTZIA scabra

Devil's Walking Stick: ARALIA spinosa

Devilwood: OSMANTHUS americanus

Dioon, Chestnut: DIOON edule

Doghobble: LEUCOTHOE fontanesiana

Dogwood, Alternate-leaved: CORNUS alternifolia Cream-edged: CORNUS alba 'Argenteo Marginata' Double-flowered: CORNUS florida 'Bay Beauty' Double-flowered: CORNUS florida f. pluribracteata Flowering: CORNUS florida

Giant: CORNUS controversa

Gray: CORNUS racemosa

Kousa: CORNUS kousa

Pale: CORNUS obliqua

Pink-flowering: CORNUS florida f. rubra

Siberian: CORNUS alba 'Sibirica'

Silky: CORNUS amomum

Stiff: CORNUS foemina

Western: CORNUS nuttallii

Yellow-fruited flowering: CORNUS florida

f. xanthocarpa

Dove Tree: DAVIDIA involucrata var. vilmoriniana

Dutchman's Pipe, Calico: ARISTOLOCHIA elegans

Ebony, Green: JACARANDA acutifolia

Texas: PITHECELLOBIUM flexicaule

Elaeagnus, Cherry: ELAEAGNUS multiflora

Thorny: ELAEAGNUS pungens

Elder, American: SAMBUCUS canadensis

American Red: SAMBUCUS racemosa ssp. pubens

Asiatic Red: SAMBUCUS racemosa

ssp. sieboldiana

Dwarf: SAMBUCUS ebulus

Elfin Herb: CUPHEA hyssopifolia

Elm, American: ULMUS americana

Cedar: ULMUS crassifolia

Chinese: ULMUS parvifolia

English: ULMUS procera

European White: ULMUS laevis

Globe: ULMUS minor 'Umbraculifera'

Jersey: ULMUS minor 'Sarniensis'

Moline: ULMUS americana 'Moline'

Rock: ULMUS thomasii

Scotch: ULMUS glabra

September: ULMUS serotina

Siberian: ULMUS pumila

Slippery: ULMUS rubra

Water: PLANERA aquatica
Winged: ULMUS alata

Wych: ULMUS glabra

False Cypress, Hinoki: CHAMAECYPARIS obtusa

Moss: CHAMAECYPARIS pisifera 'Squarrosa'

Nootka: CHAMAECYPARIS nootkatensis

Sawara: CHAMAECYPARIS pisifera

Farkleberry: VACCINIUM arboreum

Fatsia, Japanese: FATSLA japonica

Fetterbush: LYONIA lucida, PIERIS floribunda

Fiddlewood: CITHAREXYLUM berlandieri

Fig, Common: FICUS carica

Creeping: FICUS pumila

Filbert, Beaked: CORYLUS cornuta

Chinese: CORYLUS chinensis

Purple-leaved: CORYLUS maxima 'Purpurea'

Turkish: CORYLUS colurna

Fir, Algerian: ABIES numidica

Balsam: ABIES balsamea

Blue China: CUNNINGHAMUA Lanceolata 'Glauca'

China: CUNNINGHAMIA lanceolata

Cilician: ABIES cilicica

Douglas: See Rocky Mountain Douglas

Fraser: ABIES fraseri

Greek: ABIES cephalonica

Korean: ABIES koreana

Manchurian: ABIES holophylla

Momi: ABIES firma

Nikko: ABIES homolepis

Noble: ABIES procera

Nordmann: ABIES nordmanniana

Rocky Mountain Douglas: PSEUDOTSUGA menziesii var. glauca

Shensi: ABIES chensiensis ssp. salouenensis

Silver: ABIES alba

Spanish: ABIES pinsapo

White: ABIES concolor

Flamebush, Mexican: CALLIANDRA tweediei

Forsythia, White: ABELIOPHYLLUM distichum

Franklin Tree: FRANKLINLA alatamaha

Fringe Tree, American: CHIONANTHUS virginicus

Japanese: CHIONANTHUS retusus

Taiwan: CHIONANTHUS retusus var. serrulatus

Fuchsia, Cape: PHYGELIUS capensis

Garland Flower: DAPHNE cneorum

Gallberry, Large: $\boldsymbol{L E X}$ coriacea

Ginkgo: GINKGO biloba

Glorybower, Bleedingheart: CLERODENDRUM thomsonae

Fragrant: CLERODENDRUM philippinum

Harlequin: CLERODENDRUM trichotomum

Glory Bush: TIBOUCHINA urvilleana

Gold-dust Shrub: AUCUBA japonica 'Variegata'

Golden Bells, Korean: FORSYTHIA ovata

Weeping: FORSYTHIA suspensa

Golden-chain Tree: LABURNUM anagyroides

Golden-rain Tree: KOELREUTERIA bipinnata,

$\boldsymbol{K}$. elegans ssp. formosana, $\mathbf{K}$. paniculata

Goldflower: HYPERICUM calycinum

Gooseberry, Chinese: ACTINIDIA deliciosa

var. deliciosa

English: RIBES uva-crispa

Florida: RIBES echinellum

Granite: RIBES curvatum 
Grape, Fox: VITIS labrusca

Muscadine: VITIS rotundifolia

Mustang: VTIS mustangensis

Grapefruit: CITRUS $\times$ paradisi

Green Screw: ILEX aquifolium 'Crispa'

Groundsel Tree: BACCHARIS halimifolia

Guava, Pineapple: ACCA sellowiana

Purple: PSIDIUM littorale var. longipes

Gum, Black: NYSSA sylvatica

Cider: EUCALYPTUS gunnii

Formosan Sweet: LIQUIDAMBAR formosana

Manna: EUCALYPTUS viminalis

Mountain: EUCALYPTUS dalrympleana

Sour: NYSSA sylvatica

Sweet: LIQUIDAMBAR styraciflua

Tasmanian Snow: EUCALYPTUS coccifera

Gumi: ELAEAGNUS multiflora

Hackberry: Caucasian: CELTIS caucasica

Chinese: CELTIS sinensis

Common: CELTIS occidentalis

Spiny: CELTIS pallida

Hardhack: SPIRAEA tomentosa

Harry Lauder's Walking-stick: CORYLUS avellana 'Contorta'

Haw, Black: VIBURNUM prunifolium

Nannyberry: VIBURNUM nudum

Poison: VIBURNUM molle

Possum: ILEX decidua, VIBURNUM nudum

Rusty Black: VIBURNUM rufidulum

Southern Black: VIBURNUM rufidulum

Hawthorn: Blueberry: CRATAEGUS brachyacantha

Cockspur: CRATAEGUS crus-galli

Common: CRATAEGUS monogyna

Dotted: CRATAEGUS punctata

Downy: CRATAEGUS mollis

English: CRATAEGUS laevigata

Green: CRATAEGUS viridis

Indian: RHAPHIOLEPIS indica

Littlehip: CRATAEGUS spathulata

May: CRATAEGUS aestivalis

Parsley: CRATAEGUS marshallii

Pear: CRATAEGUS calpodendron

Pringle: CRATAEGUS pringlei

Thicket: CRATAEGUS intricata

Yeddo: RHAPHIOLEPIS umbellata

Yellow: CRATAEGUS flava

Hazelnut, American: CORYLUS americana

European: CORYLUS avellana

Purple-leaved: CORYLUS avellana 'Fusco-rubra'

Heath, Cornish: ERICA vagans

Spike: BRUCKENTHALIA spiculifolia

Spring: ERICA carnea

Heather, False: CUPHEA hyssopifolia

Scotch: CALLUINA vulgaris

Hemlock. Canadian: TSUGA canadensis

Carolina: TSUGA caroliniana

Ground: TAXUS canadensis

Sargent's Weeping: TSUGA canadensis 'Sargentii'

Weeping: TSUGA canadensis 'Pendula'

Hican: CARYA illinoinensis $\times$ C. sp.

Hickory, Mockernut: CARYA tomentosa
Sand: CARYA pallida

Shagbark: CARYA ovata

Shellbark: CARYA laciniosa

Water: CARYA aquatica

Hobblebush: VIBURNUM lantanoides

Holly, American: ILEX opaca

Appalachian: ILEX collina

Attenuate-leaved: $\boldsymbol{I L E X} \times$ attenuata

Beadle's: ILEX beadlei

Box-leaved: ILEX crenata

Broad-leaved: ILEX latifolia

Burford: ILEX cornuta 'Burfordii'

Buswell's: ILEX buswellii

Canary Islands: ILEX canariensis

Carolina: ILEX ambigua

Caucasian: $\mathbf{L E X}$ colchica

Chinese: ILEX cornuta

Coin-leaved: ILEX crenata 'Mariesii'

Creeping: ILEX rugosa

Dune: ILEX cumulicola

English: ILEX aquifolium

Evergreen Long-stalked: ILEX pedunculosa

Farges': ILEX fargesii

Fig-leaved: ILEX ficoidea

Furin: ILEX geniculata

Georgian: ILEX longipes

Highclere: $\boldsymbol{L E X} \times$ altaclerensis

Horned: ILEX cornuta

Hummock: ILEX cumulicola

Japanese: ILEX crenata

Kachi: ILEX purpurea

Koehne's: ILEX $\times$ koehneana

Kurogane: ILEX rotunda

Liukiu: ILEX liukiuensis

Long-stalked: ILEX longipes

Luster-leaved: ILEX latifolia

Mochi: ILEX integra

Mountain: ILEX monticola

Myrtle-leaved: ILEX myrtifolia

Northern Mountain: NEMOPANTHUS mucronatus

Okinawa: ILEX dimorphophylla

Peach-leaved: ILEX purpurea

Pontic: ILEX colchica

Sand: ILEX buswellii

Sarvis: ILEX amelanchier

Sikkim: ILEX sikkimensis

Suwanee: ILEX curtissii

Topel: $\boldsymbol{I L E X} \times$ attenuata

Tsuru: ILEX rugosa

Waterer's Gold: ILEX aquifolium 'Watereriana'

Wilson's: ILEX wilsonii

Yellow-fruited American: ILEX opaca f. xanthocarpa

Yellow-stemmed: ILEX leucoclada

Yunnan: LEX yunnanensis

Hollygrape, Leatherleaf: MAHONIA bealei

Oregon: MAHONLA aquifolium

TeXas: MAHONLA swazeyi

Honeylocust, Common: GLEDITSIA triacanthos

Japanese: GLEDITSLA japonica

Spineless: GLEDITSIA triacanthos $\mathrm{f}$. inermis

Honeysuckle, Amur: LONICERA maackii 
Cape: TECOMARIA capensis

Everblooming: LONICERA $\times$ heckrottii

Fly: LONICERA xylosteum

Japanese: LONICERA japonica

Morrow's: LONICERA morrowii

Privet: LONICERA pileata

Standish's: LONICERA standishii

Tatarian: LONICERA tatarica

Trumpet: LONICERA sempervirens

Winter: LONICERA fragrantissima

Yellow: LONICERA flava

Hornbeam, American: CARPINUS caroliniana

Columnar: CARPINUS betulus 'Fastigiata'

European: CARPINUS betulus

Heartleaf: CARPINUS cordata

Hop: OSTRYA virginiana

Japanese: CARPINUS japonica

Purple-leaf: CARPINUS betulus 'Purpurea'

Horse Sugar: SYMPLOCOS tinctoria

Horsechestnut: AESCULUS hippocastanum

Double-flowered: AESCULUS hippocastanum

'Baumanii'

Red: AESCULUS $\times$ carnea

Hortensia: HYDRANGEA macrophylla (Hortensia group)

Huckleberry, Black: GAYLUSSACIA baccata

Box: GAYLUSSACLA brachycera

Hydrangea, Climbing: HYDRANGEA anomala ssp. petiolaris

Garden: HYDRANGEA macrophylla (Hortensia group)

Oakleaf: HYDRANGEA quercifolia

Peegee: HYDRANGEA paniculata

Hydrangea Vine, Japanese: SCHIZOPHRAGMA

hydrangeoides

Indigo, Mountain: AMORPHA glabra

Indigo Bush: AMORPHA fruticosa

Inkberry: ILEX glabra

White-fruited: ILEX glabra f. leucocarpa

Ipil-ipil: LEUCAENA leucocephala

Ironwood: BUMELIA tenax, CARPINUS caroliniana

Persian: PARROTLA persica

Ivy, Algerian: HEDERA canariensis

Boston: PARTHENOCISSUS tricuspidata

Caucasus: HEDERA colchica

English: HEDERA helix

Gloire de Marengo: HEDERA canariensis

'Variegata'

Italian: HEDERA helix var. poetica

Japanese: HEDERA rhombea

Marine: CISSUS incisa

Poison: TOXICODENDRON radicans

Shrub: HEDERA helix (Arborescens group)

Tree: $\times$ FATSHEDERA lizei

Jacaranda: JACARANDA acutifolia

Jasmine, Angel-wing: JASMINUM nitidum

Blue: CLEMATIS crispa

Cape: GARDENLA jasminoides

Confederate: TRACHELOSPERMUM jasminioides

Crape: TABERNAEMONTANA divaricata

Italian: JASMINUM humile 'Revolutum'

Orange: MURRAYA paniculata
Primrose: JASMINUM mesyni

Star: JASMINUM multiflorum,

TRACHELOSPERMUM jasminioides

Winter: JASMINUM nudiflorum

Jessamine, Carolina: GELSEMTUM sempervirens

Double-flowered: GELSEMIUM sempervirens 'Pride

of Augusta'

Night: CESTRUM nocturnum

Yellow: GELSEMIUM sempervirens

Jetbead: RHODOTYPOS scandens

Jujube: ZIZIPHUS jujuba

Juniper, Alligator: JUNIPERUS deppeana var. pachyphlaea

Andorra: JUNIPERUS horizontalis 'Plumosa'

Chinese: JUNIPERUS chinensis

Creeping: JUNIPERUS horizontalis

Golden Pfitzer: JUNIPERUS $\times$ media

'Pfitzeriana Aurea'

Ground: JUNIPERUS communis ssp. depressa

Hetz Blue: JUINIPERS chinensis 'Hetzii'

Hollywood: JUNIPERUS chinensis 'Kaizuka'

Irish: JUNIPERUS communis 'Hibernica'

Needle: JUNIPERUS rigida

Pfitzer: JUNIPERUS $\times$ media 'Pfitzeriana'

Rocky Mountain: JUNIPERUS scopulorum

Sargent: JUNIPERUS chinensis var. sargentii

Shore: JUNIPERUS conferta

Single-seed: JUNIPERUS squamata

Kadsura Vine: KADSURA japonica

Kaki: DIOSPYROS kaki

Katsura Tree: CERCIDIPHYLLUM japonicum

Keaki: ZELKOVA serrata

Kinnikinnick: ARCTOSTAPHYLOS uva-ursi

Kiwi Fruit: ACTINIDIA deliciosa var. deliciosa

Kumquat, Meiwa: FORTUNELLA $\times$ crassifolia

Round: FORTUNELLA japonica

Lace Shrub: STEPHANANDRA incisa

Lacecap: HYDRANGEA macrophylla (Lacecap group)

Lantana, Trailing: LANTANA montevidensis

Larch, Dahurian: LARIX gmelinii

European: LARIX decidua

Golden: PSEUDOLARIX amabilis

Japanese: LARIX kaempferi

Laudanum: CISTUS ladanifer

Laurel, Alexandrian: DANAE racemosa

Carolina Cherry: PRUNUS caroliniana

Carpathian Cherry: PRUNUS laurocerasus var. schipkaensis

Cherry: PRUNUS laurocerasus

English: PRUNUS laurocerasus

Grecian: LAURUS nobilis

Mountain: KALMLA latifolia

Portuguese Cherry: PRUNUS lusitanica

Sheep: KALMIA angustifolia

Laurustinus: VBURNUM tinus

Lavandin: LAVANDULA $\times$ intermedia

Lavender Cotton: SANTOLINA chamaecyparissus

French: LAVANDULA stoechas

Leadwort, Cape: PLUMBAGO auriculata

Leatherflower: CLEMATIS versicolor

Leatherleaf: CHAMAEDAPHNE calyculata

Leatherwood: CYRILLA racemiflora,

DIRCA palustris 
Lilac, Chinese: SYRINGA $\times$ chinensis

Common: SYRINGA vulgaris 'Purpurea'

Cutleaf: SYRINGA laciniata

Double White: SYRINGA vulgaris 'Alba Plena'

Hungarian: SYRINGA josikaea

Japanese Tree: SYRINGA reticulata

Late: SYRINGA villosa, S. villosa 'Crayton'

Persian: SYRINGA $\times$ persica

Lily Tree: MAGNOLIA denudata

Lime, Australian Finger: MICROCITRUS australasica Wild: ZANTHOXYLUM fagara

Linden, American: TILLA americana

Bigleaf: TILIA platyphyllos

Littleleaf: TILIA cordata

Mongolian: TILIA mongolica

Silver: TILIA tomentosa

Locust, Black: ROBINLA pseudoacacia

Bristly: ROBINLA hispida

Clammy: ROBINIA viscosa

Ship-mast: ROBINIA pseudoacacia var. rectissima

Water: GLEDITSLA aquatica

Loquat: ERIOBOTRYA japonica

Magnolia, Bigleaf: MAGNOLIA macrophylla

Florida Bigleaf: MAGNOLIA ashei

Fraser: MAGNOLIA fraseri

Goddess: MAGNOLIA sprengeri 'Diva'

Kobus: MAGNOLIA kobus

Lily-flowered: MAGNOLIA liliiflora

Mexican Bigleaf: MAGNOLIA dealbata

Otama: MAGNOLIA sieboldii

Saucer: MAGNOLIA $\times$ soulangeana

Southern: MAGNOLIA grandiflora

Star: MAGNOLLA stellata

Umbrella: MAGNOLIA tripetala

Mahogany, Swamp: EUCALYPTUS robusta

Mahonia, Chinese: MAHONIA fortunei

Cluster: MAHONIA pinnata

Japanese: MAHONIA japonica

Laredo: MAHONLA trifoliata

Maidenhair Tree: GINKGO biloba

Mallotus, Japanese: MALLOTUS japonicus

Mandarin: CITRUS reticulata

Maple, Amur: ACER ginnala

Black: ACER saccharum ssp. nigrum

Bloodleaf: ACER palmatum 'Atropurpureum'

Chalk: ACER saccharum ssp. leucoderme

Colosseum: ACER cappadocicum

David's: ACER davidii

Devil: ACER diabolicum

Drummond: ACER rubrum ssp. drummondii

Dwarf Hedge: ACER campestre 'Nanum'

Evergreen: ACER oblongum

Florida: ACER saccharum ssp. floridanum

Full-moon: ACER japonicum

Golden Colosseum: ACER cappadocicum 'Aureum'

Golden Full-moon: ACER japonicum 'Aureum'

Hedge: ACER campestre, ACER campestre

var. leiocarpum

Hornbeam: ACER carpinifolium

Japanese: ACER japonicum, ACER palmatum

var. palmatum

Laceleaf: ACER palmatum 'Dissectum'

Manchurian: ACER mandshuricum
Manchurian Striped: ACER tegmentosum

Miyabe: ACER miyabei

Montpellier: ACER monspessulanum

Mountain: ACER spicatum

Nikko: ACER maximowiczianum

Norway: ACER platanoides

Paperbark: ACER griseum

Purpleleaf Sycamore: ACER pseudoplatanus

'Atropurpureum'

Purplebloom: ACER pseudosieboldianum

Red: $A C E R$ rubrum

Redvein: ACER rufinerve

Schwedler: ACER platanoides 'Schwedleri'

Shantung: ACER truncatum

Siebold: ACER sieboldianum

Silver: ACER saccharinum

Striped: ACER pensylvanicum

Sugar: ACER saccharum ssp. saccharum

Sycamore: ACER pseudoplatanus

Tatarian: ACER tataricum

Trident: ACER buergerianum

Velvet: ACER velutinum var. vanvolxemii

Marine Vine: CISSUS incisa

Marjoram, Pot: ORIGANUM onites

Matrimony Vine, Carolina: LYCIUM carolinianum

Chinese: LYCIUM chinense

Medlar: MESPILUS germanica

Mesquite, Western Honey: PROSOPIS glandulosa

var. torreyana

Metake: PSEUDOSASA japonica

Mezereon: DAPHNE mezereum

Milletia, Leatherleaf: MILLETTIA reticulata

Mimosa, Catclaw: MIMOSA biuncifera

Mimosa Tree: ALBIZIA julibrissin

Minniebush: MENZIESIA pilosa

Mock Orange, European: PHILADELPHUS coronarius

Monkey Puzzle: ARAUCARIA araucana

Mu-oil Tree: ALEURITES montana

Mulberry, Paper: BROUSSONETIA papyrifera

Red: MORUS rubra

Weeping: MORUS alba 'Pendula'

White: MORUS alba

Myrtle, Common: MYRTUS communis

Dwarf: MYRTUS communis 'Microphylla'

Sea: BACCHARIS halimifolia

Nannyberry: VIBURNUM lentago

Nectarine: PRUNUS persica var. nucipersica

'White Glory'

Neillia, Chinese: NEILLIA sinensis

Nightshade, Brazilian: SOLANUM seaforthianum

Oak, Arkansas: QUERCUS arkansana

Bartram: QUERCUS $\times$ heterophylla

Basket: QUERCUS michauxii

Black: QUERCUS velutina

Bluejack: GUERCUS incana

Bur: QUERCUS macrocarpa

Chestnut: GUERCUS prinus

Chinese Cork: GUERCUS variabilis

Columnar English: GUERCUS robur f. fastigiata

Compton: GUERCUS $\times$ comptonae

Cork: QUERCUS suber

Daimyo: QUERCUS dentata 
Darlington: QUERCUS hemisphaerica

Durmast: QUERCUS petraea

English: QUERCUS robur

Georgia: GUERCUS georgiana

Italian: QUERCUS frainetto

Japanese Evergreen: QUERCUS myrsinifolia

Konara: QUERCUS glandulifera

Laurel: GUERCUS laurifolia

Nuttall: GUERCUS nuttallii

Oriental Sawtooth: GUERCUS acutissima

Overcup: GUERCUS Iyrata

Pin: QUERCUS palustris

Post: QUERCUS stellata

Red: QUERCUS rubra

Ring-cupped: GUERCUS glauca

Sand Post: QUERCUS margaretta

Sargent: QUERCUS $\times$ sargentii

Scarlet: QUERCUS coccinea

Shingle: QUERCUS imbricaria

Shumard: GUERCUS shumardii

Southern Live: GUERCUS virginiana

Southern Red: QUERCUS falcata

Spanish: QUERCUS falcata

Swamp White: QUERCUS bicolor

Tanbark: LITHOCARPUS glaber

Turkey: QUERCUS cerris; QUERCUS laevis

Ubame: QUERCUS phillyraeoides

Water: QUERCUS nigra

White: GUERCUS alba

Willow: QUERCUS phellos

Old-man's Beard: CHIONANTHUS virginicus

Oleander: NERIUM oleander

Variegated: NERIUM oleander 'Variegata'

Olive: OLEA europaea

Autumn: ELAEAGNUS umbellata

Holly: OSMANTHUS heterophyllus var.

heterophyllus

Russian: ELAEAGNUS angustifolia

Sweet: OSMANTHUS fragrans

Tea: OSMANTHUS fragrans

Orange, Chinese Box: SEVERINLA buxifolia

Hardy: PONCIRUS trifoliata

Osage: MACLURA pomifera

Satsuma: CITRUS reticulata

Seville: CITRUS aurantium

Sour: CITRUS aurantium

Osier, Green: CORNUS alternifolia

Red: CORNUS sericea

Our-Lord's-Candle: YUCCA whipplei

Pachysandra, Allegheny: PACHYSANDRA procumbens

Japanese: PACHYSANDRA terminalis

Pagoda Tree: SOPHORA japonica

Palm, Cabbage: SABAL palmetto

Canary Island Date: PHOENIX canariensis

Chinese Fan: LIVISTONA chinensis

European Fan: CHAMAEROPS humilis

Jelly: BUTIA capitata

Needle: RHAPIDOPHYLLUM hystrix

Queen: SYAGRUS romanzoffianum

Sago: CYCAS revoluta

Senegal Date: PHOENDX reclinata

Windmill: TRACHYCARPUS fortunei
Palmetto, Dwarf: SABAL minor

Saw: SERENOA repens -

Paperbark Tree: MELALEUCA quinquenervis

Paperbush: EDGEWORTHA chrysantha

Paradise Flower: SOLANUM wendlandii

Paraguay Tea: ILEX paraguariensis

Parasol Tree, Chinese: FIRMIANA simplex

Partridgeberry: MITCHELLA repens

Pascuita: EUPHORBIA leucocephala

Passionflower, Red: PASSIFLORA coccinea

Pavonia, Spearleaf: PAVONIA hastata

Pawpaw: ASIMINA triloba

Pea Shrub: CARAGANA arborescens

Chinese: CARAGANA sinica

Peach: PRUNUS persica

Pear, Alligator: PERSEA americana

Asian: PYRUS pyrifolia

Birchleaf: PYRUS betulifolia

Bradford: PYRUS calleryana 'Bradford'

Callery: PYRUS calleryana

Common: PYRUS communis

Evergreen: PYRUS kawakamii

Leconte: PYRUS $\times$ lecontei

Nashi: PYRUS pyrifolia var. culta

Sand: PYRUS pyrifolia

Snow: PYRUS nivalis

Pearlbush: EXOCHORDA racemosa

Pecan: CARYA illinoinensis

Bitter: CARYA aquatica

Mehan: CARYA illinoinensis 'Mehan'

Stuart: CARYA illinoinensis 'Stuart'

Peony, Tree: PAEONLA suffruticosa

Pepperbush, Sweet: CLETHRA alnifolia

Peppertree, Brazilian: SCHINUS terebinthifolius

Pepper-vine: AMPELOPSIS arborea

Periwinkle, Common: VINCA minor

Double-flowered: VINCA minor 'Multiplex'

Greater: VINCA major

Persimmon, Common: DIOSPYROS virginiana Chinese: DIOSPYROS sinensis

Japanese: DIOSPYROS kaki

Texas: DIOSPYROS texana

Phillyrea, Narrow-leaf: PHILLYREA angustifolia

Tree: PHILLYREA latifolia var. media

Photinia, Chinese: PHOTINIA serratifolia Japanese: PHOTINIA glabra

Pignut: CARYA glabra

Sweet: CARYA ovalis

Pine, African Fern: PODOCARPUS gracilior

Aleppo: PINUS halepensis

Australian: CASUARINA cunninghamiana

Austrian: PINUS nigra ssp. nigra

Chir: PINUS roxburghii

Digger: PINUS sabiniana

Eastern White: PINUS strobus

Himalayan: PINUS wallichiana

Italian Stone: PINUS pinea

Jack: PINUS banksiana

Japanese Black: PINUS thunbergiana

Japanese Red: PINUS densiflora

Japanese Umbrella: PINUS densiflora

'Umbraculifera' 
Japanese White: PINUS parviflora

Jelecote: PINUS patula

Knob-cone: PINUS attenuata

Korean: PINUS koraiensis

Lacebark: PINUS bungeana

Limber: PINUS flexilis

Loblolly: PINUS taeda

Long-leaved Indian: PINUS roxburghii

Longleaf: PINUS palustris

Mexican Stone: PINUS cembroides

Pitch: PINUS rigida

Pond: PINUS serotina

Ponderosa: PINUS ponderosa

Red: PINUS resinosa

Sand: PINUS clausa

Scotch: PINUS sylvestris

Scrub: PINUS virginiana

Shortleaf: PINUS echinata

Slash: PINUS elliottii

Spruce: PINUS glabra

Swiss Mountain: PINUS mugo var. mugo

Swiss Stone: PINUS cembra

Table-mountain: PINUS pungens

Totara: PODOCARPUS totara

Umbrella: PINUS pinea; SCIADOPITYS verticillata

Virginia: PINUS virginiana

Weeping Japanese Red: PINUS densiflora 'Pendula'

Western Yellow: PINUS ponderosa

Yew: PODOCARPUS macrophyllus

Pinxterbloom: RHODODENDRON periclymenoides

Pipe Plant: AGARISTA populifolia

Pipe-stem Wood: AGARISTA populifolia

Piratebush: BUCKLEYA distichophylla

Pistache, Chinese: PISTACIA chinensis

Texas: PISTACLA texana

Pittosporum, Japanese: PITTOSPORUM tobira

Plane, American: PLATANUS occidentalis

London: PLATANUS $\times$ acerifolia

Oriental: PLATANUS orientalis

Plum. Beach: PRUNUS maritima

Black-sloe: PRUNUS umbellata

Chickasaw: PRUNUS angustifolia

Creek: PRUNUS rivularis

Damson: PRUNUS domestica ssp. insititia

Mexican: PRUNUS mexicana

Purple-leaf: PRUNUS cerasifera 'Atropurpurea'

Wild Goose: PRUNUS munsoniana

Wild: PRUNUS americana

Plumbago, Chinese: CERATOSTIGMA willmottianum

Poinsettia: EUPHORBIA pulcherrima

Pomegranate: PUNICA granatum

Dwarf: PUNICA granatum 'Nana'

Pond-spice: LITSEA aestivalis

Popinac, White: LEUCAENA leucocephala

Poplar, Carolina: POPULUS $\times$ canadensis

Gray: POPULUS $\times$ canescens

Japanese: POPULUS maximowiczii

Lombardy: POPULUS nigra 'Italica'

White: POPULUS alba

Yellow: LIRIODENDRON tulipifera

Porcelain-berry: AMPELOPSIS glandulosa

var. brevipedunculata
Porlieria, Texas: PORLIERIA angustifolia

Potato Bush, Blue: SOLANUM rantonnetii

Potato Vine: SOLANUM jasminoides

Prickly Ash, Southern: ZANTHOXYLUM clava-herculis

Princess Tree: PAULOWNIA tomentosa

Privet, Border: LIGUSTRUM obtusifolium

California: LIGUSTRUM ovalifolium

Chinese: LIGUSTRUM sinense

Common: LIGUSTRUM vulgare

Japanese: LIGUSTRUM japonicum

Swamp: FORESTIERA acuminata

Wax-leaf: LIGUSTRUM lucidum

Weeping: LIGUSTRUM sinense 'Pendulum'

Punk Tree: MELALEUCA quinquenervis

Quince, Chinese: PSEUDOCYDONIA sinensis

Common: CYDONLA oblonga

Flowering: CHAENOMELES speciosa

Japanese: CHAENOMELES speciosa

Japanese Flowering: CHOENOMELES japonica

Raisin-tree, Japanese: HOVENIA dulcis

Redbud, Chinese: CERCIS chinensis

North American: CERCIS canadensis

Redwood, Coast: SEGUOLA sempervirens

Dawn: METASEGUOIA glyptostroboides

Rhododendron, Carolina: RHODODENDRON carolinianum

Chapman: RHODODENDRON chapmanii

Fujiama: RHODODENDRON brachycarpum

Piedmont: RHODODENDRON minus

Rice-paper Plant: TETRAPANAX papyriferum

Rose, Apothecary: ROSA 'Officinalis'

Baby: ROSA multiflora

Banks (double white): ROSA banksiae

var. banksiae 'Alba Plena'

Banks (double yellow): ROSA banksiae

var. banksiae 'Lutea'

Banks (single yellow): ROSA banksiae

var. normalis 'Lutescens'

Blackberry: RUBUS rosifolius 'Coronarius'

Boursault: ROSA $\times$ lheritierana 'Gracilis'

Bur: ROSA roxburghii $f$. normalis

Cabbage: ROSA centifolia

Champney: ROSA 'Champney's Pink Cluster'

Cherokee: ROSA laevigata

Chestnut: ROSA roxburghii f. normalis

China: ROSA chinensis

Confederate: HIBISCUS mutabilis

Crested Moss: ROSA centifolia 'Cristata'

Damask: ROSA $\times$ damascena

Dog: ROSA canina

Fairy: ROSA chinensis 'Minima'

Father Hugo: ROSA xanthina f. hugonis

Four Seasons: ROSA $\times$ damascena var. semperflorens

Green: ROSA chinensis 'Viridiflora'

Himalayan Musk: ROSA brunonii

Hungarian: ROSA gallica 'Conditorum'

Japanese: KERRIA japonica, ROSA multiflora

Kazanlik: ROSA $\times$ damascena 'Trigintipetala'

Lady Bank's: ROSA banksiae var. banksiae 'Lutea'

$\mathrm{M}^{\mathrm{c}}$ Cartney: ROSA bracteata 
Meadow: ROSA blanda

Memorial: ROSA wichuraiana

Monthly: ROSA chinensis $\times \boldsymbol{R}$. gigantea

'Old Blush', ROSA $\times$ damascena

var. semperflorens

Moss: ROSA centifolia 'Muscosa'

Musk: ROSA moschata

Noisette: ROSA $\times$ noisettiana

-of-Sharon: HIBISCUS syriacus

Pasture: ROSA carolina

Persian Yellow: ROSA foetida 'Perseana'

Prairie: ROSA setigera

Prickly: ROSA acicularis

Provence: ROSA centifolia

Red Cherokee: ROSA $\times$ anemonoides 'Ramona'

Rugosa: ROSA rugosa

Scotch: ROSA pimpinellifolia

Seven Sisters: ROSA multiflora 'Platyphylla'

Striped Moss: ROSA 'Oeillet Panachee'

Swamp: ROSA palustris

Tea: ROSA $\times$ odorata

Tidbit: ROSA gallica 'Conditorum'

Virginia: ROSA virginiana

White Rose-of-York: ROSA $\times$ alba

Yellow Rose-of-Texas: ROSA $\times$ harisonii

York and Lancaster Rose: $\mathbf{R O S A} \times$ damascena

'Versicolor'

Rosebay: RHODODENDRON maximum

Mountain: RHODODENDRON catawbiense

Rosemary: ROSMARINUS officinalis

Wild: LEDUM palustre var, diversipilosum

Rosewood: TIPUANA tipu

Rowan: SORBUS aucuparia

Rubber Tree, Chinese: EUCOMMIA ulmoides

Sage, Autumn: SALVLA greggii

Mexican Bush: SALVIA leucantha

Russian: PEROVSKIA atriplicifolia

Sago, Queen: CYCAS circinalis

St. Andrew's Cross: ASCYRUM hypericoides

St. Peter's-wort: HYPERICUM stans

Sandheath: CERATIOLA ericoides

Sand Myrtle, Box: LEIOPHYLLUM buxifolium

Sandweed: HYPERICUM fasciculatum

Sapote, White: CASIMIROA edulis

Sassafras: SASSAFRAS albidum

Satureja, Winter: SATUREJA montana

Scarlet Bush: HAMELIA patens

Scholar Tree, Chinese: SOPHORA japonica

Scuppernong: VITIS rotundifolia

Senna, Bladder: COLUTEA arborescens

Ringworm: CASSLA alata

Sequoia, Giant: SEQUOIADENDRON giganteum

Serviceberry, Downy: AMELANCHIER arborea

Service Tree: SORBUS domestica

Seven-sun Flower: HEPTACODIUM miconioides

Shadbush, Allegheny: AMELANCHIER laevis

Common: AMELANCHER arborea

Smooth: AMELANCHIER laevis

Sheepberry: VIBURNUM lentago,

VBBURNUM prunifolium

Shrimp Plant: JUSTICLA brandegeana,

J. californica
Silk Tree: ALBIZLA julibrissin

Silk Vine: PERIPLOCA graeca.

Silk-worm Tree: CUDRANLA tricuspidata

Silver Vine: ACTINIDIA polygama

Sissoo: DALBERGIA sissoo

Skimmia, White-fruited: SKIMMIA japonica

'Fructo-albo'

Skyflower, Creeping: DURANTA repens

Smoke Tree: COTINUS coggygria

American: COTINUS obovatus

Snailseed, Laurel-leaf: COCCULUS laurifolius

Snowball, Chinese: VIBURNUM macrocephalum f. macrocephalum

Common: VIBURNUM opulus 'Roseum'

European: VIBURNUM opulus 'Roseum'

Japanese: VIBURNUM plicatum f. plicatum

Snowbell, American: STYRAX americanus

Bigleaf: STYRAX grandifolius

Fragrant: STYRAX obassia

Japanese: STYRAX japonicus

Texas: STYRAX platanifolius

Snowbrush Ceanothus: CEANOTHUS velutinus

Snowbush: BREYNLA disticha

Snow-wreath: NEVIUSIA alabamensis

Soapberry, Chinese: SAPINDUS mukorossi

Florida: SAPINDUS marginatus

Texas: SAPINDUS drummondii

Sorrel Tree: OXYDENDRUM arboreum

Sourwood: OXYDENDRUM arboreum

Southern Plume: ELLIOTTLA racemosa

Southernwood: ARTEMESIA abrotanum

Spanish Bayonet: YUCCA aloifolia 'Marginata'

Spanish Dagger: YUCCA gloriosa

Sparkleberry: VACCINIUM arboreum

Spindle Tree, European: EUONYMUS europaeus

Japanese: EUONYMUS japonicus

Winged: EUONYMUS alatus

Winterberry: EUONYMUS bungeanus

Spindlebush, Wintercreeper: EUONYMUS fortunei

Spirea, Japanese: SPIRAEA japonica

Reeves: SPIRAEA cantoniensis

Vanhoutte: SPIRAEA $\times$ vanhouttei

Willowleaf: SPIRAEA salicifolia

Spruce, Alcock's: PICEA alcoquiana

Black: PICEA mariana 'Doumetii'

Colorado: PICEA pungens

Colorado Blue: PICEA pungens f. glauca

Dragon: PICEA asperata

Dwarf Alberta: PICEA glauca 'Conica'

Norway: PICEA abies

Oriental: PICEA orientalis

Serbian: PICEA omorika

Tiger-tail: PICEA torano

White: PICEA glauca

Spurge, Allegheny: PACHYSANDRA procumbens

Japanese: PACHYSANDRA terminalis

Spurge-olive: CNEORUM tricoccon

Staggerbush: LYONLA mariana

Stewartia, Japanese: STEWARTIA pseudocamellia

Stranvaesia, Chinese: STRANVAESIA davidiana

Yellow-fruited Chinese: STRANVAESLA davidiana

'Lutea' 
Strawberry Bush: EUONYMUS americanus

Strawberry Tree: ARBUTUS unedo

Sugarberry: CELTIS laevigata

Sumac, Fragrant: RHUS aromatica

Shining: RHUS copallina

Smooth: RHUS glabra

Staghorn: RHUS typhina

Winged: RHUS copallina

Summersweet, Japanese: CLETHRA barbinervis

Pink: CLETHRA alnifolia 'Rosea'

Woolly: CLETHRA tomentosa

Supplejack, Alabama: BERCHEMIA scandens

Japanese: BERCHEMLA racemosa

Sweetbells: LEUCOTHOE racemosa

Sweet Box, Fragrant: SARCOCOCCA ruscifolia

Sweetfern: COMPTONLA peregrina

Sweetshrub: CALYCANTHUS floridus

Sycamore, American: PLATANUS occidentalis

Tallow Tree, Chinese: SAPIUM sebiferum

Tangerine: CITRUS reticulata

Tara Vine: ACTINIDIA arguta

Tarflower: BEFARIA racemosa

Tea, New Jersey: CEANOTHUS americanus

Tea Berry: GAULTHERIA procumbens

Tea Plant: CAMELLIA sinensis

Tea Tree, New Zealand: LEPTOSPERMUM scoparium

Thimbleberry: RUBUS odoratus

Thorn, Jerusalem: PARKINSONLA aculeata

Washington: CRATAEGUS phaenopyrum

Thyme, Common: THYMUS vulgaris

Tipu Tree: TIPUANA tipu

Titi: CLIFTONIA monophylla, CYRILLA racemiflora

Tobacco, Tree: NICOTIANA glauca

Toona, Chinese: TOONA sinensis

Torchwood, Texas: AMYRIS texana

Torreya, Florida: TORREYA taxifolia

Japanese: TORREYA nucifera

Tree-of-heaven: AILANTHUS altissima

Trumpet-creeper: CAMPSIS radicans

Chinese: CAMPSIS grandiflora

Trumpet Vine: CAMPSIS radicans

Argentine: CLYTOSTOMA callistegioides

Blue: THUNBERGIA grandiflora

Tubeflower: CLERODENDRUM indicum

Tulip Tree: LIRIODENDRON tulipifera

Chinese: LIRIODENDRON chinense

Tung-oil Tree: ALEURITES fordii

Tupelo, Ogeechee: NYSSA ogeche

Turk's Cap: MALVAVISCUS arboreus

var. drummondii, M. arboreus

var. penduliflorus

Turk's Turban: CLERODENDRUM indicum

Varnish Tree: TOXICODENDRON vernicifluum

Vase Vine: CLEMATIS viorna

Verbena, Lemon: ALOYSLA gratissima

Viburnum, Birch-leaved: VIBURNUM betulifolium

Buddleja-leaved: VBBURNUM buddleifolium

Burkwood's: VIBURNUM $\times$ burkwoodii

Cinnamon-leaved: VBBURNM cinnamomifolium

Double-file: VIBURNUM plicatum f. tomentosum

Japanese: VIBURNUM japonicum

Korean-spice: VBBURNUM carlesii
Leatherleaf: VIBURNUM rhytidophyllum

Linden: VIBURNUM dilatatum

Manchurian: VIBURNUM burejaeticum

Maple-leaved: VIBURNUM acerifolium

Philippine: VIBURNUM luzonicum

Round-leaved double-file: VIBURNUM plicatum

var. rotundifolium

Sweet: VIBURNUM odoratissimum

Tea-leaved: VIBURNUM setigerum

Tree: VIBURNUM sieboldii

Walter's: VIBURNUM obovatum

Withe-rod: VBBURNUM cassinoides

Yeddo: VIBURNUM bitchiuense

Virgin's Bower: CLEMATIS terniflora

Walnut, Arizona: JUGLANS major

Black: JUGLANS nigra

English: JUGLANS regia

Japanese: JUGLANS ailantifolia var, cordiformis

Persian: JUGLANS regia

Water-pine, Chinese: GLYPTOSTROBUS lineatus

Waxberry: MYRICA cerifera

Wax-myrtle: MYRICA cerifera, M. heterophylla

Dwarf: MYRICA pusilla

Wayfaring Tree: VIBURNUM lantana

White Wicky: KALMIA cuneata

Willow, Basket: SALIX purpurea

Black: SALEX nigra

Contorted Hankow: SALIX babylonica 'Tortuosa'

Desert: CHILOPSIS linearis

Goat: SALIX caprea

Gray: SALDX cinerea

Niobe: SALIX $\times$ blanda

Virginia: ITEA virginica

Weeping: SALXX babylonica

White: SALLX alba

Wingnut, Chinese: PTEROCARYA stenoptera

Three-wing: TRIPTERYGIUM regelii

Winter Hazel, Buttercup: CORYLOPSIS pauciflora

Chinese: CORYLOPSIS sinensis var. sinensis

Fragrant: CORYLOPSIS glabrescens

Winterberry: ILEX verticillata

Ambiguous: ILEX ambigua

Japanese: ILEX serrata

Smooth: ILEX laevigata

Yellow-berried: ILEX verticillata f. chrysocarpa

Yellow-fruited Japanese: ILEX serrata 'Leucocarpa'

Wintergreen: GAULTHERIA procumbens

Wintersweet: CHIMONANTHUS praecox

Wire Vine, Matbrush: MUEHLENBECKIA axillaris

Wisteria, American: WISTERIA frutescens

Chinese: WISTERIA sinensis

Japanese: WISTERIA floribunda

Witch Hazel, Common: HAMAMELIS virginiana

Chinese: HAMAMELIS mollis

Wood-vamp: DECUMARIA barbara

Woodbine: PARTHENOCISSUS quinquefolia

Yaupon: ILEX vomitoria

Mexican: ILEX vomitoria var, chiapiensis

Pendulous: ILEX vomitoria f. pendula

Yellowhorn: XANTHOCERAS sorbifolium

Yellowroot, Shrub: XANTHORHIZA simplicissima

Yellow-wood: CLADRASTIS kentukea,

SYMPLOCOS tinctoria 
Yerba Maté: ILEX paraguariensis

Yesterday-today-tomorrow: BRUNFELSLA australis

Yew, Canadian: TAXUS canadensis

English: TAXUS baccata

Florida: TAXUS floridana

Golden: TAXUS baccata 'Aurea'

Japanese: TAXUS cuspidata

Plum: CEPHALOTAXUS harringtonia

Yulan: MAGNOLIA denudata

Zelkova, Caucasian: ZELKOVA carpinifolia

Japanese: ZELKOVA serrata 


\section{Selected Bibliography}

Adams, W.D. 1976. Trees for southern landscapes. Pacesetter Press, Houston, TX.

Apgar, A.C. 1892. Ornamental shrubs of the United States. American Book Co., New York.

Ashby, W.C. 1964. A note on basswood nomenclature. Castanea 29:109-116.

Bailey, L.H. 1923. The cultivated evergreens. Macmillan Co., New York.

Bailey, L.H. 1933. The cultivated conifers in North America. Macmillan Co., New York.

Bailey, L.H. 1939. The standard cyclopedia of horticulture. 3 vols. Macmillan Co., New York.

Bailey, L.H. 1949. Manual of cultivated plants. Macmillan Co., New York.

Bailey, L.H. 1976. Hortus third. Macmillan Co., New York.

Batdorf, L.R. 1989. Checklist of Buxus L. Boxwood Bull. 28(3):43-49.

Batson, W.T. 1984. Landscape plants for the southeast. University of South Carolina Press, Columbia.

Bean, W.J. 1970-1980. Trees and shrubs hardy in the British Isles. 8th ed., 4 vols. John Murray, London.

Bean, W.J. 1988. Trees and shrubs hardy in the British Isles. Supplement. John Murray, London.

Blackburn. B. 1952. Trees and shrubs in eastern North America. Oxford University Press, New York.

Blakelock, R.A. 1951. A synopsis of the genus Euonymus L. Kew Bull. 2:210-290.

Boer, A.F. den. 1959. Ornamental crab apples. American Association of Nurserymen, Washington, DC.

Brickell, C.D., and B. Mathew. 1976. Daphne: The genus in the wild and in cultivation. The Alpine Garden Society, Lye End Link, St. John's, Woking, Surrey, England.

Brickell, C.D., A.F. Kelly, F. Schneider, et al. 1980. International code of nomenclature for cultivated plants. Regnum Vegetabile. Vol. 104.
Brown, R.G., and M.L. Brown. 1972. Woody plants of Maryland. Port City Press, Baltimore.

Brummitt, R.K. 1987. Report of the committee for spermatophyta: 31. Proposal 660. To reject Rhododendron japonicum (Blume) Schneider (Ericaceae). Taxon 36(1):72-73.

Brummitt, R.K., and C.E. Powell, eds. 1992. Authors of plant names. Royal Botanic Gardens, Kew, England.

Chadbund, G. 1972. Flowering cherries. Collins, London.

Chadwick, L.C., and R.A. Keen. 1976. A study of the genus Taxus. Research Bulletin 1086. Ohio Agriculture Research and Development Center, Wooster.

Chamberlain, D.F., and S.J. Rae. 1990. A revision of Rhododendron. IV Subgenus Tsutsusi. Edinburgh Journal of Botany 47(2):89-200.

Coker, W.C. 1917. The laurel oak or Darlington oak (Quercus laurifolia Michx.). J. Elisha Mitchell Sci. Soc. 32:38-40.

Choukas-Bradley, M., and P. Alexander. 1981. City of trees. Acropolis Books, Washington, DC.

Coville, F.V., and O.M. Freeman. 1932. Trees and shrubs of Lafayette Park. American Forestry Association, Washington, DC.

Cronquist, A. 1988. The evolution and classification of flowering plants. 2d ed. Allen Press, Lawrence, KS.

Dallimore, W., and A.B. Jackson. 1966. A handbook of Coniferae and Ginkgoaceae. Edward Arnold, London.

Davis, P.H., and V.H. Heywood. 1965. Principles of angiosperm taxonomy. Oliver and Boyd, Edinburgh and London.

De France, J.A. 1932. A study of the broadleaf evergreens of the southeastern states. Unpublished thesis, Cornell University, Ithaca, NY.

Dirr, M.A. 1983. Manual of woody landscape plants. 3d ed. Stipes Publishing Co., Champaign, IL.

Dudley, T.R., and G.K. Eisenbeiss. 1992. International checklist of cultivated Ilex. Part 2. Ilex crenata Thunberg ex J.A. Murray. National Arboretum Contribution Number 6. U.S. Department of Agriculture, Washington, DC.

Duncan, W.H. 1975. Woody vines of the southeastern United States. University of Georgia Press, Athens.

Duncan, W.H., and T.M. Pullen. 1962. Lepidote rhododendrons of the southeastern United States. Brittonia 14(3):290-298. 
Eckenwalder, J.E. 1980. The taxonomy of the West Indian cycads. J. Arnold Arbor. 61:701-722.

Egolf, D.R., and A.O. Andrick. 1978. The Lagerstroemia handbook/checklist. American Association of Botanical Gardens and Arboreta, Las Cruces, NM.

Eisenbeiss, G.K., and T.R. Dudley. 1973. International checklist of cultivated Ilex. Part 1. Ilex opaca. National Arboretum Contribution Number 3. U.S. Department of Agriculture, Washington, DC.

Farr, E.R., J.A. Leussink, and F.A. Stafleu. 1979. Index nominum genericorum (Plantarum). Vols. 1-3. Bohn, Scheltema, and Holkema, Utrecht.

Fernald, M.L. 1950. Gray's manual of botany. 8th ed. American Book Co., New York.

Flink, K.E., and B. Hylmo. 1966. A list of series and species in the genus Cotoneaster. Bot. Not.

1 19(3):445-463.

Fogg, J.M., Jr., and J.C. McDaniel, eds. 1975. Checklist of the cultivated magnolias. American Horticultural Society, Mt. Vernon, VA.

Galle, F.C. 1987. Azaleas. Timber Press, Portland, OR.

Gensel, W.H. 1988. Rhododendron subsection Caroliniana. Rhododendron Notes and Records 2:111.

Gleason, H.A. 1952. The new Britton and Brown illustrated flora of the northeastern United States and adjacent Canada. 3d ed. 3 vols. New York Botanical Garden, New York.

Godfrey, R.K. 1988. Trees, shrubs, and woody vines of northern Florida, and adjacent Georgia and Alabama. University of Georgia Press, Athens.

Graves, A.H. 1956. Illustrated guide to trees and shrubs. Harper and Row, New York.

Green, P.S. 1965. Studies in the genus Jasminum III. The species in cultivation in North America. Baileya 13(4): 137-172.

Greuter, W., ed. 1988. International code of botanical nomenclature. Koeltz Scientific Books, Koenigstein, Germany.

Halfacre, R.G., and A.R. Shawcroft. 1975. Carolina landscape plants. 2d ed. University of North Carolina Press, Raleigh.

Hansel, D.E., ed. 1970. Handbook of hollies. Am. Hort. Mag. 49(4):150-334.

Hara, H. 1983. A revision of Caprifoliaceae of Japan. Ginkgoana 5.
Hardin, J.W. 1957. A revision of the American Hippocastanaceae II. Brittonia 9(4): 173-195.

Haworth-Booth, M. 1959. The hydrangeas. Constable Publishers, London.

Hopkins, M. 1942. Cercis in North America. Rhodora 44:193-211.

Hu, S.-Y. 1954-56. A monograph of the genus Philadelphus. J. Arnold Arbor. 35(4):275-333; 36(1):52-109; 36(4):325-368; 37(1):15-90.

Hume, H.H. 1953. Hollies. Macmillan Co., New York.

Hume, H.H. 1955. Camellias in America. J. Horace McFarland Co., Harrisburg, PA.

Isely, D. 1973. Leguminosae of the United States: I. Subfamily Mimosoideae. Mem. N.Y. Bot. Gard. 25(1): 1-152.

Jaynes, R.A. 1988. Kalmia. The laurel book II. Timber Press, Portland OR.

Jefferson, R.M. 1970. Crabapples of documented authentic origin. National Arboretum Contribution Number 2. U.S. Department of Agriculture, Washington, DC.

Jefferson, R.M. 1976. Differences between Ligustrum japonicum and L. lucidum. Amer. Nurseryman 144(1):22.

Jefferson, R.M., and A.E. Fusonie. 1977. The Japanese flowering cherry trees of Washington, D.C. National Arboretum Contribution Number 4. U.S. Department of Agriculture, Washington, DC.

Jones, G.N. 1946. American species of Amelanchier. Ill. Biol. Monogr. 20(2).

Jones, G.N. 1968. Taxonomy of American species of linden (Tilia). Ill. Biol. Monogr. 39.

Judd, W.S. 1984. A taxonomic revision of the Ameri can species of Agarista (Ericaceae). J. Arnold Arbor. 65(3):255-342.

Kelsey, H.P., and W.A. Dayton. 1942. Standardized plant names. 2d. ed. J. Horace McFarland Co., Harrisburg, PA.

Kobuski, C.E. 1951. Theaceae in the United States. J. Arnold Arbor. 32:123-138.

Krüssmann, G. 1981. The complete book of roses. Timber Press, Portland, OR.

Krüssmann, G. 1984. Manual of cultivated broadleaved trees and shrubs. 3 vols. Timber Press, Portland, OR. 
Krüssmann, G. 1985. Manual of cultivated conifers. Timber Press, Portland, OR.

Li, H.-L. 1952. A taxonomic review of the genus Actinidia. J. Arnold Arbor. 33(1):1-61.

Li, H.-L. 1963. The origin and cultivation of shade and ornamental trees. University of Pennsylvania Press, Philadelphia.

Liang, C., and A.R. Ferguson. 1986. The botanical nomenclature of the kiwifruit and related taxa. N. Z. J. Bot. 24:183-184.

Little, E.L., Jr. 1979. Checklist of United States trees. Department of Agriculture Handbook 541. Washington, DC

Liu, T.-S. 1971. A monograph of the genus Abies. Department of Forestry, College of Agriculture, National Taiwan University, Taipei.

Loesener, L.E.T. 1901. Monographia aquifoliacearum. Part I. Nova Acta Leop. 78:1-589.

Loesener, L.E.T. 1908. Monographia aquifoliacearum. Part II. Nova Acta Leop. 89:1-313.

Mabberley, D.J. 1989. The plant-book. Cambridge University Press, England.

Mazzeo, P.M. 1966. Exotic and native ornamentals in the Shenandoah National Park. Am. Hort. Mag. 45(4):419-421.

McArdle, A.J., and F.S. Santamour, Jr. 1985. Cultivar checklist of English oak (Quercus robur). J. Arboric. 11(10):307-315.

McClintock, E. 1951. The genus Rhaphiolepis. J. Calif. Hort. Soc. 12(4):176-179.

McFarland, J.H. 1980. Modern roses 8: The international checklist of roses. The McFarland Co., Harrisburg, PA.

McKelvey, S.D. 1928. The lilac. Macmillan Co., New York.

Meyer, F.G. 1976. A revision of the genus Koelreuteria (Sapindaceae). J. Arnold Arbor. 57:129166.

Meyer, F.G., and P.M. Mazzeo. 1992. A new whiteflowered form and cultivar of Illicium floridanum (Illiceaceae) in the southeastern United States. Sida 15(2):285-287.

Meyer, F.G., and E. McClintock. 1987. Rejection of the names Magnolia heptapeta and $M$. quinquepeta (Magnoliaceae). Taxon 36(3):590-600.
Meyer, F.G., and J.W. Hardin. 1987. Status of the name Aesculus flava Solander (Hippocastanaceae). J. Arnold Arbor. 68:335-341.

Miller, G.N. 1955. The genus Fraxinus, the ashes, in North America, north of Mexico. Agricultural Experiment Station Memoir 335. New York State College of Agriculture (Cornell University). Ithaca.

Miller, R.B., and F.G. Meyer. 1989. Identification of the heath-leaved cypress, Chamaecyparis thyoides 'Ericoides' (Cupressaceae). Baileya 23(2):57-67.

Morley, B., and J.-M. Chao. 1977. A review of Corylopsis (Hamamelidaceae). J. Arnold Arbor. 58(4):382-415.

Murray, A.E., Jr. 1970. A monograph of the Aceraceae. Unpublished thesis, Pennsylvania State University, State College, PA.

Nicely, K.A. 1965. A monographic study of the Calycanthaceae. Castanea 30(1):38-81.

Nooteboom, H.P. 1975. Revision of the Symplocaceae of the Old World (New Caledonia excepted). Leiden Botanical Series Vol. 1. Universitaire Pers Leiden, The Netherlands.

Ohwi, J. 1965. Flora of Japan. F.G. Meyer and E.H. Walker, eds. Smithsonian Institution, Washington, DC.

Ouden, P. den, and B.K. Boom. 1978. Manual of cultivated conifers. Martinus Nijhoff, The Hague.

Radford, A.E., H. Ahles, and C.R. Bell. 1968. Manual of the flora of the Carolinas. University of North Carolina Press, Chapel Hill.

Rehder, A. 1940. Manual of cultivated trees and shrubs. 2d. ed. Macmillan Co., New York.

Rehder, A. 1949. Bibliography of cultivated trees and shrubs. Arnold Arboretum, Jamaica Plain, MA.

Reveal, J.L., and M.J. Seldin. 1976. On the identity of Halesia carolina L. Taxon 25(1):125.

Santamour, F.S., Jr., and A.J. McArdle. 1982. Checklist of cultivated maples I. Acer rubrum L. J. Arboric. 8(4):110-112.

Santamour, F.S., Jr., and A.J. McArdle. 1982. Checklist of cultivated maples II. Acer saccharum Marshall. J. Arboric. 8(6):164-167.

Santamour, F.S., Jr., and A.J. McArdle. 1982. Checklist of cultivated maples III. Acer platanoides L. J. Arboric. 8(9):241-246.

Santamour, F.S., Jr., and A.J. McArdle. 1982. Checklist of cultivated maples IV. Acer saccharinum L. J. Arboric. 8(10):277-280 
Santamour, F.S., Jr., and A.J. McArdle. 1983. Checklist of cultivars of callery pear (Pyrus calleryana). J. Arboric. 9(4):114-116.

Santamour, F.S., Jr., and A.J. McArdle. 1983. Checklist of cultivars of North American honeylocust

(Gleditsia triacanthos). J. Arboric. 9(9):248-252.

Santamour, F.S., Jr., and A.J. McArdle. 1983. Checklist of cultivars of North American ash (Fraxinus) species. J. Arboric. 9(10):271-276.

Santamour, F.S., Jr., and A.J. McArdle. 1984. Checklist of European ash (Fraxinus) species. J. Arboric. 10(1):21-32.

Santamour, F.S., Jr., and A.J. McArdle. 1984. Cultivar checklist for Liquidambar and Liriodendron. J. Arboric. 10(11):309-312.

Santamour, F.S., Jr., and A.J. McArdle. 1985. Cultivar checklist of the large-bracted dogwoods: Cornus florida, C. kousa, and C. nuttallii. J. Arboric. 11(1):2936.

Santamour, F.S., Jr., and A.J. McArdle. 1985. Checklist of cultivars of linden (Tilia) species. J. Arboric. 11(5): 157-164.

Santamour, F.S., Jr., and A.J. McArdle. 1986. Checklist of cultivated Platanus (planetree). J. Arboric. 12(3):78-83.

Santamour, F.S., Jr., Shan-an He, and A.J. McArdle. 1983. Checklist of cultivated Ginkgo. J. Arboric. 9(3):88-92.

Sargent, C.S. 1920. Notes on North American trees VII. J. Arnold Arbor. 2:119.

Schneider, C.K. 1907. Illustriertes Handbuch der Laubholzkunde. Vol. 2. Gustav Fischer, Jena.

Sealy, J.R. 1949. Species of Sarcococca in cultivation. J.R. Hort. Soc. 74:301-306.

Shaw, G.R. 1914. The genus Pinus. Publications of the Arnold Arboretum 5. Riverside Press, Cambridge, MA.

Shuery, A.G., and R.P. Wunderlin. 1977.

Rhapidophyllum hystrix. Principes 21(2):47-59.

Sleumer, H. 1968. Die Gattung Escallonia (Saxifragaceae). Verh. K. Ned. Akad. Wet., Afd. Natuurkd. 58(2):1-146.

Small, J.K. 1933. Manual of the southeastern flora. (Published by author), New York.
Spongberg, S.A. 1974. A review of deciduous-leaved species of Stewartia (TheaceaeL_ J. Arnold Arbor. 55:182-214.

Spongberg, S.A. 1976. Magnoliaceae hardy in temperate North America. J. Arnold Arbor. 57: 250-312.

Stafleu, F.A., and R.S. Cowan. 1976-1988.

Taxonomic literature. 2nd ed. 7 vols. Bohn, Scheltema and Holkema, Utrecht.

Stewart, R.N., F.G. Meyer, and H. Dermen. 1972.

Camellia +'Daisy Eagleson', a graft chimera of Camellia sasanqua and C. japonica. Am. J. Bot. 59(5):513-524.

Stritch, L.R. 1985. A revision of the genus Wisteria Nuttall. Unpublished thesis, Southern Illinois University, Carbondale.

Swartley, J.C. 1984. The cultivated hemlocks. Timber Press, Portland, OR.

Thomas, G.S. 1962. Shrub roses of today. Phoenix House, London.

Thomas, G.S. 1981. The old shrub roses. Phoenix House, London.

Thomas, J.L. 1960. A monographic study of the Cyrillaceae. Contribution 186. Gray Herbarium, Harvard University.

Trelease, W. 1924. The American oaks. Nat. Acad. Sci. (USA). Memoirs 20.

Treseder, N.G. 1978. Magnolias. Faber and Faber, London.

Tutin, T.G., ed. 1968-1981. Flora Europaea. 5 vols. Cambridge University Press, Cambridge, England.

U.S. Department of Agriculture. 1990. USDA Hardiness Zone Map. U.S. Department of Agriculture Miscellaneous Publication 1475. Washington, DC.

Vander Kloet, S.P. 1980. The taxonomy of the highbush blueberry, Vaccinium corymbosum. Can. J. Bot. 58:1187-1201.

Vertrees, J.D. 1987. Japanese maples. 2d ed. Timber Press, Portland, OR.

Vines, R.A. 1960. Trees, shrubs and woody vines of the southwest. University of Texas Press, Austin.

Watkins, J.V. 1969. Florida landscape plants: Native and exotic. University of Florida Press, Gainesville.

Webber, C. 1964. The genus Chaenomeles (Rosaceae). J. Arnold Arbor. 45(2): 161-205, 45(3):302-345. 
Welch, H.J. 1979. Manual of dwarf conifers.

Theophrastus Publishers/Garland STPM Press, New York.

Wigginton, B.E. 1957. Trees and shrubs for the southern coastal plain. University of Georgia Press, Athens.

Wigginton, B.E. 1963. Trees and shrubs for the southeast. University of Georgia Press, Athens.

Wolf, C.B., and W.E. Wagener. 1948. The New World cypresses. El Aliso, vol. 1. Rancho Santa Ana Botanic Garden, Anaheim, CA.

Young, R.A., and J.R. Haun. 1961. Bamboo in the United States: Description, culture and utilization. U.S. Department of Agriculture Handbook 193.

Washington, DC. 

$-$ $-$

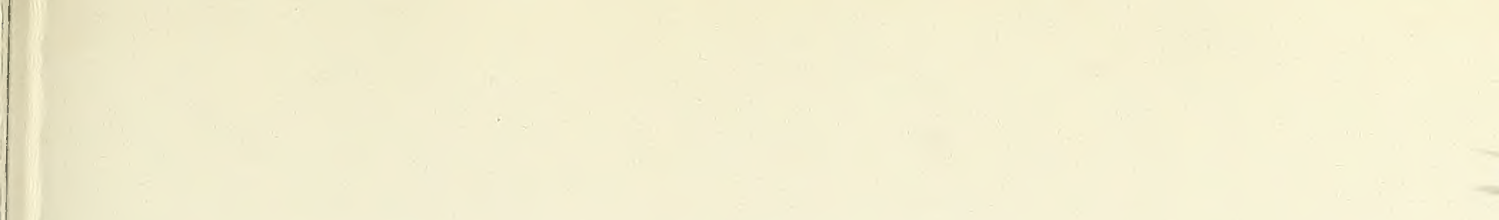

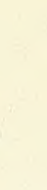






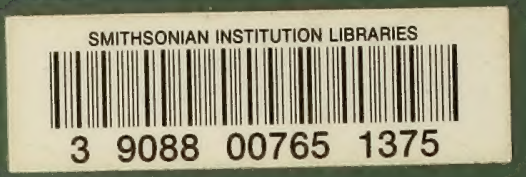

